


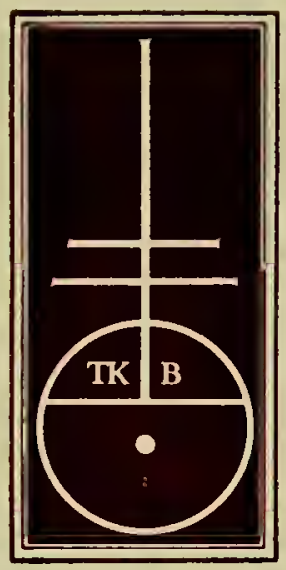

JOHN CARTER BROWN LIBRARY ACQUIRED WITH THE ASSISTANCE OF THE T. KIMBALL BROOKER SIXTEENTH-CENTURY

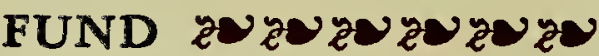





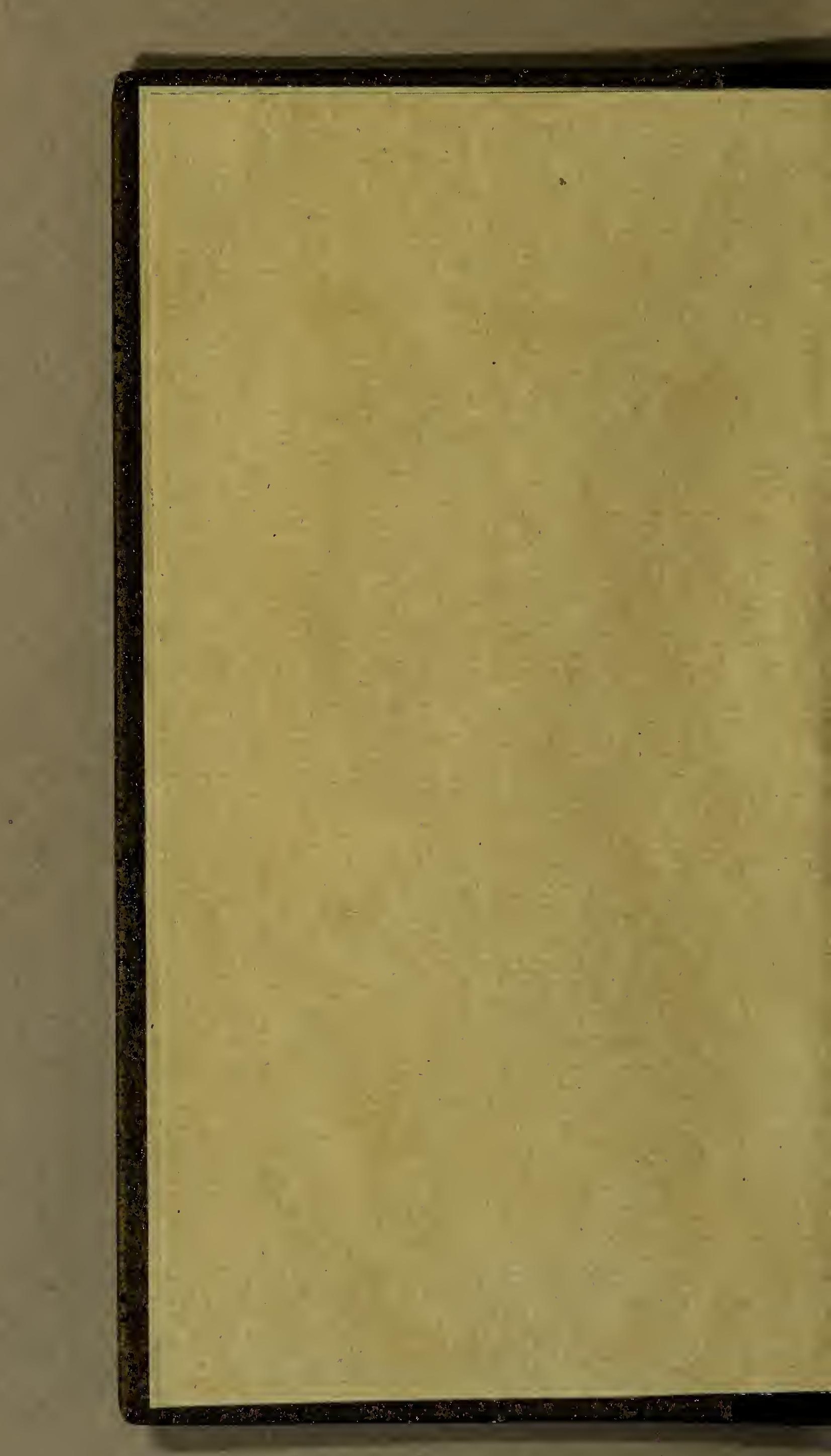




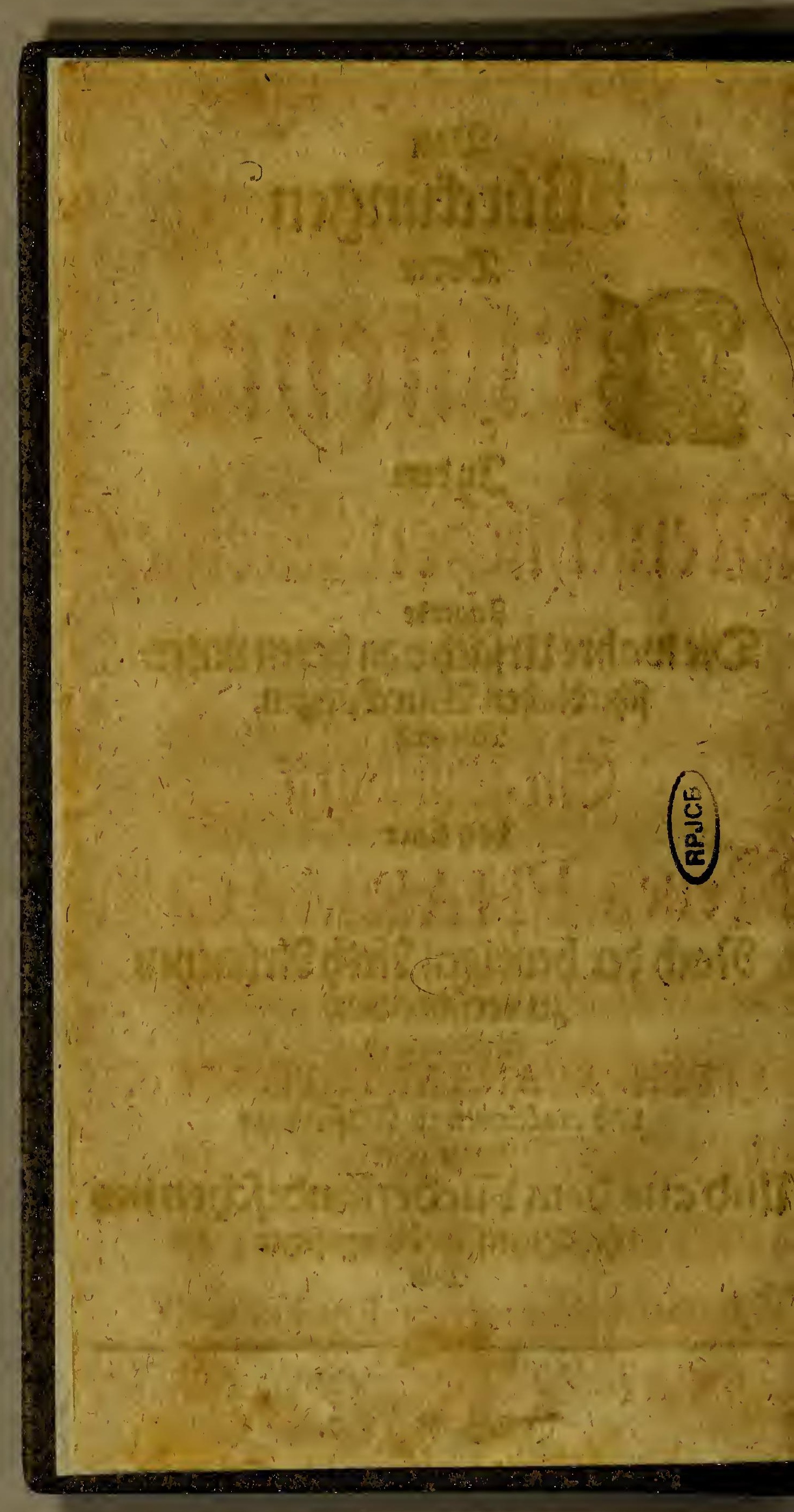


SGtanternauf die Sraf fenuno Srethe \&Sgege zu legen/Damitfie/ Güllfe zubaben/son Denen oor = beygebenden einigen Shathempfangenmoib: ten. LInd iffioldsezgeftalt Diefe founftintigen 2 (nt= fange/nad) DežHerodoti? und Galenifeugniż fe ht robe unungeidaffen ge=4 welen; bíffie aus bielent curen uno \&nmertumgen zu einen / wienobly $8211=$ 


\section{(0) 18}

QInfangs unformilicben Sorper angerwauffen/ womit man bat muiffen bergnigf fenn. \&lber gleid)wie alle sounfte in iften \&lnfange ungeftalt und unpolitef fino / 10 inerben fie bod mif ber Zeitineinebefere g:orm uno zaeftalf georadit, menn nach Serfoderung Der aseadie und Setl) bendigfeif / imer ettwas bengefiget oderaud ab $=$
13 
(0)

getbanwird. 28 leidem fato nun ift aud unfere unft / weldbein SIGar= beit Die grófte unterallen Rounften/ unterwuirffig gervefen: :erinder bor: treflicbe Hippocrates, uñ nach iffm Galenus, nebft bielen andern/Gaben fot de nidft allein in eine bef: fere \$ronunggebradjt? fondern Der Experientz audf Die Ration benge? finget. 
被 (0) 憵

23. erwi uno allo auds in Der Medicin, ofine LItradie und Riegel gefotiefet: Diefer 2abicht bat nach viel bunDertiábrigen Sudjen/ bornemlid) zu Diefer un= fererzeit/erft anfangen bellezu leudten. Sigaz

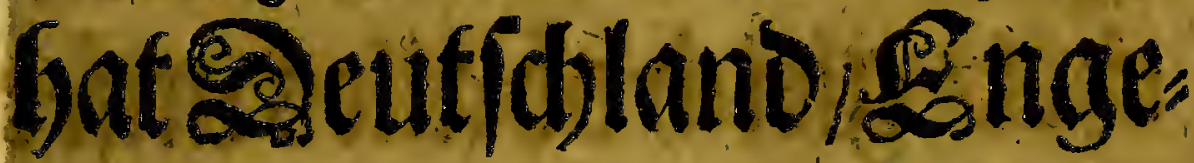
lano und andere Detfer unżnidft 60 2Experimentacommuniciret? Sfrab Gatoas? Petuer/Sgajier/

$$
\text { a } 4 \text { \&ังt: }
$$




\section{(0)}

Egergroflerung $3=22$ la $\bar{\beta}$ und die fluge \&gernunffe ung nichtentoedet? iff Gip auf Den beutigen Stageine Sabande ge: 10ejen/Dajun/ere founit fo beroundtelt blieben/ unto bat Das gemeine \&golde unz billich betlddet/ gleidb ob alles unjet

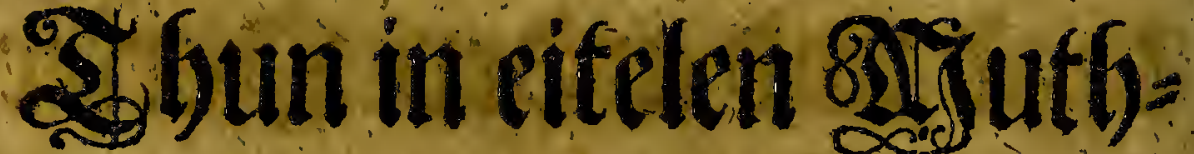
maffungen beftuinde. EB if Dannenbero uniere SGítichtgenefen/ Die Me- 


\section{(o)}

dicin auf einen gemiffern unvin berivabren Philofophie gegrumbete g: zuffellen: Sieferbaben allbeteit biele for mit auşzufúften gefucbef/ worunfer sornebmlids Der Songelán dif́de Thomas Willis Ju tedfnen/ alš weldger zu feiner zeif grofie sigifienidbafft ge= babt: sellein weil cin 8MJann nidfe alles tbun fain/ welde b focine grof as a fe 


\section{故 (0)}

fe foumf zur zolltomt menbeit bringenfan/ 10 Gabeich meinen ebemals getbanen şerprecben gemáš miḑ berpflidstet gendjef/ biebon fo biel

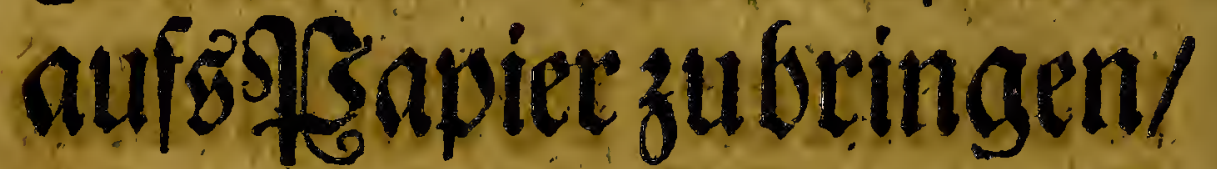

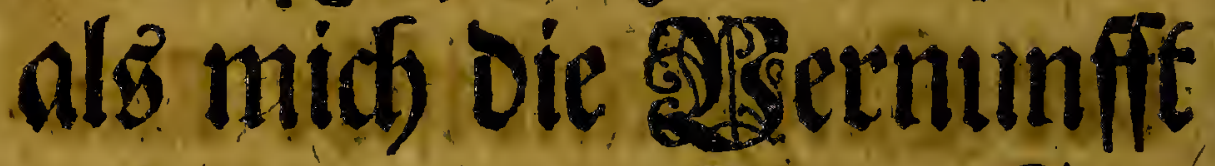
gelebret bat. 8MJein 216= pebeniffnidht/Dem ferfoldbeganjzubringen/ alżob çื inallen Stúten phne 8ysungel wåte/ weil ids gerne betenne:

$$
28 \quad D a k
$$




\section{称 (0)}

Daki id fo woblal alsa ande: re gljenjoben itren fan; fonoernich offerire allein

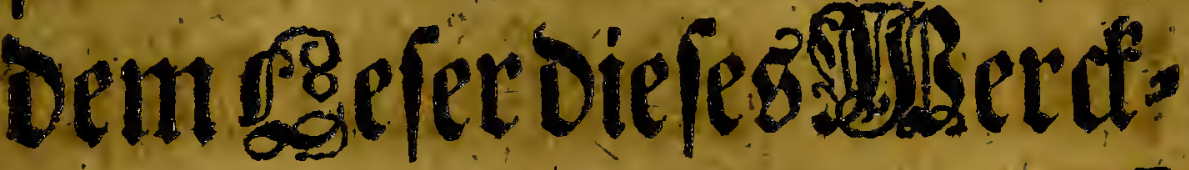
genfo gut idbs babe/ if ientand weifer fommen/ wirb en fids fobuldig wif

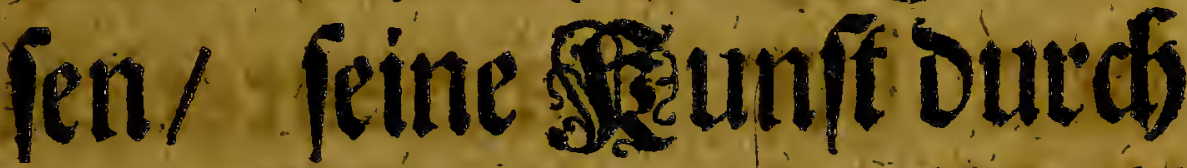
Den surut Der Srgelf mifzutbeilen: Sennbiel SBlaubern und nid)ts tbun/ iff blofier \&giabn uno 82jeid / itonit nie manden gedienef wito;

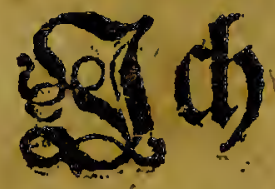


10. (0)

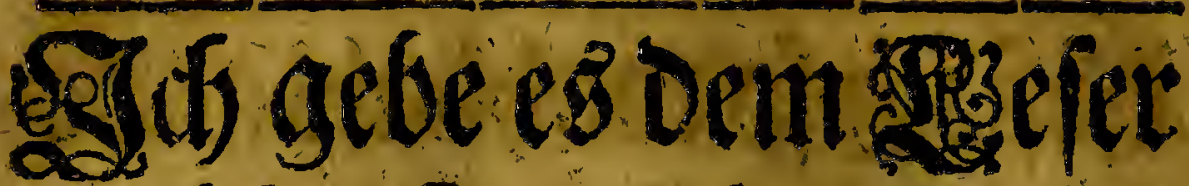
umbjon/t/ uñ fudjenidft Den geringlfen SSTrofit Damit. Safofoter Diefer seretat befler móge berftandenwerden/poift Dnz son Det Fermentation geffbrieben / notfig zu Durd)le[en/woraub̉man Die inerlidbe \$3ervegung Der sorper berfteben Iernet/ obne tueldie SISir fenidorff manoie arsur: fiung 


\section{勫 (o)}

fung Do Medicamenten nicht wol begreiffentan/ Denn eร̃ Gánget cines an Dem andern/wie Die siedereiner Sotete.

getsernerift ofnlängft gejdeben / Dafich eine Sateinifife PHARMACOPOEAM, DOib obne 8gjabmen/ Geraurgege = ben / weilaberibrerbit sulffen Daj es son mir fontmen/ Grbenfiemid

$$
\text { erfus }
$$




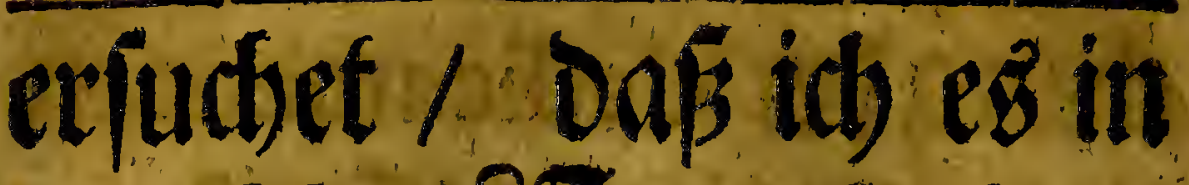
Deutidjer asspradje folte wieber auflegen laffen/ Damit Die / meldhe Derz

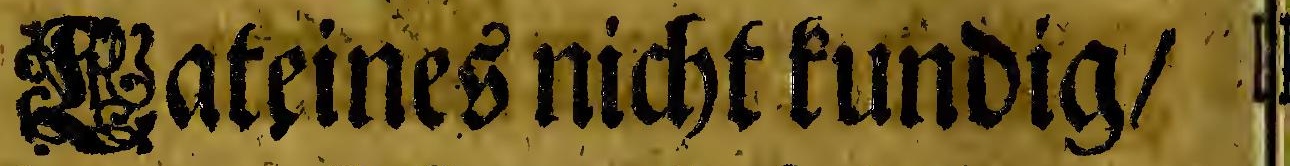
Deren fido audb bedienen fonten. Sg iff nur ein roher SIEertet / Dấ ich meiffentbeils bor midf felfofentworffen/beffe Gende aub ganz andern Compofitionibus alรี $\mathfrak{0 0 2}$, Diefermim 28. ebraudf ge= welen. ged babe eine 


\section{* (0)}

andere Methode $g e$ Graudft/ nadb Der Sfgeif Deret Doctorum, weldse íbre befler gegrindefe Praxin wertfffellig ma= dent.

SE nefime Detotue gen Der \&Jerthe Diejes Secter $=$ - 3 eldentigenan/ und (ev) berfidoert/Dáp folan= ge nteinfurker sefeng: Serabenwábret/ id alle. geiferweifenwerde/ Daf idi) 


\section{被 (0) \\ id) lenn wolle ein \&jer mebrerund 2liserbefferet unferer unft. Bec idf bin und bleite}

wher gilde cuet his

Im Jabrebrifti 1690. dens. Jan.

\section{GunftigerDiener}

Steph. Blankart,P.\&.M.D. uno Praet in Anifterdan. 


\section{0. (1) 5}

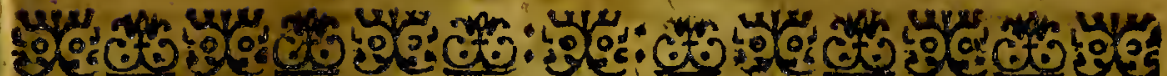

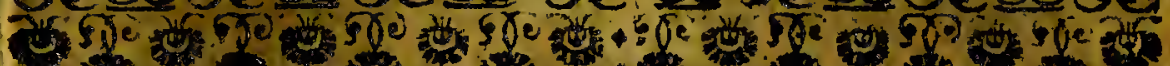

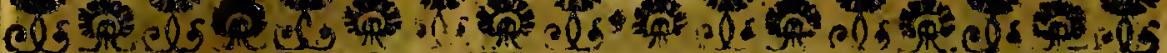

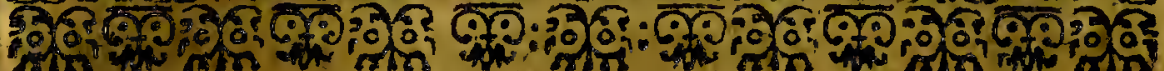

SonderOperation soev

\section{Moirtututg}

Dir Medicamenten

$9 \mathfrak{n}$

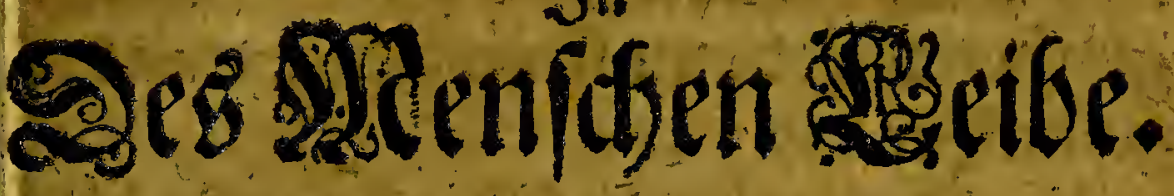

\section{Das̆ I. Stapitel.}

23on ber 93 intefung ber Medicamenten insgemein/nebf einer furken Befdreibung Des Magens unt Deróntmer.

1.

C. S baben viele fo fwobl alte als G) jiunge Authores von der 0 peration oder 2 úriffung (0) ber Medicamenten in the ferm \&eibe gédbricben/meilas ber bevse stidis nidst vergmigen fónten/ ro ift of meine Pflicht/ zu fageril was ib savori 


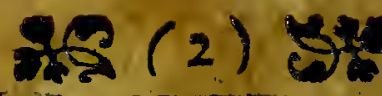

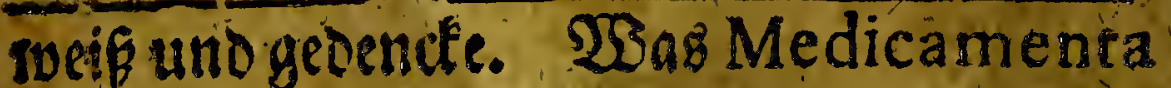
fine/ if einen iesen befant/ netomlich/ foldhe Dinge/wellhe oienen unfere verlobrene. (S3e: funobeit/ fo viel fich thun lift/ swieder za bringen; Gnoem aber oiefe sefdrreibung meitlauffiger if / als id gefinnet / Diefes 20ercif zu tractiren / fo svill ids nur aflein von dem bandeln/ rwas meiffens die Pharmacie uno Chirurgie angebet/ Dennids

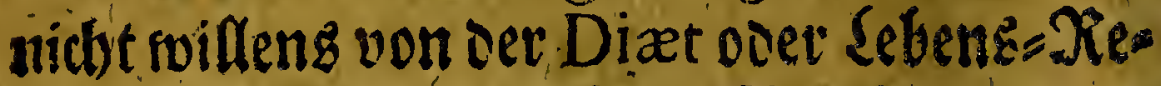
geln mit unter denen Rrandébeiten etribas zu geoencten.

II. Damitaber die Fstrirdung oer Medicamenten wobl tosne gefaffet nerDen/ muiffendie 23 ege uno (Siefáfie/rworinnen fels bigegefthebet/ befant fern/ uno weil unfer Worbaben iff / crfflid von senen purgier

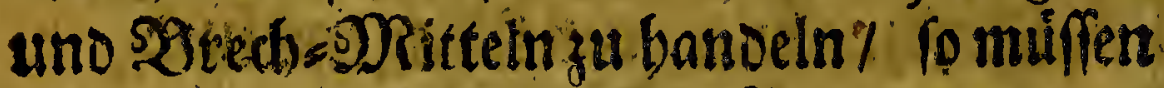

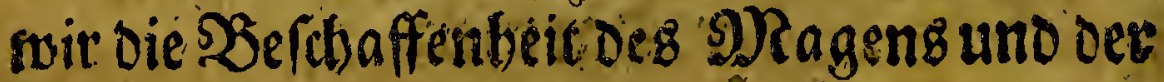
Dirmer etwas befchreiben: Denn swie fas

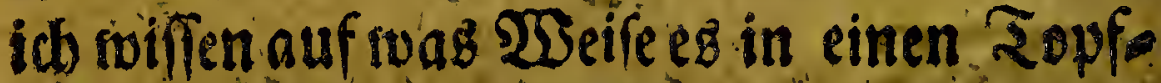

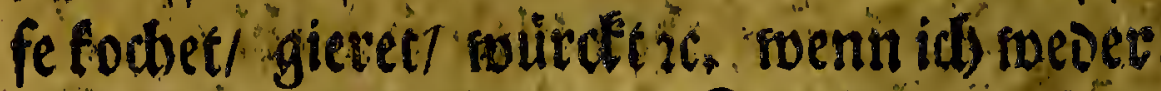

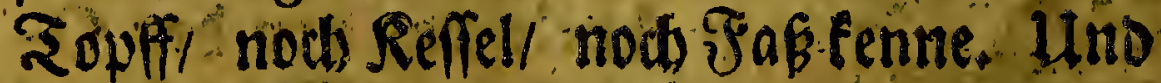
wienoblidb in theiner oeturfosen uno lateinie fotien Anatomie heitláufftig gentung oas. songedacdit/ beliebt miv bot) sine furge (E) zeblung oavon zu thun.

III. Sleidurie es nunberant ift / sag die Sperin 


\section{$\sec (3)$ ste}

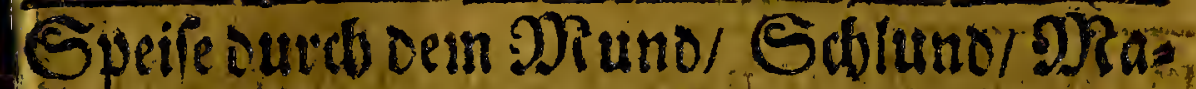
gen uno Dármer in cie פDilds - (Sefâffe gee bet / alfo emufingt man audb die Medica-

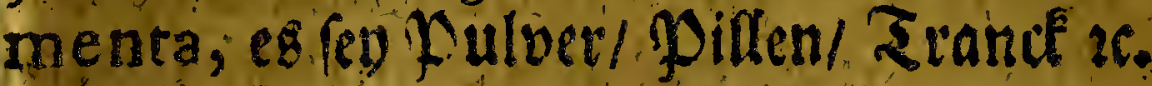
mit oem Drundel aus sweldsen fie curch oie Reble uno Schluno in oen Dragen Coms.

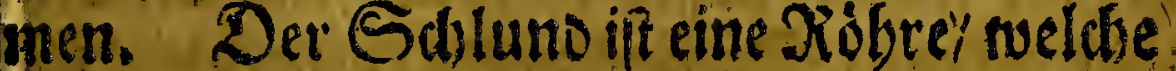
bon ser Reble ourch oen \$als uno \$ruft binter det (Sjurgel nads tem NRagen gebet/ dingefebranderthalbe Spannen lang/ ies Dods rach proportion oer Perfonen. 2 berit fie bls an oos fuinffte Wuirbelbein oer \$Bruft tommt/ musfie/ ser/ aus Dem. Serten fom menoen Sdblag-2lees/ nidft im $25 e g e$ zu

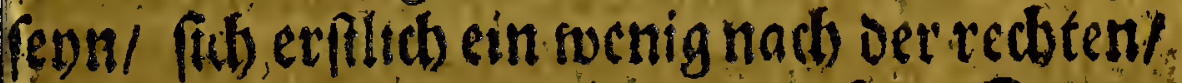
uno denn wieber nad dei lincten. Seiten beigen/ ivornufie gerades 2Seges ourds Das Buerdofen in Dem linctell D) ?agen. Xunolauffit.

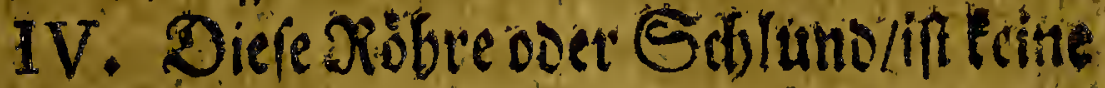
einfadie PS feiffe doer Diofre/ wie etwan die DSlev=:SSiefier madjen / fonvern es if ein bo: Ker-Mufculus, aus mufculofen fápen bes itebende 7 die einander auf uno obnarts Eretregeveife outchidoneioen / unb einen inu-

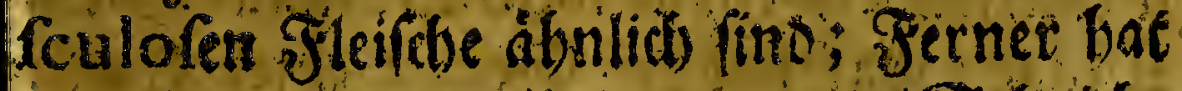
fie audh von auben als innen etruas Setivids.

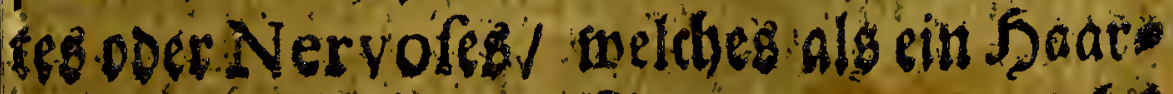
2. revists 


\section{$\sin (4)$ sts}

tond a ift/rooourd vie Jleifbigten Jafen auf uno nieder gezogen nersen. Die innendige Site ift-liber differ not befleibet mit einet orufigfen 5at/ wooutd continuirlid ets

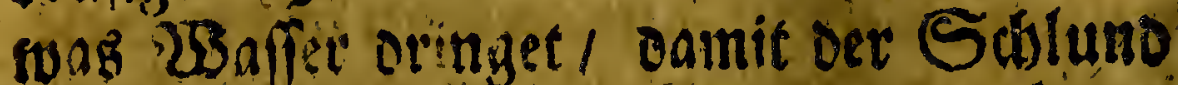
modbe feutste bleiben / senn fonft wuiroen die trotenen Spiren uns Orkseneyen nide Ginunter rooflen $/$ soie man an oem פ) bermetten far / onf bey groffen Durft bie troctenen Speifen nidbt zu Salfe geben/uno folmeden/ (rvie manzuresen pfleg(/) noie ein Robl=Strunce. Diefes fo ingenere genung von dem Scflunoe.

V. 2Benn offegetacber Solluno surdi dielinde Seite deg Znerd)= Fents/ (mit defo ren Siutgen ex fo wobl oben als unten vere einiget if / samit er fambt dem NRagenan Deffelben Servegung Theil baben módbel) binunter gelauffen/, phantset er fids in oem linclen Dragen-9)Runo. Dex SRagen lie. get in oer lindern Seiten ees Unter=52aud ches / weil die seber / fweld e in dev cechten Seiten lieget/ fo viel laum einnimmt/ oaf et gerade in oer 5) Ritter zu liegen nicht plafs ftnoet/fonoern efwas meichen mus. Diefer Dagen bat faft die. Sseftalt eine: Sacts

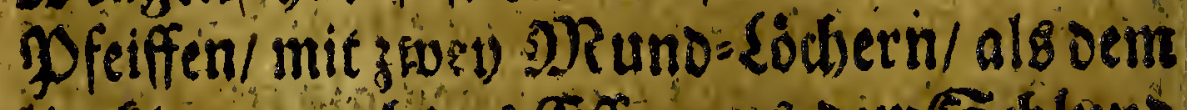
lincié/foosurh ons Effenaus demeshluno 


\section{Is $(r)$ bes}

in oem S) Ragen gebet / und oem rechten/ frelo

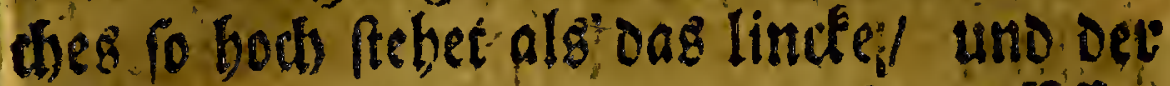

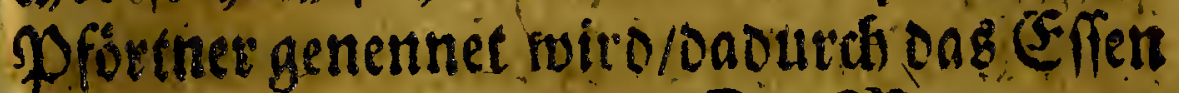
in Die Dármer fímmer. Der פRagen nun iftaud eine 2lit cines Mufculioner Dauß/ einen Sndt oner SBlafe gleicheno/ heftebenoe aub Fleifdsidten Fafen weldse unterforieo lich lauffen / renn weil in dem S) Ragen une terfobiedene Siensegungen muifen verrid) tet

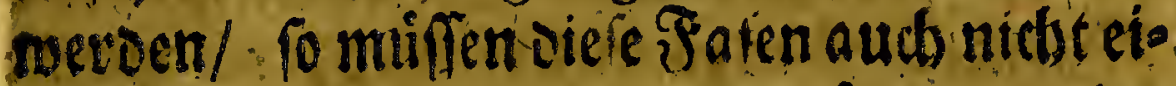
nerlen (S)ang balten/anoers witrse nur eine DServegung gefdeben fornnen: Dic auferfen lauffen Ringmeife quer umb oeñ) Ragen/bie ancern unter biefen geten von bem linctent DRunbe fojrads nad oem rectiten und endigen fid unten gegen oem?50De deg DPagens; werm oiefe abgelofet/ finterfido nods cie oritte Sorte/ sweldee ben lincten DRuno unbfan gen/ uno gerade oben aufoem s) Ragen nach Den Pfórtner lauffen/ uno wetnr fie folchen aud umb fangen/ endigen fie fidl umb oeffen Deffnung. Diefe mufculofe Jafen/fino inrvendig uno ausiwendig mit znery Feflen/ Șátgen/oder vielmefir-sierven/befleidet/ weil foldbe fonderlids Wrerctigeuge Det. DBe negung fint. Die intwenoige nervofe Saut ift vof Xungeln uno mit eitnen ouns

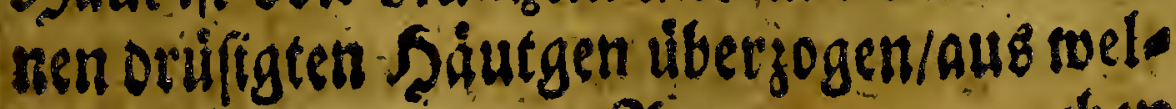

$$
\mathfrak{A}_{3} \text { bet }
$$




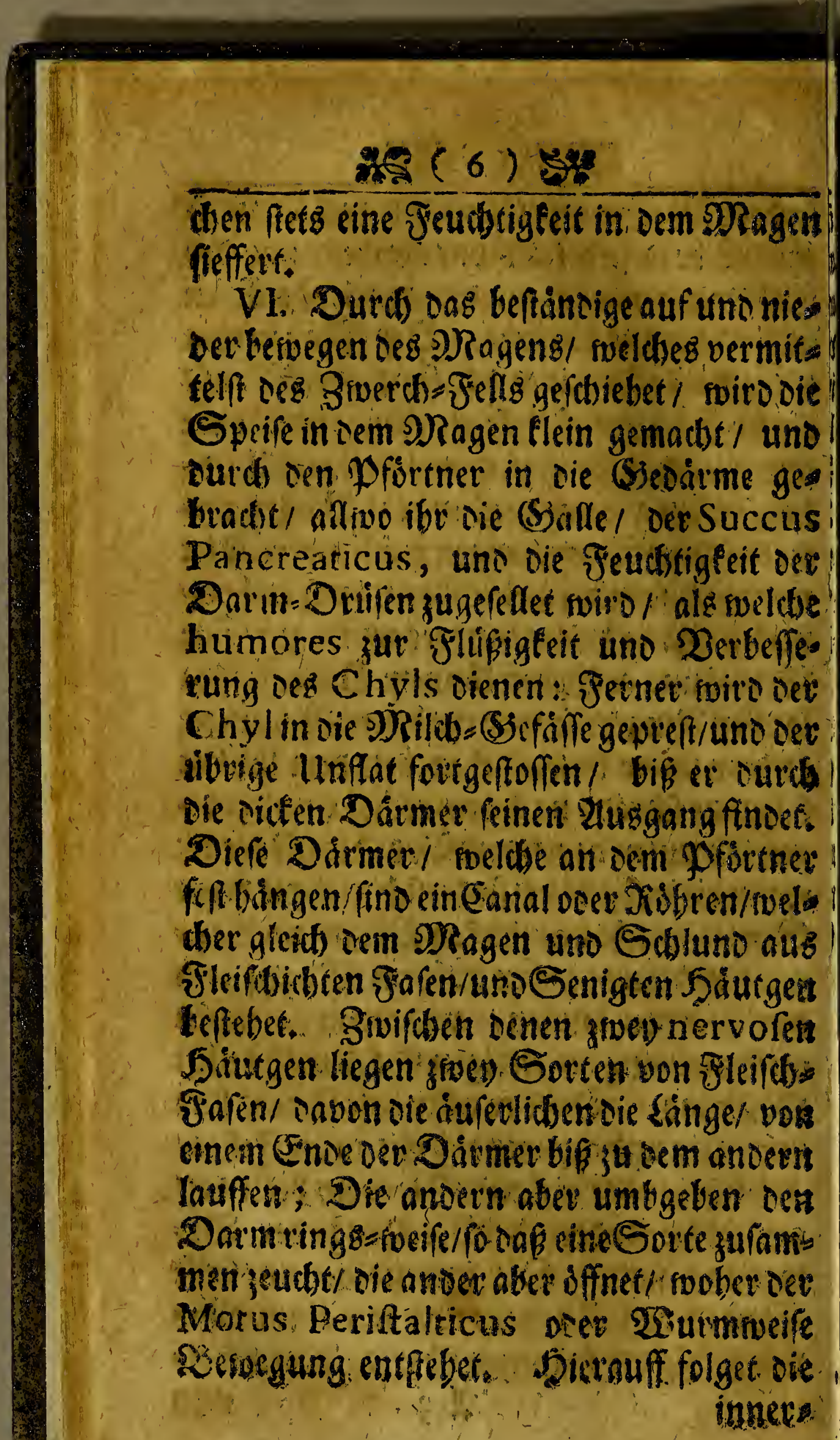




\section{0 (7)}

innerfer fertigte faut/und if mit einen fobleis migten/ Drufigten 25 efen wie oev s) Ragen uno Sdbluno úbergogen/ roourdstriglid viel ZFeudlige eit aus Dem Slutbe gefondert/

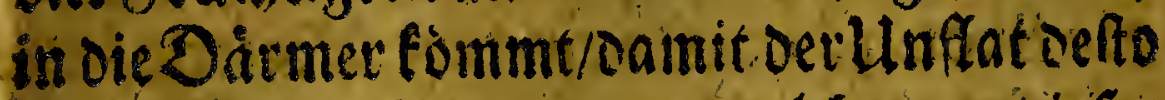
leidter fortgetet; oenn melche an Diefer Jeudstigleit MRangel Leicen/fino bartleibig. Diefes fev genung von oiefen $₹ \mathrm{~b}$ ilen/. met mebr su wiffen verlanget/ oer feflef fid in meis ner A nato mie umb / oa wirc ev eine volfe Commere Szecdireibung antreffen.

VH. Die Medicamenta uno Shilffis

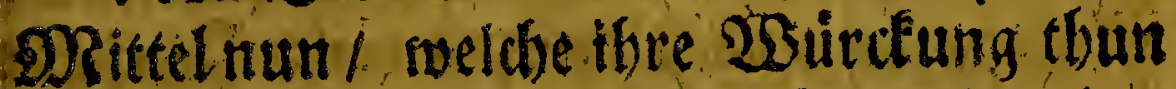
follen/ fin entroeder innerlidfe/ uno geben

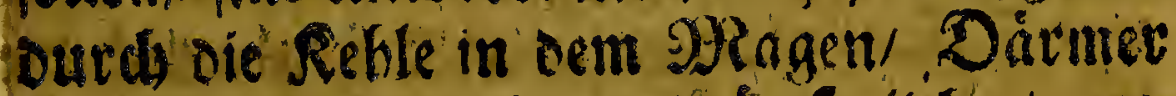
uno S) Rildb-(Stefáffe; ooer állfertidide / uno werden auf die \$aut gelege/in die aldernges

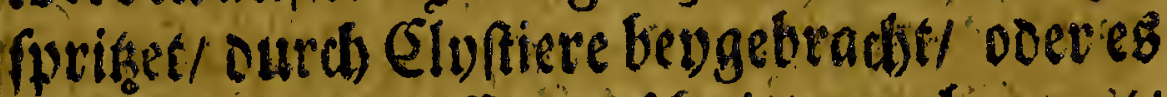
swiro Riluth gelaffen/gefdynitten/gebrentel uno fo, fort. Die Wrurclung biernon zu iviffen/iftnothig/ Daf man oie Fermentation und Effervefcenz, oost langlame uno folnetle (Sicrung verfefe. Siervon ei=

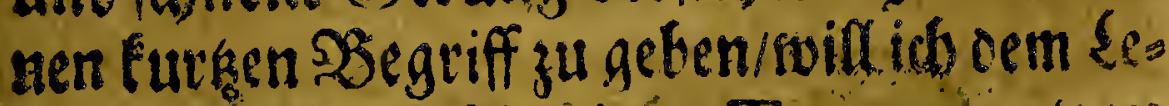
fer vethoifsn nads meinen TRACTAT bur Der FERMENTATION, weleber unlíng(t beraus fommen; altea eine Erfántniz vers (prodjen wiro von oren) Principiis over an. 14 fins 


\section{as (8) Sos}

fángen/ als meldhe Simpliciffima, ober Cinfältigften unter affen fino. Das erfte if Die Prima Materia, ocer erfite NRateriel sicht uno 2 Eervegligteit; bas anoere Materia Secunda, Doer andere ग) Saterie/ eint $25 e$ en aus gribern uno runoten F beilgen. beftebenol foodurch oab sidbt zu uns oringt ; Dáz oritte ift / nuelcber Das sidht miederumb bukget ooer augloffhet / uno beftehet oeffent 2Sefen aus vielerten S beilgen/mie sie Chymie offerbabret/als on fino (sseifter/ 23 afo for/Sals! Sauer/Del/ Erde/2c. aub oet 20stroegung uno Preffung ciefer ₹beilgen gegeneinander/entfebet DieF ermentation uno Effervefcenz, uno folgbarlich bie

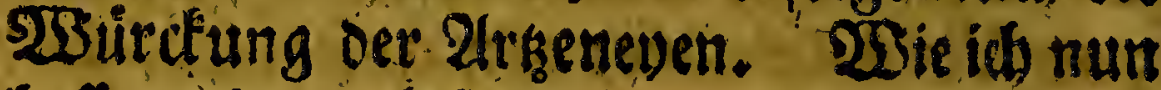
boffe onßz Der Eefer diefen meinen Tractat swiro ourdiggangen doer die Philofophie des Cartes geleren baben / fo swill ich fort fabren zu extliten auf swas Te eife bie Medicamenta in unfern Seibeib:

15. 2Butrdungen ver. vidjeten.

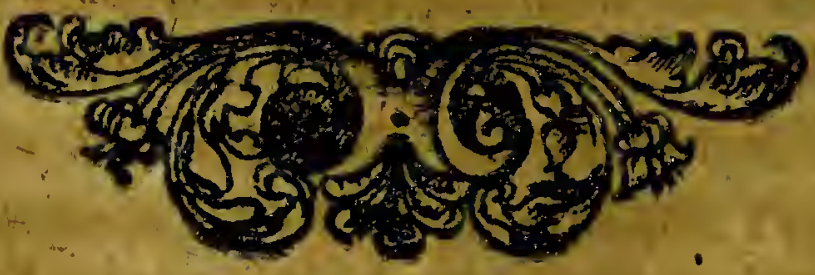




\section{$28(9)$ \\ Dav II. Sespitel. \\ 3on Purgieren / Cinftitern uns Stul-3ápffigen. \\ I.}

(27. Ts Purgieren if niduts anders als 28. Sein ourd Dic Runft gemaditer vermetberter slogang/ es gefduebe son obenoder unten / oamif tricts allein Det Unrath aus Dem' Deagen unc Dármen j̧ bringen/ fonoern andh alles bófe aus dem

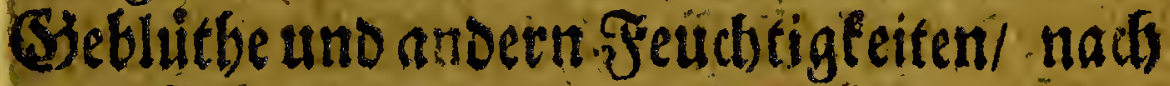
Dem Sammel = PViaks. Des meiften Unflats/ nads oenen Dérmeern zu zieber. SSier módb: te iemano fragen/ wontit man berweien fins.

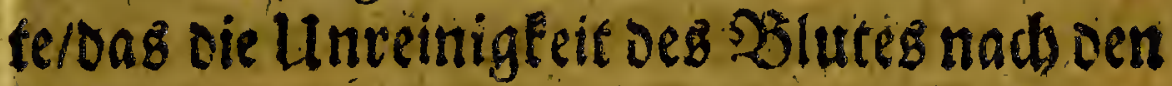
Dármern gienge/uno nithic surdb die SBlarel boer fon/t abgefíbret suritoe? Demirellen

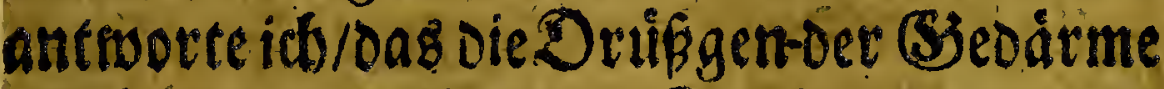
am bequemften fino oie Fendstigteiten aus

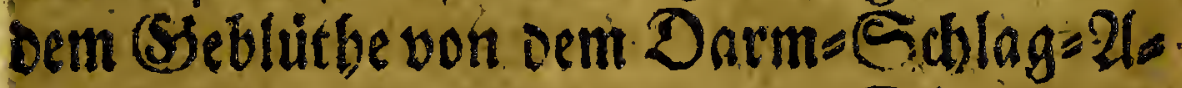
Dern eingunebmen / uno in Die Dármer z̆ bringen. Denn gleich ivie bas (SSebirn aftein bequem if die rubtilen (șeifter/oie Sbruiftedie 2) Rilch zc.zu fondern/fo fino auch diefe Drifs.

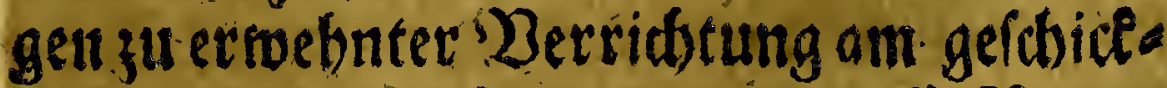

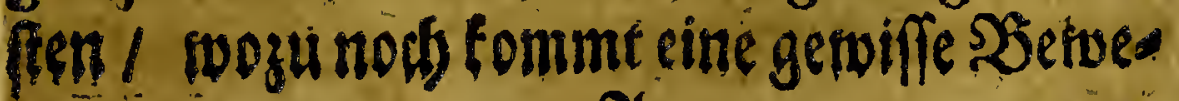




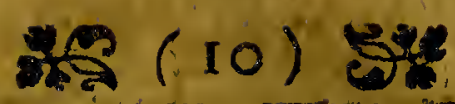

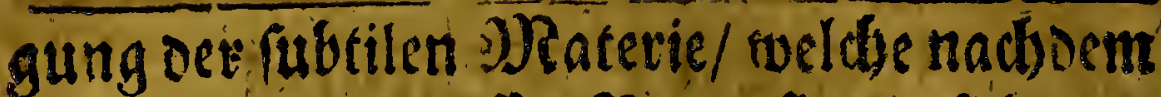
fie fleinen ooer groffen Xiaum finoet/fids uns cerfdsiedidf beriveget/ swie fermer foll angezeis get neerben.

II. Dic purgierenden Mitel suerben aus allerband Dingen gemacht/ Die meiften fino aus Vegetabilien/ tornig aus. Mineralien ! uno fo viel mir beivuf feine von TGieren/ eb. mulfte הenn de Srafle eine Purgation abgeben / miervobl ids sabon feine Erfabrurighabe. Diefe bringet man nut

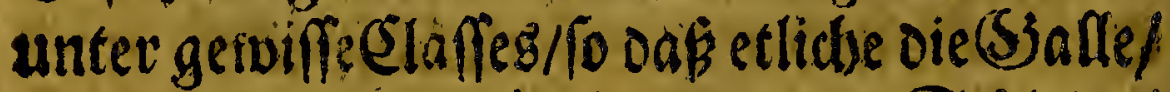
etlidfe oie Melancholie, andere Sulleim/ swaffer $2 x+$ abfuibren. Daf foldues fo gav fpecial zugetbet/ swie die allen fict eingebils Det bahen iff falfot). Denizes fino mebr ₹ beila gen im 23lute als Siane/ Styleim/Melancholie, Waffer ze. Denn gleidstwie die pues

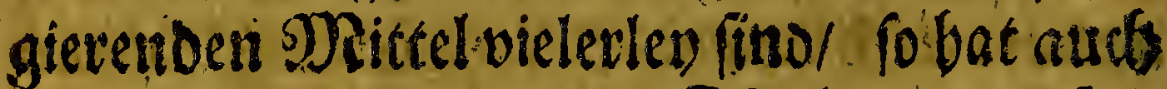
iedredes feine befontoerne S. betlgen uno Fis guren/vermittelfit oeren fie sine unterf(d)iedes ne SBerwegung in Denen Feilgen Deg S3lus

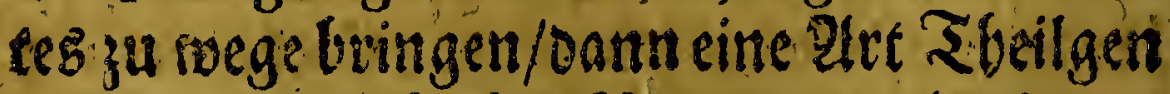
mache inmer farcteresberwegung als oie ans. Deret roeldbe oem SSlute mitgetbeilet/ verut s

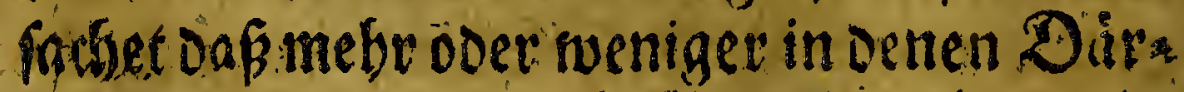
men evacuiret nird. 'Dsan En aber nicbe 


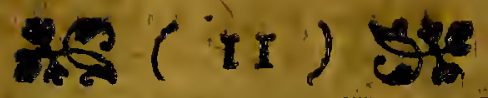

fagen/diefes purgietet Sauer/Sals/berbe? Suleim uno dergleidben.

III. Qlud fint eclidbe Specifica, diesas Saupt/ Seber/ Mills ze. purgieten foflen;

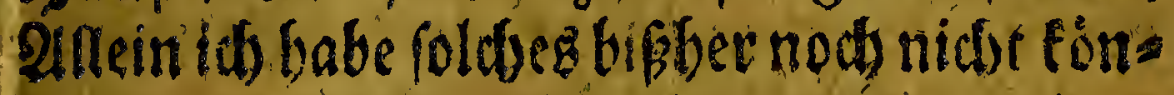
nen warnebmen/saber id ocrmeine/ oapsoie Purgier Drittel nicht in einem SpecialFbeile allein/ foncern in ser gantsen Mar-

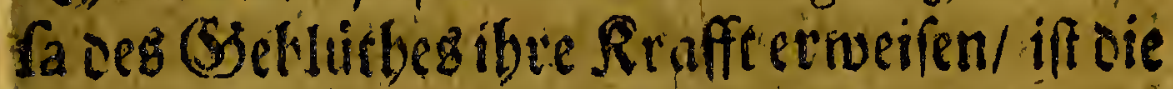
ganthe Mafla gereiniget/fo muffen notbrene Dig aud oie Special-Sbeile fauter roetben; Darumb baben fie auds nidot unbilfig etlidbe purgier= SDRittel/ Carholica; oder alnges

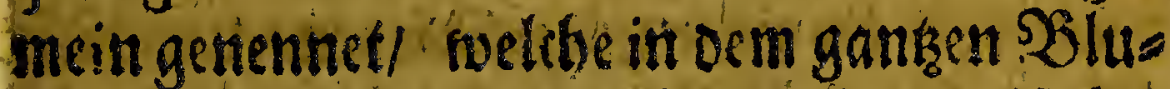
teibre Operation ausuben. Uno allo bat nan nummebro/ Da man es beffer nueifi affe Specifica berworffen/ uns ridstet fein $216 \%$ rebet a llein auf oiesllgemeinen/uno auf oem Umblauffier Siffer.

IV. 'Eg werben sie Murganken gemeis. niglid in ored Sorten getbeilet/foeldye nidbt fo roobl iben. 23 efen nach als gradu uno was oie 25 lirutung befrifft/oneneinanoer un: terfbieven/in cet erften Gotte fino oiegans gelintoen/alsManna, Caffia fifula, Pruna, Cry falli tartari, Elixir Proprietatis \& c. Zum anoern etwa ffardere/als Rhabarber, Aloë, Setrez=Slihter/Mechoa- 


\section{$35(12)$ s 35}

choacanna, Agaricus, Turbith \&c. Drittenz die gar frardin/ welche aud mandomablbrecben machen/als Jalappa, Gutta gamba, Scam monium, Elaterium, Euphorbium \&c. Ez ift zruat wabe/ Daf fids gerwiffe gradus madien laf fen/ affein wenu man fich genau dacan balo ten folte / muiften oie unter sinem gradu ftes benoe SRittel auts cine gleidge Dofin bas ben. Sum Sximpel/Scammonium nno Euphorbinm, Deren Dofis ood uns gleidh; Darumb fan man fid an oie Gradus nidht binoen. Inlle fino voneinanoer mebr oder foeniger unter foieden/ uno laffet

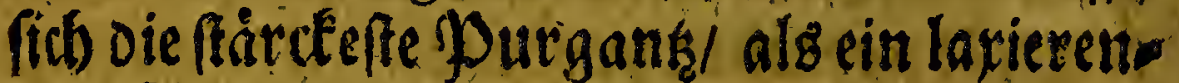
Des Drittel/ felbft beb Rinoern gebraudien/ wenn nur die Dofis vermindert noito/ sol? d) ${ }^{3}$ id felbft in meiner Praxi uno an meinen eigenen Rinvern erfabren 1 . Da id an ftate Der Manna, fo id nidbe beo Der Sano bates gr. jj.Scam moner gegeben. 5 mus leon/ Das in einem Dinge mebz wurgierenoe S beilo gen zu finoen/ als in bem andern. Dafert ficb aucbeiniger mofen gradus macben laf fer. Bum Exempel/ fwenn in $3 \mathrm{ij}$. Manna als einer Dofi, neunbuttbert uno fectogig Sbeilgen fino vie purgieren I miffen notb. nenoig in 3 j. Rhabarber, ideldjes auds ei- 


\section{$39(13) .5 \%$}

ne Dofin giebt/ eben fo viel wurgierende

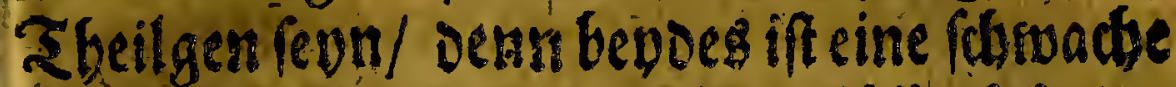
Dofis; Uno nuf foldbe Weife läfet fidb eine

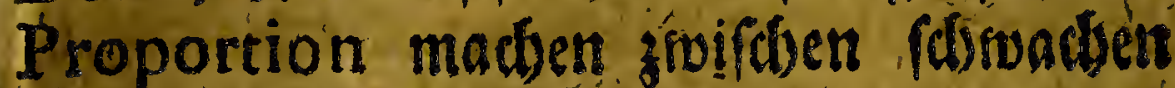
uno fiáclen purgier $=2$ ) Rittelin/ ro. onf man

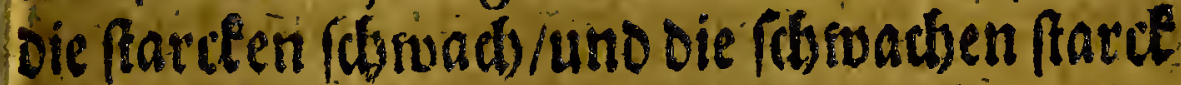
madien fan. Feerner baben soir nidit fo vies leclev Purgantia vonnótben/ fonoern es fan die Sadbe mit suenigen vertidbtet swer. ben/ nur blop roeil etliche SOatienten yor Dem einen Edel baben/fo muf mebr benneines bev der Sano fenn; wie oenn mandoer die Jala ppa nidbénefmen fan/' bern mus notb. wentig/ wo er fol purgieren/etwas anoers gegeben weroen.

V. Wiele Me dicamente bie in ifreer ge. bòrigen Dofiftarct folutiten/: thun mandse mabl suenig ooer nichts/ wo fie nicbt mit eis. nem bequemen vehiculo gegeben twerden; e. g. oie Refina Jalappæ in SBranderwein gegeben/ thut nodf eing fo viel als foofit mit 23raffer SSier uni dergleidsen genommen

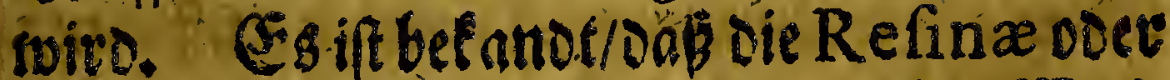

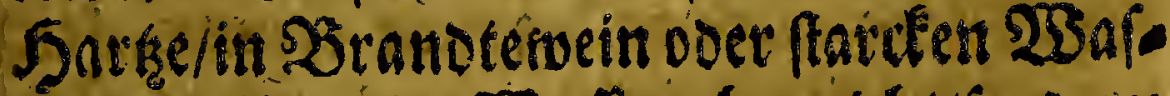
fer/fdomeligen/im 2 affer aber nicht/fondern fallen zufammen/Das ein Tbeilgen nadb Dem

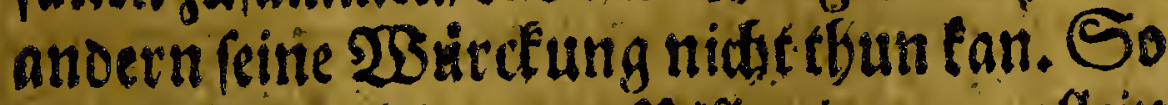
geber es ards mit benen Boiflen/ wenn allein lauter 
lauter bar $\frac{(14) \text { Diget }}{\text { Dinge zulammen gemilabe }}$

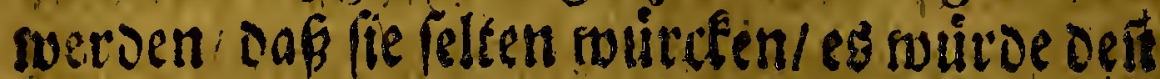
S3ranotervein oarauf getruncten / roovon fie idhmeligen/oder ettwas onzunter gemenget/

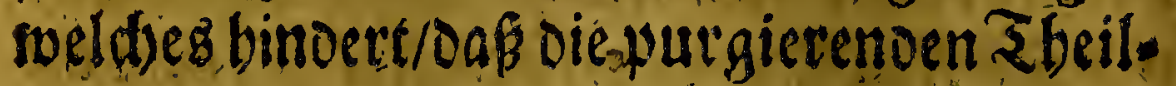
gen nicht zufammen lauffen; Uno aus dies rex U? rache bat man auf oie Præparationes, sobl Ldbung zu geben. (Ez gefdsiebet auch) sodbl oẩ iemano auf eine Beit feinen / und auf eime antocre vielen 2 liggang von einerled, Medicamente bat; sueldbes fidb leiduclict;

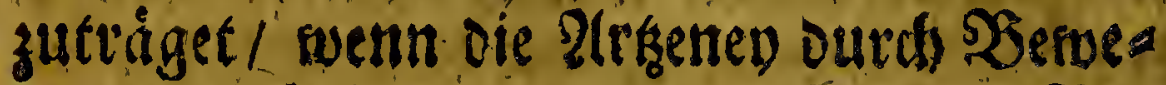
gung oes Seibes gefdirvinoe aus oem 2) Ras gen getrieben swivo. Sngleidben fan eg fenml Daß Det SRagen uno Daitmer zu einer Beit mebre alszu der andern mit Drufene Saffe angefuiftet/Denn Die Sarteibigfeit und Ders fropffung/rubret von sem (Ssebreds getonds. ten Soffteg ber/ oa im (Siegentbeil ein flúsi? ger Seif aus seffenuberfú fommet/if mebe Sdbarffe oabev alz fubz geburet/ lo entfebet gar ein Duzdsfall.Ssleichergeftalt verbailt es fids mit vem purgierentifif viele/zu2luflifung Dez Medicaments bequeme Feudtigfeif vorbamen/fo geber viel von unen weg: Im (sjegentheil/ wo feine Teuchtigfeit zugegen/ aveldse die Diffen oier eine andere. Purgati on ouffifet/erfolgen wenig poer feine Sruls gang 


\section{me (is)}

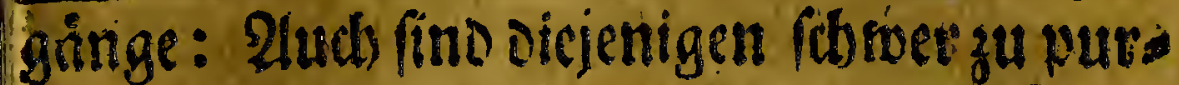
giecen / weldse viel fauere und iroiffste ?heiJe in ibren Humoribus baben/ indem die groben Sbeile mebr Xube baben/ oreer friffe Viegen/ als oie simnen / fo von oer fubtilen Materie leichter berweget wertien; Darumb babe ich worbin gefagt / cap die refinofen

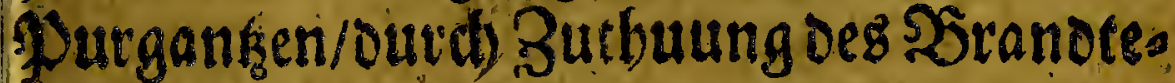

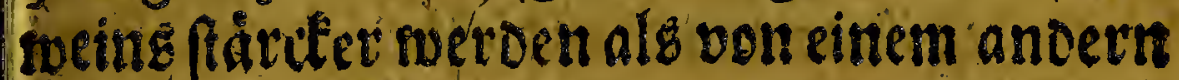

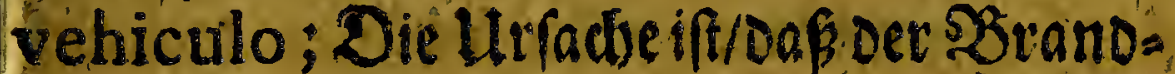

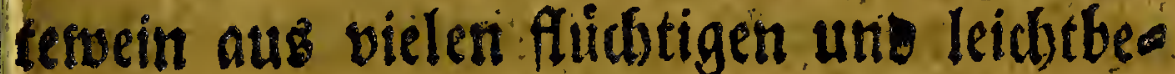
sweglidten Zbeilgen befteber / Die nudb) leidse anoere entbindern und zut SBerwegung brime gen, Darumb giebt man auds nade Einnebmung oer Purgation, SBiev = Suppen?

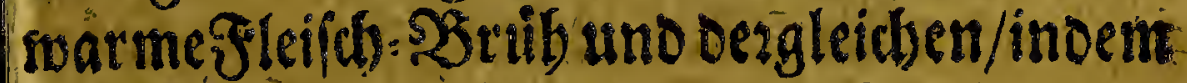

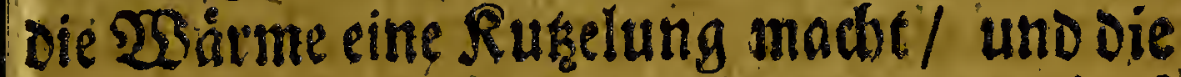

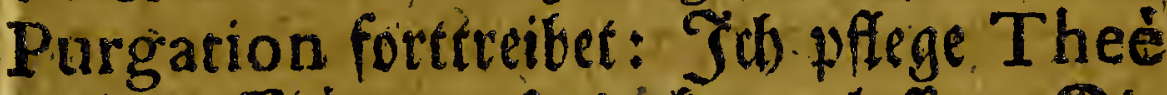
boer Coffeé darauf triniten zu laffer. Die Speife/ melche vor Eimnetmung der Pur-

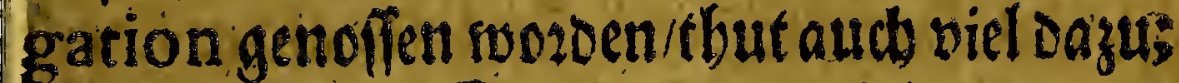
Denn trodtene. Epecien uno wentig getrun Eten/verfiopffet bie Dármer/oafí oie Purgation zu swircten micte Raum uno Saffi ges nung bat; Demnes if zu wiffer/ oaß bermitrefif bes Safferes bie vurgierenten Theils

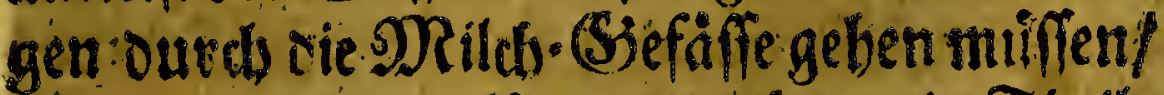
imangelt oiferer/ fo fónnen autes spenig Tbeilo 


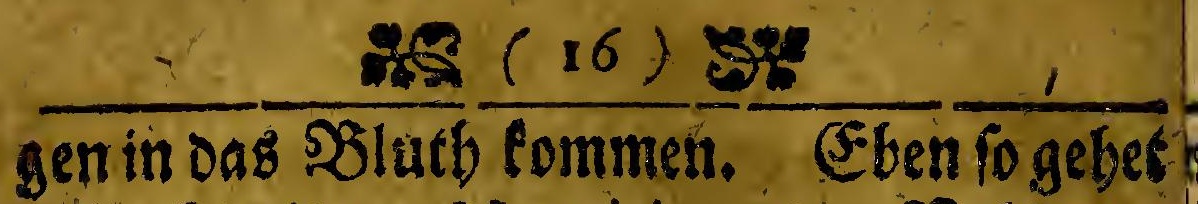
eह aud mit der Nutrition poer Nabrung ber / we r wenig trindt uno viel troctne Svei. fen iffet/ oer woro wenig gensibret/ wer abec beo trovenen Speifen viel trind' / oer be commt viel Rabrung.

VI. Die Uriactsen swarumb man purs gieret- fim folgenoe: Erfflich/ menn eins: bartleibig if roiefnobles de Ur fadbe de? Dets ftopffung nidberwegnime/ fo bilffe es bod oa Die Dírmer nidst gan's verftepffet swerden/ worauz groffes unbeil folge rotiroe/no nich mit einem (Elyftier fonte gebolffen nerden. Bum andern/ Die linreinigfeit/ tivelche viel fauerel falsigte uns ansere grobe Fbeile in fidb bat/aus oem (Siek hitfe nads oenen Dars men zu bringen. Dritteng/eine SBenegung zu machen / soeldse in theilg Bufällen/ alg Sdblag und andetn Sdblaffe Strandibeiten Godbfint nithig iff.

VII. Feenter if es beffer linse als zu farce purgieren / insem ftarcfe furganksen/ seib. reisen / Rotbe = Rubr / Convulfiones Dbnmaditen zc.veruzfacten/weil in etlidben Oerfonen fo viel Rraffe nicht ift/ bergleichen Suföllen zu wieverftehen. Singleicben mus

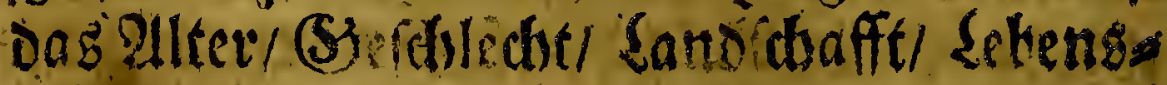
grit uno bergleiden angefeben nerden/ oem 


\section{s.}

ein Bauter ber farce atbeited foll wobblores bis viermabl mebr tơtbig baben! als eit Denich Der wenig tbut/ uns fid mit wenig uns leidten Speifen Gebilfft. Denn ein SBauer der grobe Speifen iffer uno frárcte arbeitef/ bat allemabl mebt Rraffic/ poeinet Purgation zu noiederfteben/ als cin foldbex 3artling. Flud if ratb/amer / orewmabl mápig wurgieren / alşauf einmabl mic (Se) forale urito Sdraden / inoem inzfuifdsen oie 5) atienten outch genoffene S?abtung swiever gu Rráffen fonmen. Dafs ftetige uno vie It purgierent tauge nichte / Denn viele fwerdent Daourdi Rraffilop/ uno befommen die $25 a$ a perfudbe/ Darte/ Sdbartbod/ gelfe Suds uno sergleicjen 3ufáfe am 5als.

VIII. Tbeilg seute belommen Stul gang von Dingen oic anoere ftopffen/ als Caftanten/ Mripelm/ Sdleen-2e, weldhes fos feltfam fobeinet, 2Allein swie man bavot Gialt sas die Rhabarbar ftopffer unto nut nacb ibten wenigften Tbeilen purgieret/ mie Die Runft uno ibr anbaltenter Sufmad be geuget/difo fónnen audb in fopffenben Din gen Sbeilgen fern/meldbe den einenlaxiezen/ Den andern aber fo fárcter uno bartleibiget int Popffen: Ta Die Caftaniest follen allein Stulgang maben, nean fie rosm geffent 


\section{He ( 18$) 3$}

wetoen/ fo fern die oarinnen cuedh oas Fets. exzur Serwegung gebradsen Theile/ ibre Szerwegung in unferm seibe continuiren/ welches: fie fo leidste nicht thun / swenn fie wieder enle worden.

IX. DRebe Detroundecung madte es/ Dafe etlidbe ruenn fite nut eine Purgation fes

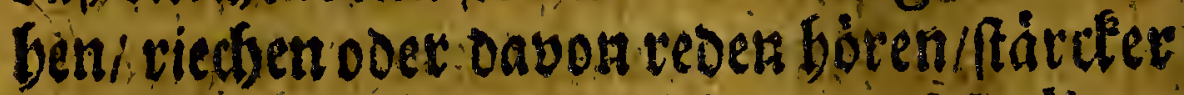
purgieren/ als oec/ solcher oab: Medicament genoffen. Jier if zurwiffen/ons foldhe seute eineu groffen (sulel uno albrdbeu Davor fabent uno rwenn Deffen Dencébilo/ (Idrea) ibnen ourds bie Sinnen vor fommel swiro der ganfege (Sorper alteriret uno bendes get, Wenn nun oie fubtile Materie naç foldber Idxa in oas SBlut uno Säffe operiret/ fo fan nidts anoers als Qtbgang et: folgen/ nacboem oas gangs: SBlutb ourch Die Szervegung alfo difponirer suDEDRM DAF es peine Unreinigleit vonfiç lâf. Dergleidten erzeblen auth oie Capitaine/ Daf Das Bolde vor Sieferung einer Siblaht/ megen SBans

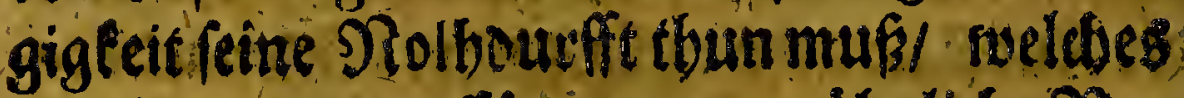
Durdb eine oem Conper ungerwobnliche SBetwegung geritheben muiffel morauf oie afles ourdfiftrablende fubtile Materie ibre-23ste ctung thut. Jab babe ebentalen einen Sertw zu Sranetfer getenc/ deme von eimem P rofellore 
tos (19) 58

feffore sourgier s Dillen georbnet worsent

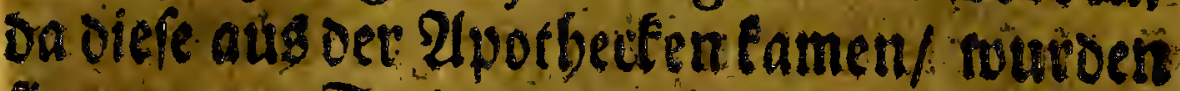
fie von ser Sodster angenominen und oev Detutter úbergeben/ norauf bev se ourdy oic Citibilcung un oarauf erfolgte - Alte ration, melopurgievtem als oer 20 ater felbit: Der die:Pillen getraudbet batte. Totb neig cine Jungfrau/wennibe bon Schnurigfeit twegen(S3elo, Sachen gefaget toiro/alte riret fiefrobalfo/ daf fle mus zu Stublegeten: Cinte anoere/ roenn fie purgieren nill/getbet nux in die 2lwotbecten/ sa fie von dem (Sje. mude alo afficiret noiro / oaf fie efliche Stubl: (Sange savon betimmt. Sold)e ano oergleitben Ekentwel gieht es viel. Die Mirfache / if eine grofle cinbilcung/ worauf eine farcie Setwegung oer (Siefáffe uns

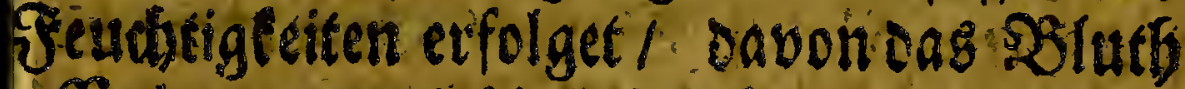
Afficiret/ uno bie Unteinigleiten in die Dår. per bäuffig niecergeleger werben/tweldbe sa. git angefultet genotbigetfinto/von folthein Inrash fich zu entlaften.

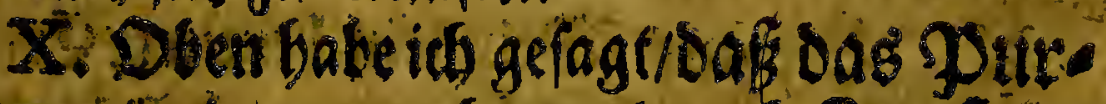

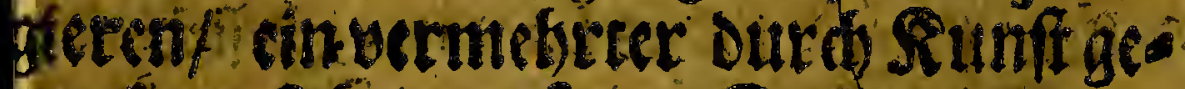
hadter a6gang for. Diefes etfocines pug tem eirtgenommenen Medicament, tus sem oarauf folgenden Effeet ooer.

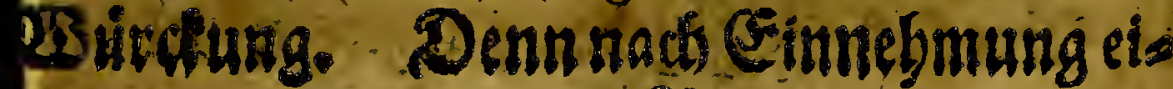

$$
252 \ldots \text { ner }
$$




\section{3. ( $(20) 38$}

net Purgation, wirc man offeris mebrttn: flat lop/als. fonftin einer gantsen. WJoche. So Inftung nun feben/ soorinnen sigentlich oie Srafft Der Pourgier= 2) Rittel beftebet. MRatt. fieber dabin alle Purgantibus cine Suljàzfe. fe fern mutfel oie cine mebr als gernobnulidje: Serwegitng nichtallein in Denen Dármern/:

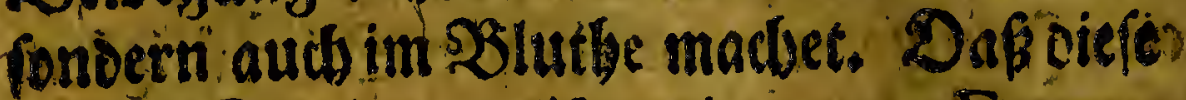
Pbuarfe Theilgen meiftens in Denen Extra= etender: Dinge entbalten/ nud mit anoern Fbeilgen in getwiffer Proportion miffet vereiniget feon/erforbeinet Daber/Daßs afte Ex-t? traeta von Jalappa, Rhabarber, Senésa? şlátted/Scammoneo, Aloë, Gutta. Gamba, Elaterio, Helleboro \&c. aus

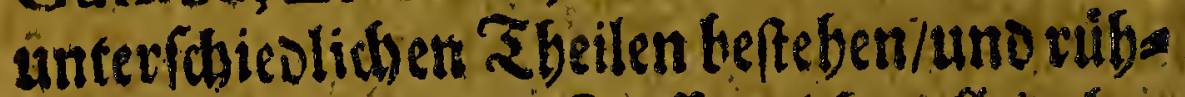
cet die purgietenoe Rrafft nidbt allein ber von einem Alcali, Sauer/ Eroe doer ber gleidsen/Dern nenn Diefe Tbeitgen ourds Die: Retorte voneinander geidobieden / befinber mantoá iedes aflein vor fith felbp feine Purgation abgiebet.

WXI. Die aus 23 eimftein bereitete erv. ftaflen/ fino ein linder Laxativ, beftebeitse aus Sals/: Sauter unvertrab Del/ roelitites

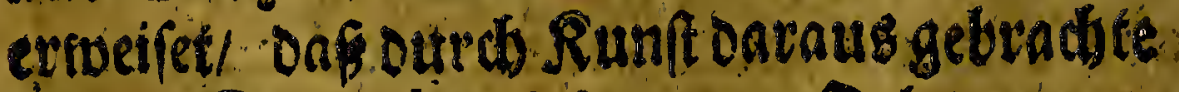
Sals/Sareter Spiritus uno Del/veren a berfeines affeine gegeben/pargierets weldhes जel 


\section{$20(21)$ s*}

aud in anoez purgiezenoen Ditteln zu obferviren ift. Wir muffen sannenbero wifs fent onf ieoribedec Sorper feine befoncente Betmifotung bat/ uno aus unterfdbiebenet 21rt6 Sbeilgen beftebet / cic afle nadh Proportion ibret (Sirose uno Dereimgungles. nen grofen doer lleinen 3 wifdoen s Daum (Interftitia, Poros) haber / nath on fien 2Beite ooer Snge/ die fultile Materie mebs ober fueniger ibre R rafft oarinnen zu etrbeis fen vermag. Dieneil hivit nur viel Dinge baben/sic in iber Decmifdung uno Bersis nigung bon oenen Purgantibus untets fobieden/ soie die Chymiebezeuget; fogea

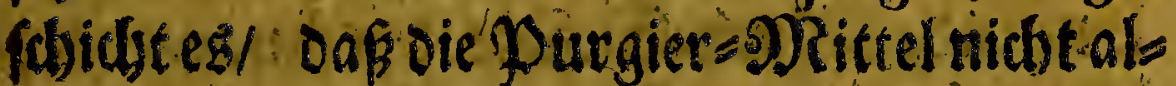
Iein oie Dámer angreiffen $/$ fonoeinfelbf

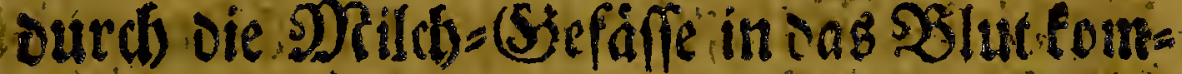
men/ndldeg $25 / u t b /$ bon ser fubtilen Materie die Defoegung nad 2lutb oer purgiciet. Det Drittel empfangende/zugleidb eine-foldte 23ervigung befommt/alBzu oer Excrerion oser Lbgang notbig if. Ubev siefer frto Die Xibigen der Darm=Dripgen alro hes fobaffer / oafs fie folgenos sec im silutlie ge= madben Difpofition, meto Fiudstigfeicen ats dem Slute empfangentuns in vie Ders

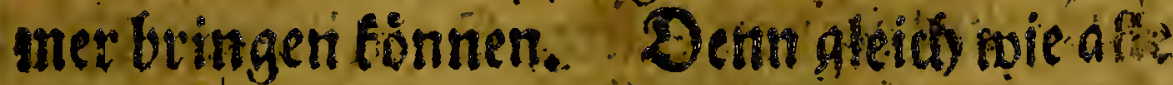
grobe Sbeile ound eintlates Sirbuidu ge 


\section{*⿰冫欠 ( 22$)$ )}

ben/ wenn es nicht gefduittelt roiro te fo fan Dag SBlutb and nicht fo vicl Jeudotigheiten in sie. Dírmer nieserlegen / pusnnes nitbe surds ein purgiev: DP ittel affo porbereitet

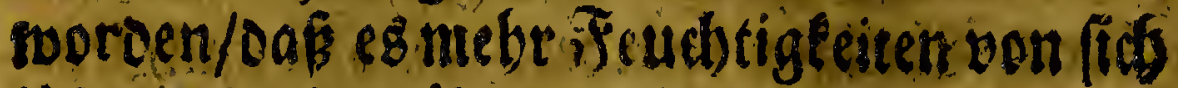
láflals fonft ordinar gefphiebet.

X11. 2Renn vie Purgansen in zu grof fer Qvantitit/ vornebmlid zarten Eeuten gegeben suerden I erfolget ein uiberfüisiger albgang / bey oenen (Siricden Hy percatharf tis, o. i. Utber Purgierung genonot/ speldtes nidstrallein D y fenteria m ocer $\Re 0$ the Piur verurfachet/ menn ourd die uber

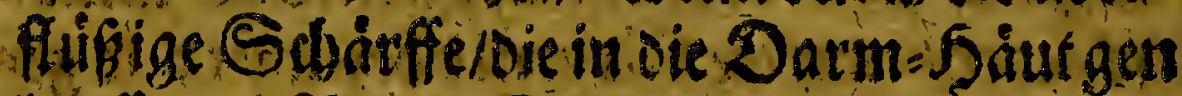
lauffende SBlutb=(S)efáge ourdbnaget nees Den: fontern es formmen Convalfiones

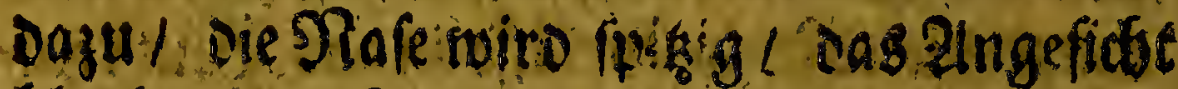
bleict 1/ Die Alugen eingefunten/ wie aut Srampff / Dbnmadocen une or gleichen/

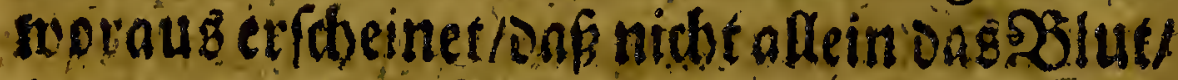
fonourn nuth oie fo uthtigfeisen/ welche bon Dem (Sebirn ourch die Senen geben/ angegriffers toerien: Soldses bat man audi be: funven/curdbinfprisen wurgierenter Din: ge/ in die Zloern eines Sundes: Der Setr Antoni de Heide, Practicus zu @ittels butg/hat die von Gutta Gam ba mit 2 af. fer gemadye Tinctur in oje Sgein-2loec

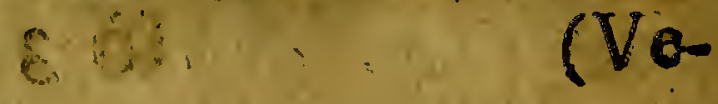




\section{Is $(23)$ है⿴囗十}

(VenamCruralem) gefurişet/weldfer al fobalo Convultiones fotommen tino ges

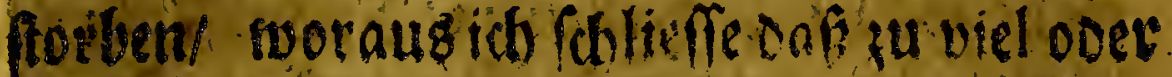
zu tale gerweren; Denneb nefrefr Herde. berfuchte ea miteinen Extract von Senese SBlattern, Da blieb oer funt lefen. Wor unteridbied liche mit gutenSucce fs in Drenfobengetbane Experimenta hegibuet/ Der lefe die Acta Philorophica in Engelanol bas Journal de Sçavans saug J Jrancticif utto den Inflang heom Sculteto.

XIII. Sieraus if zu fdilieffen / onfole Purgier =פRittel in groffer Q vantität our bie 2reern in oas SBlutb gefpriset/Demfelten Effeet tbun/alz menn fie ourch oen D) Rumo genominen nerben. Ingleidben erftheinet

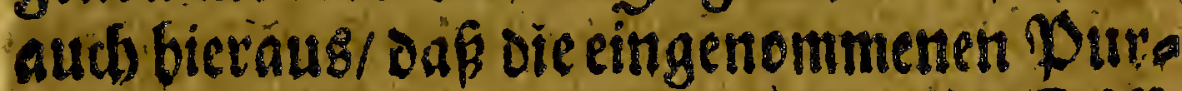
ganken/mebr in on s3lutb uno oeffensiff. te/als in die Darmer muicten: Rudb ráf fie aus febrifdarffen Fbeilgen/von Sauer uno Sals befteben miffen / twilde mit anoern (f) arffen Tbeilgen oes SBlutbes vereiniget/ Rotbe Xubr/ 3udtungen X̃. erivedfen/nie Detien begegnet / rweldbe Arfenicum ge nomimen.

XIV. Sier foute iemans einnorffert uns

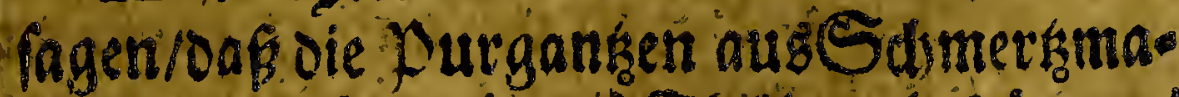
dhente (Rodentibus;) Sberlgen beftuinden/ $\mathfrak{B}_{4}$ 
$\frac{4}{\text { Dic den }}\left(\frac{24)}{\text { Ragen uno Dátmer angreiffen }}\right.$ Convulfiones, Rneipen uno Wrinie mon.

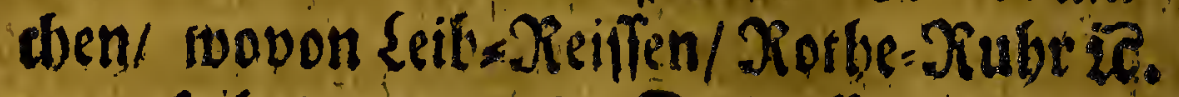
verurfaibet sweroen? Darauff antworten

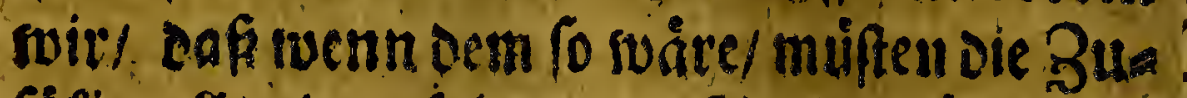
fálle allezeit erfolgen ; 2luch müften oie Spannifchen Fliegen eingenommen / pur. gieren/Die boch cen S. Die Dlafe angreiffen. Dákein pobarf Sals. in ienen Purgantibus vetborgen/ if be= fanot/ man finiet es aber audb in Rorm Sleifth/ Fifhen id. Darumb if zun niffen/ Daf oie folorffen Fbeilgen alfo mit oniern verbunnen uno vernvidele fine/ basp fie benen Darmern fo viel Servalt nicbet thun fonner. XV. Nun frage ficts/ swober bodbie Pein ober(Sirummen fommel fo nad) ein: genommener Purgation in tem seibe gea fíblet wito? Biel gehen oer Schirffe des Medicaments die Sduto/mober audb die Csprubonbeit entetinnenCorrectoria sabey ju thun, 叉or wenig Jabren war eine Jrau bo u mirl oie hatte gebourt can man die Stiets gin aus cenen Senes.52linttern fudbe/ weil fite Ruken madte: Sh antwortete ibe aber

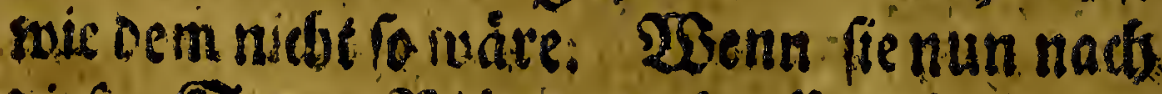
dirfen S.ntze Blitter vertauffet/ bat fie oie Etielyen allemabl beraus gefucpt/ bif fie oes 


\section{축 (25) 54}

sen cine gute Dart gefammelt/ Davon fie ein Sotb mit Wa affer infun diret/Dar in fufum ourd)geocudt/nuthtern auggetrunden/uno ibrer Ergeblung nadb/Davon linoe purgieref. Dos (Simmen/ Xeisen 2 . if alfo nicht fo robl dem Medicamente, als denen fdaarfs fen Feudbigleiten beyzumeffen/ die fauew/ falsigic. fiuo / mit oem Purgante vereiniget/in oie Dàrmer ffefern/uno oafellfte einige

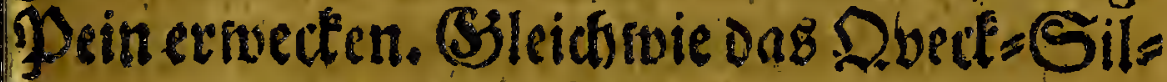
ber an fidf felbft fein (Sifft/med) Salivatorium nod Purganis ift/ Denneg befebet aus zunoten Theilgen/ wenn es aber mit unfern Saffer fid bermifdet/ werven Deffelhen Sólgen ooer Pori uno zarte Riobrgen / mit Säure uno anoern fdbarffen Theilgen allo angefúflet/Da enolich ein Corrofiv daraus soiro 1 wovon in dem 2Runce und auth off? ters in Denten Darmen groffe Ungelegenteit uno Sommersert entfrebet / swie mir dent in Der Chymie erfabren/ Das Duectfilter mit Saly, Salpeter uno Vitriol fublimiret/ ein tootlicbes (Siffe nuird/ ob fobon feines von Diefen Dingen vor fidh gifftig ift.

XVI. Die Purganken madjen allo in

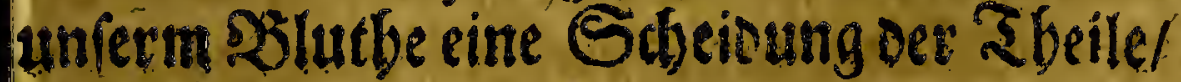
weldhe roegen iffer Figur bequem fino ane

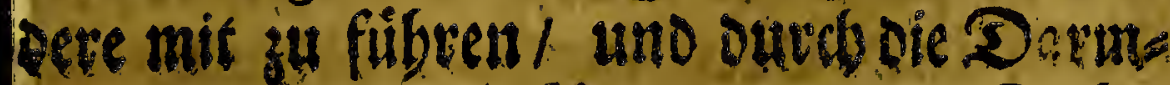

$$
\text { S5 } 5 \text { Drif }
$$




\section{5 (26) 5}

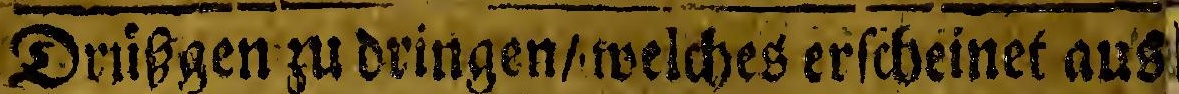

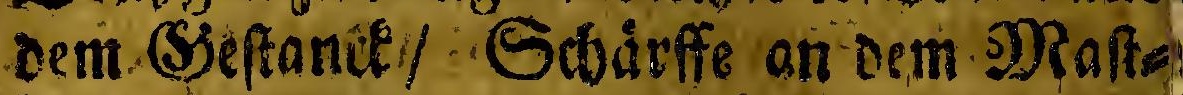
Darm/ uno Rineipen in Dávmern / ourchs. sweldse S djarffe Convulfiones, 2hufblad

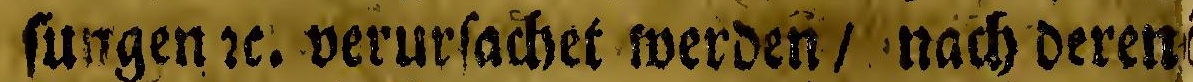
2ublaffung Evieidserung erfolget/ umb Deswidlen thut man 23 inofreibence DR ittel zu denen Pourgangen/ foldce diffo mebr zu befóroern und fortzatre eiben. Fis befeptiget. audi unfere) Reinung Hippocrates fagen De: 28 enn man nads Dem Pourgieren Durff befomimt / fo bat oic Purgation Das itrige getthan; 2liz inolte er Fagen/ swent viel fertidstigkeiten surch oic 2loetn uno Sdblags 2loern in Die Dairmer ausges

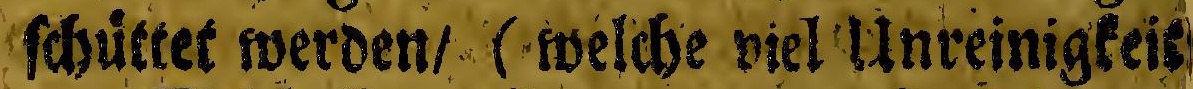
uno Sdbärffe mit fiti genommen) fo teident geoadiste (Siefäffe an J"eudstigfeit WRangells

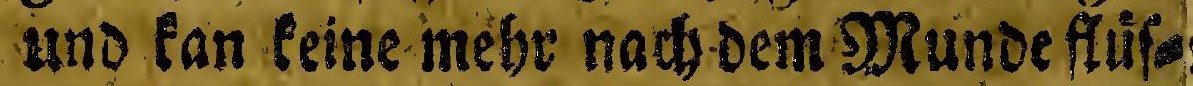
Fent Daber 'Froctne uno Zevlangen nacts Seurbtigfeit entfetebet.

XV11. ßiel thun ben die Nourganthet Correctoria, bemfelben ibre bjfe 2Urth łu benebmen. 2ukein es if meines 23 ou undens.

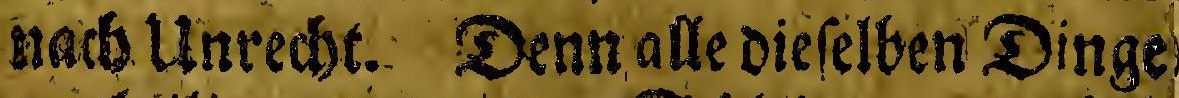
gertheilen entiveder DenS dbleim uno treiben

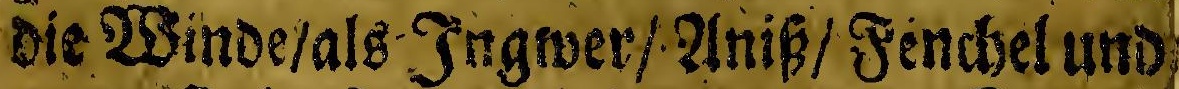
veren Del. Doer temperíren Das Sauev 


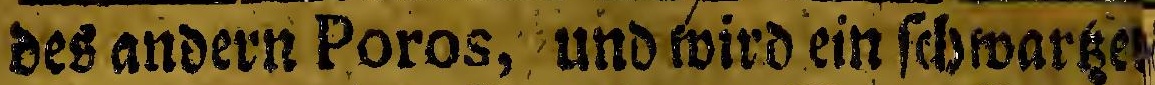
Liqvor Dacauz. Sft alfo das 2 Bein=Steins:

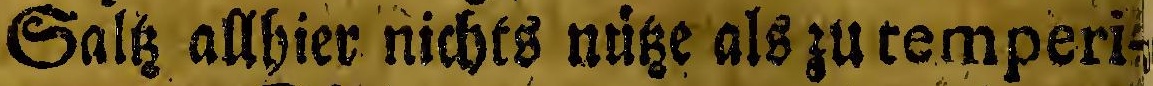
rung oer Since.

XVIII. Es roerdenaut nobl Tama - rinden / 2 seinftein / p flaumen ?c. zu Deit Senes=:SIattern getban / es fino aber fein Correstoria, fonsecn machen einen gelind Den libgangl oaourd oie Unreinigfeit befto gefdrwinder uñ bequemer ausgefibet roirb soie Deinn diefe Dinge alein gersoffen/Den offen balten/nidst anders alis bev viel setiten Die S2utter= Drild uno MRolefen tbut, 20 sonn man frifbe PPfaumen/ $\mathfrak{W}$ sin s rau ben uno ) Soft zu viel braudbt/befommt man nicht allein oen Durds Jall/ fortoenn wobl gar eine tosclidse. Rranulbeit Choleraged nand $/$ nidj anoers als ob maneine ftatcle Purgation genommen;. Dergleichen iof audf von So utter Drild trincten/ gereben/

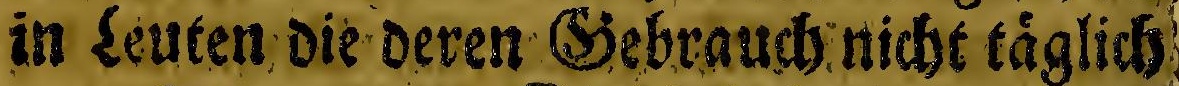
genobnet maren. Diellufache if Diefe/ Dafe Die activen falgigren uno fauth Sbeilgen/. welche vorbin nod miteinander berbunder

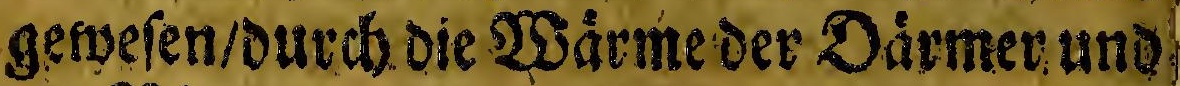
Des SBlutes rege gemadic roerdet $/$ uno oag Sjlutb alfo difponiren / oaf es viel for arfe Sbailgen in oem Magetuno Dàmeraus? 
Dffet / morcuf oer Unflat uaterind oben brtgebet/ ro tas oer Patiente mit Convulonibus ino Dbnmadeen úberfallen/ is Ifobalo toptlidy frand wiro.

XIX. Fetnet tragen mandje Siesenz

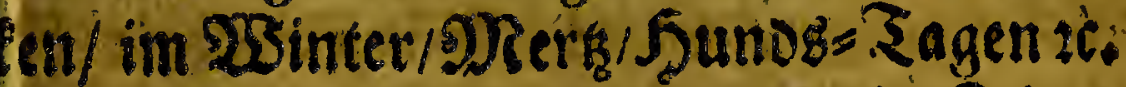
I wurgieren/ allein idb babe zu Diefen Beiten ichtmebr Ssefabr oabev gefunoen/als zu eier andern: Sft iemano farcl bey Rráf: en/ uno oie STotb erforoert es/ toatumb fol hanibn/ unangereben der Zeit/ nido purleten; ievod foil bie Sotb allemnbl nidse b grof als fie gemadit niro/ fo Darff man es en fo offte nidot purgieren. Der einige Sicupel if obbancen 1 dofs in oen 5umbse Sagen ber seib oon groffer 5 itge febr abges. hattet/uno viel fluchrig Sals verlobren bat: billein vieferiff oorjufommen/ment oetpas iente fid in einen fublen Logement aufa. bâlt/ uno cine limbe Purgation aug Aloë, Mechoacanna, \&c. lieber zowevmabl mi STuken/ als eine facte einmabl trit Sobas ben gebraudt. In Dem Winter ift cine war me Stube zu erweblen/ weil die Ralte die Saffte forwer uns träge madis toaf oie Purgation eber ote Perben angreiffy uno su sungen neturfadoet : Sf alfo fiepliob im 


\section{0}

Friblingel weldbe Zeit weber fu warm on of zu falt / am fequemften zupurgieren.

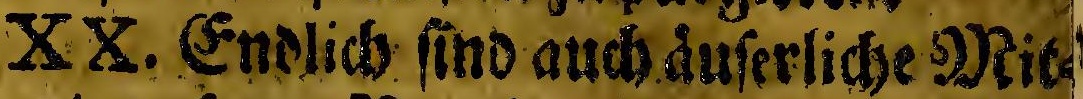
tel/ oie auf bem SSaudi geleget/ purgieren. Bum Exempel/ Daß Unguentum de Ar. thanita, soeldbes denten findoein auf oem Rabel geleget/ Stubl: Ssing madhet: In alten Seuten aber bat es fo viel Rrafft nidyth

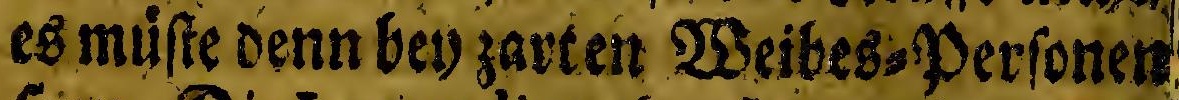
(ciun. Die Ingredientien fo vazu foinment muifen ourch sie engen sidfergen Der 5 auth in die umblauffende Säffele/tmo von oar ins SSlut oringen/ tweldes won diéfen Tbeilgen ouf gleiche 2Seife difponiret swird/wie oben won sener pourgantrien gefagt roorben.

XXI. Zubem Purgieven geborten audo

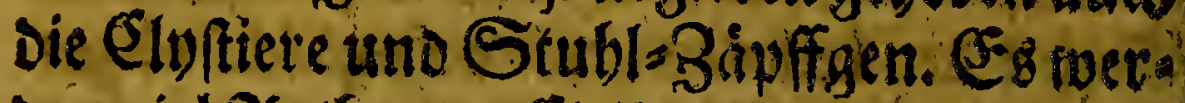
Den viel 2 2retben ber Elyfitiere von denen $A$ althoribus befdorieben/wir rollen allein von Denen purgierenden fagen: Sie weerbern ges. meintiglich von gerwiffen Deroetis, 25ier to. gemadit/ mozupurgierende Dinge unto zate sweilen Olitadser geétban wersen. 25 enn man aber betendeter rivas oie Intention iff / caves

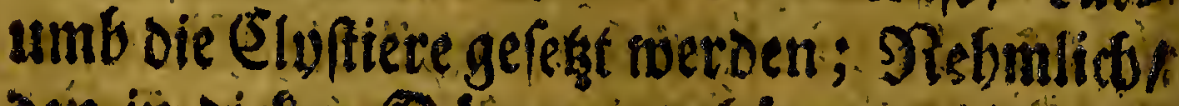

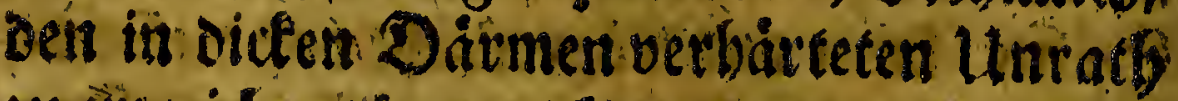

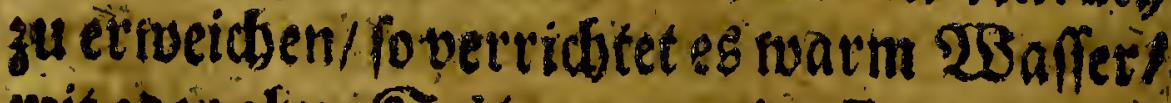
wit oder obne Sals: Dent sine Purgatioa 


\section{$\sec (31)=$}

on unfenbengebradbe/ gelonget nid in oie

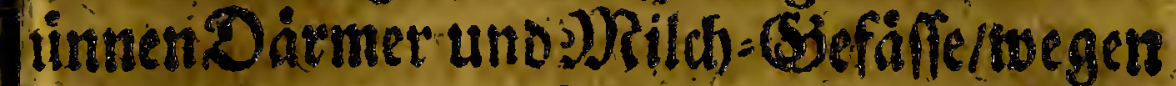
er zrvifhen dem oicten uno ounnen Där. netn befindichen Valvol h; uno alfo fomme e aud hichting SBlut / - twierwobl man mey.

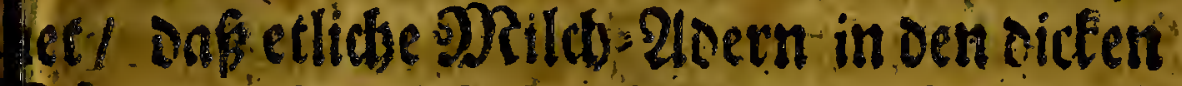
Dámern feon follen / fo foerden ibver ood penig feut/ indem die viclen Dármer feinenthylum, fondern nur die feces cation em. fangen. Sdjeinet allo oas 2abreben des Eloftierfergeng afleingu feon/ sem verbirtes

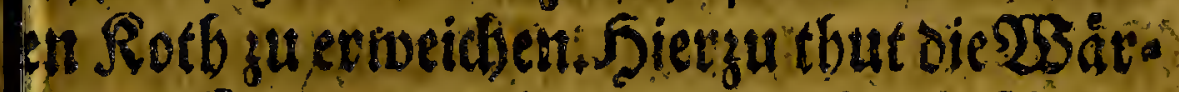
pe Deg Clyftien's viel/ indernes allezeit SSlut:

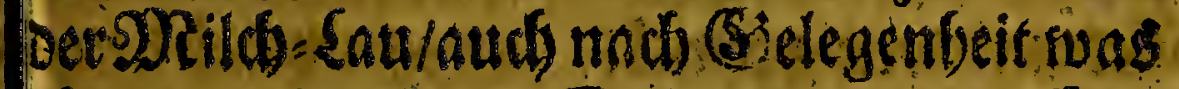
bärmer gefegt noviro. So man zu denen (Sli): ieren/nodb einige fotharfe Dinge/als:Sols/ purgantia, Urin 20 . tbut/madientie in oent parmern ein Rusigln/ uno folgharlidi eine drdete s3eweging / moourd oer Linflat locb imebr fortgerrieben noiro.

XX11. Die: Sarte Seibigfeit fommt handimabloon 3) Rangel oer (Sialle/ ruelde. icht allein oureb ibre Suärffe die Därmet 4 mebee: Qbfdyeisung oer Saffec irritiret! abonser Rotb fohliwffrig mirs; lonvern fie

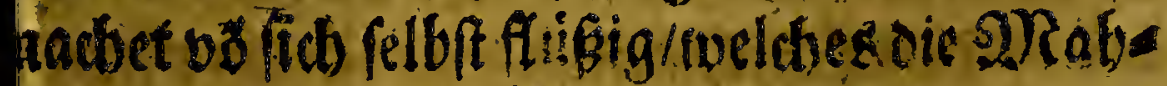
se coiffen/menn fie(S3alle unter dieJnrbe mi ben. 2Senn nun dic Giafle mangelty to 


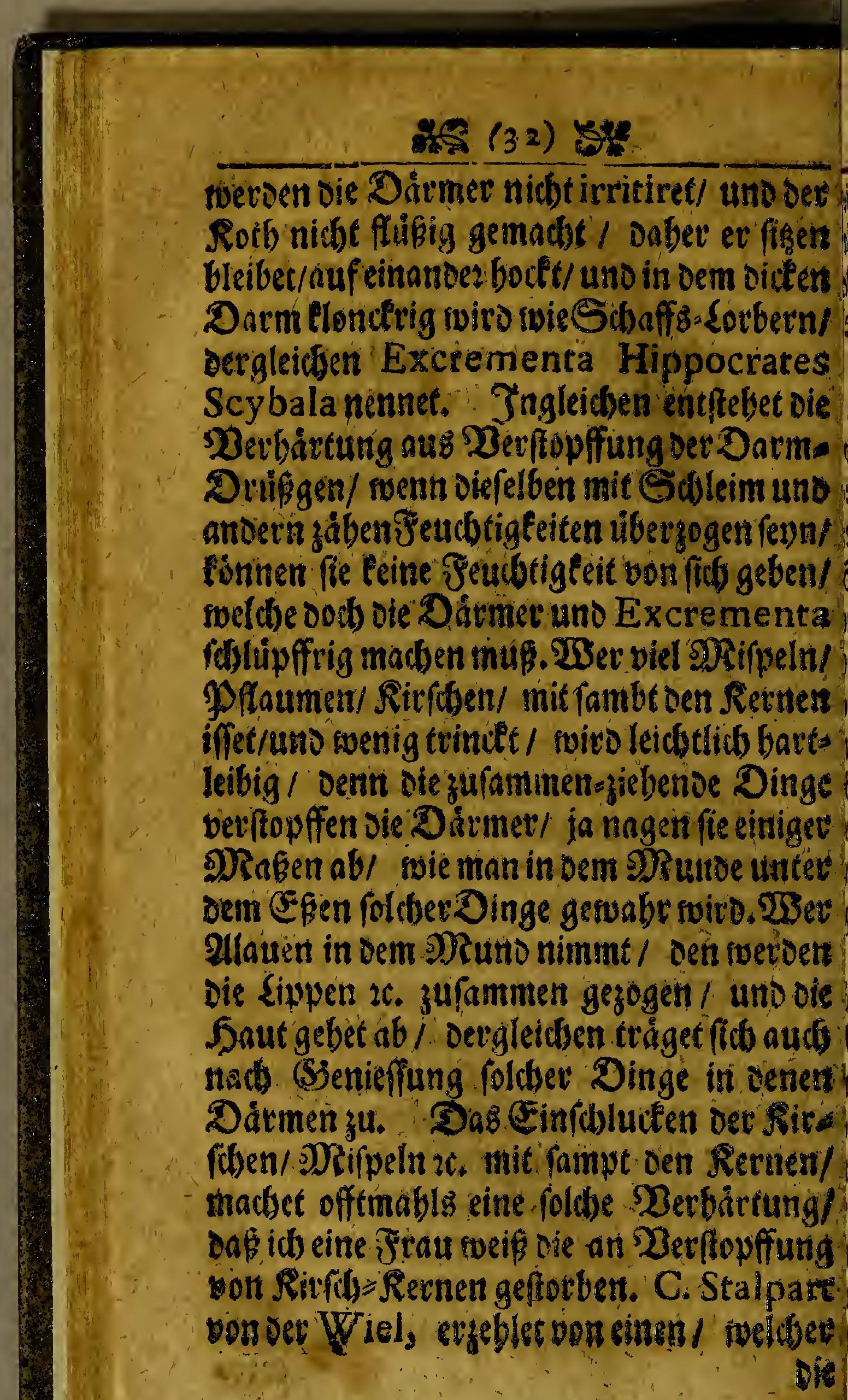




\section{$25(34) 35$}

Set als bartgeloctiten Jonig uno Salist

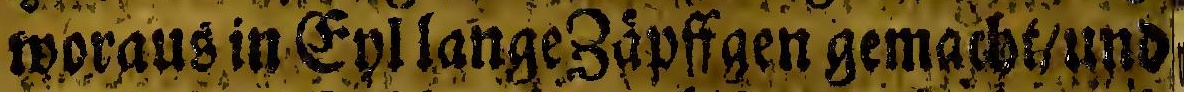

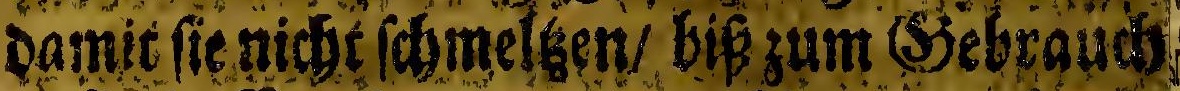
in Del affervirec merden. 2lnoere nebment sflein ein ounnes Sichtgen mit. Del beldimics) ref// poer fotmeiden dergleidfen nus Seiffen 22. rolidse Dinge pon unten in dem Darm

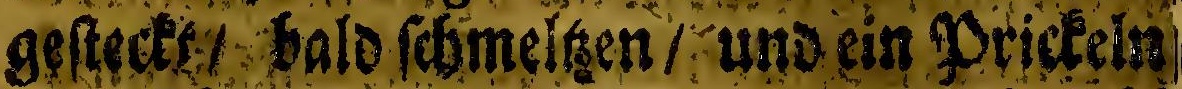
oper Rúkeln madjen / foornado mandsmobs Der Stublgang erfolget.

XXV. Tot mus nocbetruas gepentert won cenet übeln Compofitionibus, beren viel fo wol vanoenen Doctoribus veridtries ben/ols bon denen 2/potbectern bereitet nete Den. 5 sic noffen erftich oem R habarber-

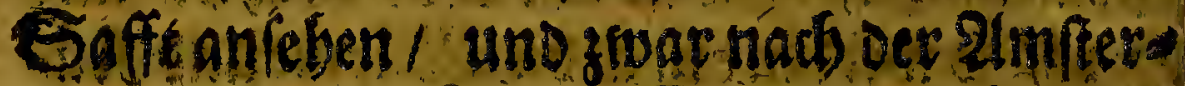
Dammiffen Officin. Diefer Syrab icteis. net alle Qvalitóten as baben/ inoem ex bey

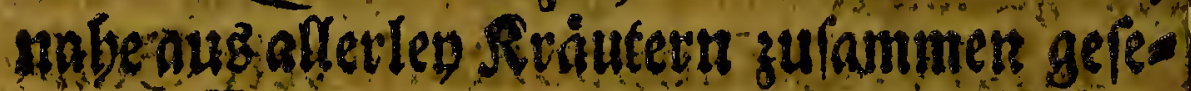

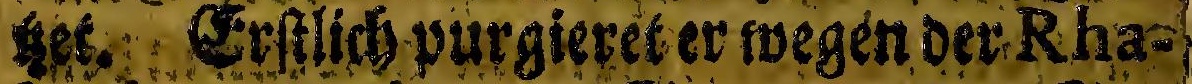
barber treibet ocm STrié ourd die Ju० Den- Rirdden fúblet megen der Lactuca Jubret die Siall ab ourd dem Erovaud

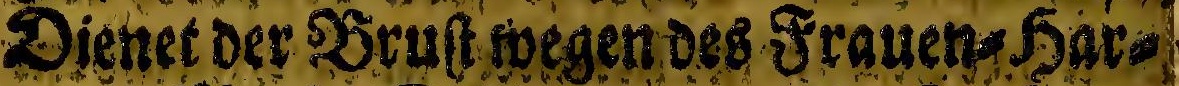

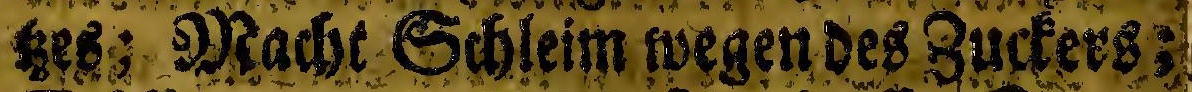
Gudfinet vermittelf oer Sinoleufft Spats

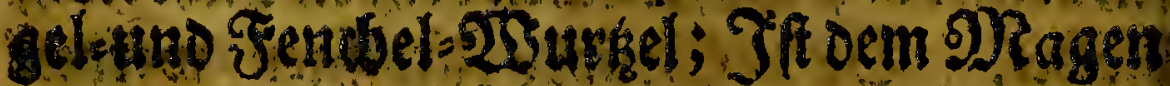

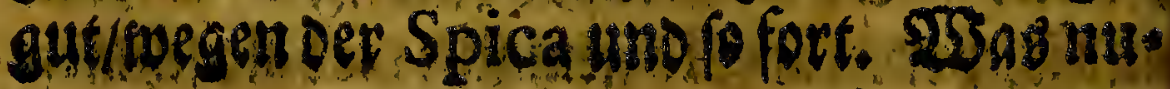

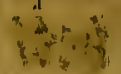




\section{is $(35)$ s*}

In fo viel Sachen in einer Compofition; an mat nicbet etruar cinfadyers and De: habarber madren; Sifioie Spica bier ein orrectorium? $2 s$ as madjot diefes thethe Ding Davinnen unter fo vielen geringen patben? (Siebi unan nidit vielmabl biefen Daffic Denen Rinoernvor SSrechen uno ver. bleimten 2) Ragen / twag madf toenn fo viel

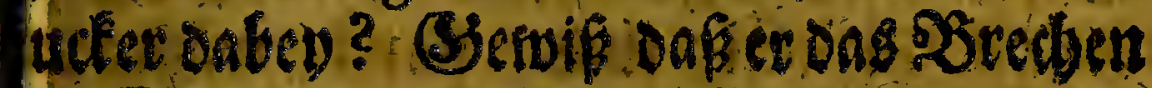
1o. Solleim sermebren fort.

XXVI. 25 artumb thut man tilds betm offen Agaricum unter Dem Syrupum lacnicu, tob Gacimantótbigoie Trahifcos zu nelomen / Daruncer allbeveic $3 \mathrm{j}$ :

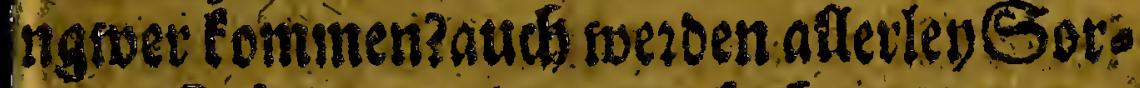

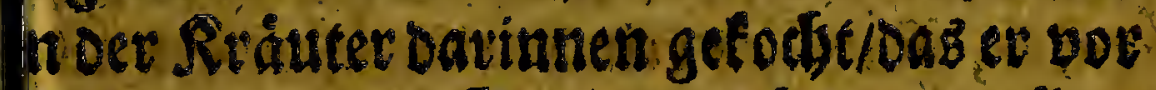
Rerbain gut if; Tngleidhen fonmen allers no Purgantia Darein/ Darumb purgieres efer Syrup alletley humores, twie bie. ingelebriteri ooer zum menigfen viel obne ation zufagen pflegen. Der Syrupus ofarumcum Senna if nas einfactiev 10 mag nodb fo bin gelfen/itwentm manja affe Hrupeigebraudien mufs. Das Benedifum Laxativum ftopffet rwegen set abfe 1D vurgietet aud. 2quein es if jubeflas H/eaf in fo cine grofe Compofition nut te drachma Sal gemme fofft/ on man

$$
\mathrm{S}_{2} \ldots \text { in }
$$




\section{กิ $(36)$ है:}

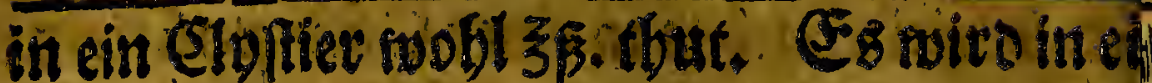
tien foldsen Compofition fo viel belferzaly in Fupfigen in einen SBraus Reffel. Di Confeetio Hamech, bat aud oie Rraffic allerlev humores zuptrgieren. SSerwip. Derseib mus tooblangegriffen sweroen/weth et bon aflen Drien fein $23 a f f e r / C 3 a f l e$ Soleim 2 . bergeben foll. STun purgiers - Diefe Confectio linde/ ftarch mápig un fopfft auch sugleids / Dentn alle diefe Qvali

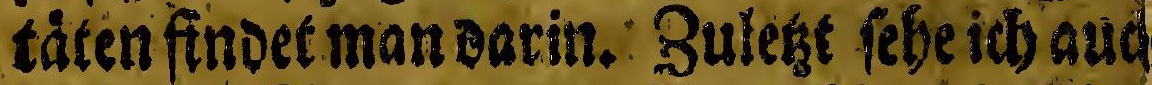

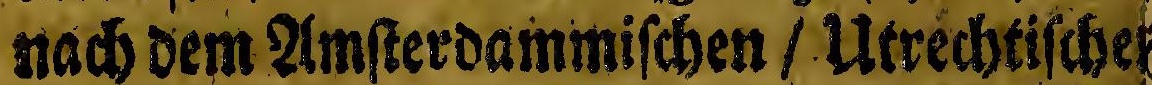
und 2 intwerpiffen Difpenfatorio oa8 Se men Spica nardi indic ; $216 e r$ in $V$ a lerio Cordo, oer Sondiffen Pharmaco pca, finde itb fein Semen fondern allei Spica nárdi Indica; 2llo oak Die enfte Drev einander swie die Schaffe nadigefolge .25ab fie num vor eitmen Saamen fubftit uy sen/ modste ids toobl soiffen/: weil tein foldye Saamen zu finden/ aud leiner in bern $C_{\text {a }}$ thalogo Simplium angezeiơnet. 200 madjet Das Sifje 5ols in Dem Diacartha mo uno Diacatholico? 25atumb nto

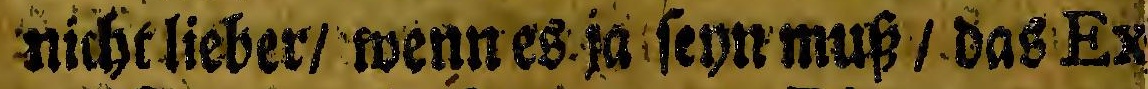
tractum, gleidonie zu dén Diapruno ga nommen. Das Diaphanicum if ein Compofition aus Dattela uns grat Ui 
$-15(38) 3$

ten/Tenefmus, Rrampff/ Buthugen a

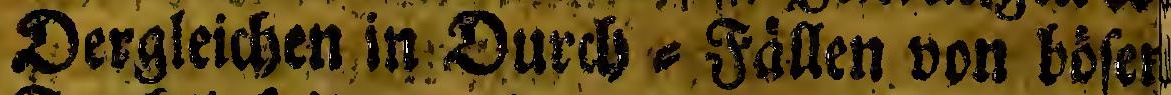
Ferditigleiten entfanden/ ober in ber Cho lera zugegenfino. Diefes alles ribertved

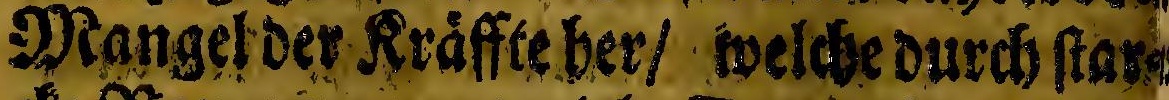
de SBervegung uno biele Excreriones get

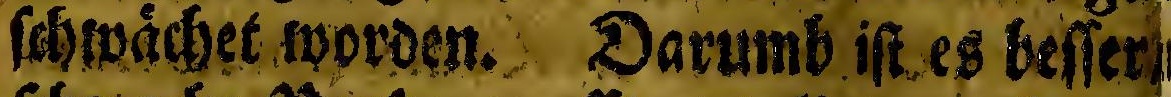
forbacbe perfonen off atno linse / als ats einnab! zu viel evacuiren. 538 elde bied robarfe Seuditigleiten ben fitb baben/betoms menalle Sthdrffe auf einmablmit Shaufet in dic Daime: Darumb fiebectnan es niche gerne/Daf Die/ weldie Den Sdjarbodt/3diba rung 2e. babent Durd $=$ Fafl befommen Denn ibre Rráffic berfobsomben nicbt alleim fondern die Dármen wevoen toot gebifen. worauf oer 5 Bratio / uno alfo der gefdrwinted Foor folget. Ca ift befanor da B Die fubrite Materie oie Fleilgen Der Purgation all

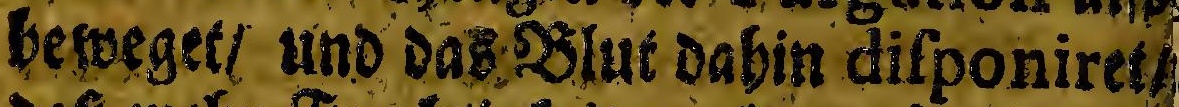
Daf mefo Feudtigfeiten:als ordinar ged

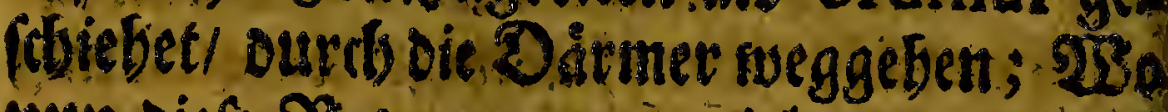
tutn viefe SBetwegung zu viel uno zu fard ift roivo die $T$ extur uno Dereinigung Des SBlutes mit andern Sáffen oufgelsfet.

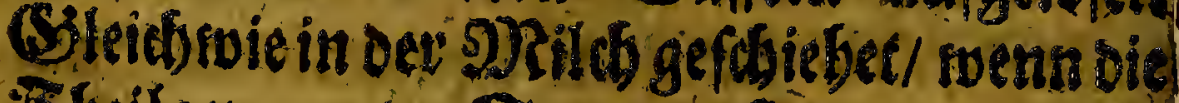

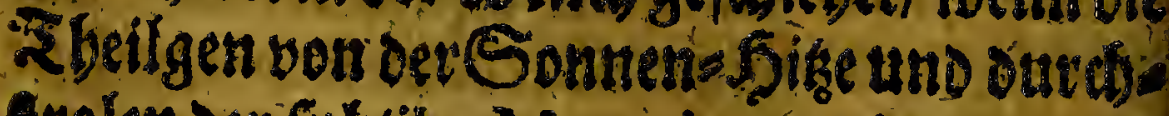
fralen dex fubtilenMaterie voneinandor ge 


\section{$25(40)$ है:}

Jiezu fommt Soblucten/ meldies cin bifes Beichen if/ alloiefueil oag gankse (Sebirn und Genen volfer folcher fobarffen Sbeilgen fió befinden.

XXIX. In vergleidien SEegetienbitel giebe man ftárckende uno anbaltende DRite tel; als 3immet/SReglein/SDulcaten-SDlu-s men/ DR ufcaten=s Süfe und oergleidhen/ oes nen oas Opium, als das vortreflichfte PQit? tel in diefen Fall / bengefeset wiro; Die evel fen ourbovingen noggen ibres fubtilen 5 beilo get alfobalo oas Sglut/ uno oie feetedtigleit

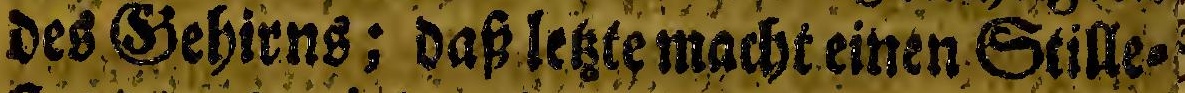
ftand/uno minoere/ ober zum ivenigften ars Dere uno der vorigen contrare ssenegung. Denit ons ourcb oas Opium eime s5etver guing gefojiebet/ can niemano miederfpre: chen/ fonft toarces unmoglicb/onf foldes in 10 furger Beit uno wenig Drinuten feine 2Surding thtm fonte. Davon foir bey Det 5anolung von Sdolaff-S Ditteln agen rool Jen. Siebe aud oavon die Sdbriffen Sertn C. Bonteke, iscldier biet uno dar genung von dem Opio gemeloet.

XXX, 24s Detren S5ted a DRitfelin

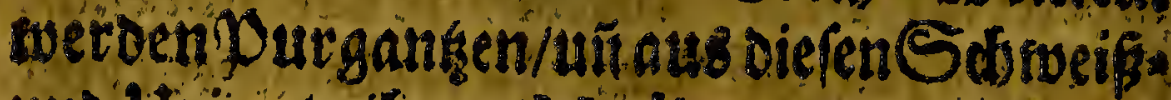
und Utrin treibenoeMedicamenta. 25enn

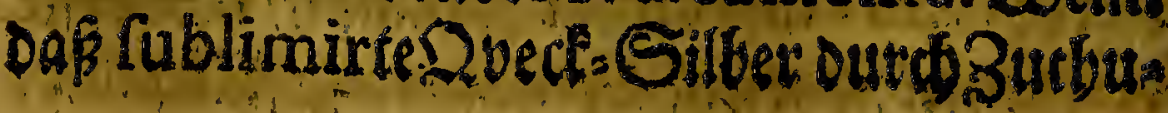




\section{so $(42)$ st}

cet oem calciniren gebraht if/oder aud tut

- ófen Caput mortuim: Solcher ibet bliebene Sdgrefel/ niro gedfen Theila wait Dem darauf gegoffenen Bitriols (SEeift odev? Del toot gebiffen/ oafer hicht thebe fofara muircien fanalsguoor/ uno ods grofte Tben aér Maffa Alcali von (Slafe uno Acjdum Deg Vitriol-Delg if/nelobe Den J) Ragen unt Dácmet neniget angreiffen uno oabcrmo purgierett.

Das III. Sapatiter.

2tuf twas 2Beif bie 2Brect)-2) Ritter in unfern SJagen witrden te. uno wie Dus Breden auf unter foredido oseife mebr becurfact toito.

I.

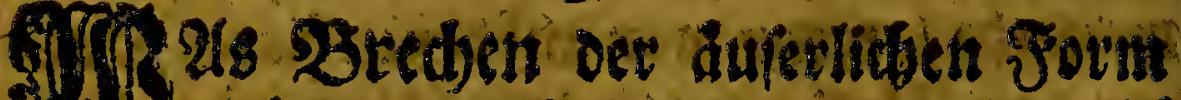
*es nach /ey/ reip ein iede/ toic uno auf

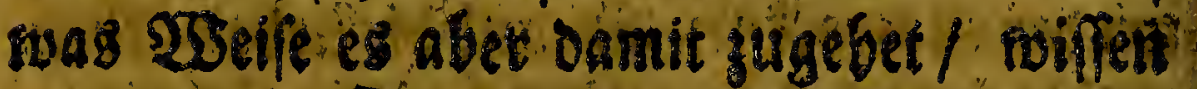
niche alle. Dif untendaiebene mufculos?

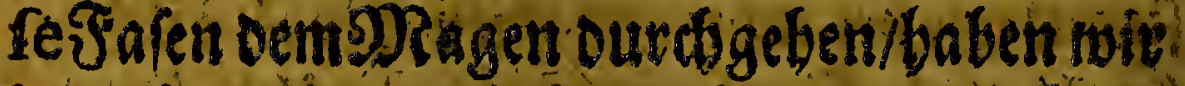
in unferer. Anato thie angefolefen uno biets oben etlidset DSafen erzeble? 'Wentuth

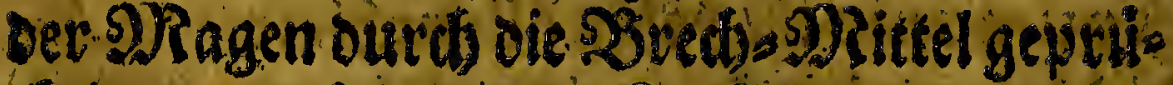
deltuito / folget tine Budung over Con-

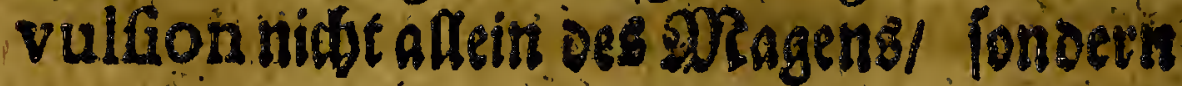




\section{$x$ (}

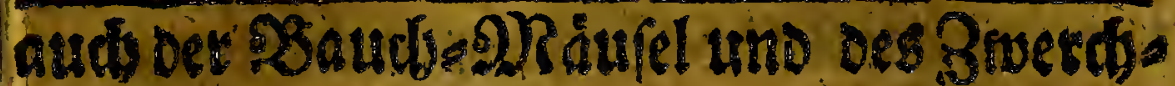
Fel's dent es baingt bermittelf der Membratien ooer 5 äutgen eines an dem atoern. Znoem nun eine Con vulfion etfolget/ges fobieber ein fardier Einftup oer (s) eiffer oder Sirne Saffes in die Prerben des Mragens uns anoerer Fbeile; Siedurds rwerben bie Fibra mufculofa ober fleifdiditen Ja

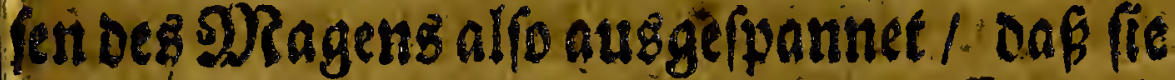
alles suas ffe in fich entbalten; mit Werwalt cugftoffen uno gleidifant augfprisen. Dent

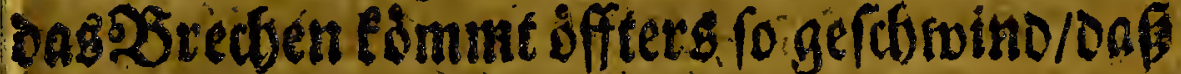
(s zu SDaule uno siafe beraus fobieft/ ja es

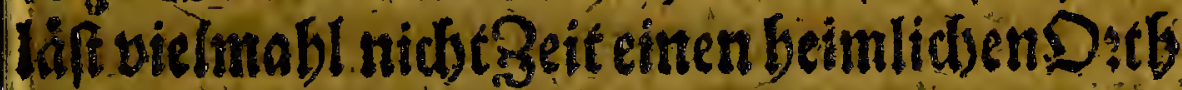

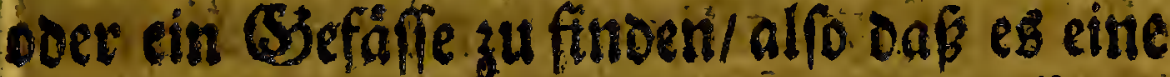
gefdrintioe Convulfion uno alusfofiung Deffertift was der . Dagen bey fids bat. Denn

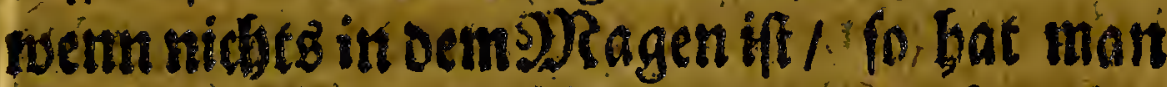
tobl antrieb zum S3redsen/ es gebet aber nichts for l morauf oie Seiten oez DRagens

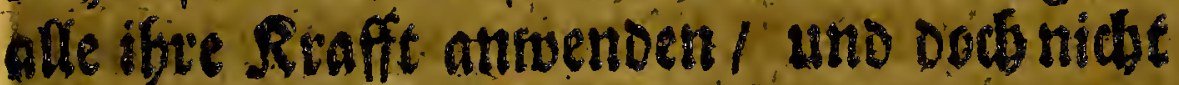
cinarser texubren fonnen / fo er folgec oem eine ubermápige Sserwegung/ wornad mandiniabl negen groffer Preffung oevDJlut (sefäffe/ ein wernig 23 affer beron gezisungert roirs.

II. Ee fragt fid ob nidbe atdid bie Däts. mer afficiret iveroen/ inoen man um off 
tecn viel (Sall uns Sauery sweldeg aus oe:

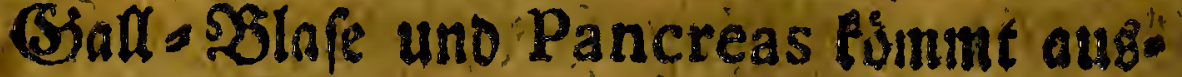
bricbt? Da mú man swiffen Daz oer Dragen nidit allein ourd feine eigene Fibras, fonbern aud ourd bie S2audb. DRaufel ged yreft roiro/ bon Denen aud oie andern Sina gerweioe uno alfo ote S3alls 3 lafe uno Pancreas gebructe nerden/Die fid nabe bet dem DRagen entledigen / To ons.leidftids etroas Sälle uns Sauer binauff in Dem Nagen

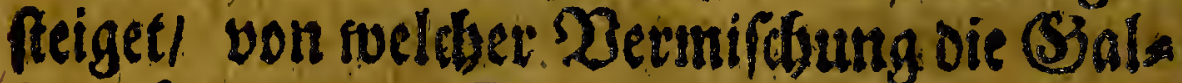
le grune friro; Daber oit Exute fagen id Gabe lauter gruine (Sinfle aus getrodien. Darmenbero mus man in oemsisteden niós allein auff Dem Xagen fondern audi auf oie

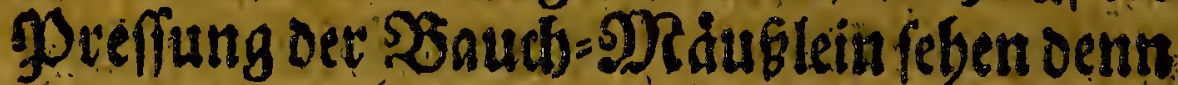
eines fan obuc bas ander nichtfeom/ swer fid bridst/giebr etroas aub/ uno weil feine ses oigfeit ooer Vacuum in oem Shagen (eitt En fo preffet dic aus gegebene. DCaterie die Suffe gegen oem south / weldeb leibt zu beneifen roennich mido wolte in vis Phy fica einlaffen; Uber viefes fersen etlinge nodb vic Sarmo in tie Seite umb oeftomebo nsuef fung zubaben.

III. Sbeils seutefino lebr leidhtzum bre den zu bringen/andere ongegen fobnew oef fenllefade ich zu fevn bermeinel Daf Det 


\section{sis $(45)$ st:}

DRundoes DRagens in einen metor gefchlofo reen als in bem anderm/ boer alfo berchaffent

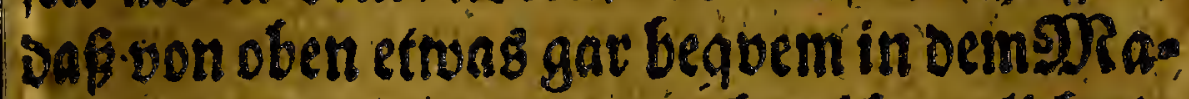
gen tommen/ son unter aber fosmerlich efe twde oben beraus geben fant. Bum anders

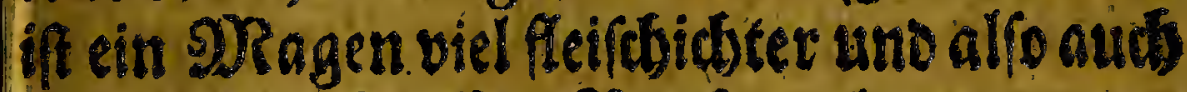

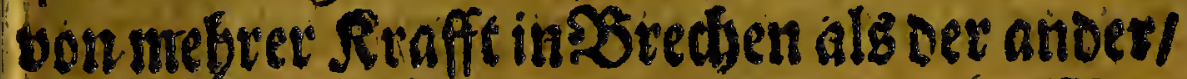

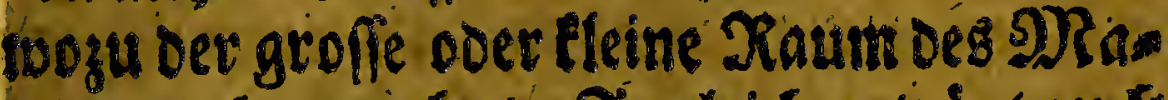

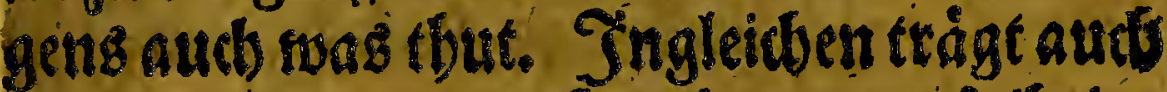

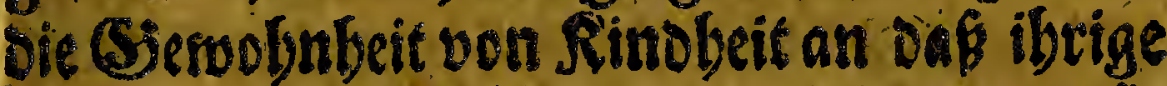
bey/ Denn viel Rinber brectern fich fo lange fie faugen/ baf ibre DNagen befien gendobut werven bifisine alter/ teidstlids/ uno wenn fie twothen von fid fellbe zu brectien. 2alfo fage

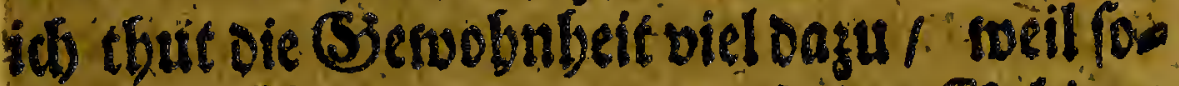
Denn oie fibre carnex ourds Dem (Stebint Saffet leiditer aufgefpannet rwerden/eben als

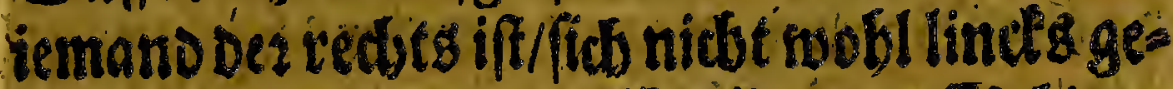

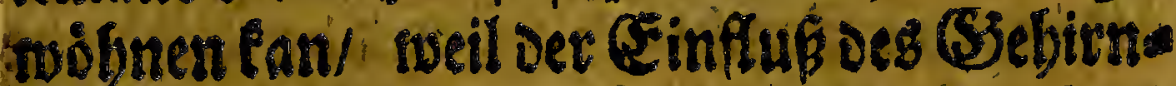
Saffes die Xisbrgen der SRerven bez redten alrms alfo errweitert bation af gedadter Saft ourd Diefen viel burtiger lauft als ourdo berm tinident.

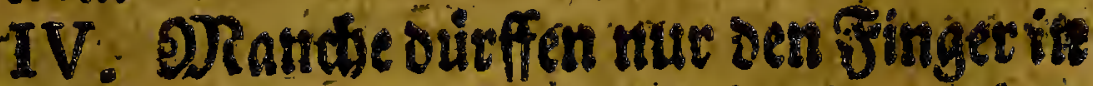

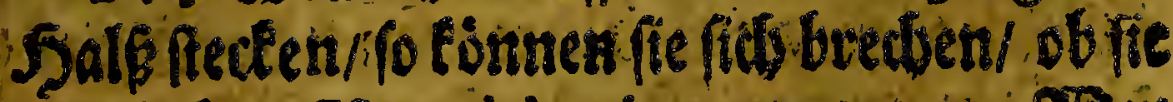
gleid fein Vomitiv eingenburnert; 2Seit ind der Anatomie nidis erfabren/ Dem forit Diefes fremiboe sor/ samaber bie Structur 


\section{is $(46)$ be}

Det Tbeile befandet Bem if afles flar. Dare umb ift ju wiffen onßs Dag adget Paar SRers ven/ Par vagum gemand// feine. Bweige Dem Saluno uno 2Ragén mitefbelet/went nun Der Schluno mit Dem Finger geflişele wird foerfolget eine Convulfion, meldie wegen Connexion der Nerve Den Nsagen mit betrifft / nicht anders als nie mit einen Striel viel Solocken zugleids finnengezox gen fwerben; Doer fwic an einer gefpanneten. Säiten/swenn fie autb eines) Rélle lang waire/

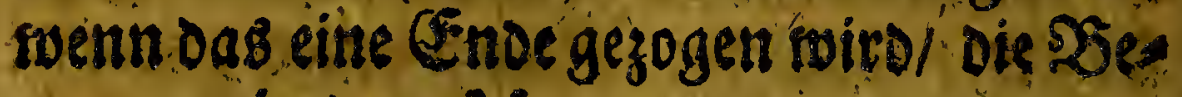
suegung in oem Moment an Den andern Enoe gefpuibuet mird/alfo/wenn eine SRerve bey ibren Principio geprielelt twito/ em pfinden es afle die unterften Tbeile: Wie manfiebet in gefäbrlichen 5aupt- 53 unden! Da Das SBredben nirgenos anders berfonmt! alsoas die Seudbtigfeiten in sem (SE)birms

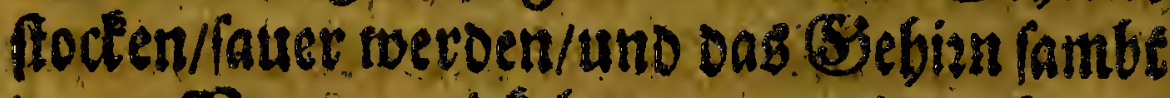
benen SRerben prickeln/on Denn die nads Dens SRagen gebenoe SRerven convelliref ner. Den/woraus ein sstredien erfolget.2llfo gebet es audb ber in Rierento2356/Colica/Darm:

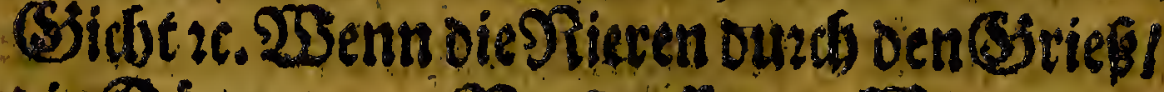

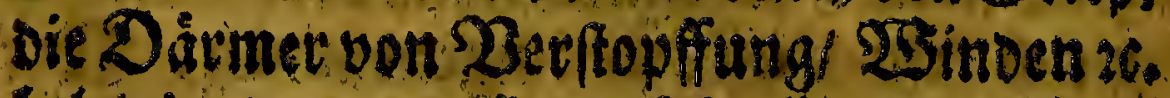

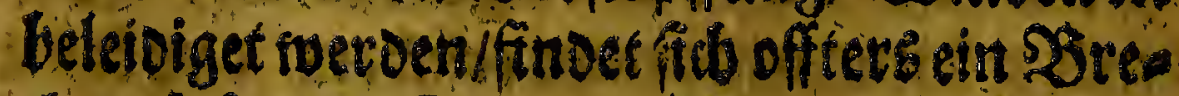

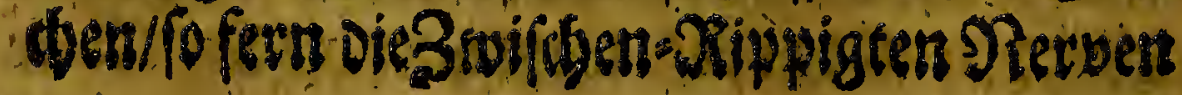




\section{6 $(48)$}

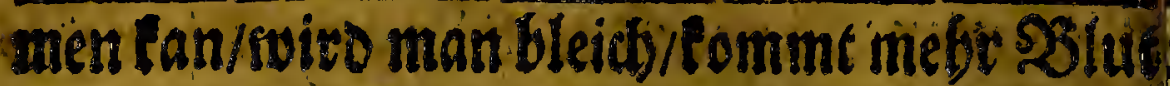
in oas 2ngeficit/ als ourdb die zugerchloffenen 2loern wieder fan zurticle geben / wito

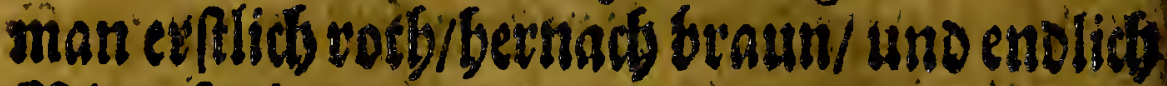
DSlen farbig.

VI: Das Soebert der Sipwen ift theils yout Der (Siemein/d)affi Dex Membranen obeth Sáutgen nomit oet Dragen uno oie sippent belleider fino/theilg von Sparten der Senen) an Denen pie SBerwegung Des einet Cinoes? fich bis an oas ander erfitrectet/ als manfiebet an Denen Sniten uno ausgeipanneten Stricten/ beren cines Enve nidor fo ged forwino gerubret soiro / es besweget fic oab atber aud ; uber diefer fino oie Nevbent uno Membranen soll Safft, swenn num

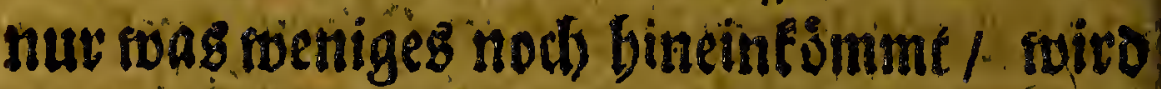

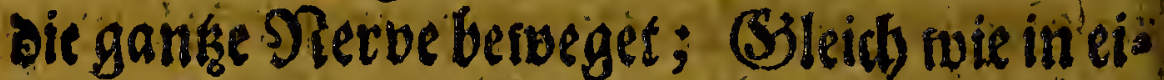
Her mit ssaffer gefulten Sprize gefdrictet.

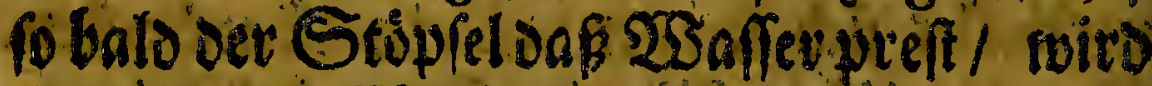
porvobl ons 23 affer an bem anoern Endel.

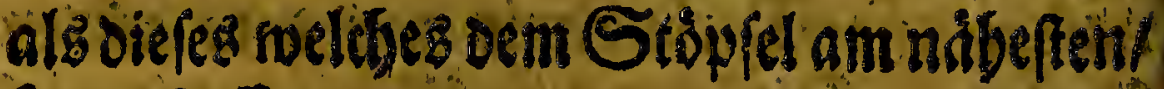
forfgeftoffer.

VII. 2us eben biefen Fundathent,

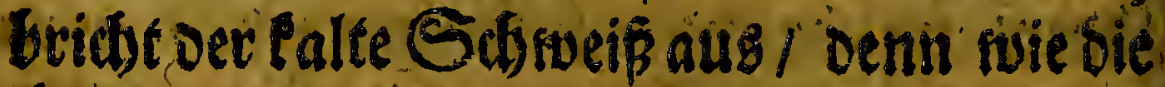
Sippen berwe get werden 1 . alfo swiro autd dié ganse Saut benseget/ denn soell mann is

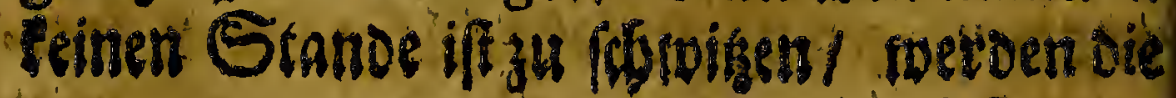

Jึeนt) 
$\frac{\cos (50) \text { ses }}{54}$

Stiugen im NRund batten / onourdo oid Rerven/Membranen, Dríen id mege berweget uno geprefétwarden / oaffie mejul Jeuchtigfeiten alo fonftent in Dem DN uno ga? ben. Inter dem esen troutener Syeifen/ befonme man mebr feuchtigfeit in bem DRuno/ als soo man nidftiffet/ oenn fo oes

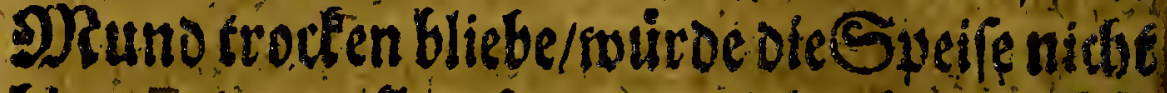

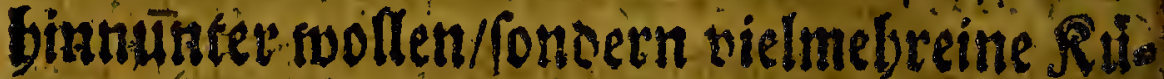
6elung mactien uno mic edel aubgebrodsen swerden; Eben mie idf von fecten ors Fins gers in oie Rele gelage babel ober fwent man ein Saar iu den 5 als Reclis madjet es sine folche Rúselungioas man fith oes sorechens nicbe entbalten fan/ oarumb iviirge man/fid oud ruent ein Saar in ber Speife iff. X. Neancbe miffer / wenn fie gesuime

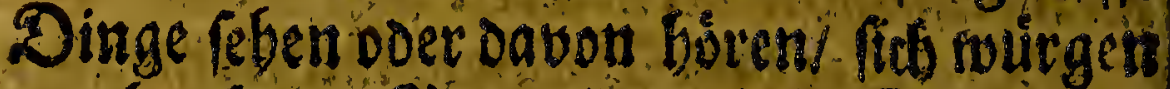
uno bredgen; Ta es fino etlictue Speifen die

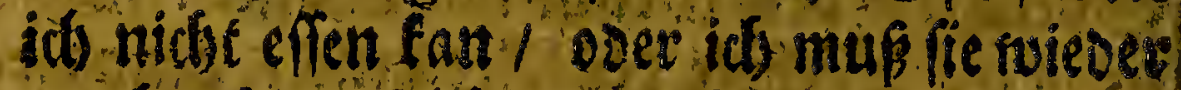
soegbreden / Foldhe entfebet afrein bon dem Durdfgatge oer fubtiten Materie, sollowe mit ser unfergen nidhetiferein fomme/ oar

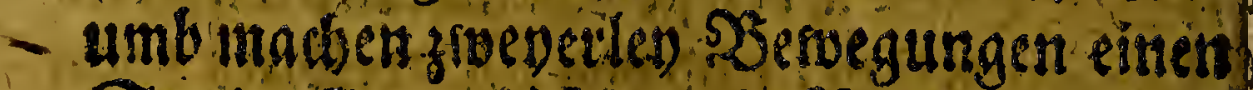
Streit/ Convulfion uno Szredien: SSent aber diefe Spsile (ebr jung fino/begegnet mit

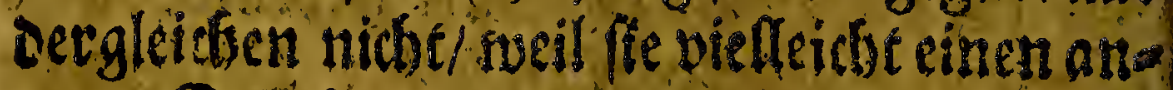
Defn Durdigang Der fubtilen Materie bah 


\section{*}

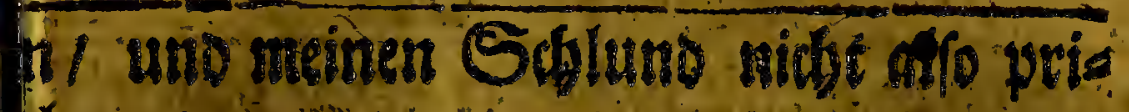
In.

X7. STev fdruangetn 28 eibeen if in 15ange ein continuirlicbeg \$5tedhen zu fbren/ Denn Der beygebrachte gRannlidje anmen / if ourdsoringlids/ uno madiet in n 28 lute cine gants andere ssenegung fo 3 viel Salleim in ocm DR agen folft unto

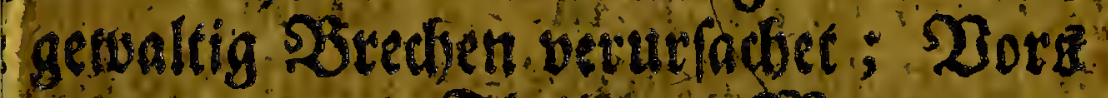
pere sverden die Jbeile oer Mutter uno

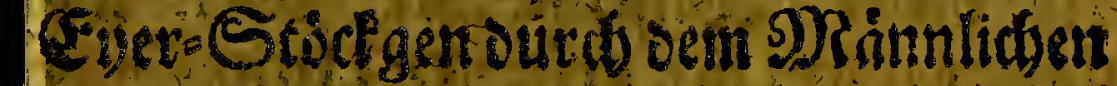
hamen gletdfoim inflam miret/ uno weil - DPagen ourer Säurgen uno Bivilden. pige Senen mit diefen Fbeilen Semein: affe bat/ Eanleicur cine Co nvulfion unt Eedhen verurfacher hotben; Drittens

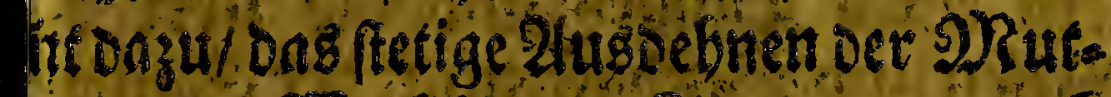

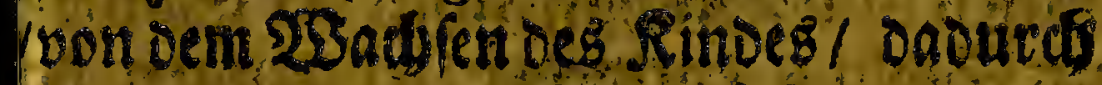

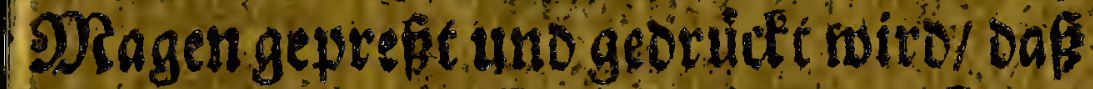
gen ber getringften (Selegenbeit/ Dic Siau Sstredien antomint.

XII. Die nidbt viel zut Sec gefabtem bé nie man zu redent pfegt/Sieferande

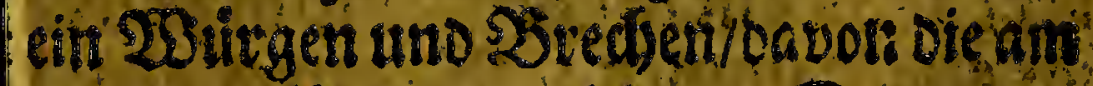
en zertgen formen/ twel dise zur See gerve. Ino einen Dertano van der Sadbe bas

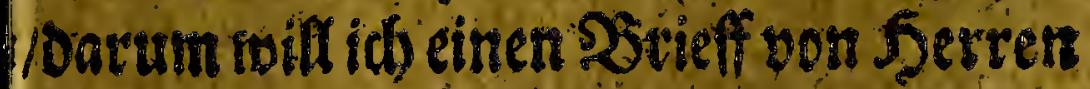
onytio von der Sterre aus Curacáa 


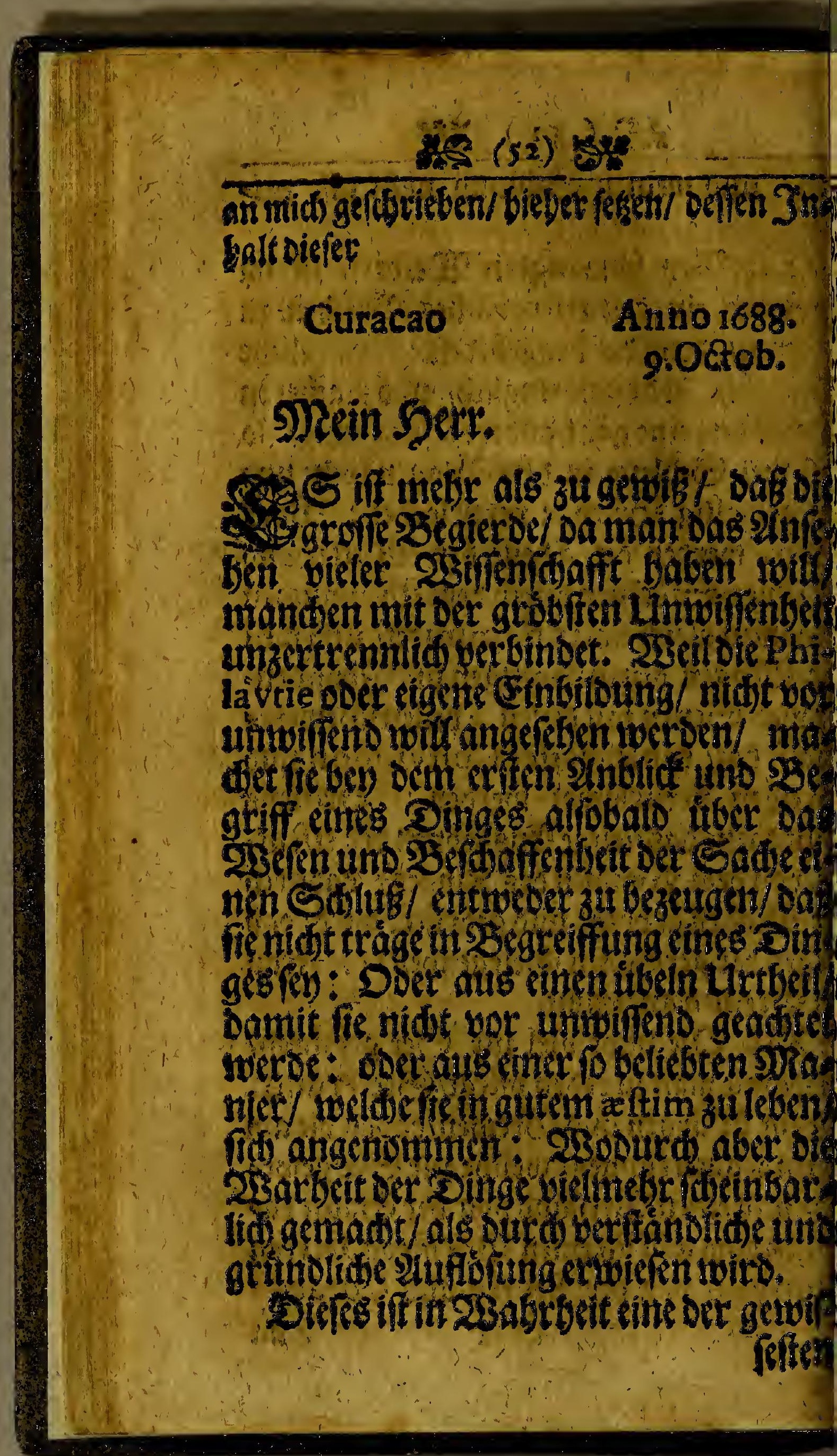


fen Urfatien/welde die Lungetiben in cter Unmifenlseit etbalt/ inoen fie vise

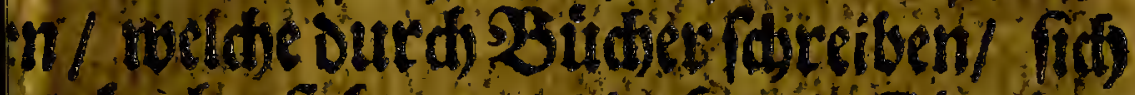
be boderfabren ausgeben/ Blaubat comefien/ Daburds fie at cinen unvors. ominenen Judicio gelangen/uno mit es: In Prejudicis angefitlet weroen/uno Slabartid Die Urfoden vieler Dingel

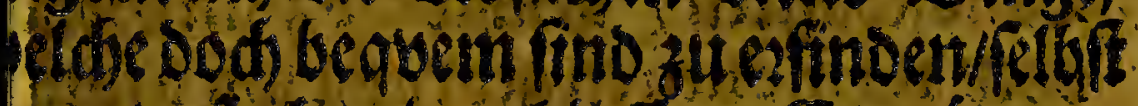
finterfiden/nablaffen. Go ware zat Gnofden / oas nur oife/ uno nidit ofr offe S beil oet Scribenten felbof folden dfler an fí batten. Dartumb if es Gid / Daf man dic Prejudicia verláft dan nidts Ginder bie Limbftande or orfâle alle zugleito felbf genau un

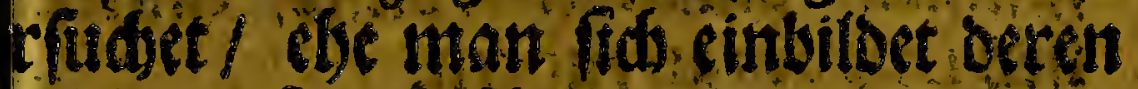
indig 34 mon: snders mird man zu at/ oser gar nidft erfabren/ twie weit moer 23 abrbeit abgewidien woroch/ ud folbftin foldsen Dingen/ oir voln opa. inlinerfabenffen gefaffer werden.

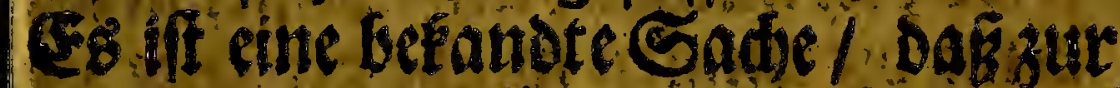
be in denen Sdjufen/ie mebr foldto auf m 23 affer bin ino wieder gevorffen

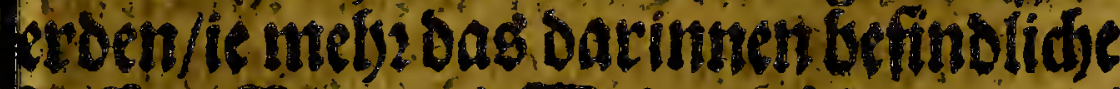
Baffec/ Sier und OBein turbiret wird/ rauf cine merotlige 23eranderung

D. 3 Dira 
Derfelben zu erwarten. Mlan bat gerim

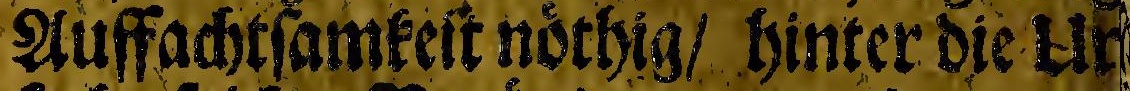

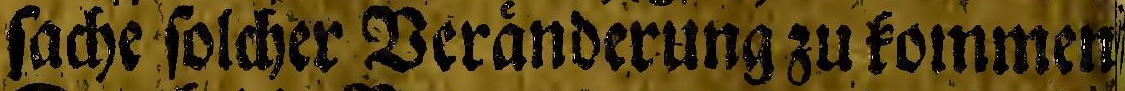
Durd die serbegung twird Dasjenigh

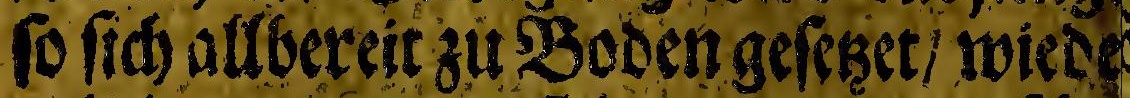
mit Dem ganken Liqvore bermifase wooturds oerfilbe (fondertid in beife

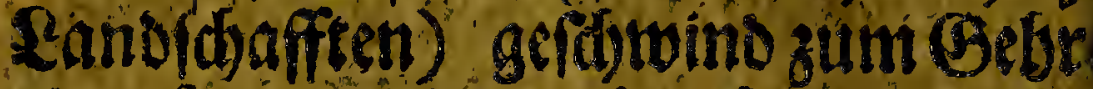
Doer fermentiren gebraditivír unt e

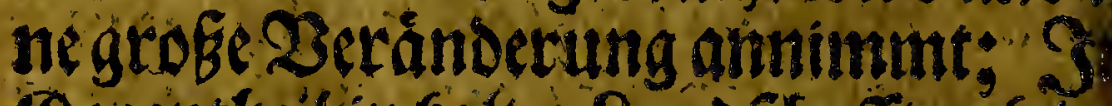
- Begentberl in falten \&and/thofften feim - Der festr wenige und langfame Berä Derung teidet. Es if Denen Datu

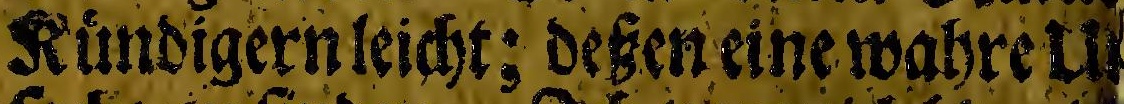
fadie zu finden. $S_{d j}$ vor mid bin ni gefintinet/antangende diefes/ sineneig ven Difcurs zu futfren.

EE begegnet-aut betten sRenfit) uns sbieren wegen getwaltiget Setw gung oer Sdjiffe/ eimige 2 eränderut uno ungentads. Die 2sakrebeit ber? wiro von niemand in 3weiffel gezoge? Denn die meiffen wadthe ber Sec ung wobbetectempfinden eime groffe Betáno

tung anithren Qeibe/ wetlde is nen ats

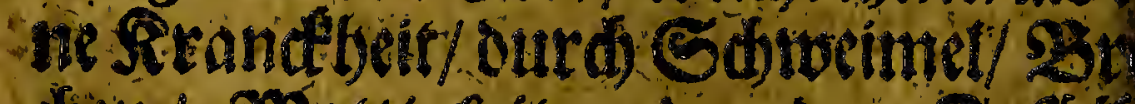
d)en/ SRattigfeit uns andere Zufíl ungelegenfeit genung berur 


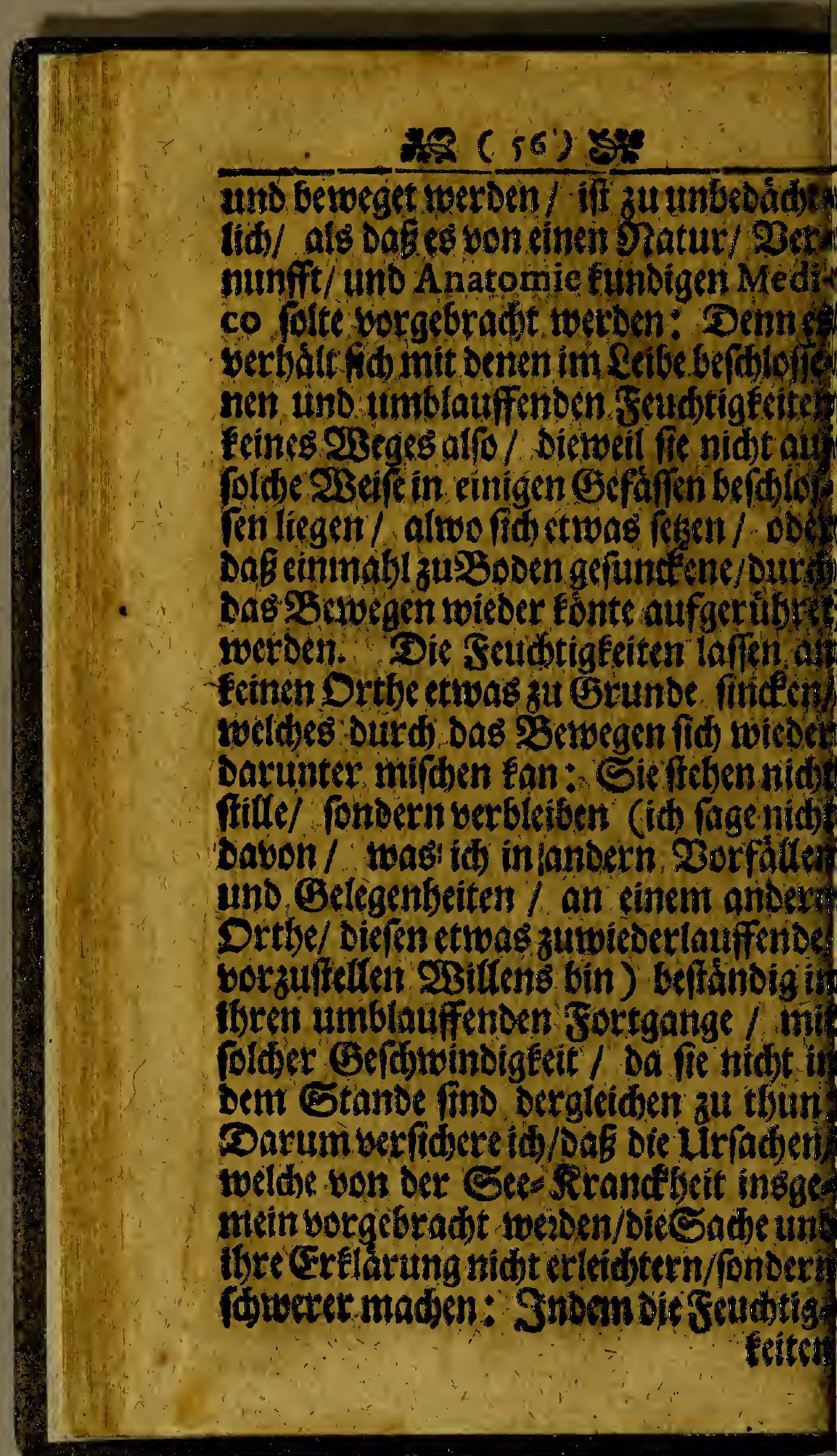




\section{$-6(57)$ st:}

Eeiten in dem Ecibe/cine gank unterfwics

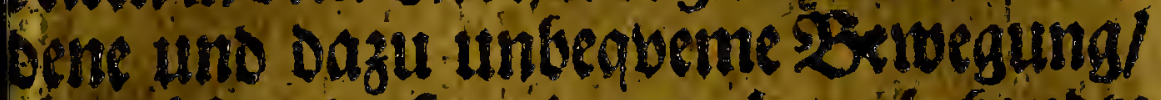

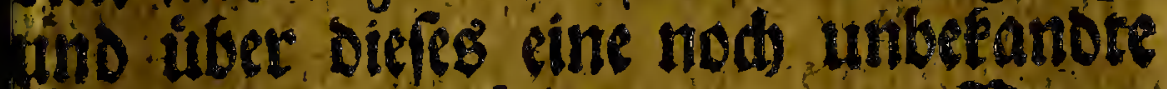
Uerfach zur Zeranderung uno servegung/ ingleiduen die Gefáfie Eeine 23 eguentigereit/sildoung uno Gleidgeit Sazuk habent.

1. Dic Crfatrung yon Der Seessabet jat inidf folches befier gelebret, Demnats 36. mi fauf cin nadi Curacao in 233 eifs Sindien lauffendes Sdiff begeben/ uno wir mit guten 28 inde faum in Sie ges Laufien/ mulfe id nad groffen Shiwe mel Des Saupecs tmo sotorángnis oes

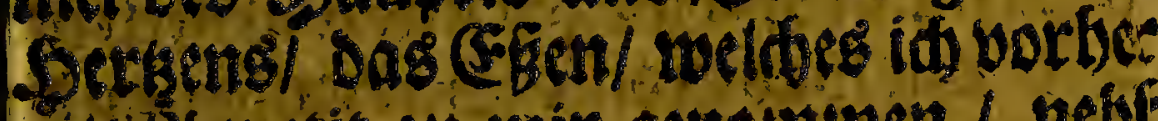
nitt sippetit zu mir genoumen/ neff: Garlund andern Seudtigfeiten/ Durd Den SRund von mir brecten.

Diefes Deffo Dentidider uns grimoli

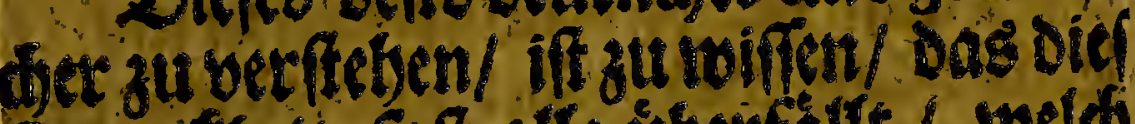

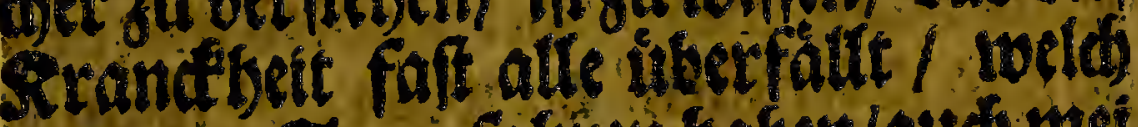
nidst zur See gefabizen haben/audo met fiens alle dieferben / fo metiemablenau Decm 23 affer gefagectn/ fonderlich wew feceinige Zeit fifile gelegen ober am Ran De geween/und mit bartes: 23 inde wie. Derin Seegeten/ oder audilange untet

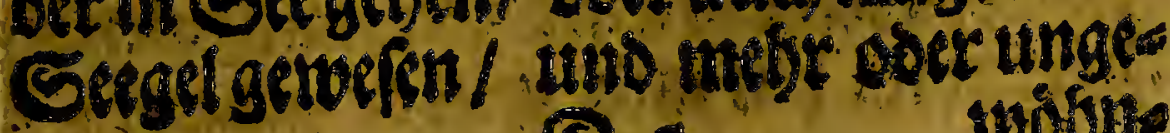

$$
\text { Ds wain }
$$


$(58)$

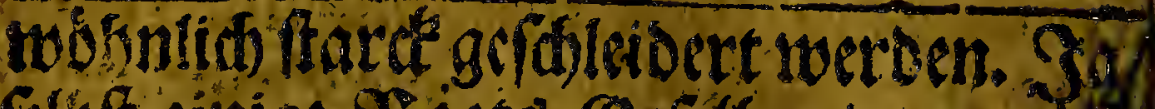

filf cimge Bovts= Sefellen/ worn

bey Ungetwitcer auf oem DRaft frige

uno fin mit megr Sorgfalt anbalten uno borfesenmutfen als unten in bem

Sobiffe/ werden Damit geplaget:

Ser Sce- Rrandbeit fino aud un

tcrworffen dicjenigen/ Ioldo mit weni ger Segbenligetiffe beteffen eonnen

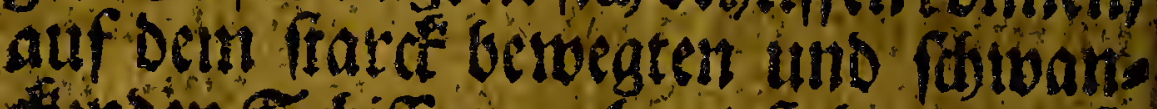
anden Sdiff zt geben/faben oberfi. Bent.

Nadisent id mit genouter suffitute feung/ soerd ertwo gen und begriffen baaen/ mit was grofler SBefremiboung Die

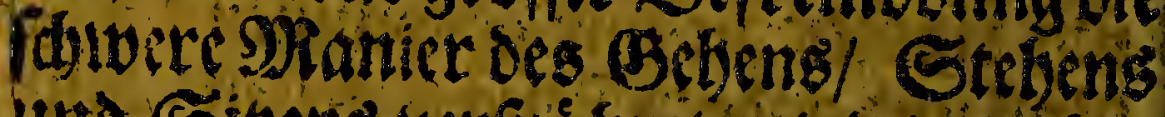
uno Sikens berincithert wire twober Dicfe fomm / tho was bor alteration ber Rauff der Fendtigfitten in unferm

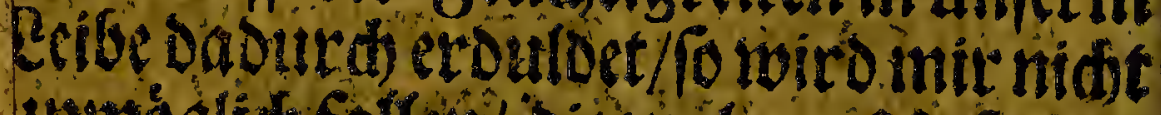

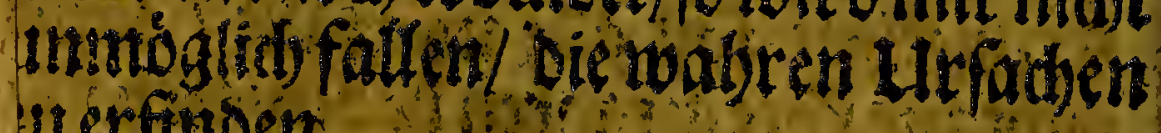
unerfinoen.

$23 \mathrm{em}$ bie ber Sdiffrafert unigetwolns

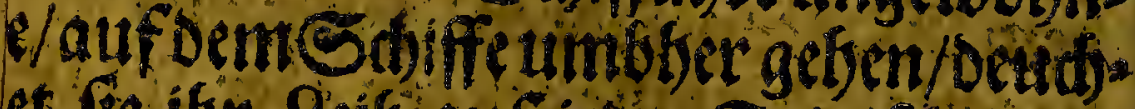
et fre itor seib auf ieben 3ritt iftuerer itrwerden/ fo twobt wass Den obern Ibeil als bie 3 beinte betrifft/ oie ned z bers big orrynabl bin uno toleter gefene / ro 
Iteiff/ lo /diber und fo mate werderi/ oaf man fie nitht beatgen tan / nod fo viel

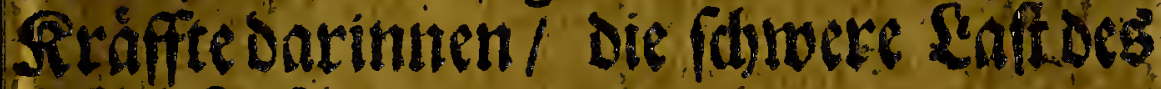
Dect \&eibeg zu tragen.

Der Reib focinet fotweter zu lest/ (is fern die Mufeuln, welde Denfelben. tragen/ betwegen unt fortbringen fols

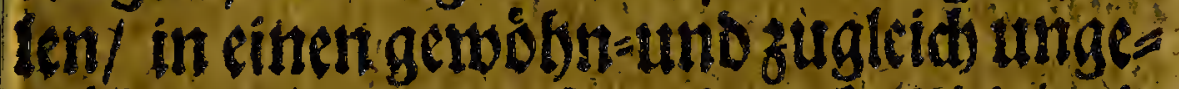

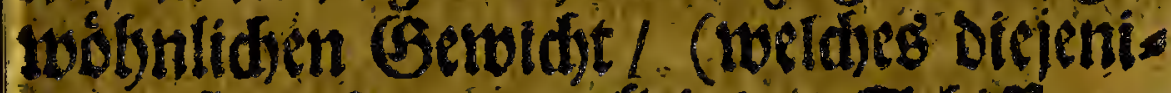

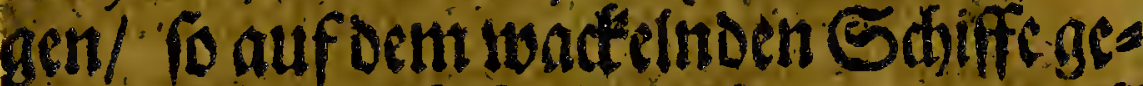
beri/ balten muffen ) nidbe genung uns bequem find oct \&eib zu berbegen/geben/

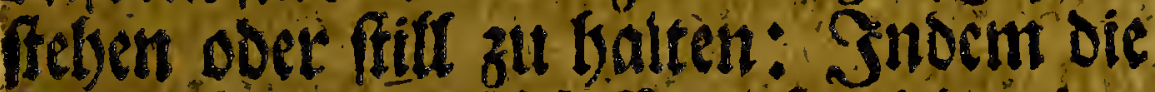
Saregung beg Sdiffes/ bev led weder: merdididen Saleidering / nebf senen

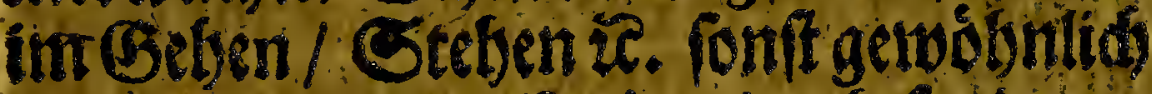
braudibaren Mufcutn eine befonderne Epannung nod anderer Nzersen etfo. Dert / Damit Die anfangende LGerwies gung/ welde in Dem bengen bes Shif? fis auf oie cine Seite/ gefpitbret thits/ modte in ein Aqvilibrium obir(Sleids) twidgtigfeit gefiradt uno ctrwan in einet. perpendicularen Seffigfecit erbalten werden/auf Das bet Leíb perpendicular tuben fonne / auf Dem Plase nit we th detr er au form betwegten Sabife nicdet Eommt. Zutweldier 2 ertiótung man 
$\frac{20}{\text { zugleid andere und nothige Mufculn }}$

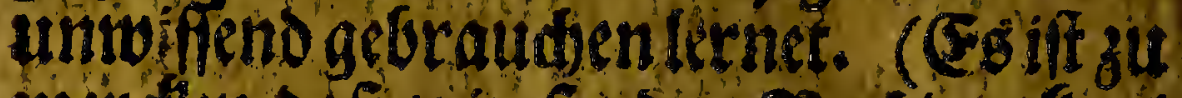
mercen dab nir fonder Narbiendét Doer Yuffadtramert anf Die im geben brandsaren Murculen stofung zin bas ben/ fortgegen: blof geonféno wie wir bon cinem orthe gll dem anderit commen noolden: sobon bieferdits ein

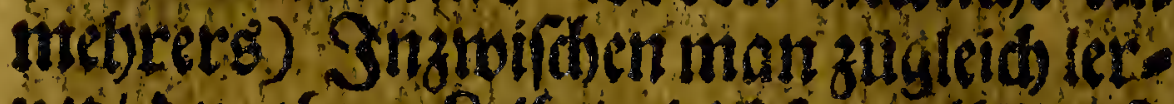
net/ Den obern \&eib/gleidfam allain/ fo gureden/Durdi dic Gedanden perpendicular rutben zu lafien/ Init Suilffe cet Múrculn, tollde Dem obern Reib auf oes nen Gebeinen Der Fufe ober untetn Theile / Damit ber Reib das bewegende Sdiff beritgret boer fid Dacauf ftams met/ erbalten. Viditungleide eimen auf einer radel fiebenden Com pas, neliber ungeadtet oes fowounctenden Sdifies? unberieglich oben uno in einer redtens Sládje poer Gleide bleiber/ ob gletdo oie Mabel mit Der Berbegung des Sdiffes/ fid meretlid ouf sine Seite leget. Sit

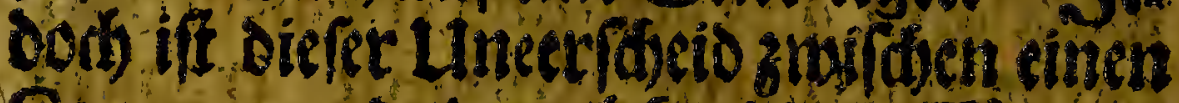
Compas uno cinen lebenoigen Corper has derCompas fonoer Sob be oder 3 ieffe in Gleidutwiditigerit auf bie 3radel gea madt if/ oamit er umb fo biel beffer fon- 
attein bie Meanblein unt Teridines ber aufertichen Náubletin/ Sonbern autd) (ode bey at beofiadten die Sorge bor Der Sel: Gefabte uno falfen) Die Mufculos det Singetweide un innerliden Sbeile/ (refbit Das (Sebirne) raldie zu Denden/ Surdtet tino Stummer Dicnen/ betwegen/ twoburd / wie id mefrmabl bezeiget / Die Fcutbtigfeiten alts Der Circumferenk Des Leibes/nach oenen intwentigen Shetlen getrichen / unto Durd Deffuunget fort unt ausgeffoficn werdent. Snzwi reten aud twiederumb cinige andere in ungethoriger Menge zu bee gewoobntis d) en Bermifosung athlitfen/ rooburd einige Lunrube unto Seorángnts entffe bet/ onoon das barauf folgenbe Stretien und alle bas andere erfolget.

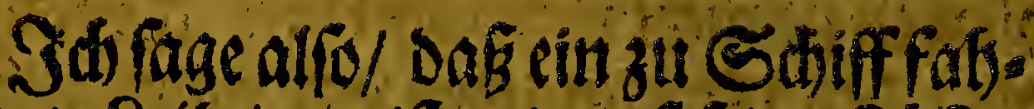
render Rcib/ meiftens auf /cite Füb/ iebo d aud zugleid auf alle andere sheis le uno (Gebeine fid frámmen mus/ gleids cinem Compas anf feiner Padel / fid mandimabl auf ein / mandimabl auf keboe Beine ffellende/als im Geteen uno Eteben (oder aud) dem reits im Gerwid) zubalten/afle bie obern abeile zu Soulffe neb: 
domende) Damit das oberfie if cinct editen Balans getbalten werde.

Der Doer $\Omega \mathrm{cib}$ / welder allegeit auf

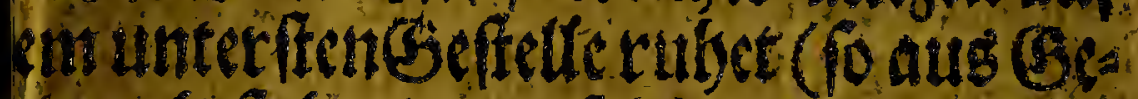
entri beftebet/ vonfoldher Conformaon, das immer eincs auf bas andere b) lieft) Woird wie bef and und gefaget iff/ urd) Sillfe feiner Máublcin att ge

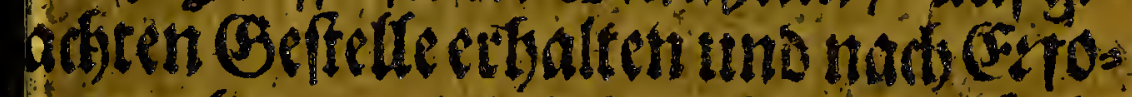

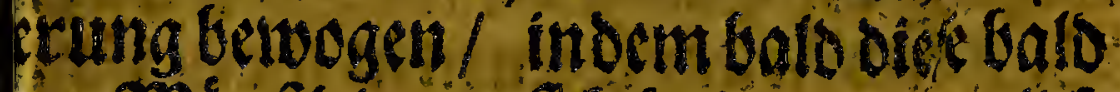
he SRáblcin on fid bolton/ ainklidy adtaffen/ mit andern attgleids wirden/

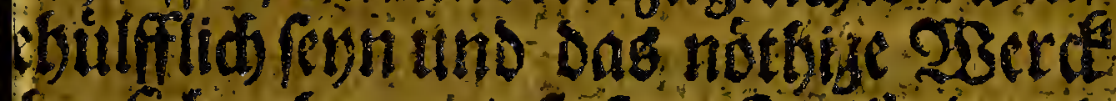
(ber fid nebluten / fo fern fe getralt uno egoem fino/sureh ibre 2sircenth/oem

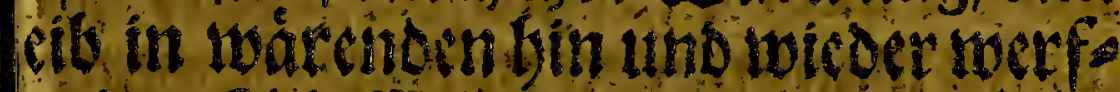
on Des Sdiffes/ in Gerwidt und itber d) zu balten. Denn antere batmant otbig zu ffelsin unb zugchen/ andere

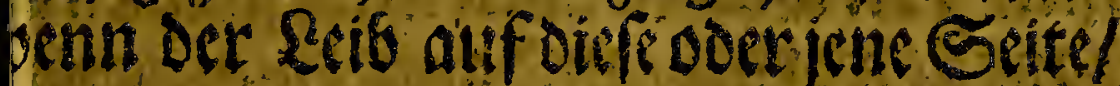
Hoere went er vor oder binterwerts

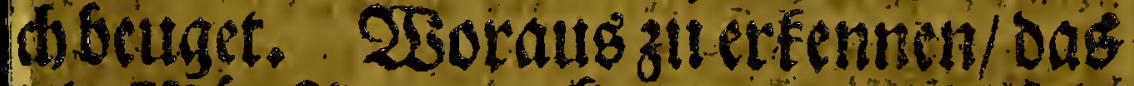

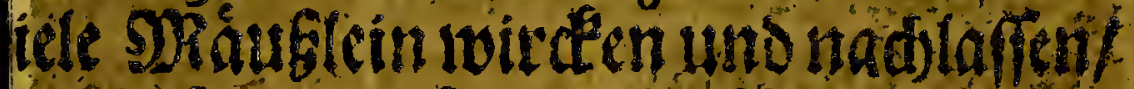
ginit fo unterithicolide serwegungen

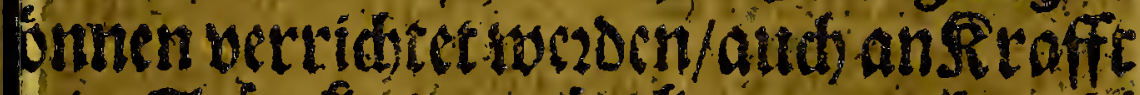
ino Stâcte in witrden mandinabl anklid ab uns zunetmon. (csiff oud

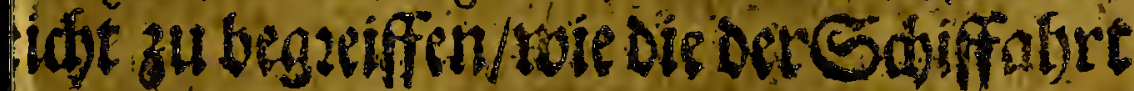




\section{is $(64)$ ses}

gant ungewoobnte/mit fiberflibiger arth

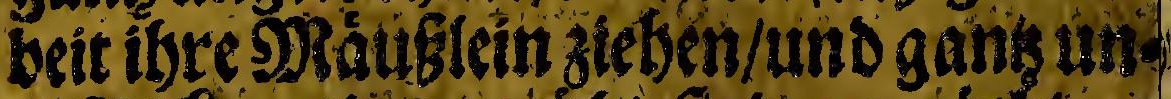

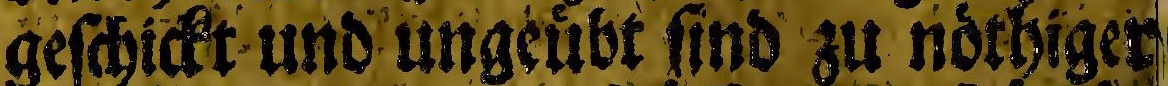

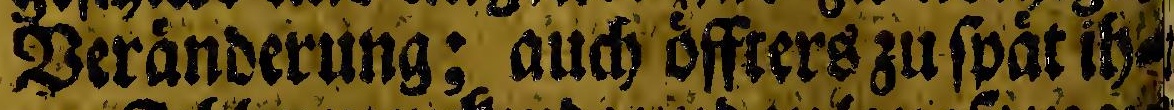

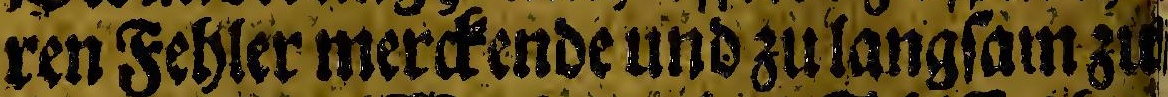
einer andern 28 endung bes Sdiffes be reít fentidel/zu Soden fafen.

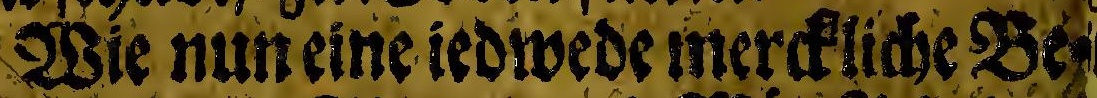
begung Der Sinnen uno 9Ráu Blein dos

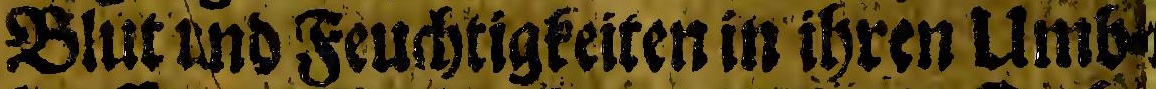
Tauffertweder in boer aus einem Drth forttreibet/ twirb einer gewabs/ went man nad) Deffnutig esiner 2tDer am 2ich then / Die Sand zubridet ober Guffet; tweldjes aud biejenigen verffeken/ food twiffen twazunb eine mábíge Setuegitng Des feibes gefino if : $23 a r u m b$ eing

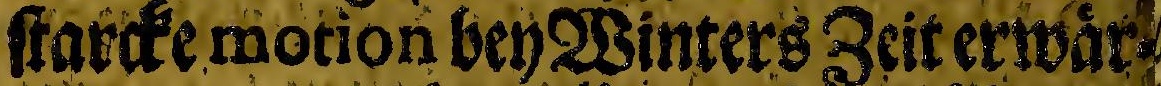
met/ won tweldhen allen mebrimabilen ge

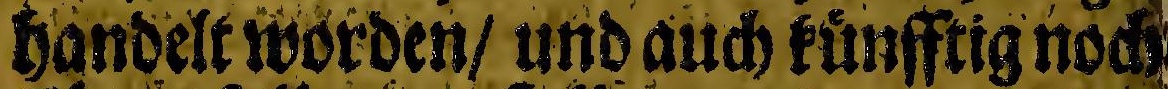
flarer for worgeffetfet werden/ in bes Sanditung von Urradien/ wartuts nad groffer Gemuttgs-sBetwegung fo merte? lide zeranderungen ent feteber/ und of

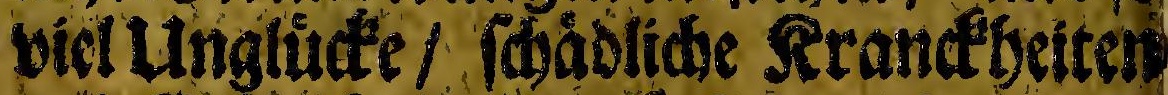
umb (Bebreden veturfíadet wer Den.

Sid vermeine nun gemugfam befanise

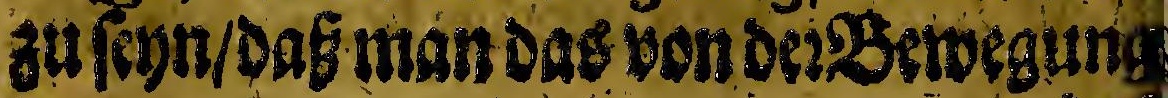




\section{5 (66) 8}

den; Eten als wie bet fontelle fortgang Der Seurbtigfeiten in einer lange-um ffersgezogenen plumpel Feine Sesung verfattet bon Demberwegten un fow wam ifender Sdaiffe.

CEs iff alfo alteine bas Spannen bet Senen tmo ungewobntiche Bieken bed SMauflein/Daburds das Sdjif cime?zen ánoertung in bem Rauffe Der Feudtigke ten surd Preffung oer Gefábe serurfal deet/und Daf Diefelben uberfitigiger in Do Gefäbe uno nadb denen (Fingeweiten z lauffen/Gelegenteit giebt; Wobon cin

- unterfidiedide Bervegung / uno folg barlid) Bangigleit/ 2angif/ 2Bürgens Bred)en uno Sdw weimel entfebt. Do Dagen ift oer Drth/ Da alles otefes ber ftamintet/und woolger man Die gentiffeffer rationes bolen fan: Sodag dier Al teration Deg \&eibeg nidit berfommt an: einer Betánoerung Der Seutitigetite ooer bes Síutes im Ser'sen/ als viel thebte wegen einer úteln $23 e r m e n g u n g$. fo in Dem Mragen angenommen wiro. und ob audb bem Slute oder ganizen $2 e$ be/ etroas von bem Lbel mitgetbeile wirte / bat es bod vondar feinen lte forting. 


\section{$28(67)$ sैe}

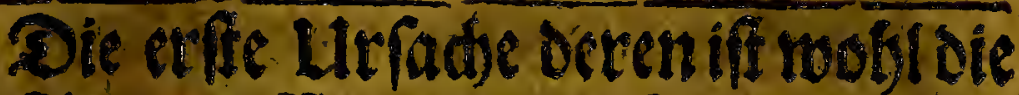
un Ste und 23 ino veturfadte 25 erves ing ves Sdbiffes / ourd und mit welin Sdbiffe der Seto / und nfo zugleids sarimen entfoltent sendfigfeiten beget werden. Senn die Bentgung - Solfifes/ wiezueradten/ bringet ine undroentl. Motion in cenenfseuds. feciten zu wege: fortoen die ssive Ing tno Spanntug oer Befase/(Ivelpallein Dienten den Reib in G clotd te zu Iten/) inadjet cine fonellere und ubere fige Servegung det Seuttigeriten 10089 . Sfutlys nad) cinigen innerfiden ferten/ivelde zu Saltung ocs Keqviliif fober oder garnitit zu bringen/ of B ofere nun oiesentigftiten uberfin: er in unt ottro for loffer paffren: 30tauf folderstidtigferten bie Grafise ber anfillen uno aufpanner//unto dis

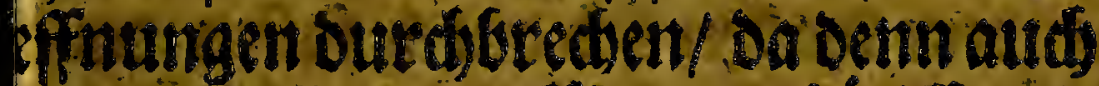
ins umb sen STagen bátfigere uctigfeiten auzgefofen mertoen/twok i) wegen groffer uno zugleidf tutor. ntriber SRenge und itbelen $23 \mathrm{erm}$.

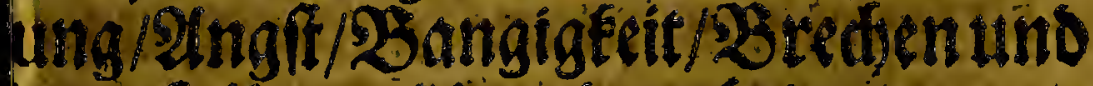
e borfatlende Ungelegenteiten futwe geforadt: weroen.

$$
\text { E }, \text { Det }
$$


Der Sdwwinder und Sopff Sdimet

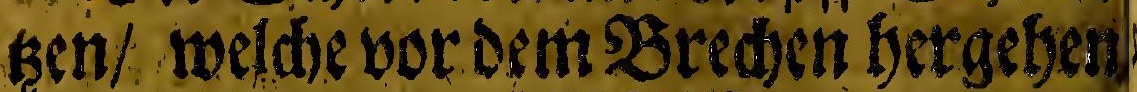
und folder vergefellichafften/ vermum Dern uno verticeen fids butrob Dafieloes affietweil Die Lurfad)e DeB Ungemader und uberlaftes/weide bie Fafen/5oint gett und Senen Des Magens atteifeten

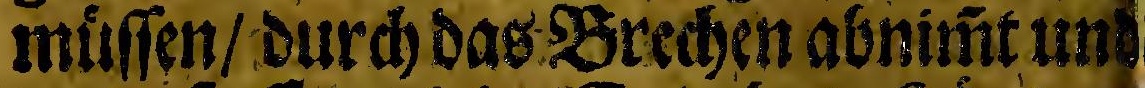

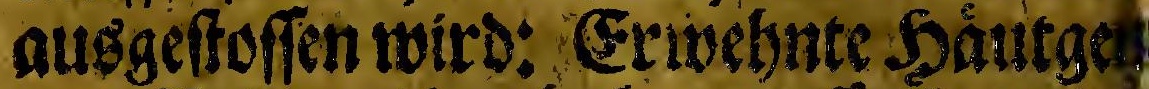
uno Nerben aber/baúen groffe (Bemein? fhafft mit Denen Jirn - Säutgen unh Dem Gebirne feltoff/ wief fe oenn in viele: Bufälen auf gleide 23 eife benvege weroen.

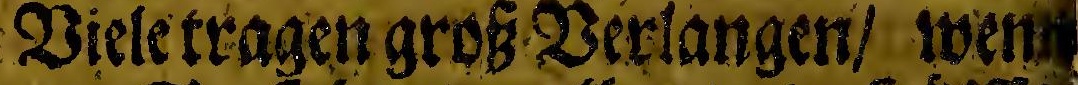
fiezur See fabren : wollen / cin Soúlffe Mitter Davor zu betommen, Sa ctlidy Medici mennen ctwas Davorerfunder 3ubaben/ aflein es bilfft ityren Seute

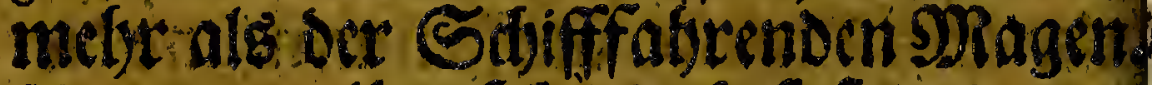
2ndere soolfen fid butrd faften babon befreben/ aber fie betrigen fid forwols? als Dic erffen. (Etlife Eatten einige Spe cereven/ weldseg audf fomit wenig bilfft

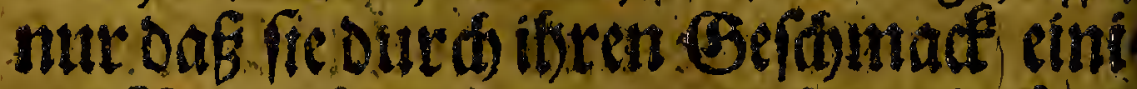
ges 2 ergnirgen gebert/ aud zugleids et wab 23ectindertung in of Setudtigferiter und GeDanden bringen/ woburd oce?

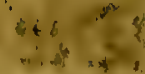

कर Reb 


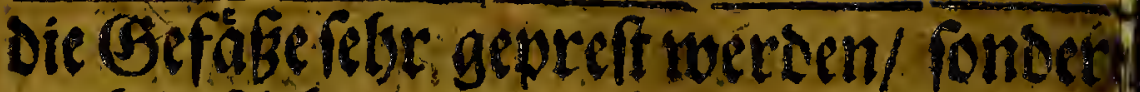
attd in Qt them bolen inne balten und hil gleichfam vergeffen.

Daf Diefe Sirandetbeit auda nidted Der Sec=\&ufft berrubset/erfitseinet bau

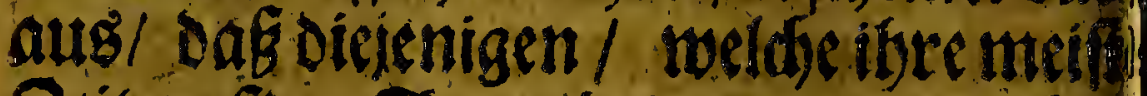
Beit auf oer Seezubringen / nict)t fret Dabonsleiben/ wenn fe mur wieder ci Bciclang anf ocm \&ande gegangen/ gt franden/gefefen und gelegen baben/ni oaf fee nidte fo fefor damit geploget net Den / toenin fre mit fanffen 23 inde ur fitler-Sce fabren/oder ben etwas barte

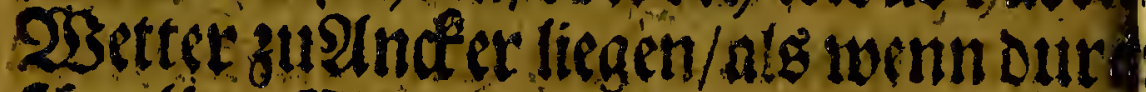

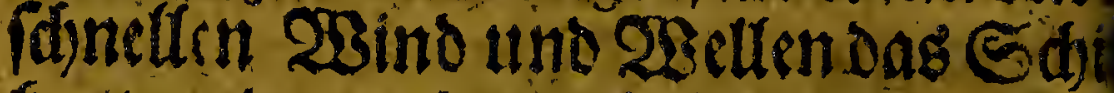
furs uno unorderntlid getrieten uns gs

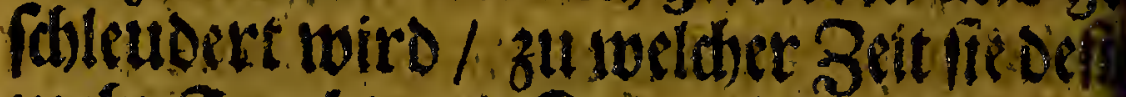
mebr Jurcht uno siummer/ audi met Lberlaft uno Mritbe in gethen/frefen/ sen uno liegen/fid zu balten/babon.

Sa/ cebeffertfid / twent Die Surdh uno Summet vor der See uno fafle verfidshinder/ twenn man des Sdjwand if ens/Gebens/Stchent uno \&iegens go wobnet iff / und als untoiffend und au

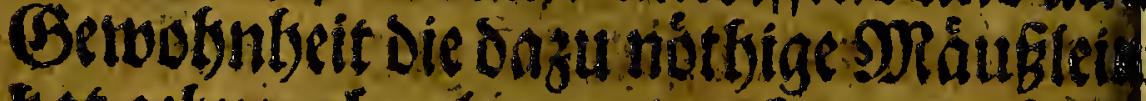
bat gebratuden lernen/ gad mit mebre 9huth fid oben anf oas Sdiff in of se: 


\section{$80(71)$ s:}

uffe begieber/in sureben oer Seelauge $\mathrm{en} / \mathrm{freti}$ in ino lnuffen.

Dif if oasienige/ toas id atts eigenet no derjenigen/ welthe neten mir auf kin Sdiffe von diefer Serandéteít moAntretworden/Grfalsung/mitgroflet Infadtfameeit vorgeftellet babe. So otmeyne auth bas ce folde rationes no/tweldbe von denen ain beffen verftank en torden/ bie fid den getwotgnliden

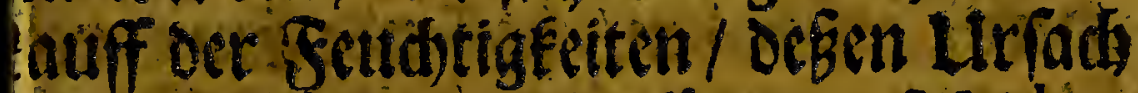
ino Berninoerung fonten Mathehatice borfictfen / und Daber auti effere rationes zu geben wifien als bie. higenll weldes mit Dem 28 ort Natur/ y mparhie d uno andern ocrgleident Detrimantetin ser Unwiffenbeit $/$ aften

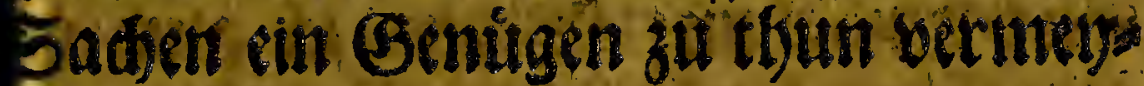
en.

Sob Gatte mandmabl sie Srevbeit! thidhe See-eeute zubereben und gu vers. fandigen/Daß́ mebr Der Rummer/ un equemligecit/ uno Lingewobntyeit Ut:

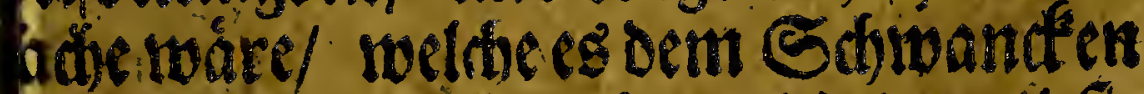
ls Sdiffes zuditieben; Lnt weil fie urd oiefes 23 or Urtbeil oder Prajudifum eingenommen toaten/ fonten fie brortid auf sine anoere DRennung ges (E) bradst 


\section{แล $(72)$}

Eradit werden: Dabod// wenn fie beth

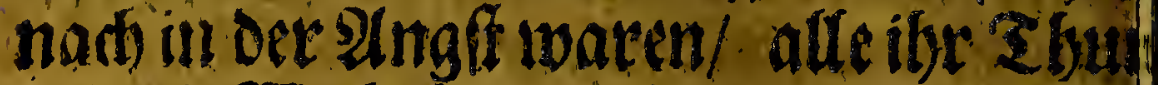
mir dic $23 a b$ hbeit genungfam oarffetle te uno fie zur Sceảntnis bradte: S2m Dere twiedsrumb/ adteten eg fich vor cing Sileinmitbigfeit uno Padbetbeil zu ferin wernn fie von Ungetwobnbeic boren fols

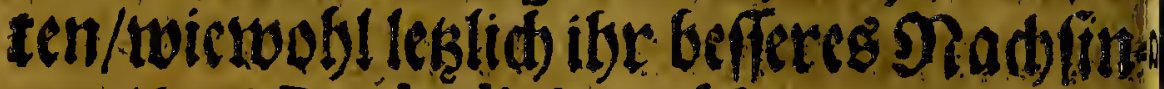
nen/ Das Prajudicium úteraeugte.

Siermit nerde id / fets geebred Sorrt abbreden/ boffente (Gure grofita 2Begierde/ mit nod) inctye netuen Dingen

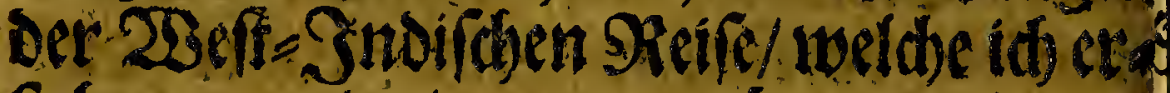
fotben werde f zu vergnugen/. aber es. wirs bielleidht die (Srobe (Euetes und bieler 23 erlangen nidge erreidjen. 283 id merd'wirdiges gefeben babe/ will id Fud funt thun: $23 \mathrm{el}$ (b) if and wea gen Fure fondertidien Prigung une Seliebung zt was netuen mid odite fouldigfon; berbleibe

\section{Mein Sou}

c. C. B. 5:

Dionyfius bonber Sterre, Med. Doet.

XIII. 


\section{HE (73)}

XIII: So neit borgedadter. Sert. Fer. hev bredben fid Diejenigen vont bem Toback audien I fo besen ungerwobnet oie es abee Jenobut fino/miffen nidats bavon. Es iff bea

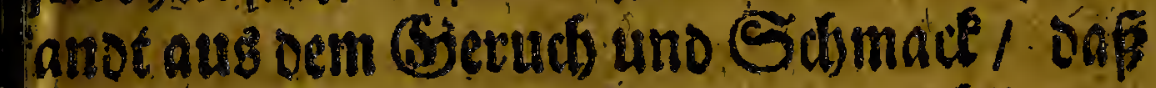
ev Tobad aus forfffen uno prictelmoen Sbeilgen beftebet/ wenn mannun oen Tos ad'senaud fauget/ zenditi man Das Dot tho fichtige Sals aus Demfelben in Dem Diuno/wueldies grope. Sighe machet/ worauf ine farde 'S Servegung Der Sadutgen uno Rervèn Deg Diundeg / welche mit Dem Stbluno uno DRagen (Ssemeinichaffit thas en/ enffebet. Dake eime grofese SBenuegung arauf folget $/$ erfdbeinet oub einen Experinent am einer Ra (e nurde mit Del bon Tobauk beftrictsent bovon Das Fbier foldre Convulfiones the

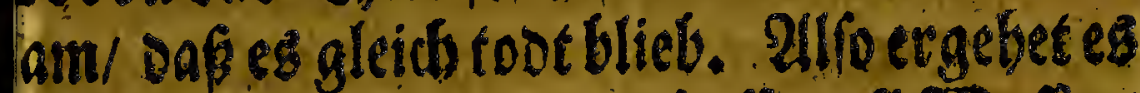

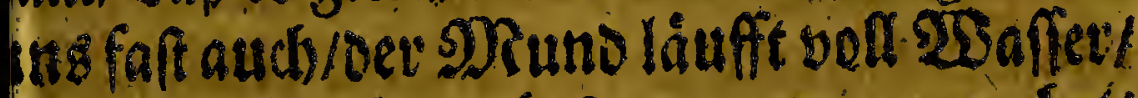
han wiro bleich/Die Suft zum reben vergebet/ ex Sdineig bridis affentbalben ats $i c$.

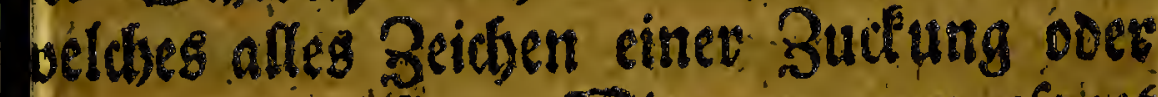
Donvulfion ii wenn oie Membranen ooer Jiutgen no Senen oes SR andes convelliret/mu olglbavidid audi Dev5DRagen gesogen werden/ velfer aftes soeggiebt twas ar bey fich bas:

$$
\text { (5) }
$$




\section{as $(74)$ sts}

Es tware demn oaf es suieder ulberbin gienge? swenn man in oie suffe tảme ober barauff

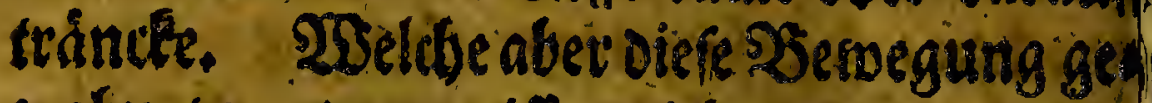
soobnet footdern/ wiffen nictes barbon/ senth

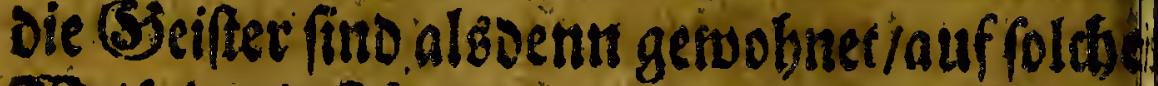

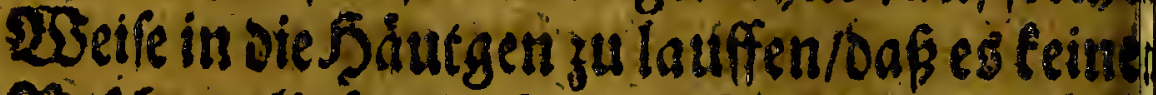

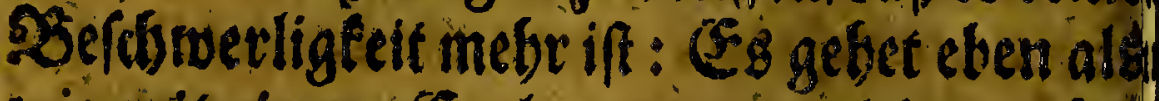
twie thit einem (Eoelmanne/ woidher muth lernen pfligen und oreforen/ sg folte ibm 2ins

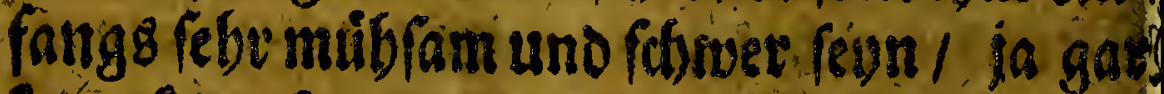

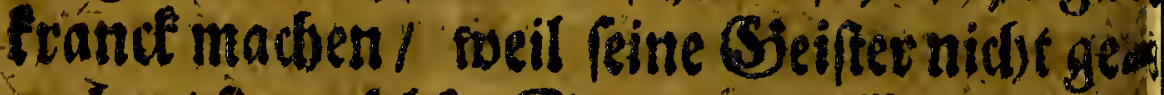
nobnet fino/ poldie Senen uno werctireuge. auffurparmer/ alg zu foldber 2trbeit nutbige suenn abee die Senen oaffelbe genobnest. meiper von feiner Ungelegenbeit. XIV. Cetlide baben eine Runit $/$ biel

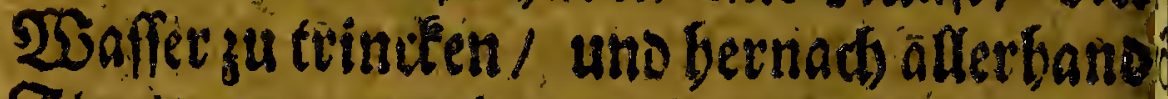

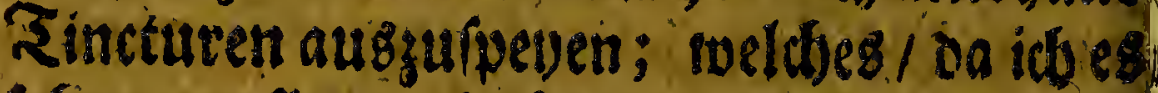
fafie uno affes nobl betractstete entoedice ids Das Spiel. Eg gebet zu nie mit oencri sent: tein 7 weltae die gegefferte Speife in orme פ) Runo bringen uno swicoer fauren/ welches

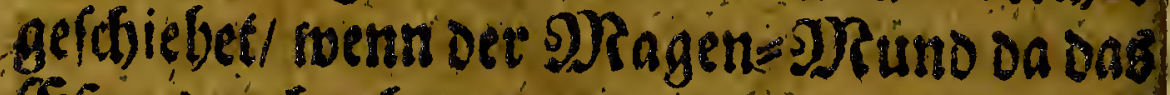
Esen ourdigebet/etwas gezaum iff/uno mats

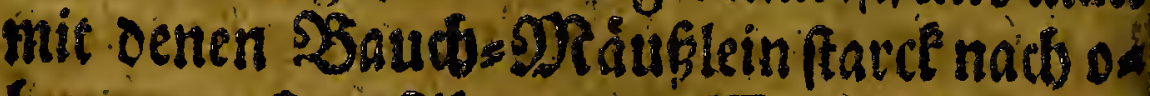
ben zu pref. 2aber diefer Isaffer=-Spenges mufte/ als ec /eine funfertwieg/ cinten lectert - Dragen baben oamis ogsienige fo er aus

fwiefet 


\section{6. (75) 5x}

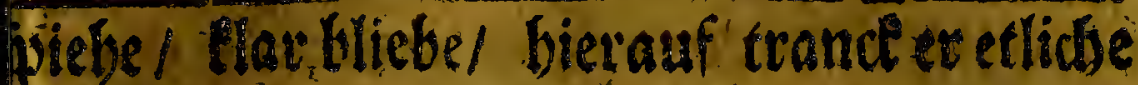
SHIáfer voer fo viel er fonte laulicht WJaffer

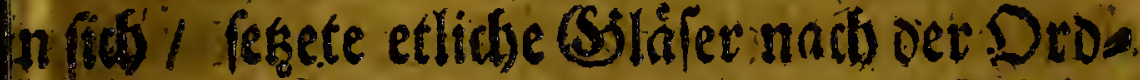

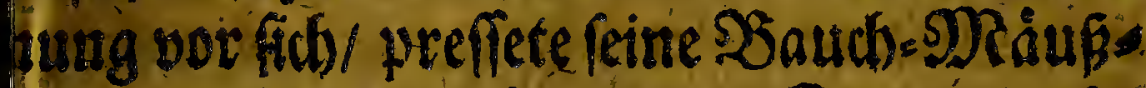

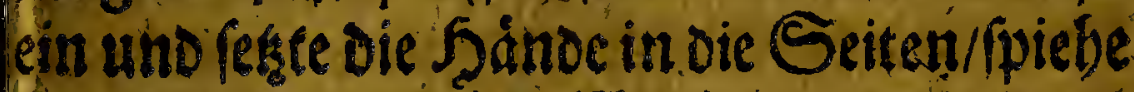
niedeg (Slap etrong Waffer/ welches von unterfobiebener Farbe war/ weil er oljne Bweiffel in iedes @slap einige leidbr fobmels semoe Farbe getban battel die allobalo dem b3affet feine Sinctur mittbeilete. VISenn - iemano molte probiren uno fict oarz̧u ges

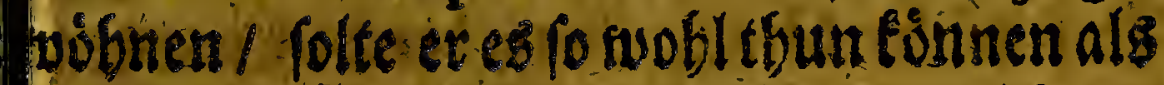
biefer DSann/aus oenen Lir/actsen/weldthe ies

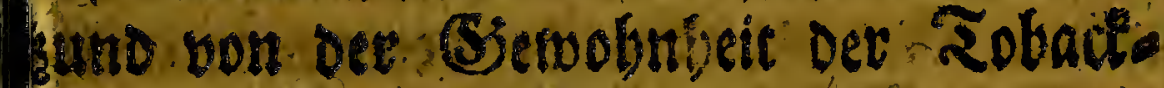
S tinder fint artgefibret tworoent.

$\mathrm{XV}$. Es war nod ein anberer 2 affers Stremer/ ber aflethano diftillirte 5saffer

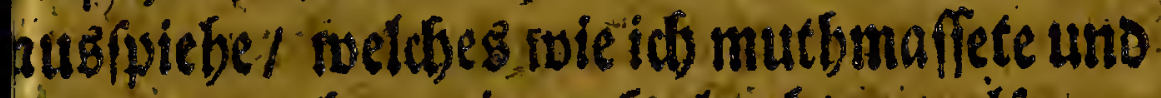
38 andere nebenmit audiglaubten/ alfo zus gleng; Er batte viefleidht eint Fiffel an dev Rele/ oie bis in ben Salf gieng/ Darein er eis he bleverne Xobbre geftectel/ wovan er lesets. the Canale fonte fefte madien/ fo bif geget Das. Ditteltbeil feines Keibez reiducten/ stto Intertmit lesernen Sidefgen verfeben was. ren mit allerbano diftilliren 2Baffeth ans befillet; wennee fich nun zum SBrechen an: Selfeter oridote er mit feinen in bie Seite gez ftim: 


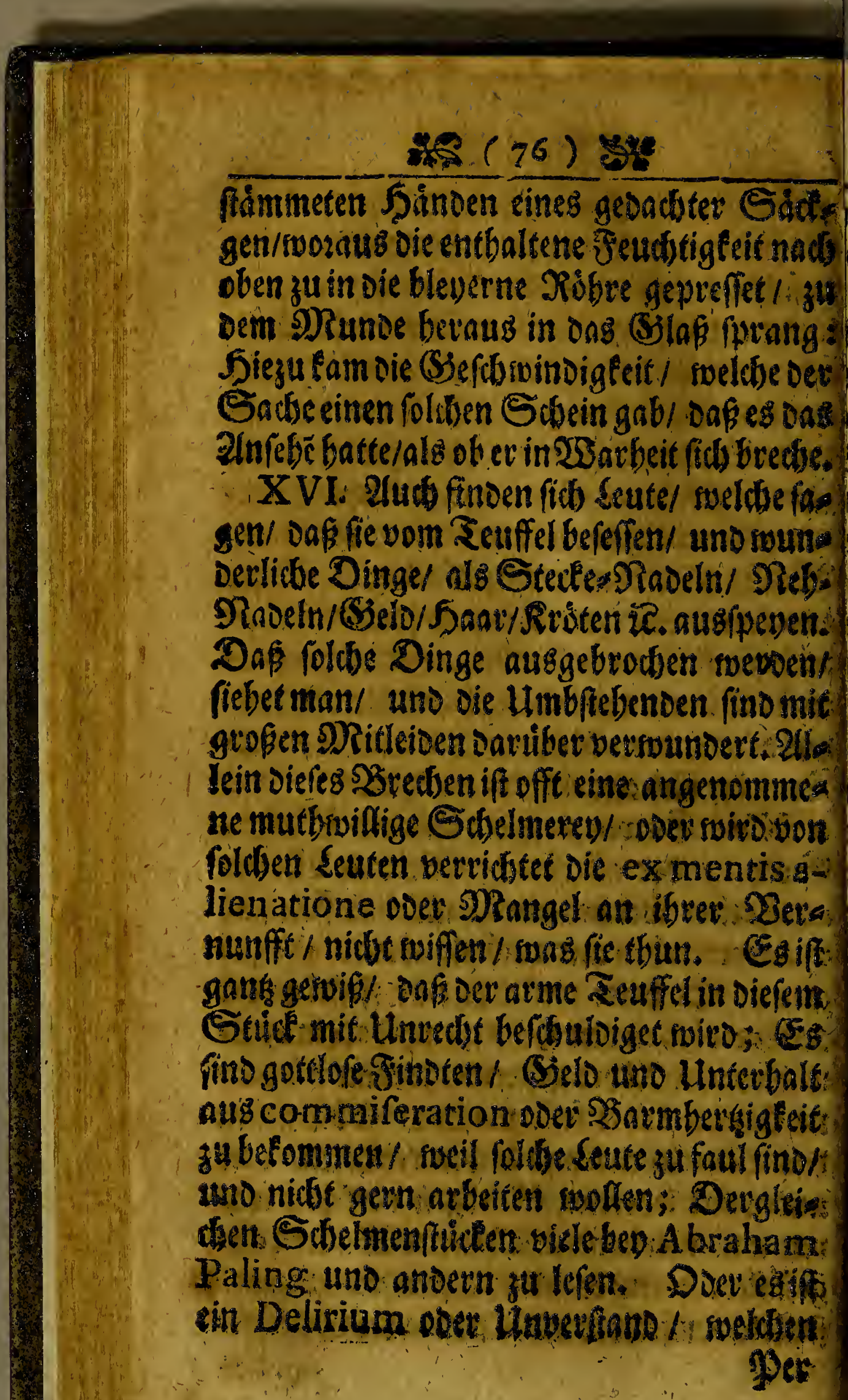




\section{As $(77)$ (4)}

Derfonen es bov gut za balten. Soldje \{eue verfolingen entwecer erzeblete Dinge/ os er fallen fie in vem פR unde ver borgett/uns

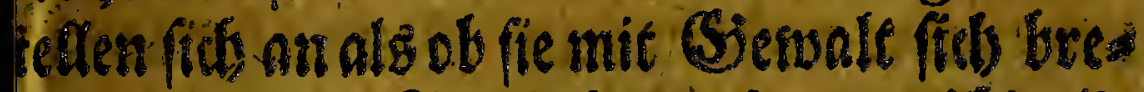

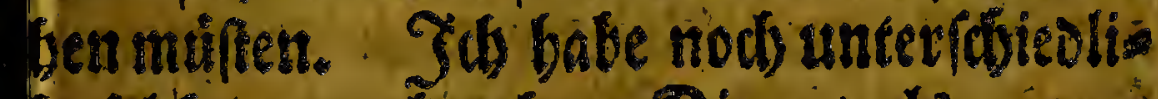

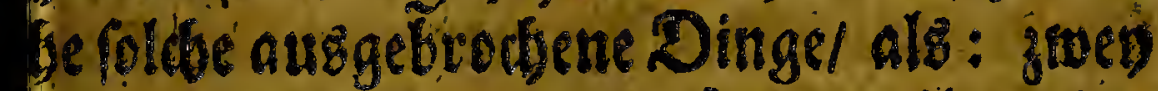
Burabantifbe Dertgen/ Ereukswaife anteins

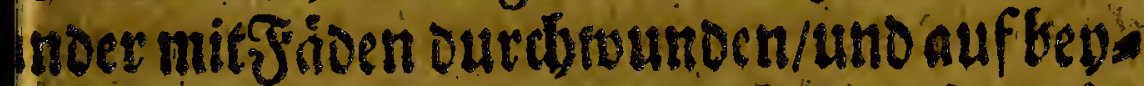
en Seiten ereursweife mit SaDeln beffedt"

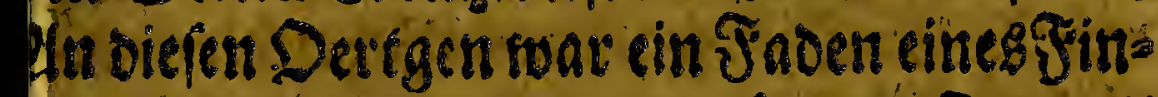
fers lang/ woran wieserumb z'ves) Dertgent Infgleidje vesefe fefte bierigen/ uno diefes ven bis viecmabl. Ingleidjen babe id Rett. en von frumb getogenen unt ineinander ge.

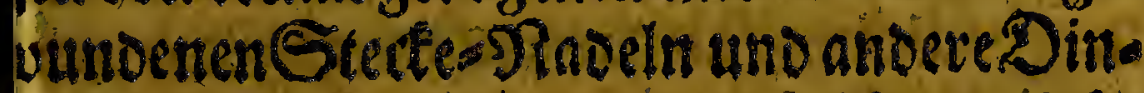
re mefor gefeben; unter andern bat diefellie

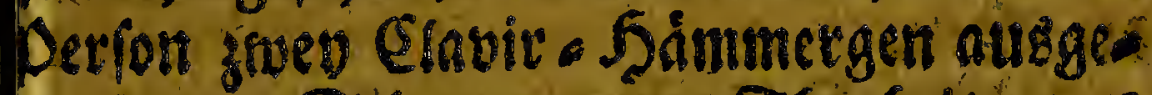
rodsen. Sebet/ nas vor Shorbeiten ons.

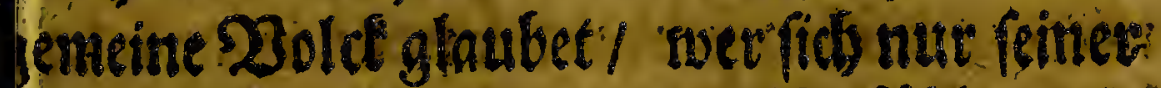
Bernuniff gebraudbet / oer Ean füblen tub reiffen oaf sies Sibelmerev if. Dermivie

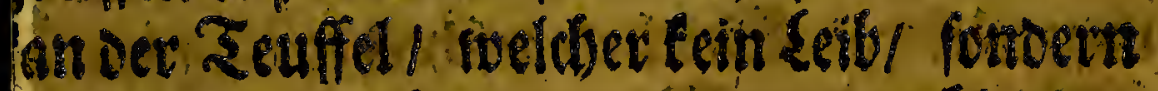
an Steift if/Die Dertgen aneinanber bintent

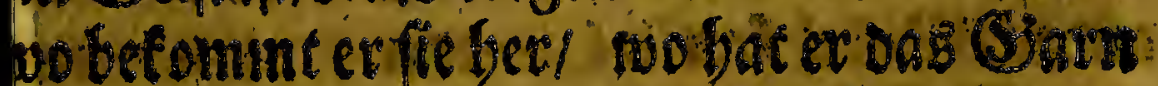

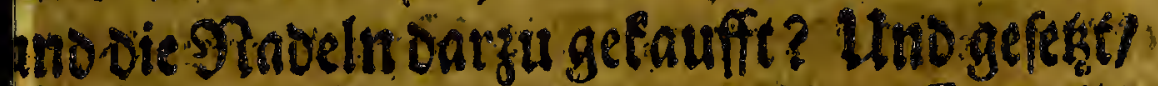

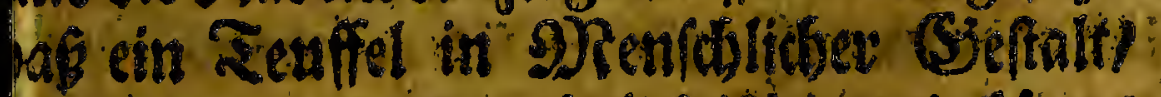

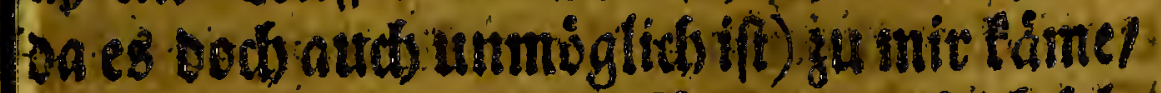

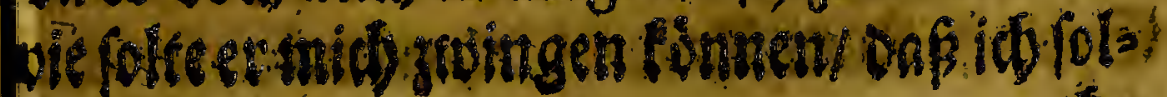




\section{me $(78)$ sis}

de Dinge mifte in midf fresen? Tob babeh

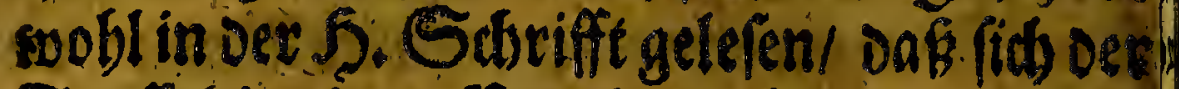
Feuffel in einen Engel oes sidhts verwans

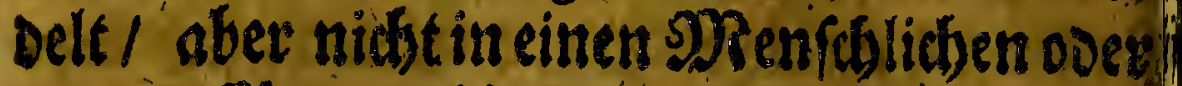

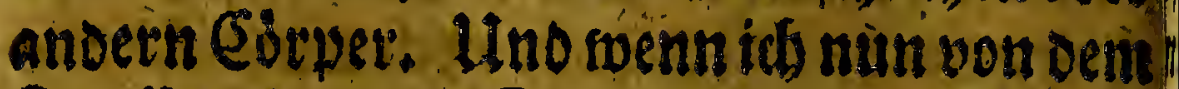
Reuffel alle foldse Dinge annebme/muife id es felber berfoflíngen/o arumb bàtte id es ges than uns rodire folgbarlidf vor Der Dbrigteibn firaffällig. Jass vor mids bin oer DRevnungh Daf fold : Seffigen uno SBez̧aubern eitet: Schelmerev ift/uno weil oas gemeine Bolde? ez nicht werfebet/, swito es leidst betrogent forengen es in Dem gartisen \{ance aus / Dafis fie ez gefeben / uno die meiften פ) ?enfósen mit Præjudiciis (20ruttbeil) angefiullet/mebs men egleicht an / Damit bleibert die sigen in aer rselt.

XVII. Radboem nir ziemlids toeit abges

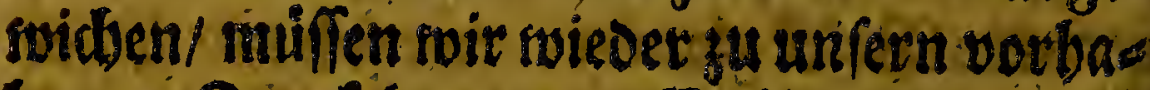
benoen 3 weul fommen. Es frage fich/ wats umb une in was vor firand beiten Das 25 tes dien nothig? Solches geffhiebet nut iwent Schleim in oem D) Ragen/oen man ound fein ander \$Rittel tan lof soerven; aud wem einer (S3ifft poer bergiffteteSpeifen/wiffents lidfoser uneviffentlids zu fid genomimen. To tem inlangivievigen Srandbeiten/al 8 oa if:

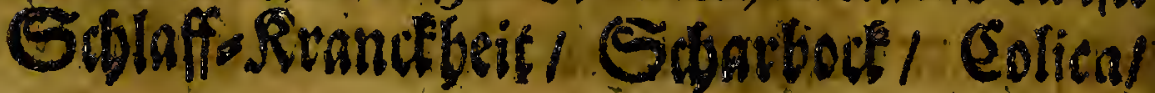




\section{$38(80) 38$}

Den von ibrer langwierigen Dvanl befrefert Eg folte eine groffe Runft feyn ourch sised dien oas grieber zu bertreiben/ wiero obl mant - azumab Inicht roufte was die DSefchaffenbert

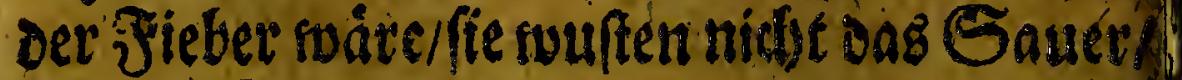
Sals/ Soblein ü. Dier fimpigten/ und fold:

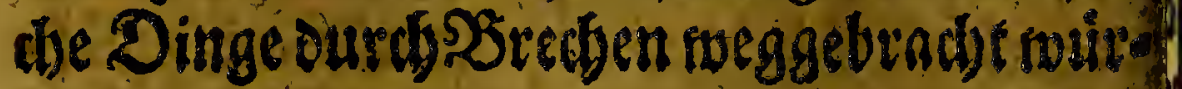
Den.

XIX. Niel Sufâlle verbietben sas stred. dien/zum Sxempely soenn oer J Dagen anc Dairmer inflammiret foácen/oa nuitbe Das Ubel groffer gemadit/ oas Sieber/ Solymev fen uno Siske vermebref werden/ ingleidbat: in Entyinoungen Deg Znerd) fell/ Sauds.

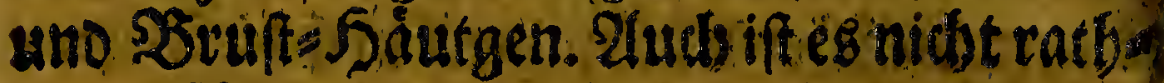

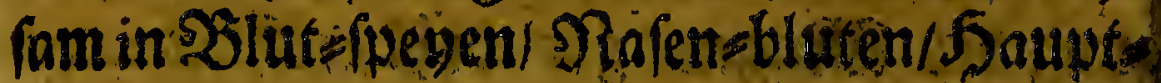
SEtuftouno 23 aud = 25 urden/ bornebmlidg/ sonen ote innerlidsen Tbeile bertesef fino. Niobt wetriger if vorfichtig um 3 zugeben init zangeren Perfoner/es write. Denn/Daf fie fid

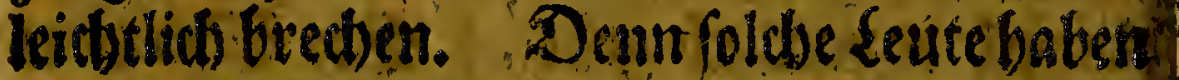
soerig Ridfte/ uno foltenfoabgematte soet Den/oaf fie gatrigon fict felbft fimen: Drane: we geben senen nidbt gerne jubredben/, wels de lange Salle faben/aber es thutwenigy zur Sache 1 uno bábe (d) in ineiner Praxi feinen Unterfofeiometuet sónnen. Dem

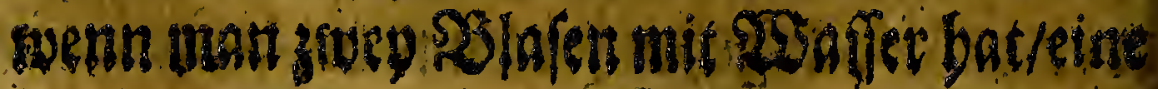

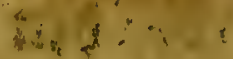




\section{$2(81)$}

It einem langen uno oic andere nit einem

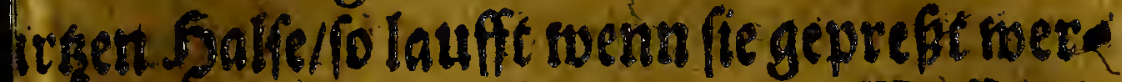
In has oev langbadfigten oas 283 affec fo 61 als cus ber furkbälfigten/dependiret

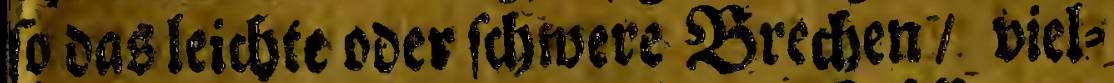

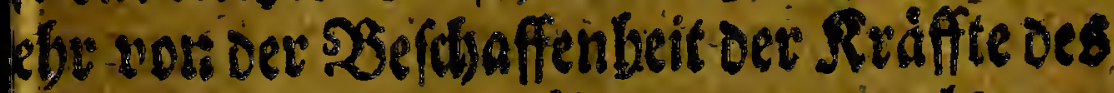
Pagene uno der Medicamentet/als von ren fursetr oder langen 5alfe.

XX. Uter diefes daf in dem reibe eine ele Conftitution fevn fas / sueldje das

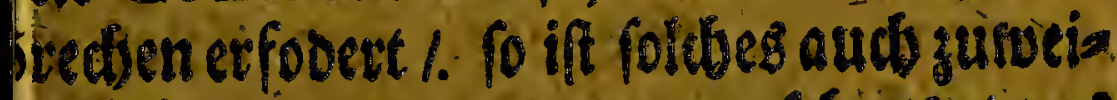
insthig i swenn man unverfebens etwas Iftiges gefen / als: Champignons, Fbetling :28ur le, snoworn C. Stalpert yon der Wiel, trien ZGeils 43 . 2mmerdiung gevendetet. rent NRiureapoulver/ A uripigment. ablimar und anderes (Ssifft mebr; Sto eiden wem einer zu viel Opium einge-

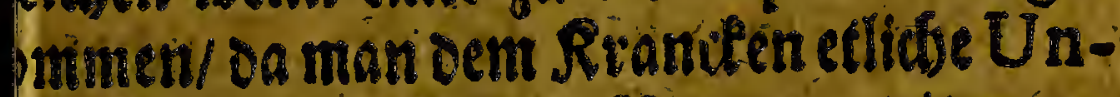
en Del ober zerlaffene Şutfer/ mit nate

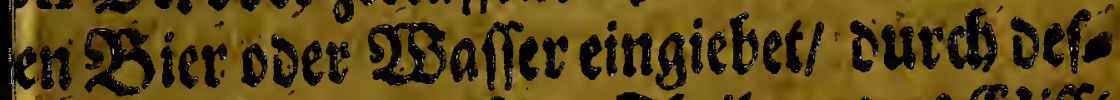
Pramofe oder zactigfe ? beilgen Das Ssiff

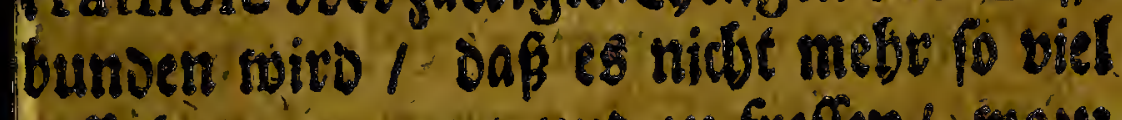
raffibar zu nagen uno zu freffen/ - mojs

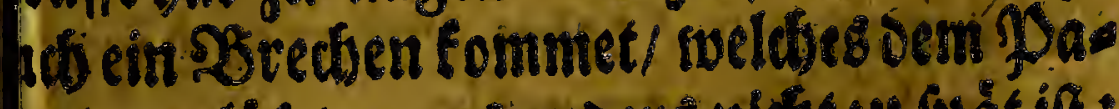

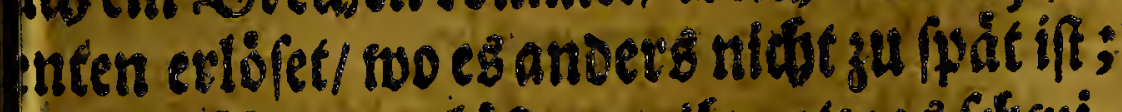

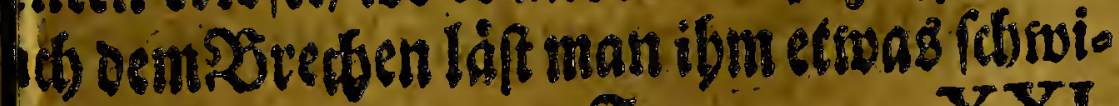




\section{ती $(82)$ और}

XXI. Wiele madjen eine $25 a b l$ unte?

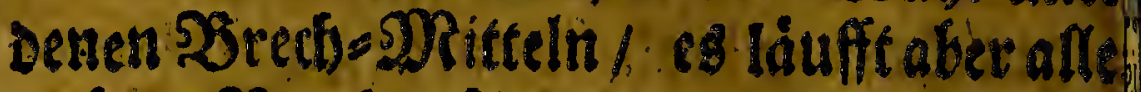
aufong Sjredien Ginaus/uno finoe id teine

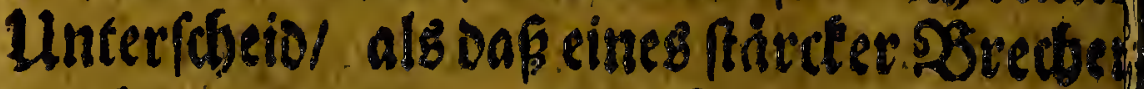
madot cent das andere. "SGe idb aber weited gebel miffen wir gedenden oas oreyerle S5redb-5Rittel (eun/ nemlid) aus Minera

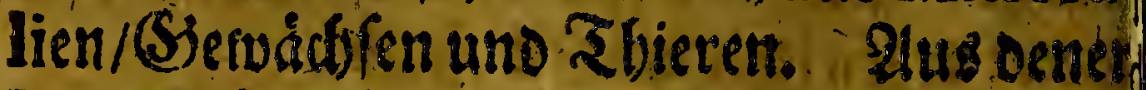
Mineralien/bat iman die Silbersund Rupf fer= Cruftallen/ blauen Ditriol/ Regulus Antimonii, Gotomeffel uno Sslö nu Spieg=Glap/Crocus Metallorum,Sgre denmachencer Wein=Stein/ rotber Pra cipitat, Mercurius vitz, Sublimat. Turbith minerale, Hercules Bovid Ditriol.Sals/ uno oergleidien mebr. Un ter venen Vegetabilien oder (Servidhfem) if deb Sabaut / Solitter bon Sard.25nus Gratiola doer Sottez Sinad/Nux vomi. ca, Nuces Staphilodendri, Frudht bor

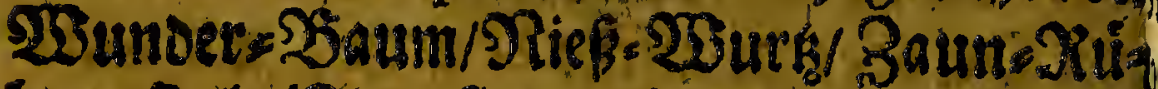
ber/ Del/ ic. Jiergu commen Die S5reds. Drittel bon Thieten/ als S5utter uno Gett in Drenge cingenommen/foldge/ bornebum lich roo man fein gett yeroauen fan/ linoes

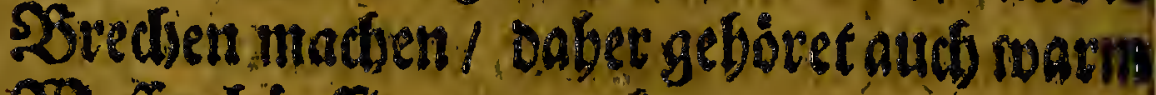
23 mfer bsiffig getrumden.

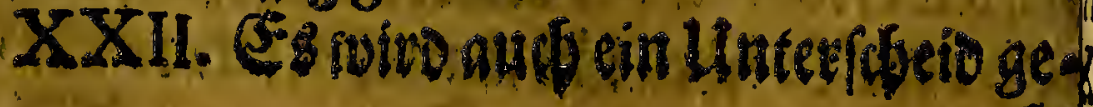
th,,$:$ madis 


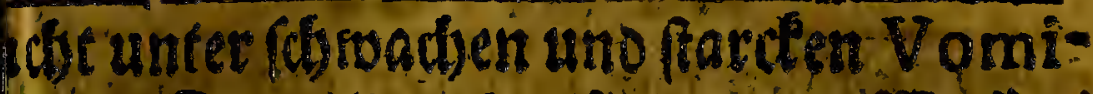
hiis. Die gelindeften frno marm 23 offect

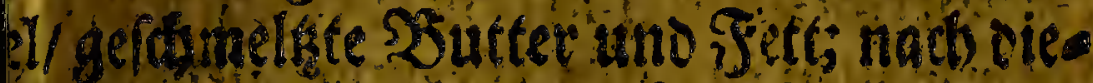
folget der Saffo oderinfufum uonisioto (Senad 5 a felinuns/ Crocus Merallor - Tartarus emericus, unter dieffite

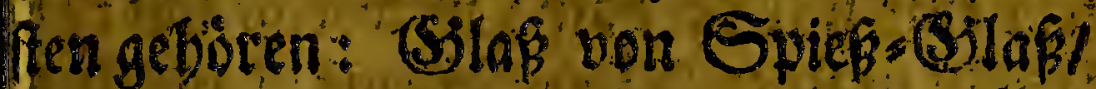
blimat, Mercurius vitz, Regulus timoni, Niefinut/ Turbith minee, Tobact $x$

XXII. Unter Denen Szrech ondittetw egen ettide zugleid and zu purgirent nebmith ment etroas viel auf cimnabl Jeber wirs / anter vieren fino Jatappa, un Ruben boer Brionica, Gutta Gam- Tobná iz and foldse Medicamenta

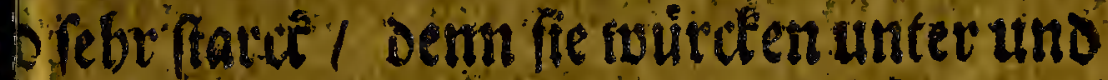

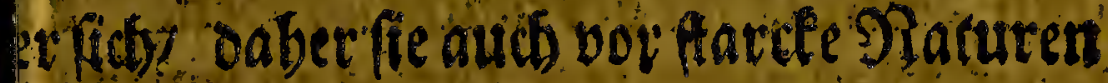
Het. Sie participiren nidat affeinuon Pen purgierenden/fornoern auth sjrecten. denoen Fbeilgen; eben nie die Nibabar: zugleids fopuffet unt pnrgieret / als mat in diefe Dinge S3redsen uno Stublong'

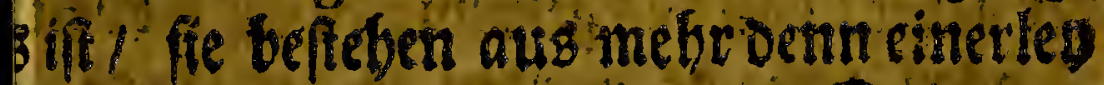

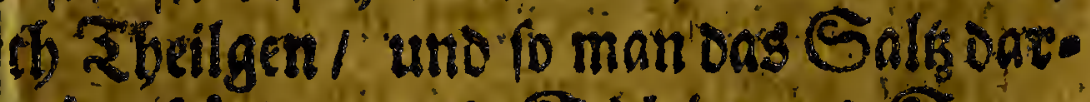
- zogeibátte man eir Sallein und Snuer mperirenbes MRittel.

XXIV. 26er zo unfern Snet zufom. S mew, 


\section{$20(84)$ set}

men/ miffen nir extlaren auf mab weil

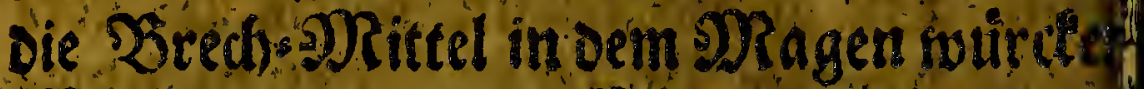
SBSir roollen zu eiren Ejempel nebmen od Vitrum Antimoniipors@ilaf aus Spie:

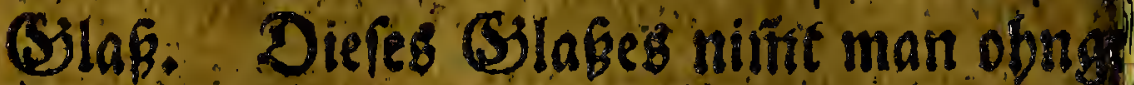
febr eing uno ein balb Dventlein/ láft es ef STadt in einen Xisiner Weirs: ( Welches mancomabl getban babe) giebtes oes $\$$ (?) gertsein / uno bringet alfo ein SEredgent? Dege. Diefes Silás bebalt fein Servidy obone nus bavon zu verliebren; wierwohl is midb erinnere daf ein geniffer 23 uno 2 tit uno als mich Deucht I JPeifter Jan Siva Zalgr. mit ein Stuctgen Antimoniev

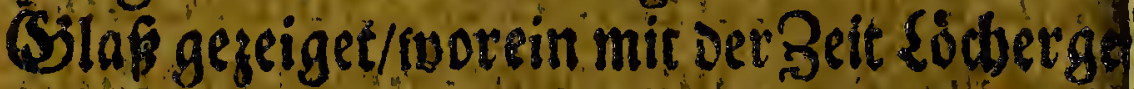
sefeegen nocet/ nadscem loldses zu oergl doen Broec larige gebraubr fordoen. D

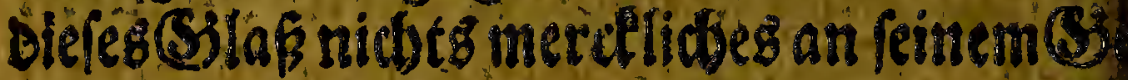
toidhte verliebret / baben neb/t mir toufen seute angemerctet aber oafs ez aud nit of Beit ourd die Saure des șeines refolv. ret niro/ift gan getwis. Wer Bsiten bon Der fiebet / Dap das gemeine (Stlaf soovon of Fenffer gemadjet twerben it bex Srbe ze foleuft von Rifze wirs/ uno wegen Du delfeit einem (Silafe nidjt mebr abnlid of bet; warumb folteoas Sing von Antim nio in SDein geleger/ nidh gleidet Buf 


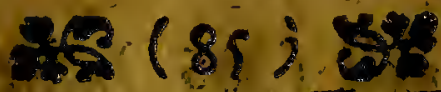

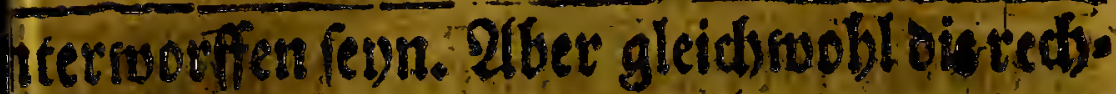
2Babrbetit 3 u fagem / ro if es fo ein sweniges/ ac oas Sauter des 23 eine von derm. Alcali

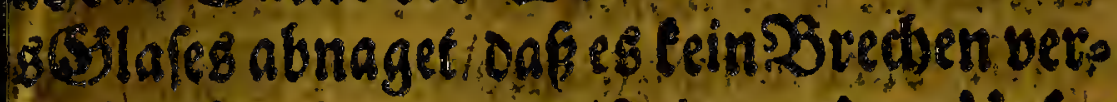
fadsen fan fondern es if ceime andere Urias

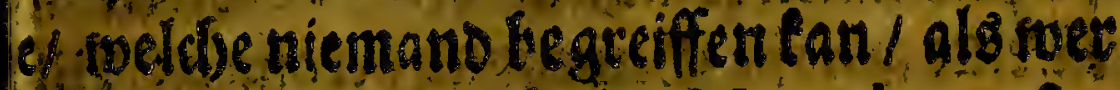

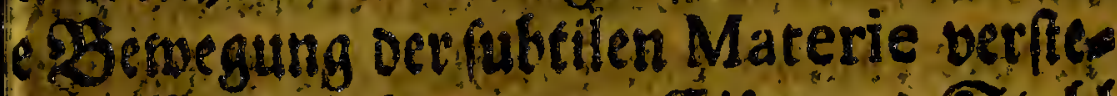
Es in befande sab Eilen und Staff 1. Co ne

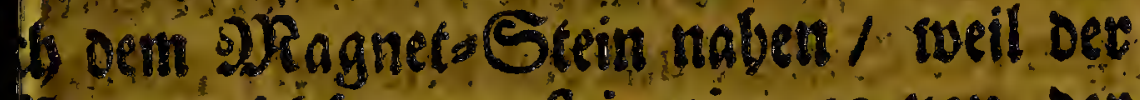
Ragnet folde tran fpirationes vout oes btilen Materie fan ourdilafien/ Die in die oros doer Letber on pes Eifens oder 5tables/zergleidsen Einflus madsen/Dag fie efe nad ocin DRengnet ju fragen und prefs 7. Liber Diefesl twird ourdi geoadbe effluia oem Stabl uno Eifen eben oergleichen Benegung Der fubrilen Marerie mitgetbets ti als in Dem MRagnets Steine wat / uno pourdi etlange man die See = Compas.

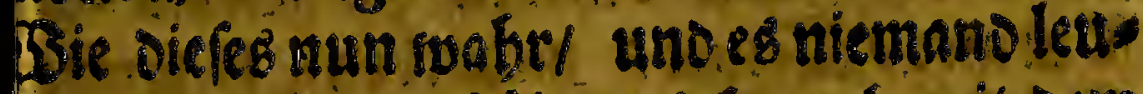
Men fan/ fo verbólt es firds auds mit Ben

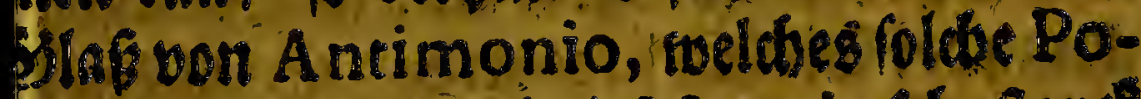

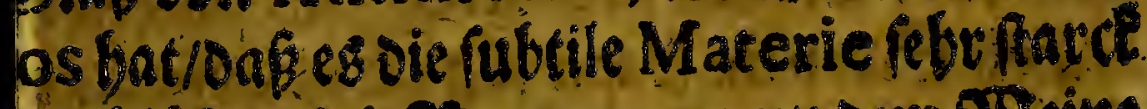

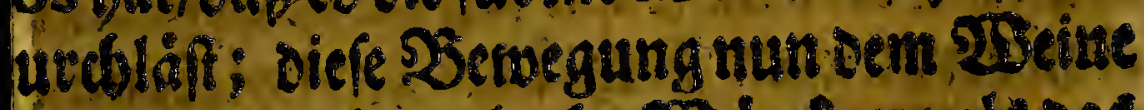
hitgetbeilet/ tbut glectbe 2osurctung als Das

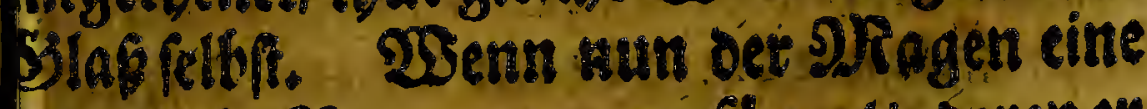
buberlicbe SBerwegung empfänget/ berenes: ingerwobat / uno weldse mif feinen Durds:

$$
\text { F3. Finf }
$$




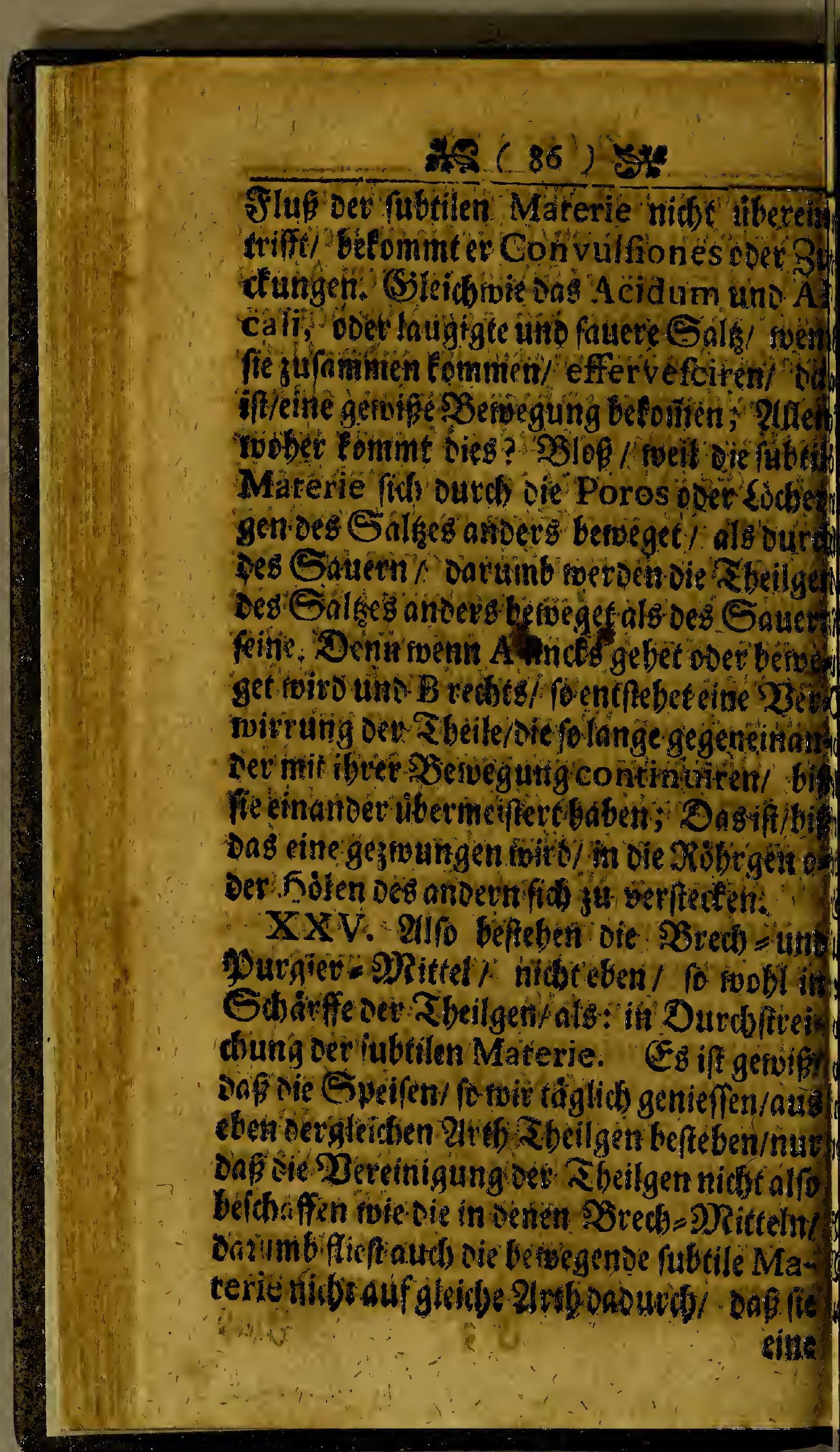


ne Convulfion in oem S)Ragen erfocten Snte. Tdo vermenne oaf oer Sefer/nocldse c Sechunff uns fein Prajudicium ooet Bowallitbeil beaudieilgenunglam verftebet

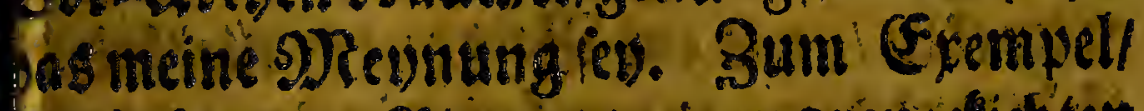
jab babe einen King uno einen oreyedicten bower/idj fede fe bevoe incinander/fo muif In notbousnoig orey intevftiria oner 3 sul

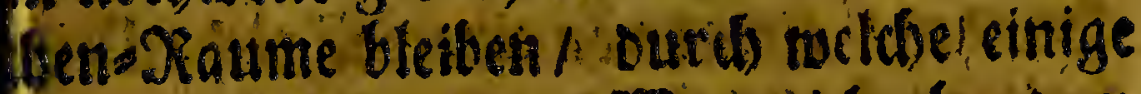

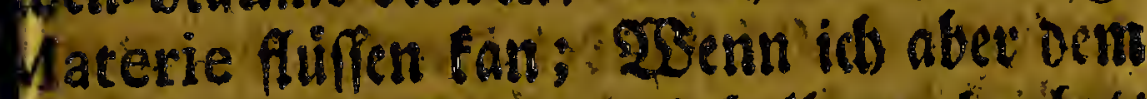
Ring mit einee Rugel / vie balk curdigebet ugfine ftuffectiobts caourds: fo mun eis

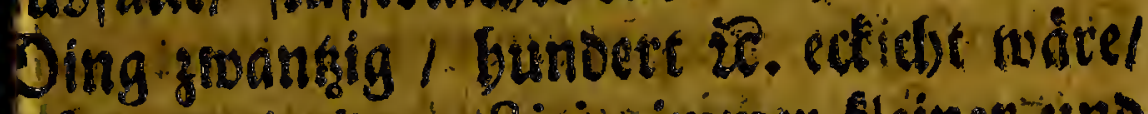
burbet de interftitia immer fleinec uno leinerfosis tho biecimen beftebet oer the etrdeio der grofent fleinenober gar feinen

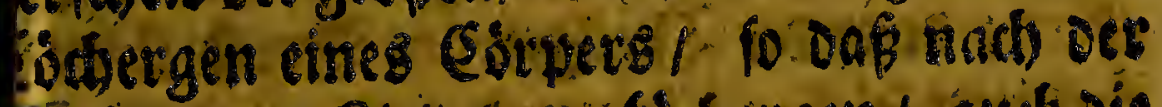
Srofe over Rleme oev \&doergen / atrib oie Ibtil Materie fid inebu unt mebr serbeict / uno nad foldser Detboilung ift vie soe Hegung langfamer ooes fobnetter. (Slciof) bie es fidi num mit oem (Silafe oon Antimio-

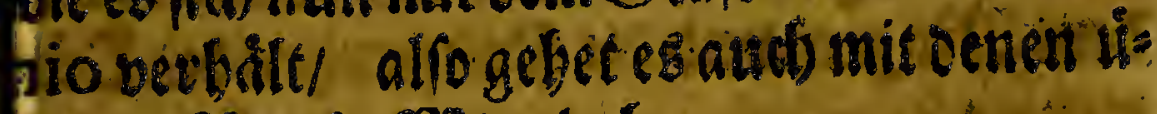
brigen Sted $=$ DPitteln ber.

XXVF. CE frage fid fetrie / ob oie

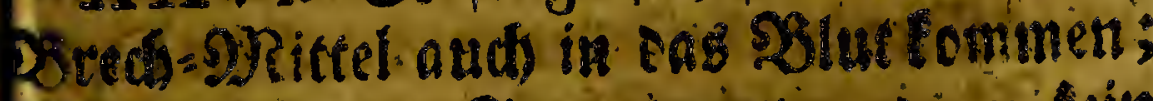
Sorauf id mit Ta antrobth 1 benn tein utoboringeno Medicament fan fo lange 


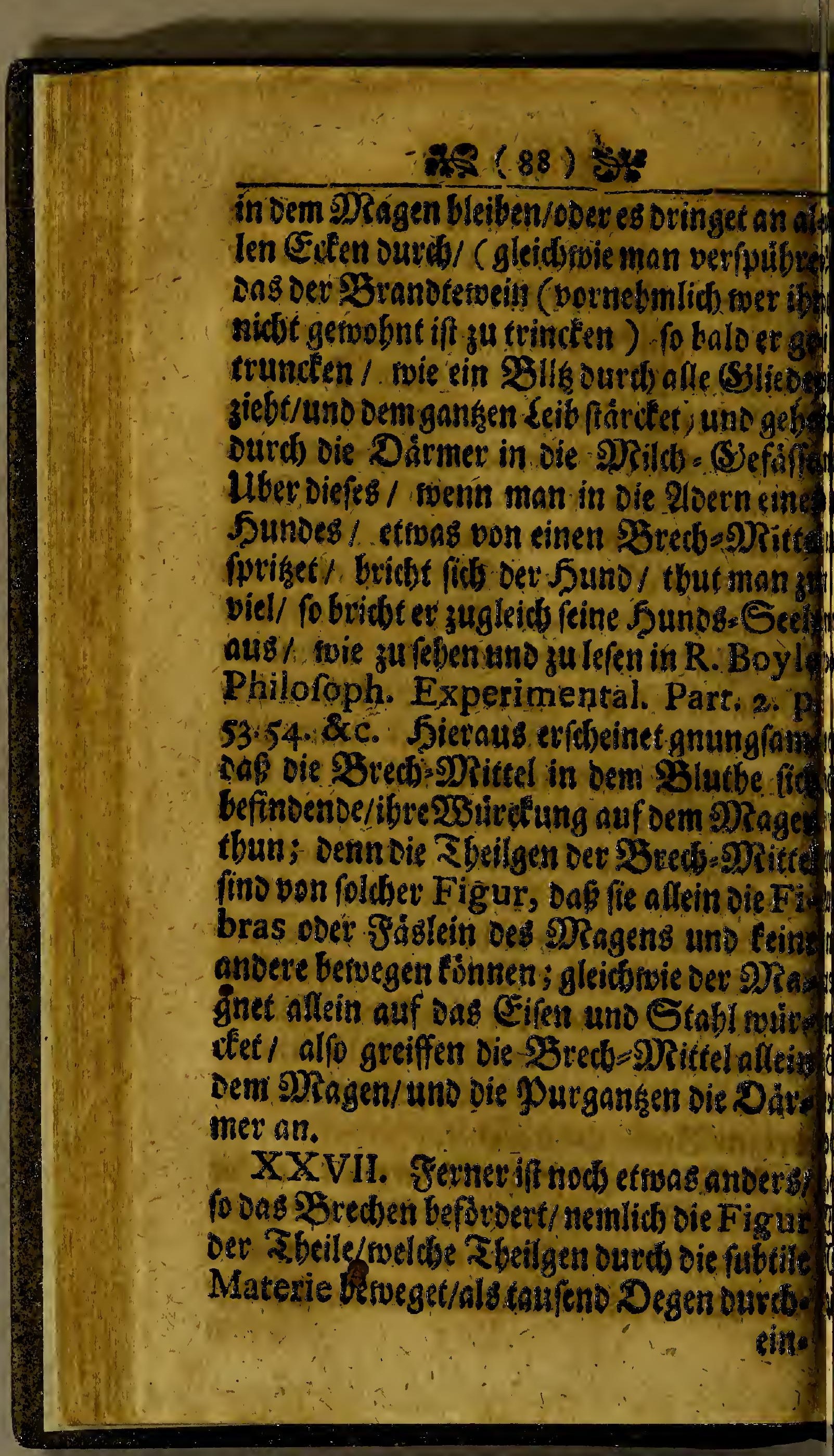




\section{8 (89) 5}

hatider fiegen/ und alles inas fle antreffen/ rferken uns zerftiditen/and foldes begiebt

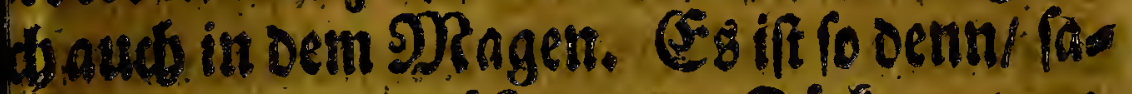

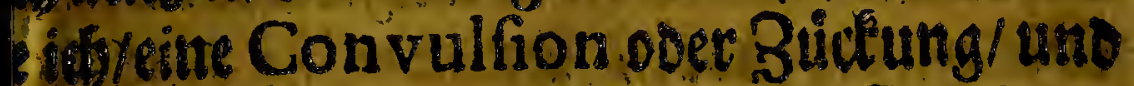

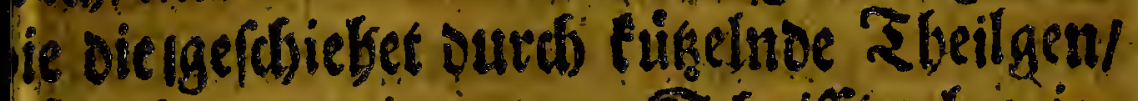
the id efrwan in andern Sofriffien bermies 1. Inofurs za fagen/man betradbse rove feiner regularen ooer ordentlicben Seemee

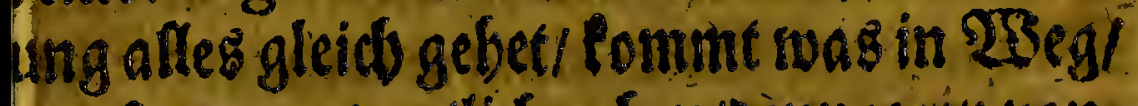

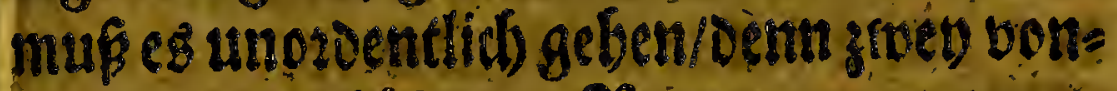
nanver unterfbiedene SSengegungen/ mas en cirre Ungleidubeit.

XXVIII. Die Purgier = SRittel fino

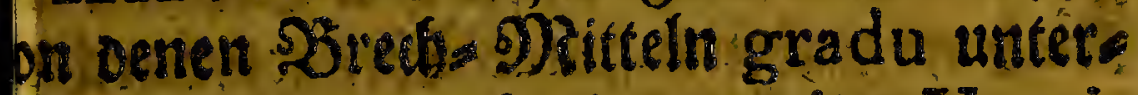
bieben/uno oarumb swiro ous einen Vomive eine Purgank/ mie in demt vorigen erfert ayitel if gesoabt wovoen: Ess fbeinet uns

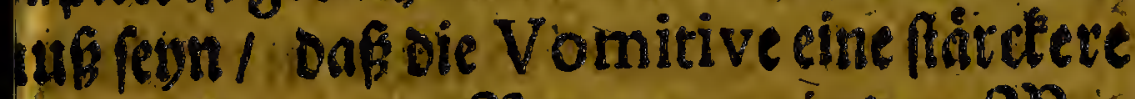

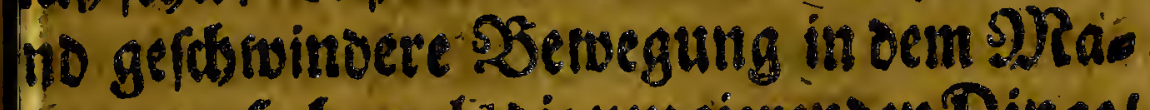
ein verurfacten als oic puagievenden Dingel no miffen poncinander differiren/ als Branofervein und $23 \mathrm{e}$ ein/ Denn man bes et: en 23sirclung eber füblet als oes léğten/

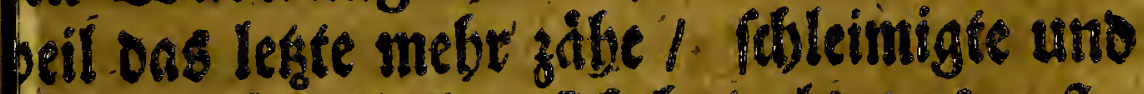
palprige Zheile bev fid bat ale bas exfe.

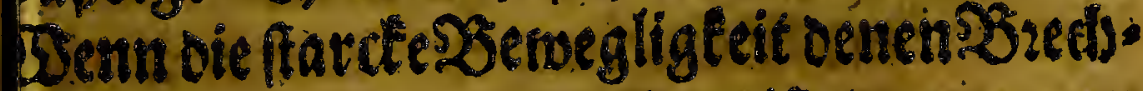
DRitteln benommen iff/ (Dasif/ / wetrin man ie Figur ibrer Sódergets 1 ba ber Durchs?

$$
85
$$




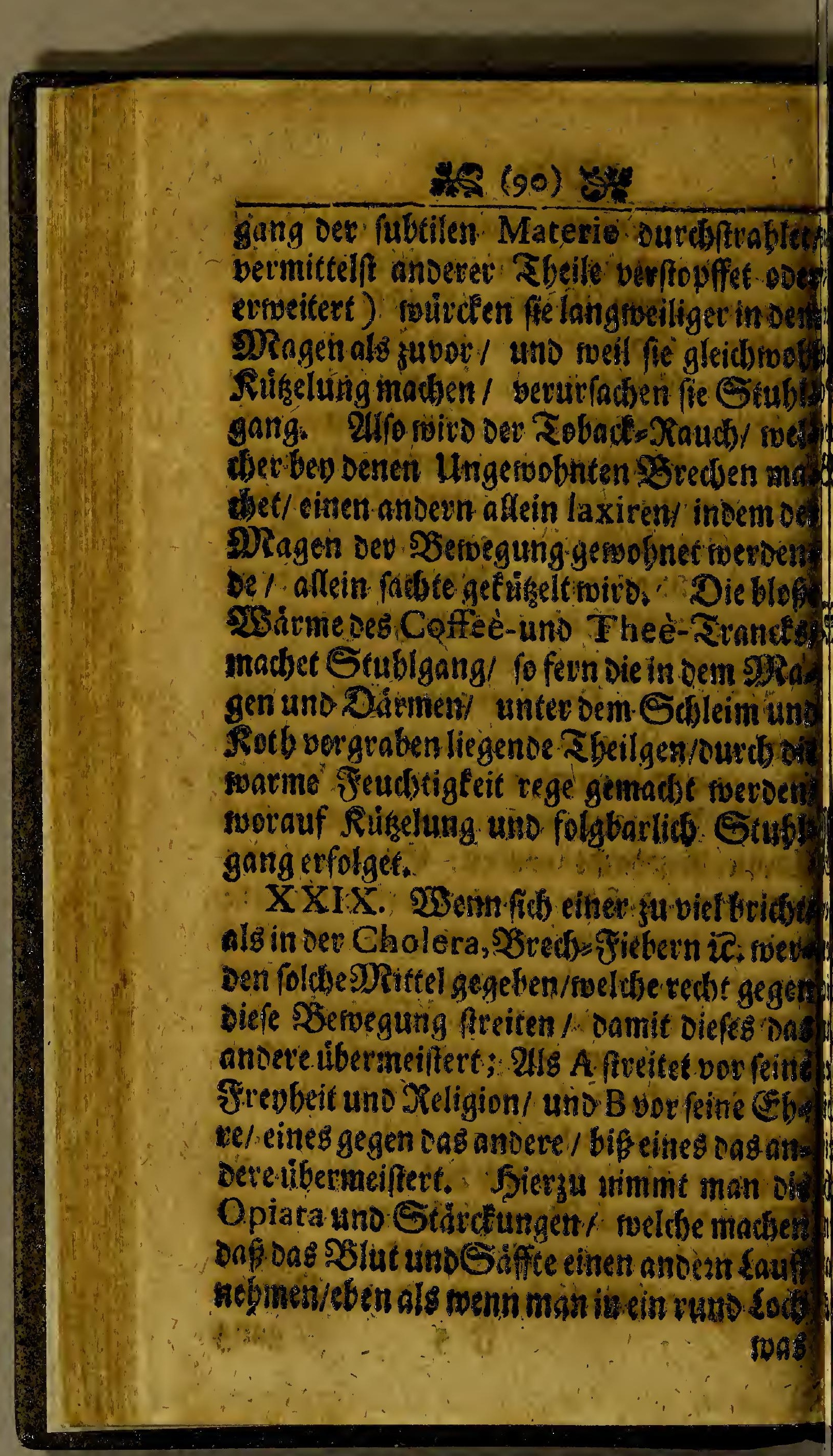




\section{Af $(92) \times 3$}

Sanct. Sanctorio, welchernachgeforfds bat / toie piel ein STenfos einen Kog lans ourch die 2uspoimp fung sec Saut los swird 23embeliebet/ der fan genenneten A uthos Davon nactirblagen. Diefe unbermerth Shrogmyfung ift fo notbwendig/ oas weni serte frand meroen/ez mangelt an oerfelbey. man folte berftem/ soenn oie Dünte teinen, 2tusgang batten/ oetn too 23 sirme uno vieh Seuchtigfeit ift/als toie in unfern Szlutbe/ on miffen $D$ ámpffe fevtn ifo nut unfer soib tois

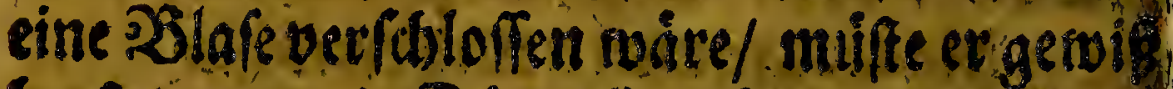
berfen ooer die Dámpffe miften jumsintun Debraug/ swic Der Raud aus oem Dffen! IDeld bes oes 233 inters ober gefdiebet/soeil oir Qlugosimpffung vezmimoert iftuno nadi tor stir uns mebr ober mebe erroarmen/ if bi Alusatbenung vermebret oder verminoert/ f. Daf Diefe SUformpffung ein Effeet un/ Szerunobeit if.

II. 2thein watumb bratdot man in benest Rrancibeiten fóneiftreibenoe Arfanenen (5 gefoiebet aus feinet andern Urfadsel als oamit Das SBlut cinen anpern Durngand von det fubtilen Materie baben mobrel uno foldbes vornebmlicbin Fiebern/ ba oa: SBlutb einen langramen sauff bat 1 foldses Seftall oaf tas Alcali uno Acidum, obe 


\section{$35(93)$ 3\%}

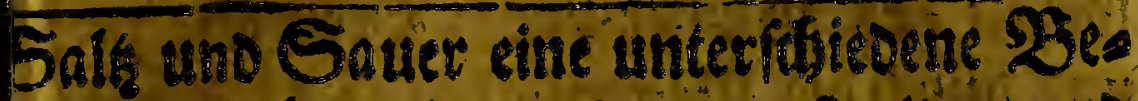
Degung baben/gegen sinantber frteiten/ und

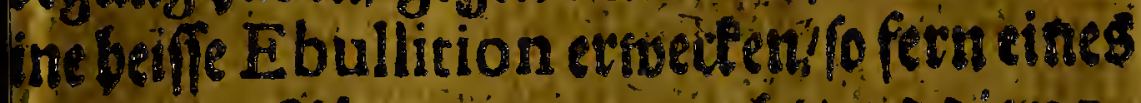
Es anteern Tis rgetr enger madit/und oie poitheiner werben/ saber mebr fubrile Ma-

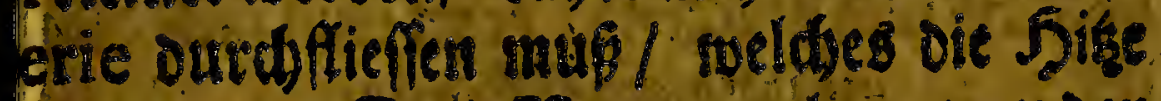
Emelinet. Diefe Dertwandeling nun be: Sheile/woelde audb oic interftitia boer 3wis

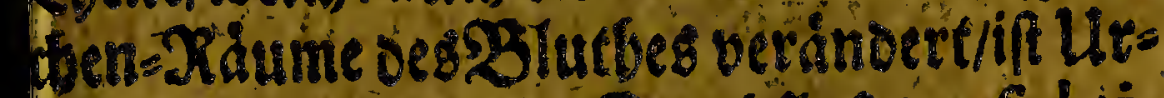

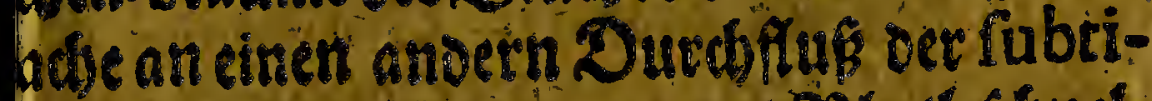

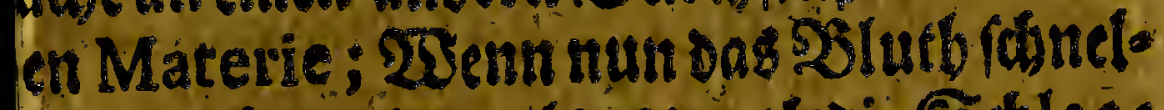

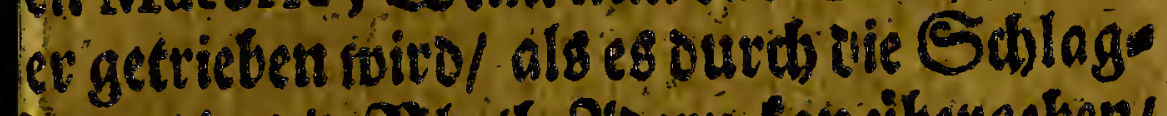

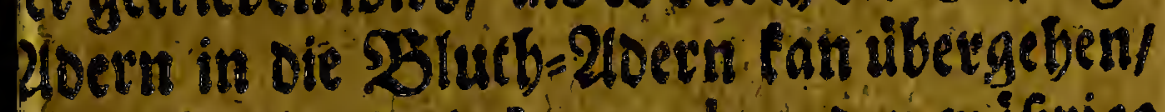
Hgleidfen outs oie Ly mpha ober waíprige Ferudstigfeit/fo mus ein groffes Tbeilgeudh.

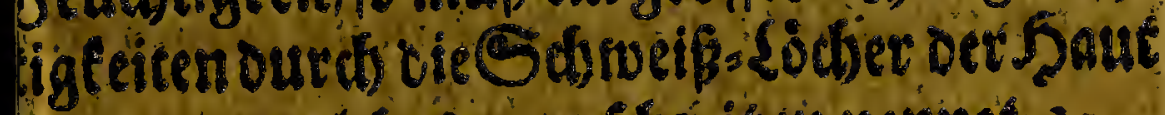

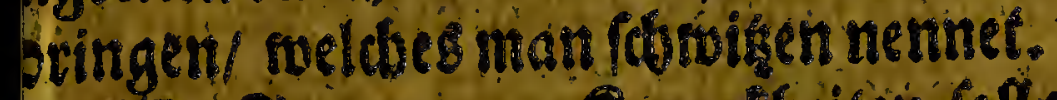

IIf. In was vor Rrandbeifen foof man

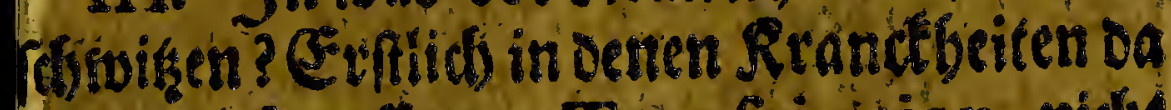
Die RluBdampfifung/Tranfpiration, nidst fwobl von tarters geteet/ als in allen bie mit

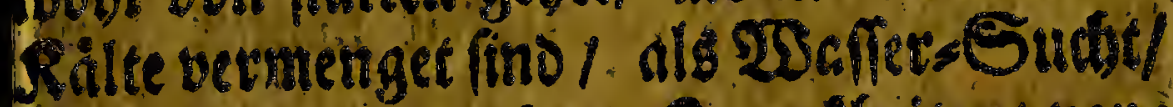
fund aflen folleimidsten Rrantclbeiten/ wors

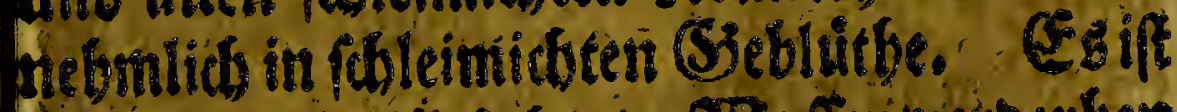

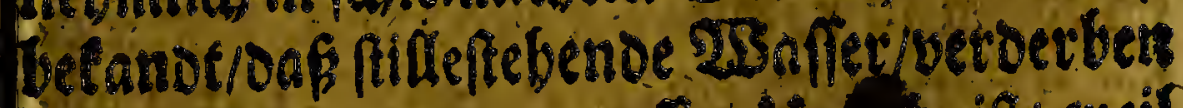

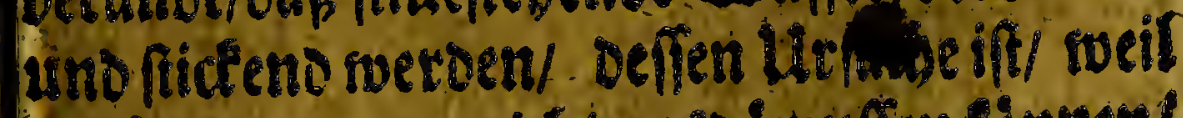

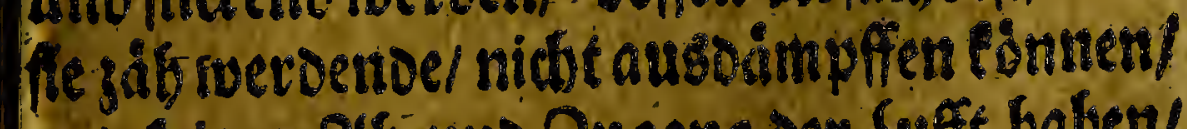

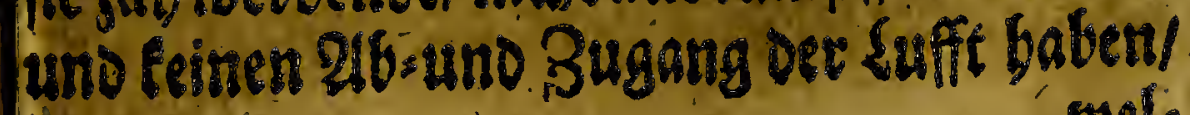




\section{काषे $(94)$ है}

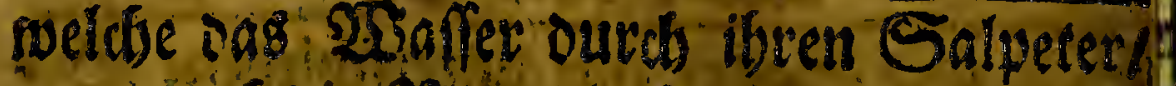
uno our w oie Soervegligfeit fo Darinner if

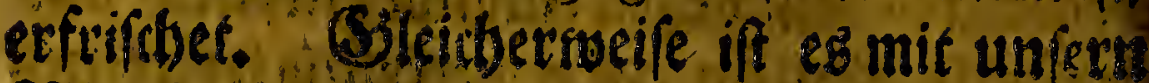
SSlut beronnot wenn es betbindert noiro/ ir tranfpiriren ooer Durdo ompffen/ wivo es foceno uno finckeno / Dent esiff sch / uno betomme eine lathglame 55 évegung. Sn fols

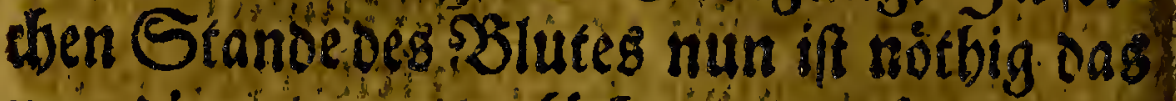
man die poros over sjctlergen veranoert/fo. thanig Dap ein giofferer Durdbgang oet fubtilen Materie erfolgetl sooourd mebr Servegung in ons. SElut gebracbt wito; in folchen 3uftanoe roecoen oie Feuctigteiten ourdh alle rubulos ober Xobrgen oes ́eibes ftórcer gepteft uno weil ole Jeuchtigfeiten negen ichnefles. Seroegung in die zufubren. Den Sefâfe fatroter einlauffen / als fie bot Denen affibrenden fonnen eingenomment suerden/ ro begiebrftef / oas thebr Jeuchtigs feit Durch oie Saut getrieben mits/als in oet ordinar Xusomimfing zu gefdeben rifte. get I uno oiere Teudhtigfeit nentiet man Sifroeif. Durch oiefe serwegung veratt: Dein fid auch oie pori oes SSlukeg//10 oag det Sdoleim allmáblich veranbert uno reiffer os Der etwantorts einige Someip=sicher ab gefubret nirs / ro das Subeif - treibende Dittel eine foldse(Sifalt ber Sidgergenpo. 


\section{W $(95)$ s}

tram, inoems blutbe madben/als in Stane oer (seftut beit erfodert suito.

IV. Jeuner dienet Das Sdomigen/ment ne Ebullition oder Alufwallung in oenen eudtigfeiten if/ als in Fiebern/Peft/ unt en Malignis ooer \$bogarthigen Rrande iten / alliwo daz Szlut nicht allein zu dict। Heern auds aufwallet uno beis if : Dents 13 Alcali uno Acidum Salgund Saue betsint negen gebemmeten \{auffeb/ (Se: genbeit mit einander zu ftreiten/ melches die oros des. Sglutes in eitren folden Stano inget/ oas oie fubrile Materie ftard ein inget/ und weil fie forwerlids noicoer can leas to men/bleibec ein sbeil oavon in cem den-25lutb) Darauff wiedec andere folget? is diefes madot oen Seuten Sise unt G̈ieber. Darumb tnus man Dureb begume Mittel e. Poros in eine antorte Figur oder (Sieffale ingen/ Danit Die Iuffwaflung verfolswing

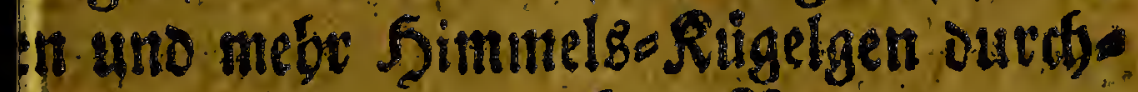

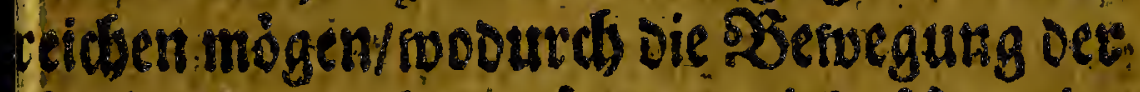
btilen Materie verámbert noir Biftoie Utradbe des Sthrwitgent.

V. Ssiebr man aber denn die Sduneifs.

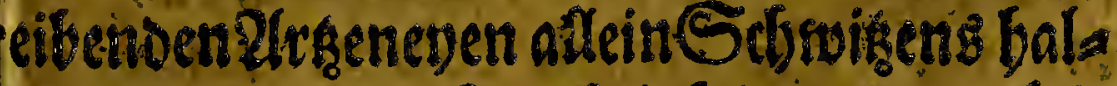

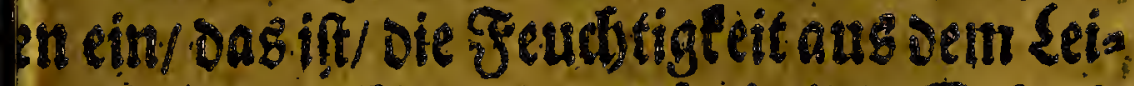

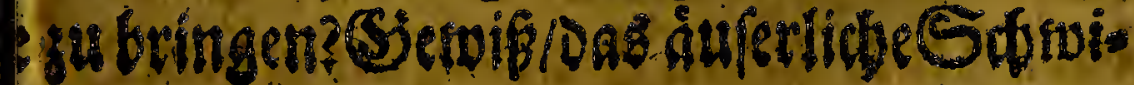




\section{the $(96)$}

gentbutmenig ben der Gadiel fondern do abreben ift/ ons die Pori des Sslutbes un Deffelben Safte forfen berandert suezonn/rool

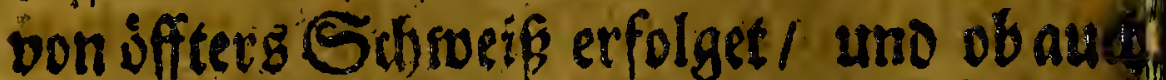

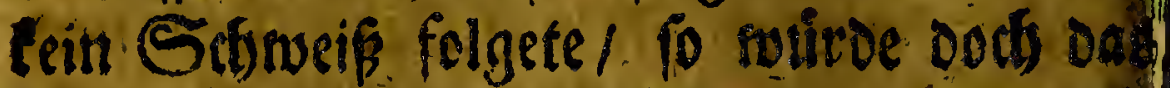

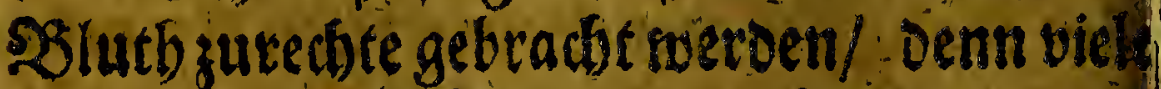

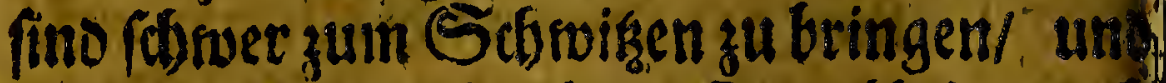

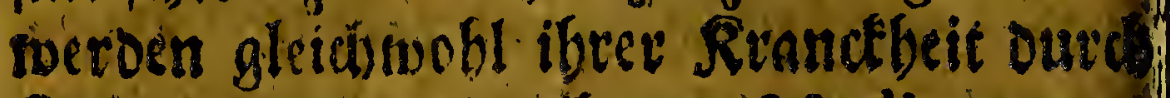
Qjebraud formeintrabenoer Medicamens.

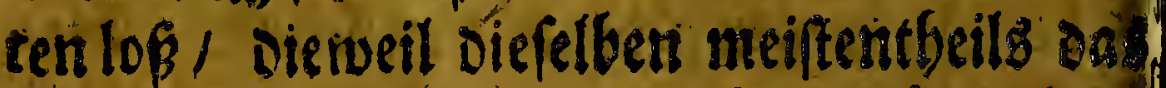
Saure temperiren. - Dex sufeclichs. Sonneif thutalfo nidhts zur Sadge/ uno if nur ein Confequens ober cifolg von oes. 25 urcelung in sem PSlutbel benn gleids wih man bey gefunden Stande allein cine gut Tranfpiration ooer 2lusoàmiffung mul

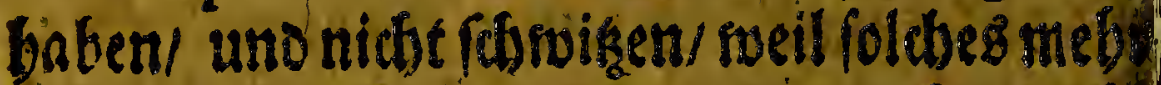
als Zluroaimpffen iff fo bat man eben nich) notbignach gegebenen Sobroeip-treibendent

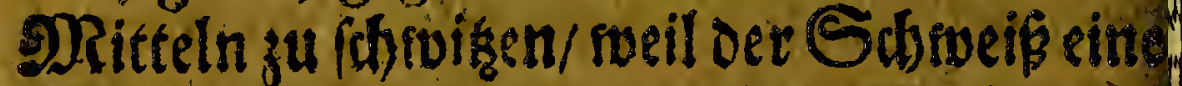
Sorte von lluin if I uno fo toenig barnes.

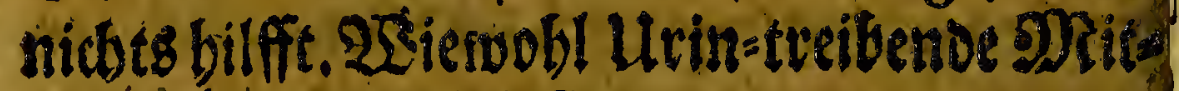
rel auch febr dienlich fitts.

VI. Tos babe geragt sás etlidbe leich robwisen/ ansere aber fobswerlich sarzu ged langen. Die Urfache belebet Darins/ oaf tinen oer 5 arn leichter abgebet/ uno folg? batid nide fo viel Jend tigteit in oents lut 


\section{$40(97)$ B.}

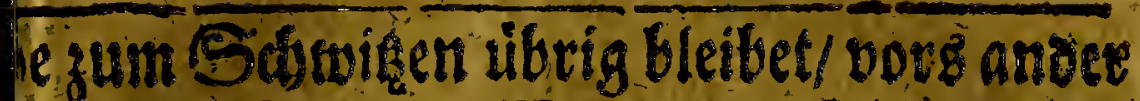

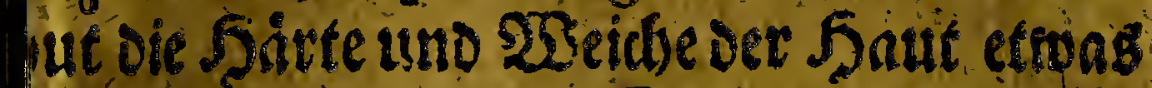

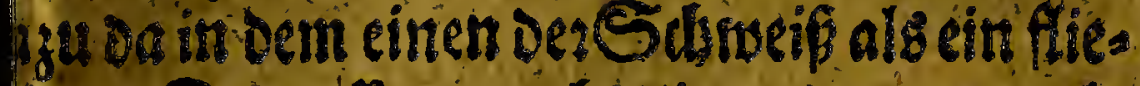
toer Dampiff weg geber/in Dem andern als 123affer liegen bleibet. 2uch if mand

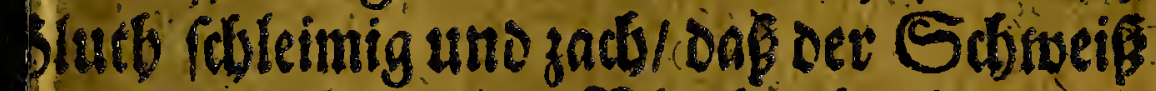
dot teifjetlid von semgluthe abzufondern. De Enge uno 2seite der Róbrgen / uns ren Derffopffungen tfun audi viel Dazu. netlicben if sie' $T$ ranf piration ober2us?

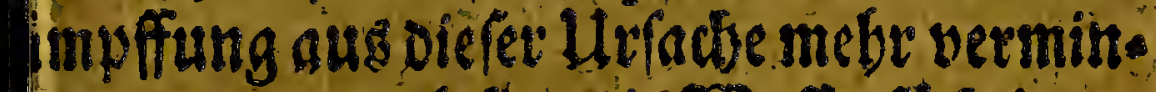
It uno oarumb find Die WSafferfüdstigen/

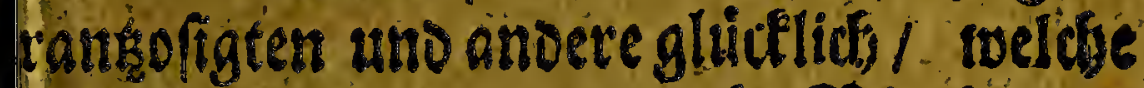

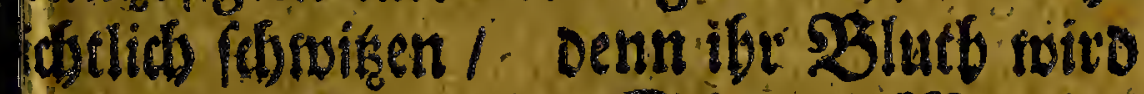

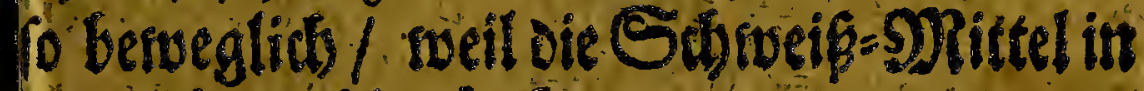
len nidtyt twobl nuivclen.

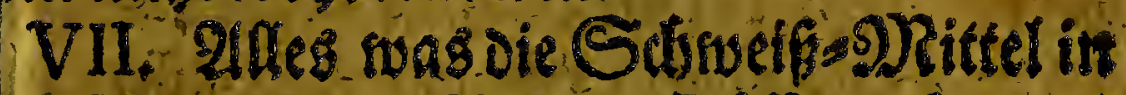
nn Slutthe uno ubrigen Sáfften tbun/ if dots andeers als dấ pie die poros verán th/Damic ein anderer. Durdigang Der Suffi no fubtilen Materie in sem?2lutbe zu wes (gebraches serbe/ eben als mie der 'zeig

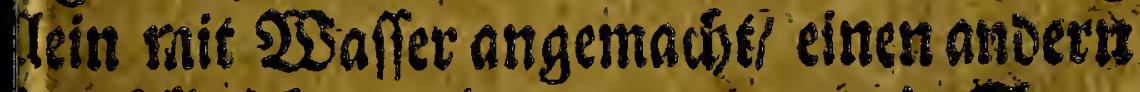
burdyflü bat/ als fwern ein twenig Sam. - Teig doer Sefen darunter gerbantift:So ubalt ifths mit Dem Blutbe and 1 in wels en nath proportion Des Ferments, bers bichen oie Medicamentáfino / die porí 


\section{और $(98)$ गैं}

fich verandert/ wie oent nuch ein Fermen inebr treibetals cas anoer: Soldbes fiebs man lelb/t in oer S) Rildb/ die/ nachoem ein ber bas anoet Sauer Daunter gegoffe birol auf unterfobiedene neife gerinnet.

VIII. Da fino nun unterfabiedne?trtbe von Santisen/ als ourd Baven . Bero gung / ourd Dampff von Branderiveit cuiferlidbe $2 B$ arme / beisgetcuniten The Coffeè \&c. Wâfer/ eingenommene $M$ dica menta \&c. Alnlangende vie $B a ́ d e$ fommen fie entroeser aus oer croen! bel uno mineralifal gleidgroie gu Qlfen ut andern Drtben zu feben : Doer fie toerde Surch funft; mit oder obne 3utbutung as derer Dingebereitet. Daf vornebmfte bo ibret Rraffe ift die 23 arme Dez $2 W$ afferg un Der Tbeilgen die darinnen fino. WBenn our - Dasissaffer uno die wárme die Saur et

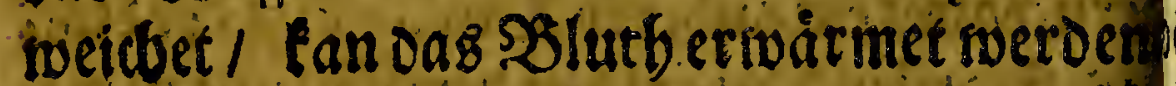
uno biel mineralifhel ober aromatifo Tbeile oer Rrauter ourdboringen / fooburd Die pori oes S3lutbeg veránoert/ uno o Riandfbeiten/ nornebmlid ivenn Rraff

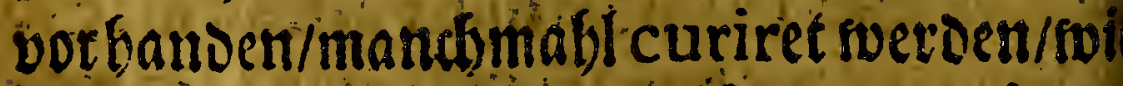
fooblfie offeers l toenn fie foleinen curiret? feoth / roieder fommen utro oen. Soot mit it: bringen. Dieje 20 arme in ons şlutb fom 


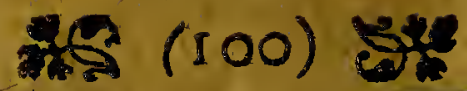

(roie fonft mebr erwiefen) oa ež nun in eing Stunde etlidbe mabl ourd oie Sunge pafl ren mü / foir o es continuirlid angeftede fo oafenolid) ade Treudstigfeiten in Dem Seit

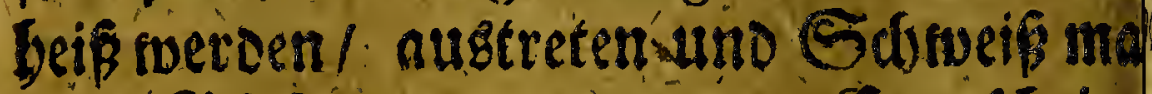
dien. Id) fage von austreten. (5. crfobein Diefes zur (Sienugel wenn man eine Flafa)

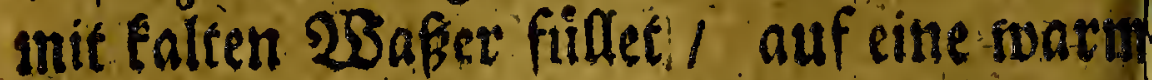
Stäte ferset / foláufft cas 2 Saffer oben aus neldoe 2lufiteigung von ber Wiörme berru ret 1 uno ift oas ausgelaufféne 23 affer des Sdoneife gleidy/Der ourd die Rlobrgen un feter Saut oringef.

X. VBenn man bif auf oem Sdirveis g. arteifet/ over ftard gegangen / /o /chruik man nods mebv/ nachoem fich einer nieder/s

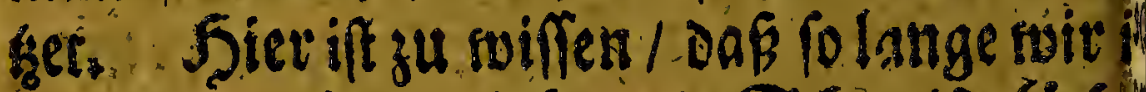

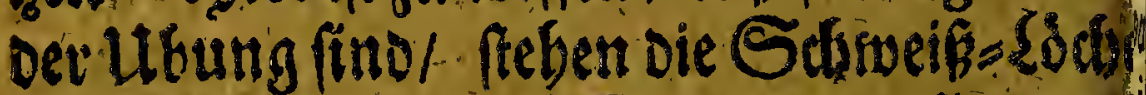
sweit offen 1 und de Sdyntis berflieget on ein fóneller unto unfichtbarec Dampff/ nuif

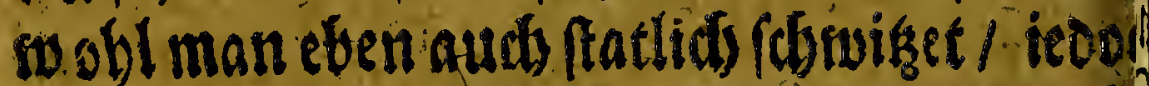
biroman es in Stillefísen mebr gerwab

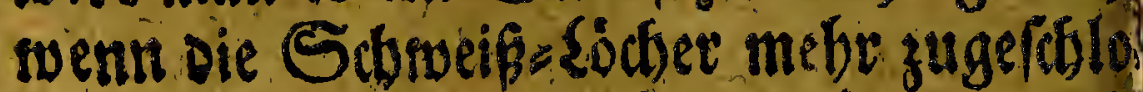
fen meroen / Hno oex Dampff untertieif yon oev calten Suff geftebet/ fo fuird et

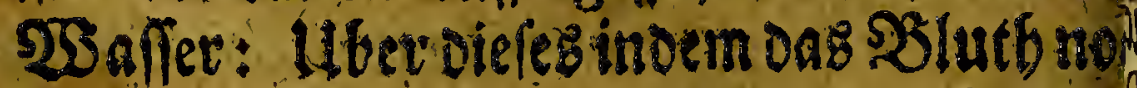

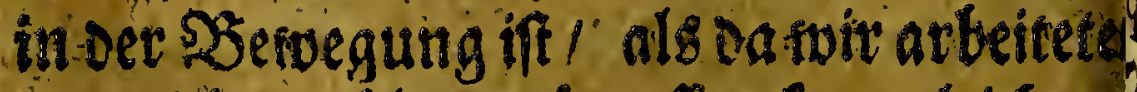

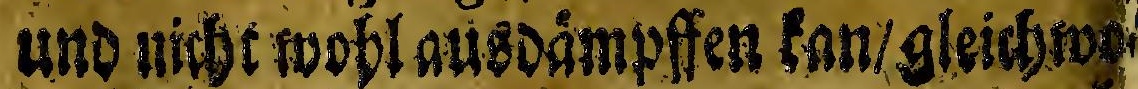




\section{0 (101) 5}

bet ausodmuffen mug/ fänget es ain aufzus wakent (oenn wir reerber beifer;) Diefes

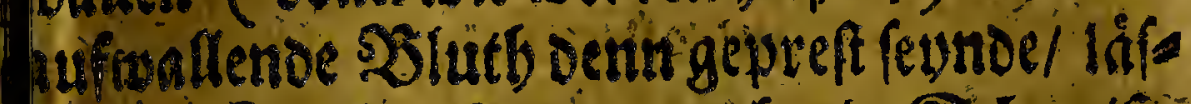

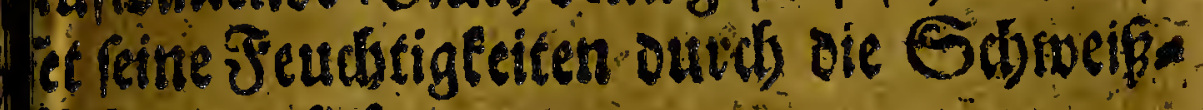
Sedeer ausfiefern.

XI. Jielmabl folget Sthineis auf ons Euludien von Theè, Coffeè; twatme

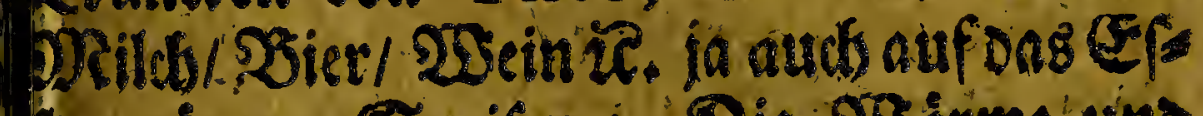
ent oinner Speifen; Die Fbatme uno Feudtigfeit der Syeifen/ erriatmen das

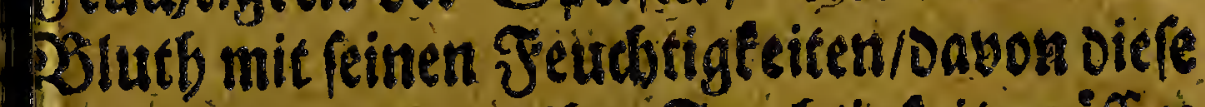
hudb austrecten utuo ibre Greudstigfeit muifen pusfifiefern lagen/indem ro biel nidbt tan zautiofe gebern als berzu fuiffer.

XII. Utber Diefes das de rearmen Feendis.

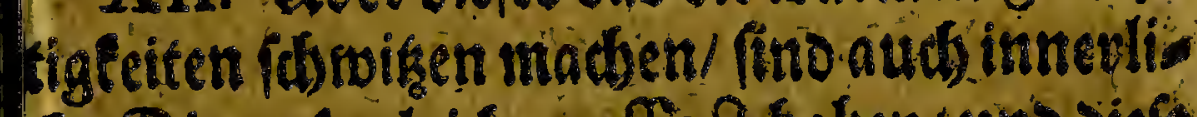
bie Dinge fo gleidien effeet baben/ und oiefe

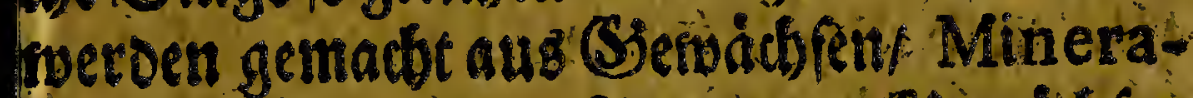

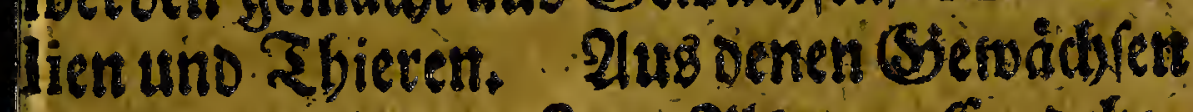
Hitadit man Extracte; Mis bon Carbedice medicten/ Seordien/ Ingeliclel Saffrant Contrayerva, Opium, Eorbeern/ DR Raan/

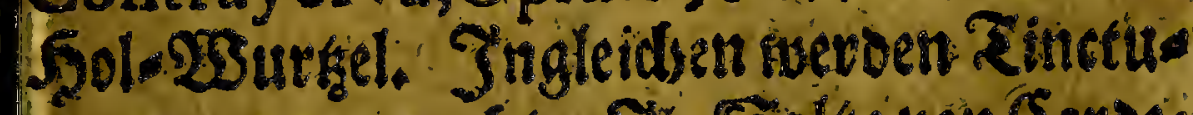
Een davaus gemadt: Tt. Salse von Cardebentedieten/2Bermutb/Scordien/ Rauten/ 22. Ifus oenen Mineralien ba mant etlidse-

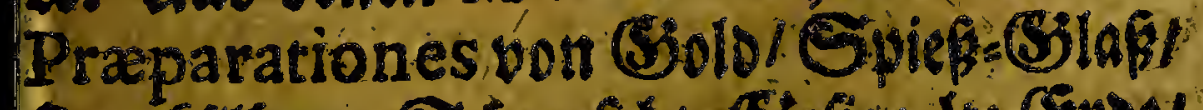

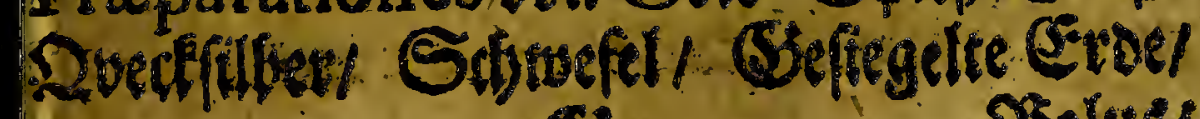
(अ) 3 Solubt 


\section{$x \in(102)$ set}

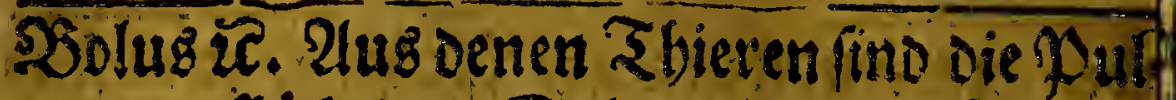
ber uno fludbtigen Snikel als von Sublant gen/ Rroten/ uno affen andern Sbicren und beven Sbillen/ als Solutb/ 5arn/ Sialles Drift ze. SEezoar=Stein/St)mein=Stein 20. Diefe Sorten werden entrweder allein. einfacts eingégeben/over Deren unterfobiedens outcheinanoer gemifdset sie denn die altes: (bren Sheriac/9) Retbridaat/Diafcordium, 2C. baben. Anein neil diefe Compofitiones groften Theilz unnuike Tevn I uno ang vielen Dingen befteben oie zur Sadsenidst geboren / fo verroinfft man siefe billids uns machet mit soenigen limb fảnoen beffere.

XI1I. ante oiefe Dinge// fie fenn nie fie fewn/ mactien fhrovigen/ oorsebonlid went oas Opium sazu fomme/ Denn fveder She? riac/nodb PRetbribat/noch Diafcordium,

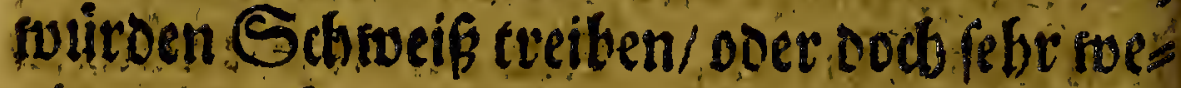
nig/tuenn fein Opium oabey fwáre. Dent Das Opium aflein 1 ooer sie zuglecid benges Fergen QRittel fino von folcher Figur uno Durdigang/-oaf oas szlutb einen gants ant Dern Ourdbfuf ore fubtilen Materie mue befommen/ uns exlanget mebr 25 satme/ one ourch es ausgeftoffen woiro / uno mebr in die anbringenden (Siefábe fommt/ als ourch oic furtidbringenoe fan verfubbet necoen/ uno

weil 


\section{$29(104) \div$}

Get / Denn viel Rinder firto mit fchionikendet Trufent gevlaget $/$ fino aber uno bleiben ib Lebenlang bis ing bobe aller gefuno: Zlut

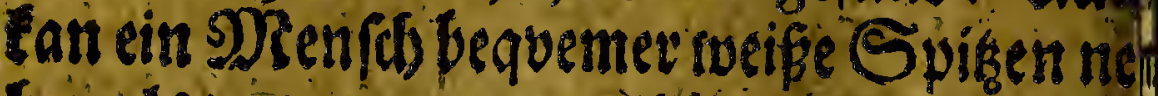
Gen als das anderer meil êines sánce mebs

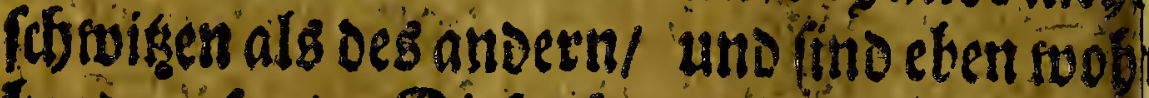
bevde gefuno. Diefes fan gefdo ben/weil ded

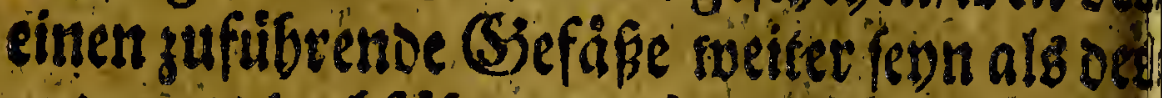
ansern/ Die abfibtenoe aber enger fino/ oed balben mefr Jeuchtigfeit zu = als abgefübr suiro / uno mus alfo continuirlict efrom Jeuebtigleit nusfrefern / weldves. Schncith if:

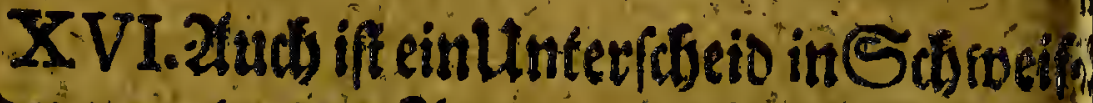
fe/Denn unter den Ormenl aufoem Saupte. an Dem Jtifen/ beimlichen Dertern/ if oet Sdhmeis von unter rdhiedenen (Sterud). Die fes rubtet alkein oaber / oá bie Druipges

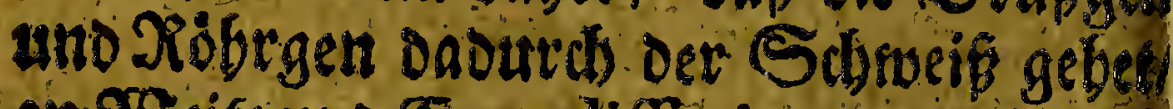
an 2 eife und Enge differiren/ uno allo an cinen Drte fleine an ben andern groffetes FGeilgen ourchlasen. So if auth Det

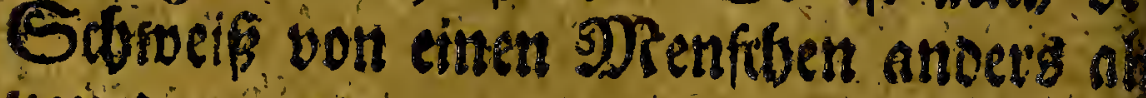
yon bem andern/ inden ber eine weitere Schineip = \{otber bat als bet andere t uns Darumb finder Paulus metbr als Petrus. uno lener bat findendere Fis als diefer weil an Denen nistafein viel Sfineisifon 


\section{$\frac{1}{25}(106) 3$}

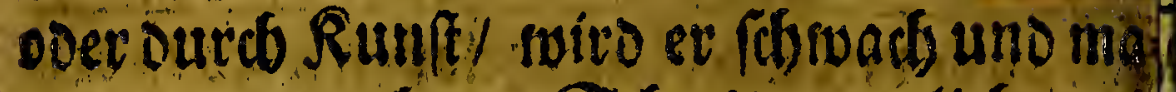
ger/ Denn ourds o,as Sdbmisen verliebret en ner viel Rriffe \% uno oie Rafrung geb el sweg/in er verfället in zebrung uno wsaffers fucht. WBenn oas SBlut feiner Alidytigen Tbeile berauber ift/mizo ez dite uno untraf tig. I woraus viel Rrandebeiten enffeben. Dannenbero die an Jranks ofen curiret nove Den/ uno die Cur gebubrlid ausgeftanoet!

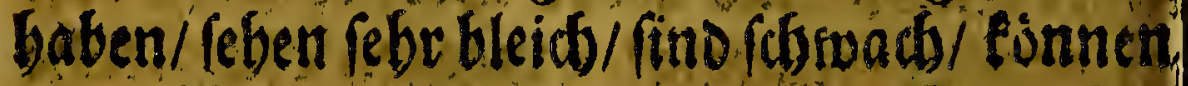
fibsuer fortfommen/ jafie meroen Sdsarbo dícht uno las.

XIX. 2Bsenn man eine Durgantseinge geben/oúrffen leine $S$ dssueiftreibenoe DRit tel gebraudbet rueroen/ bebor die Durgan

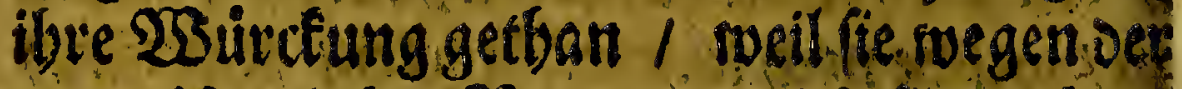
unterfhieolidben 23 ervegung/ fo fie mach an cinander binoerten / in es folte nobl bon tei nen die Wrututung bonftateen geben / benn gleidsuie oas cine von affen Sbeilen nad Den Dámern filguet/ fo gebet oas anoere in (Siegentbeil pon dem Centro oder IRittenl nadj dee Circumferen oder Hmbfange Alfo mus man autb Das (Siegentbeil nidf thun/ ieoods fonnen fie bevfammen fteben: tonn oie dofis oer Yurgantis noch einmab lo grof if oaf fie oie Dberband bebalt; Dent menn eine Durganis nicht gebovig folirces 
Tho in oem seibe bleibet, fol get nobleinduse. Alen Des Saars Darauff/ oie Ringel rwerden hwarks/ an oem gantsen seibe fabren 23 afs 1- \$2lafen auf/ die enolich einen gruinoigen Ddiurff unacben.

X X. Tadh vielen Schrwitgen folget: eidbrie auf Das Pur gieren Durft/weil oas Slut oet Feudatigfeiten beraubet if 1 . uno 18.oemelben nicht genungramer Speidbel oen \$) Runo fommen tan/ wovont Scocine is al io. Durft entfebet / oazu Theè uno offeè wegen ibver $2 S$ àrme oienticber fino s ander fúbles (Sietrandic.

\section{Das V.Sapitel.}

Bon Denens Sarntreibenden Medicamenten i Spadanifden 23 affer und Defen unterfibicoenen

\section{Gráften.}

I.

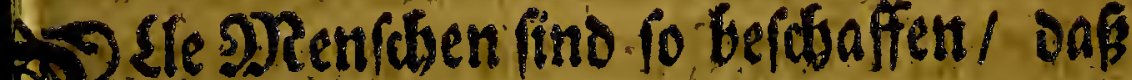
At an cinem Dutbe die groben/ an einem been die duinnen Excrementa ausges beet werbett. Die olinnen fo uiberffüsig bem Slutbe fino/ nerden contin uirlids und Dic PRieren abgefondert//nus Deren tubuliṣ 


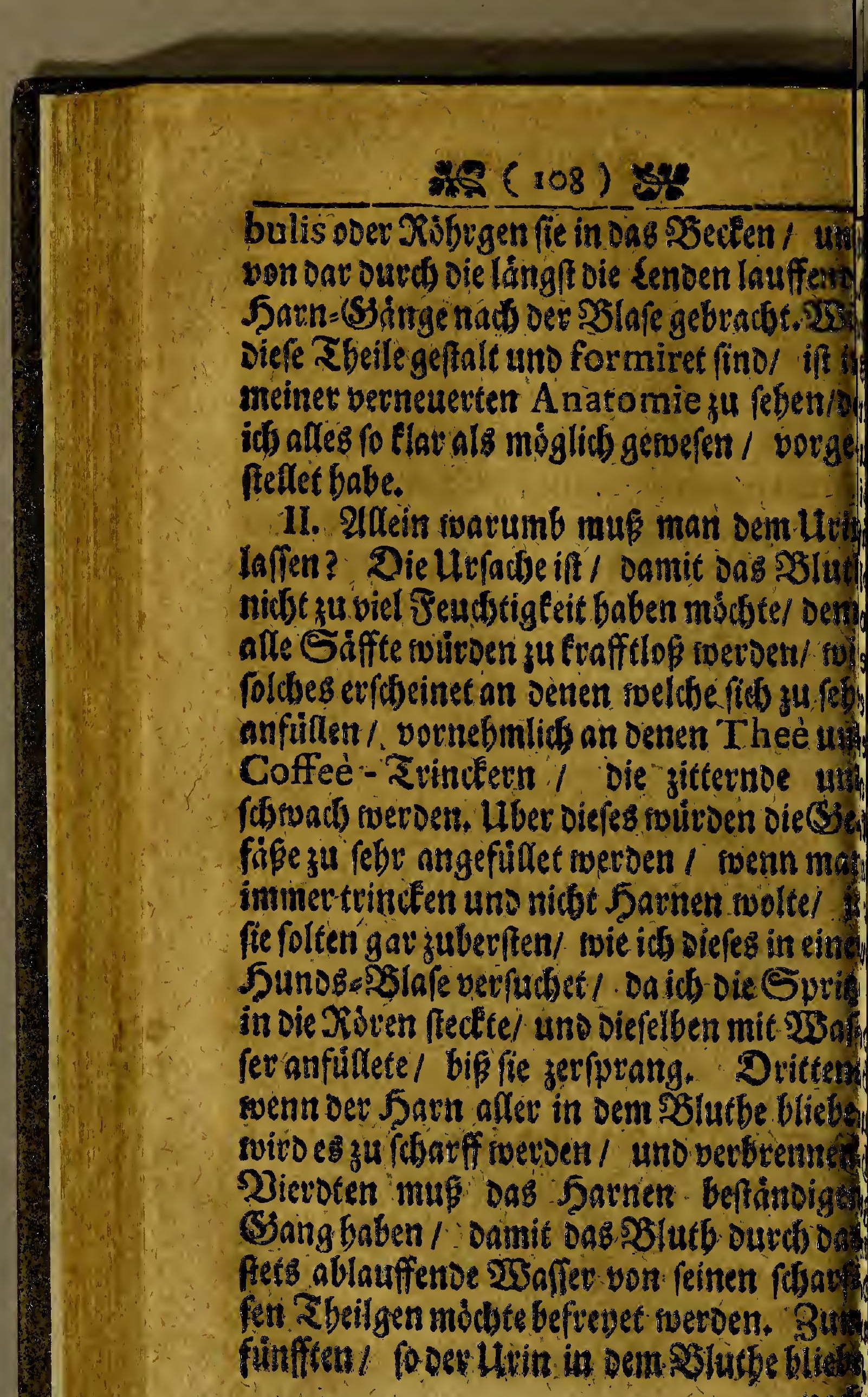




\section{Iิ $(109)$}

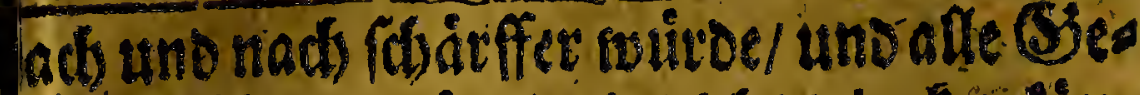

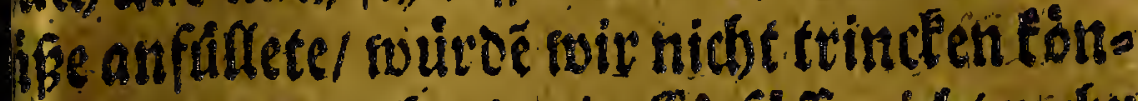

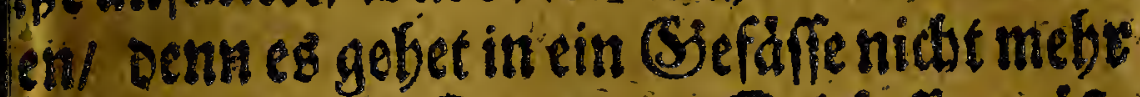
Is Paum bat; Durds bie Sabárfe muffe er Speidfel fojarff und falfigig weebden/wors us Durfentffunde / und gleidjerobl fointen bit nichts neinbringen. Sedffeng fo bie (Se) äe allezeit mit Jarn angefúfet blieben/wo Dlec Dern oic Nabrung in sem Sslutbe ber. ommen. Sietendes ivurbe die infenfibee tranfpiration ober 2ubsoampffung it

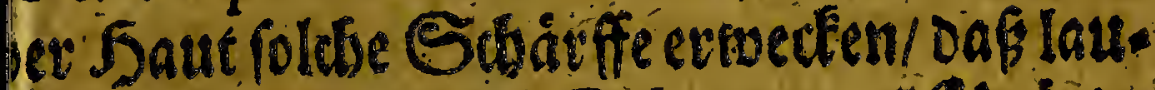

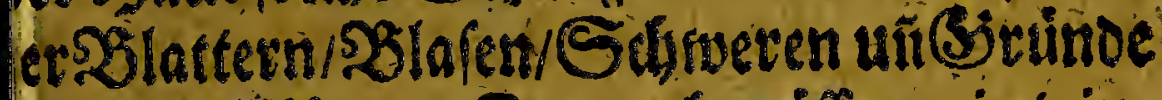
atan auffilibren. Darumb uniffen swir trin:

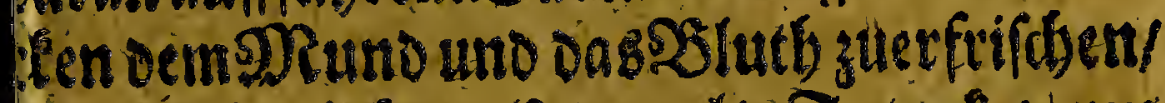

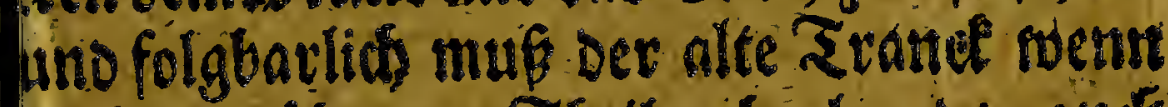
xx feine nábrende Tbeile abgeleget/ aur burds bie Jain=-Siange rweg geber.

III, Es fraget fich fertrer was eigentlids

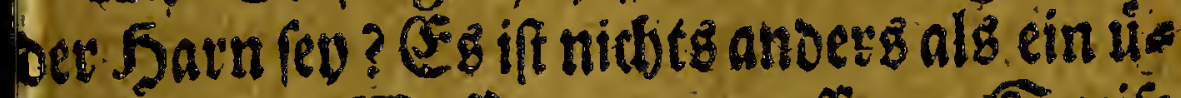
berffipiges 23 afier yon gentofiener Speile und ₹rand: Denn weent wiv zu (5)afte ges

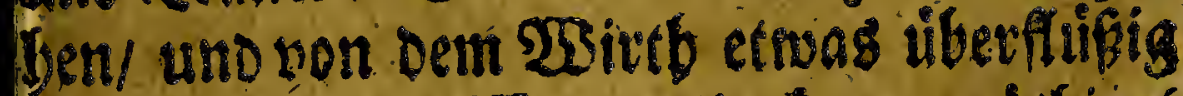
sum şier boer 2 Sein trindelen senúthiget fuerben/ fo iff Das erffe mabl oer litrin gelbe!

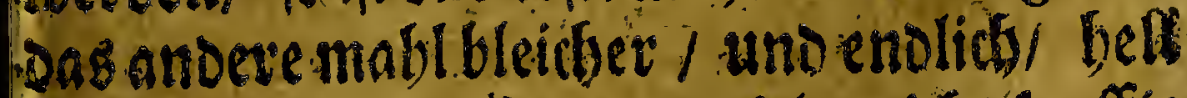
and Elar rive $53 a$ affer/man folte niddic fo offec

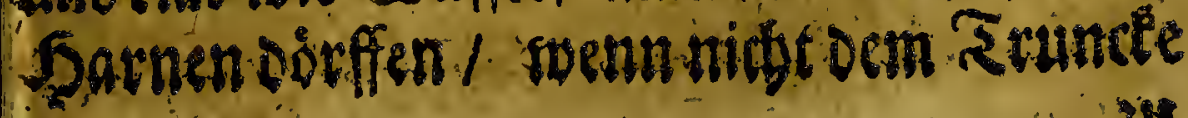




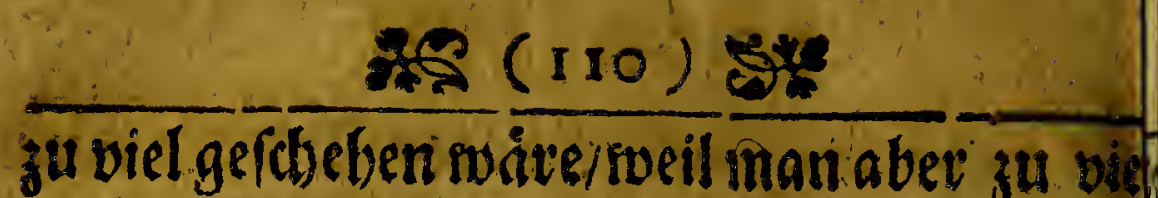
getrunden/Ean nidbt alles in Den SSefáped bebalten mezoen/saiumb laufftez contin u.

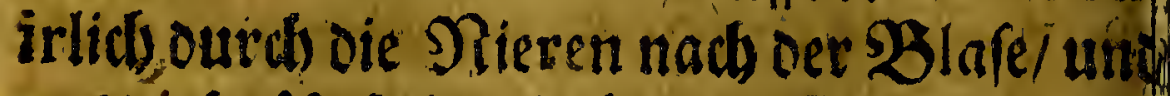
aus oiefer Urfactsen baben die Såuffer grofd

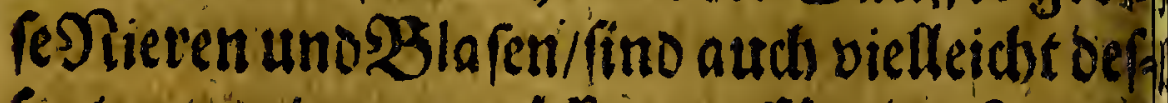
fentwegen in oem geoffneten Eórper/5errem Doct.C. Bontekc.diesieren fo gró

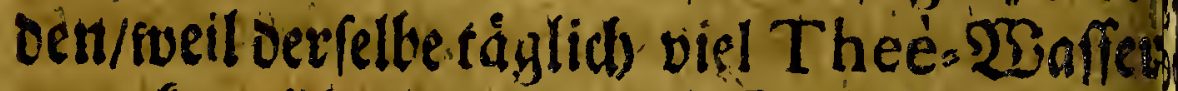

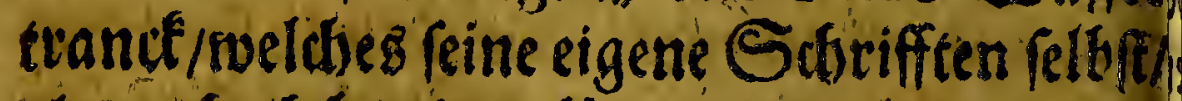
als audb th bezeigen fonnen/ift alfo oer Utrin Die überftüige Jeudhtigfeit suelche aus Dent 25lutbe abgeionoert niro.

IV. Disfe Teudbigfeit if gemeiniglick

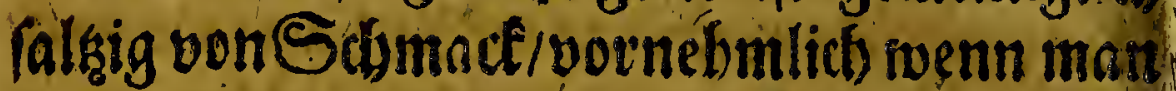

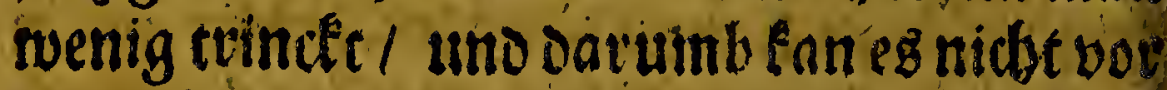
ein recbtes Serum oder 9rolcten/mie ez ing gemein genennet swiro paffiren/ fonoem allein vor $\mathfrak{W} a f f e r / b e n n$ rod einer viel getrunclen bat/ ift oer Urin noie gefagt bell uno flai uno obne S3efhmadt mie Xegen = Waffer. DabSerum oder 5 Doluten if in (Siegentbell fuffe uno gans anoers als $W_{3}$ affer. Liber viefes noiro inzgemein davor gebalsen/ oaf Det Utrin fo obne vieles trinten neggebet/ ein us

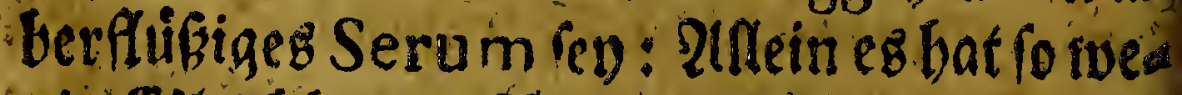

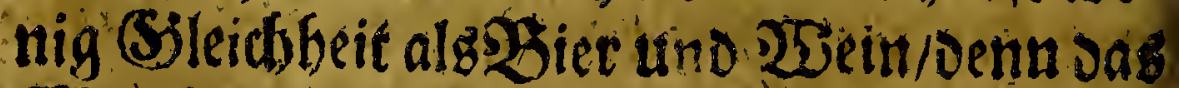

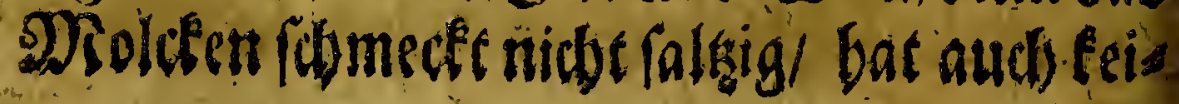




\section{at (i1i) ste}

en(Seruds noic Salmiad / giebr aud fei en foldebri burds oringlicten Spiritu m nie et Sart. Cg if alfo oer Urin genteinigs 6) ein Sals= 2Baffer beldies in oer Speife uno Trande ift/ la arget

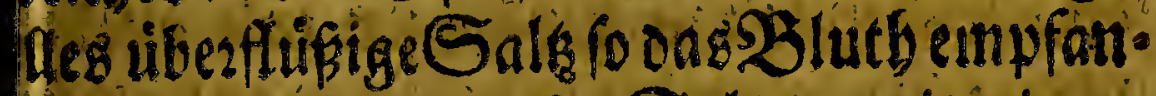
en bat/auz/ uno ciefes Sals/Damit wie ge-

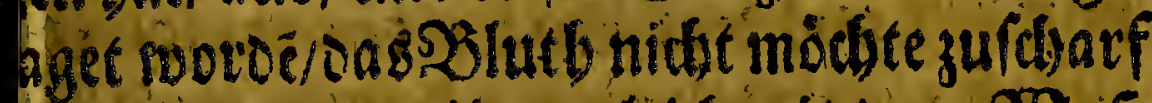
berden/ swito alfo jugleids mit Dem $2 B a f-$ er ausgefúbret. TSseñ sir aber zu viel naffes

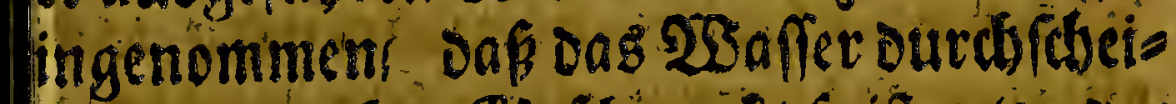
lig befl und obne (Siefdimait/ fo if entriveser

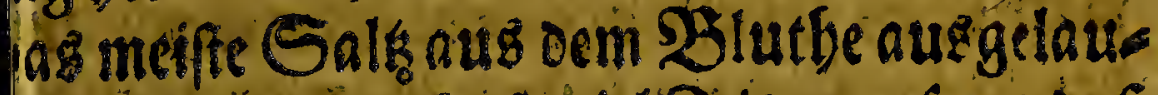
Jet/ ober es ift nidst fo viel Zeit getween / bafs ines mit oem anoern fidh bat veteinigen sinnen.

V. Eg if eine Jrage/ of oer Sarn went han viel tritutesurdo oem Dragen/Daimet

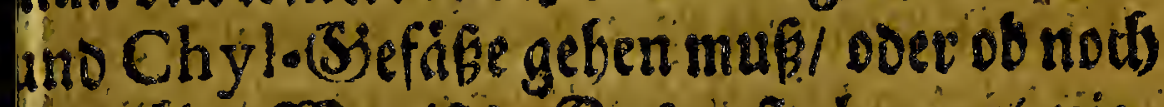
in năber Wseg ift? Das erfte leugnet nies hand/bienveil es genvis if ong oerChyl nidht bone 25 nffer fan flipig fevn/uno Dag oag/el

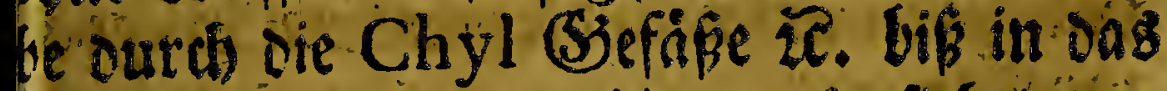
Bjlutb gebradbe ivird. Und oiefes fiebet man augenicberinlict); 2nlangende aber vas ande=

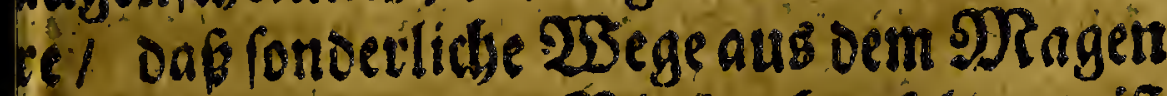
und Deirmern in die SBlafe geben folten 1 ift

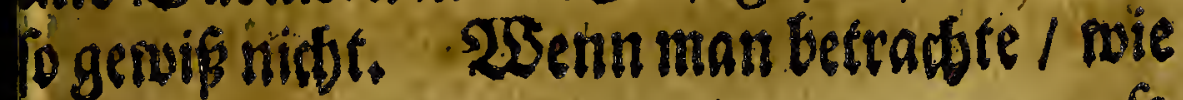




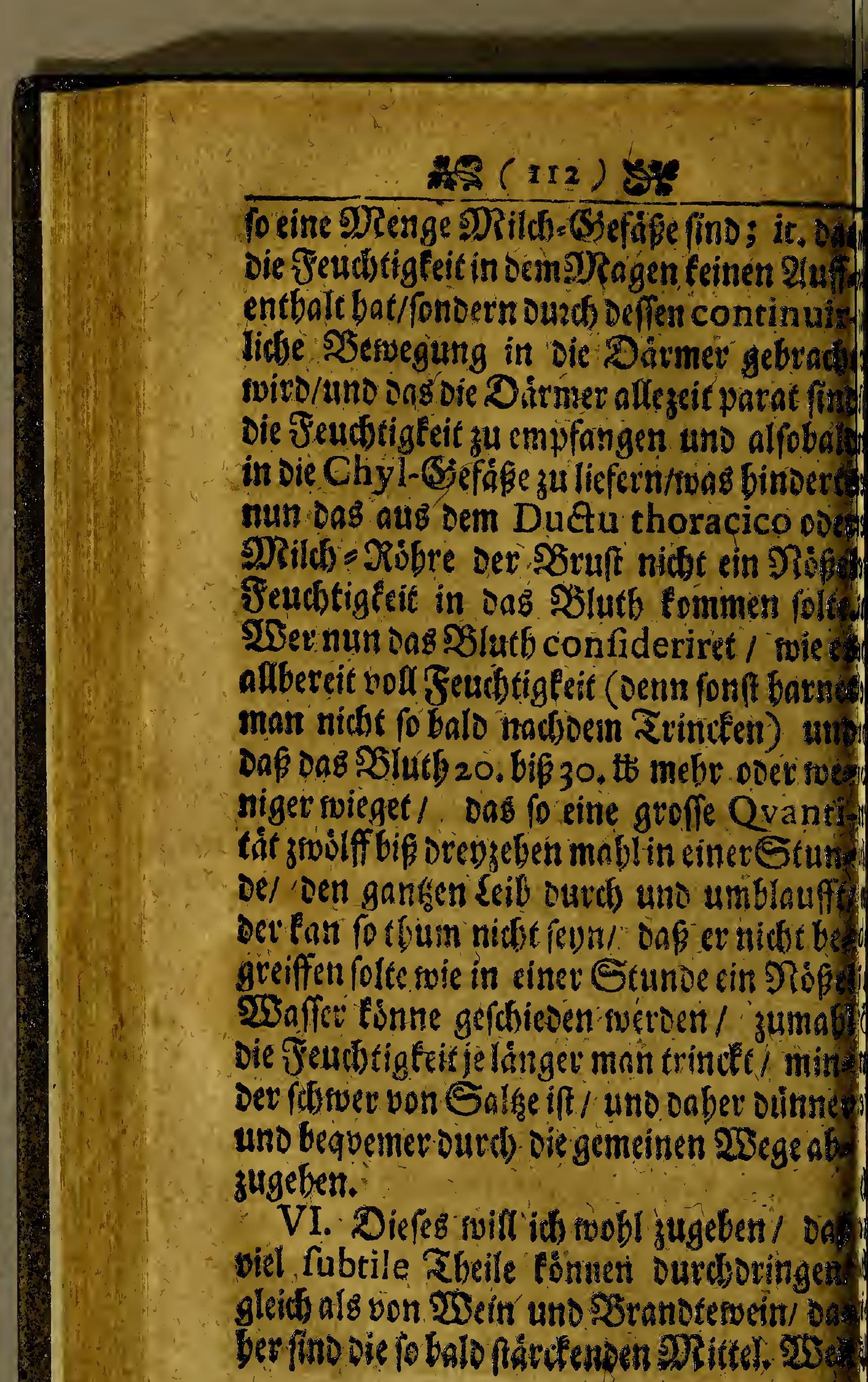




\section{$\mathrm{NS}\left(\mathrm{Ir}_{3}\right) \mathrm{Sw}$}

an oie Structur des \$R

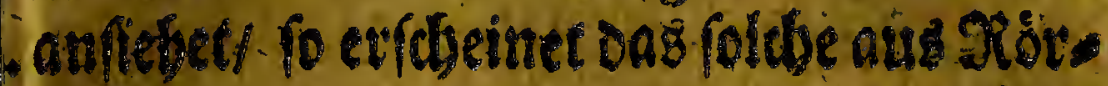
an Safen ve, beffeten: Uno meilfein Cirs

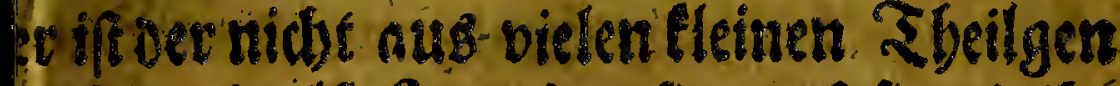
fithel/ bie allo formiret fino bas fie mit ib

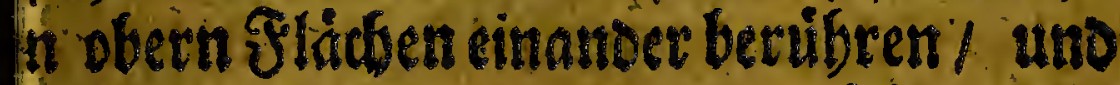
eilffe von aflerles Figuren/ muifen nothe enoig interftitia bleiben/. eben als wenn ey Rugeln alfo geffeffect werben daff fie einboer beribuens / fo bleibet geswis ein Swb-

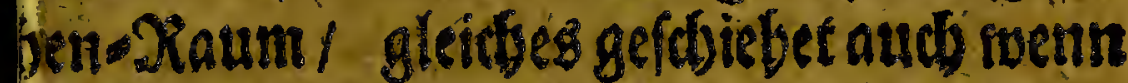
el Sugein einander betribsen. So if nun

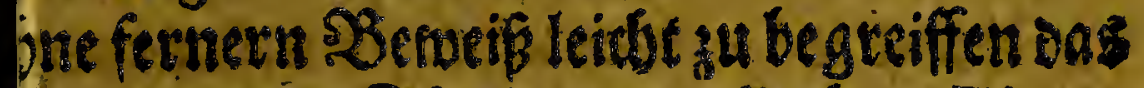
vifden benen ? beilen von afletbanoFigum/Znifesen= Zaum gelaffen wito. Uno ale bat audf unfer keib oer aus afferteg T beile en beffebet; biel poros, baburds etlitbe dese abtileften Theilgen unmittelbarex 235 cife hoagsbituth gelangen fsinnen/gleich als wie Stanotervein/28affer 26. Durds Die poros mgeteinten Papiers geben/ Jadurdo oute Fesdthtigfeiten nitut formmen tosnner.

VII. Det Sam gefunder Keuteif unters bietlith von frarbe unt (Steruds. Fsent

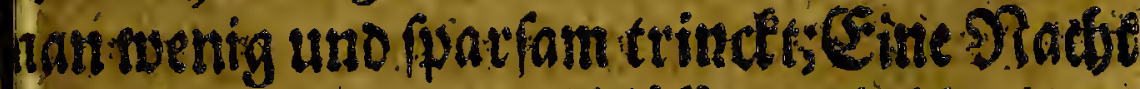

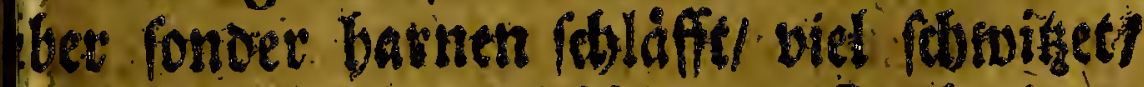
bind das 23afferviel bisfer an garbe fennt?

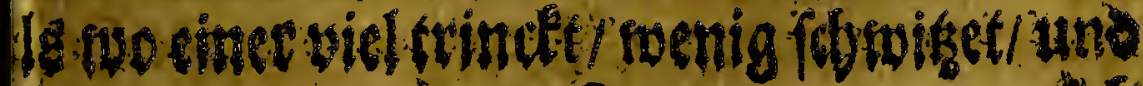




\section{$\sin \left(\mathrm{rr}_{4}\right)$ )}

fich wertig betweget. IBenn wenig Jeudistigh

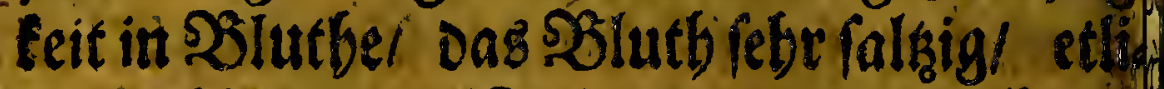
de mabl in einer SRadst muß umblauffents bermenget fich mebr Sal's untet das 23lutb. als no oas Siegentbeil fid beftroet; Uno mebr Salszugegen/ je boher oer Utrin bo. Sazbe ift/mie aus oe? Saugen vonpot - Ir d be Doer Sein = Stein Ealkzureben; Dieris mebr fie mit Sals angefiflet/ie bober fie oos Jantbe unc contra. Dieneil aud in Dem SIlutbe viel fette uno wbligte Sbeilgen fins. eveldfe ourd die fetige 23 ärme mit ori Sals uno Waffer vereiniget fuerden/ frit)

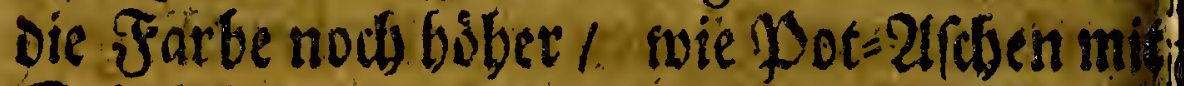

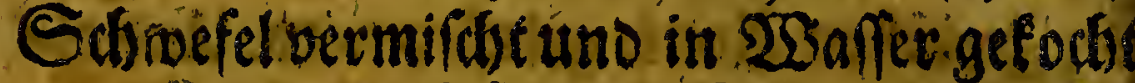
sine rotbe Farbe zu soege bringet. $D$. Stante fo mandimabl in oem Urin gefund Selute gemeritet toiro / fơmmt gemeiniglid bori genofener Speire ber. Bum (Stempel) wer Spargel iffet oeffen Sarn roird in eine Galken Stunde Darnach ziemlich ftindens Die Urfacte ift / ons oer Spargel auf feif

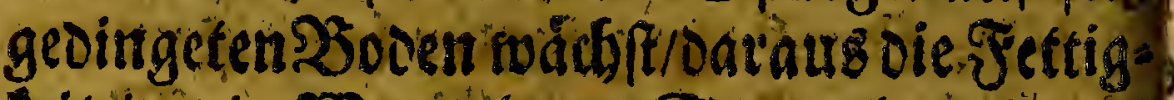
feit itt Die 23urkel Des Spargelis orittget

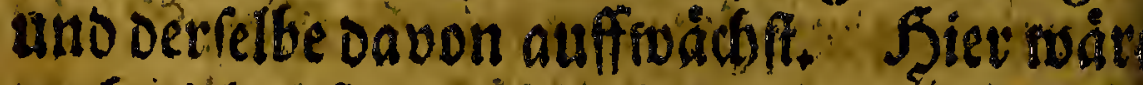
hocf viel qu lagen vont oem Urin oer Rrant dentl aber es iftniche bet Drtboazu/ noe berlanget von oenens Setuigereven ser 5ुart 


\section{6 (in) 8}

Octortano Satn Dropbeten zu lefeti: Der febe ock2Sulj foD.Joh. vanDiluerén ge jrieben.

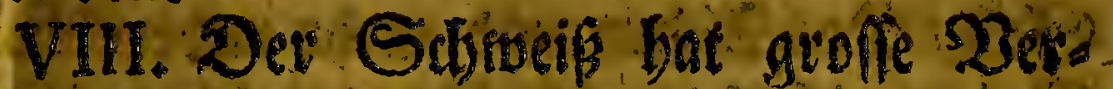

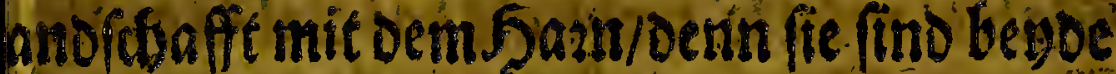

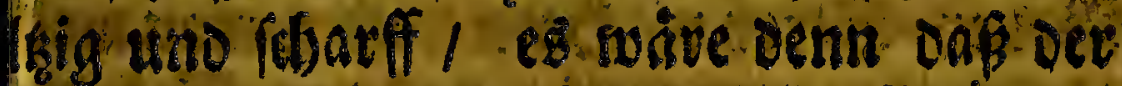

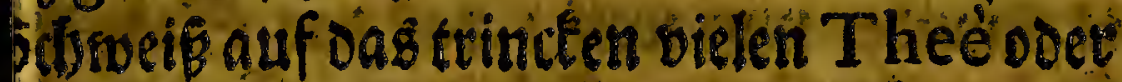
- ffee 2Daffer exfolgte/iff deffer Sclimiact B Regen=2raffer/ eben nie vertuin. Son

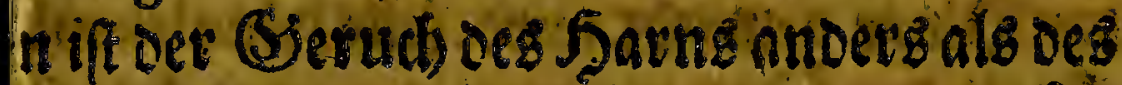

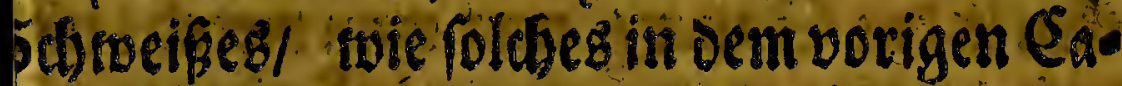
tel enllatet novden/ roeil ber 5arn oureb bere Drifgen gebet alz ber Sdjweif: bie differiren aud ontin/ bas ein (Sefun:

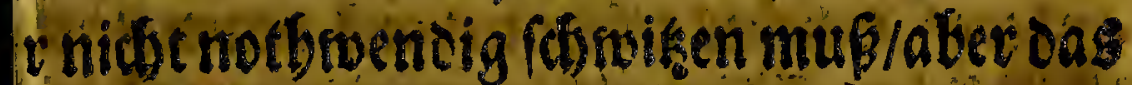
arnen fan nidje unterbleiben/ ooer die

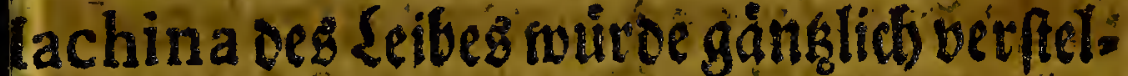
t fwerden. Denn bas Schrwiten if nut eit. ufallobin zu vieler. Jikg/ gleich twie tas yies Samen ein 3ufain ift refolvirten (S) uitbeg.

1X. Dis Sdincignteibenden Medicas. ente folbft differiren wehig von benet

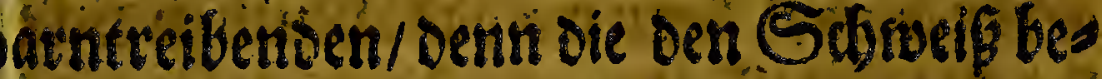
derm/ treiben auds sffets oen Jarn/ uns man zu benen meiffen Sarntreiberiben

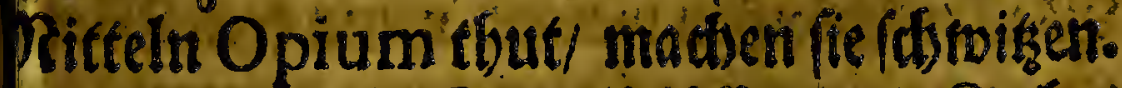
un Exempel. Tob gebe offers die Rrebs: 


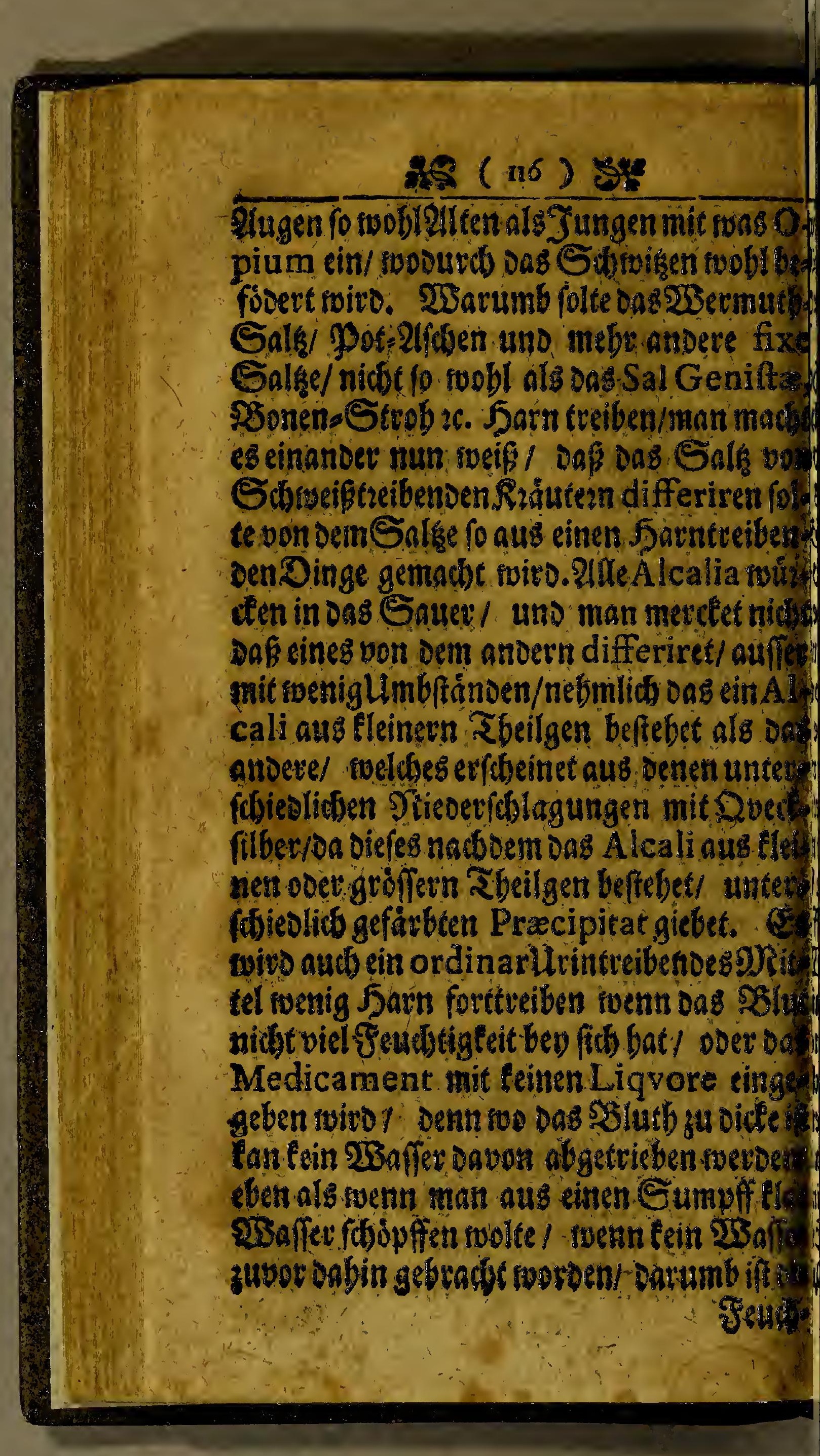




\section{is (ni) 84}

Seudfigfeit der Jubrinann/tno oie Sarno

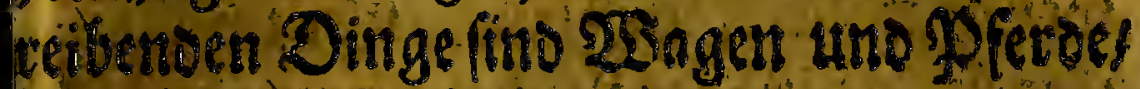
he oem trith fortbringen.

X. In theils Rerantbeiten gebet oct Darn nod fort $/$ in andern nichet ober sodis

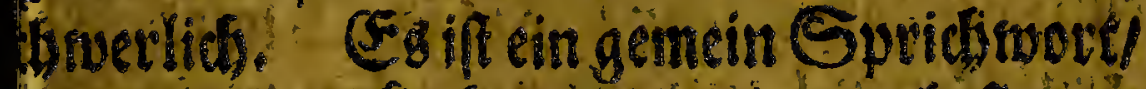
ber viel trindet/barnet biel/ uno beftnders 8 a ud bie Zrinder integenein allo: Das begentbefl inus allo aud wabz fenn/oastoes benig trinfe/aitd wentgharnct. Theils ente barnen wobl uno viel/weil ibe SBlutb/ no afle die s beile oaburd oer Sarn geber sobl berdabfen fino; Es fino aber auds eini(e ole offt uno swenig Urin laffen/ foldes veto

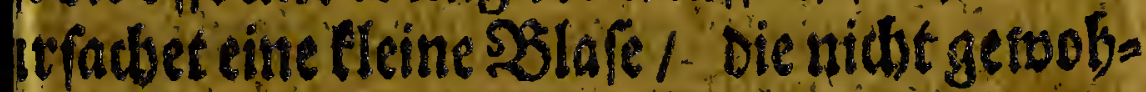
let if viel Feuditigfeit simzunebmen/ wetm e nur ben Stelegenbeit mefremufarght als de beberbergen fan / wito fie gegnungen!

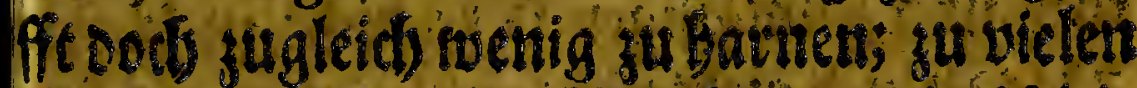
Baffern/geboret sin silutb öas nidbe folle nig if/ in bem das W3affer nit Sdleimuet:

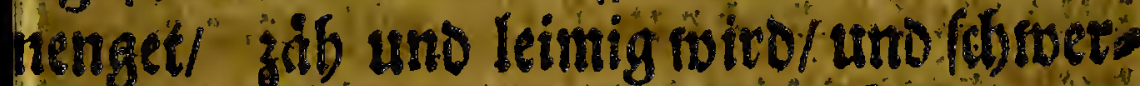
d6 swifl und tan ourd tre! Mieten ges en/ fo swenig als Lein.2Baffer ourd ein atreg Sibb oder ungelemt Papter wity ot ingegen vein $25 a$ ffer burtig ourd laufft.

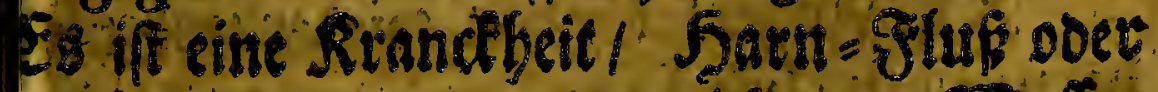
Diabetes genanof/ in welcher ons $23 a f$ fet 5 3 mit 


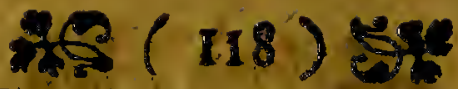

mitbauffen ablauffi / fige von (Siefdrmad sobev groffer Durft uno aufer zroeiffel fart. ces Steber ift/ Dergleichen ich an einen Dors Diger gefeben 1 oer weit mebr harnete als

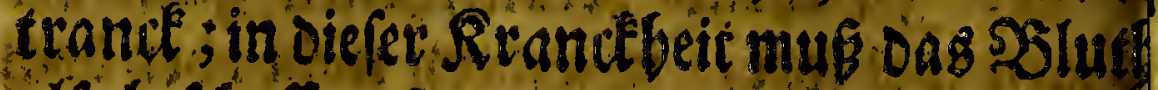
alfo béchaffen fenn/ oasesfid mit bem ge: stundenen Liquore nidge fan vermicherth nebmlich zaib uno mit anoern Seuditigfeites unmenglar $/$ nich tanders als nie seim out bâlb zu feiner Confiftens gelocbt ficf nid

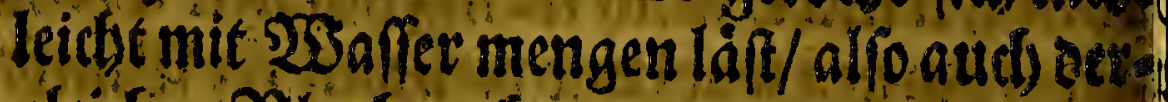
gleichen Slutb noch natm fennoe/ vonsem Enlten Setrándte nibbt fojmelset nod fio Damit vereiniges/ oecobalben oie S3efáfe mik folder Jeudstigteit angefullet jo efelbe our Die Pieren muifen continuirlid loffenals lauffer uno menn oie patienten nidht trite deti iniffen fe bue luffuallung oeg Szlu thes uno unmaffiger Sikge nidit zu bleiber Das Sllutf giebtnidot genung amen Speis

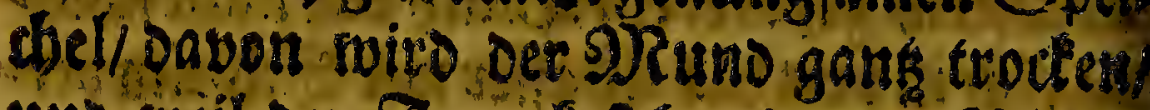
uts neil bet srance fich mit oem Slutbe nicht bermenget / geber et wieder in form cines ungefocten (Sbuls ooer Elaren SR ol

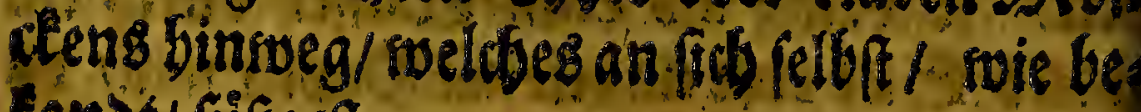
cantot frige ift.

XI. 2noece fontett toieocrumb nid Gannen/eugutradge ong oiestietensslarez timin 


\section{2क (120) $\mathrm{Bg}$}

gen / SElafen uno Róren cifie Berfowftut macjentome.

XII. Jor nenig Sagen wurbe mit us Chrift. Trawen practicirenoen 23 und Shrste zu Dronthem in Siornegen folget Detrater Sufall uberforieber.

Sin Jungergefen von 30 . Tabuentoet Tabr mit (Sirief gevlagt oemeft befom lings eine gónticbesderbaltung Desurin soldbes acbis) Rablzeiten lange/fonder ein gen Fropffen zulaffen soabrete: Daraifi Das 23 affer wiever oren Sage gegangen/um Denn wieder gwey হageftiffe geftanoen nal Diefem wiederzwey Zage gegangen/ uno not Dev gned Tage aufgebalten 1 nod moble.

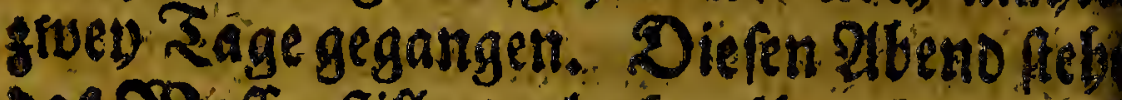

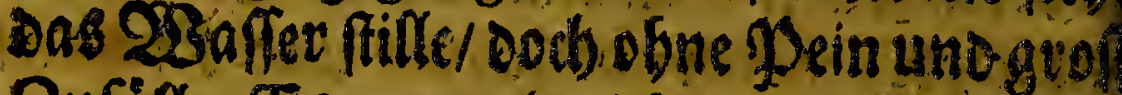
3ufálle. (Ez mangelt nidst ant Dersslafe ad STieren/ondetn woblmeiftens an Dem SEl thex. Seffrieben aus Dronthem 168 (10) 20. Fèbr.

(5) muf notbroendig fon das Sdlein

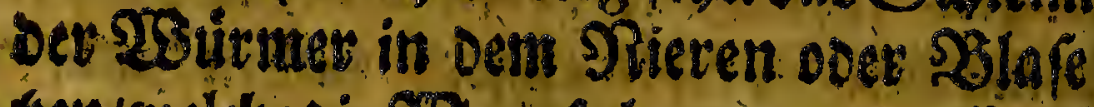
gen/meldge die 283 ge folonge verforffents fie vor bem Drang oes $23 a$ fer libertse

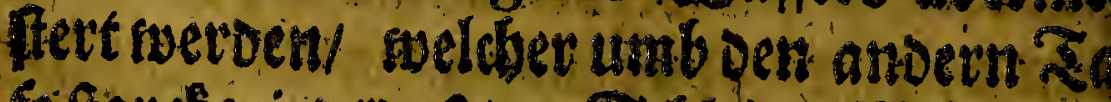

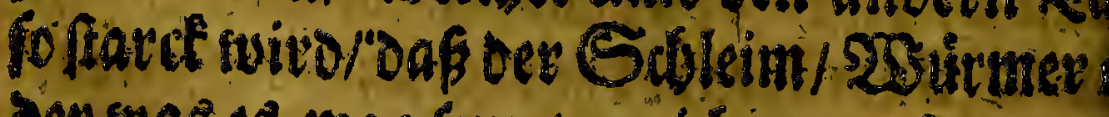

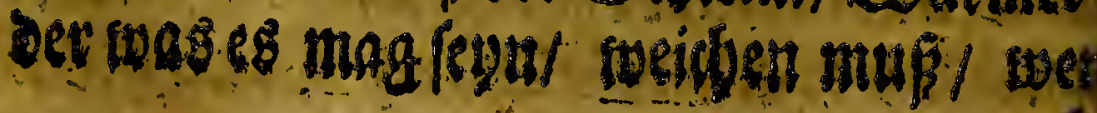




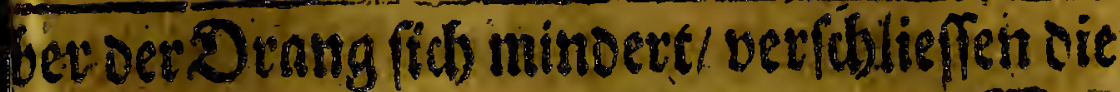
Bsirmer/S dbleim ooer Polypus aen 25eg bieter. Tob babe biec ju 2imferban nud men Drann gefennt / Der nobl zrwolff bif lertgeben Inge fein $25 a f f e r$ liês / unt gleids tren (Siefunben obite Sobmersen blieb) leidjmobl aber ftart. Weldies eben yon etgleidgen Uriadje mur getwefen fegn/ bent.

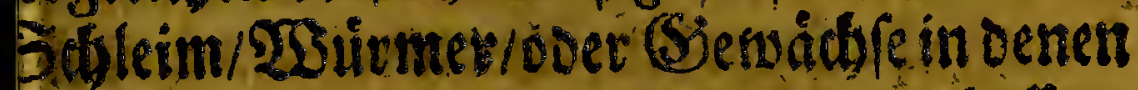

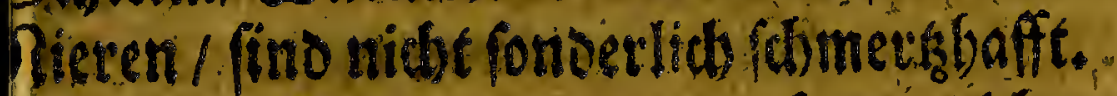
XIII: 2 siv soollen nun ferner feben. boraus die Jarntreibende Dinge beftelyen; Sie milfen viel Seuditige eiten beo fith bas en. Ssleido oem Spadanifden/ Coffee-

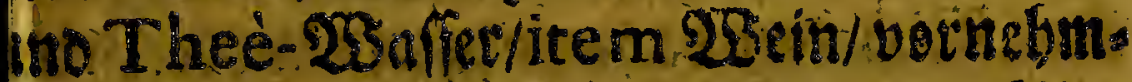

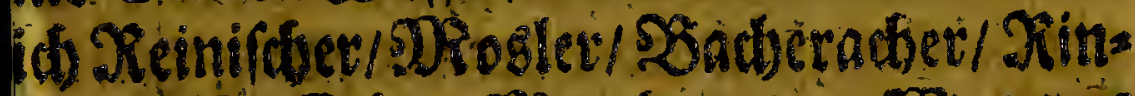

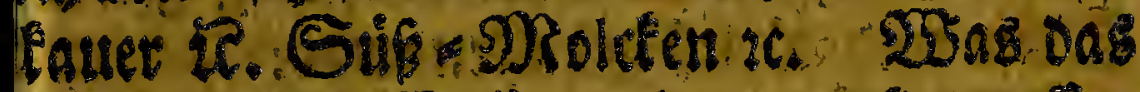
ppadanifobe ssaffer anlamget $/$ fino beffet inter/biedene Sorten/Denn oa fins vier bes uffene 83 runnew als : Pauhon, Geronter, de Savoniere und Tonnelet. Dis 23afer. (folgenos Der 53 efficibung son. Edward. Brown) bat feine befie seitin Aem beifeften uno trodineften Sommet/ano wenn es im Winter ant bartefen ficietet/ zo

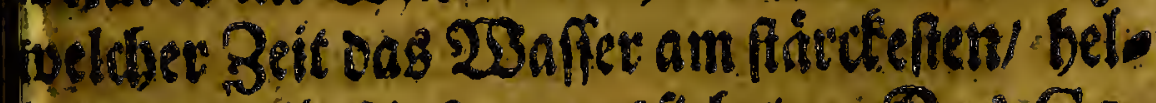
(ifent uno tuitigften ausfiebet. Das Ge-

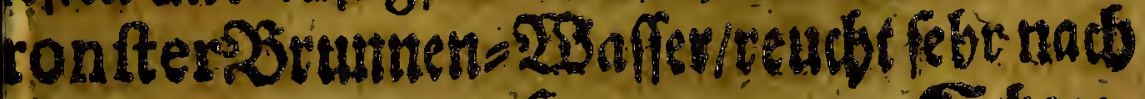

$$
\text { 5. } 5
$$

Sowp 


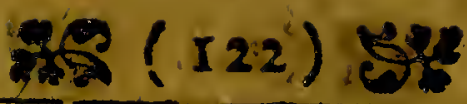

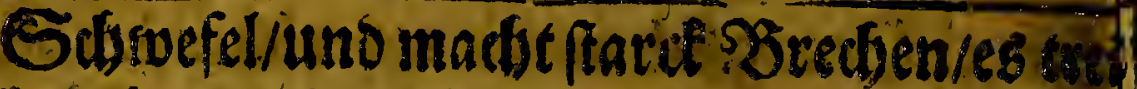
bet aber audo treffliob ben.Utin/gleidsoie f Diefes meiftens miteinander gemein babem foent (Siafle Qlepffel Dareiti getflan rwerdent

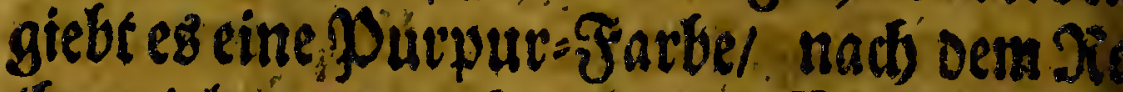
then fielende mebr als oas Sengelándifs Wrafer zu Tunbridge. 2iuf oem (Srut De Deg SJodens ift die Materie lichtblau/ of andern Drtben aber fiebet es wie eim moort. Potbeg. Sicht sueit bot oiefer Doelle nodseine andere / weldge gleiche Alrth bout pteflet.

XIV. Derzu Savoniere bat fóarfflic SSaffer / nicht fo fatch als das zu Geron Iter. PRidst fert Dabon liegt cin ander.Dscuit: nen/ Diefen gleidj/ weldber vor gut gegen Dent Stein uno Sries gebalten nite.

XV. Derzu Tonneler, for Galpetris ger fentrals die oorigen / oetun bas $23 a f f e$ madjet groffe Ratte in bem 9) uno uno D? gentoaf man es faum truinder fan.

X.VI. Son oem za Pouhon, boblt mol ons meifte 23 affery fo berfdidet twiro/ viel leidfet weil oiefer Stumten in Der. Stad liegt/uns die antoert nupen. SE faseinet oap Diefe Duellen meift aub Dittiol beftebent

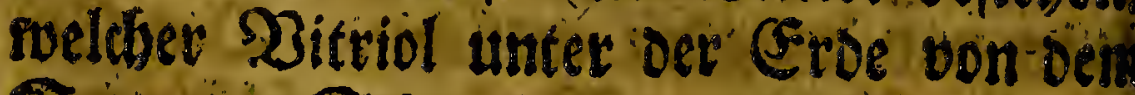

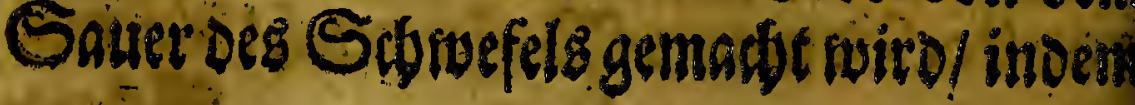




\section{$28(123)$ 5*}

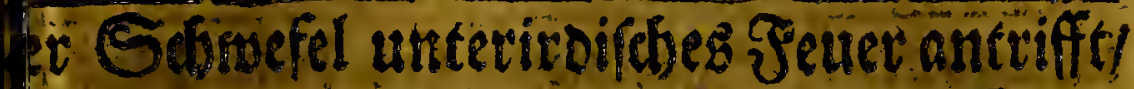
ho rneme er zum brennen Eoñen/ein Saus

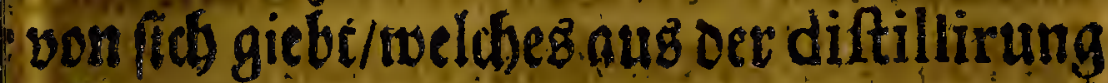

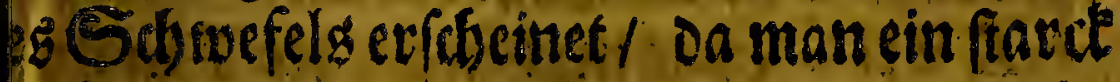
cidum betomme. Weenn fid) nun geonds? \& Saure unter bas Waffer vermenget!

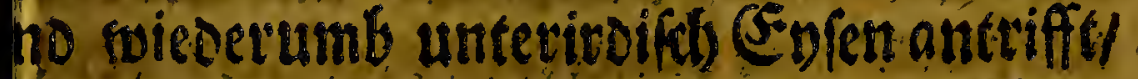
unctet uno efferverciret es mit Bemfellben! diche fobrinefflidbte 2hufflofung des Evsens hen Vitriol giebt/menn sas 28 affer davon inut; fo lange es im $23 a$ affer bleibet / ma? etes ein 2 ittriolifds $23 a$ affer mit Sdywe? LS Dunff vermenget/'svoraus ein Sauers Brunnen twiro: Dais nun in eflicben Sals. ster ift/ tan swobl feun neenn es ourds ein. to andere 21oer gehet/ und methr unterivoia ben Salpeter mit fid roblept.

XVII. 23 as foll nun mefer waffern mas

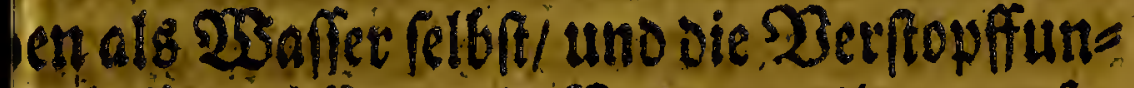

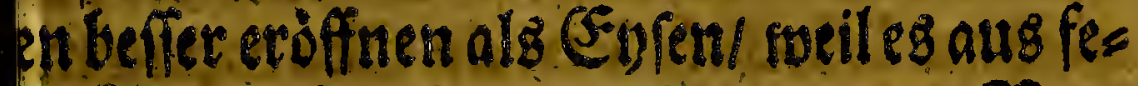
en Fbeilen befiebet/ welche gegen die Ders

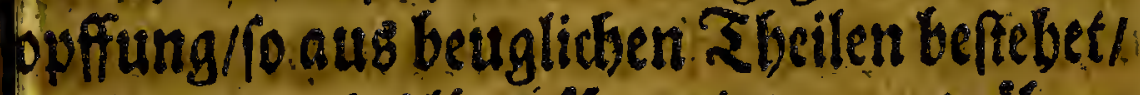

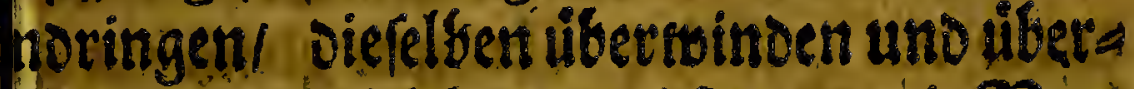

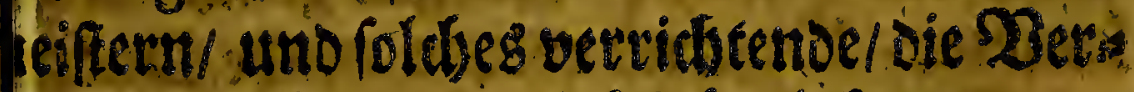
puffung offenen/ uno folgbarlids einen ge? ahnter $253 e g$ vor die Säffe madien. Siers Hnen beftebet Die gantác Rrafft oes Spada=lifber 23affers. Ez frbeinet dabl po viel 


\section{axis (124) 84}

idf aus oer S3erdreibung 5n.Joh. Majo meruen Can/ Die WSaffer vor Bath nic ob fen einige (S)meinfobaff baben.

XVIII. Was nun ferner bie Theè u

Coffeè anlanget/ gieber un savort die tă lidje Srfabrung SRadoridot uno fóer oavi notbnenoigen Inteiridgt begebret / see b peloe Secan Corn. Bontekoe, uno oie Tras ctation melde ids felbft von der Cóffé pie bliciret. Daf det 2 Sein Satnmabtum

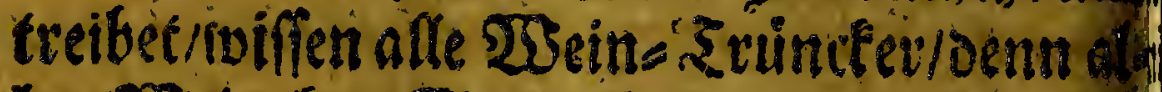
Ier WSein bat Sauer bey fich / uno all

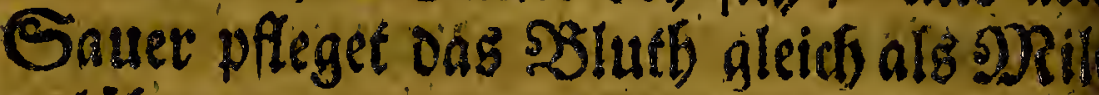

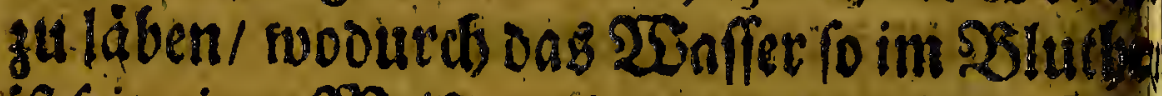

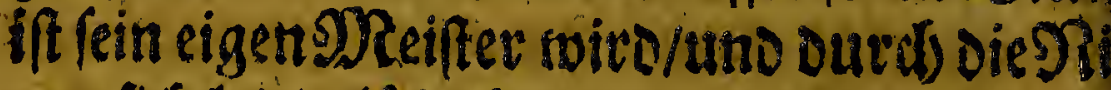
ren fids leidge laft abfondem/ oaber man bo cinem Nóbel WSein /, leidt 2; Tópel Sav los roiro. Daf farve obfondern des Seri ge flitebet befer yon magern Wieinen/als Xes níforn/ Suroblet/ Vin de Champagrie, Vin d'aix \&c. als fetten sseinen/ in toe? Gen oas Saute fo merulliob nidf iff fonoen ourd biel zactidste sbeilgen gleichfan ge bunoen. Darumb oronen etlicle Docto res Spanifben 23ein Sect/ DRufatelle

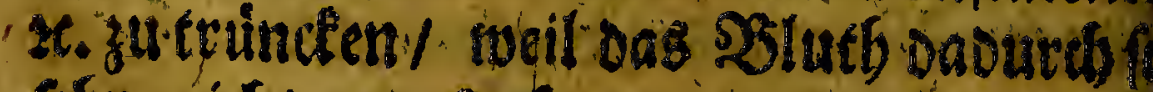
febt nidg angefect wino als not dent muetr. 
XIX. Pimnegen an ber 53 al / , bat vien tagetmein Mol genant/ ein timnes. etronde 1 meldies Darumb febe gelobet ro/meil es trefflid fublet uno treibet/ oat ib manc oie Patienteti viel truincien lâft. iefer Treiben balte id sovor / beftebet it r Duimnigleit bon 2Saffer/ ong mit cin mig Salpeté bermifduet iff / sweldfeg feine Alte ussiveifet/siefe bevoe miteinanoec vet. higetiruenn fie in oas SSlutb fommen/ vers innen foldbes/ indem cie Nitrofitát bè die Birme fommet / fids ausbreitec uno einen popen:Raum verucfactset / wie vorlängit um Carthefio berwieferi ; diefe Expanfi-

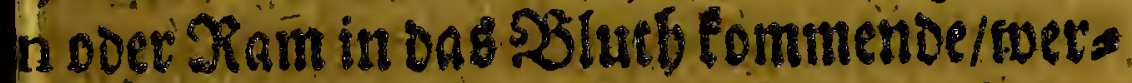
in alfe Sbeile lo 1 . Dap oie Jeudigfeiten iditidh ourd vie Sieten ablaufen.

XX 2uger oem 23 affer uno 23 ein $6 a$ an nod vid faute Dinge/ die ben Sort Wreiben/l gebraudot werben/ ale Da fino: piritus Salis, Nitri, Saffe on fauert. Beinbecten! Simonien I Ipffel Tramit Tartarus Nitratus uno Vitriolatus. piritus Tartari, Spiritus Succini \& $\mathrm{c}$.

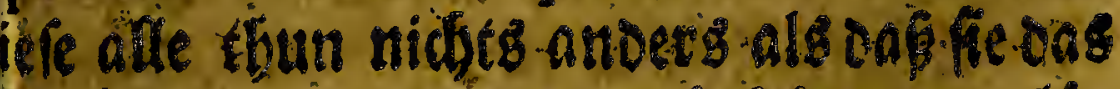
Slutheinige mafen pracipiticen otet lo

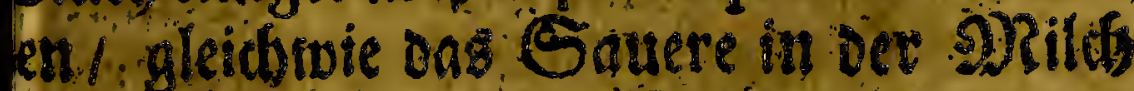
but. Denufpenndes] 


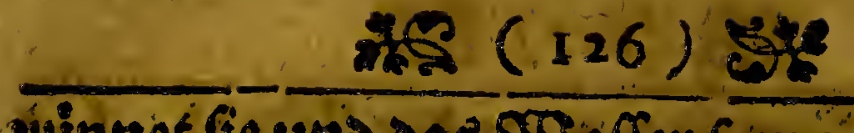

tunnet fie uno oas 2 Baffer fammitet fich afted ne; Uno ment bergleithen in SSlutbe get

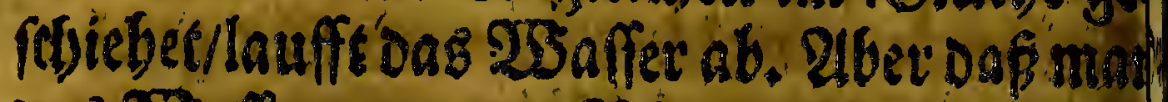
Das 25 affer von dem 58 lutio præcipitiret

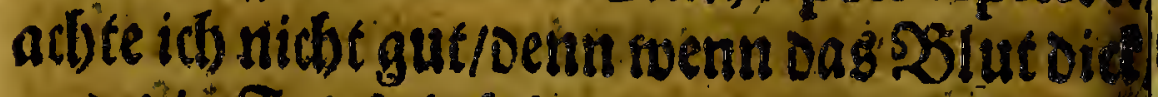

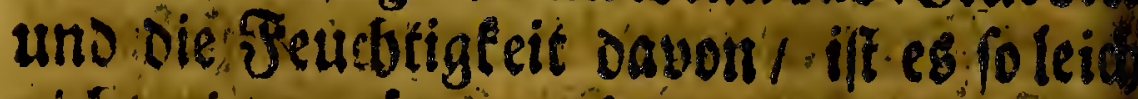
nidhe rvieder suinne zu friegen/gleidjinie mat

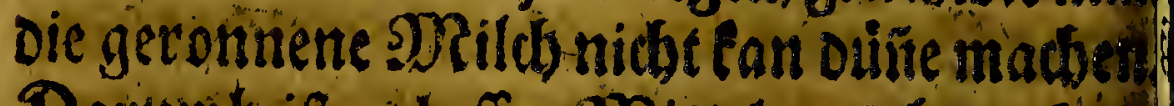
Darumb ift es beffer De ittel zu gebraudden, Die oag SBlutlbetsunnen/ uno soenn es oub ne/ Die Feudbrigleit Dutrobie Sieten und Solafe ablauffen unabenl ebert tose man olo Duinte SRilch fán oidte machen/ swenn Durd ein geleime Papier lauffe/ oentr ald bleibet alles dicte in iem Dapier/obne ons fit geninnet/uno foldbe if teidst robed olinte? macben/ wenn nur nos Jecuchtigfeit ons gethan nirs. Sif es alfo beffer feine faues. Dinge zu gebiaucben / fonoern liebev diefer

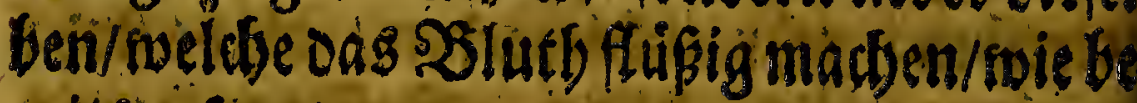
veitżgefaget.

XXI. Antere 5arntefeibende Ding fino sweld be nus eineri fixen uno Alcalin fchen Salke beftetent als oa ift: Snge bol 25eine ben-2fchen/ von genifta, SSonem Strob/ und vergleidben mebi: SEeinfeim Sals/Wermutb=Salk/ Ralie bon getrant:

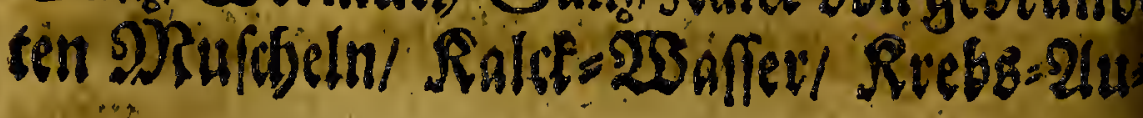




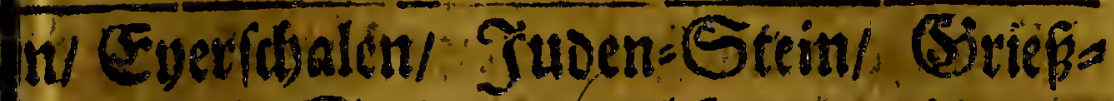
tein/ oie Steingen sweldbe man it oem

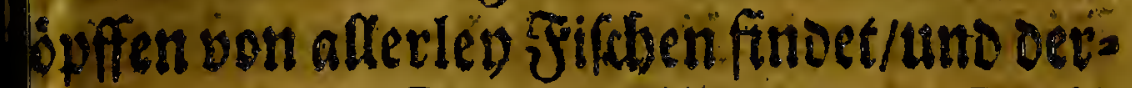

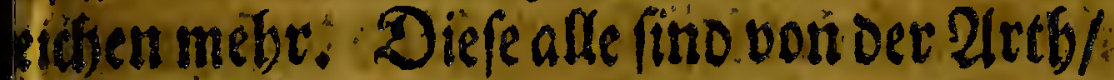
Ef fie bas Sauere abforbiven / in fid jies. 1/ swie id zu mebrenmablen in meinenvo: en Sobriffen betwiefen babe. 233it wiffer (B) ourch das Sauer Das S3lutb dick und.

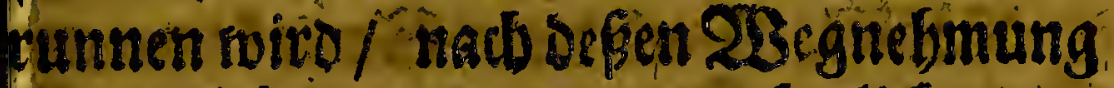

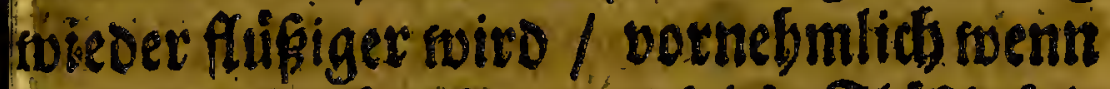

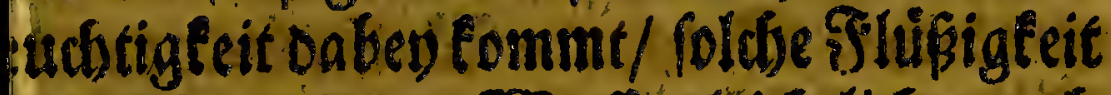
Urfactse oaf ba\& 23 affer léichellids ourds Dietenablauff. 2hiser bent aber daf

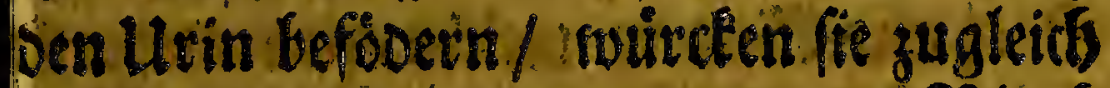

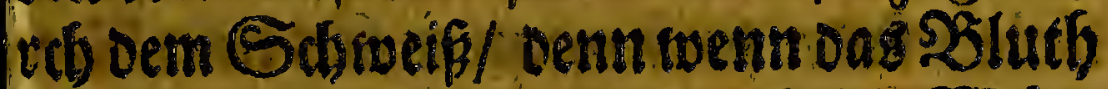

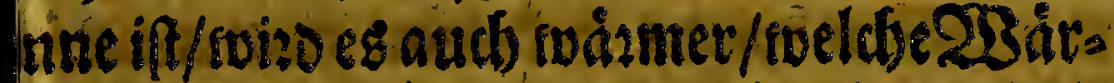

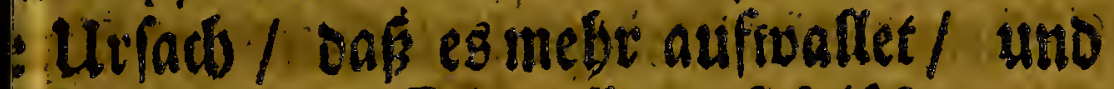
gbarlicf mebr Daunff von fict läf.

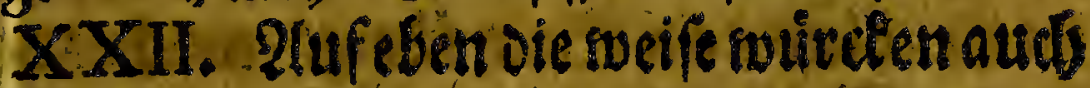
frudtigent Salse/ unter Denen find Flo:

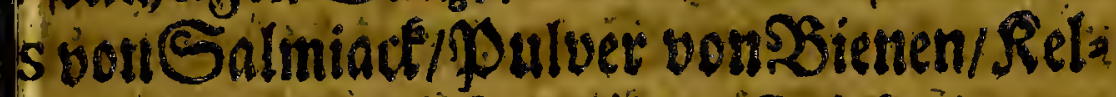
(chaben / Conchenilia, Spiritus von avn/ Spiritus und Salk aus Şirfit:ben/Sparnidfte Fliegen uno oergleidbert

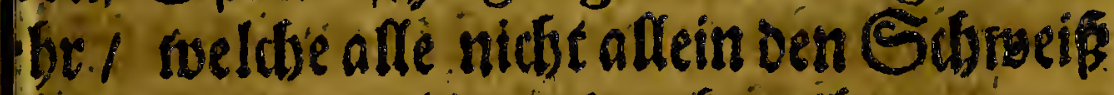
been audo ben Urin farefé treiben / benn

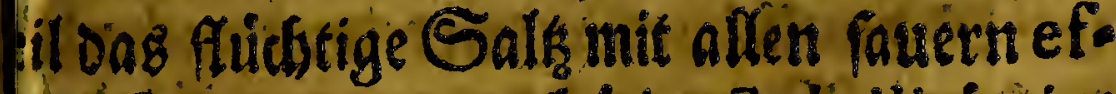
rverciret/ uno ourd feinte Subtilitát eint refo- 


\section{ำ $(128)$ ํำ}

refolution madbet / if es Utrads oaf ons SIuth ounner uno ouinner soiro 1 uno Sbeilgen mebr Raumbabende/ laffen leidgd

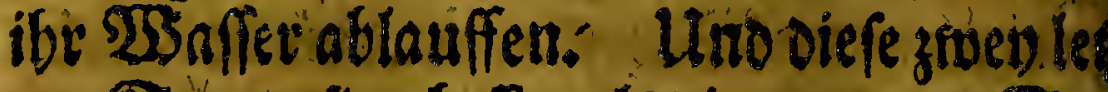
ten Sorten fino beffer alc die man auz $S$ a: ex bereitet 1 Dent fie verberben Das 38 Iut: nicht nie Das Sauer / forbern Isfien es? feinen 2 Befett.

XXIII. Nadis diefer Gorte comment Salpettigen Dinge/ unter fueldben Die D? necalifben Salpeter. 25 affer fino/ uno Salpeter felbfimit allen ruas onvongemat miro. Der Salpeter beftebet aus fixe Sals uno Sauev/ sueldo miteinander ve ciniget find Denn wern er aus einet Xeto te oifillicet fwite / befommt man einen fel flidbtigen ourdbouinglid riedbent uns fdom venden Spiritum bertiber uno in ber 2 torte $/$ bleibet ein Salsubrigl neldes oer fixen Salse gleidget Sieraus etfde net auds/oaf oer. Salpeter nidht brennet for gen feines Sdifwefels / wie etlidbe gemetn baben! fondern rogen oer zarten FGeilga DeB (Satters/ welches die minfe Szerv gung fuiblenvel mit fambt dem feften Sal fvegfiegt: Denn neil man in Diftalirent:

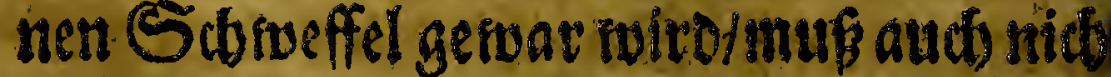

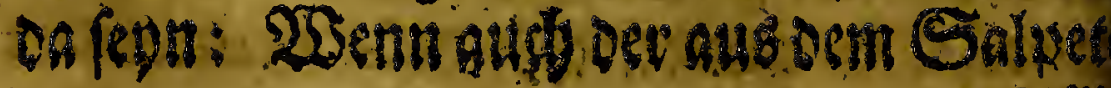




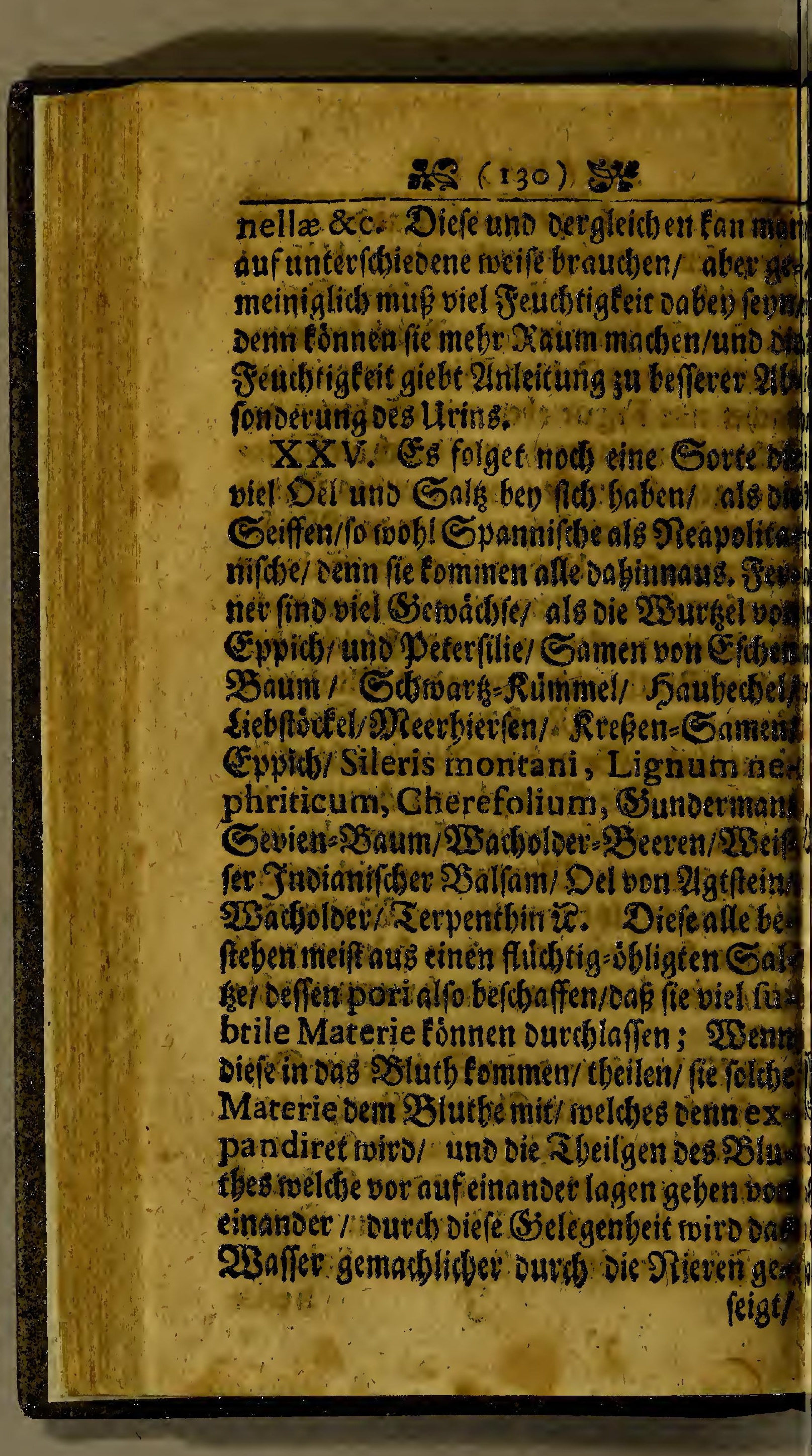




\section{te $(131)$ st}

ge/ moerauff oenn vieles Jornen etrolget. ie obligten Dinge madbenaud oas SSlutb Wipfrigh uno barumb. balten ifere viel Das Rarvel und . Oliven Def vor febr gut. Det biricus von Soffel Sraut / uno Rettigen athen viel 5arnem in felbft ber Brandes sive weldje afle rwegen ibrer Durdjoring feit/uno expandirung oes SBluthes/mas * dasper farn abgebet. Sodas alles inns

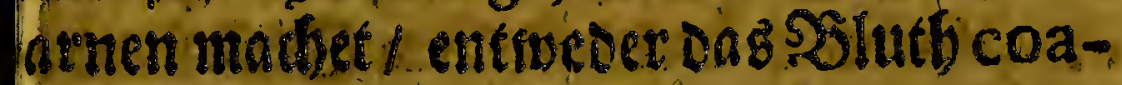

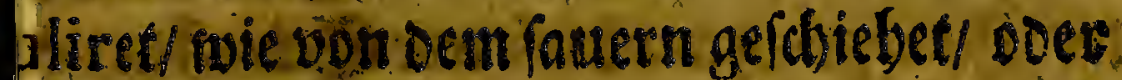
madyen é flúpig ourd oag ?3affer/ ober. terbuinnenes:

\section{Das VI. Sgsapitel.}

Ron Sdilaffinadienter uns Sdomers fallenden Noittely/ wie aud von oem Opio, sabact 2 .

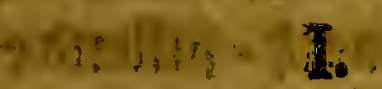

Tre Siv nollen sweiter geben uno ung za

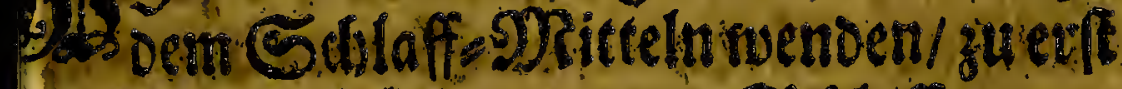
de tirtslids befeben $\%$ soas Sultaffen uno Bacheri if. Das.2Batbeniftuiduts anters Beinfetiger Durchlauf Der Jim S Siffet

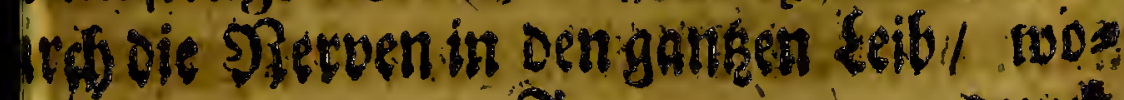

$$
S_{2} \quad \text { out }
$$




\section{$\operatorname{me}(132) 5$}

Durd alle Xiskgen uno pori genungfam of fen gebalten uno aufgefpannet weroen. 23 e aber ein groffer ₹beil oerfelben confumir ift fonnen die Rizgen nidht mobl aufgefpant

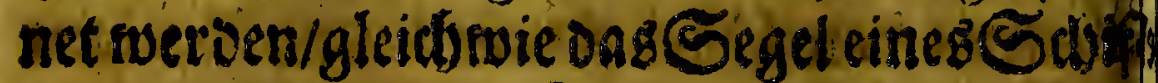
fes einen langlasten Fortgang machet/rwem rein oder wenig 28 inoift alfo weroenuly

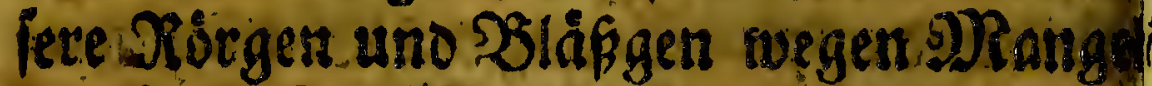
des. Sirn=Caffierebr wenig oser gar nid aufgeppannet/ welcheseinen Stifteftano an

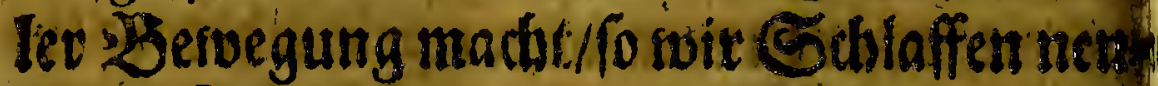
nen. Detmourds nenigen Sinfufs neroe: Die Xörgett enger uno länger 7 netunficabe aufgeblafon/furcher/eben wie cin aufgeblofes nev Darm oder Siofe thistsec uno rundets wetoen. Diefer fer nur fur blich gefagt/ toe is julang meroen folte) alle vie 3 orfúlle wiecerboblen/ soldbe id in meinen Inftiru tionibus meffens befidrieben Gabe.

II. DRan bat unteridhieotidie Soblaff

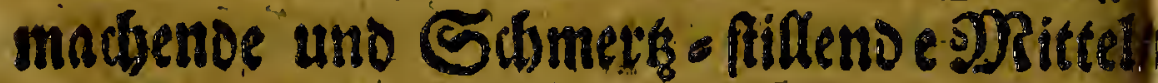
Deten eines fiảrcter if als oas ander / als ift oas Opium, oer gemeine bie zu San soadbrenoe SRobn/Deffen Samenumo RJp

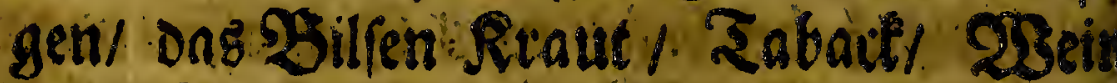
Parde Bier undrergleichen: Dä Opius ift kin Saffit fo aut oer Suruter fommt/sa unter ons on Theben sas vonnebmfte on 


\section{Is (133) Bis}

ehn idine Sorte beffer als cie artorte iff. Das mater notro von oem auggep? fen Saft

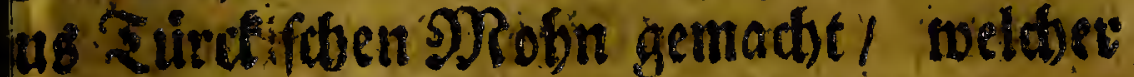

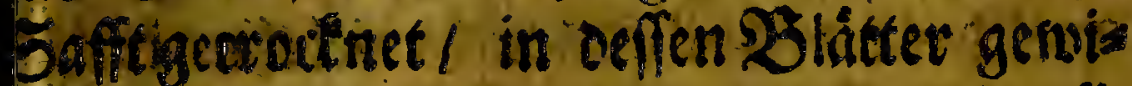
aft uno berfteret soiro/allein ez if nidbt all. Hauber/fonoern volutureinigteit/ weldbes enungfäm etfobeinet / roenn sman eg mit Peanoterbein extra hiret/uno Das liberbliea ene trodent I fo finber inan viel Stide volt Blättern als ein Bcidyen oaf oer Soffit nidbe ar zuhobligereiniget abict: Man findet bet nod eine andere Sottel toiclde geram: t wirb aus vetwinieten IRabns Ropffeh/ bratus ein neiffer Sofft läufft। vet Daran ustrodenet 1 uno bernads gefambilet wird eldies das fauberfe. Opium ift/(ons fo viel kiftals Saff.)

III. In unfern Sanbe giebe es tooblauds Drabu alleituer if vill gevinget vout Sraffy thu geget cinen gran Stredidyen Opir, Thg man des Sodätoifden wo l 20. gran mgeben. Der Hyofciam us beer SBilfen biro bier wenig gebraudbt/als allein ber Sa: reni uno beftebet feine Rraffe falt thenin oet Surctung als oas Opium in oen es Dadaffenmadbet. Eg if am beften siid, 1 gebrauthen/. Weil ittan es nidst nótbig bat/ mo viele tmboen fols gebradot; Sint gut

$$
\text { I } 3 \text { Sdilaff: }
$$




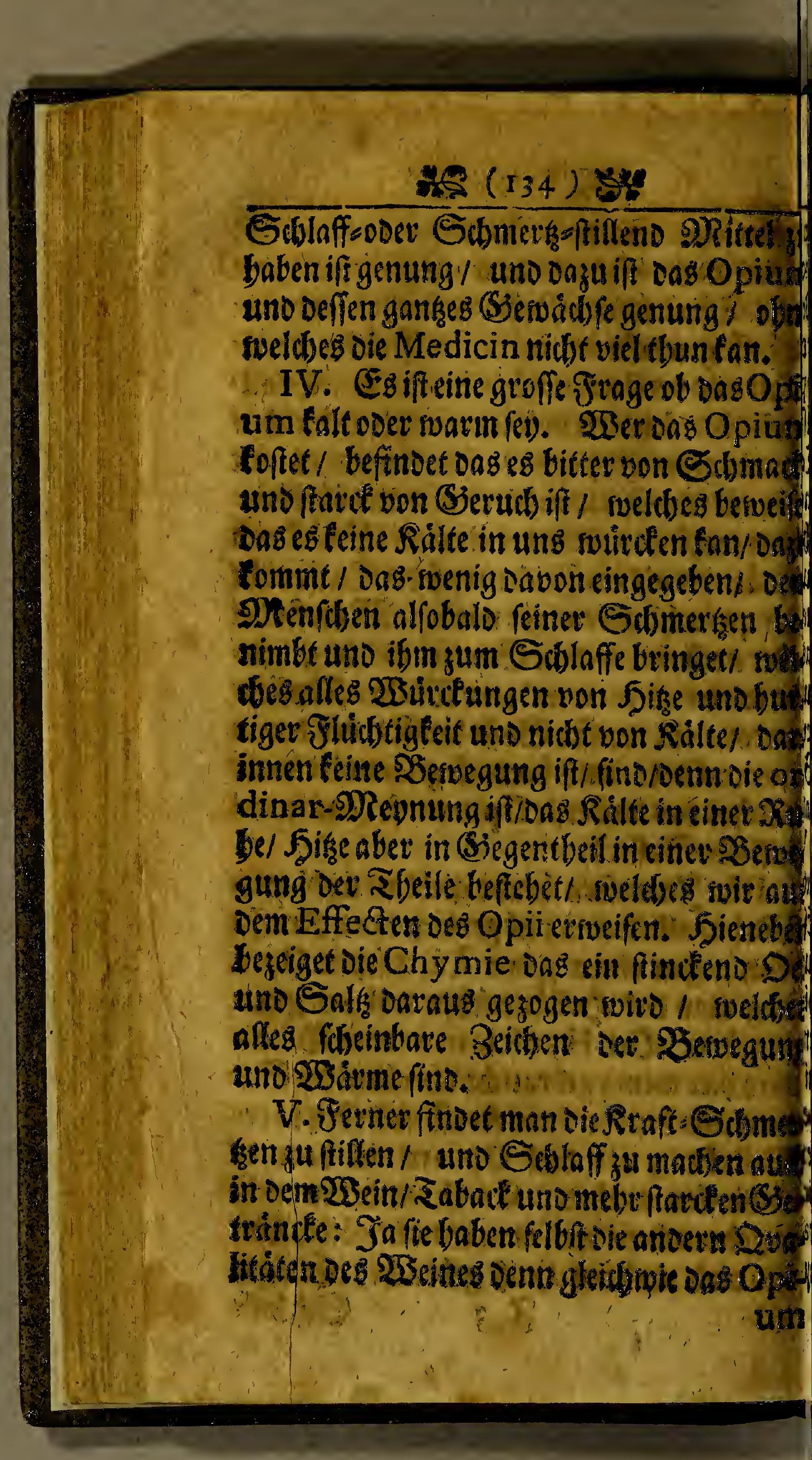




\section{$\sec (135)$ 3ै}

mi die Surden trunclen matbt / uno afle Etaurigteit vertreiber; fo tbut aüd oer Bein uno Tabauk oags fte alle miteinander roffe SSemeinfduaffe baben/ uno gleid toie Dium zu viel genommen/Sdylaff madbet! (fo thit auds oev uiberfupige 2 sein unt Labad. Denn gleitb no Wein uno 2 as. hod uno unterfdiestere Derfonet/ unter

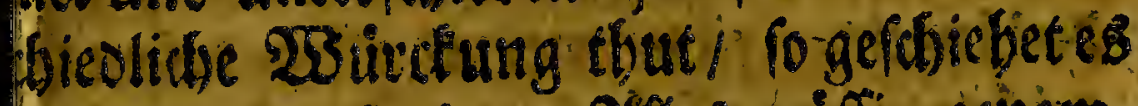
udi mit sem Opio. afles ináfíg genom: hen bringet viel (Seifter zuroege / soourd as SBebirn uno Senen vol von (S)eiftert ufgefpannet werven/allein wenn fie entroes

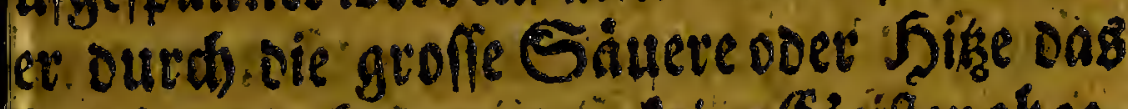
Blutb veididen/meiden feine (Sseifter abge. onvert/uno oie Da fenn verfugen.

V1. Das Opium wito auf alletiants dethen prepariret / als zum Sxempel mit Gfigl Davinnen sas Opium aufgeldet/ urd gegoffen uno roieser eingetrodtret bire. Invere verrichten foldbes mit Qvits ens Saffe tweldoes beffer ifi / nod beffer aber hit 28 randetwein/Dab Saure verderbet und lePruiret/ note betant/alleg mas cin Alcali ev fid bat/gleids rwie rns O pium. Aud if

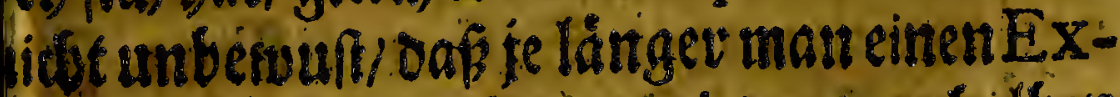

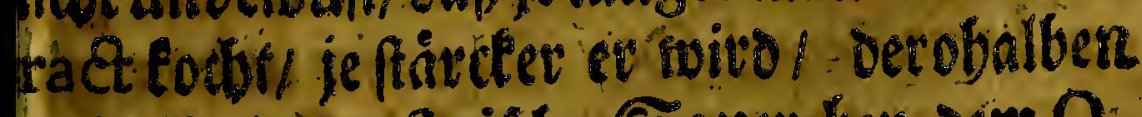
hup dif corrofivilde Gauev bey dem 0 -

$$
\text { Ү } 4 \text { pio }
$$




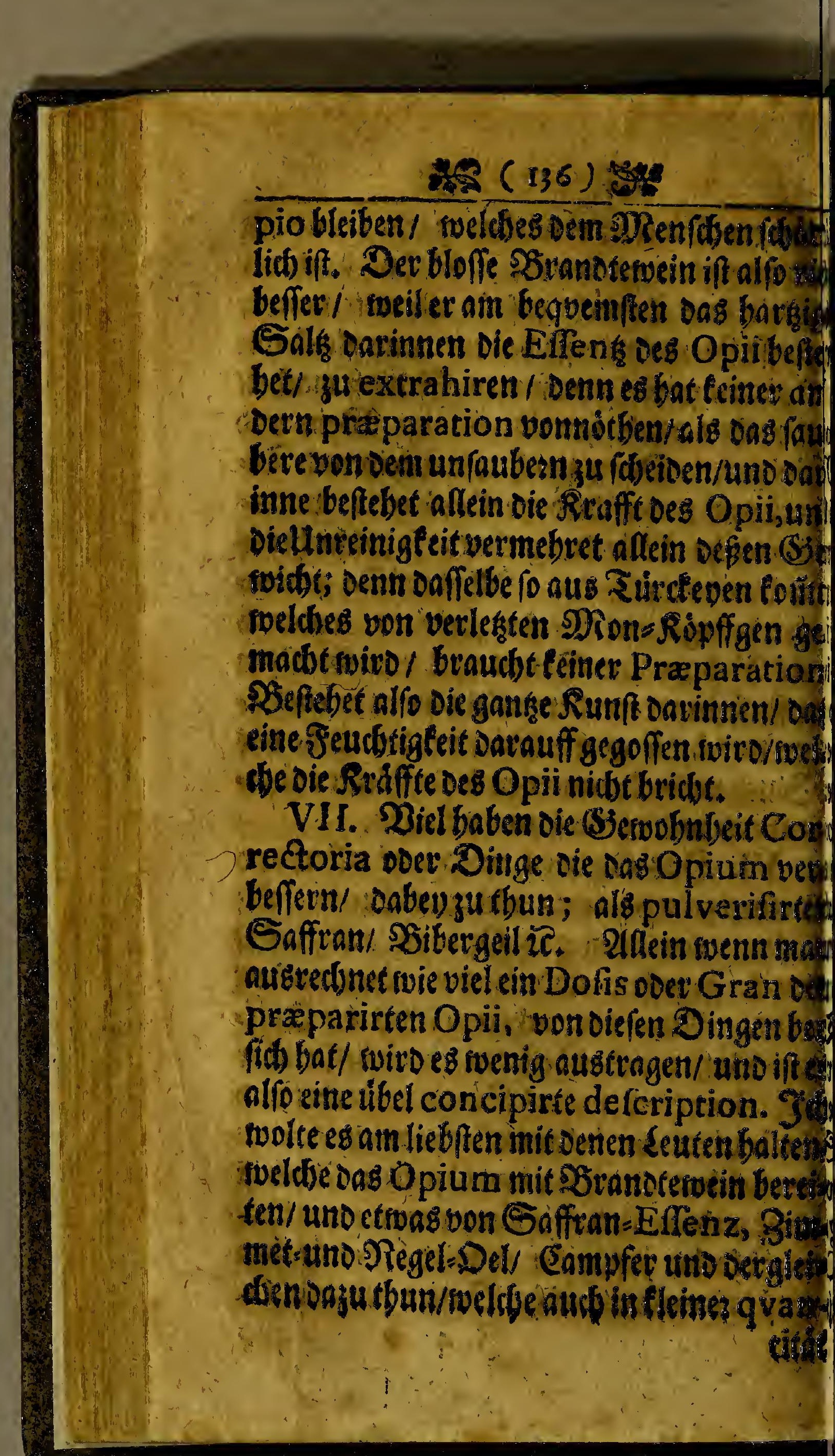




\section{$6(137)$ है।}

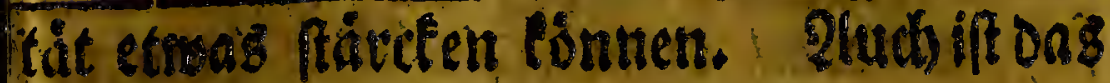
údstige Sals von Siricbliorn Dazu getban/ idsefchtimin. It. Ambra, Morchus und ergleiden.

VIII. Ferner if auds ein Unterfdseis wifden oenen Narcoticis poet Sdblaff= DRitfeln/ uno Anodynis ooer Sdumers.

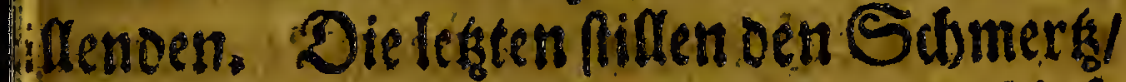

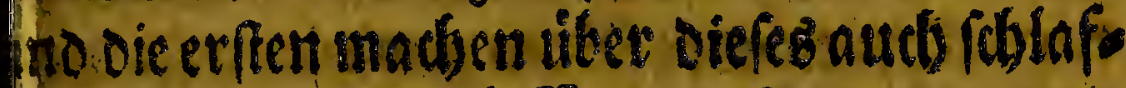
1n. Derobalben differiren fie nut ut mais \& minus. Denn oag getrodinete O. ium madst nenig fdtlaffen/ itzgleichen oas pmit 23ramberwein ausgejogen if / weldbe

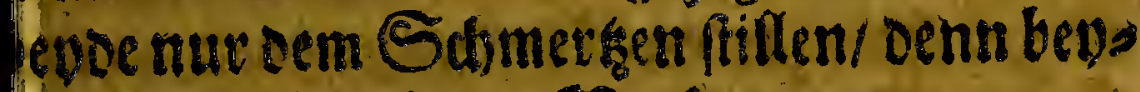
eet Riafft beftebet in Derànoerung oet poorum unt Werfentoung oce Sbetlgen / oie n einert anocis befusaffer als in Dem andern? aber die fubtile DRaterle in einem fadrcter furchfecidstals in Dem andern. Dennoa butb) 2lustrodinung uno Dluszithen einige Sheilgenabgeben/ mupéeine Derdnserung ffolgen / gleidb wie gebrande 50 olf in Roh:

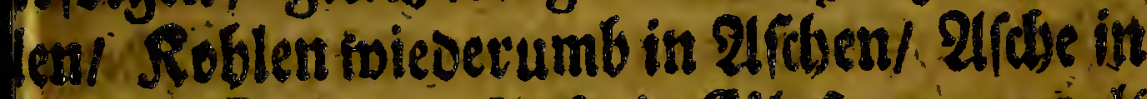

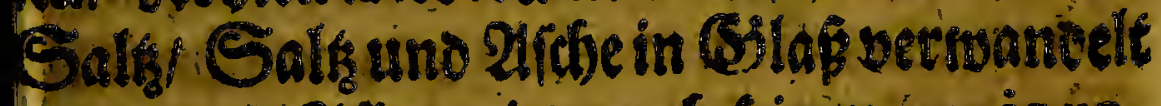
percen. 2ifles foiro mady feinen poris reguliret/ foldbes ffebet matimoer Analy f Chymica Dez Opii, menn affe Theile von

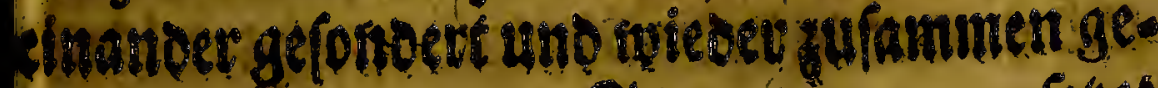




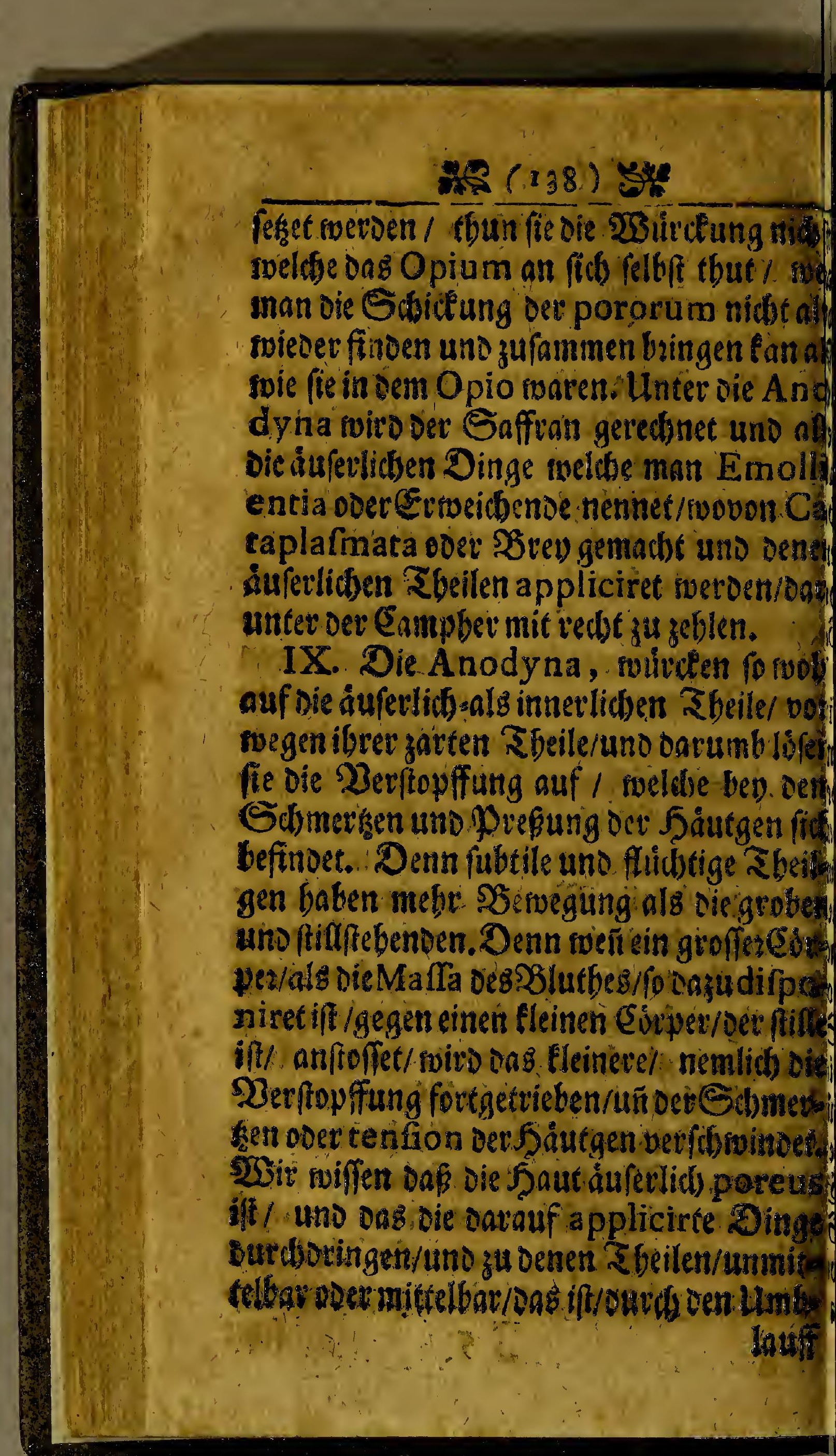




\section{Is $(1,39)$ s*}

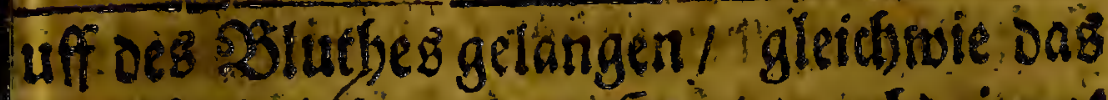

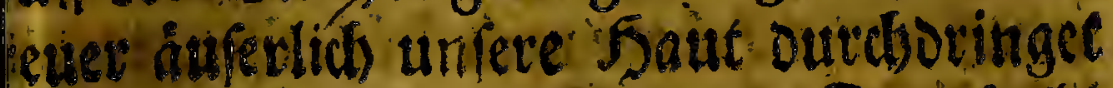

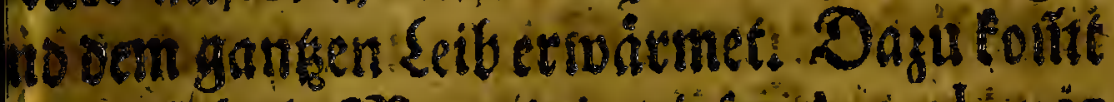
10 Die fubtile 9Raterie in diefentanodynis Itoe Poros findet/ dié bequem fino serglei-

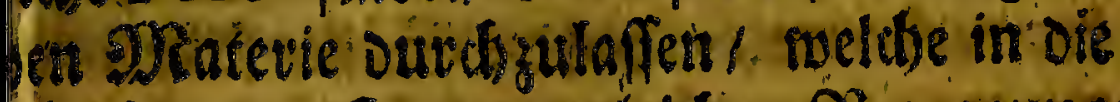
abulos Dec Saut Dergleidien $23 e r p e g u n g$. betbringet als zu Stilluring Des: Sctumér ents nòtbig ifli gleichivie Dag brentende

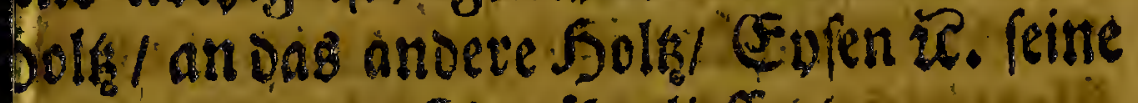
Berwegung uno Sigge uiberlieffert.

X. Albet auf nas

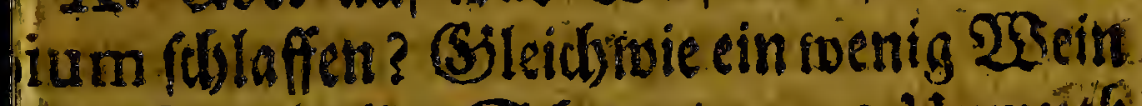

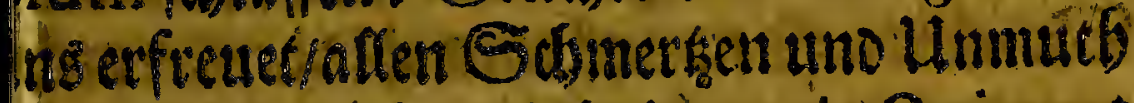
pegnimme/ fo thut ands ein wertig Opium; penn man aber bevoenz zu viel thut/ madbe es imifos uno trunden. (Darumb swerben ie Surctenvon sem Opio trundern) Riunt han zu viel/ madben fiz Sulloff und fo man tods mebr nimme / fdslaffe einet den ervigen

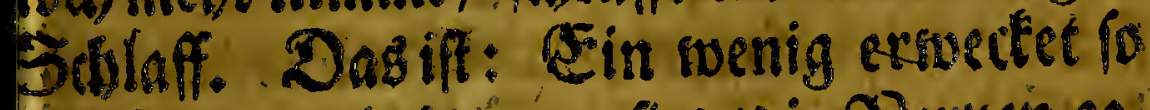
Diel (Sieifter/als bequem fino/ oie. Sierven ges Abutids) aufzufpannen; bey oem andern wet: en fie nteby aufgefpantet/fo daf mebr in oas Stebirn fonme als geborig / une oureb sie Sobduffe eine Sorte vor Convulfiontr: wectenhworaus unozoentlidie Phantafmaa entfpringen/ano Die 3unge reder mebr alg 


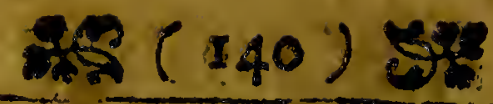

fie felbit roci fo oaś roeder Sánoe nodb Jühécibre getwó lidse Pflicht tbun. So man nort mebr nir twevoen die Säffe gleids fam bevoidét/(gleic)

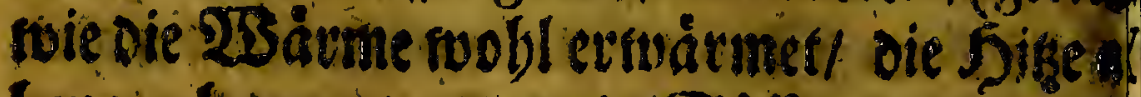
ber verbiennet / Das die Saffite ftiffe ftehes. wie man im verbiennen fiefet) oap bicte - Saffte in Das (Ssebirn fommen als zubou bieraus entfetet foslaffen turo fdinarche uno well truncten if / fáftet nisoer noie efit

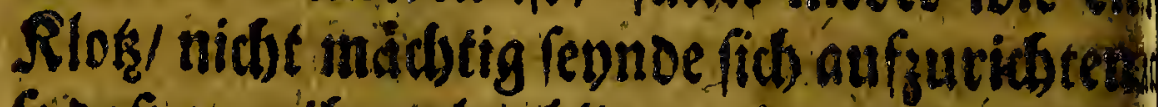
fo bas man ibm plak blino voll/wie man fagh madb Saufe umo zu soette foblepven :mu 2330 aber iemorno auf einmabl 3 j. oder mebs: Der ez nichs getwobnt/Opium einnimt/ ooe

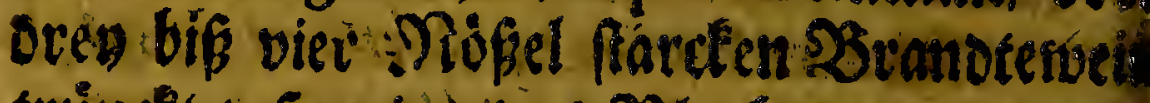
trundel / fo mirt Das s5lutb togen grofer.

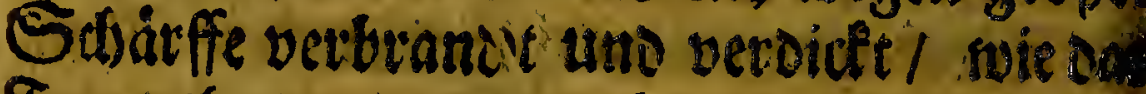
Feuer ffut rvenn nuir ibm zunabe fommen.

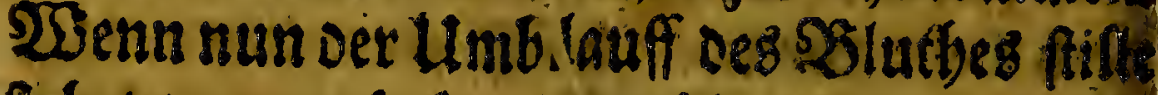
fiebet / verurfactet fo . wobl oas O pium all Der 25 sein den Toot. (Soldiergeftaltift a

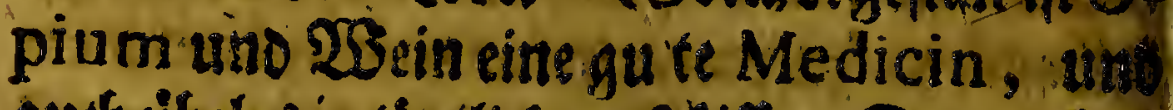
aud ubeles in tootlidbes (s ifft: Darumb; jungen Doctoribus, dienvenig gefeber ba ben/(Denen guten Medicis nicht ju trabe ge redi) roenig in (Sjebraudb on :s Opii zu trar

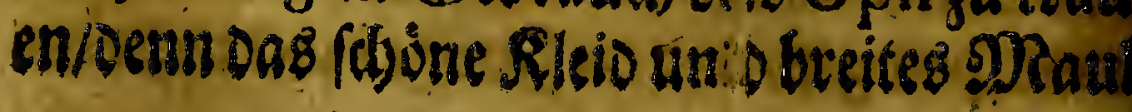


mit oog gemeine Dolet bezaubert foitrol adbetemenDoetor, ob ex gleidsfonfrein rlider Diann feun fon: Es fino niditalles

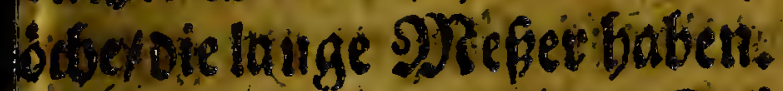

XI. 20iter bat was Opium, gleids kbeniabls von andern Medicamenteniff foget fwordert/ feine befondentne poros vobes. Sbrgen / nodurd die fubtile SDR aterie bes

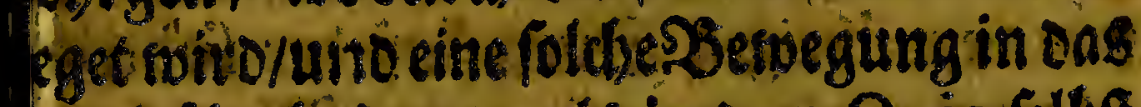
flutb utierbringet/ alb in oem Opiafelbft ar ; Stleidhoie Dag Jeuer feine Szerve-

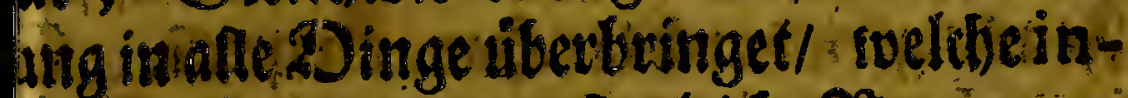
ammabel: fino/ oas fie gleidie SEenvegung g oas Seuer felbit twlangen. So dos OpiIn máfig eingegeben nivo / if bie Serwes ang mánigh uno foldtes madtit uns linoe

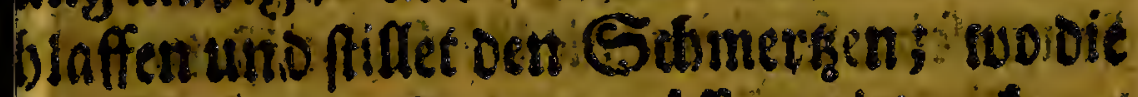
betwegung groffer uno grôffec fwiro/ toms

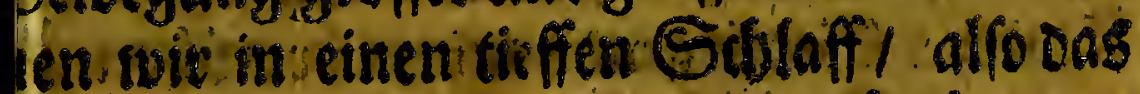
Whete. Theilgen in Diegarten tubulos Des

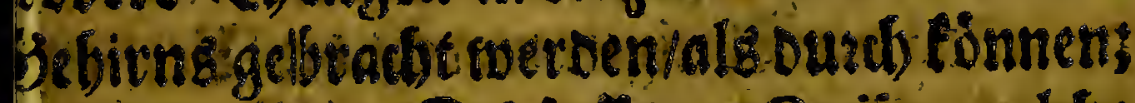

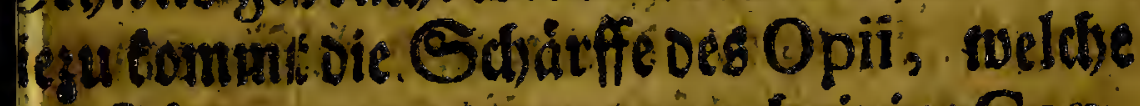
Ife છing gen naget/ savoriaucheinige Conulfionesindem Sebirn entfeberi/ toef ntrivegen audb Dag (Ssebitn feine plictst idte clum fan / 10 dáp fie in bem tieffen

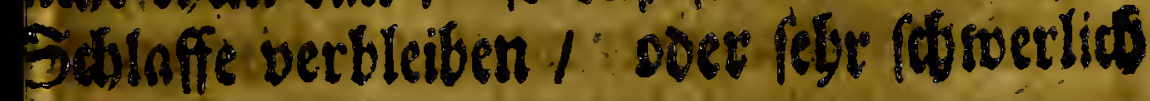
hantet iberoen.

XII. 


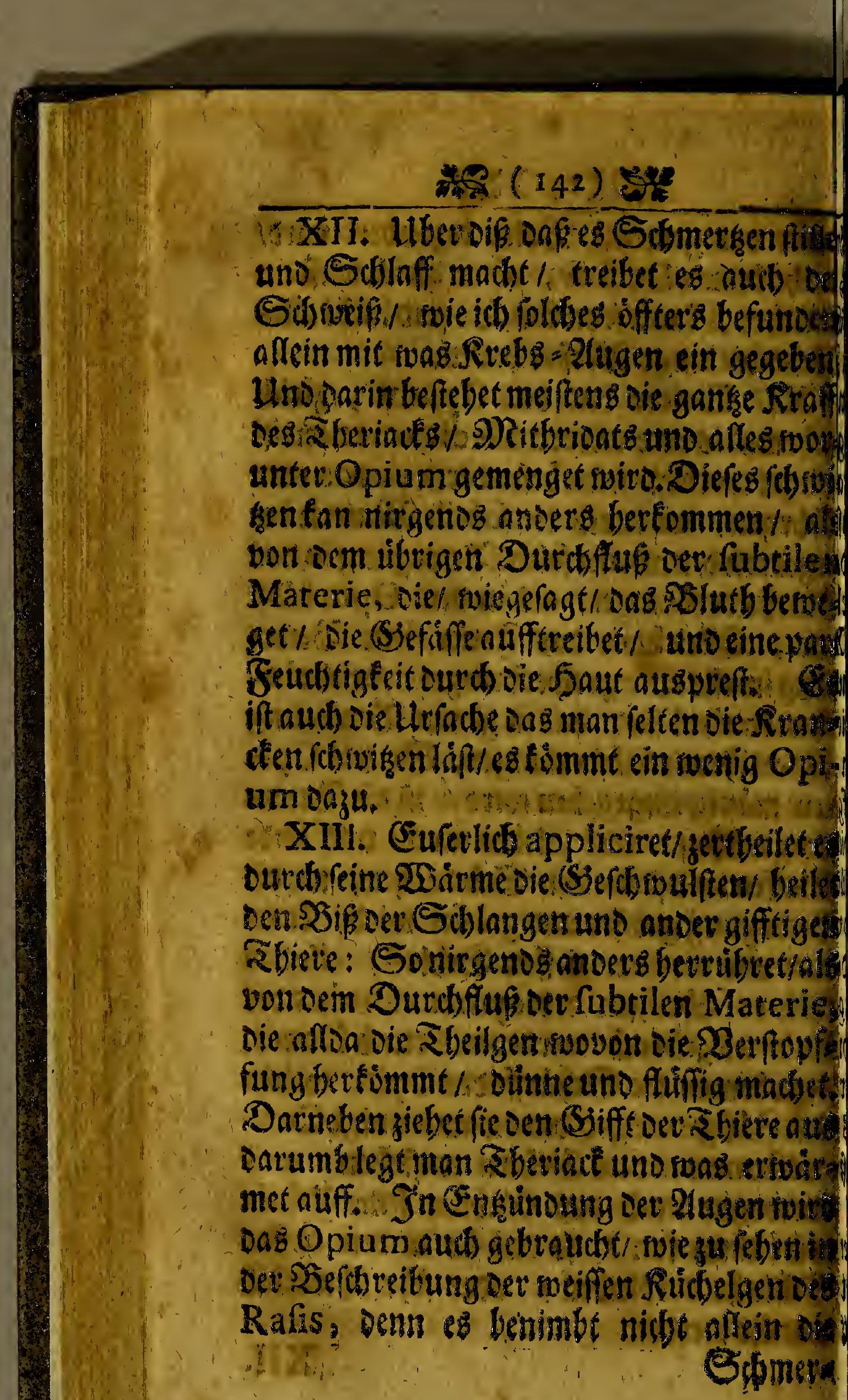


çmerken Der \&ugen/ fonoern jertreibet id oif Entzintoung. 2his oer uriachen

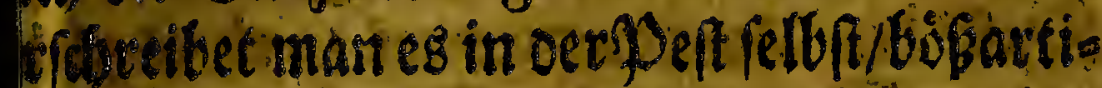

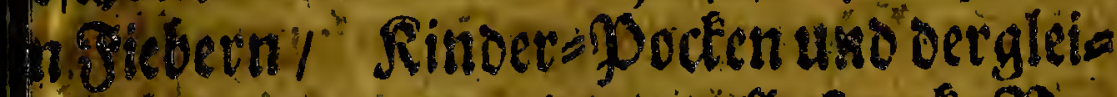
to es linvert uno minoert alle farde 53 . guugen unfers reibes/als in Sdimerfsent ampfi Jutfen/ Durdbfafl / Dumbeit? beren 20 . Co vertreibet die Bieber nuel bas sBlutf flilfig maitit / weldses in beten ebern oite iff uns rottet viel fauers aus. XIV. Es frage fid swie ond oas Opium

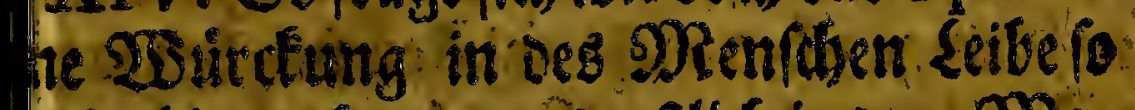

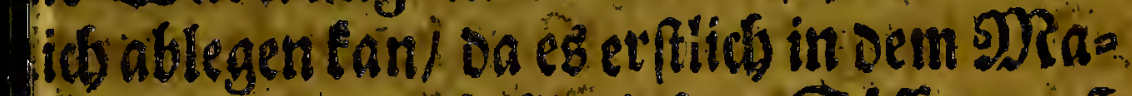
1 surd bie sa befindidien Safffe muf

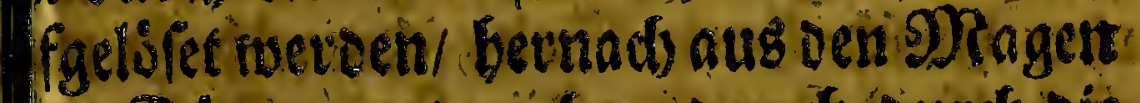
bie? Ávimeruno nady uno nach Durch Die:

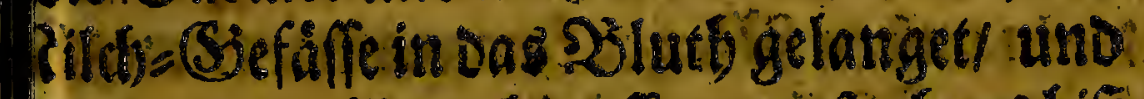
o efliche mabl umblauffen muf ebe es bif bas (Sicbirn fonmt? 2Illeine eine Jrage. gaman wobl imit einer J?age beantswortent bulids wie bers 2 rand tervein/fo bald er nut

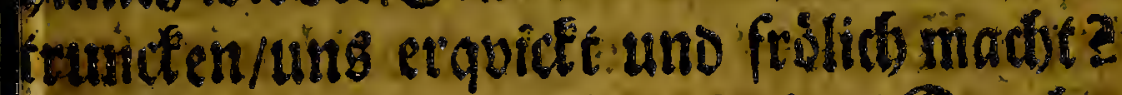

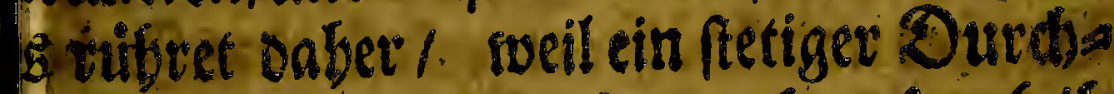

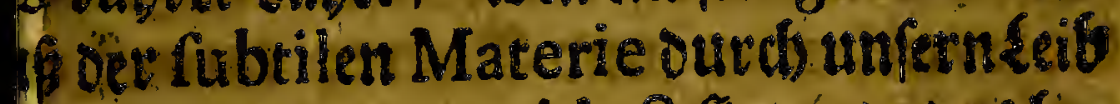
und diefeltbe ein Subject findende das lie:

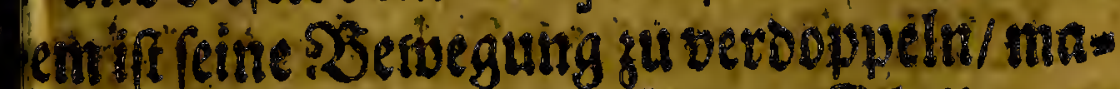

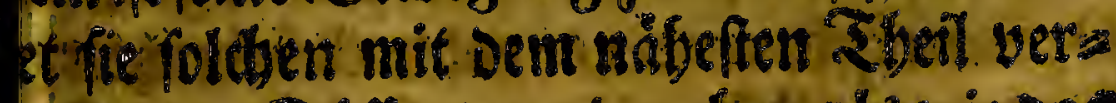

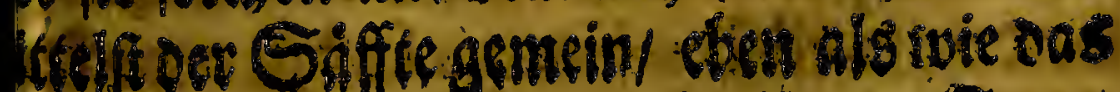
groute: 


\section{(5) $(344) 3 \%$}

Setiex uns marm madjet of eb. foson I eingenommen wiro / alfo fáge ids wiro of Rrafft Des Medicamentes. / ob es gleth noch in 2) Sâffenmitgetbeilet/ fonf if teine Uriad

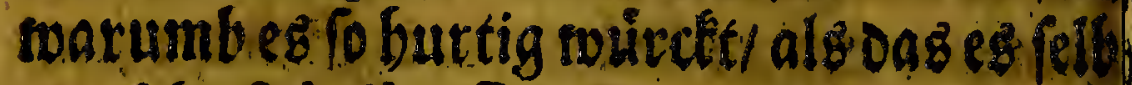
aแs fabr Rubrilen 2 beilen beftebet.

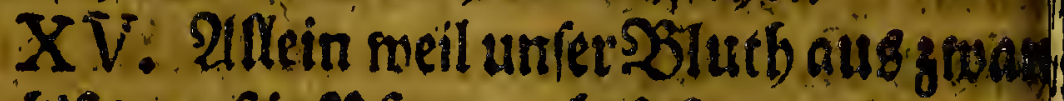

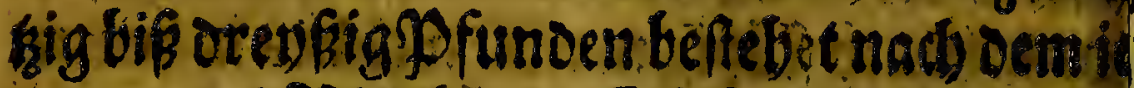
mand viel SBluth uno Saffte bat $F$ swiel denn ein Medicament in fo meniger D van

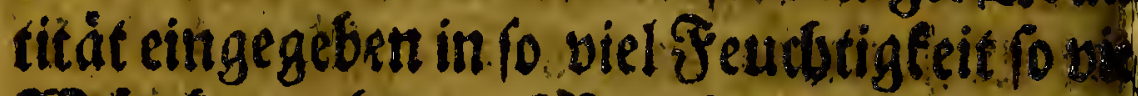
28titckung thun? 2)Ran bedende abertiol viel Jeuthtigleit cin Trappffgengimmet:

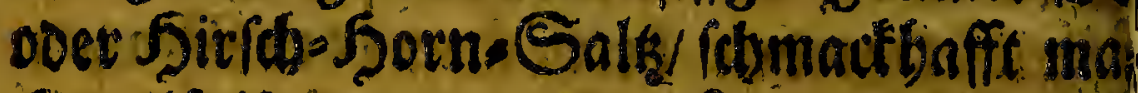
ijet/alfo iff ez mit der Tinctura Opii att Die in unfern 2) Ragen fommeno/unto aus of brileun FGeilgen beftebeno die ganşe Mal fam Des SBlutbs mic ibrev Rraffe inficire

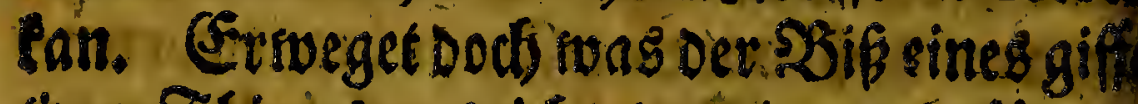

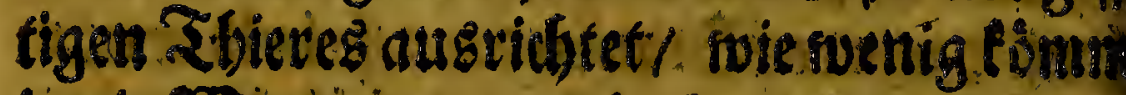

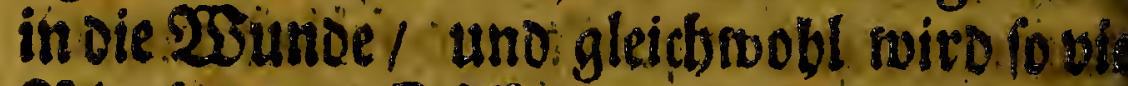
DSlutber und Siffte angeftecti / Das Jiebrt Convulfiones und Diaferey orrauff at folget:

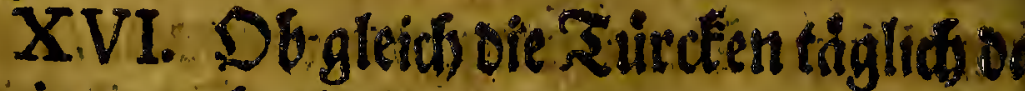
Opium gebraudben/ oadurdb befiertser:

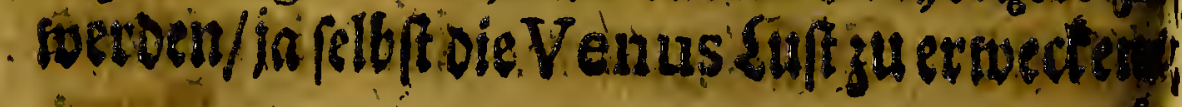
it: U. 


\section{$28(145) 5$}

bat man aus relation, Daf fie aflbano Jáffrig uno ouñ von Detftato merden; Wein tenen Diefes if bon sem Wein und standetepein zuesnatten/ bent wetn fie

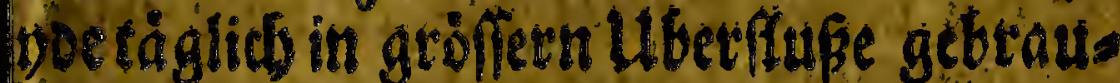

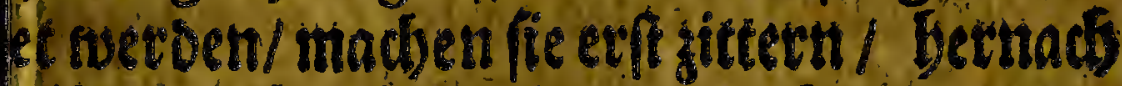
ffált Das@seoddbtnie uno Judicium ano trben bie Seute nie Rintbet. Diefes ges

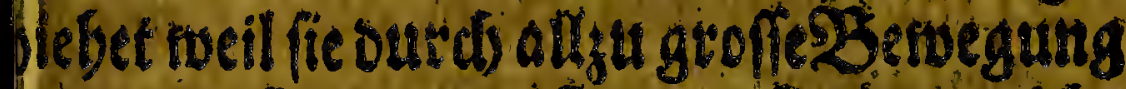

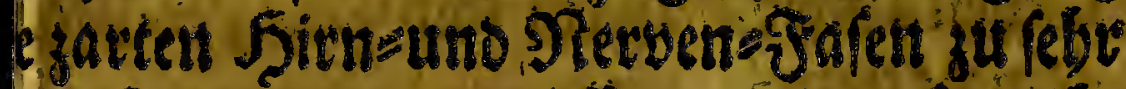
Isbelnetr la gar setreiffen/ Daourdi grobe kudhtigteiten/Dic zu Doubringutrg gefortse 15 2 errichtungen unbeguem ino / outeds. chen/ toldbes mit bem Opio, 23seit/

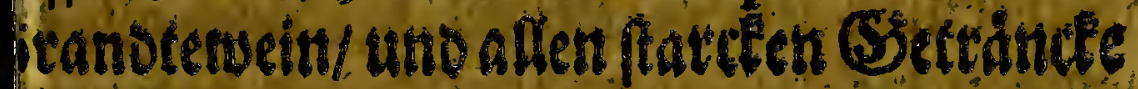
rosiebet:

XV11. Etlidbe babett Experimenta thadst mit oer Infufion bes Opit, in bie Dev eines Suntes I weldes infufum pbalo nadi bem (Siebirt circuliret / Deti ano gleido fobláffrids tnactit and ood cben

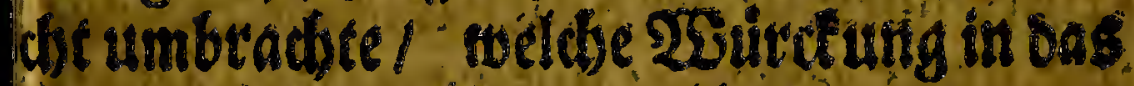
Jutb auf gleide 2Deife gef hiebet/als itsent. ourdis oem PRun eingenonimen tovotcen

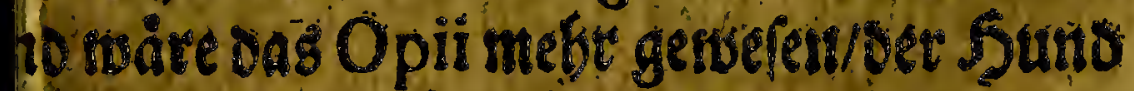
tefo gut geftorben fegn als went ef es get. slien biatte.

XVIL 2Altin ob Das Opium, fortin es 


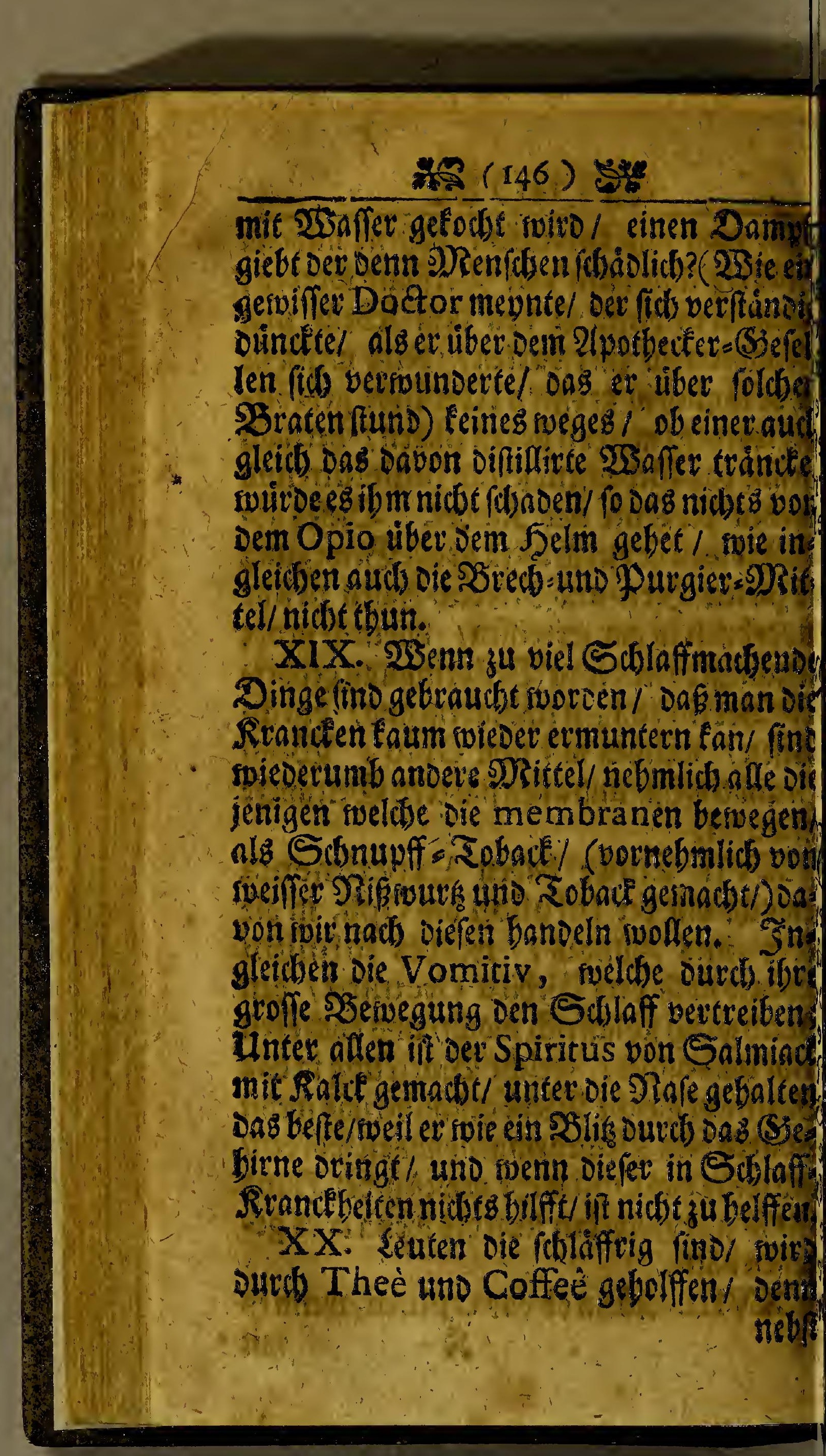




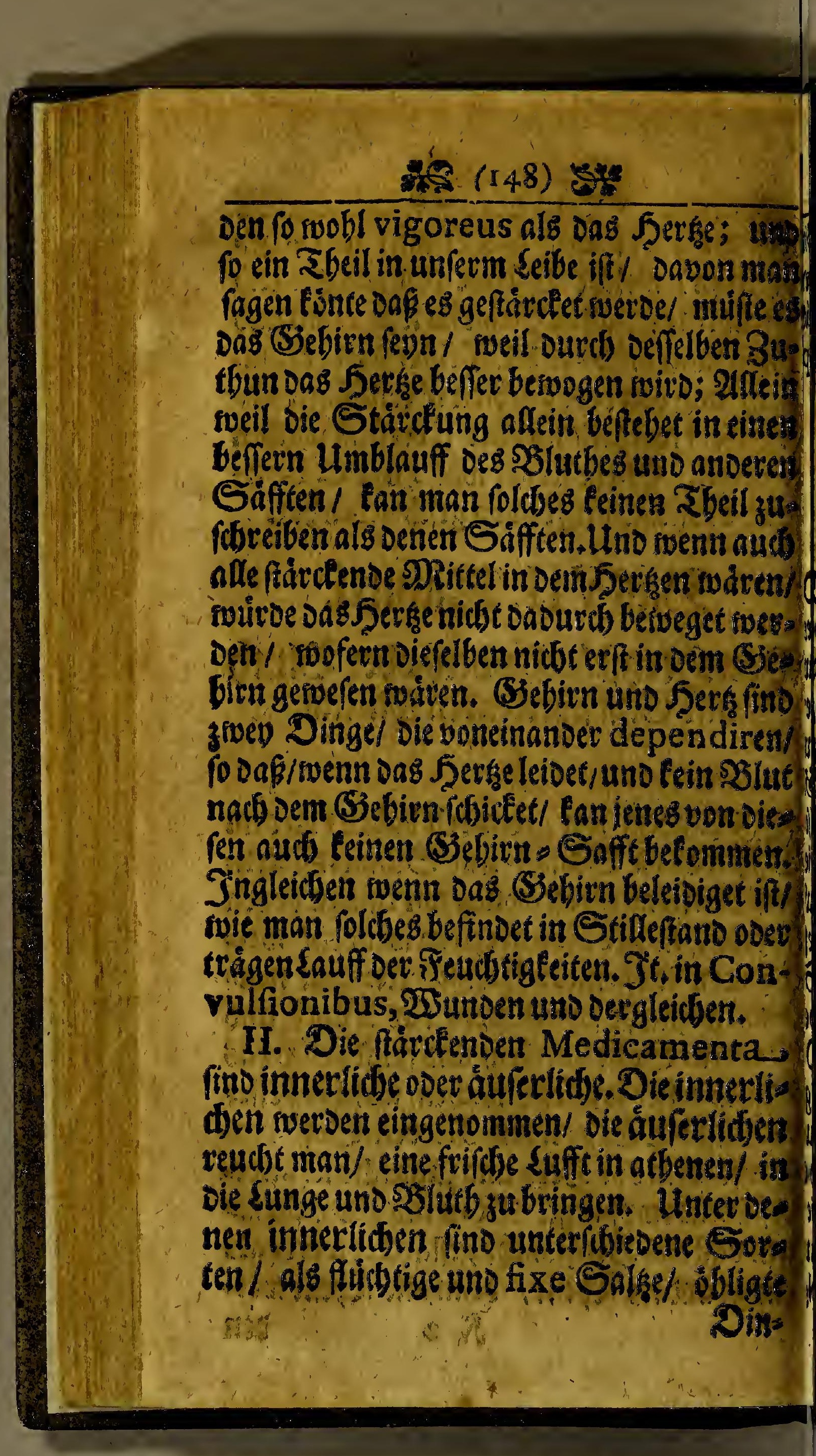




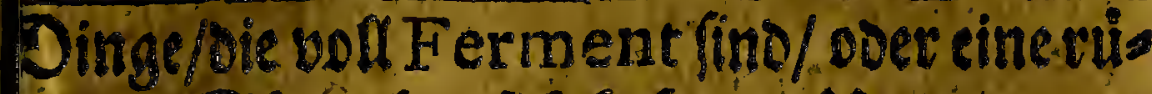
bende Saute bey fieb baben. Itnter oenen uiditigen Salken finden (ith)

piritus Salis ar- Seiff von Salmis

moniaci,

Corn. Cervi,

Offium,

Urinæ,

Fuliginis \&c. ade.

Sirfdsborm

Soeinen. $\rightarrow$

Sartt.

DFen $=$ RuF Xe.

Diefe dietien swenn iematto Convulfiones es 5erkeng uno (S3ebirns bat/uno oer Uim= auff Der Feudbtigteiten gebemmet ift. Denn erpdoen fonde/madjen fie eine grofe 2Serwes ung ber Membranen, sweldje mit oem Sobirn (Semeinid)affe baben/ movon diefes Yro afficiret wiro / Daf, alfobalo die Sáffie jie ein S3lis ourd bie Nerben flupen/ vars uff die Convulfion nadblaf. Utno foldbes efindet man bornebmlich in 5atupts Bdomersen / sweldser alfobalo veridowinoet benn man an biefe. Dingereudit/ meil bie Durdsoringligfeit Diefer (S)eiffer Die Fetuds gleiten alfo antreibet/ dap fie mit (Sjemale urch oie 2 erftopffungen binoringen/ wels be Hifache dez Sojmerkents war/Denn netn ie verftopffende Jetucbsigfeiten aidst forts auffen toment sweroen fie van denen nads: blgenoen gepreft/ Davon die Săutgen auf= 


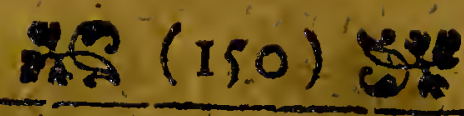

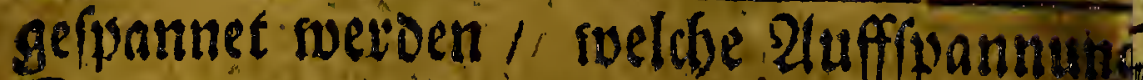
Sdbmersen berurfachet. Diefey dienter alt Iein zu einem Dorbilde / daf foldbe Sieifle ooer fuchtige Salge rine grose SSenegu berurfacben.

III. linter oenen Alcalien ooer fixe Solken/frno etlide aus Steinen/als: vol Stabl/ Rtebg=2lugen/ Sverfablen/, pet len/ Derl=s) Rutter / Salia fixa \&c. die cotircten nicbt aläbalo / fortoern nadj un nad) / imsem fie nicht gleids ourchoringen

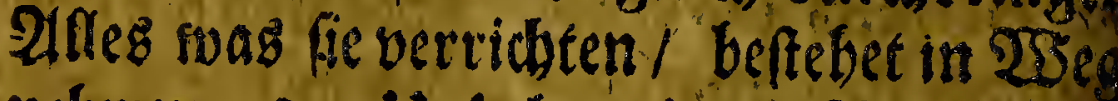
nebmung oet Ulvachen de oas SJlutb in fet nen lumblauff dick uno trage madgen. Uim. riefeziftallein bas Saure/ wie id in meine borigen Scbriffen mandimabl beswefent NBentnun rab Saure aus oemsolutbe bin

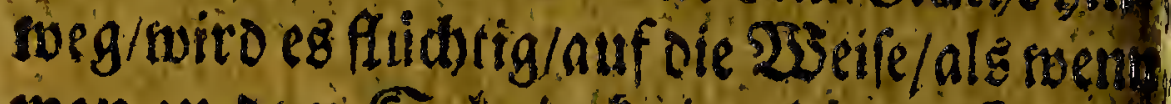
man zu ven Salminu ein Alcali fixu thut/ro zeuche dos Al cali alles Snuer in fich uno ons fludbrige Salás nizo fein eigen SRet frer/wie vicjenigen beftroen soldhe dein Sp ritum Salis armoniaci macben. Suth nt: Dem if cev Ourchfrom oet fubtilen SRate rie po gef doict/ oaf fie oem IJlutbe cine gan andere Durdifteblung uno folgents ein

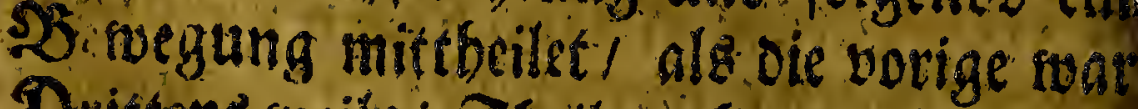
Dritten weil oir Sbeilgen bart uno unbieg 


\section{$15(152))$}

c. 4.) rwiro von cinen andern beneget: Corper bleiben fitfe lisgen/bif Das ein ander

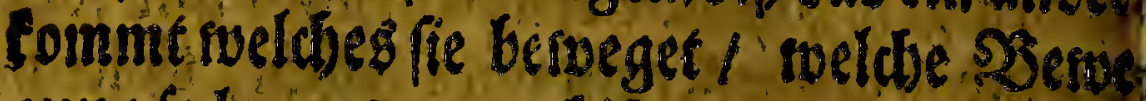
gung lo lange Dauert/bis eine anderelirfady fommi die der SEerwegung entgegen gefots (siefes num/Das siefe flidicige Aromatifo 3.beilgen in SEervegung fino / fo bersegen auch ondere, eben als mie Die Fbeilgeti

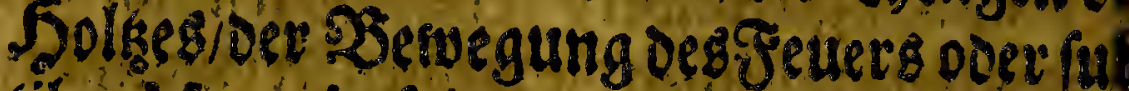
filen Materie folgen / fo rage ido/ werbe auds Die Fbeilgen der fobleimigten uno zâbe Jeurbtigleiten berweget melche Sierwegtwi

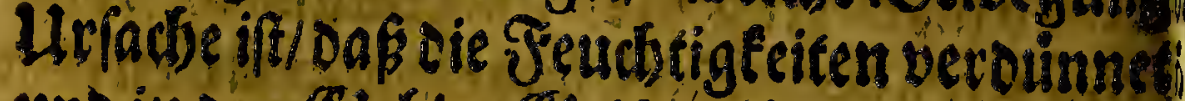
und in sem (Sibirn(Sieifter abgefondert wet

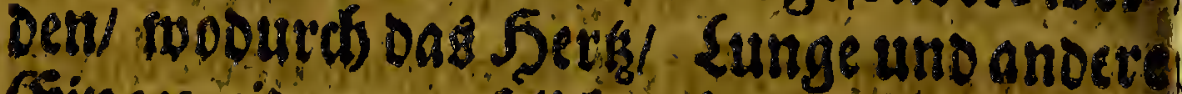
Cingerveioe gemadblidber beswogen sueroen solches vie vorige Sdjwndbbeit negnimun uno une far a madjet,

V. Dafs viel fubtile Materie durdfleu?

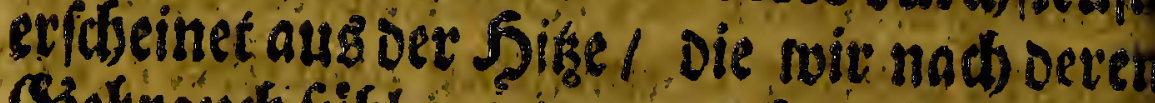
(s)ebrauth fublen / Denn es brentiet auf or

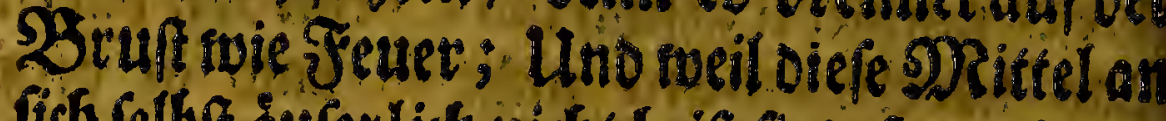

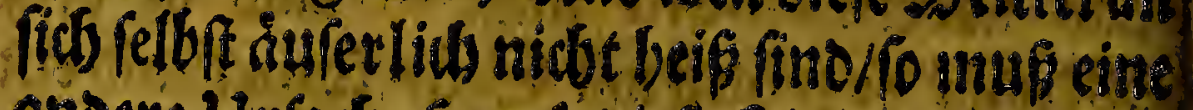

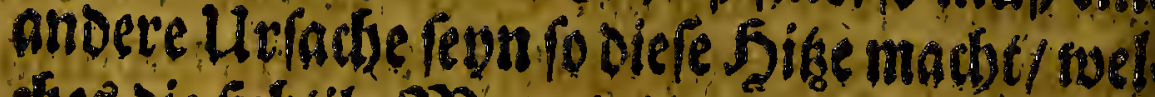

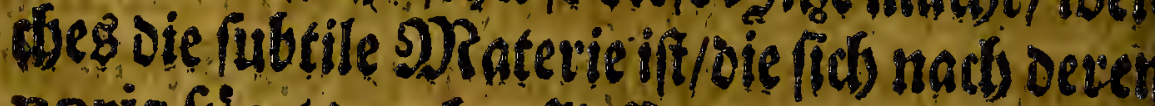
poris figet ourdyzuflieffen vermittelf : wel diser'servegung Die zazteften sbeilgen fortge

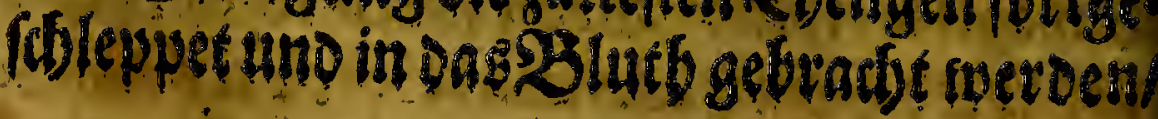
gleics: 


\section{을 $(154)$ हैं}

if/ Dab Der Dutchfug ber fubtilen Materi Diefer Dinge/ nidfitibersinfommets mit s nen poris ibrer (Saffe / woourd en dis order in benen Siffer/ uno folgbarlia Denen feften Sbeilen entrebet/ Denn fit wieoerwabtige Servegungen geten eine 11 oronunglinoein eine Species oer. Ifeile on Ders berveget wiro als Die anders. Gien. den offers alfuftarce. SEeroegung/ nd

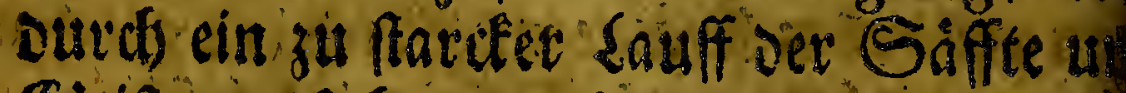
(S)eifter entebety weldbenadi Denen and

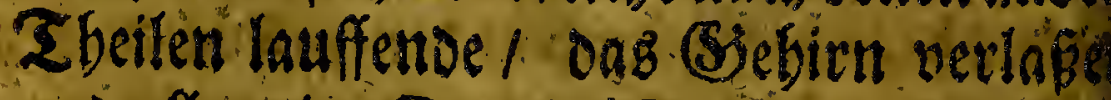
und alloa eine Con vulfion erwectertoiet nent Albricu von foldben Dingen gebiever Darumb baben foldbe Seute licber Sadjen o oon groben uno záben sbeilen fino/ alz Da Affa foetida uno Caftoreum, weldie gefdroinde nidb efurchigeben/ fonderti neg iljer groben Sbeile fich langer verweile sulerwobl fie in stnfefung anderen Sbeil flidyrig genung fino.

VI1. Daf ourds foldse Aromatin Dinge ein fràterer Trieb oer Saffe bo fommt/ ift surd Das Gxempel cintes geturíti Dredigerg/ unto anoerer befanor / sueld

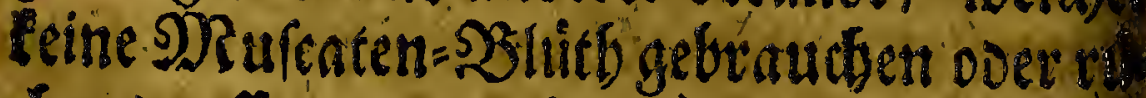
then ourffe/ ober es fteng ibm tie Sare an blutben: 25 ane feine farcefe SEeneguh 


\section{$\operatorname{ma}(154) 35$}

mentation; eg muffes grovederle 58 st gungenfenn/ eine bon Dem Satrem/ Dis at Dete bon den Solke. Wsent man einen 10. Salberftulteh in oie Suff bringet 1 that man s das oas Innglam lauffende SElutb u: Safftelourd einen tieuen Salpeter set on einer fuichen Suffe poer falien 23 affec/ fo ne frifde Suffemachec/ bortomme/ suiever umb leichtet berumb gefübiet foiro/ Denn no dependiren fofebr von Der Suffe / Dof ht ibrer feine balbe Diertelftunde entbeber. fonnen/oser foir fino beb Jobeb/melche E\& perimenta ourds Die Anthliam Prev maticam oeg. (joelen Sertn R. Boyle ge mugfam befands fito. Dis suffift in einet fold bet warmen Rirdie ooer verfdolofene Siemads mit Roblem/ voller forsoefflidten o Der zactidten Fbeilger/meldbe alfo oie:Dber bano nebmen/safs die S beilgen/ neldje unith ceben zu unterbalten begbem fino (nan mas fie Salpetrig oder anderg nennen/) untet:

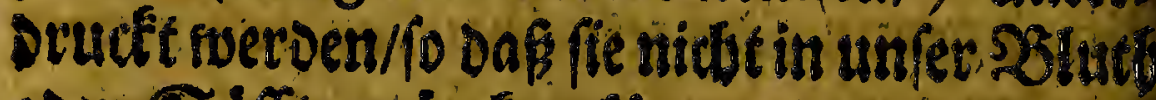

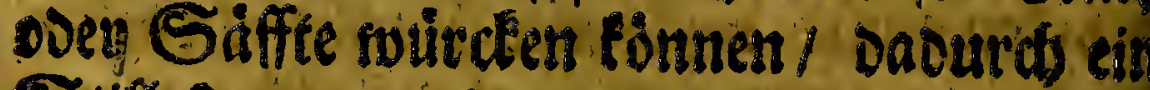
Stilleftano over langfame SEervegung bet: unfachet toirs/ woraus. Dhumaditeri/5ers. Hopffen / Spannutig Der Sirn = Sảutgen uno oergleichen entfeben: 25 ent aber ein

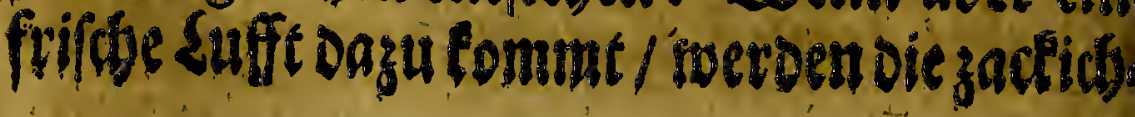




\section{$28(155) 5 \%$}

1. Fbeile ébermeiffet / uno oie fubtile $\mathrm{Ma}$ Hie der: Sufft/ befommt einen gangs anibert uadf us in unfern 23ituth uno Saffereet bor anders thefdbaffen nar. Eveil bie Protrion Des Durch= Fluffes nicht difponiwav/sas. 20tut in feinen gehorigen Stan uno fllipigteit zu evbalten.

IX. Den Êfig / Reinifhen 2Bein/ unt os nas fliddige . Tbeile unter bem Sauev *1 gebraudbe mant in fordsen SBegebentheis 1/ Daran zuriedben/ Dieweil fie auds eine

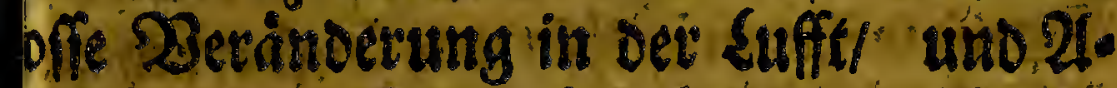
intolung madien/ fafteben wié bie frifthe

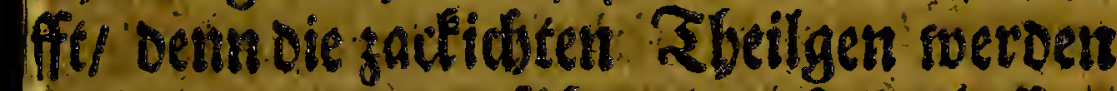
ra alein untergeovidet'swovauf eine beffeve urdfitrabiung Der fubtilen Materie ers

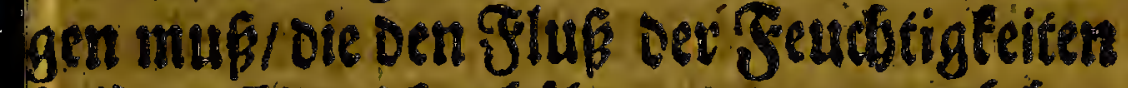
beffern (Sientidje balls / wovon das seben Seibes dependiref.

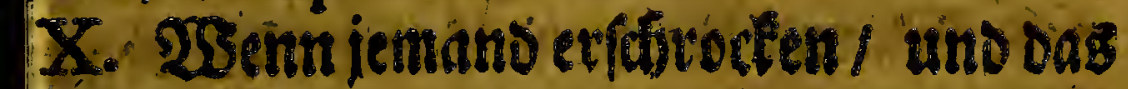
enge befleminet if / oafer ex wobf atcis in

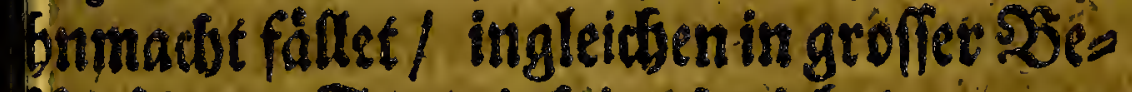

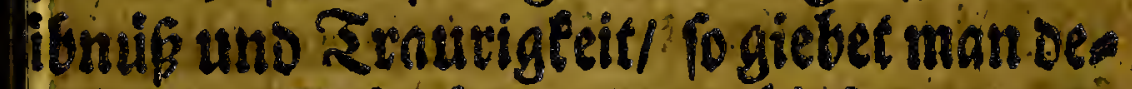

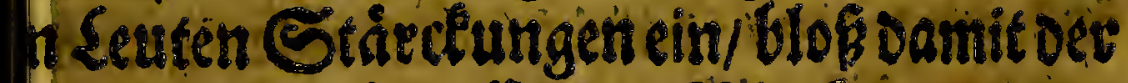
gafame umblauff des SSitutbes swiever

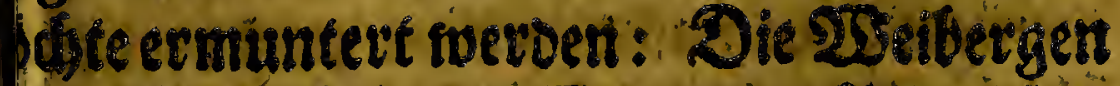

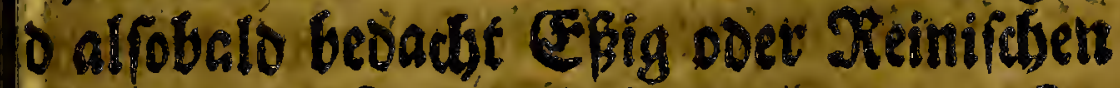
Sein vor die Siafe zu balcen I Jamit die fu- 


\section{$\tan (1.58) 34$}

beilen surdoringlidben S beilgen dine beffer

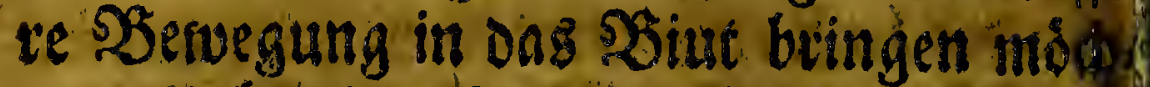
ten/ noie bereit mebrals einmablgefageif. XI. Ulber Dieinnerlicben uno duferlich Medicamenta gebrandot man-nolebefan auds fácutende Speifen: Diele flägn od fie boin Sunger Rerafflop soerden / Dafu fein beffer Sxittl wiffen als?fen zugebrom doen/ ourch foeldser garte Sbeilgen on Sluth gefarcket roiro/ bornebmlido/ fo ol

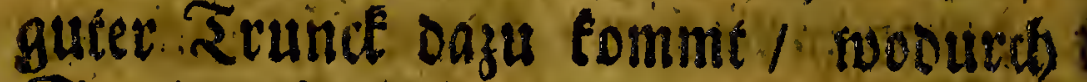
Speife mefor aufgelofet uno folgbarlid me fubtile sheilgen in oas solutb gebras fwerden/ uno die fubtile Materie beffer $\$$ berband in denen Siffén befómmt; $D$ umb befinoet man fids nad sen (Sfent nid

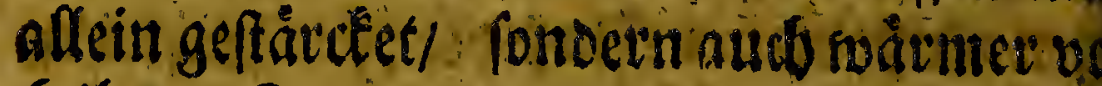
seib uno Freudtigfeiten: Die Wärme beft

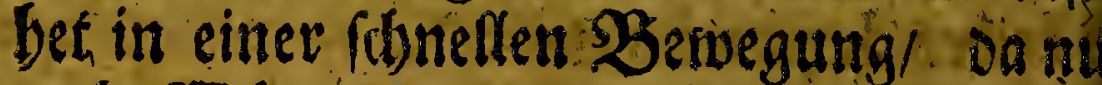
mebr 23 irme formt/fo if mebr:soservegum als zuoor / welche von Dem Durdb folefeno Simmels= Feuer ibren Urfprutig bat. XII. Anein es toico mit jemano einfoet fen uno lagen/ oaf ich ebe mablen geodely wie ein D) Renff 20 . bis 30. t6. mebr oo meniges SIlut/nadosm einer grof over bo

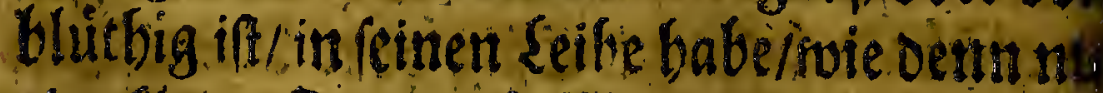

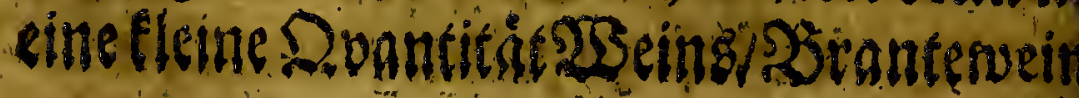
aising 


\section{$46(159) 5 *$}

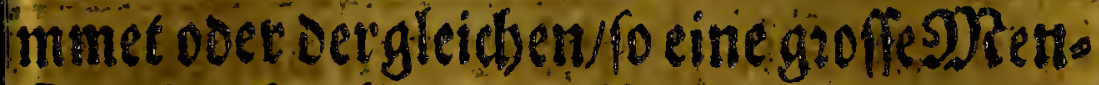

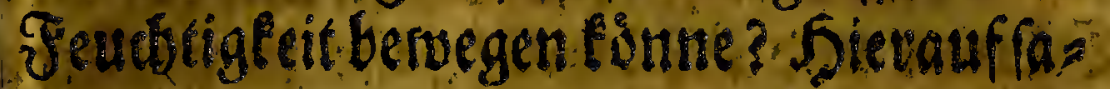
in sie eit wenig Vitrium Antimonii

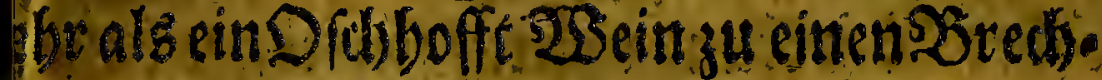
pein madien fơnne/ Jt. nie cin gr. SSEeg

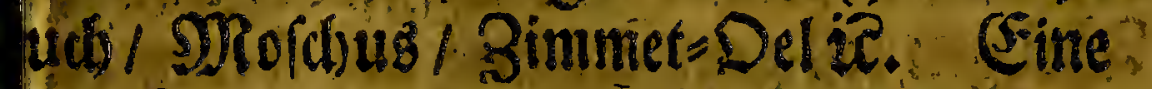
thse Rammer mit feinen (Sieruds cinnimt \& c8 ein ieder viedst. Boyle ferget cinen ropffen Simmet Del gegen mebr als vietben taufeno Tropffen weitt/ Derobalben iffen diefe ftàtulende Dirge/vor serffe aus or zarten Fbeilgen befeben / bor andere. iffen ibue porialfo geftalt fessn/ oaf oie fuile Materie in groffer פlenge fan ourcheffen/ taburd viel zarte Sheilgen bif in - Sóffe des Slutbes gefibret werdent bon in einem Alugenblitigleids als von eis n angegunderen SBidsfen - Pulver/ Die pnfticution des ganisen SBlutbes verins we suipo.

XIII. Denn oen (SSang diefer fubtilen taterie fublet einjeser felthf ourb oie Sis 10 oiefe fántenoe DRittel auf Der Bunge achen/Denn fo einer ein?3irnts? Plegel odec idégen foratfen Bimmet fautet auf oer

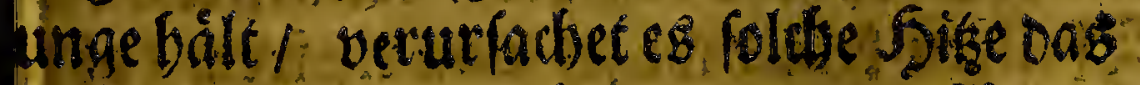
p jouno fcheinet zubremen/Darauf folget Soire Stándungen nidst ebennothig bas 


\section{और $(158) .83$}

ben in Den SRagen zulommen uno ourds Darmer uns Shilds.Sefâfe zu paffrem

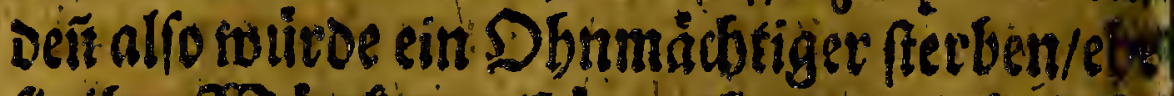
fie ibre 23 uitutung thaten/ fondern fo balo fis von bem. Speidiel fino aufgelofet uno ibu Sbeilgen dof gemacht uno auf freven $\mathfrak{F}$ geftellet roordery betommetalfobalo ois fub tile:Materie S3elegenbeit ourdb zuftublem XIV: Siec mócte iemano fagen Das oes. stibaug feften Sbeilen beftủinos wie fonte Denndieftardienven Dinge vur d oie fefte Fbeilc oringen? Sben toie die Sonthe un Decofelben DSsinme ourdis (SInB / Doer oi Jeuter S beilgen ourcb einen focbenoen' Dringet: Demn ob man gleich teine Deffrum get: Darin fiebet/ fo mus man fie oo b mit Def Serftande begreiffen / welches in Die Mera phyficä geburet. Hippocrates fat foldjel fobonzu-feiner Beit gerouft/uno die Dernum lebret es zur genige/foenn man fids nur red beoendiet Denn alle groffe esuper befteben aนв Sbeilgen tor allerbano Figur, tunb, ectidot/lang/furs id. WSenn man nun be Dendet daf unjer Setb aug fo vielerlen Sor? tén bon Ibeilen beftebet/ sunen svir dief Ibeilgen feines roeges concipiren/ (ob) aud nod to fefte an einander gepref ose verbunden waven) oder mas concipiref stw gleists 


\section{$25(162)$}

Seiber gefuno fino/ Darinnen felbiges ing

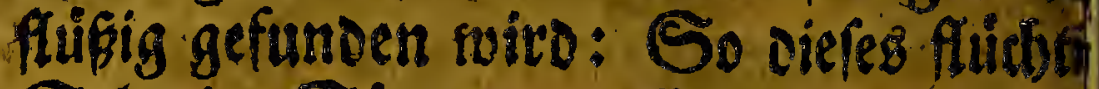
Salscine Säute antrifft wito es miee Salmiad/ Das zroar robbl aus vielen flid tigen Fbeilen Des Sarns umb Dfen-Xuk beftebet/ allein eb. if figiret ober fefte gem

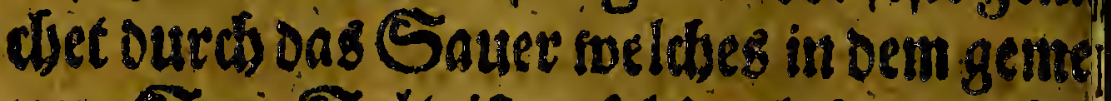
nen See = Sals if ; plobes lop sumadies tbun foir cin feftes Sals! oder Rald Dors fo swiro alles Das Sauer ourd oas fefte Sald

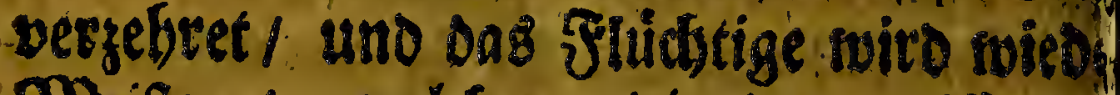
Dreifter ! weldses Dicienigen toifen. Den Spiritum bon Salmiad bereiten. ben diefer tbun siti aud in unfern selph ourch aflerbano Drittel die oas Sauk Dámyffen.

II. Dieres) Rittel fino unter/fitedid//nt toeber fefte oper fluditig. Die fixa ober fin. ften bat matn aus henen Tbieren / Groge

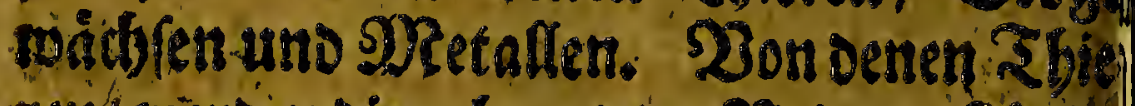
$\mathrm{ren} /$ roevoen die gebrandet SEeine / Sorth uno Steine die Danimnen gervabfen/genow unenals

OsSepia, Girussoin Oculi Cancrort, Rrebgolugen. Chela Canen, StebzeSdeten Ofrex, Conchas glufectobales. D) Ruthelichalen 


\section{$\operatorname{xoc}(164)$ sto}

blauen/ formarksen/zc. Corallen. Diefe me Den aud unter oie Alcalia fixa geteduty Ivell fie farch mit dem Snuer effervefeirs uno oaffelbe tóten/ nie zu feben in sDerfel tigung des. Màgifteril Corallorum Unter die feften fauter temperirenoe - s.

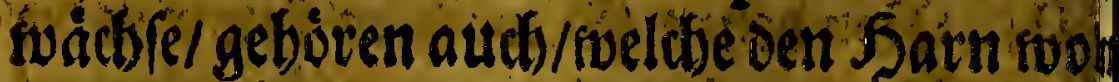
treiben/ po fern daourdo biel Säls uno Sal erg abgefubret wito/ dergleiclad fint Rle

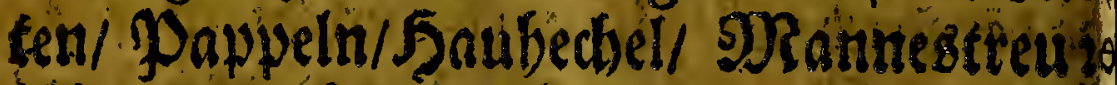
Utber diefes finden fich ibligte uno Arom

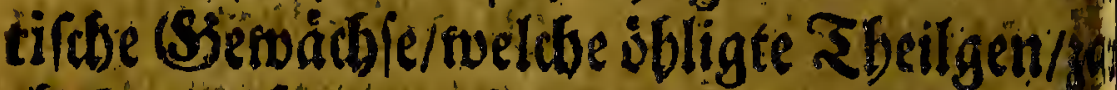
dicht uno bequem fino bie fauern Degengy in ibre Zlimen zufaffen ourd toeld e umb. faffung fie als in Sofjeioen fecteth uno nid mebr forneiden fs nnen/ Daber fie eine anto Servegung exlangen um gegen Das flud ge Salsuno Saffe un/ers Leiber/fiol gat anders berbalten ald zubor/untec ocheth on Chérefolium, Petrofelinum,

Semen Fraxini,

Lignum nephriticum,

Apium,

Levifticum,

Rerbil.

Daucus \&

peterfitien.

Samen bon fotsen=-25aum. Srifosols.

Espich. Siebroticter. Sdirbart: Ruth mel 36 . 


\section{$25(166)$}

len, Dinge/ Dic von innentig uno a usto dig oas. Satter temperiren/als ca fimo

Anrimonium,

Creta:

Pompholix,

Lapis Rubinus,

Judaicus,

Nephriticus,

Saphirus,

Smaragdus,

Tutia,

Silex,

Lapis calaminaris

Hematites,

Ceruffa,

Lithargirium,

Cinabaris, \&c.

Spief̧glas.

Rrcioen.

Nicht.

Rubinftein.

Tuoenftein.

(Ssrigftein.

Saffir.

Smarago.

Sutit.

gieucretein:

(Solmenfteith.

SElutblitin.

Blevweip:

(silette.

Cirober.

Siecunter gebsven aud bie Terre oo Croen/sls:

Bolus Armenia,

Vulgaris,

Alba,

Terra Sigillata,

Arena,

Argilla, \&c.

Itrmenifder?

(Simeiner

23 eifer

3. Slü.

Siefiegelte Croto.

Sano.

Sbon:

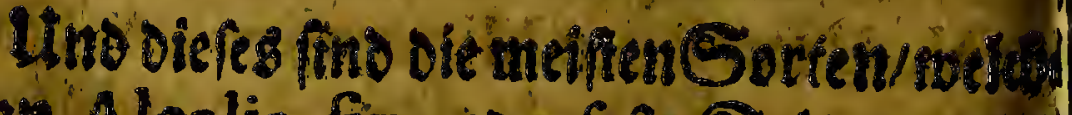
man Alcalia fixa doer fefte Salge nentu 


\section{2x $(167)$ है}

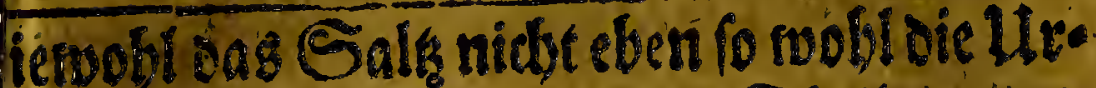
afeiftals vic difpofition oe? S beilgen uno wiffen=Xäume/swie twit bernads erweifen

oller.

VI. WBas dieflúdtigen Saltze angebet/ langen nit Diefelben meiftens aus bem

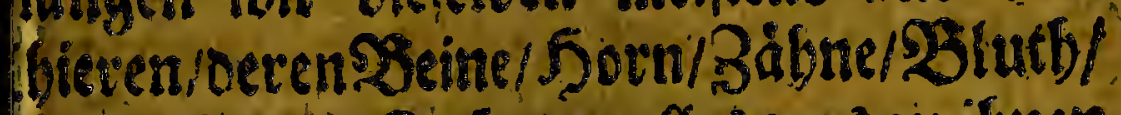
leiro Sart/ Rotburo alles mas an ibmen $/$ giebt batuffig fhidtiges Sals/crierwobl $\mathrm{eg}$ Ad)/ als gebadht/aus fixen Salken ctlan trwico. Der Dffen-Xupigiebt audb vill eldeg meift ein Vegerabile ift/ uno von It was in unfern Turf combutibelif. biezu fommt ong Salmido / meldieg boll iddigen Salseg von Utin uno. Dffen-Xuf / Daraus es groften S beilg beftebet. uno iefes fino die meiften fautr temperirende Dinge/ aus Genóden/ Sheren uno Mieralien.

VI1. 23 it wollen nut tweiter gebon uno

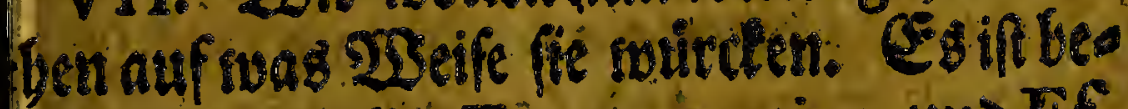
andioas in aflet Fermentation tho Ef-

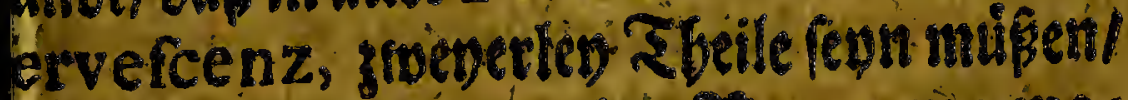
benn fe-mitemandet eint ssetpegung mos

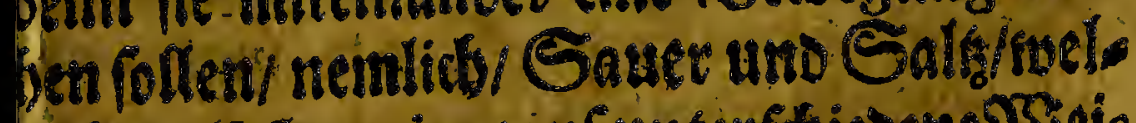
be fenoe Materien/auf unterfobiedenesseis. 3efueget rwetsen. Wetn mun diefe Sbeile vfammen fommien/ muffen fie aufeinandec 


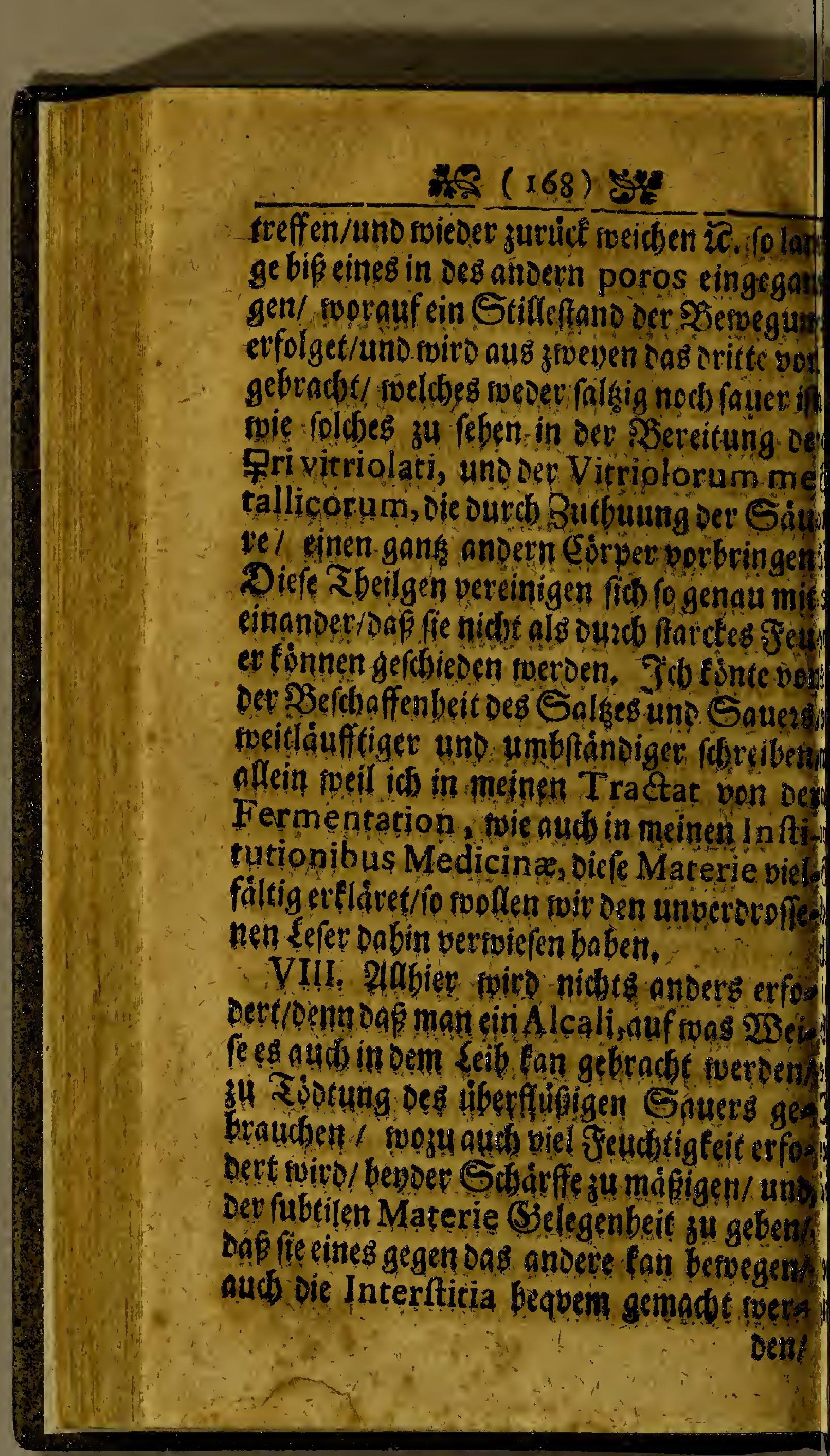




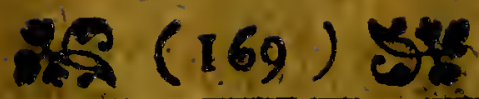

en/ Dap genunglam fubtile Materie ourcb. treichenfary Davon oie ganse \$senvegung dependiref:

1X. Die Alcalia, nebmenoas Sauen aub fleinen Degen beftebet/ in ibre tubuos barimnct fie vermabeet feynoe/bon ibret Benegung ablaffen: Diele Dereinigungf

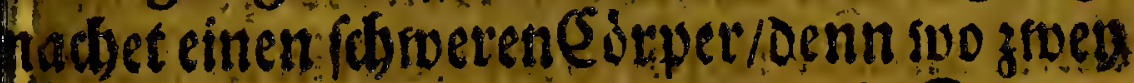
Sorver aneinanoer bangen/if mebr Sob he e ale oa ieder vor fid felbft ifte oaber die, ,jea begung nud gebentut roiro. Sleichnie die. 8. nun fich mit ochen alcalibus begiebet/ fo trbalt eg fich mit oenen fetten Dingen gants noev8. Sif genif / wie offers geoachth as alles Jett uno Dell aus jnclidten (ranofen) Tbeilgen beftebet/ welcheourd oie Bjervegung oer fubtilen Materie, ffol leictsts idi) umb die faurit und faftigten Sbeile dolingen uno oiefelben umbroinoen/ daffe hisht/ oo er oodh febe soenig.ficden uno nagen/ ben soie ein SReffer / fo in eimer Sibeioen tecte poer mit einen Sudb umbrounden iff. Daber leidetlid ju begreiffen/, oaf audb oic setten uno oblidjen Sbeile/ oas Sathete peimpfitt.

X. Das Waffer/verbienet add nidft bie

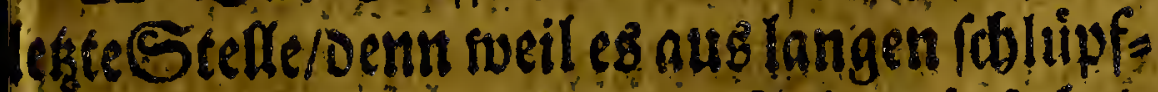
Higen Sbeilgen/gleidj Denen Algen beftebet/ \& 5 


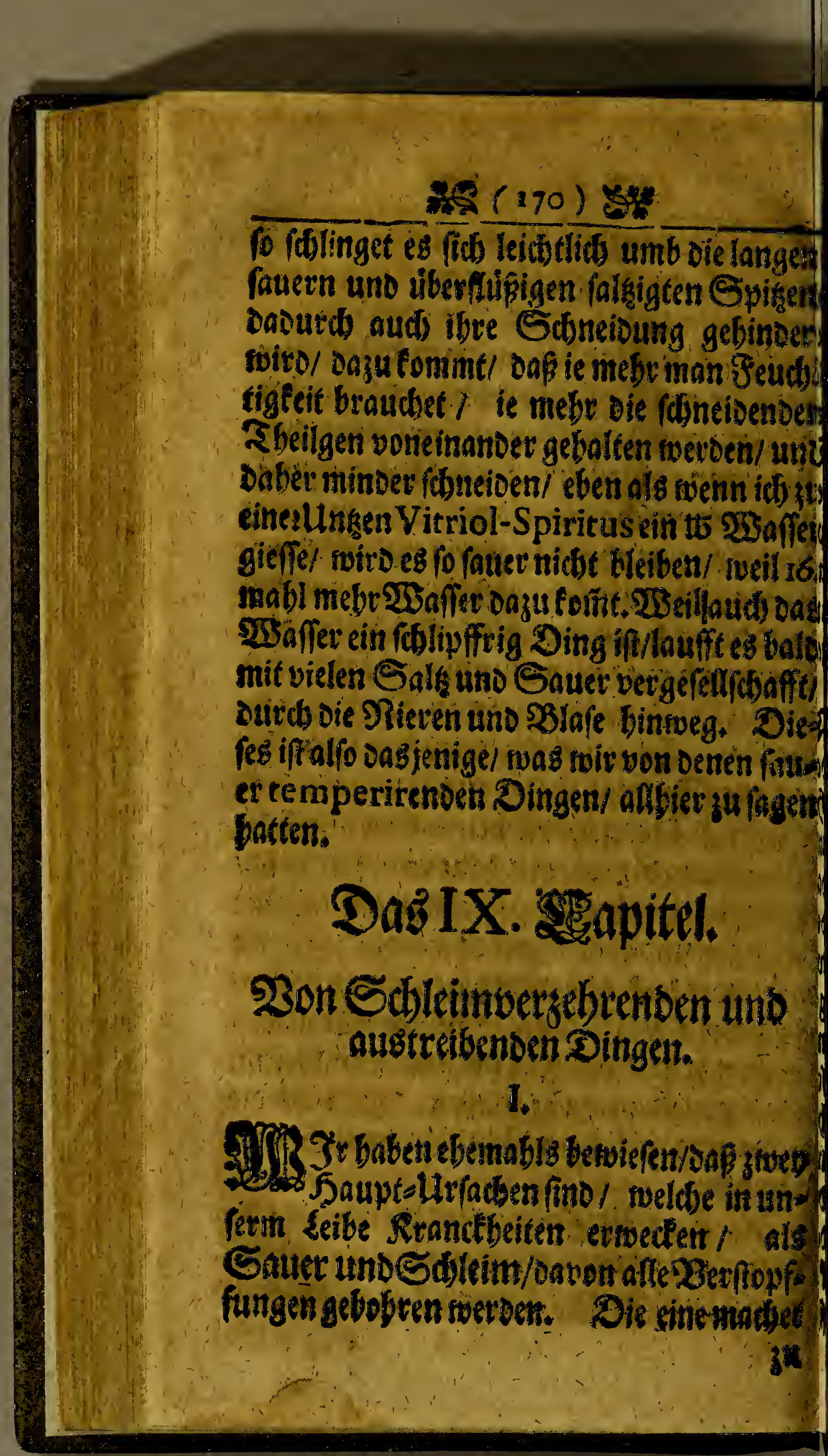




\section{xe (171)}

a viel Shageng uns Qdufwaftens / baber Soner uno 2uffivallung ertftebet; Lnt ie anorte madit eine Zübigfeiti sie alle zarte Wubegen mit ibren S Sdlkim berfleiffert/ oas de langrame Soenegung uno folgtarlids. Evadgheit uno Ralte entftehet/ ano ro fre beys ci als offter gef getiebet/zulamment tommen/. Wielen fie iber Tragcedie, uno mactien

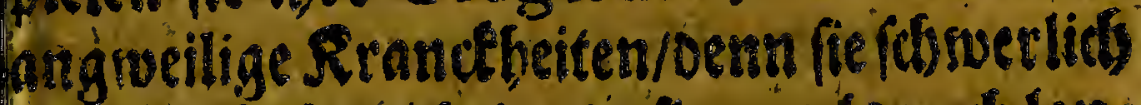
tus vem Leibe zu bringèn finol als nach lastSen (Biebrautio guter uno beqbenrer פRittel.

11. Der Salleim befetbet in ein bauffen Kheilgen fo allzumenig Deswegung babent daber fie trảge lauffen/unt soeil oie SGeilgen Sactentweife anteinandeer bangen/fino fie zad Lins tleiftrig: Dev Saffe/fo in unierm Seibe bazu am biquem/fen/fif oez neue Chyl, fo aus bei Speife borfommit/ rwelder ourds alles Dias colt if / feine SBenegung verliebrt uns gerinnet / benn alle Ralte befeliet in einet Guirtoern: Singegen afle Wärme in einer

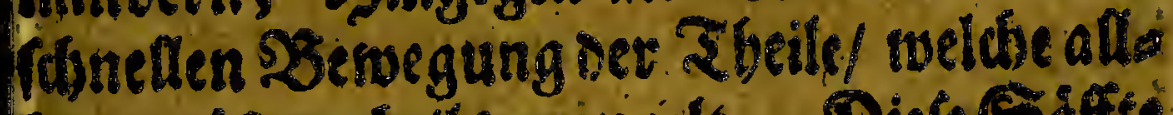
Giet groffen theils mangelt. Diefe Saffte wetben alfo ourds oie Ralte gemadit/ als ourdb falte Suffel swenn man fie einatbnet od oet fiet entblbfert; ooer ourds Benieffuns

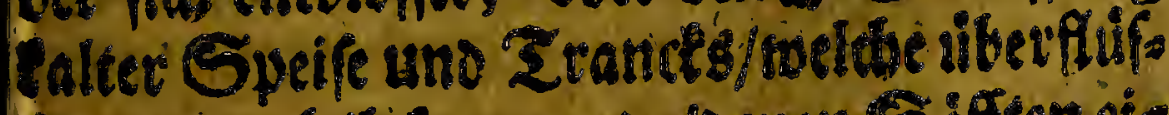
Ig in dem scib commenoe/omen Saffen ei: 


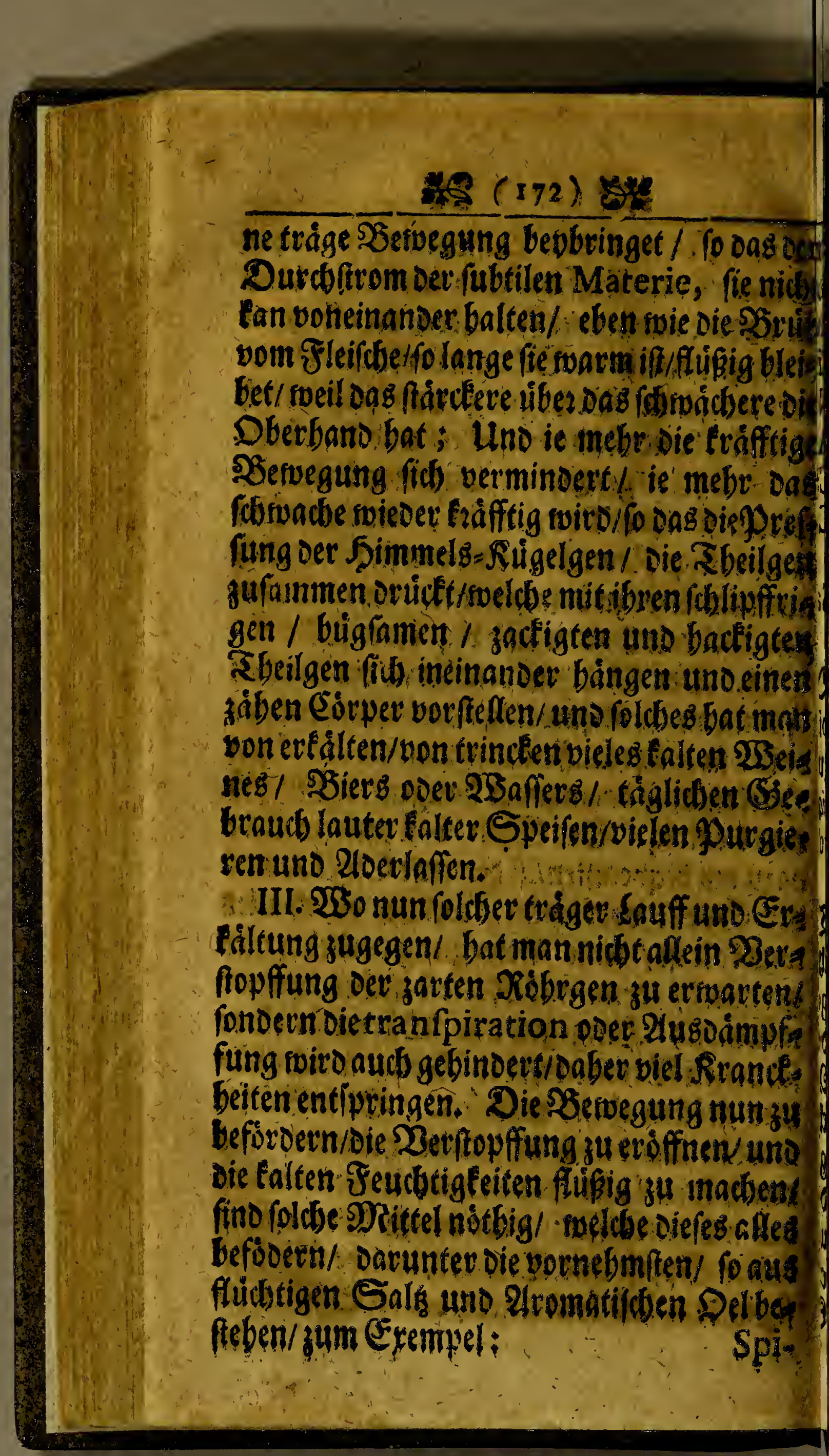




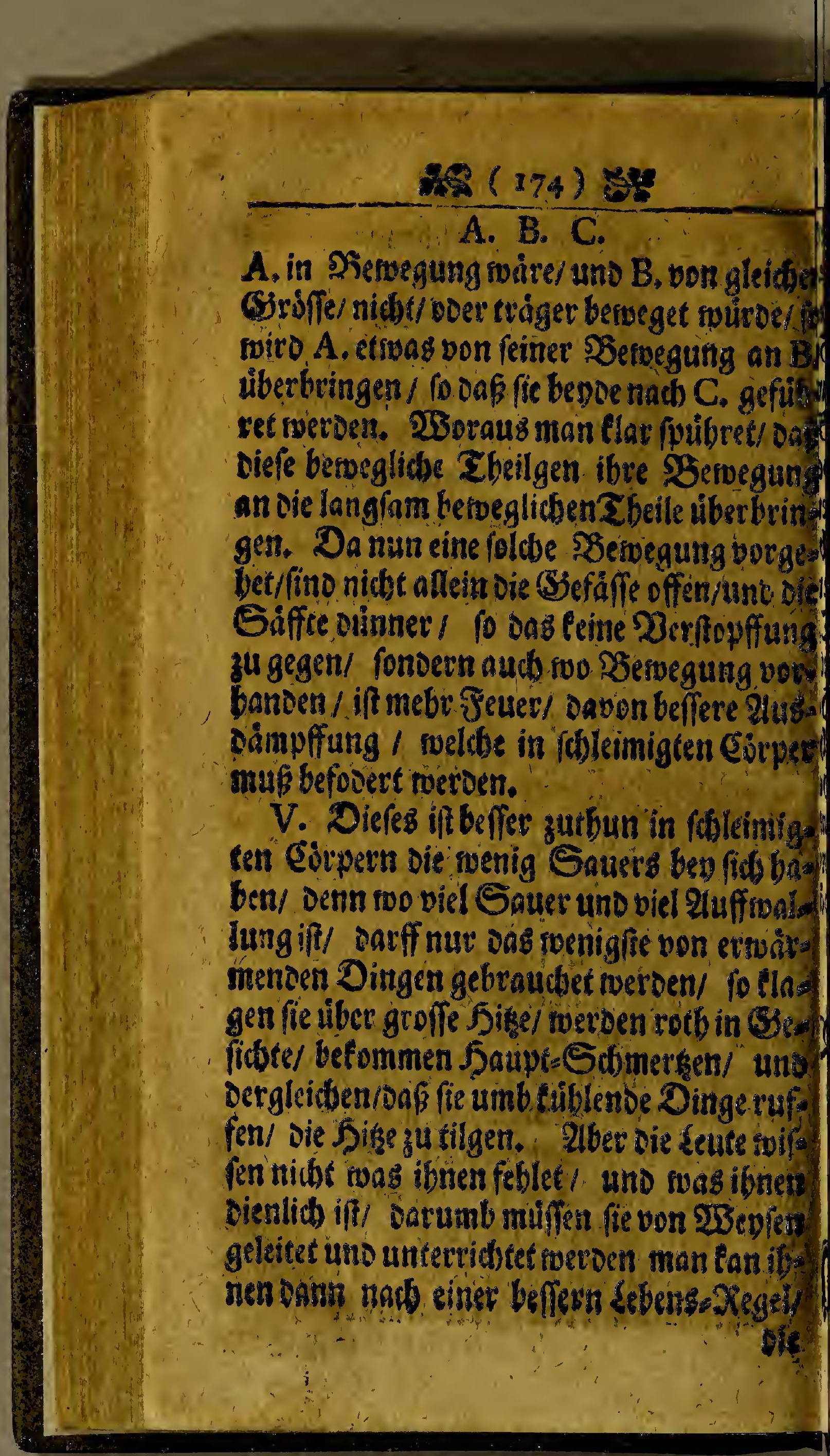




\section{as $(275)$ ses}

ie flidbtigen Salinge mit ein nenig von einers Medicamente aus Stabl beveitet/ vermifiet/ aingegeben/ woburdin nidst allein oas

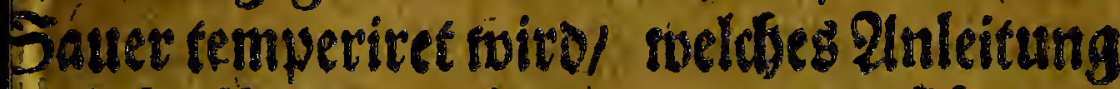
av Ebullition gab/ fonoern das fluidtige Bais/ fo aug zarten/ unto oarumb leitet bea

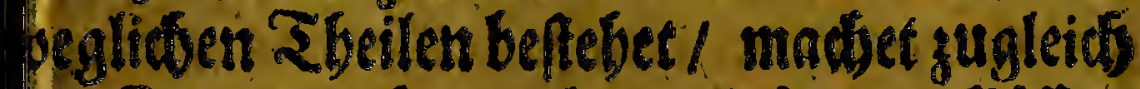
en Schleim ounme / berve gliob/ and fluffigl ber diefes beftebet ter Stabl aus felor barz en Fbeilgen / bie mit anvern bersegerioent Ebierlgengegen bie כerffopffung angefubs

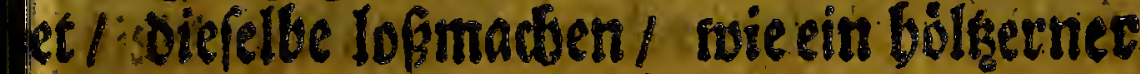

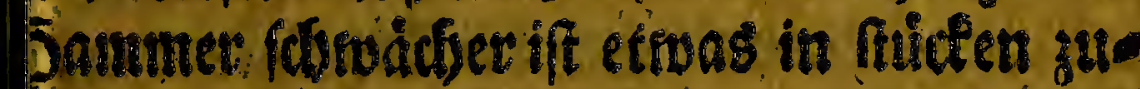

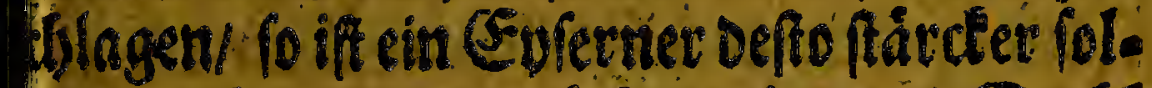
bee zu tbun / Darumb braucht man Stabl mo andere?Retalle in berMedicin : Denn bre ₹beile fino viel feiffer als, die von (Ste. bächren/ Salken und Steinem.

\section{Das X. Sgenitel.}

Bon Mercurialiften Dingend: Det Medicamenten welde aus Doedffilber gemadit werden.<smiles>[AlH2]</smiles>

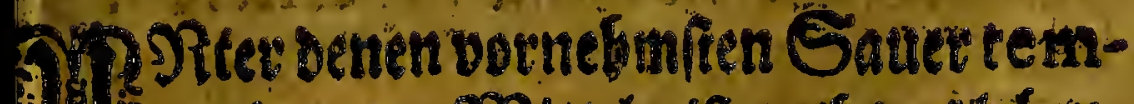
1. 


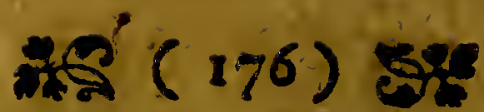

oc Soectiflber uno oeffen praparata, twei Die jenigen/ meldbe (Sieiffern oder Saliviren. folct)es genuglam in Dem DSuno uno Reblet gervabe werpeit.

11. Tob babe an andern Drtfen gefag Dấ Das Dveceffilber aus fleineri cunden $R$.

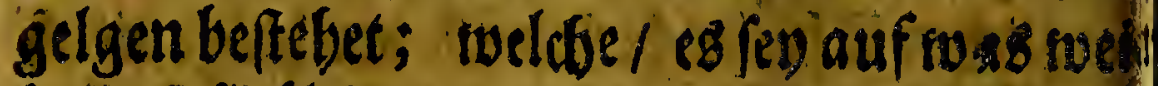

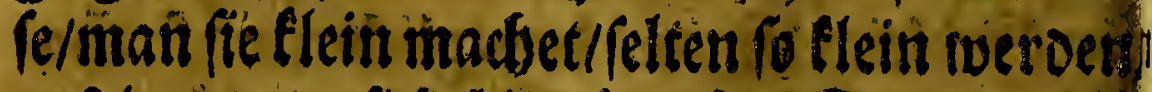
Dafi jeoes vor fidd felbit beftebet. Denin fo feds. Rigelgen didbte nneinanoer fenn / fo berub: sen fie einander allein mit ber Spissextre. Circuls / Daber múffen interftitia bleibes welche wièber mit anoern Rigelgen ats gy

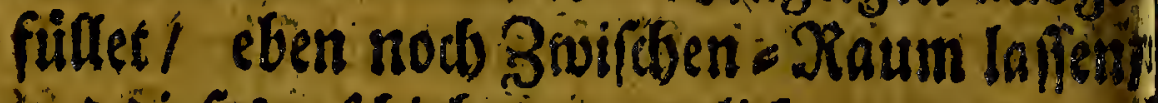
uno oiefes gefobiebet unenolicts.

III. Daßs Das Queuffilber aus theined

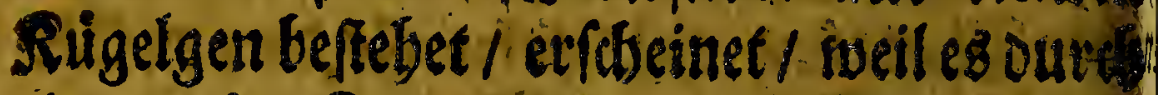
Die zarteffen. Saden roffet / inno fich nads oe? nen fleineften Deffnungen fiigen fan/ $\mathrm{mu}$ ift getwis Das der Eleinefte Eórper mus rum feon/ Denn foer orev ober vieredtidbe twáce. tan man ourdi 26birechung oer Edern/ affe. geit ein rundes oavon madsen/ fo dâs uns laugbar es muiffen oie Doectfilber= Sbeilge: runo uno unter allet vor oer Eleinefen Fi gur fenn und die meifie fuperficiem fol ben.

IV. Feff fentende unn oas die Duedeft 


\section{$29(177) 8$}

sc = Rugelgen rumo fino uno tos jedes Ris Algen aus etliden Rigelgen beftebet/ fo bal

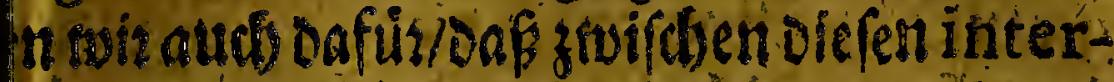
itia reon miffen/ ebent alb wenn ids eine band voll Rnitfer dier andere Rugelgen abef fo fanidb oie interftiria it (Sirofferes eh/ welde id fonft in Eleinen concipire; In die Bnidaen = 2âme ober interftitia ex Rnider/fan id sum wenigften eine SRas Il fecten over fonft was robarfes oas fids arein /ebide/ uno sarimen fefte bleibet/fino um oeven bundert/ fo werden fie cinen SDals

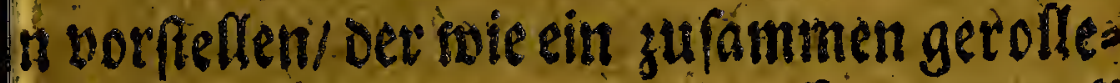
it Sgel/ oset utrauggemadte Caftanie oon

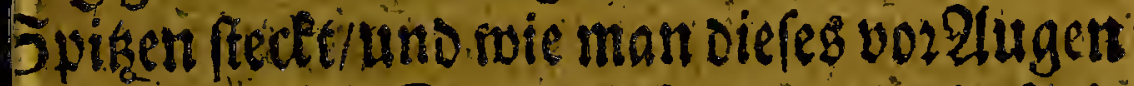
eber in grofen Dingen/ fan man es in fleis. en concipiren; $\mathscr{2} 3$ ent man nun fuppoiret t Daf bie. Doedefilber= Rugelgen ouref nfere Sáffeuno tubulos rollen uno ge: ibret weroen/ uno safs oie allerzarteftent ubuli geraum genug fino roldse zu empfant: en umo ourdo zulaffert / fo fan man leidbe be

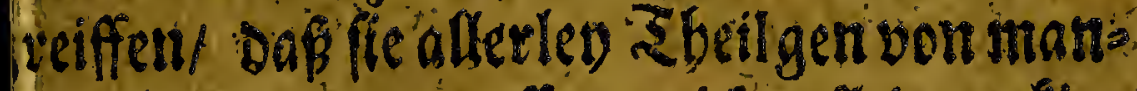
beley Figur antreffew/ nichic allein zactig: - Sdilangentiveifel rundel fondernauró

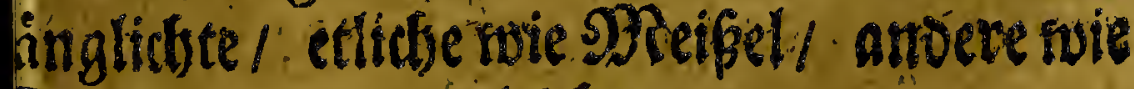
Degensc. uno oergleidjen.

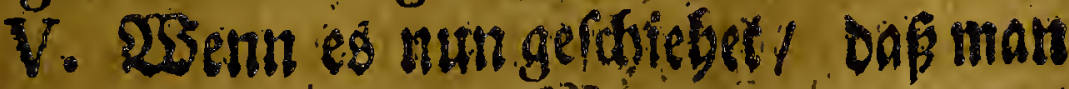
Did Das 


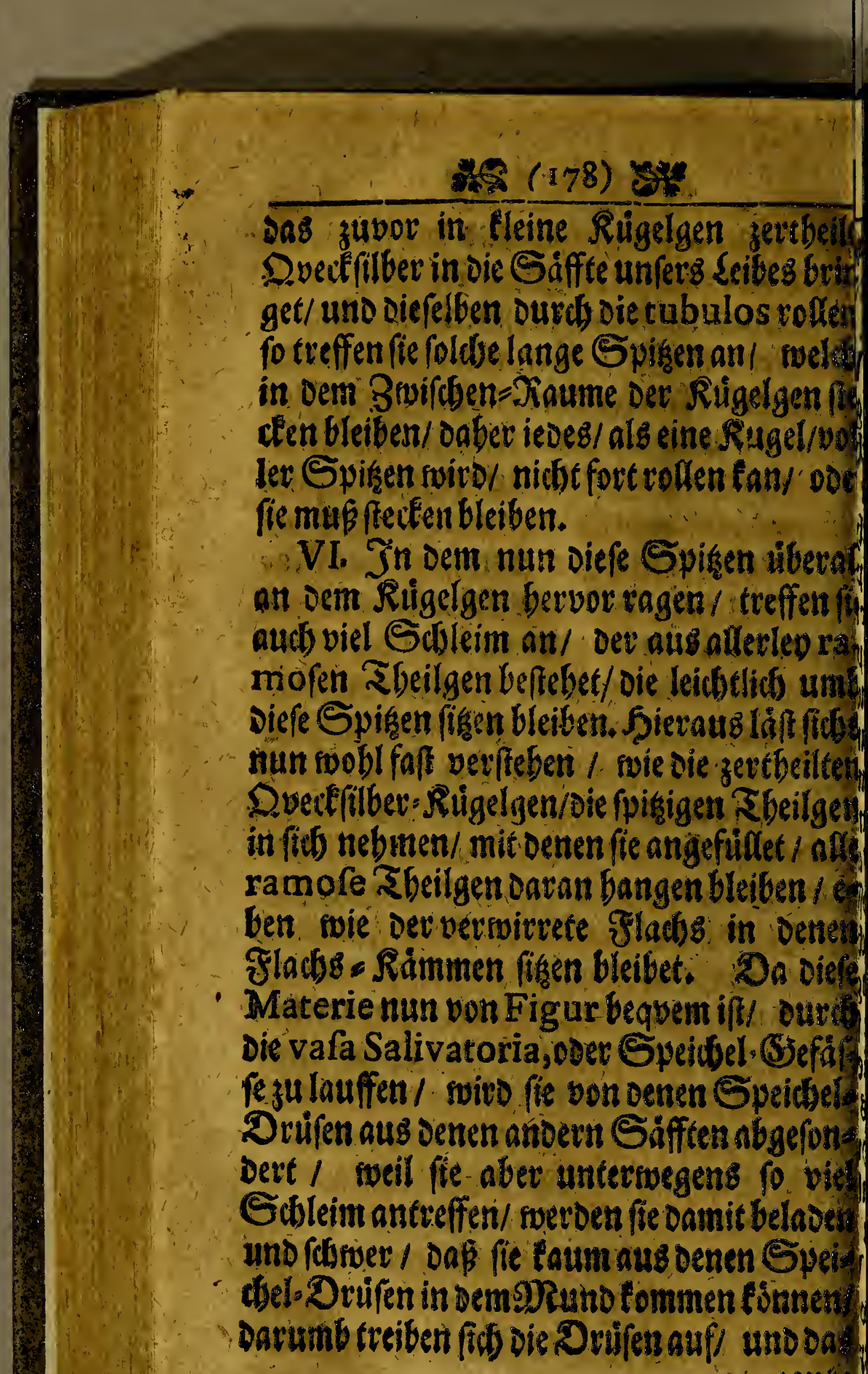

gantse 
ํㅗㅇ $(179) \div 8$

Inge Intgefidst/ ja felbf Dec DDeund/Reble no oerigleidben umbliegenoe Tbeile founela

7.

VII. Diefe alfo bon Sdbleim fesnde Ru: Igen befoiner nods immer mefre uno mebse. dsente Spifsen in fids/ Denn die fobleimige: 1 ober ramofen Sbeilgen/fino fo Ditbte the umb die borigen faueen oder febarffert pisengefdslutigen/ baf fienidittiodb inoftitia bätten / in weldje mebr bergleidien

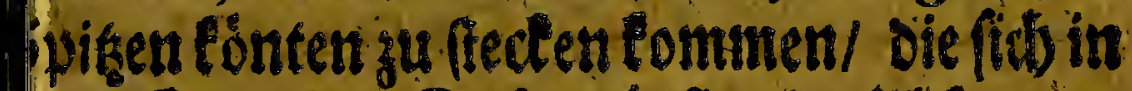
ner Speidjelis Drufen befinden/follde vet

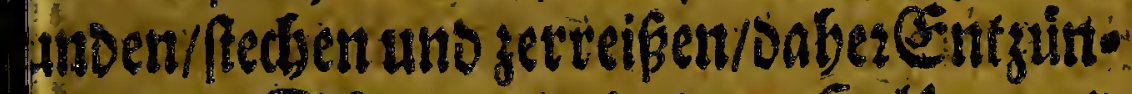
Itg uno. Sdywerung in ben 5alfel unt to umbber liegentoen Dufien Des MRuB entffebet/ Davon die falivirende sperfonent offe Ungelegentbeit baben: Sntem Der dileim in oenen vevleften FGeilen bleibets iget er an zu foulen uns zu finden/soobon

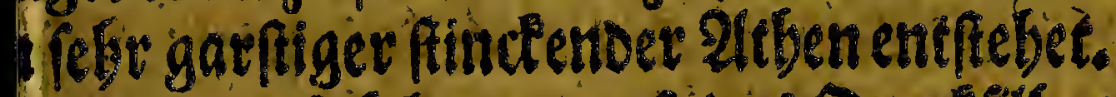
ievaus muf folgen/ Dap one Doedffitbev: - Sauer uns bem Scoleim trefflid abs bret und die Säffte bavon reiniget / battbif man nads oem faliviren fo frifd/ als gefundex 5 abn.

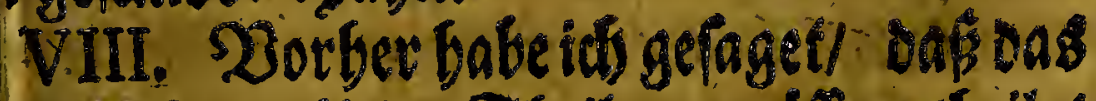
vedfilber in Eleine s beilgen miffe getbeilet woen. Diefes gefdiebet/ weil es nidje ane D2 2 Ders 


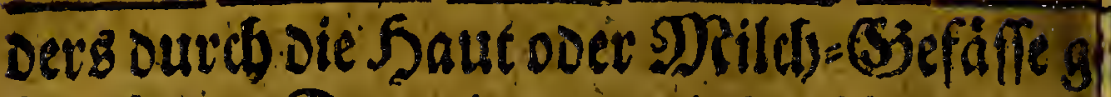
ben fan: Denn fo man sinige Unsen cin nimbt/lauffes ouz (6) Dem Dagen uno D mer/obnefith mit Deneth angetzoffenen Feurt tigfeiten zu vermengen/: Denn sueil es lobros it/fo findet es balo nads unten zu/ uno bat fei ne \$Racht siefe Tbeile anzugreiffen: Utbe Diefes bangen Die Doedifilbet = Ruigelgen. 1 febreneinanoer: sâp fie niobt als mit (Sie sole von einanoer zu balten; $23 e l d b e$ ma gervabr wiro foenn man rob Doceffither un ter eine. Salbe / gulver' boer dergleidsen mi Whet/oazuev Rugelgen nido fo balo aneinan Der fommen/ cs ruiro eines Daraus/ onf mas Drube bat fie boneinatioer zu Galten. Dàt umb thut es aud nenig Sobasen soent en mit Jenge eingenommen avito.

1X. Das Doedffilber unter Galbenge mifdst / uno die zaite 5aut der Seiber oami geformieret / madset aud faliviren; Wein

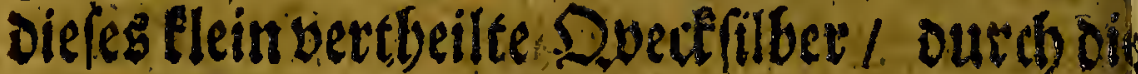
zarten Schneify soctserder 5autbif in una fere Sáffe oringet/ Darines siefelbe 25 tir ofung thut Davori oben gefagt. ". Stleiffer - foeife gebet es juntit oem Raud voti Doect filber odez Sinober/woeil Die zatten Tbeile des viaudjes leidbe ourdjoringen/ uno fu unfsul flifigen Sbeilen gelanget. 23er mebr vo 


\section{और $(181)$ एक}

ec SButrung ves Doeutilbers begebret fu siffen, lefe meine nnoen Sdiriften/ uno bos nod bier ferner folgetr móchte.

\section{Daร XI. Rgapitel:}

Bonbenen?itefmitteln/Sd)nupf: sobact und andern Durd) Mutun und $\mathfrak{T a f e n}$ Shleimabfulls renden Dingen.

\section{I.}

DPaln fiebet beute zu' zage baf viel seute

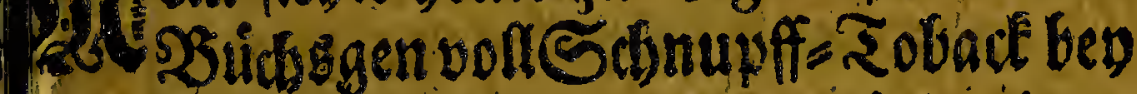
do babenl/ uno niemano fomme faft zufam: ten/Dafer nidht Sdmupff = Sobade braudst berben fictibar. Es if ein Sabr zroev bif

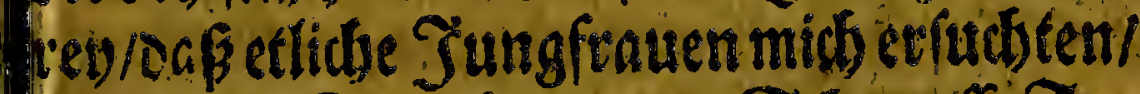
1) folte cin Tuactátgen von Sdinuyffi= To=

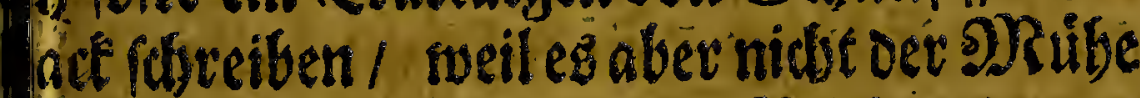
berth war ein abfonderlich sjud bavon zut: hacben/fo mebme idh iefso (S) elegenheit Davon 1 banseln.

11. Das Niegen gefdriefiet in gefunoen euten won fich felbfren / went von einer öbsirffe ber Freutbtigfeiten / ooer getroul:

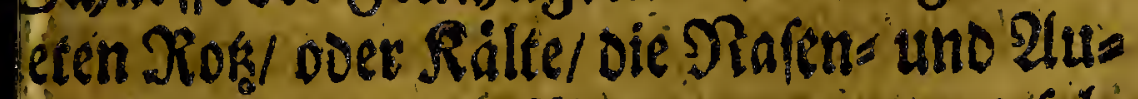
en $=$ Serven convelliret nerden/ uno fols. 


\section{$\operatorname{sig}(182)$}

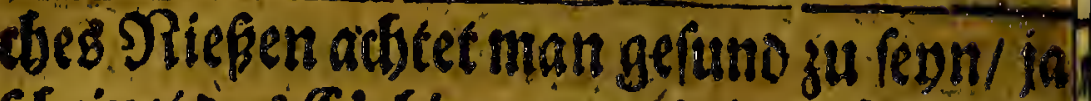
fdeinet Das SSebin von éiniger SSefdond ligleit Juevleichtern. Das Diesen finoet auds bey etlicben Rrandelbeiten/ und if ent Convulfion Der Sistrgen uno Rerve? die nacb oer SRafe laufen / von etivas fo fh selt/ecreget. Die onitte Sorte des Niefert. Davon rvir lagen roolen I. geldbieber Dur Runft/ es feo ourdo Pulver einjufdonupfer - Det etrwas in die? ?afe zuftedfen/zu fauen mierwobl fie afle gradu differiren. 203

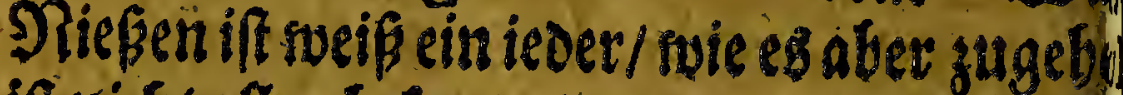
if nidat aflen befandot/wenn wir niefer/min fen wic erft tieff altben bolen/ : fonft solitoe nicbe gelicten/ bernadi folget ein ftarie.

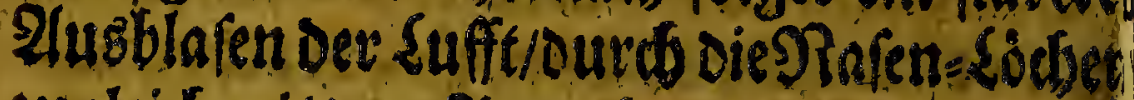

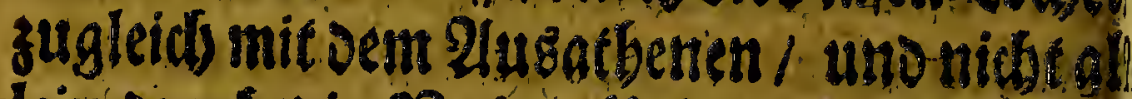
lein ourd Die SRafene Sodfer / fonvern aud

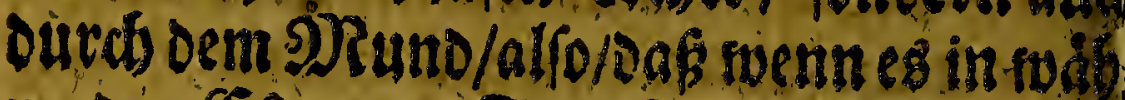

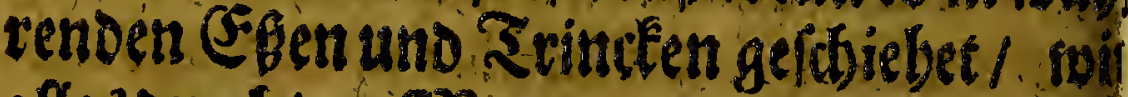

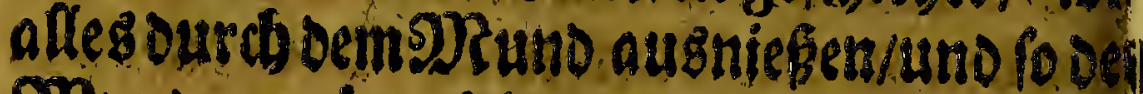

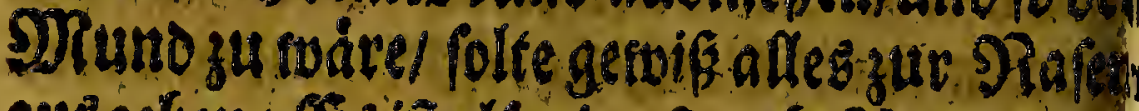

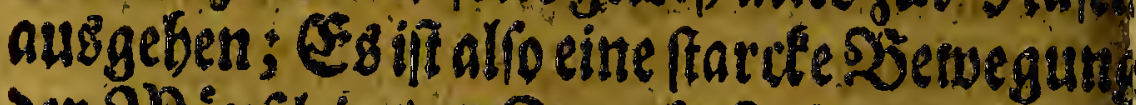

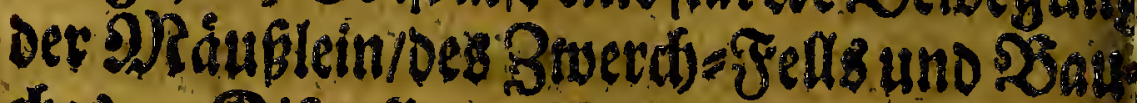
dheg. Dif alle gefdhieber neil Die Diervet

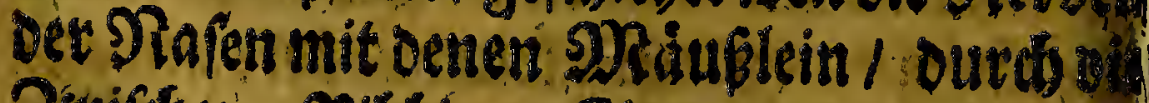

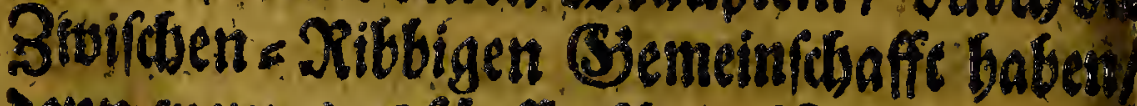

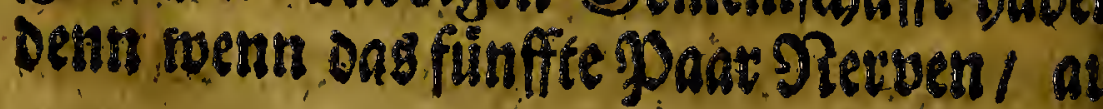




\section{He $(183)$ )}

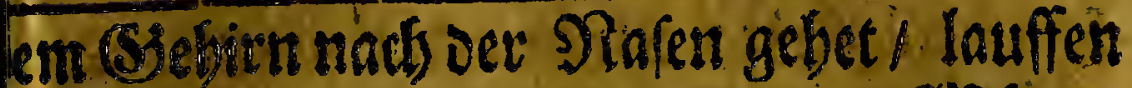
thide 3 woige nach oenen 2lugen = Sy?äus:

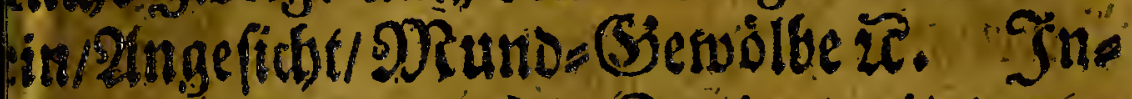
leitsen bereinigen fid) Stpeige mit Denen fineigen oes fedfifen Paars/ uno fo fort nit

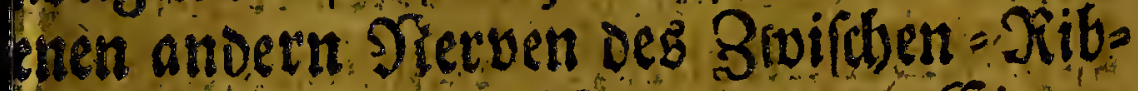
Igten Stammes/iweldyer mit denen Eingea

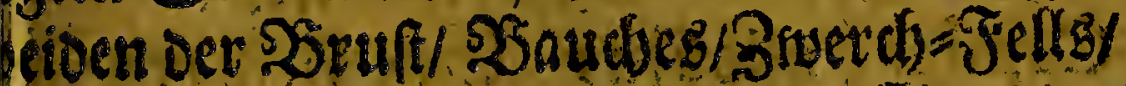
Jiäuplein oes Unters:Saudbes / (Siemeina baffe bat / oaber exidsuttern affe singe?

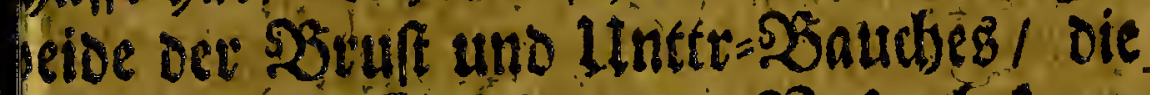
Kufculn des Sefidstes und Nafen/betome en ein andere (Siefnlel. uno oie 2uger ge: nniber. Rurszufagen / es if eime Conulfion over zhidiung vorgenenter Thei

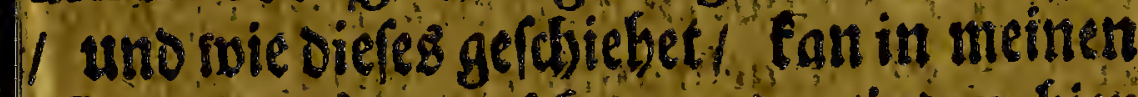
hititutio nibus gefefen spesden/indem bier

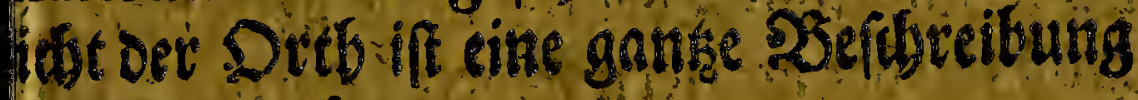
abou zu madtert.

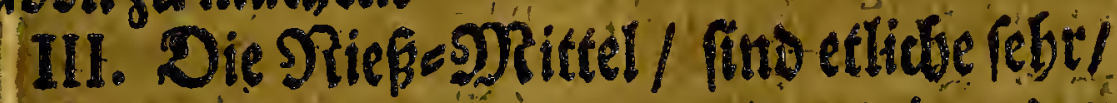
lidise riditgar zu forch / und bon einander radu unterldbieben/ Dás ifo / eines madbt Pebr Wudteln als oas anderef uno folglars. do groffere Convulfion in etruebrten

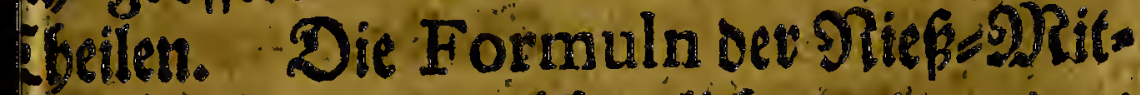
W/ fino pulver/ noeldbe etliche gart/ anoeve Mober nadsen. Diefe fino swiederumb ein. (4)/ ober bon untexfdoiedidben Dingen zus MR 4 fams 


\section{봉ำ (184)}

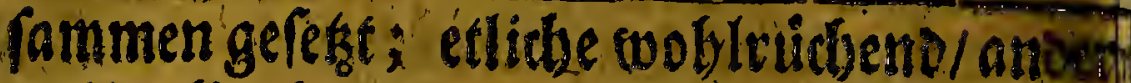
nicbt/afle aber von cinerlay \$3urctung. Dit ftsiruléferu/find folgende:

Elleborus albus, 2seiß Niefrnur. Piper vulgare, Siemeiner bofffer. Piper Brafilienfe, 25 rafilianifichespof fer.

Capfici alicujus Ein 2tetbon Eav Species,

Pyrethrum,

Tabacum,

Euphorbium,

Zingiber, \&c. Ingriev

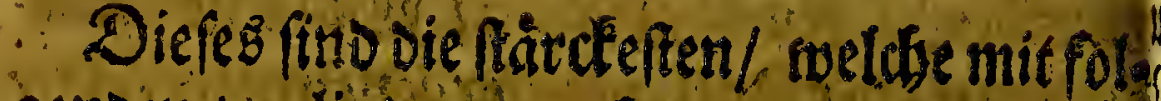
genden / gelinoe zumachen/ oder die linden.

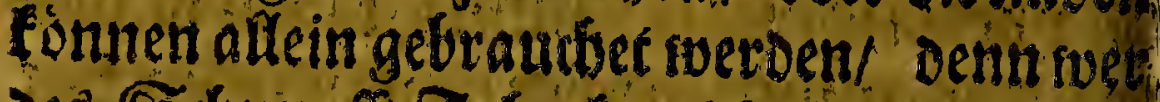
Des Schnupff= Sobacts nicht gewobnet iff mus fith oer mittlern Sorte bedenen; Uno oiefes fino folgentor:

Fol. Betonic:

Setonien=SSlatter. Lauri, Sorbeer-Slátter.

Majorane, Najovan. Rorismanni, Rogmarin. Salviæ, Salbev.

Rad. Acon, Ireos, .....

Cuboba

- Seilgen-25utret. Eubeten.

Cario. 


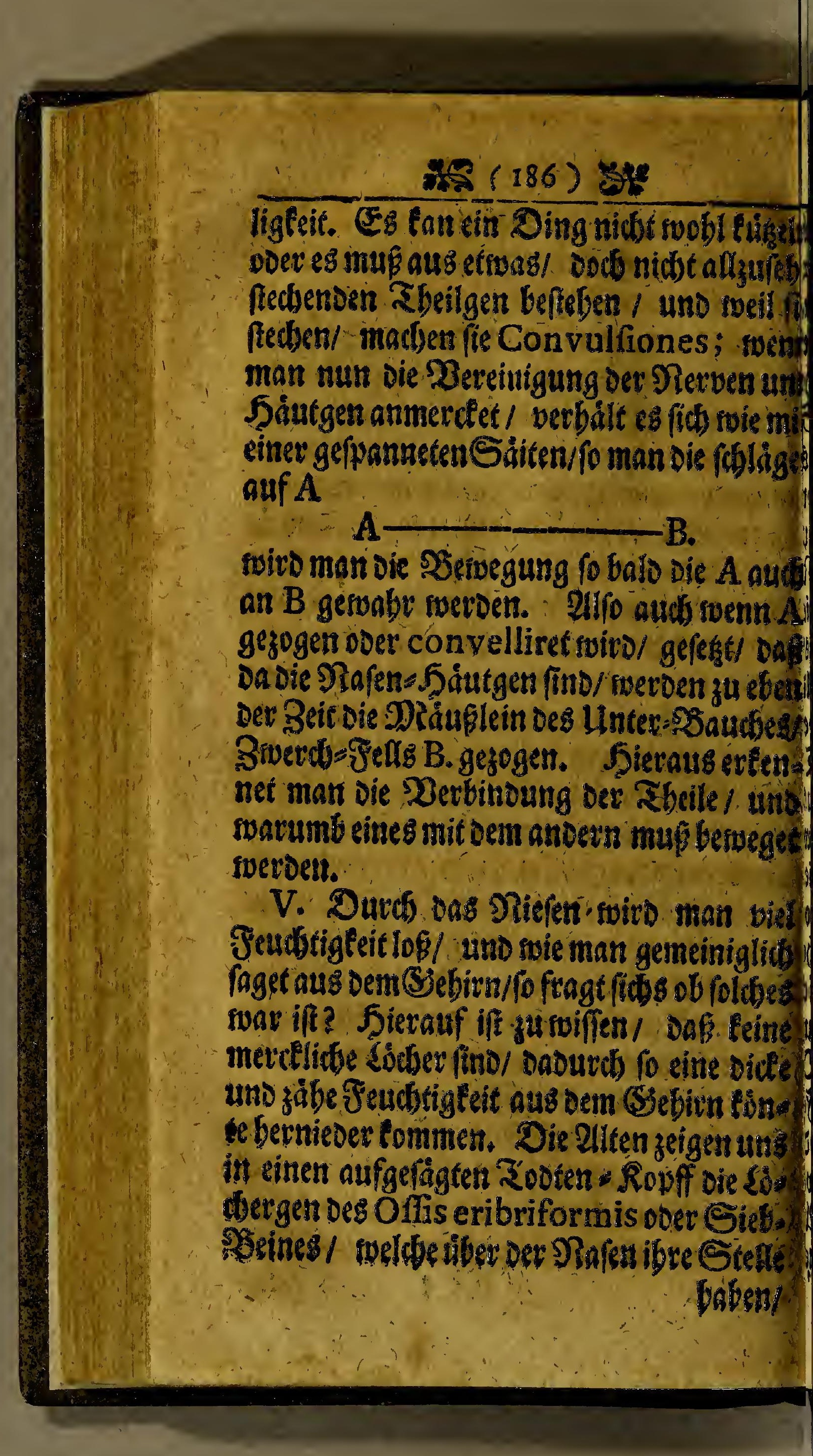




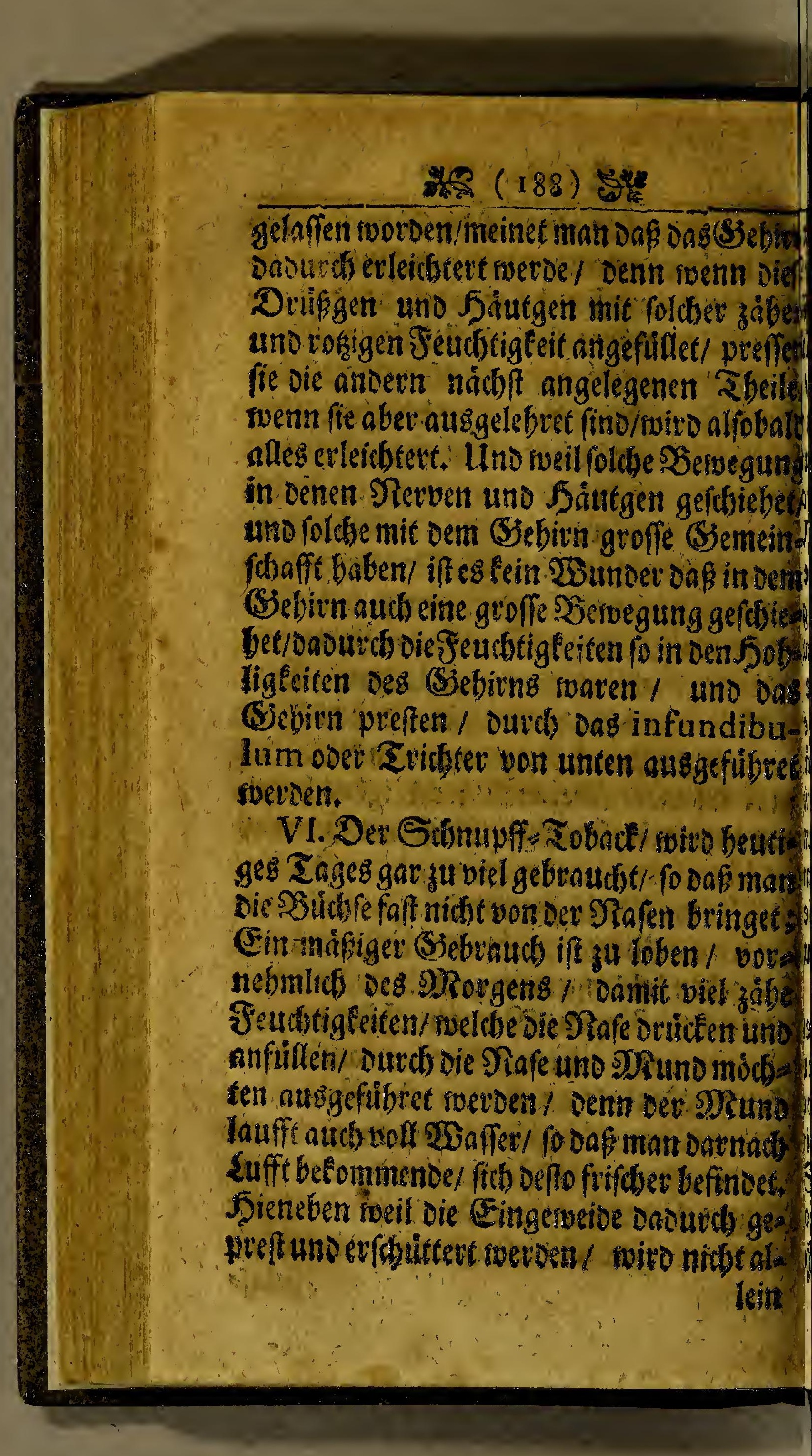




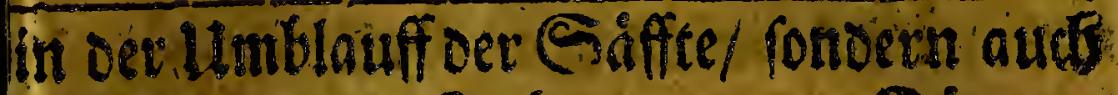

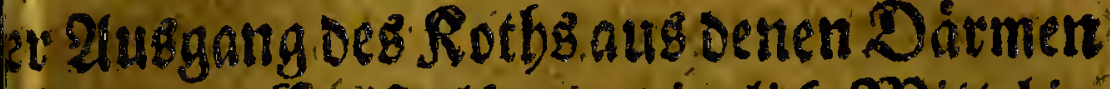
fobert. Es ift alfo ein vienlids Drittel in

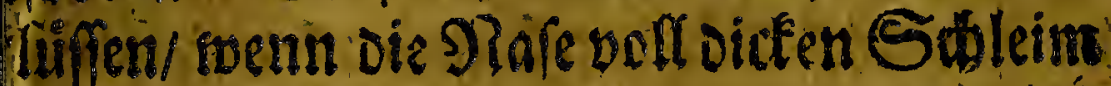

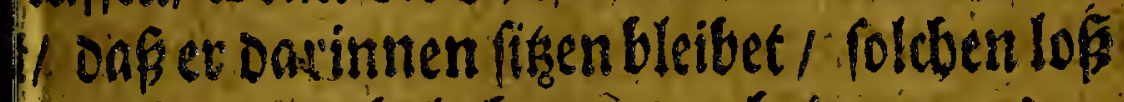
A madent uno bolo beraus zubringen / Das urdb aud oer veclobrne (Stectid wicoers. badit noiro.

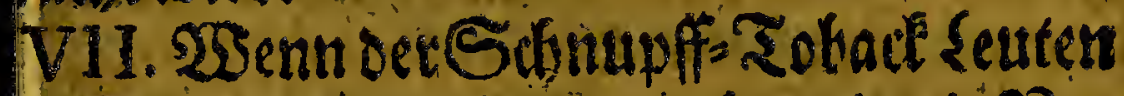

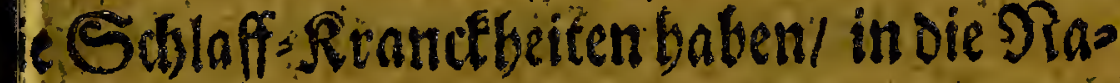
geblofen wito \% foecben fie savon munterf no rob die Scblaff-Riand beit ro grofs/ Daf enidbrnieren/ ift ce ein gefabulids Seidjen/ itweil in vem Sbebin altbeteit eine groffe

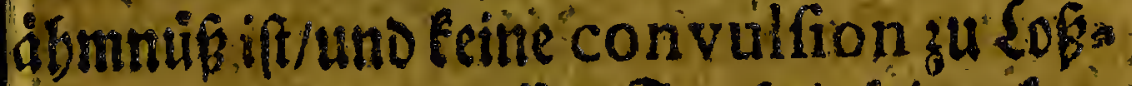
ringung oer vesffopffen Seudtigfeiten tan emadt suerben// eben als twernman eiriet atcén Spiritum Salis armoniaci unter ie SRafe bált/uno ver Rranute feine SZerive ung oavon bat/ift es mit ifm gefobsen.

VIII. WSern einev mit Saupt: Sdinew. en belasen ift/ uno foldse gerne balo will lof son/ Den la veldev die Serven/membranen uno SSea in berweget/ oasurd oie fiffefertsen und Bdbmer'sen madienoen Feutingteifen angbar gemadjet werben. 230 aber die Senditigleiten fefte fisen obet forwerlith ver ponfto 


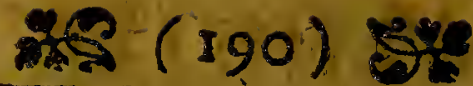

fopfftefino/ richer er wobletwas aub/abe febr wenig / indem die Sobmers madbend Materie auffer ibren (S̈efaffen if / Dareth fie nidst roieder folthen fan/fo roiro ein febarf fer (Evter Daraus / ta mandbmabl gar 25 tiv mer; 2lud if Der Sdbmextsen dffers in Deth

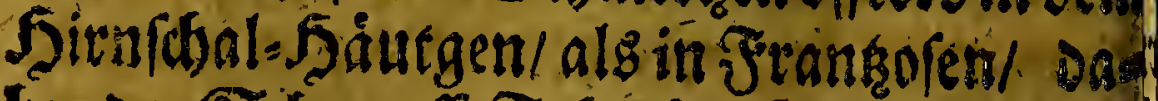

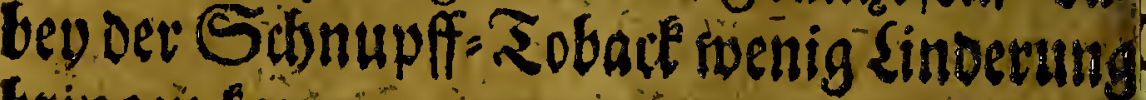
bringen fait.

IX. In $3 a b n=$ Sobmersen bat siesed Deittel unandimabl gebolffen: Detn dit

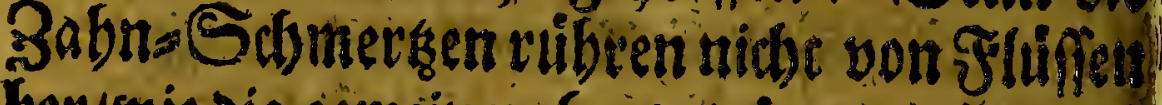
ber/woie oie gemeinen Seute traumen/fortoew bon etlidgen forffen Tbeilgen die die Siuts. gett uno Perven der $3 a ̈ b n e$ priveln/ iveldso SPerben Den gantsen Riefel/Unter-uno Dber: Babne mit ifren 20 urtseln an einanoer bin: Dem/ Darumb fublet man Die Babn=Soliner sen nidst eben in einen 3 abne 1 fonders mandjmabl in Dem gantsen balben Qlugefichs uno 3abnen. Dieneil mun oic STafen=5aut, gen uno Plecten/ mit benen andern lebr groffe S3emeinfobaff baben/ fo vafs toenti dis in ber. Safen convelliret ooer gejogeti wet. Den/ auch Die SEervegung in oie Babbire fith enfrecter / onourd die Feuditigleiten vio Die 3ábne beleioigen/ fortgeftoffen/ uno die

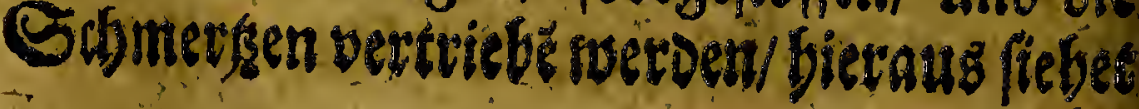




\section{int $(191) 38$}

an foble was idf oben gefagt/ oas in bem Runo viel 23 affer esimmt'। und follobes als in ourdo die (Siemeinfdiaff toer Theile/eben ie gedadgt/menn man eine gefpannete Sei han einen Enoe fobldgt/Das man alfobalo - SSerwegung aud an dem andern Enoe thimme.

X. Fent einer die fallende Sudt/ odet beve Convulfiones bat/tam man ibmeis n: Sdbnupff's Tobact in die Nafen bla-

hi Daburds die bereits gegentuartige Conalfion ourdf eine andere sertrieben mirb; ho Sebirn/ war gleidjfam obne Sifftel

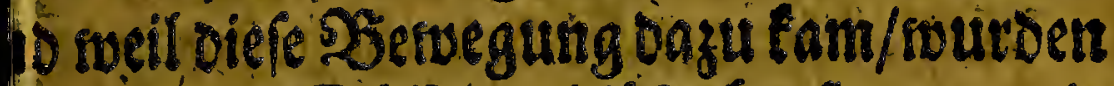
ieser mebr Sáffec dafelbfi abgefondert/ die inds oie Rerven fieffen muften / sweldie: infutus sie reftitution vorigersserwegung rnege bracite.

XI. 28 ent inat gebibrenoe Tratuet

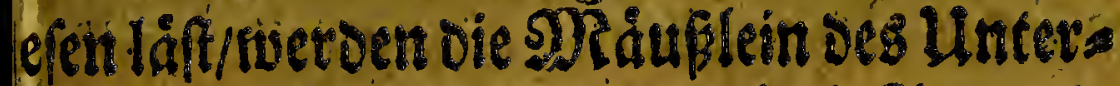

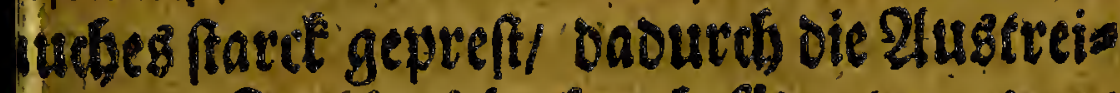
antg Det Frtuct) febe fan befsoert iserben/ min meil bie eingeatynete Suffe ftard auss

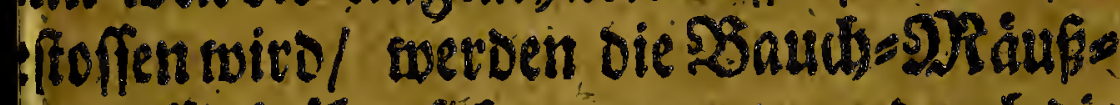
in treffliob uber fidh gezogen I mosurdo die Beiber Rrafft triegen zu beffecer Sortfes Ang oer Frucber uno siadjgeburtb: Sa

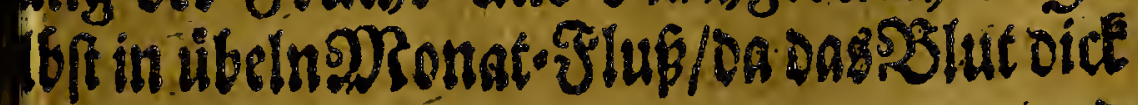
uns 


\section{If $(192)$ है:}

und Clóppich ift, Dafes nidje nobl abflefter Ean/ Dienet Das Siefen/ unD Dabergemaribt Datid-Preflung.

XII. Ferner to jemant mit Sdjlucten beladen ift/ weldes cine convulfion o DRagen: / Golunbez uno anverét Daro.

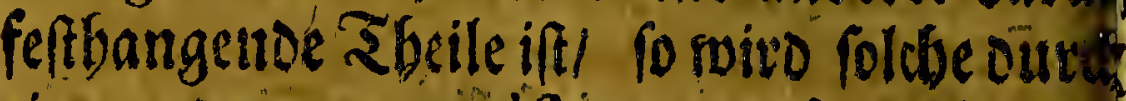
citte andorte convalfion zu rechte gebrach th uno boret auff/ clon ale wie ein groffer Somertsen oen fleinen libertábet/ all macb toiefe groffe canviulfion, von Rieles berurfadset/ Dof die leinere von Swluded verfdinitioet.

XIII. 214e gute@3ebratudse baben mand

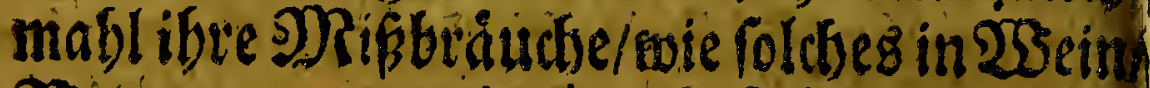
Branterwein/ Thee, Coffeè uno dergles Wen Sranu und Speife geforibet.alfo fvir auds bev vielen Nationen der Sdrnupf Tobade nicht foenig mifbraudbe $/$ diendil fis ibn fetig fontupfen. - Die Sbeile Dev $\Re a$ nerben oiefe Priulung fo geroobnet/ caf nicht mebe niefen / oarumb dieje seufe bers nach feinen 9 Rusen oavon baben/ wenn fie múffen ins?oth gebraudsen. Lber diefes fvit inter mely2 Ros berfantlet inoem die Rö gen raourch ser Jios flieffen mus/ inmer etroei. teit coerden/ gleids foie diefo viel effen un trinclen/cinengroflen Dagen/ Darme 


\section{and (193) St}

Do SBlafertiefommen: Daber beftnoen fie bftets gleidtram in Saupte verftopffti-uno he ned mebr Sdonupff= Sabact brathden

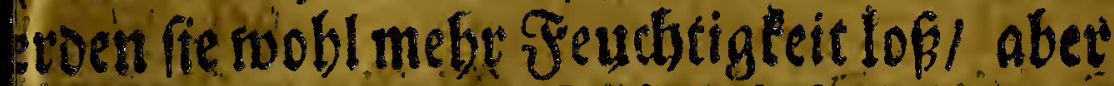
beinffe allezeit mekir 3uftug tefominien/ fo loenffe aud mebr ver fop fft.

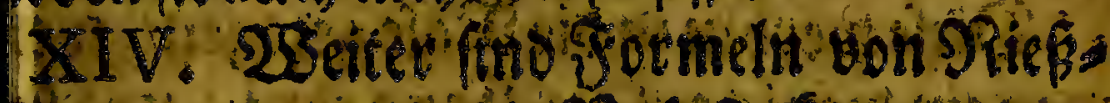

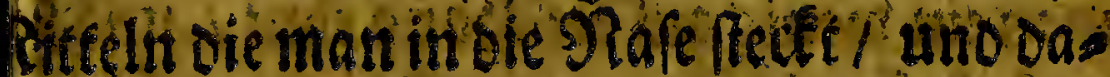

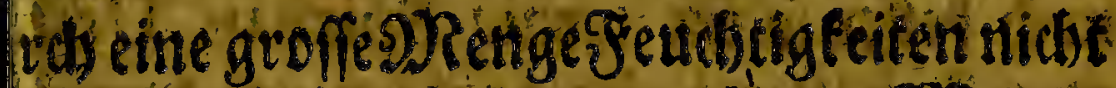

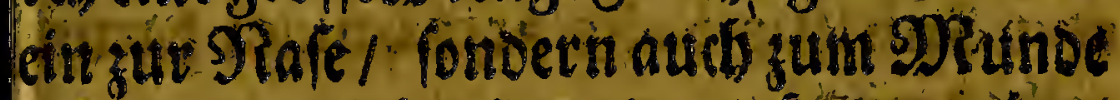
Blät/untee diefen fins oie trocketten alo: abacums tonica,

ajoratias

ftoreum,

ocus,

corus;

pryophill, \&c. Neglein ze.
Den ₹about/2etomien un andert Din-

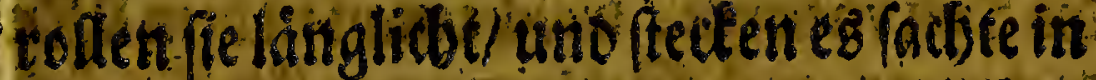

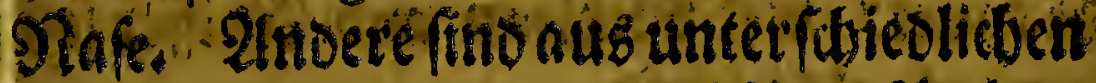

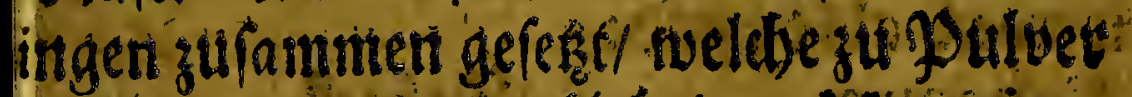
foffen/ uno mit einer Elebrigen Materie. langen 3 ápffgen for miret werber.s Mebit.

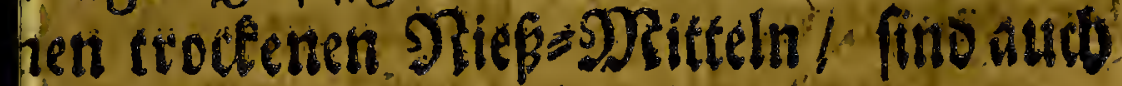
ile/ als?

iritus vini,

Iccus Betonicats Saftwon Setonien.
Skeanoferweit.

Sobract.

Shajerat:

23ibergeil:

Saffran.

Ealmus:

Reglein 2 .

Botæ,
SSetonite: 


\section{$20(194)$ set?}

Betæ,

Nicotiana,

SRangolt.

Iridis, \&c.

Sabav.

Sdhroertelnon

Bel.

SpiritusRorisma- Seif von Xopma rini \& $c$.

en 26 .

Dieferwetben ín bie Rraregezogen/ wen man ein wenig auf oie 5 ano thut / uno (in) now beffer fo ein wenig Spiritus Urin oder Spiritus Salis armoniaci Darun getban twiro.

XV. WJas angebet die 2 eife zu forl: den / fino fte nut etrivas in Riafften to.

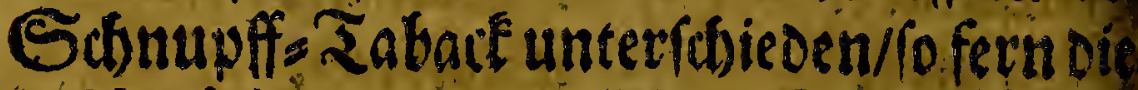

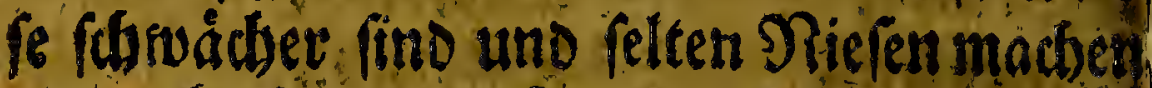
wieroobl eben fo viel Jैeuditige eic Daourch ab getrieben nito/ uno yor eben Die Rrand ten oienth.

XVI. Unier diefen Xang geboren ait die Salivatoria, welde man in dem SD w De fauch viel Sdylenn uno Striffer los soerden/als on fino:

Tabacum,

Piper,

Pyrethrum,

Zabace

Sem. Staphidisa- Souptirnen griæ,

Caryophill, Segleit.

Iofeffer.

Siertran, 


\section{1. ( 195$)$ है*}

cortus,

Calmug: :

idis radicis,

ngelica,

ubebre \&c.

Sdineetel=20sutgel. Angelide.

Cubeben.

Diele alle ruerden in vem Drunte getaus

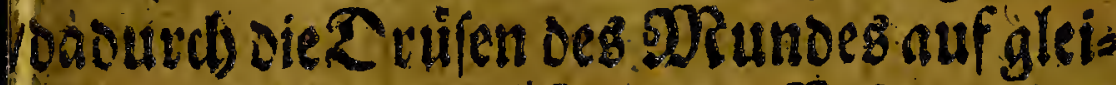
- 23 eife als bie ber Slafen/ afficiref wet*

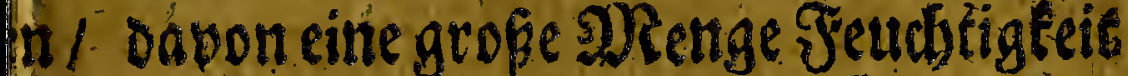
18 oem Drunte laufft. Der Tabact ift bbr das ftarcieffe/alfo dafther deffen ungea bint/ Drebeno uno tunden cabon wirb. ber sfertrnm ift vas linbefte/uno giefit teine boff Drictelung / ob wobl viel Jeudatigfeit? fen Sebraud if / vornebmlid in Sabns

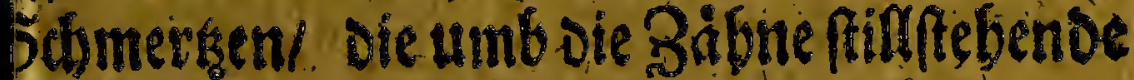
eucdigfeiten in Serwegung zu bringen: Daneben farbert es die 3 abne trefflich/ benn red) Das ftetige faliviren/ fomme vielfitin enoe Josudtigteit beraus/ Davon fonft die Sibne verderben/ ourdinaget uno fofimerts

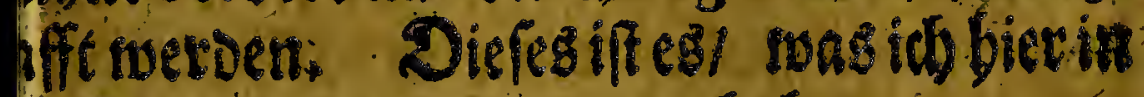
- Rirke bason su fagen gebabe.

\section{Das XII. Saptef.}

Bon Sifft und Gegen-Gitft.

$$
\text { I. }
$$

Z S fino allerbano Sorten uon Ssiffu

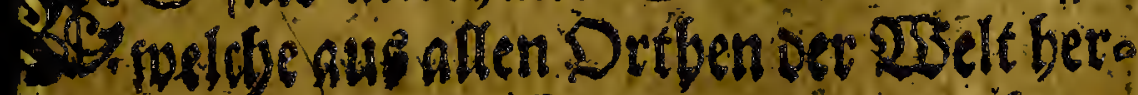
Y) 2 fom 
Eommen/ unfere Saffezu beroerbet/ un gleid) fam ftets im (Srewebr fteben gegen un. zu ftreiten. Boin benen Fbieren fomm (Siffel es ren aus ibren gifftigen Speidbet oura SBeifen/won tollen 5unoen/2Bolffent Raksen/ze. Davon das S2lutb uno die Säfs Der D) Penchen/eben oergleidben Durdbfros fubtiler Materie befommet/ als oer Sbie ift/ audb eben diefelben Sieberoen fo fie an fith

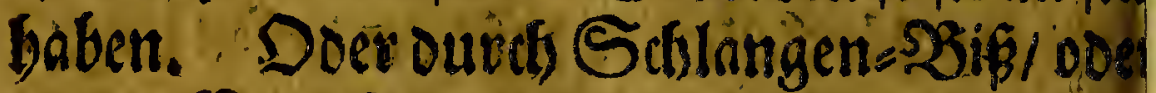
ourdb Sinnebmung einiger Sbeile bon go miffen Fbieren; (Sileidswie ben A. Kirch

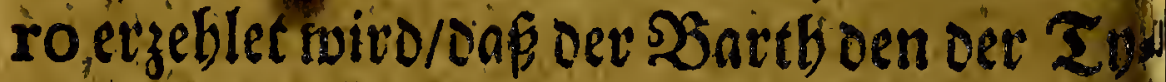
get in Bengale bat/ 10 gifftig fey/ / a / / wenth

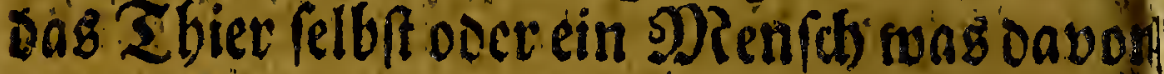
in seib friegt/muiffect fterben/man braud was vor Siegen=Ssifftman wolle. 2lnoev fedsen mit ifrein Sdrwan / als die Scors pionen/andere mit einem Stachel/ als: SOiv

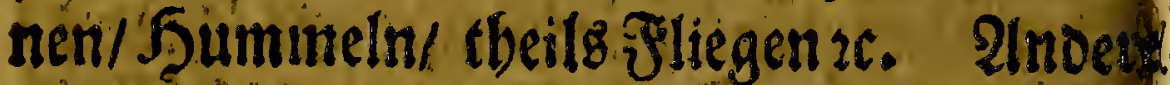
faugen boer ftechen mit oem SRaule/ als:

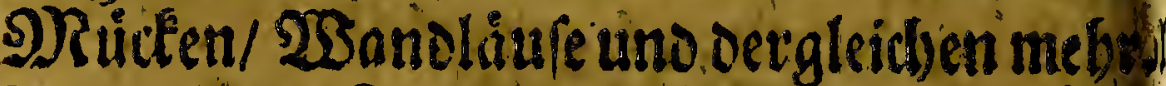
Unter dener fichen if Det Pieterman, bef

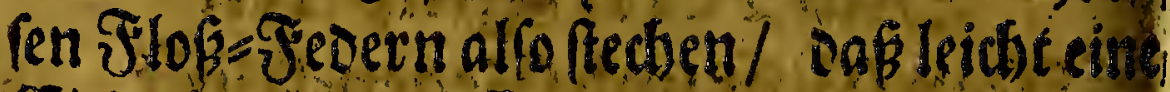
Gifterbung des (Shlieder darauf etfolget. Die Spaniften Flisgen madsen Sluth: batnen. Die Tarantula in Tofcanen madit groffe Convulliones, algauch ox, 
Srandbeit welche dic W3eiber baben/ fo auf as Seft St. Viti tantren muiffen.

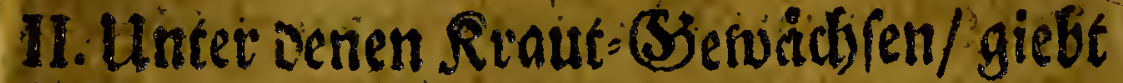
saud viel gifftige Dinge/ als oa fino oie

icutá,

enanthe cicutæ

foliis,

aftinaca filve- Wiloer Paftinac.

ftris;

conitum;

Ta americana,

ungi qvidam,

pongia,

pium,
Sdbierling.

Denantbe mitSdjet

lingebláteern.

Einfens Sutlein. Imericanifbe Dica. Entictse 2lrt SBitse. Stiriamm.

Zurcticder SNono raff.

olchicurm,

langas filveftris, 20 ilde SRarigas. arura Indi,

yofcramus; \&c. Dilfen = Renut 2 .

111. Unter betren Mineralien, funo aud Retbano gifftige Dinge/ als:

Irfenirum, uripigmentũ; andaraca grac. Aercurius Subli- Sublimirt Doect: matus, erufla, \&c. weiser

(Siellev

Notber

Sultent

Tranco.

filber.

Slenweis tc.

$\mathfrak{N}_{3}$

ซึt 


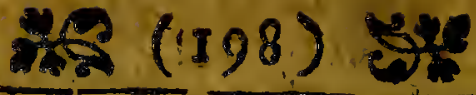

Ferner if de Suff mandhmabl beruncet

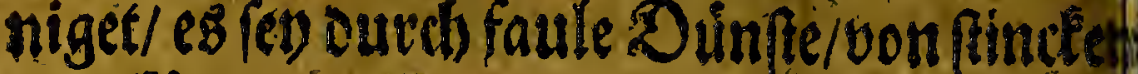
Den Girpern/23affern unto Dergleichen/ ood if ourch Sufberfung oer Jinern unD 25 ge/mit Arfenicalifden Sbeilgen erfüfer Der wenn man in einen verfolloffenen (S) mach ift/ oavinn Sdoweffel/ Sifft žr. ang zunoet worden/ober ourd Dampff vonRoh len ic. ftivbe uno enftitef einet.

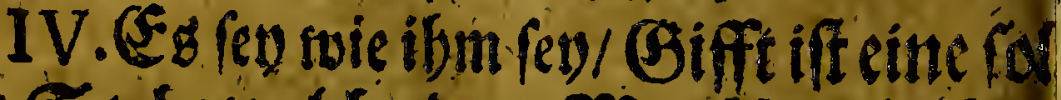

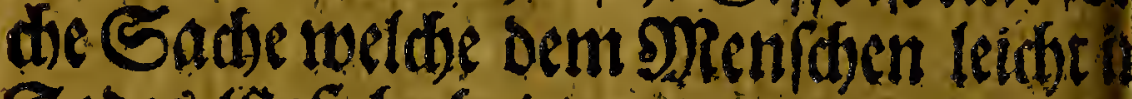
sodes sefabr bringet/. senn no feine ge Gorige Sulffe geboten tvito/mus einer umb Fommen/man mages in den S)Runo beforn metz/ einatbnen/oder einfoluden.21Me(S3if. se muffen in die Saffe operiren, Sie for men folgenoz der Bufálle oie cabery fich erei nen DaE SBluth uno Säffe zu berviden/

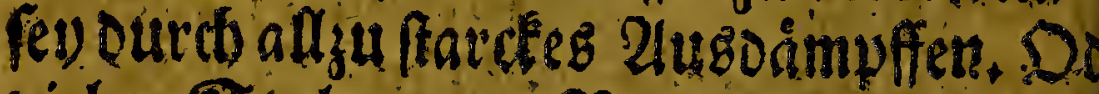
pielen Stulgang/ S2recten uno 5arner

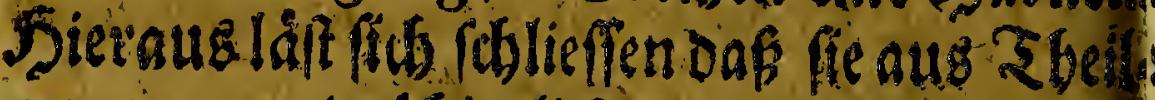
gen bon unterfobieblicbet Figur befteber.

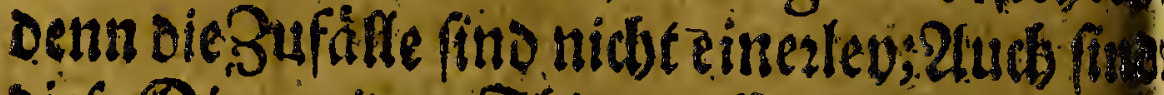
Diefe Dinge einen Sbieve'gifftig uno Dem a

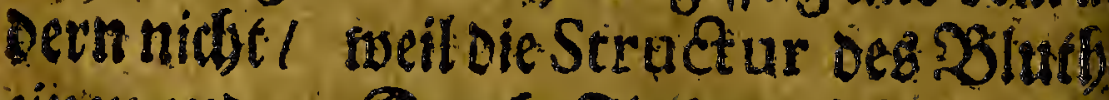

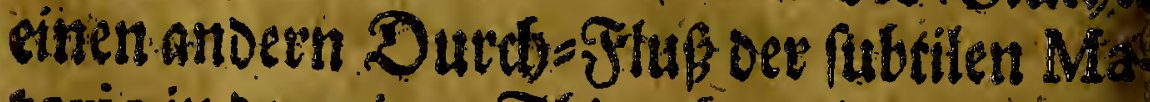
terie in oem einen Fbiete Gat/als in oem an

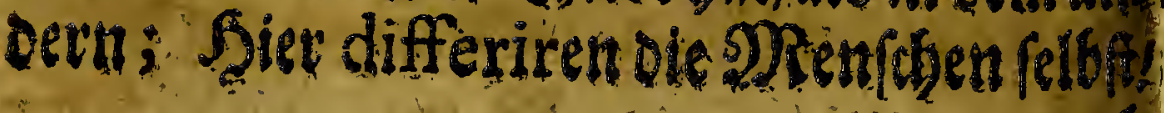




\section{Is ( $(199)$ Bus}

beldes than táglid in oer Speile uno trante febet/ Dem oeffen if feine anoere ation fugeben $f$ garambeiner bon oiefer

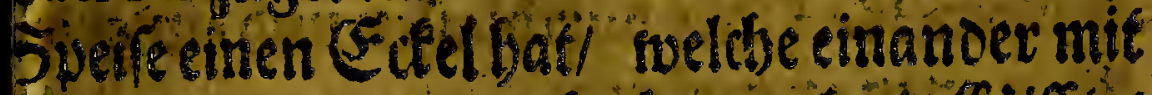

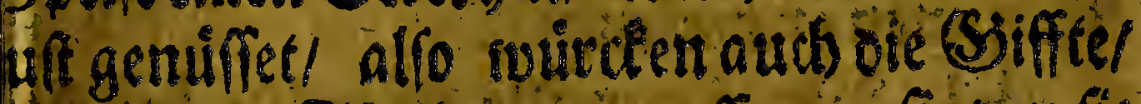
ads Dem fie Tbeilgen antreffen i finven fie did unterfobieolide poros, weldbe nadbem e bequembarin zu batifen/ fino fie aud) voor iner foldben Sórper mebr uns groffer (Ssiffe. V. Wiele Species beg Siffes machen Convulfiones, Raferev/ Tantsen/ nát.

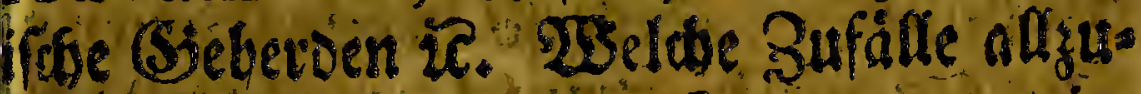
tabl unter de Convulfiones uno wies. er willen fitt begebenoe Bervegurigen gus edonen/ Deren Uirochen roir ebemabls auss cleget: TSem allbier bie Ieudbtigkeit aus enen Saffen zu bäuffig Durch oie Stieren efrieben soiro/ fwiro oag Blutf gank tros fen und bicte/ baber fommt fein Speidjel 1 sem Dumo / worchs Durfe entfeflet

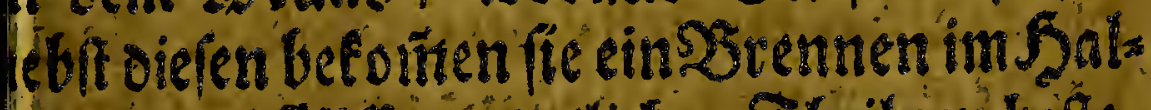

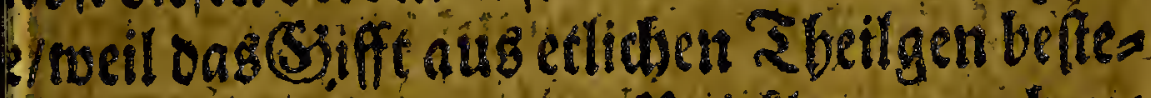
ef/. Die nidbt aflein eine Prictlung madben/

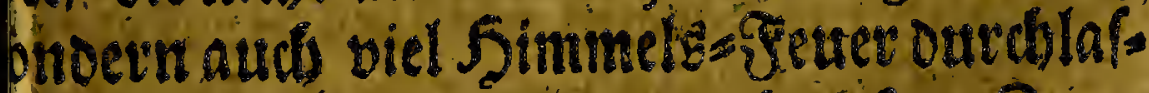
kil oergleichen Sithe man ands auf oer Zums e und in falfe gefwabr nivo/, wentseiner

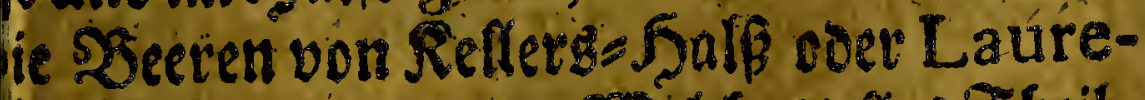
ta in Nuro nimmt; 2Belcbers afles ?beils

$$
\mathfrak{N}_{4} \quad \text { get }
$$




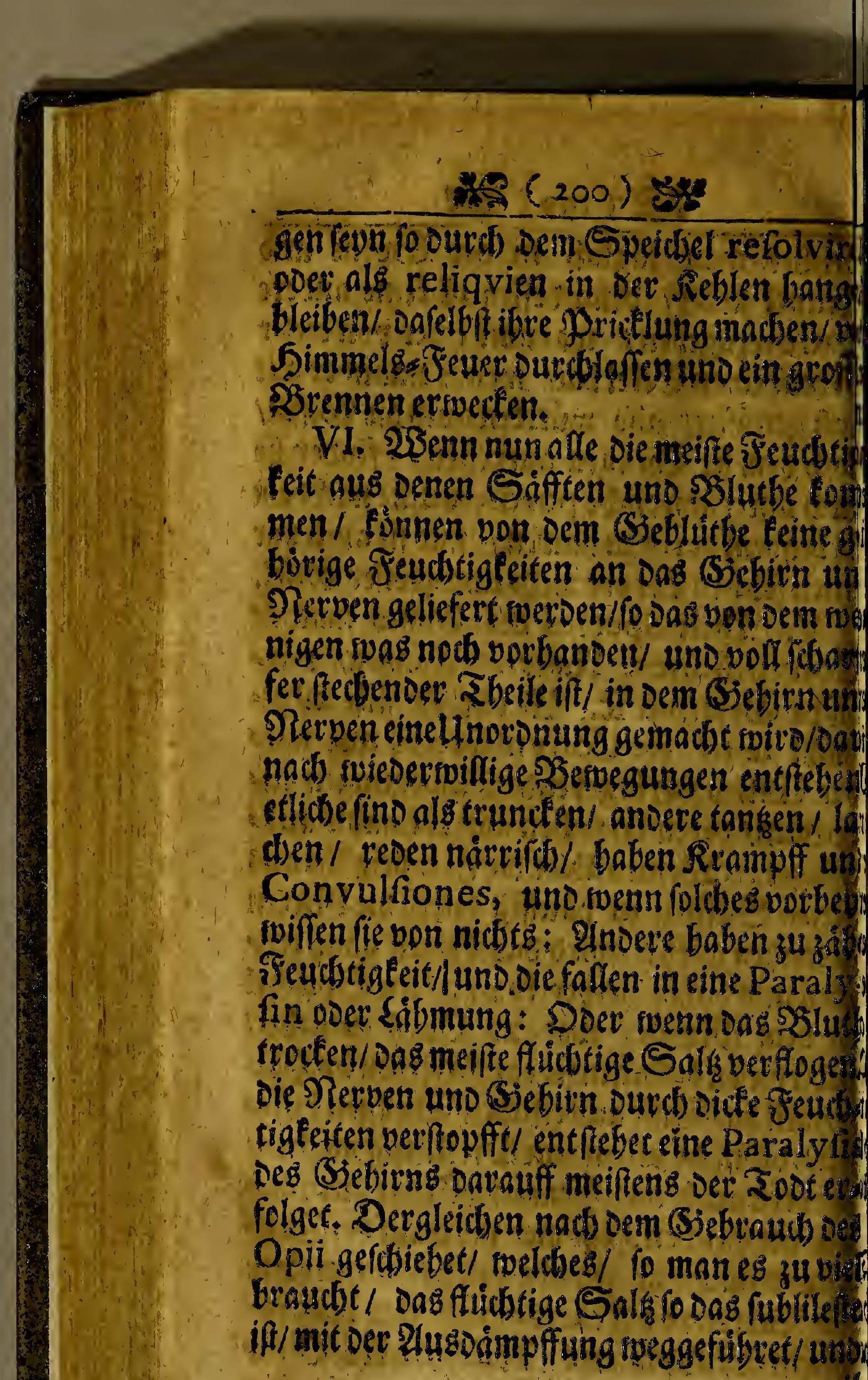




\section{is $(201)$ ste}

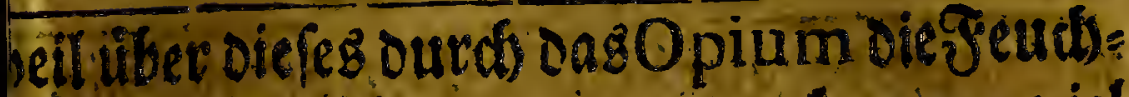
geeiten fearde berweget nerden/fommen viel

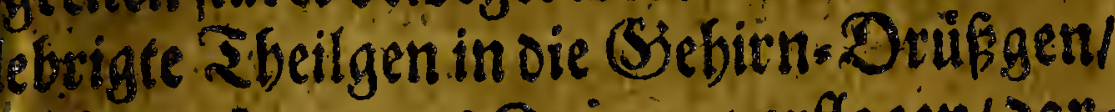
beldse nadbem oaz Opium verflogen/ sar. inenfteden bleiben/ uno eine. Paralytin in as SSebirn bringen/Darauf Der Toderfole

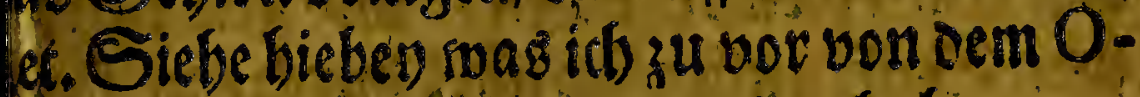
io uno Sdblaff= S) Ritteln gefagt babe.

VII. Die Corrofivididen (Siffelel fins heif Mineralien/ odev Durds Sunft oaraus eveiter/uns diefe befteben aus vielenftedsen. en uno rwisigen Sbeilen/ Die Das Szluth idot anoers coaguliren als der Esig oie DRild; Uno aufer bem ons fie die Theile ăs

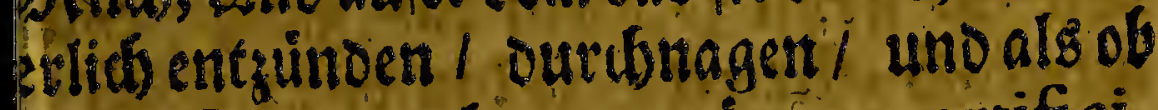
ie mit Feuer verbranor swiren mortificien/ to madben fie audb convulfiones in enen (Eingerweioen/ Ourdinagen/ourdboly en und ourdsfieffen biefelben. Ino weil as S3luth fo febr geronmen iff./ werden bie Datienten blau uno gelbe in 2ingéficht uns on andern Tbeilen úber dem ganksen Eeibel Wos fweil oa geronnene SSlutb bier und dar teben bleibet.

VIII. Durdb sas ftedien doer beiffen oet

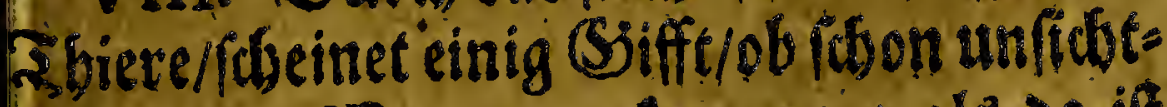
bav/ in oie 23 undo zu commen/ als oa if bon Scorpionen/ SBienen/5ounipen/Soume श25 meln 


\section{$25(202)$ bै}

theln uno dergléiciten. Don oenen Dient speifich/ Das went man ifger Stadsel th feinen SIlággen abnimme / fo finver fid $\mathrm{c}$ ? bittererSuff in venen Soláfgetz/weldben id auf oer 3unge probiret/ von ourdboringl d)er Shitferfeit befunien 1 uno gelainget fol ber Safft aufer Zneiffel burch eine flein

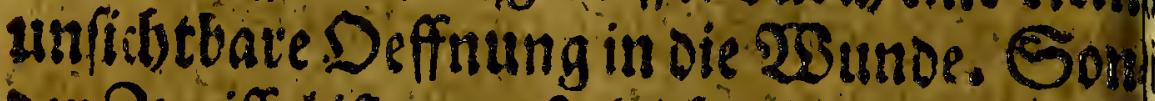
Dec Zweiffel ift es auf gleidse beife bejdbaffe

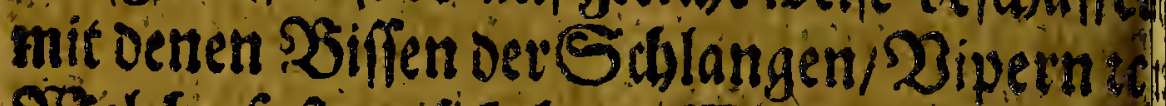
siseldbe faft unfidotbare wsunden madbeth uno cennodis bie Drenchen beffig beleidigen: 2lber gleith wie ein Tropfflein Bimmet=

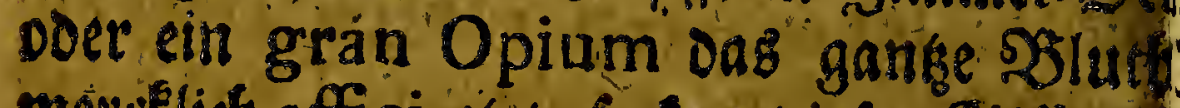
merotich afficirety fo can diefer Sifft eih gleicbes thun! dennes if eine corrof vifob Materie, dise aus foharffen uno leidht berweg lidben Tbeilgen beftebet / Die enf ons belei Digte (Silied inficiret/ oas es blau un

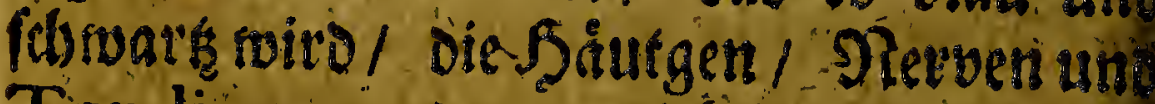
Tendines werben geprictelt uno befom men convalfiones, die borbey fieflerios Feuchtigleitety nebmen continuirlib was mitfich / uno bringen és ourch oas gants Slutb uno afte Siffe L welcheg fich atio os bon beianoerts Daf affe feine Sbeilgen ein andere Stellung und alfo oie pori, eite an: orre Eigurbefonmen/fo saboie fubrife Ma. 


\section{$5(203)$ (5)}

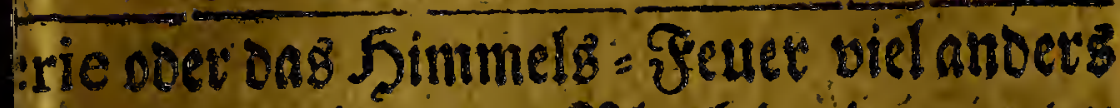

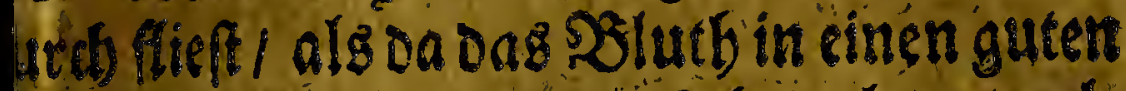

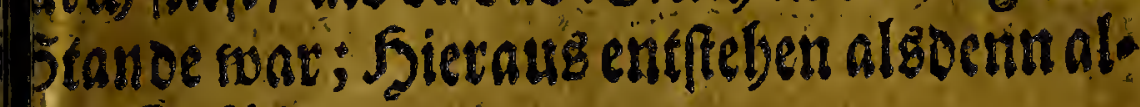
bie Zufälle.

1x. 2lber man folte fagentsie bie Zufalle unterfosiedlid fino/ oenn oer eine befindet 6) fo/ oer andere fo? Es if sammentero zu

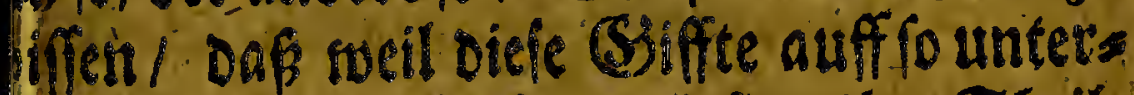
Diedene sweife surteden / fofmo ibre Theils: en uno interftitia nid) affe cineeley) 2(rtb) Ho oaber yon unterfobieolictser sienegung Ino Durdffinf oer fubtilen Materie, uno Tuf alfo oer (Siffet nads proportion feimer gGeilgen ein Sbeil oser Sslied mebr afficien als bagrandere/ Denn nadboem oic Figuen oer (Sifft. Zl Keilgen fimo/ tonnen fie aud jefen ooer jenen Sbeil mehr oder fuenigee deivigen uno diefe ooer jene zufâle er betien.

X. Die (s)iffet fo aus Jaaulung entreben,

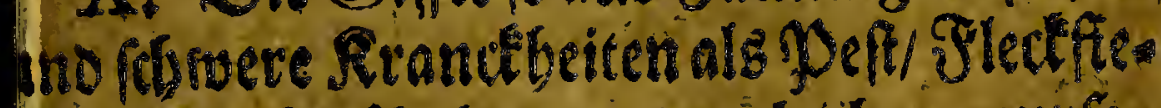
(e) I NorbesRubr uno oergleidsen veurfae ben/ beptben aus benen effuviis oer veto gulleten Dinge/ Darinten A. Kircherus

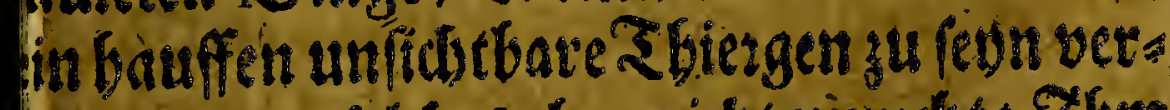
nennet/uno foldjeg eben nidst unreedt; J Gm.

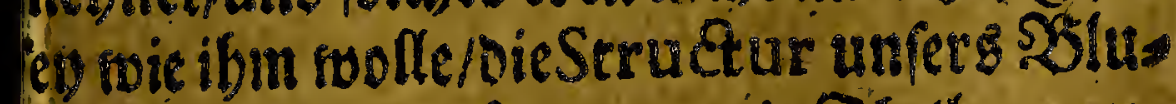
bes iviro febr veraintert / bie Rbeilgen ans cinash 


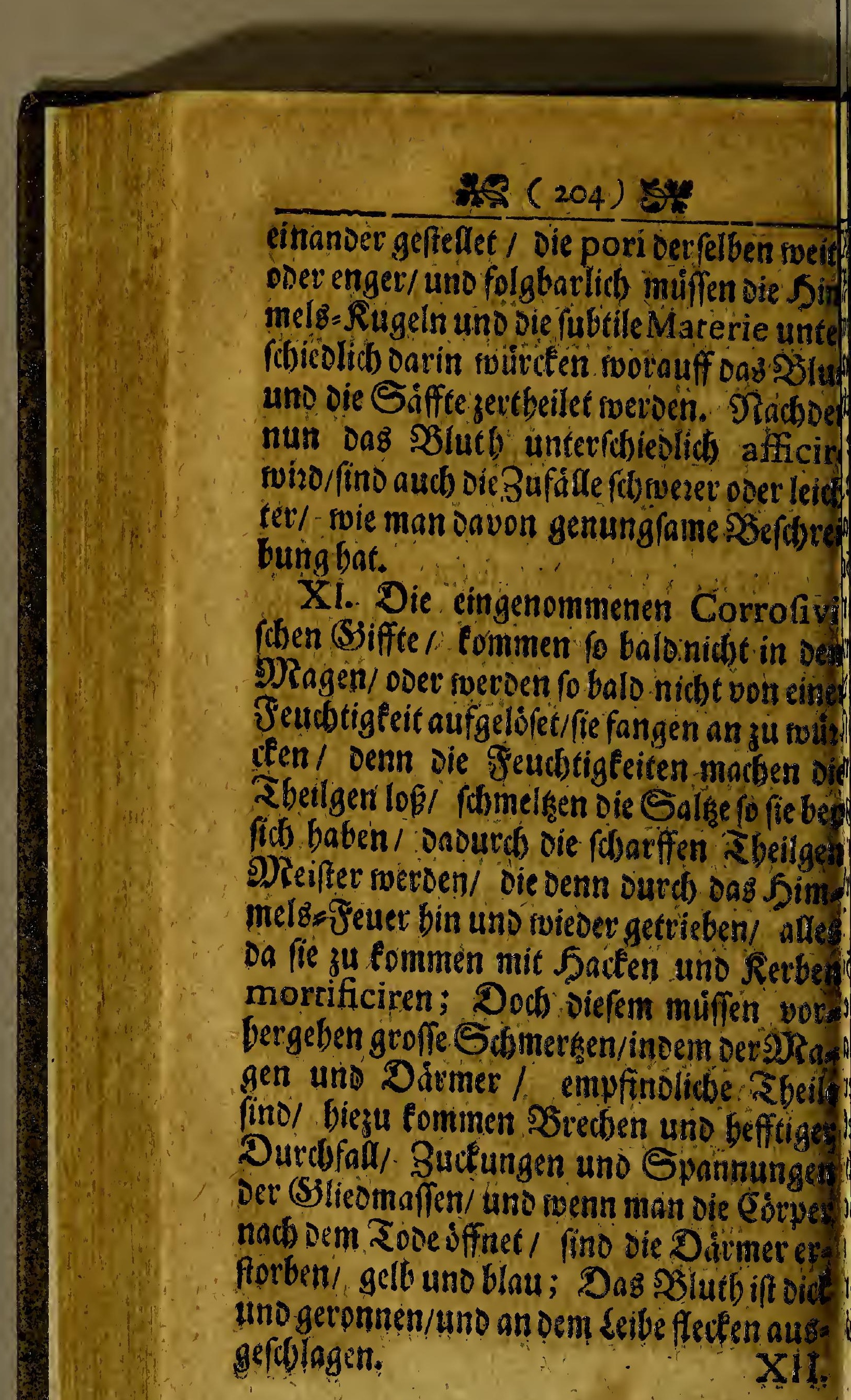




\section{xot $(205)$ 5ै}

XII. Der Subnamm iftan fich fellef fein

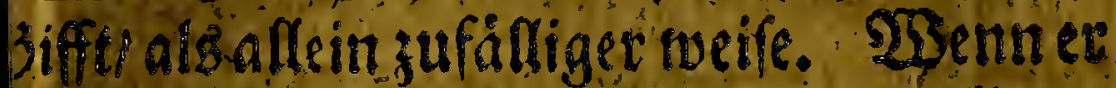
bhtict in oem Mragen fourint/ quillet es on oenen Feuditigeriten alfo auf/ oafe dot fan beraus gebradst werden/ eben wie - Railber/ourdh oag Secten der Saare/ won Rub/rudan fle raugen/einen Rlumpe oder. aar = \$3allen in oem Diagen befommen. Doldber-S5ant verftopffet aflezeit oen. 2lufs.

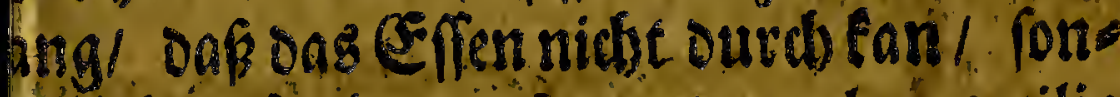
un alloa faulen mu bie Därmer gelanget/ Daber swenig uno ble Rabrung fómmet/ weil aud der \$Ras n lange vorli bleibet/ uno wegen der Unges genbeit fo fie carimnen baben/ verlieret fich iv 2pyetit/, effen roentig/verborten und erben.

(XIII. Eg fino unterfobiedidbe Pretbet

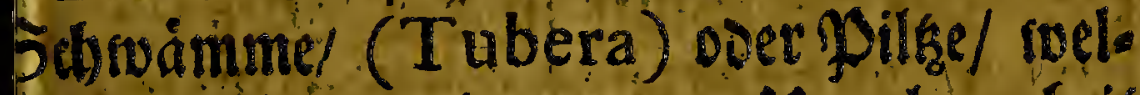
fe nenn fie zu alt worben Ungelegenbeit nacten. Denn wer ibrergeneuft/ oem wito ex Salf zufammen gezogen/ wiro rafeno/, tómmt convulfiones, ver 21tben gebet

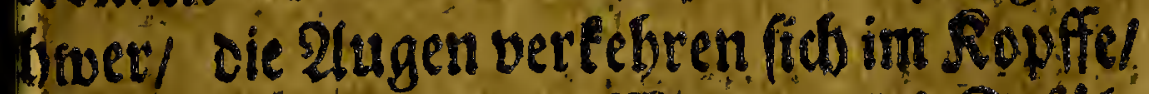
When uno orrgleidsen. Wierwol oiefe. Zufáls nidse eben in einer Perfon zugleids fino/ hoern balo alte / balo inenig t balo viel; semeiniglid meroen bie sRánflein Des 


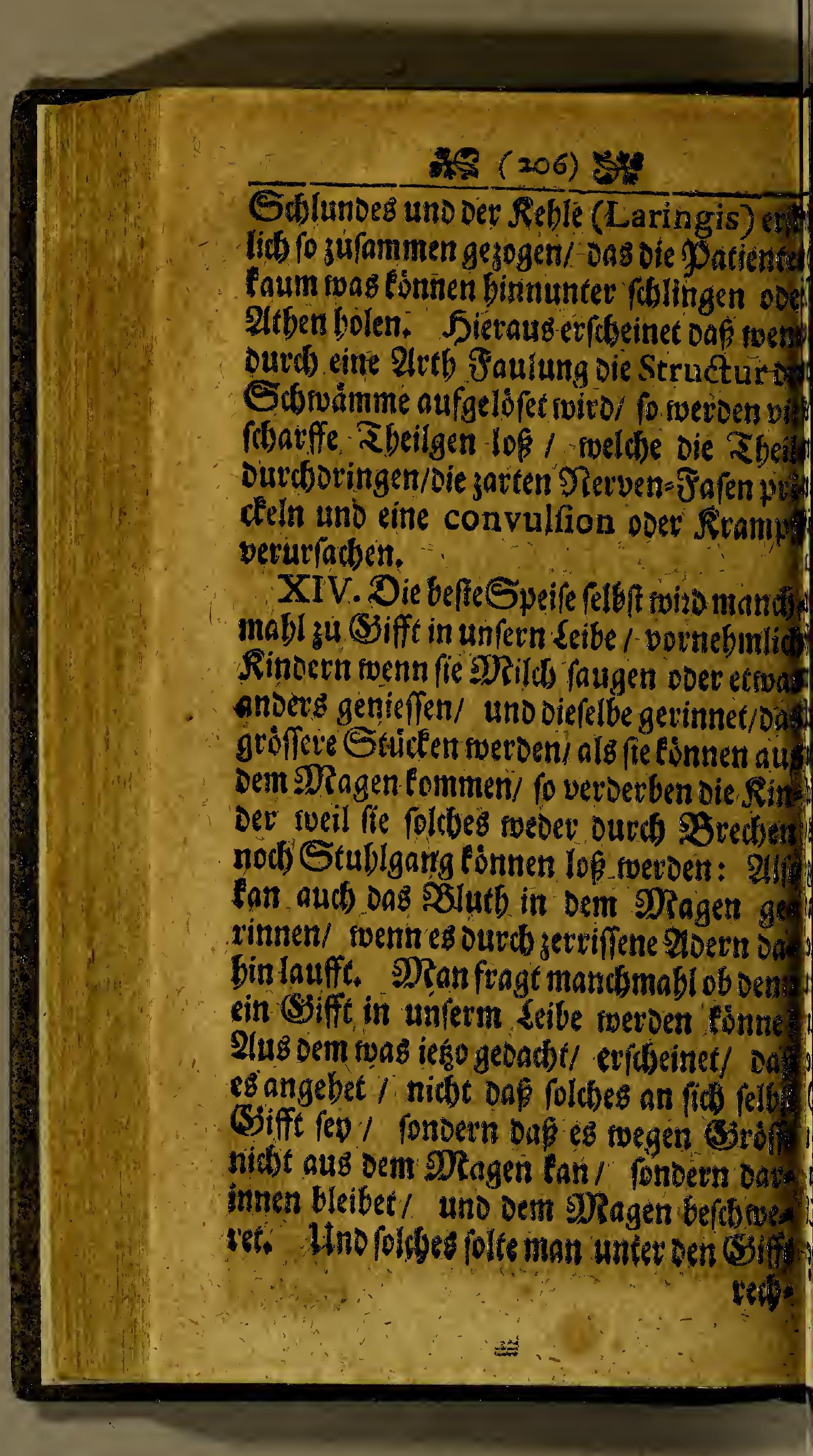




\section{xes (207)}

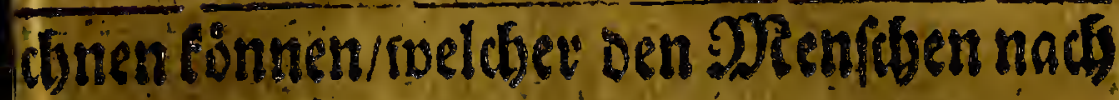
no nnot umbringet.

XV. 23sennaber iettand oen Siffe bon her Sdblangen/ Natevi Sienen/Etors

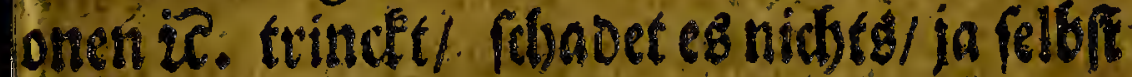
If Fleifo der Solanger mito zut Speife noffen. Dran inodite fragen narumb oenn raus nidte boles exfolgte? Siccaufif zu iffen/ oafs diefer Gifft in Dem SDagen feit Abjectum findet oarein $e$ s murcten fons. looen fo etrons thut/tbut es ooch wenig/toeil fiet Rraft gebrodien ebe es in oas S3lutb mmil uno alfo bernads oarin niobts ause doten tan. Uber diejes noiro es unmittele ter weife in Das S8lutb gebtadst uno faft idteinmabl alB cine Feudtigteit/ fonoert S ein ourdboringender Dampfil oberfeu. ge uno fobarffe Suffe; (Eben sole didit oie antze Subltans des SRànliden Saae eng dienlich ift/ ein (cy oeg 25eibes zu bes -itoten / fonoerti allein oas fubtilefte uno urdboringlichfe fan foldbes austidbten unt gantse Jrau vertoantein ober alteriren: bie Some madiet alle Saamene Sentid.

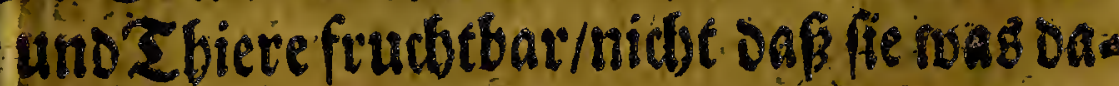
giebt/ fonbern allein fo fert fie in onten theilen eine Serwegung madbe:- 2lifo aud sundiefes Siff berpegethizo/gebet eg fort and 


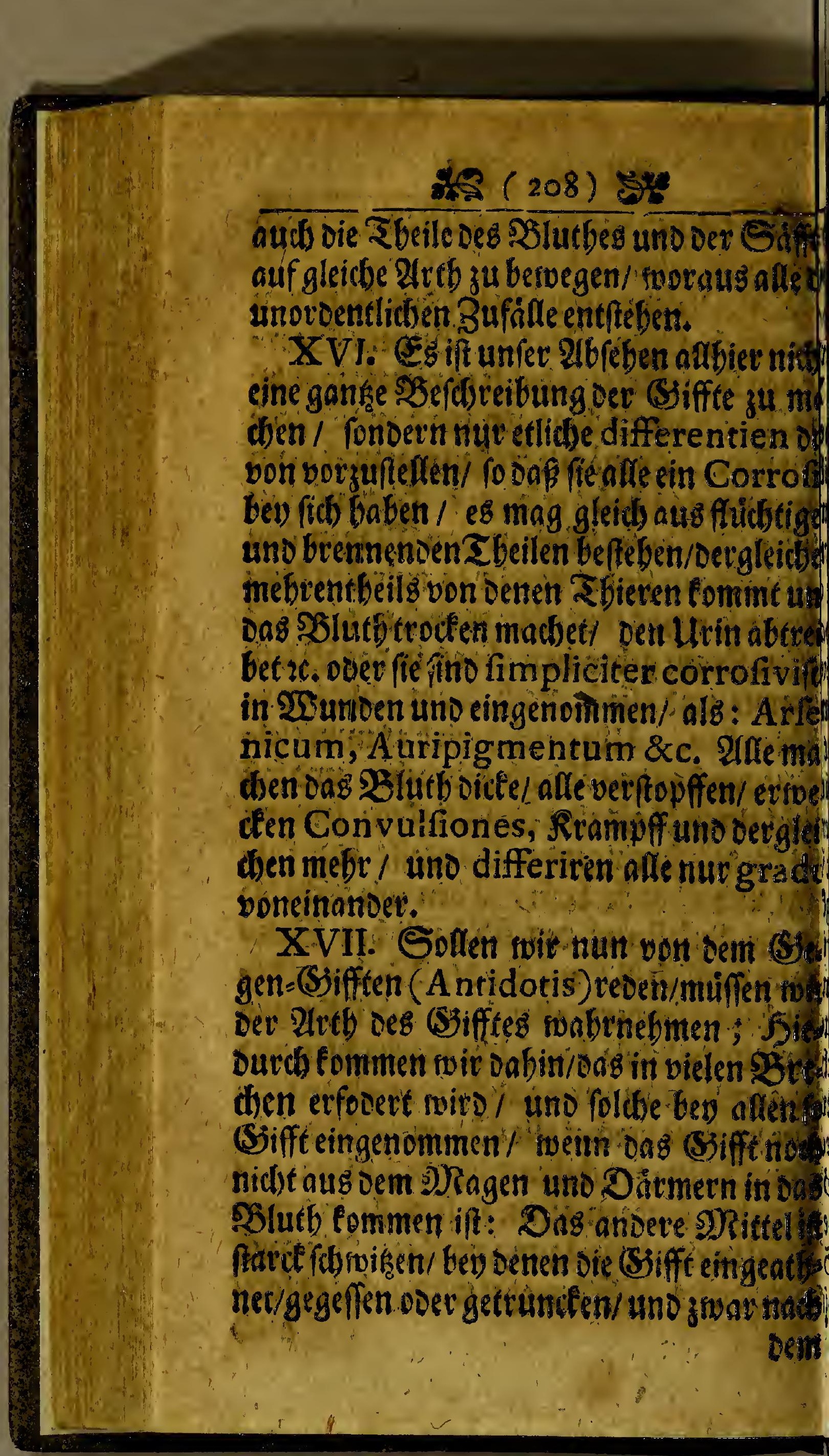




\section{0 (209) ses:}

m S25redsen/ ingleidsen wer geftodien oder ebifen/ oem if ons Shbriken bodaf dien. d) / Dabey aud auferlicie DR ittel enfodere eroven.

XVIH. DRan mus oeroniegenşzedient hes theils oic fobarffen Sbeilgen aub oem tibe zu bringen/ auf Das Die Da armer fambe m DRagen nidbet durdbnaget wetoen/ und ther Daf fie nidst ins. Blutb formmen unt / coaguliren:" 2inoers theilg Damit Die barffen Tbeilgen/ourd/ die Materie fo oas Brecien madie/ modjten umbrounben swet

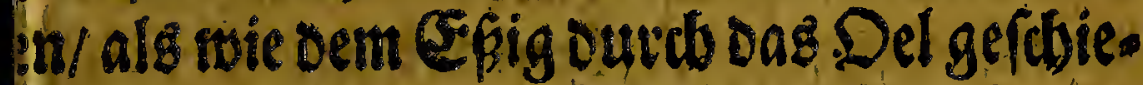
te. g. in Seveitung deg Ungventi nuiti oder Sitargietien Stilbgen. Bum DSre: sen mun gebraudjt man Del/ Sett/ Souts

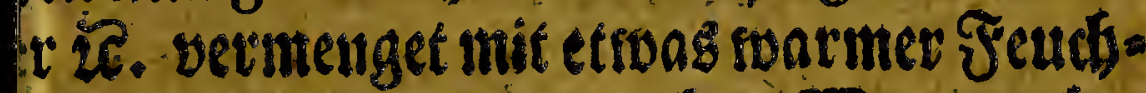
ge eit uns al fo ausgettundent. 25er nun beo viffen bat/ oafs oiefe fette Dinge aus zactig n (ramofen) Fbeilgen befetien/ serfant (id) leichtlid fids einbitben ivie biefe Sadigen (6) umb oiere Degengen (d)lingen/ uno Das ennads oie Degengen gleids als in Sdbeizen edten/ Das fie nidfts fontren austiditen.

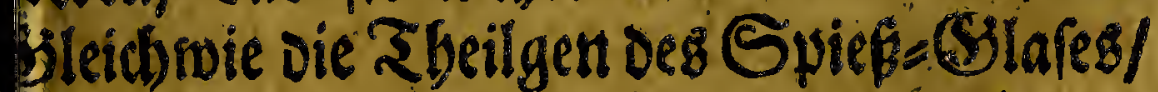
enn fie rob eingegeben roerben / teinen Sobaten thun / swern eह aber auggrwauds bird und oer Sdwefel sueggebet/ swircten 


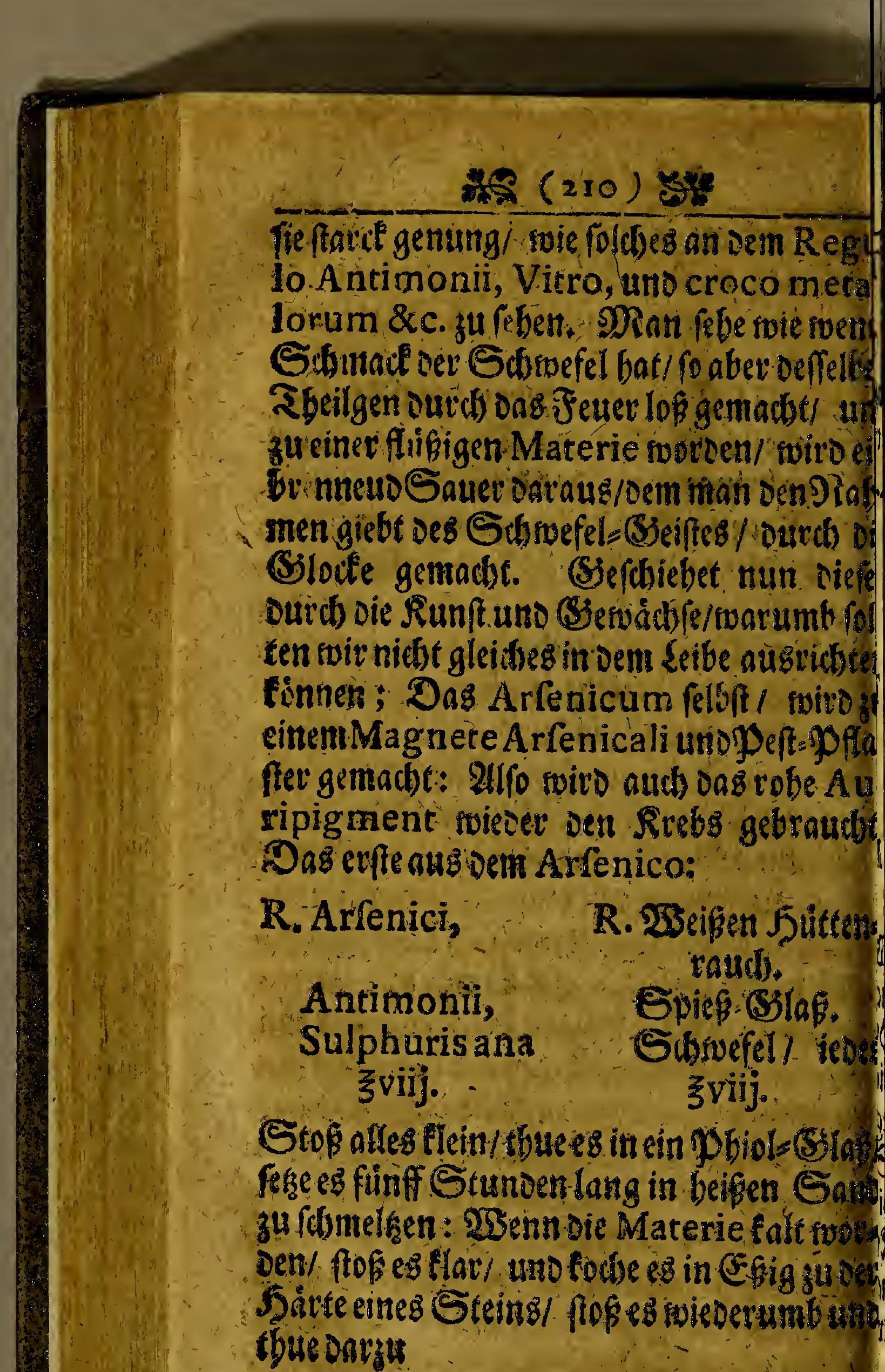




\section{$\operatorname{man}(2+1)$ at?}

Terra Vitrioli Diftiols Serdesviij. żviij.

Calcis viva tto jebentigen Rald toj.

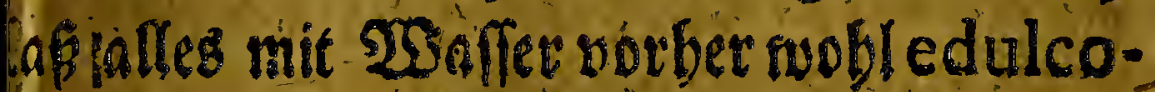
Henoser ausfuffen. Dent

R. Galbani,

Ammoniaci,

Sagapeni ana jjv.

Cera,

Therebinthan. 亏iij.

Præcipitat rubrilbj-

M.F.Empla-

ftrum.
R. Galbani. Ammoniac. Sagapen. ana ऊjv: s3adys. Setpentbit ana ziij: Rotben Precipttat Ibj.

Drifde es zu einem Plaftet.

Benn der Ruebs nidt fobeiden foill/ freut han oas Pulver oatauf/ uno gebraudbt oas pflafter. Diefez Dorbilo befdseribeid bier Hidut als ein Exempel/uno daf bev diefer@e genbeit iemano fid Deffen besienen fónte. Biebe mebe won dem Arfenico uns Auribigment in meiter Chirurgie Cap, vom Rreb: Sobetraftige id mu biemit daf tan Dieje Siffte einigermapenzabmen uno wingentat.

XIX. Sadform Sredent uno wo te 


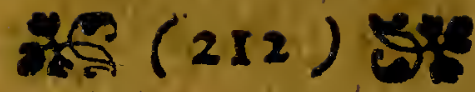

man) von einem giffigen zbiere verlet

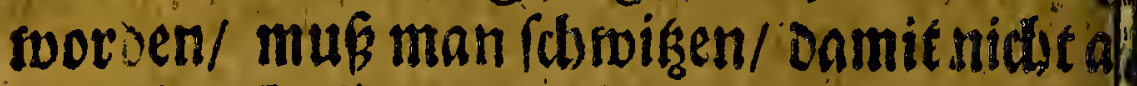
lein ons (SJiffe aus Dem Seibe gervietren we

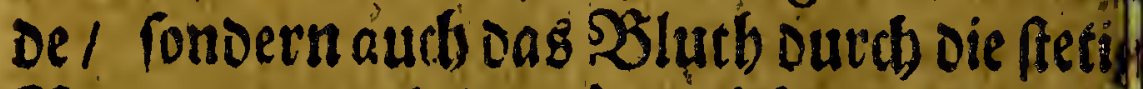
SServegung/móchte vinn fluffig uno gang bar bleiben: Shiezu pflegt man prédbtig Compofitiones zu machem/ als Den

Theriacam Andromachi,

Mithridatium Democratis,

Orvietanum,

Uno taufeno Dergleichen mefo / Denen ma: Den siabmen giebt A ncidota oder (Siegen (Sifffte : Allein menn idh Diefez allez swob. examinire/fino ce meiff erroarmenoe Din ge/ die mit Dem Opio, Darauf oas meiffe an fommt/vermifcht. DQßF DaB Opium Das vornebmfte oabev ift/, geichieffet/ weil es Dem Schneife meifteng antreibet/ uno on Convulfiones fiflet / Die einez von Den gróften 3ufällen fino; Darneben niro den Sdimer'sen/Den man mandismabl empfinoes mus / menn einige focharffe uno fobneioend: Theilgen in dem Eeibe fino. Ferner fino un ter Denen Antidotis folgenoe: Ralmush

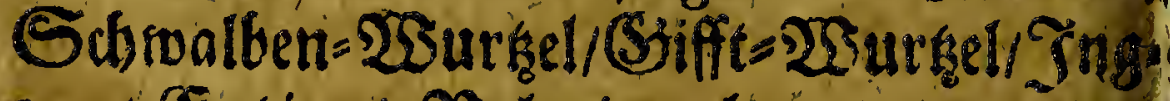
ruer/ Snķian/ Salorian/lange unt runots Dfterlucy/ Xopmarien/ Xauten/Salbev/ Sorbeet=SIlátter / Saanen von Eardentus 


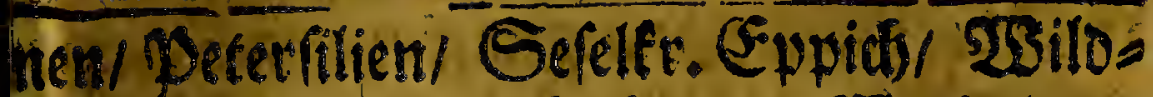
Drobren:Saamen/ Sorbeeren/ Wadiolders ecten/Saffrari/Dfeffer/ SReglein/ Diurcas

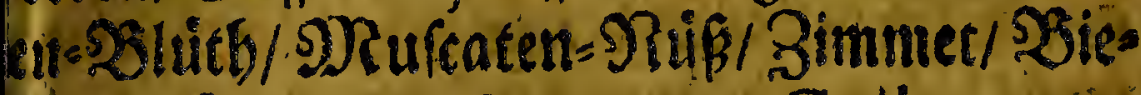
eigeil/Samelfeu/Opponax, Galbanum. Keufelé:Dredk/2)Rurrben/Eampber/Stos. ax/O pium \&c. Aller viefer Dinge Riraffe efrebet in ibren zarten Tbeilgen/ sueldbe ane afammen fino ols eir Sal volatile oleoam oder flictstig óbligtez Sals/urio foldhes efo meby / metm Effentien uno Extracte aron geinadjet roerden. Uno Darunter ges lengt

piritus vel Sal. Geiftooer Salkugot

CC.

Uriná, pleã Cinamomi, Del von 3immet. CaryophylSitraborn. Uritt. lorum,

Macis,

Neglein. Drufcaten = blitis.

NucisMofcatæ \&c. 


\section{H $(214)$}

theilet / Deven Benegung in Dem Rronder gebemmet.

XX. Es mino viel weefens gemadjt sod benen Swlangen bie in sem ₹heriac fort

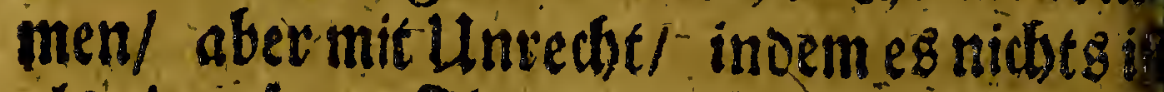

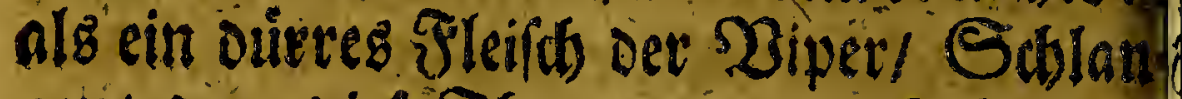
get: / Denn Diefe S biere weroen gefocbt/ oce affe Rirafte raus geber/ eben wie man Dail

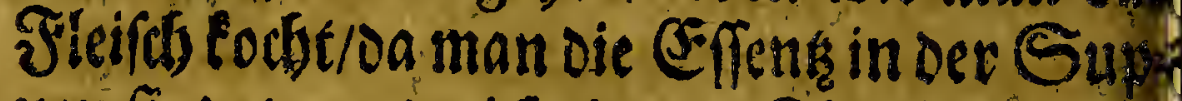

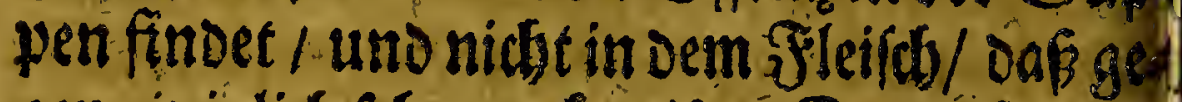
gemeiniglich febr trocken ift; Diefes SBiper

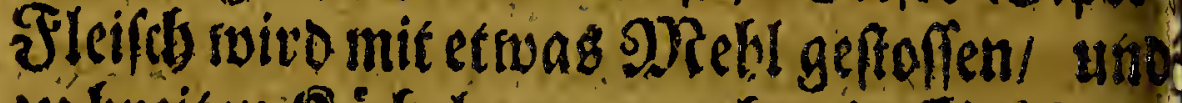

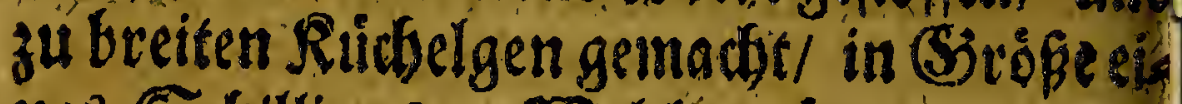
nez Sdsillings. weldbe durre uno ibter beffen Fbeile beraubse Ruitselgen / in Deen Theriac genommen rververt.

XXI. Nictot beffer gebet es mit Denem TrachifcisScilliticis oDery)Reev=3miebe

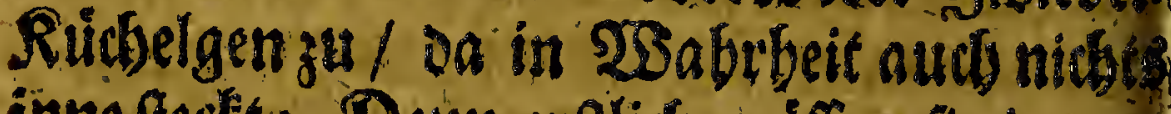

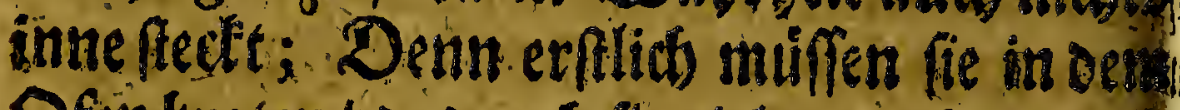
Deen braten/ o adurds fie viele von ibren ful

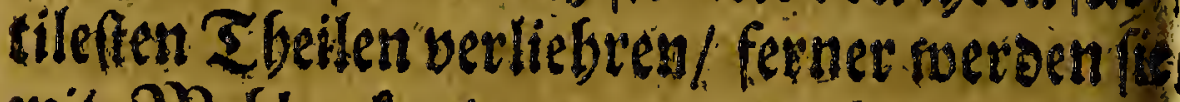
mit SRebl gefneten und oarnach getroukneth woraus denn afe Rraffit bertroctmet. Sou

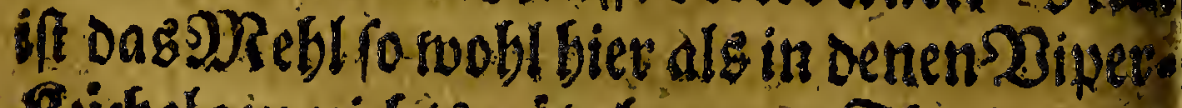
Richelgen nicbie nifge bey Den Therine/und

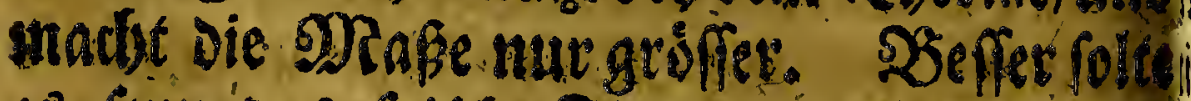

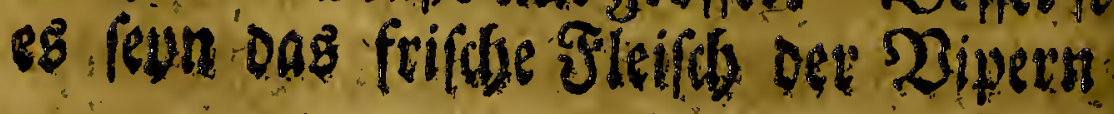




\section{0 (215) है:}

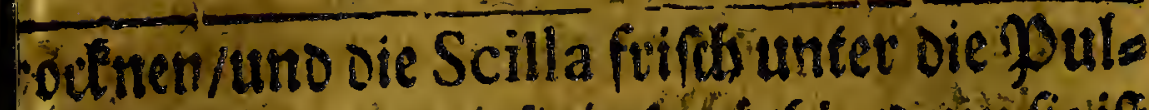
et zu foffen/ weenn fie jafo hóthig/oetriffeift

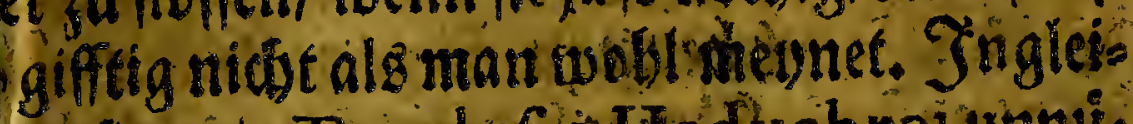
Sen fino bie Trochifer Hedy chroi unmi. - oabey 1. sueldhe Materie allemabl in die

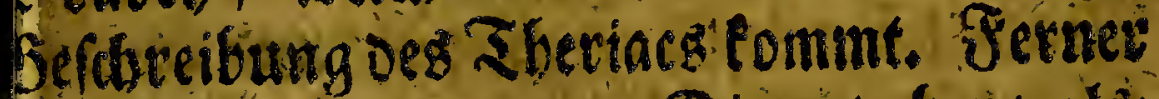
ho nocts vill ungereemte Dinge Dabeg/als:

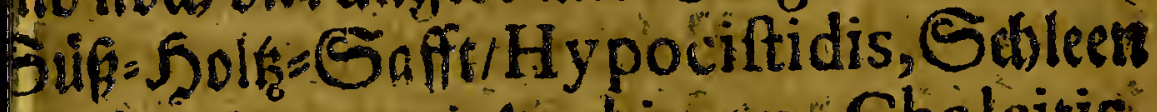
p̄affil Gummi Arabicurm, Chalcitis, haponticum, Eerdien Sdswaini/ rotbe Roren iz. Denn alle oiefe fino bequemer Dab

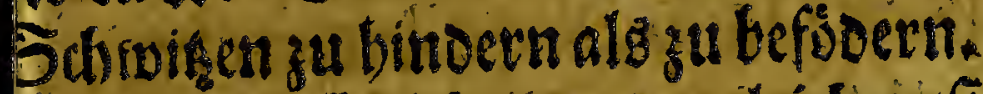

XXII. Soldte tino oergleiderif firoe ids udb in oem 2Ritfridat/uno unter aflen/Deme Baudb von einen gerwiffen vierfiffigan Sbiev icincus genant/ weldses gefralsen bieber ge= ractst wivo i tan aber nicters nutsen/ weil Das

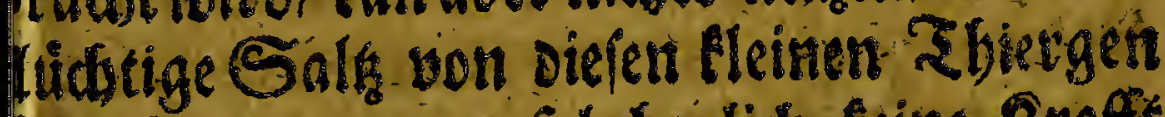
igiret' wivd / und folgbarlith feine Rraffit um fobrowigen baben fan: ; (Stleidinie das

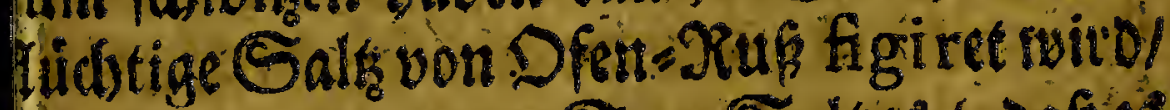
purd 3utbuung oes $S_{e c}=$ Faliges $/$ dafes

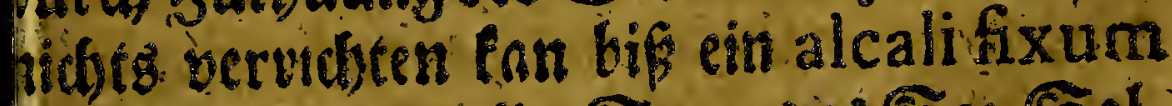
yazu fómmt/uno afles Sauter des See-Sal=

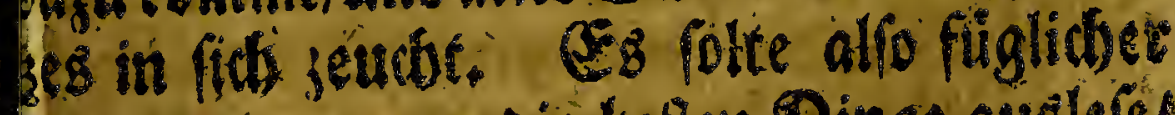

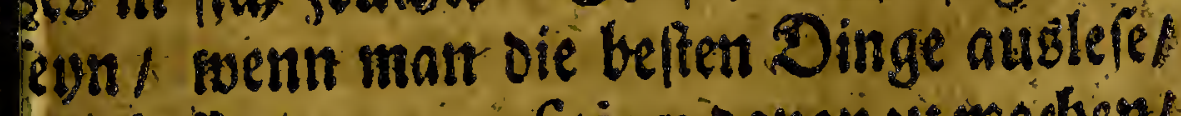
inte befere compofition oavon zu madsen! LE Denen allen nadysufolgen/ bie nidft bes 24 Dadtet 


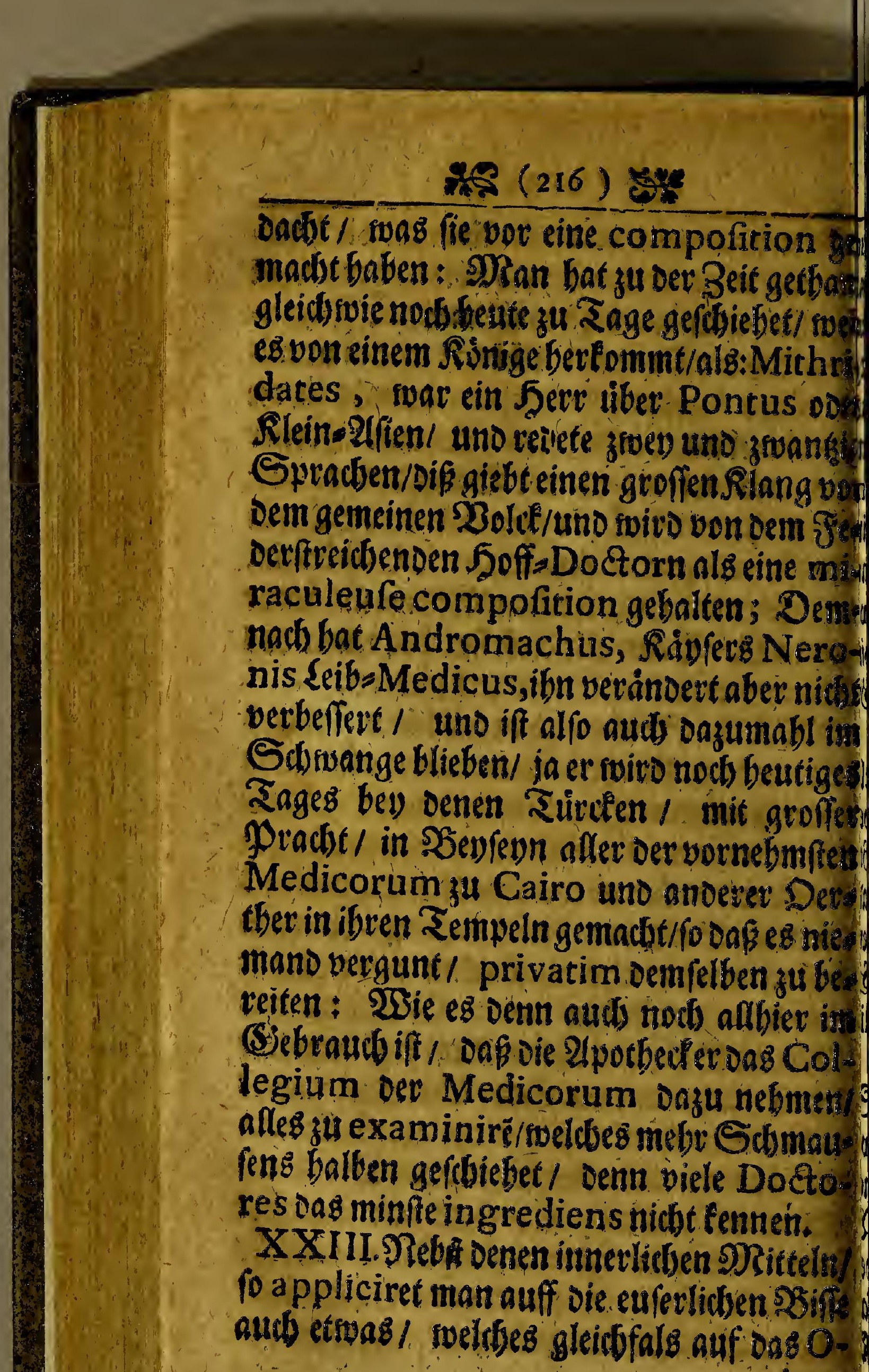

pium 


\section{Ist $(217)$ sto}

Ium uno oie vorigen fludotigen Dinge ans mumt/Daraus Salben/ SSrey ober verglei= en gentiacht uno aufgeleget werden/oiesbes egangen ineldse das (Sifft madhe Dures 68 Opium juftillen/ uno fo allbereit twas Heer die Sáffe fommen/ ourch oab Opim juodimyffen / Denn in Dem Das Opium inen Durdffus von der fubtilen Materie at/ fo wiro das (Sifffe audb allo berweget/ ins wifhen Daf foldhe S3envegung gefdriebet! bivo oaz sBluth fluffig bebalten/ uno oas Biffe Dåmpffet zugleids mit aus.

XXIV. Unter denen ćuferlicben פRits lin if audi cas Sorentren nidbe undienlidal Denn matt nuf Dem befdodoigten Dref ein Brenn=-Eyfenoder etwas Dergleidien feget/ aourd bie Tbeile ses (Sifftes niche antein

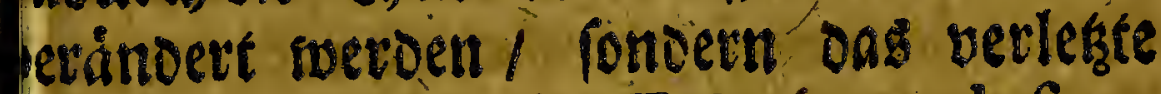
Shied wito auth bon sefferbung defendiref.

XXV.' In Dft Intien giebte es cintt Stein/ weldhet fobswars uno manchmabl was grau/turio von Figur, in oer(Sirsffe ei? Reg deuits : Diefer Stein wird mieber die Bsiffe der Sdblangen gebraudbs/ uno auff Dem Deth oa dee SBis if geleget/ Darin fo zu agen afles@ifft zeutb $/ 0$ b bierer Stein bur(b) Runft gemadht swito/roie nan glaubt/ober ob. 


\section{0 (218) 5ुर}

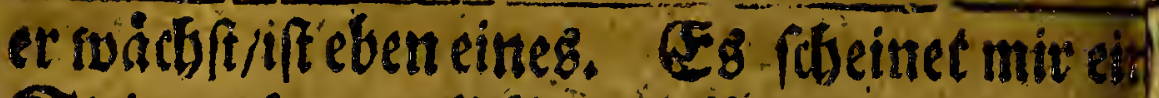
Stein zu fent vod fleinet sadbergen/ in wo: die ons (Sifft nus der Wunte getrieben misod unto det Stein auf, Der 23 unde feit bleibet eben als wie eine neue Zabacts = P feiffe $a$ Dem Sippen bangen bleibet; SSenn bes

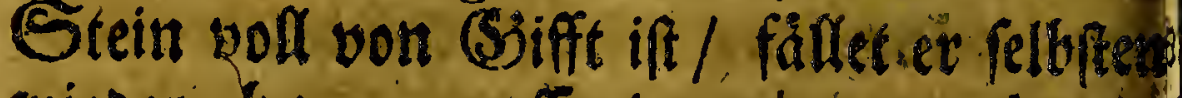
toieder ab / nocauff ein neuel applicire swizo/ooer fie legen den borigen in fuffe DRild Davori alleg Siffe beraus gebet/io zuvor bin ein gefommen war/ uno legenibn toicoer atf Dem befatiogten Drtb.

XXVI.' 2 as ang bet die Tofcanifhe Spinne Tarantula genant / uno oi Rrandebeit St. Viri, beftebet folde in 3 tu Eungen uno convulfionen, tho foito au cine fonserlidge toeife curiret/nebmliph out of tanksen nach einec gefviffen DRuffe die gan to Sub beftebet sarin/ Daf oie unorbentlito Servegung our of oas Sankse als eine anoet 20rwegung abgefebuet werde/ gleich roie oi fallende Sudt roted in Convulfion be ftebet/ ourch Pliefen berteidben nird / libe: Diefes treifen fie mit Den Tantsen bie Shwein uto folgharlid biel Sifft aus dem Seibe/ torldue fonf in cem Solutbe vethliebs: Darneben rwerden die J beilgen oed. Blus: thes ino ifre pori anoers difponiret: io 


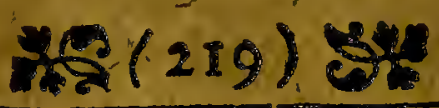

B. fie fo lange tanken bif fie zui Erven den.

XXVII. Das Ioetlaffen if bev vielen

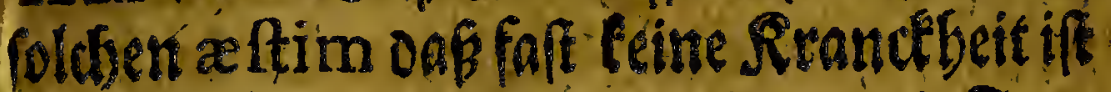
fie es nidit vornefomen / fo if nun die Sraz ob es fier nidbt auds sienlid) fey? IJenn s (Sifffe allbereit im Slutbe iff / uno mats ber lâft/ ifteg nidje anders als ob einer aus Hex Sonne Salswaffer/ein SGeil abzapffen blee/ oas úbrige zu verbeffern/uno fo wenig a diefeż gefdhiebet/fo wenig gebetez init Dem

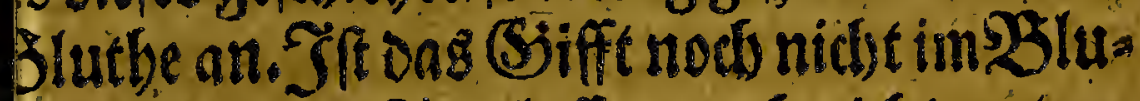

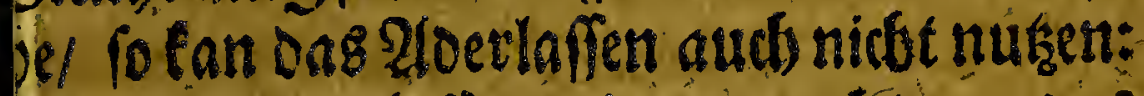
Ino in Dem gelaffen swird / zetudot man Das

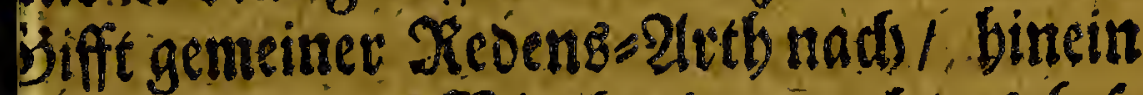
bertE/Das ift oas SBluth friro ourd das Eafs in fibler uno folgharlidh oidfev/ uno befoms het alfo Salegenbeit mit dem (Siifte metb no melir zu getinnen. Soll mañ aler ganfs. cin Blutb yon fids laften? Sa/ meinm mant

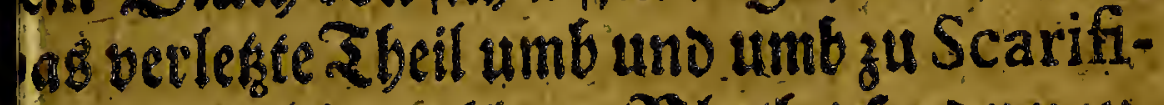

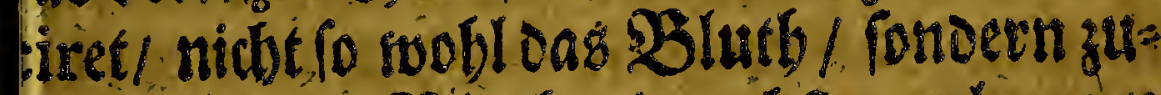
Jeid mit sem Blutbe oienediff angelegenen Siffte oie viefleicht allbereit modbten anges

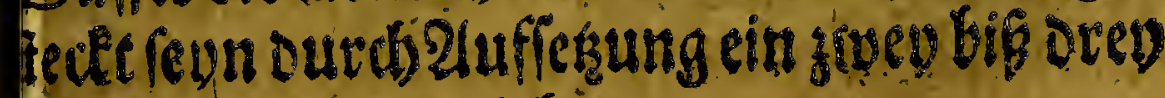
Ronffe bevaus zu zietien.

XXVIII. 2illes Sifft orbentlidi gutenta

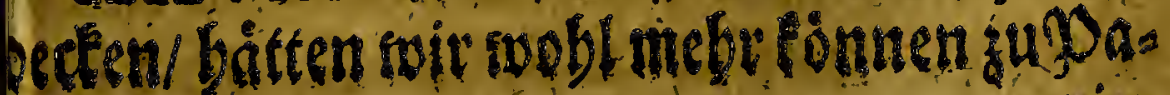
pies 


\section{piecturing $20(220)$}

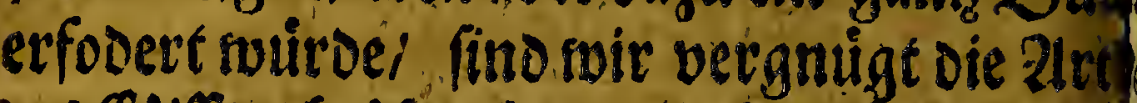

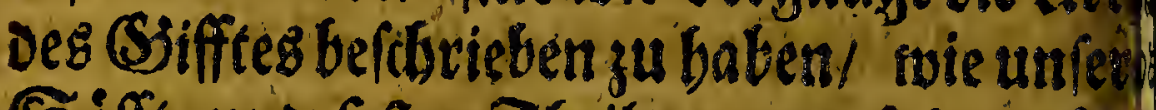
Siffte uno feften Tbeile bavon fich vevaing Dern/ uno ourdo geborige Medicamente vertrieben werden/ begeben uns oerowege 34 2165anoelung anoerer Dinge.

\section{Daร̉ XIII. Kaspittel.}

Bon Denen Gradibus Der Medi carnenten und benen Specificis weldhen

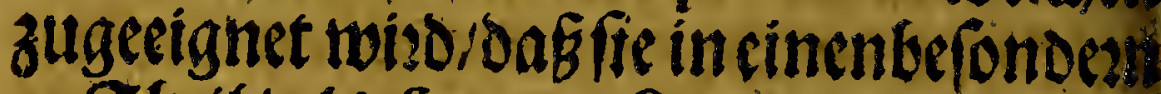

Sbeil/als Sautut/Rungen/Serk/ Magen/ Reber/Mrils/ Jiles ren ic. ífere 23 íts ctungtfun. I.

ST) Tlten baben die WRanier gebabe uno if auds nodf ben vielen in (S3e. braudb/ Oás fie unter ibte Medicamenti einige Specifica vor das eine ooer anoere Sbeil/ als 5aupt/Secber/ S)Nills uno oerglets dhen nebmetr/ nachoem aber afles gentat uns teriucht/ uno ber Sauff ber Säffe erfunden noorden/ bat man geéfenen das feine Medicinen find / reeldye iuft diefez oder jenes fefis 


\section{$\operatorname{xec}(221) \geq$}

Beil afficiren. Ezif wabe/oafids foldtes cht fo abfolut láugne/ in oem id) fagen mus salle Speife uno alle Medicamente elon unterf(bieolidien Durdhf laterie uno Preffung oer Simmete= Ruis Igen baben / uno dap foldher Durctiflus 15. Yreffung mic bem einen Sbeile beffer bereinfomme als mit sem andern. Diefes nun wobl in anfebung oes feften Tbeils/

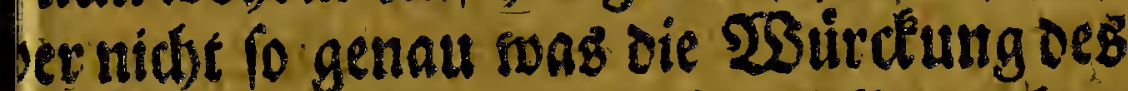
ledicaments rweldise es thun foll/angebet. un Exempel/ ids babe cin SRagensoder Ses

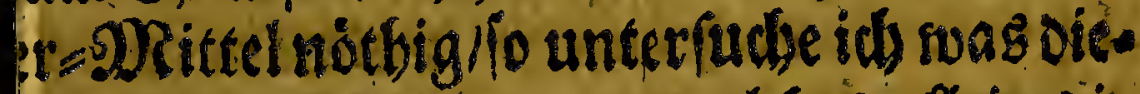
I beile beleibigit bat 1, sweldyes allein die Jäffec fino/ welchezáb/geronnen/fauer uno rgleidjen/ Diefefteben fitfe/ madben $\mathfrak{B e r}$ opfiung/ Prickeln/Stedien/ Rägen ze.

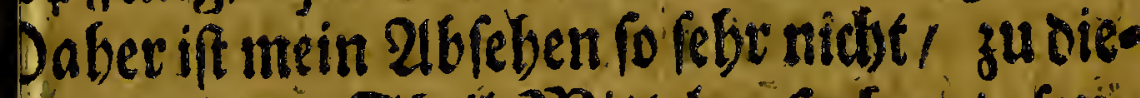
noser jenen Sheil D) Rittel zu futben / forto ein oldbe / rwelche in alle Saffee des gantsent

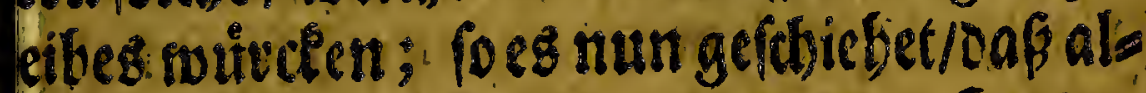
Säfte foned genung aus dem Serşen no Pulpoatoerm/ Durds afle (siefâffe uno Ifte Sheile getrieben toeroen/mup nothrwett ig oie כerftopffung/Sdbàrffe ż̃. meg fennt. Bsie fan man auch ineinem sbeile abron erlidb/obne Sieruibrung oer anbern operien? Jem/ /o idb in ein Sbeil alleine wour ot?" 


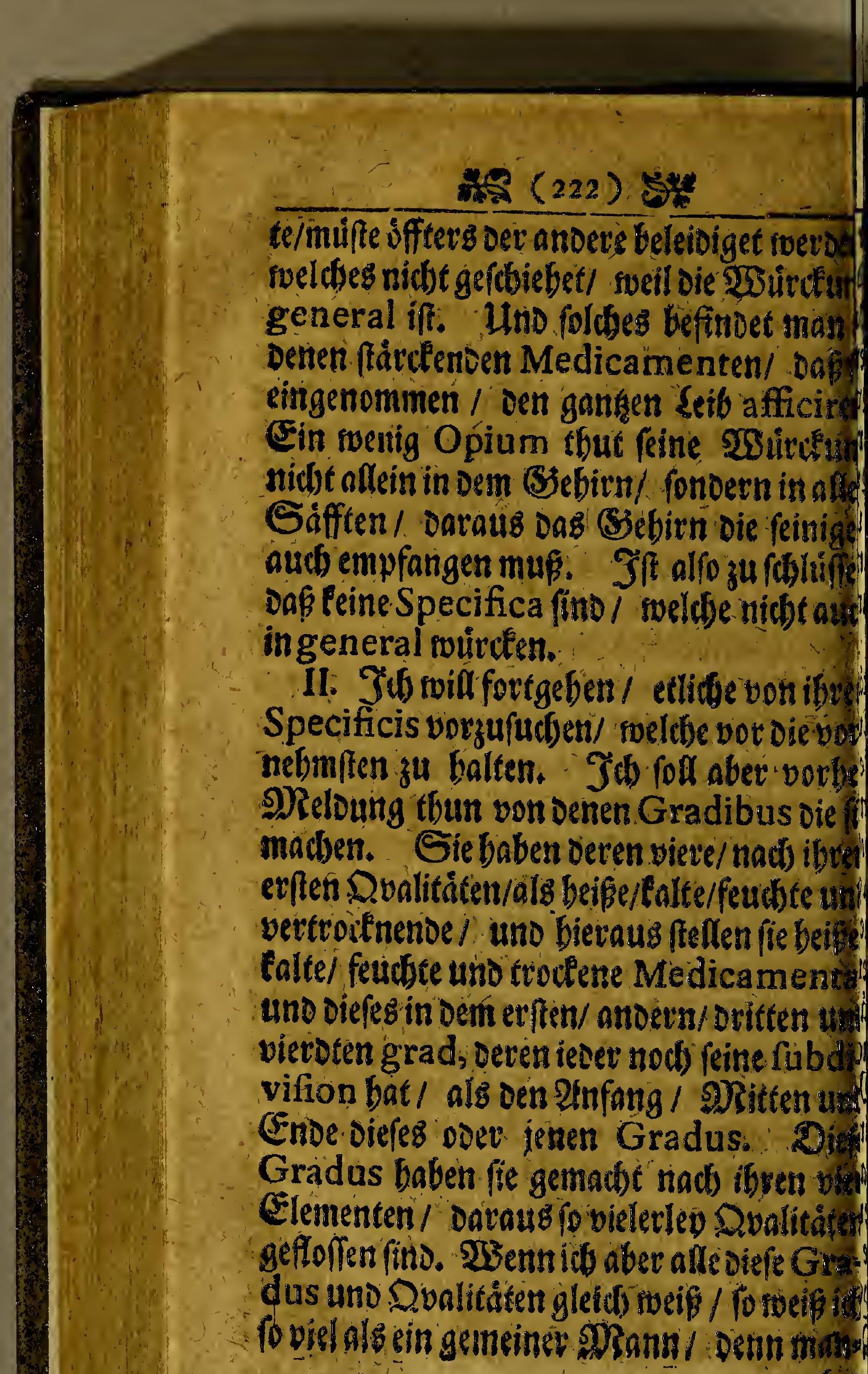




\section{as (224) है?}

IV. 253 baben wic ro biel fublende Me dicamenta vonnotben/oenn fo fie in ged ard ten biskigen Rrandébeiten unnuise fins/ Darf man fie in oenen fogenanoten Ealten Rranch beiten nidbt gebraudben/oenn fo Die Ralte $u$ facke ift / wiro ia beffer ourd rorme Ding gebolffen:

V. So mant trodenende Dinge babet muß/ iftes 23 under/ roarumb oie 23 affet? fúchtigen fo gequálet roeroen/ uno nidb ebs ibre gervuninhte (Sienefung exlangen; Detth swas dienet dex Terminus trodenend Dintge/ wonn ids rold 2 baffer nicht vet trodenen fan. Die feudtenden SMittel fino audis eben oer 21etb/foer Durft bat/fwám er nod fo tboridst/meif wob) ( S rindten zu be fommen oder zu fooerny la felbft das fleinef Rino/ welches nur zu reden anbebt.

Sis if SSunoer / Daf man fie in oenen bisigen Rrand beiten nicht giebt/Da unfer Seculunt eserfunden/ gebende Denen Rranden bei

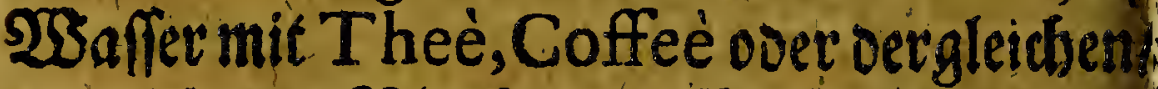

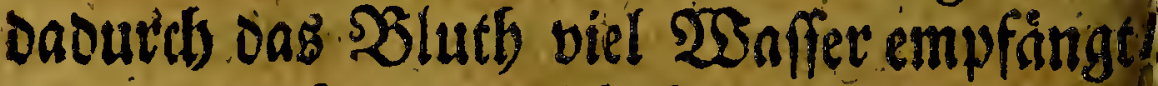
und ungeadotet man febr beis oarnach soino: soiro fich cods nach, Dem etuinden / Die Sith uno Sluffwallung oes (Sieblitbes. vermith Dern / uno oer llein robbl geben. Jeuer it beip uno trocten/ 23 affer/Sobnee/ if feude 


\section{มีล $(225)$ )}

10 Ealt/ Denn swenn man Ealt ift/ futbt man

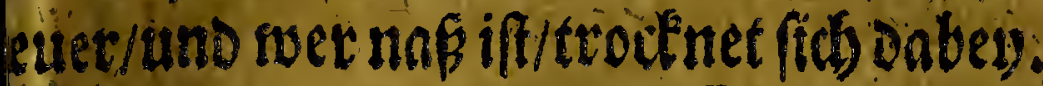

Vi. Unter senen beifen Dingen/ sweldte bem vietsten gradu fteben/ fino untevo levene gradus, oenn fo vielerlen Simplia, fo vielerley fino gradus suzunterden! to alfo aud bey denen andern Gradibus. De suarme/ falte/ trodétene uno feudite graus mit ibren ab bbeilungen zu wiffen iff nit

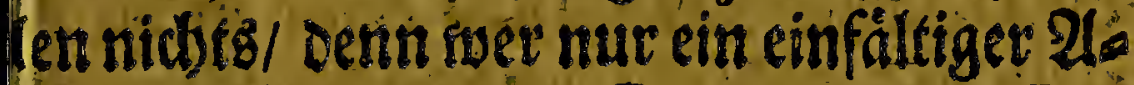
thecfer ift / uno feine Dinge verfucbt fat A viel beffer vavon zu urtbeilen wiffen / als

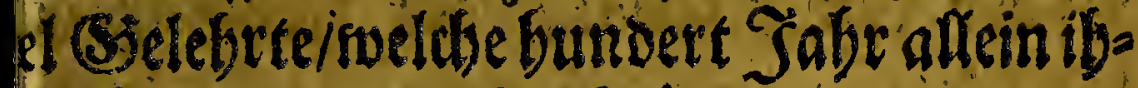
Ssuidser surdbiudser baben.

VH. Sch finte unter Denen Dingentrive! eim bieroten gradu feten/ Das Auripimentum uno Sandaracam gracoim, weldie ebe talt in oern vierd ten gradu lefefgenentet weroen/ alloieweil fie thas thaner operation in Dem gantsen Eeibe os re eiten Sbeile / ben ganken Seib ooer oas beil falt madben/ Denn es exftirbt/ uno Das bens: ร̌euer verfdoninoet.

VIII. Weiter baben fie Medicamenta, nenffie die andere Facultat von Srifften no- Qualitáten zufdreiben. Diefenennen setweichende uno verbårtenoe/ oưnn uno dimactiende/ offnenoe und fopfferide/ suiffo 
tig uno féfmachender reiffendel Jleiforith thende/zuleimende/ Wruno:20Rittel/ fhent madsende/ Seids)=Dornen vertreibenoe/ wis Der rovadjenmadienoe/ einbrennenoe/ D) uno Saamen vermebreno uno verminder De/ Suftersuedende uno benefimenoe/ Ste: zerbredsende und treibende \$Drittel/Sdslat macheno und Sthmersftillende DSitt SSlutbliflende und oergleictien. Diefe F cultâten uno Riraffte fino beffer zu admit tiren/oods nicht afle/Denn oie zurtictétreibend Dinge/ fino nicht oergleidsen/ woil folds? ivieder Den Umblauff Det Feudbtigfeite fiteitet.

IX. Wir fuofen erfflid von Denent en sweidsenden oderEmollientibus reoen/un plebe fino unterf(biedlidb/als

Wurkelin:

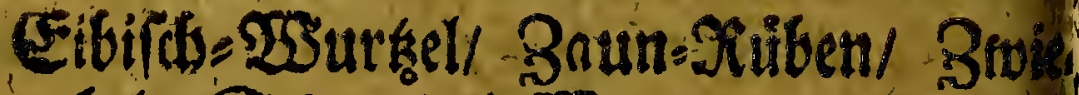
beln/Somertel=203ursel/ weip silie 2Burâsel.

Solattee:

Mangolt/ Cibifdo/Nappelm/ Zag th Sadht/Diolen=Reaut/Wsulftaut/

Sanment:

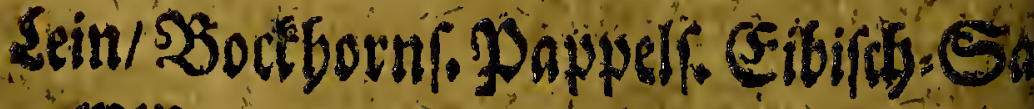
men. 


\section{ne $(227)$ sto}

\section{Şlumen:}

Camiflet/ Pappelm/Saffram.

$$
\text { Jrtidte: }
$$

ฮีeigen/ Sotuft SBeren.

\section{Liquores:}

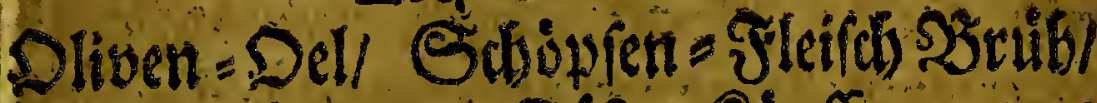
Decoctum Der Suisel Ropffe 2e. von etlidben'Sbieten/ Sein $=$ Del/ DSandels Del. Siezu reditte.

Gummui Ammoniacum, Styrax, Edjmals unterfhieolidjer Thiete.

X. Die 2Surctung bierponift/oge batte ierweichen / Derftopffangen unto derglei en/ twieder gangbar zu modien/ oas iff / oaf irdo die Rrafft eines foldhenMedicameng/oie Derfopffung/nelldoe etwan zugegen/ eggenommen meroe. Saft ung aber nut Gen nuf rwas Weife diefe Medicamentett

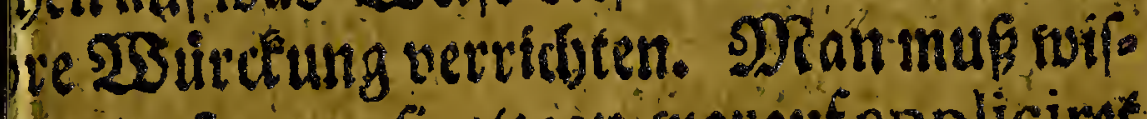
ii/ Das noenn fie etwan sworauf appliciret botden fino/ fie Durds Die iuferlidye von den

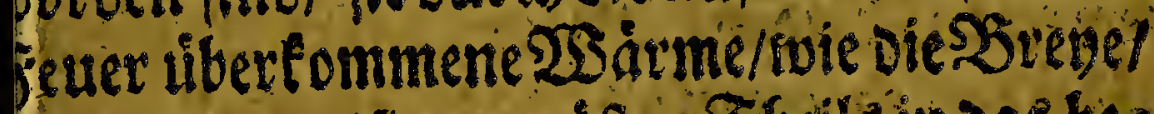
bebungen $2 c$. find/groften F beils in oas be eidigte ? be il ourdsoringen/ uno soell viele Salpetrigte uno andere zarte Sbeilgen mito efiubret nerben/ gelangenfie ztit oem barten

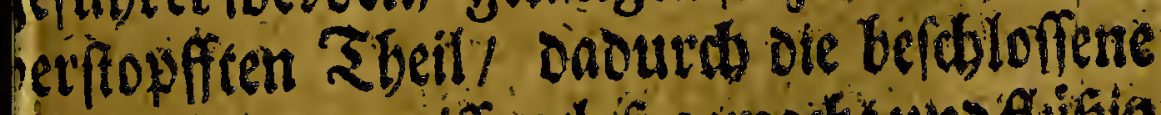
Materie angegriffen/lóf gemaitituno flipig

$$
\text { p } 3 \text { - nut: }
$$




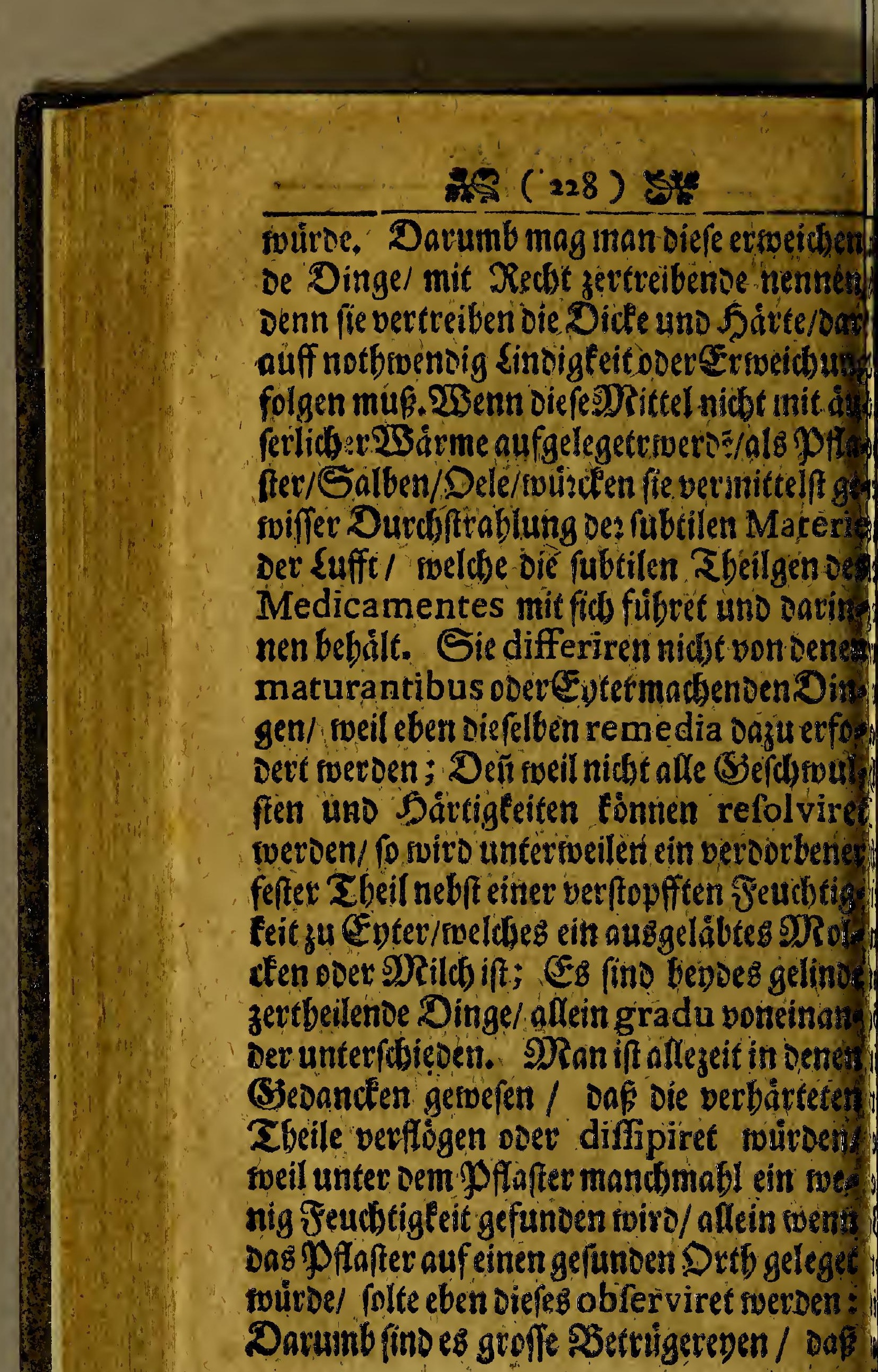
mant 


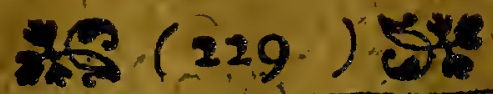

and Denen Podag ricis sweir madit/es wuiro

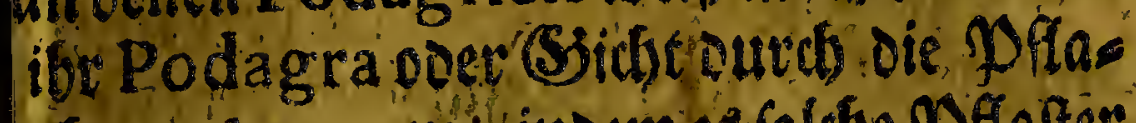
theraus gezogen/ indem es foldte PPffaffec - o oarunter Salmiace fommet/ weldses

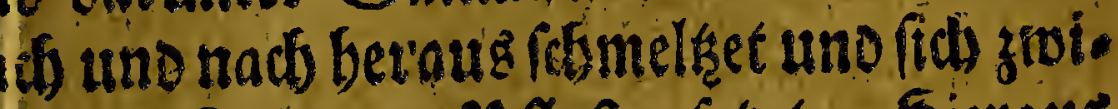

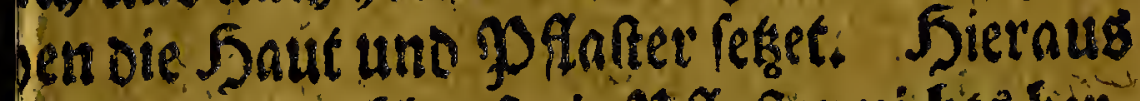

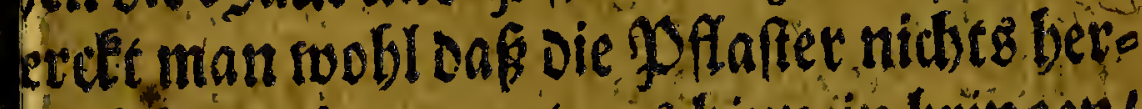
[s gieben/ Conoern etrwas binnein bringen/

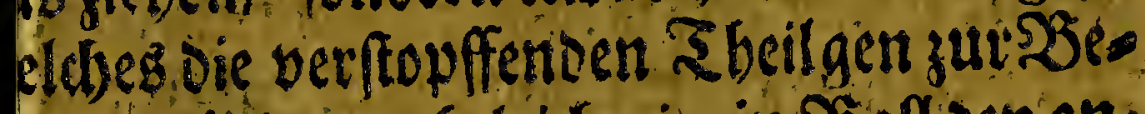
egung bringet/ (gleidhwie ein SSafl oen an= un forffóffert) Dás fie ourch die SBernegung $v$ antoringenoen Säffte leidytlids nuegges. adie rverben/ Dadurds oas aufgefpannete beill linte rwird/ rvie zuvor.

X1. Berbärtende Dinge baben swir dor nostfig/: Denn fie veruifadsen einen circhum, barte Drifen und Dergleidsetm. no folcbes geftosiebet burds applicirung

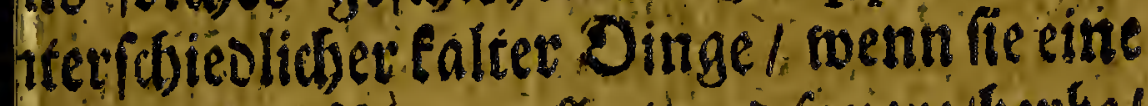

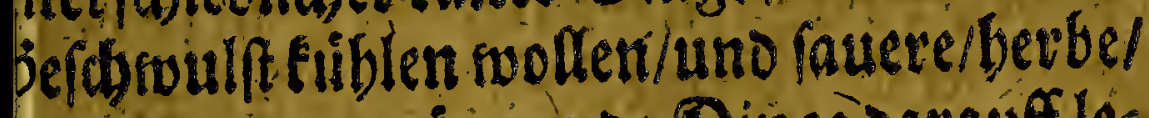
ines sneges erwármende Dingé darauff les in/ onourds oie Fendutigfeiten coaguliret

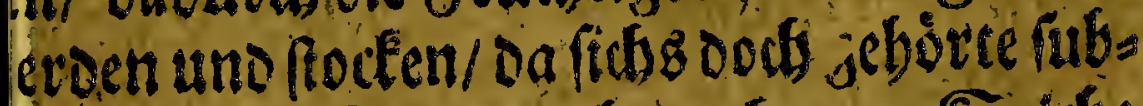
e und linde Dinge zu brauthen. Soidte Berbärtungen fwerden vielfältig gemadit on benem/ weldefe aftezeet repelliren wollen it adftringirenoen Dingen uno Dergleis ayil do fie bietten zertbeilende gebrauchen Hen. 


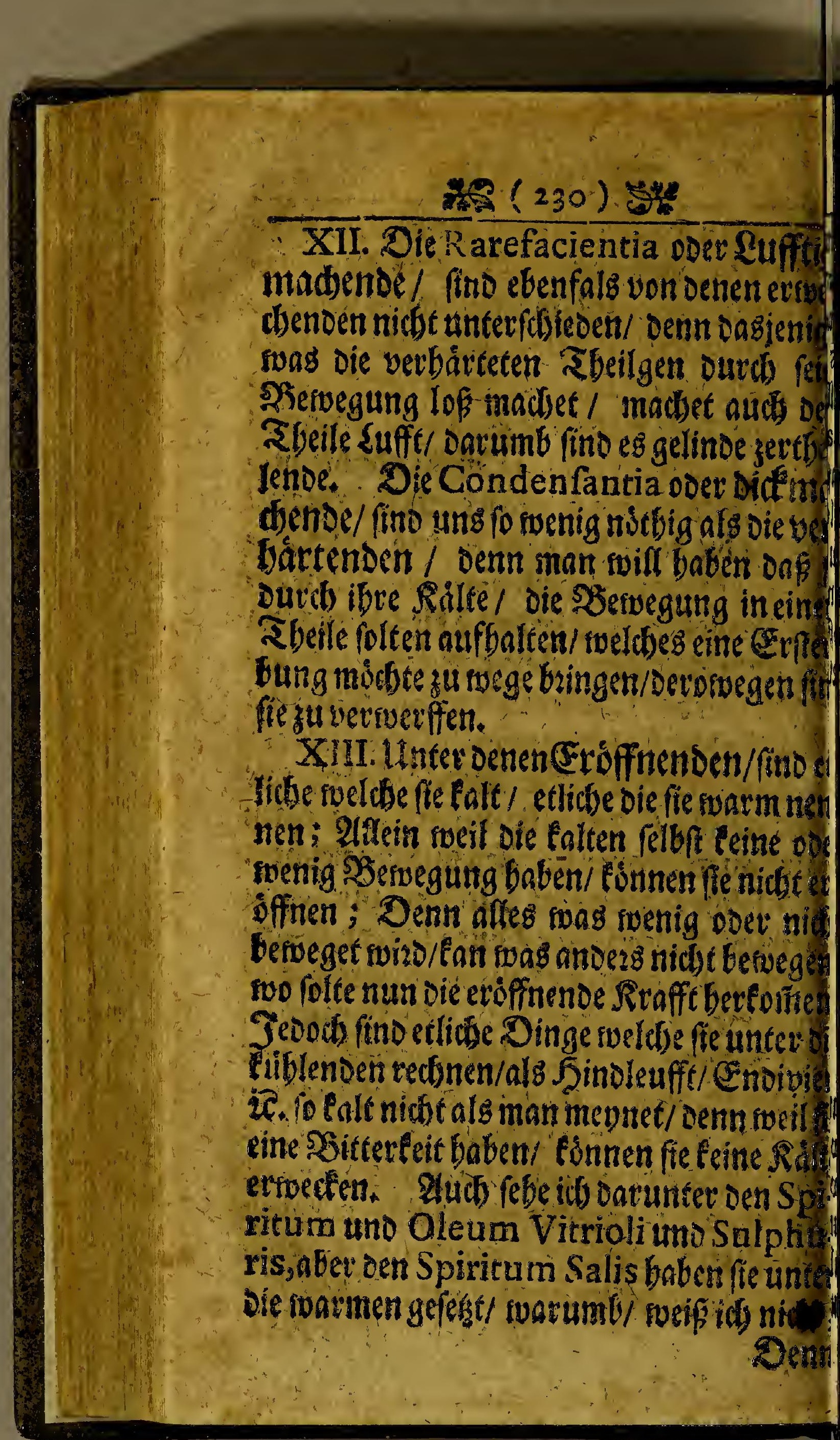




\section{Ix}

Jem als man die fogenatioten fublendett Dinge auf obe Bunge nintit/ brenten fie wie reuet vornebmlich oet Spiritus Sulphus, weldses der Spiritus Salis nicht thut. Ihe die sffnemben Dinge fino mut gelinte Laxantia, oeten Sbeilgen alfogeftellet fino/ af fie cine pridelung madben/ noenn fie auf late Draßse von der fubtilen Materie der uffe berwogen werden/als ifte pori befthafs

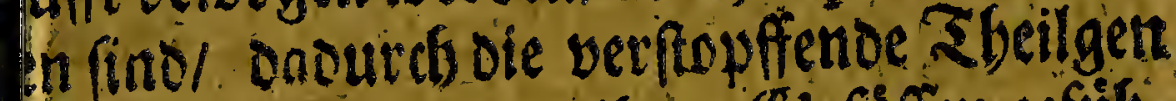
of gemadht/ und aus ibren (Siefáfen gefuib)= et fweroen/unter diefen find oie vornebmiten

\section{Wuturseln:}

Eppidb/Deterflien/ Jendsel/Sindleuff/ - Duecengrafi Spargel ż.

\section{Rerảuter:}

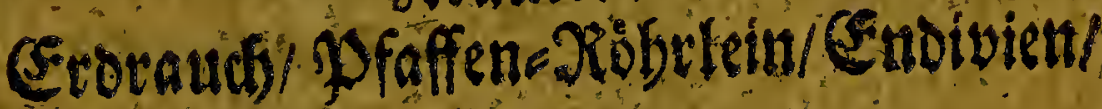
Sindleuffe $x$.

\section{Gummata:}

Ammoniacum, Galbanum, Aloë \&c.

Diefe madien eine gelinde Deffinung/oars Inter aud der Stabl geboret/ und oarumb weroen lie offnende Dinge genernet; $E_{8}$

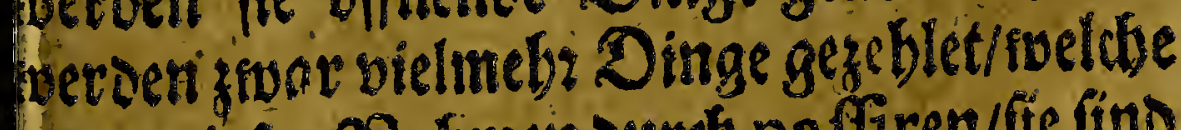
Anter diefen SRabmen outch paffiren/fie fino ber meiften diffolventia, uno múfien oar utter gereednet werion.

$$
P_{4}
$$




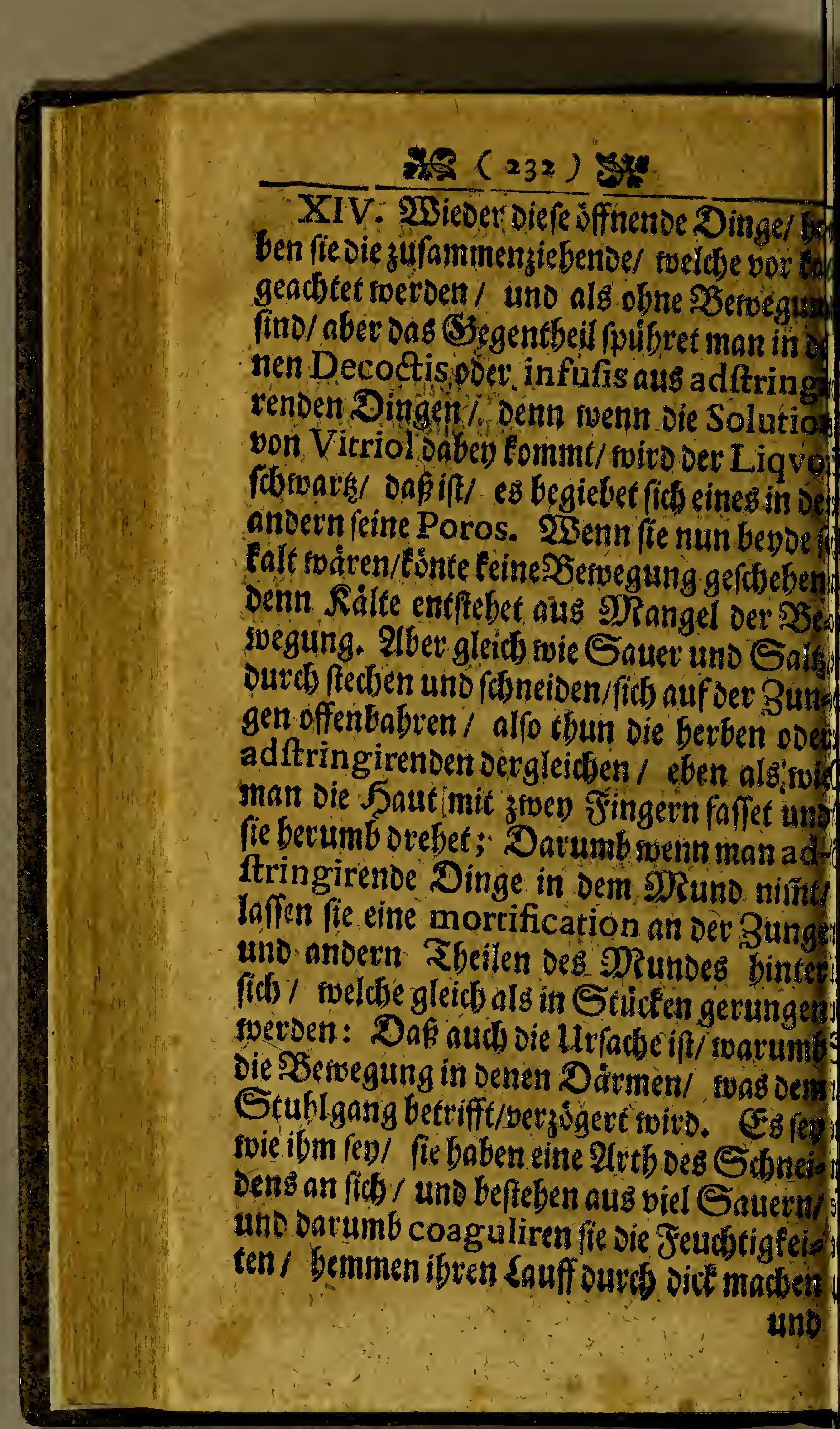




\section{the (233) हैं}

no gevinnen/ uno so cinige (Siefâfe offen no / beifen fie die Deffmung too/ utto veis blufien fie folgbarlich.

XV. Ë fino unter/dsiedlide Dinge/ Die bne Saure fino / uno gleidbrobl ftopffen 18: Rraffe=Debl/ Gerften = DRebl/ barte soer/gebrand @elfenbein/SSolug/SJlutbe cim/Eoralfen zc. alle Diefe Dingeftopffen/

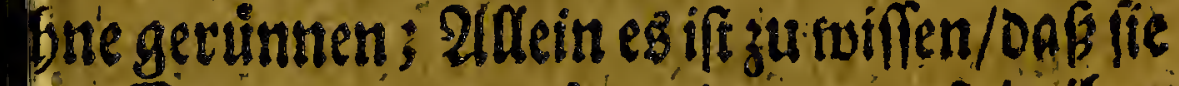
as Sauer temperiren/ uno oas in ibre pros viel /dbarffe Sbeilgenfth verbergen/ ie nadi uno nadi aus, Dem Seibe gyefübret berben; Uter diefes triegt oas S3lutb einen effern Chyl uno Derbindligleit/ uno oie Durdhftrablung oer fubtilen Materie, neb/t Preffung Der Suff $=$ Ruigelgen/afficiren Das Blut nufeine andere ${ }_{\text {SBeife. }}$

XVI. Sierauf folgen bie Attenuantia Ino ourdb/dineibenoe Dinge/weldfe alle aus lidotigen Sals miffen befteken/ Damit fie

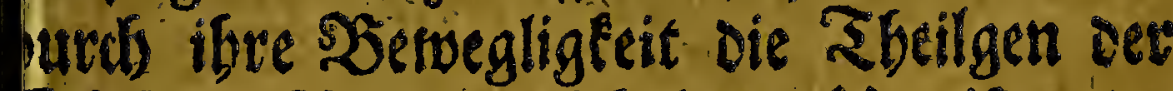
Siffere in 2jerwegung balten. Uno if unter Inoern Das twarme 25 affer / einez Dew bor rebmiten Dinge/ Darumb nuirs von denen neiften Doctoribus Das Theè- und Cof-ee-2Saffer / in allen Seuten fo getubmet/

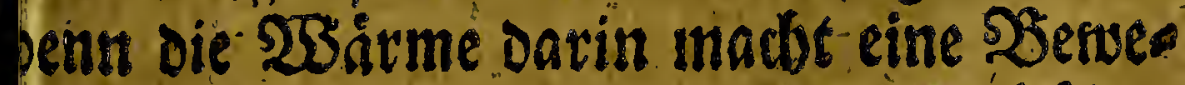

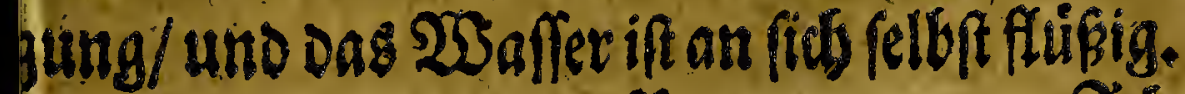




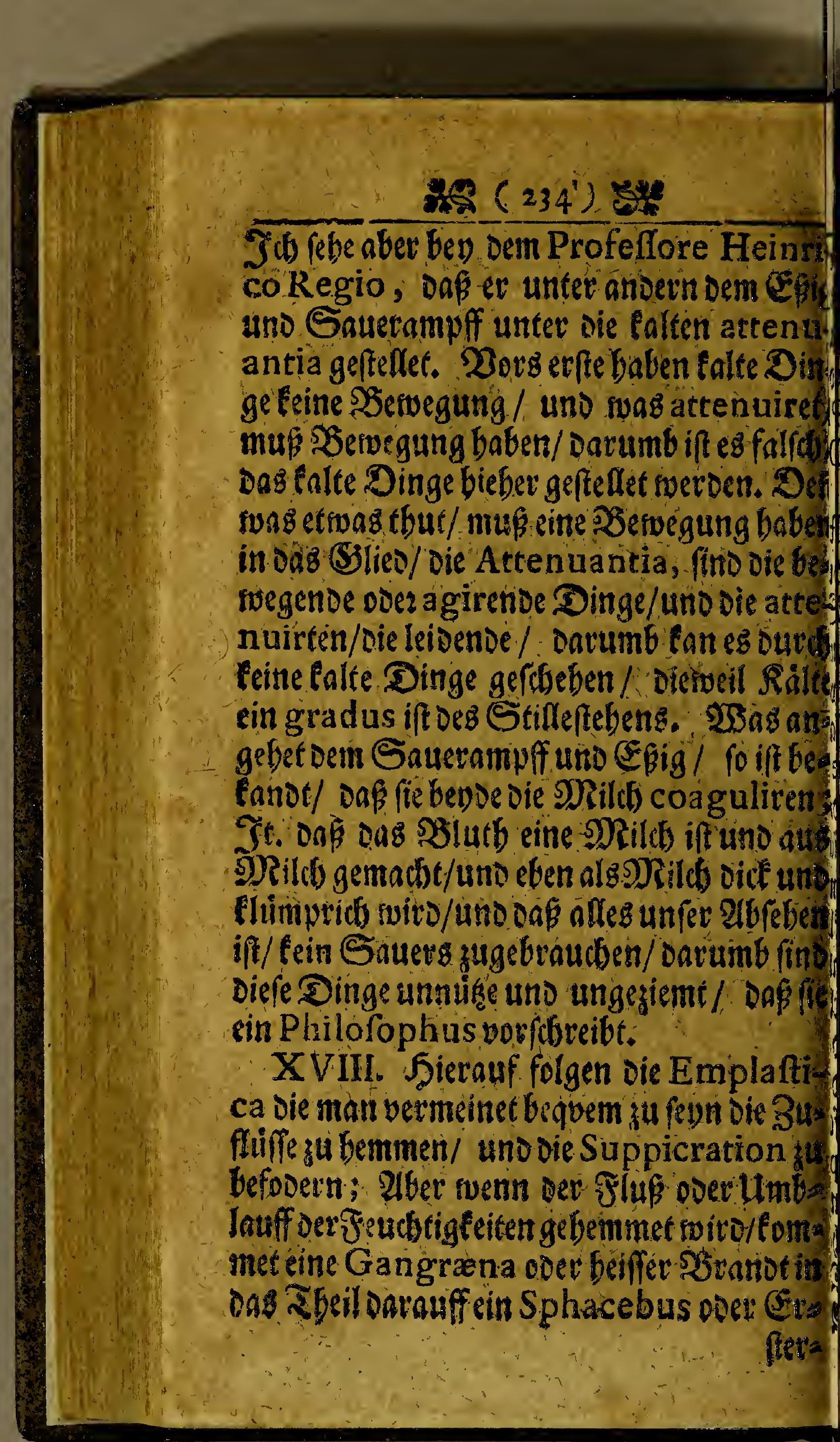




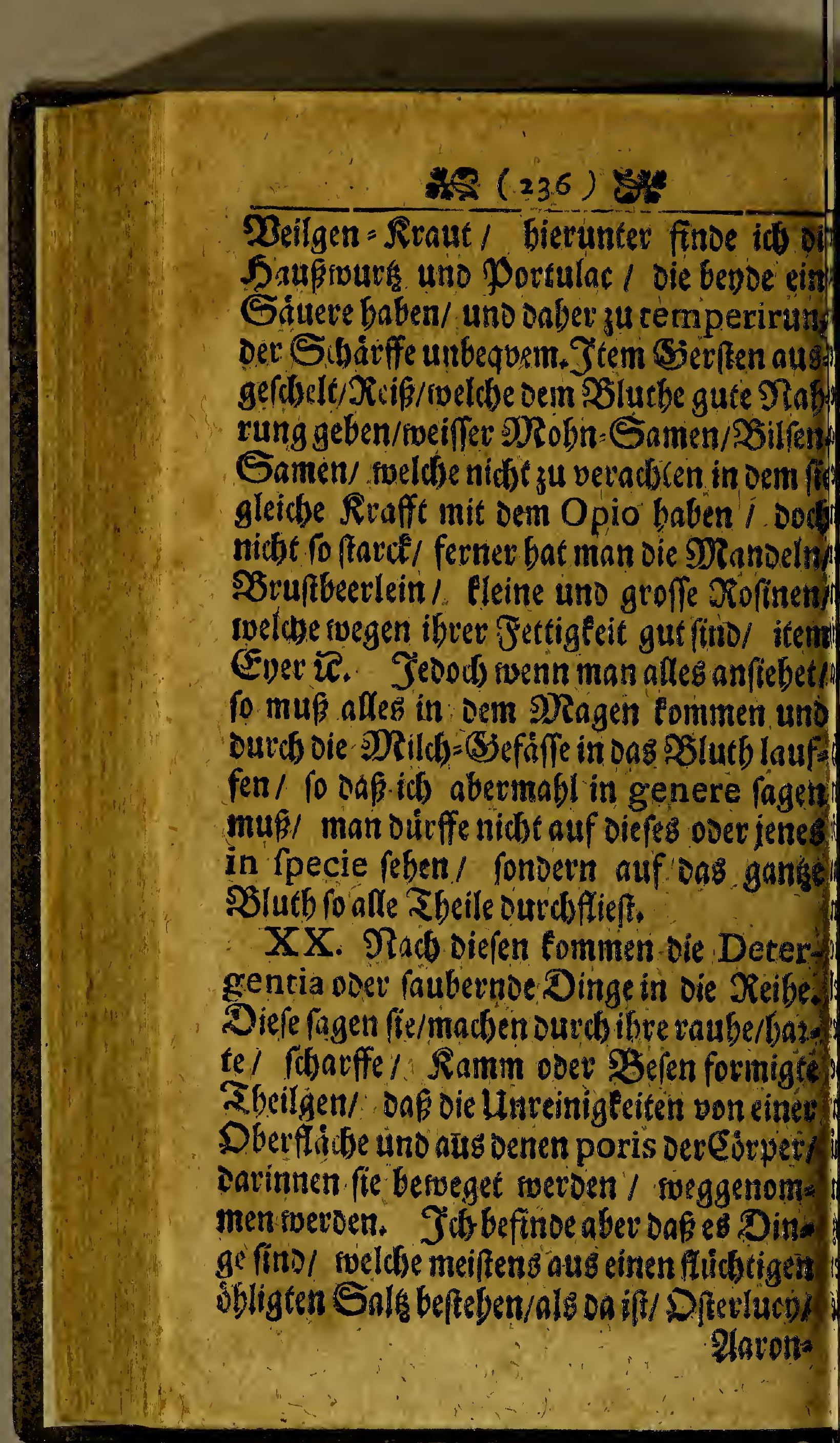




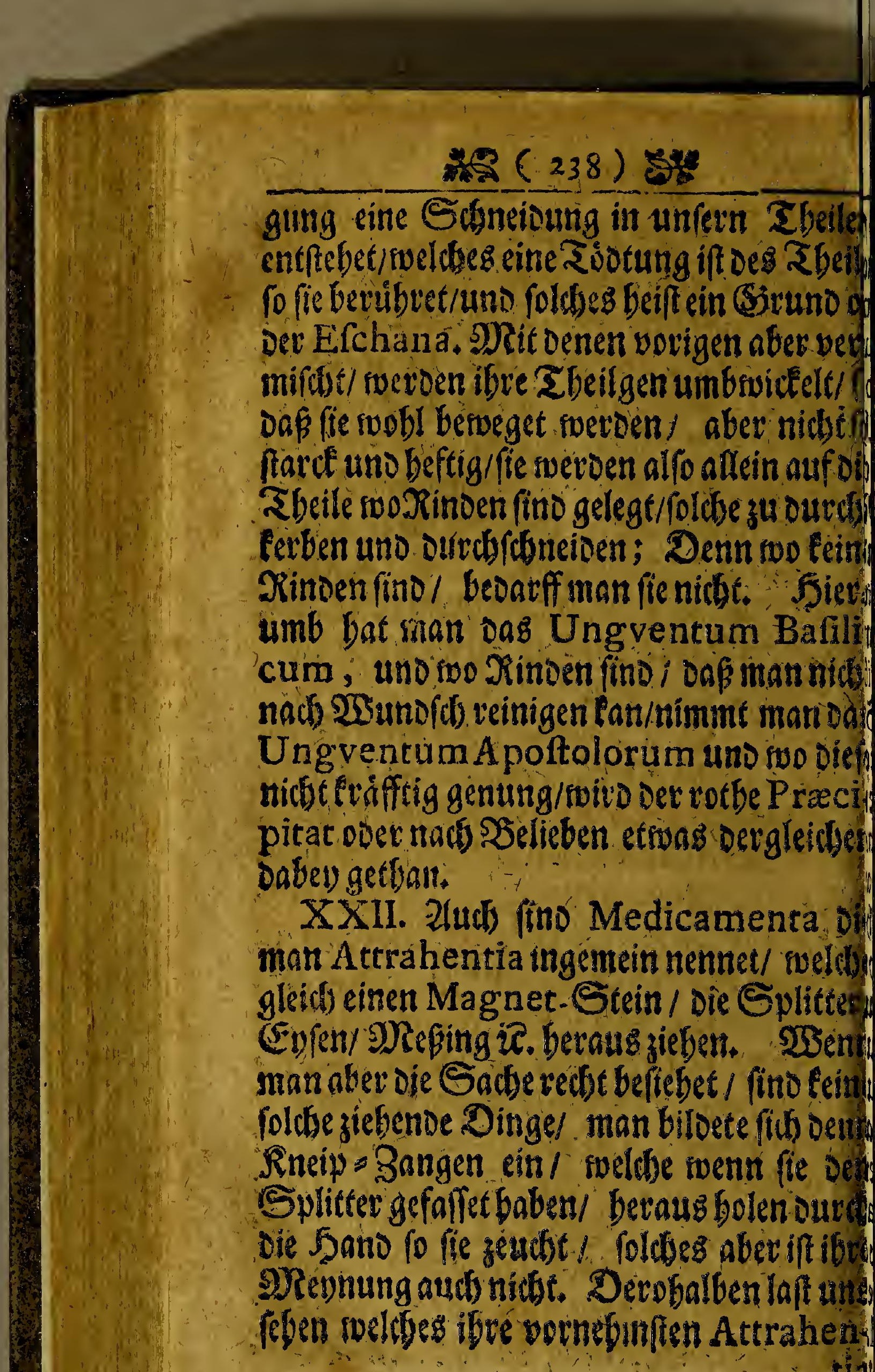




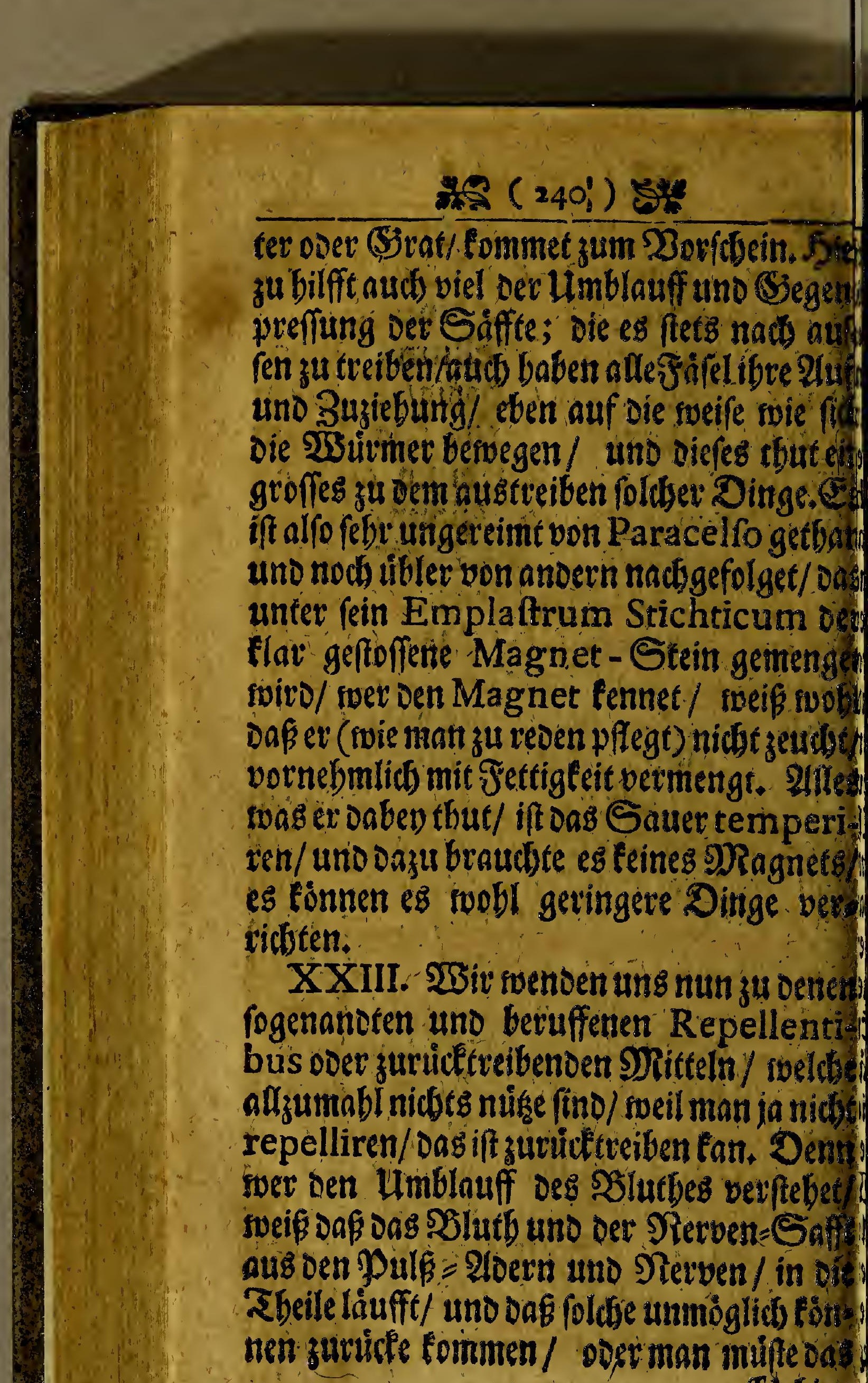

Sebjin 


\section{$x 5(242) 58$}

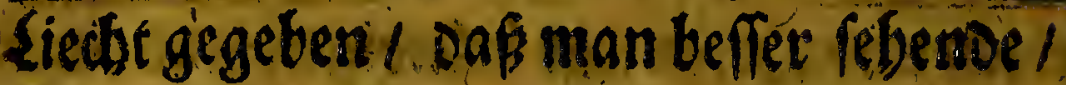
Dinge nadbliff/ mit einem 230 orte/man et nichtrepelliren. 1. Ran fan aber in foldh Bufall die Materie nobl verounmen us flifig machen $/$ es gebsoren aber viel lubtile Dinge oazu/ sueldbe Die Materie jenfchmi

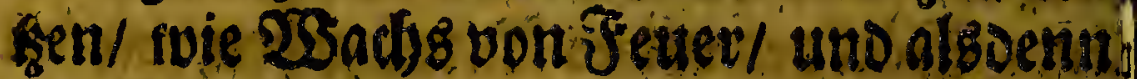
Die Derfopffiung noeg/ neldies auds exfood foiro.

XXIV. Siun solfen wir die maturat tia doer reiffende \$Nittel examiniren/ um zu fében wie fie nutircten / bietunter gebove vornebmlich folgetioe

\section{3urtiseln:}

Althex, weipe Silien/3aumuniben/Srois: beln.

\section{Althex, pappelin.}

\section{Solitet:}

Saamen:

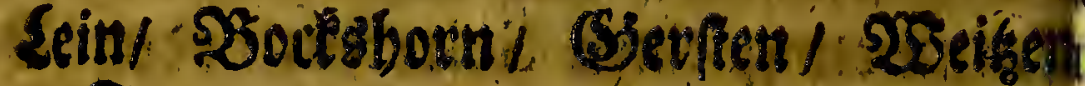
Feigen/ Gummi Ammoniacum Terbenthin, Sart/ Del i?.

Diefe fon man biel eigentlidber reiffend

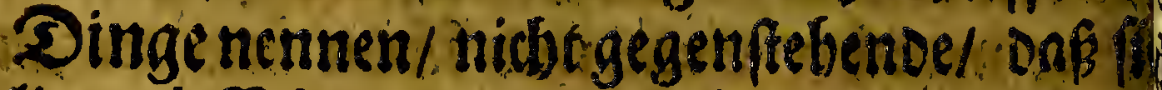
linoe diffolventia poer losinadiende Din gefino. Denn fie urachen lofs wos fie tönnen uno diefes geber mit sem Limblauff fort Daf uibrige neldbes fo weit nidye fan ge? 


\section{कर $(243)$ है}

dot forcen/ uno aufer feinen Xibrgen/ in oider ebol/ ben ntant Solet nonnef. fragt fidf/ ob oiefer (5 vter fein slutb gee fen/ ano ob oas szluth fich nidh in enfec mandelt? Su fage glatt weg nein. Dem

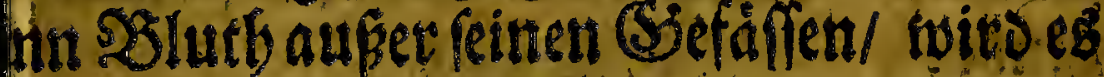

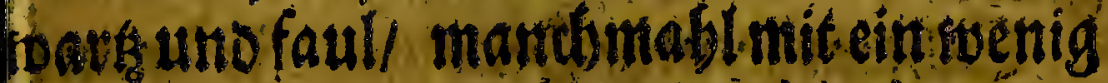
bet vermifot/ Das Solutb felbft abectwit feimen Stev/ soic in betten Contufioni-

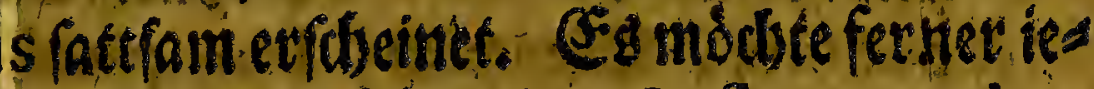
Ino fragen/ ob bev eitter Inflammation or Entzunoung fein SJlutb auset feinen efaffen / uns ob saffelfe nidst gu (s) tet hoe? Td fage nein/ wie foldacs borlángf B unfern Fundamentis enfoienen/ fons. It ezift cin Chy], cin STaburitgge Saffu geronnen unofifle febet/ soldier at e.

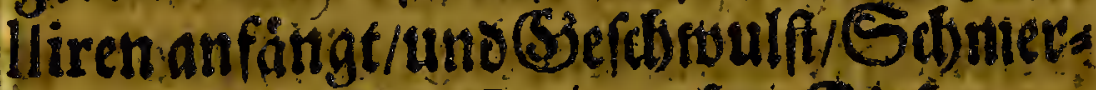

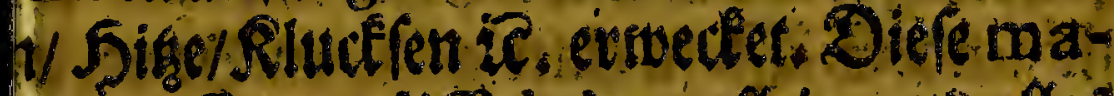
irende Dinge diffol viret alleiniuto alles s fie nidh diffolvirentlof anto flúbigma nfönten/ Sab bleibetfigen/ uno weil es bereit aug finen Stefaffen gelauffen und Gt nieber bitteit fan/ vetoechet es unbliet Saut uno umbliegenten Sheile / bipes

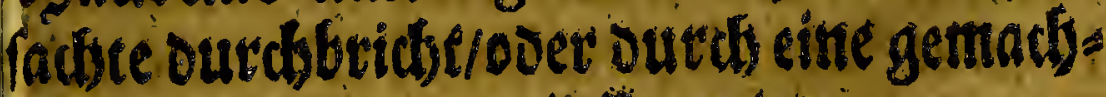
Deffnung betaus gelafiet wirt.

XXV. STun folgen die Sarcotica doet

Q2 Steiflo




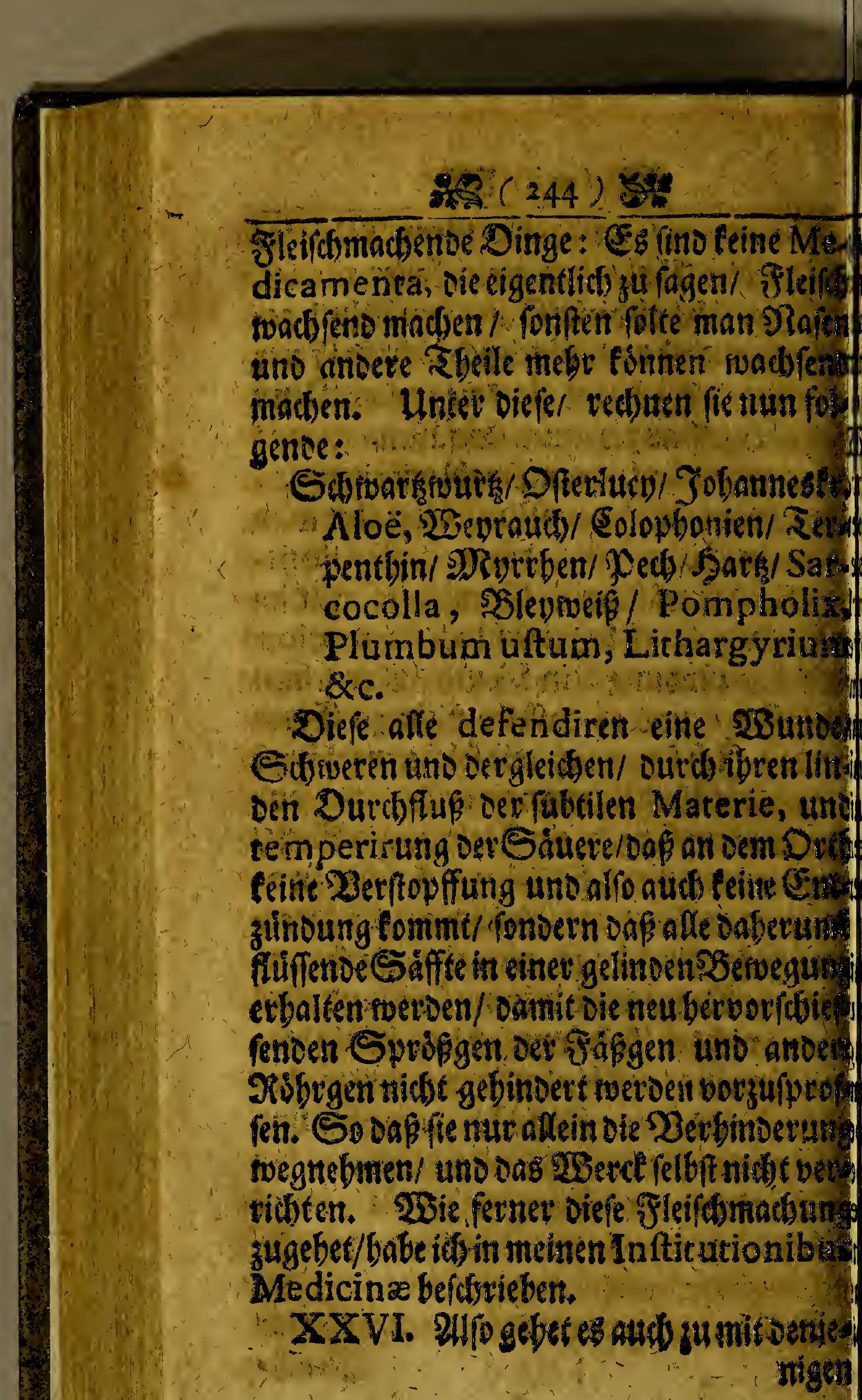




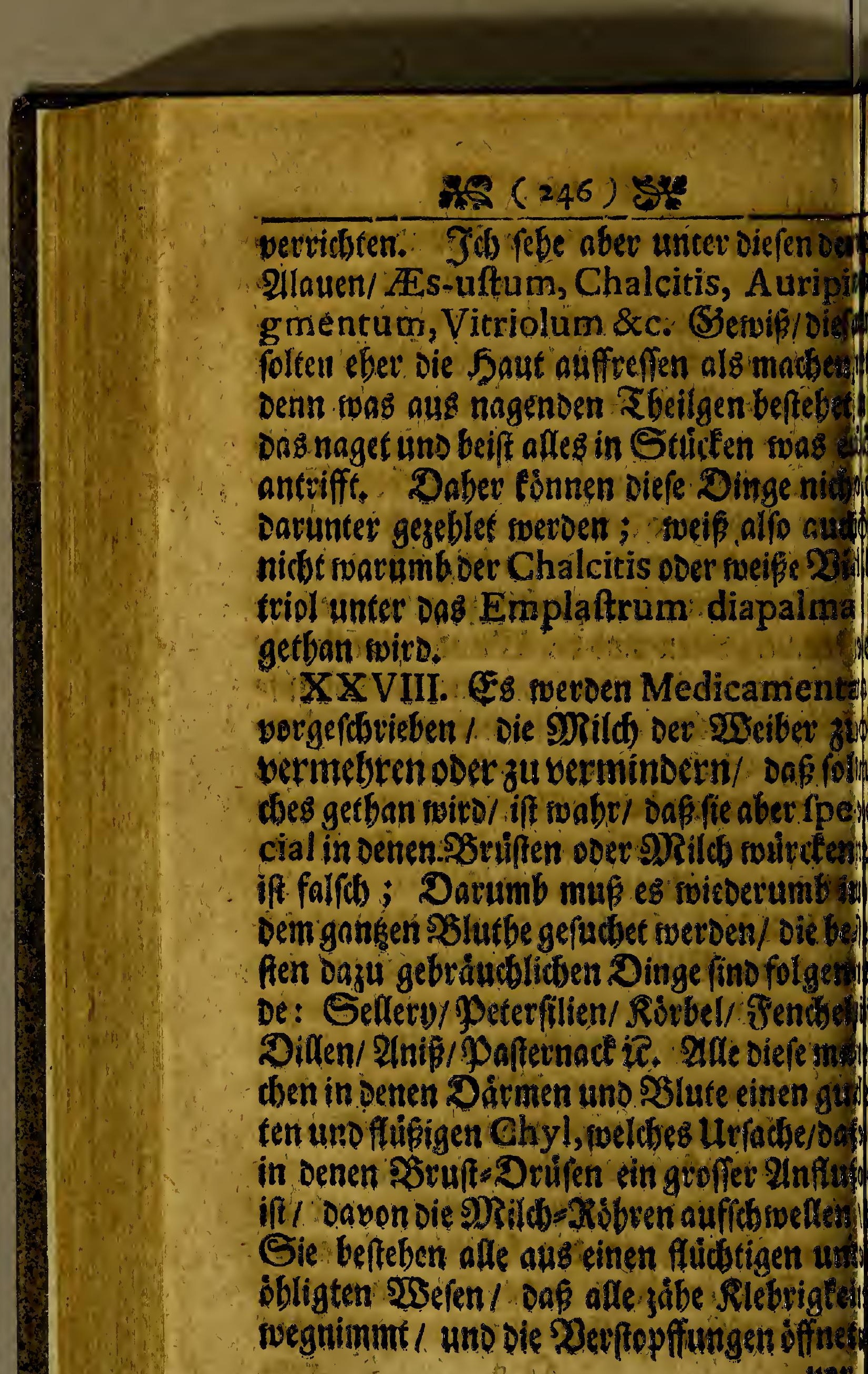




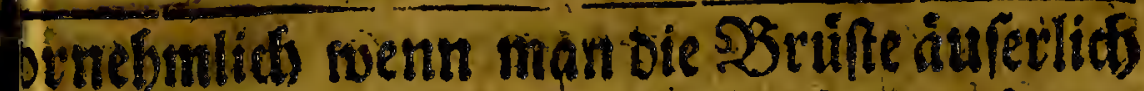
mit bebet/ fo fdineflest bie Drufen auf/ oas whoen Szlutbe uno Drild mebre (Sjeles. Shbeit binein zu fluffen gegeten noire; Sierd Diettet alle nabtbaffe Speifen uno (s): dincle) als: afles ibas man pon allerkanio

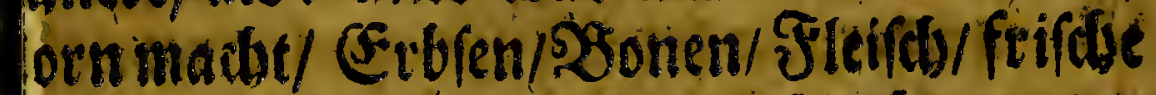
birnen/,2)Rilds/Cver Zd. diefe geben guten byl unio gute SDRildo.

XXIX. Soll die s) Rild verminoert

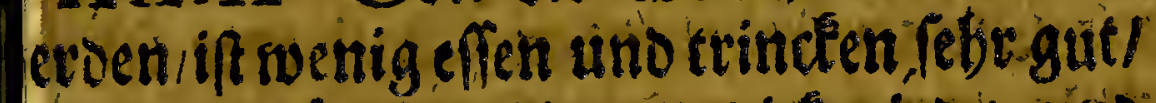
won ber Chyl wenig uno oide twird / uns pas vielweniger giebteg / neent es Speifen io sartitten swenig Pabturitg ift/. Dergleio en oie meiffen Sildse fins/ oenn fie fine balo rzebret und geben loenig Pabrung. Wiel

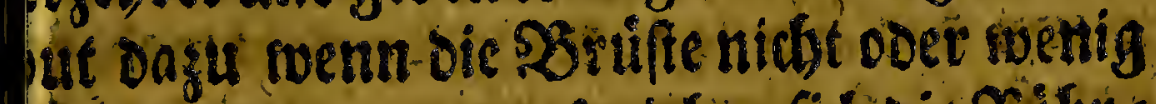

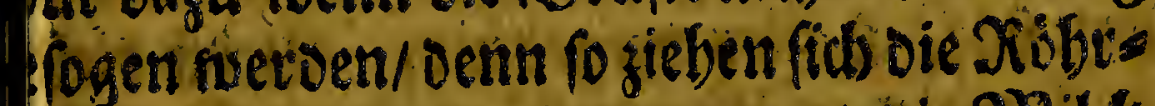

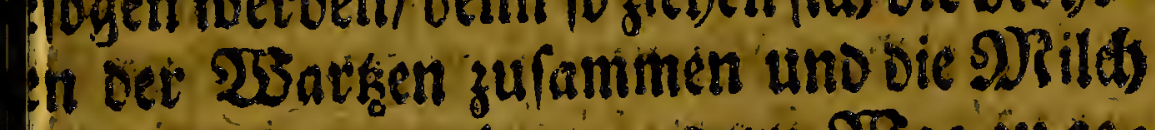

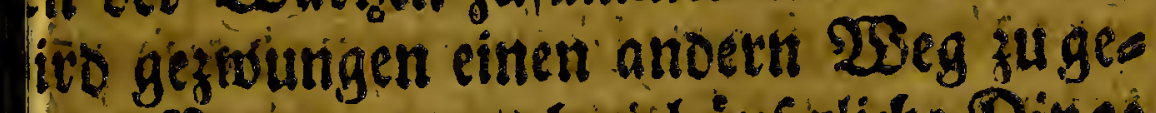
11. Es iveroen atids vill duferlidbe Dinge braudity oarunter icb oen Esgig antreffel eldien fie auf bie SStuffe legen / bas gleid)= obl ben biefert D)Rilds = Flarchen gefübstich

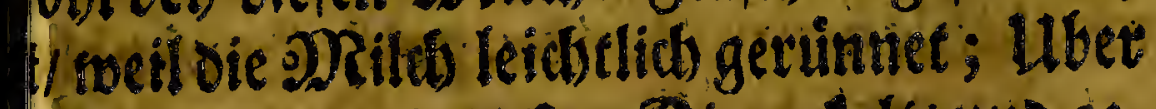
efes/ werdeen bie trapen Dinge talt/ unto ge-

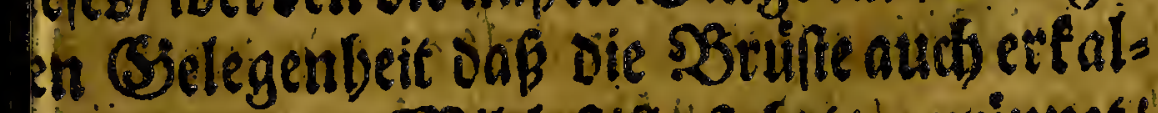
hi oavon die Drilds fitle feffet/ getrimnet/

$$
\text { D. } 4 \text { Edrues }
$$




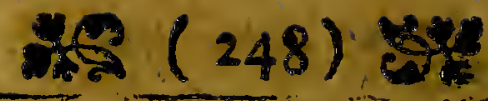

Sdisverung / Rirebs uno iergleichen entaced

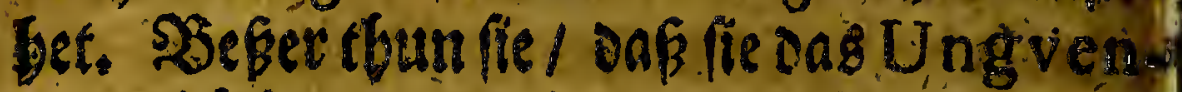
tum Altheæ̃, Agrippæ, Emplaftrunis de cumino \&c. auffegen / ondurdotoes

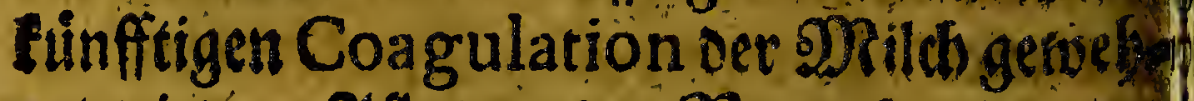

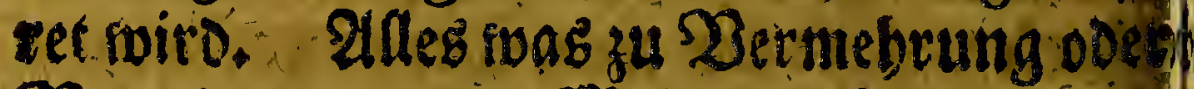

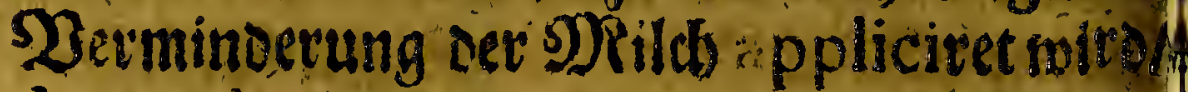
dependiret; aflein von mebretn ober wents gern Sinflúp oer fubtilen Materie, Die das SSluth nadl gerviffer Proportion difponiref / feinen inhabenden Chyl ooer MRildy mebre ooer fveniger abzufondern.

XXX. Es meroen auch zu פermet o

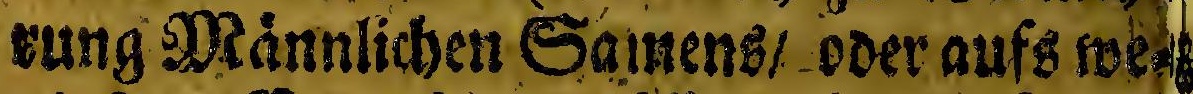
nigfe zu Errwectung gröfferer Suft: DRittel gebraucht. Siezu bilff viel cine gute Difpofition ber TGeile / mentt oie nitht nobtibed

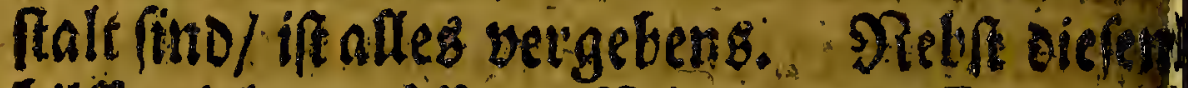
bilffi viel Daz ifftere Effen guter Speifen:

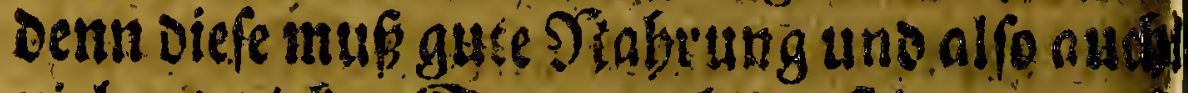
biel und outEen Samen geben. Sierunter if

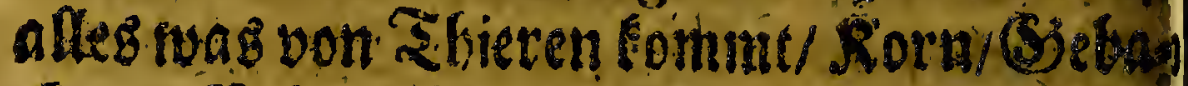

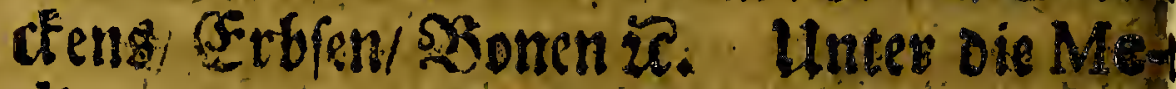
dicamenta Rentent fie Radices. Satyrio wis, Mofchusn, A mbr. geif. Saufenble. fen Examiliche Otliegen 20. Die poriante

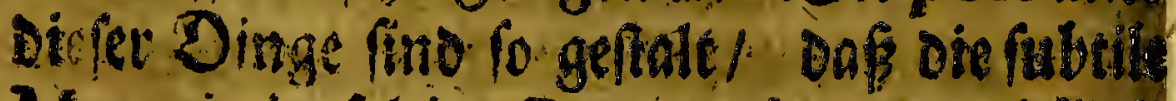
Materie in foldger Proportion curchflicere. 


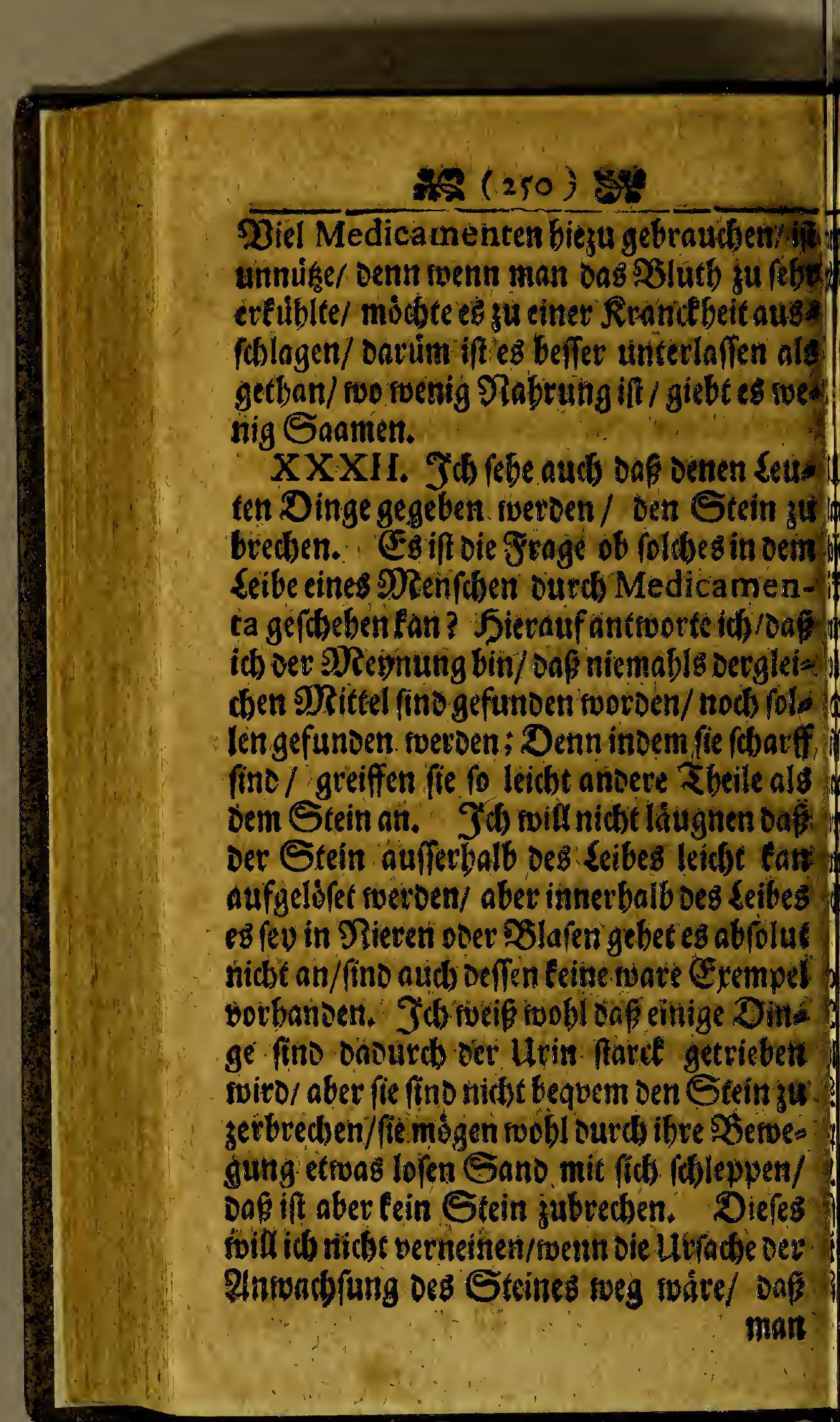


Han alsoent Qen Stein folte tonneti $a$ b. difieffer/ nads oen gemeinen Spridunort: Gutta cavat lapidem, non vi, fed fxpè cadendo

Der Xegen - Sropffen/, bobrt burd bate Rierel=Steme.

Uno oas thut fonder Spadte ber sfftere Fau

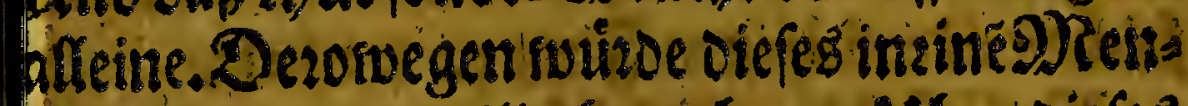
fien febr langrweilig bergeben. Uber Diefez

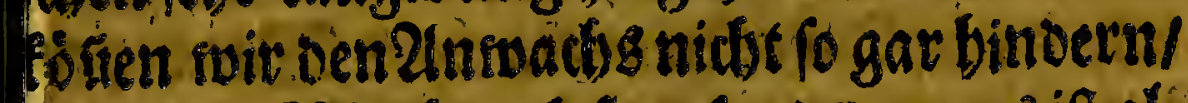
wenn oagsslutb nod fo geínd/Denn es iff alo lezeitSalsigfeit imsSlutbe/uno oic ausso'n Dige Jladie der Steineift fo geftalt / Daf oie hiffen uno falsigten S Sbile leichtlid fefte oars auf tleben bleibett. Saft Deronegen alles wergebliob nas man tbut/ Denn fo vicl mix be: wuft/fno fdiled ter Dinges feine Stcin=zerfredistos sorttel.

XXXIII. :3u denten fogenanden Specificis geboren aud oie / fo ole DR onatlidie Reinigung ersweden; untev oenent boneme lick folgende zu funden: Snoen-DSaum/

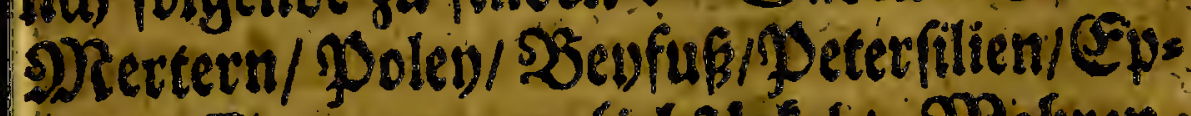
pidy Gaamen von liebfodtel. SDrobren Ruimel/23adjoloerberten/Sorbeeren/5eu. felsored/ Siebergeil/ Stabl/ Igtfeim/Det 2. Diefe alfe suricken in sem allgemeinen Solatb l uns sarumb tan thas fie auf ein s.beil

$$
\because \cdots
$$




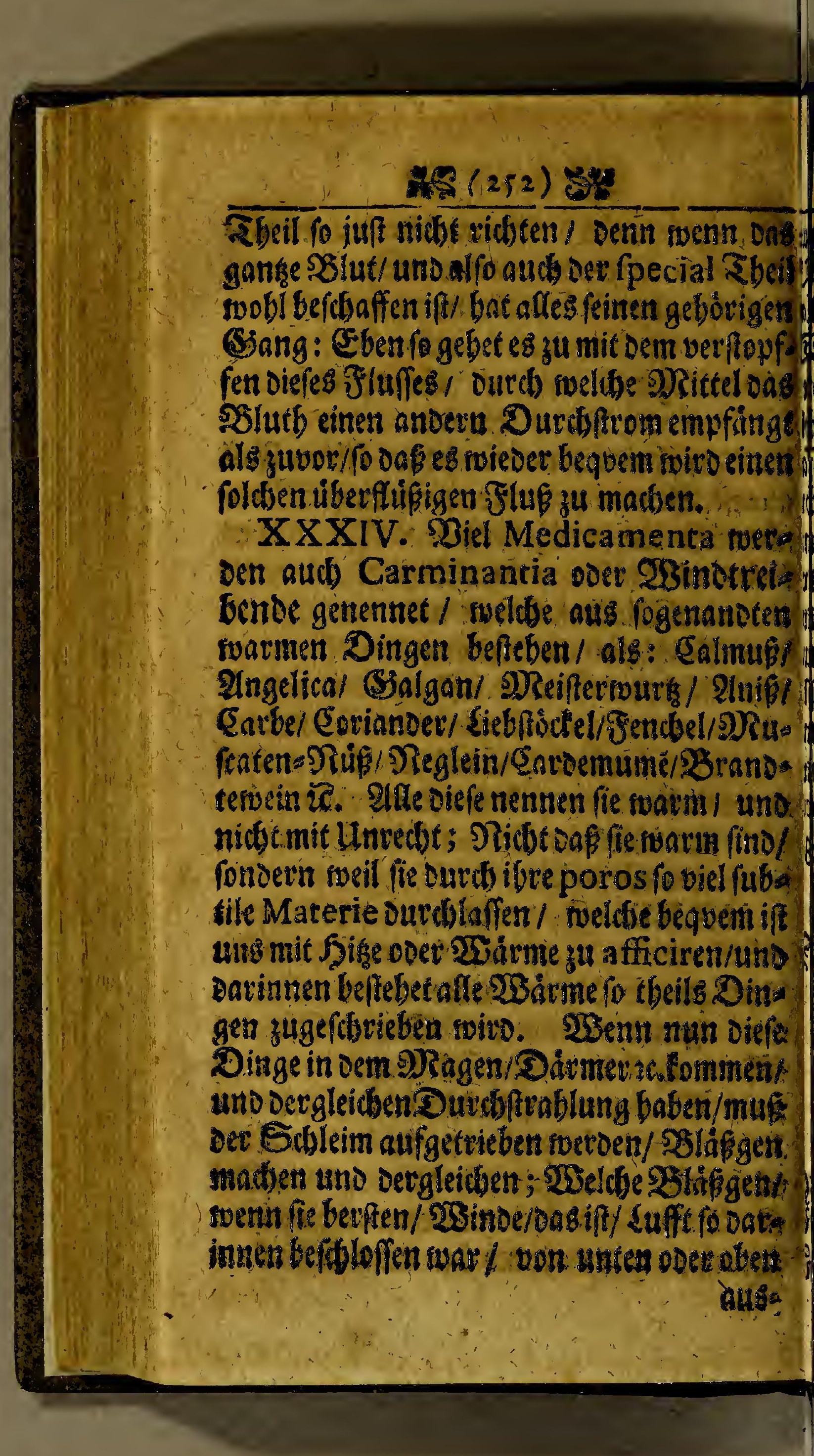




\section{5 (253) 38}

auggeben. Uber oiefes/ meil fie auzgarfen Sbeilgen Eefteben/ Dichen fie rimen beffern Cauff zu machen / oa die aufgefpantetert

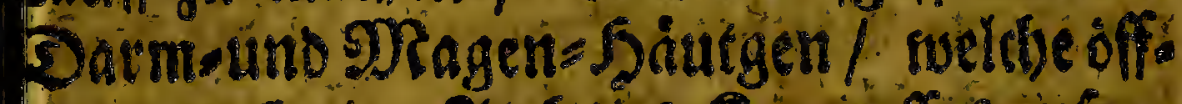
ers ourds cine - Irtb oes Rranpfes aufges blafen wetoen/ baourd fie viel Suffétifió foffen/ auggepreft werden/ uno 2 inde vor bringen, Darumb gevifter biefe Drittel. hid feben allefeit juft dien aimer art/fondeth birten felbft in oaş3lut uns Sirn. Sâfte weti oeren Saffec berbeffert fino/oec Drageit

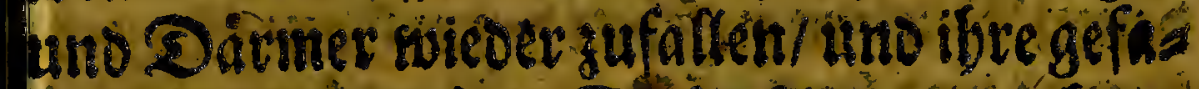

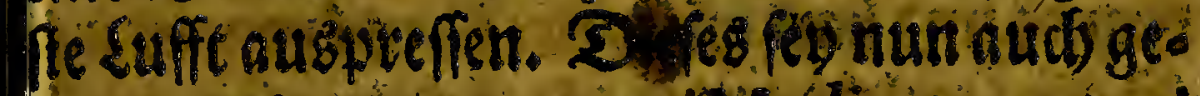
hung gefagt von oetuen Medicamenten/ welde bifberiger Jjeyntig nady/ in parti-

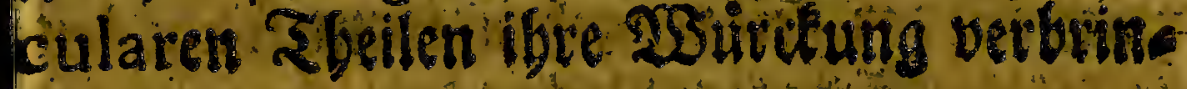
gett.

\section{Das XIV. Sapitel.}

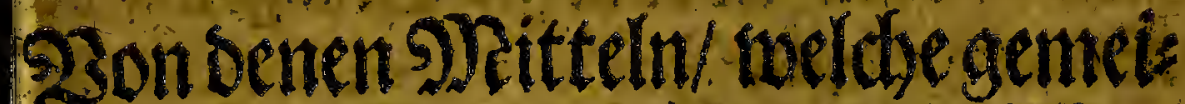

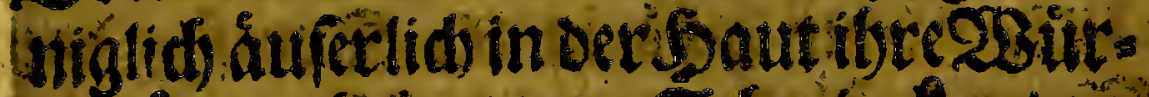
fengtfiun; von Stdmindim/

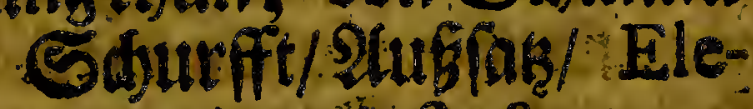
phantiafis \& $x$. I.

OกD?

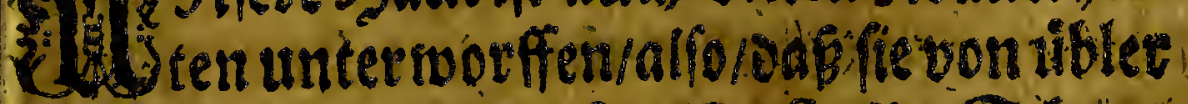

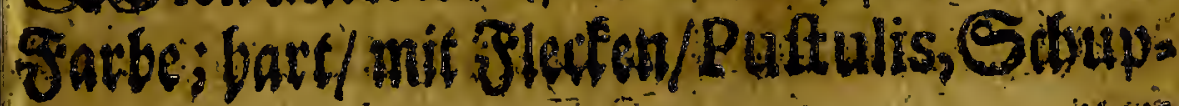




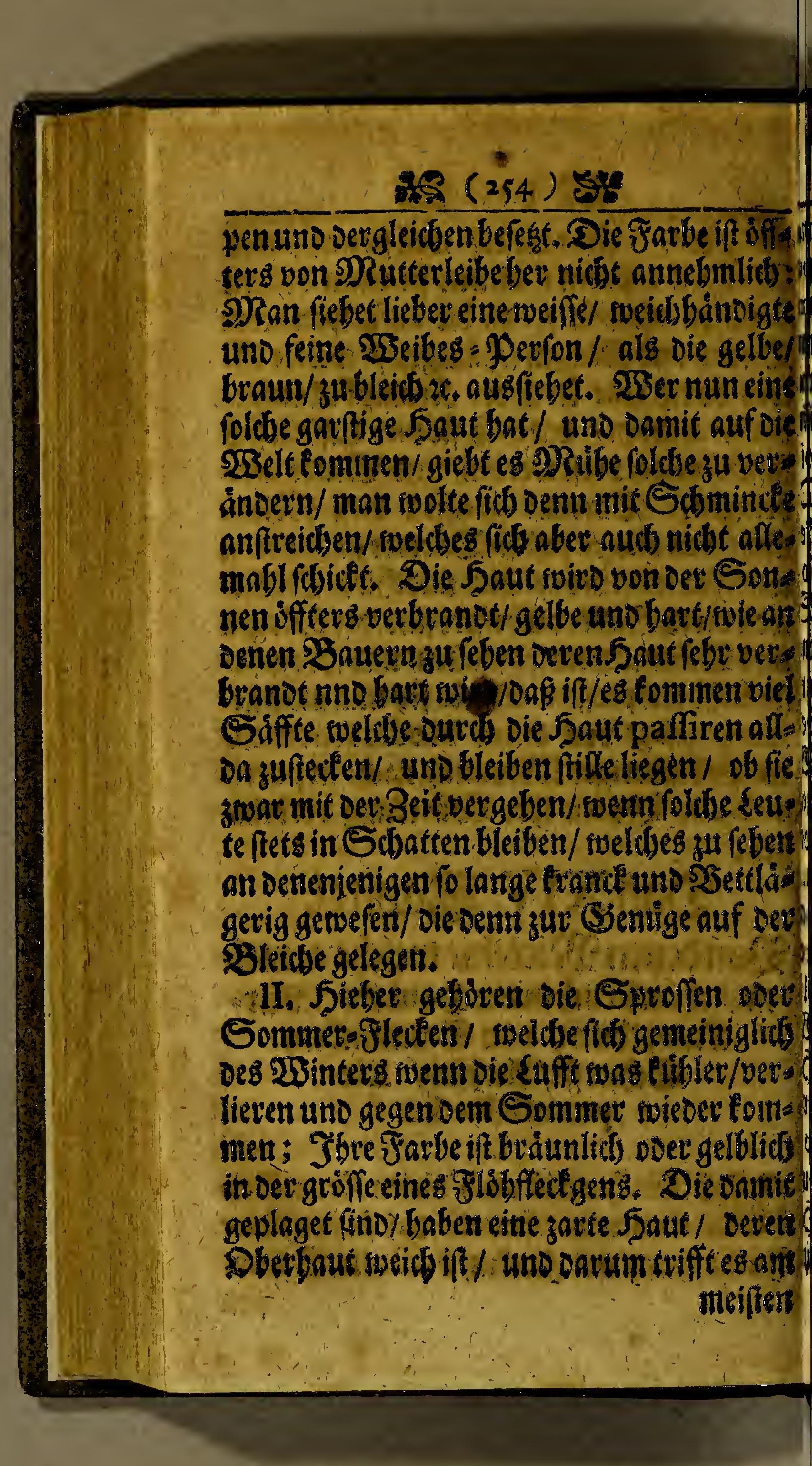




\section{the $(256)$ be}

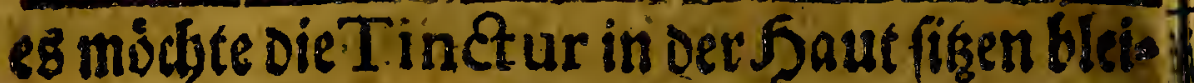
ben/unc die \{eute an fatt fobojn/gelbe foezoenly 25 enn aber oeclutin fo flat wäre niexiegent $25 n f f e /$ molte id eg-woblgefdeben laffen. (5ifino nod) anvere Singe' als 3 ald = Dell Liqvor Tartari, Seiffen/ in Scheined 20affer refolvirte Marcafita, Lac Virginis \&c. Wrelche alle foldbe sbeilgen baben/ saf fie die Sberbaut ounne madjen uno mic Der Zeit abfoleiffen/oieftets foieder anroádje uno ieu wirs; Unterweilen werden die fills liegenien geronnenen 3 roit gen weggenoms men/ uns alsbenn befommt inan eine glatte. ano unbeltecte 5 aut.

11. (Eg fino now allerband Flecken in Det 5 aut/ meldbe commen unt roieder verge ben/aufoie SBruft/,21me/ Beine us. ftch fes. senve I fie baben offers die (Srroffe eine Jano- Seller: I Eleiner uno groffer I-gelbe) rotb/ Slenfarben uno oergletchen; SDan fies. Gerfieviel in Sobarboctigten Seuten/ uno. wêlobe zarte Sautbabet. Sie differiren. mebr doer weniger boneinander I mofino. nichts anders als aus senen SSlutb. (Siefifo) Fen gefturşte Jeudtigfeiten $/$ eben ouf Die 2Beife als ment fid einet geftoffen und on

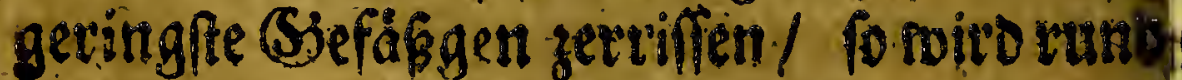
unbber ein Sleck / roldoen fo lange Dauent. 
SO bie vorber lauffende Feuditigleiten de iffebenden S beilgen mit binneg forlewpen/ Ho alBaenn verfdominoet der gange Fleut. Fe swevoen felten siel Mpittel Darrwieder ges roudit t w weil fie ruenig ins (S) fichte formmen. à man aber ja was ibun suift miffen ce lola S Dinge feun/weldbe die getunnene Jeudb-

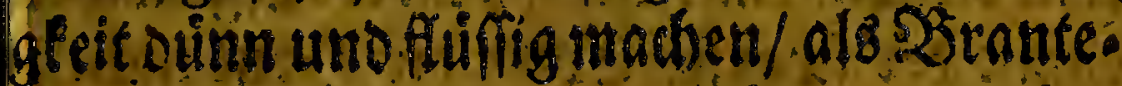
ein/Campler/Spiritus Salis armonia- soomit de Saut euferlich genafosen und erieben miro/ Damit geben oie Sbeilgen IoB/ no werden bef fo eber weggefubret. 2 Beil as - teine (Siefabr daben / wiro telten Path in gepflogen/ uno fan man es nut bon fich lof laffen liberbin getben.

IV. 2luf oergleidsen neife twetben auds leden in Deftilenkialifden Fiebern I. DRa ni Poden id. nebmlich von einer coagurten Feudhtigfeit/weldbe ourdh einig (Sifft/ bus der suffer unfern seib fomsnen/uno (b) ausoámpffen fonnen/in ber Saut bleis infiffefteben / Dafuorallen in oenen Do or en zu feben/ oa die gange Sout wie eine inoe nivo/ Sals/ Scllund/ MRagen uno Dirmer befekget. In diefon Suftande fuerdent - Patienten ruarm gebalten/samitviel von im (siffte augramplffe unt nug bem leibe smme / Darumb giebt man aud biel

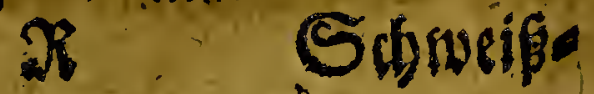




\section{2xt? $(26 I)$ bु:}

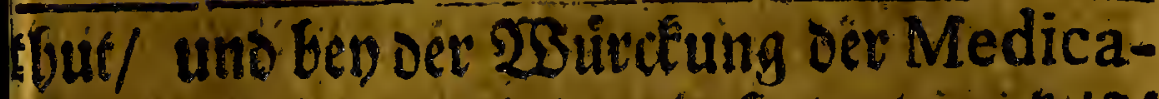
menten gemeiniglids sas ' Sauptwerce ift bbne roeldie man ben effeet nidht fpublen butcel oenn jeoes Médicament bat feirte befonderne Sorte bon Sbeilen/Die in Figur

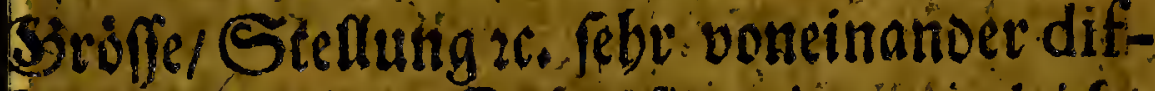
Ferirentonnen; Daber fino oie pori gleidf) dals alle unterfateden/ fo onfo oie fubtile Maerie whituf nadbom fie diefe ooer lene poos oer Sbeile antriff.

\section{Dả XV. Sapitel.}

Bon Demen Spanifan Stiegen supplantalibus, tuns affen 8 lafert mate. dhenten Dingen ivie autd von Denen Caufticis,Septicis, Ercharoticis, Moxa \&ic.

\section{I.}

13 S phegen bie seute in bielen firatid - GG beiten/ Spanifbe Jlieger ober antere Blafen ziebenoe s) Rittel / aut rolebe Dertévi veldbe fe oazu bequem adjen/zulegen. Sie ienen am meiften an foldien Detrect to Schimertsen ift/ Die poros uno Jerftopflute enzuetoffiten/ uno enen Durdgang vor mpere Jeudytigfeiten fubereiten Die Spa $x_{3}$ nifden 


\section{$-4(262)$ S*}

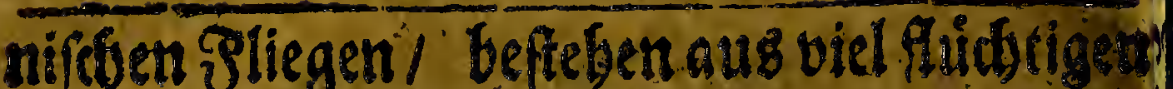

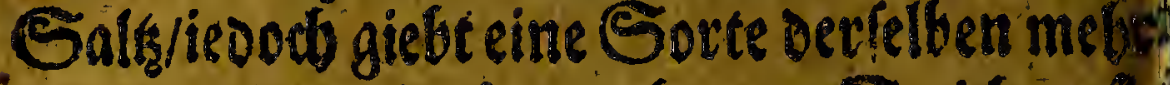
oder reniger/ nadis oem Sandes Strids oa fis bet Bommen/ die 3eit oarin fie gefargem uno nadoem fie lang oder furs fino getibsecest gensefen; Dured ibr fluidstig Sals muffen?

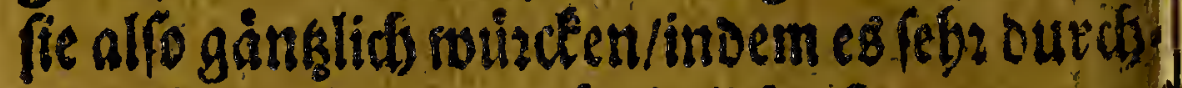

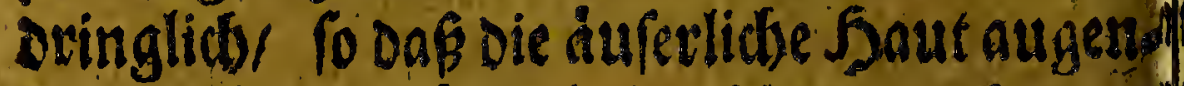

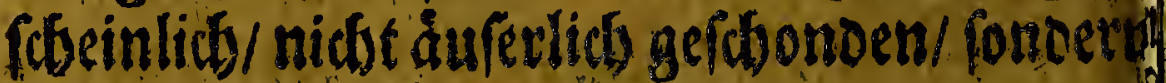
affein von der Sout yermittel/f eines autge: freetenen $2 B$ affers gefdhieden wird. Dief Sliegen ater iniffen exfi geffoffen uno mit ete mas/als Sauterceig/aiben id, rueich gemad.

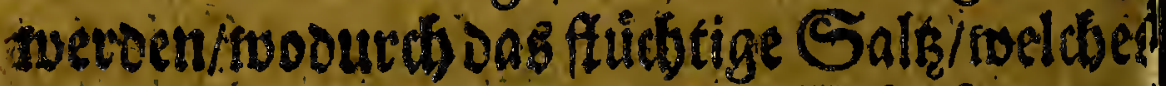

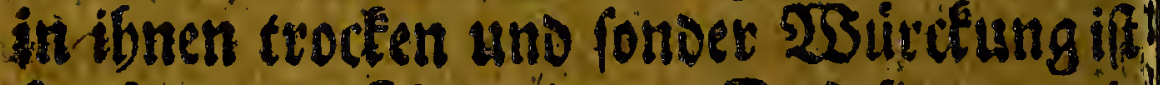
feudst uno activ swiro. Doṕf fie aus vie,

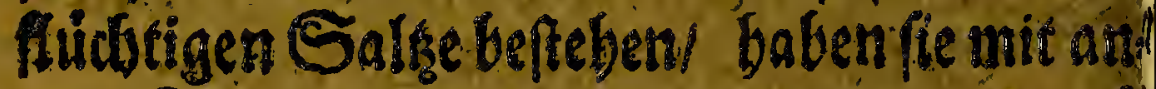
Dern Fbieren gemein/ aber bes einen if nich fo Durdboringlico als des andern 7 Denn 8 B

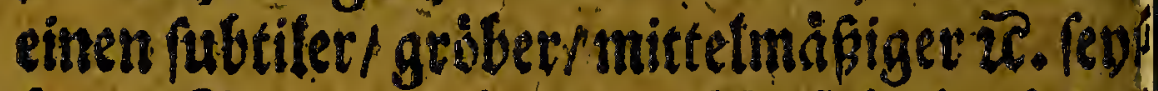

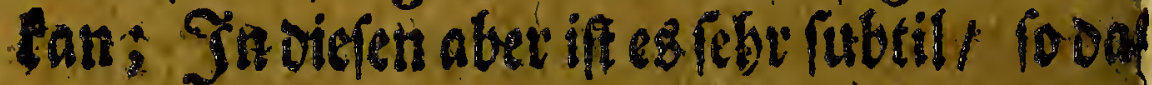

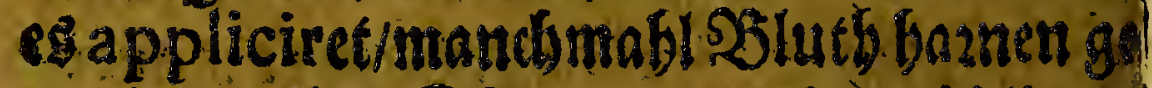

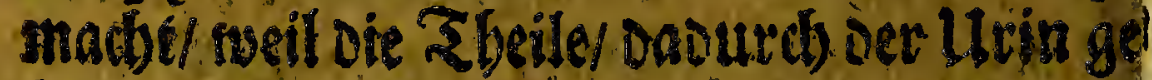
beth fellef mit oslafert angefifflet woroen/ on

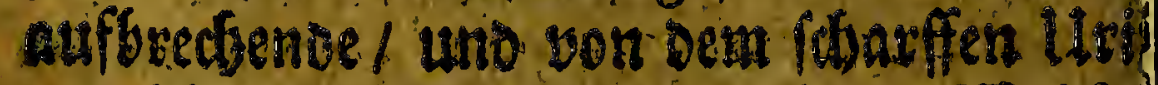

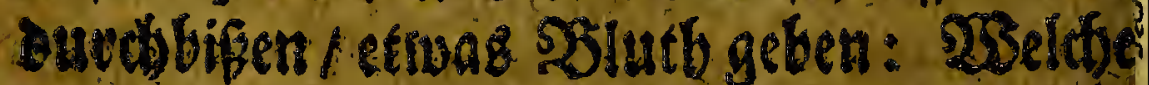
ein zectfen / oa

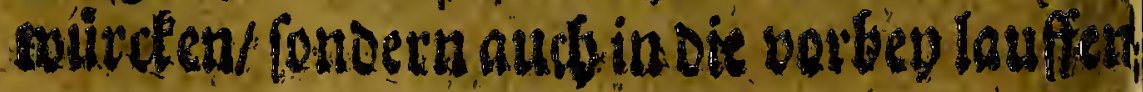


De Saffec oringen/ Danon oie Ieudhtigleit phiarf foted/ und zu denen STieven uno Sllas fen fommend/ diefelbe SBewegung bat die die Spanifden glliegen laben; Das ifl $/$ on

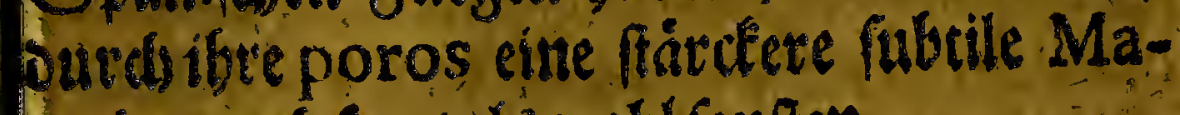
kerie ourds fan/ als wobbl fonftent.

11. Die 23eife/ mie Diefe Sflafen gemadtot werven/ mus diefe fenn: Sie swerben ges madyt/Joch viel lang gamet/gleidjwic oie von

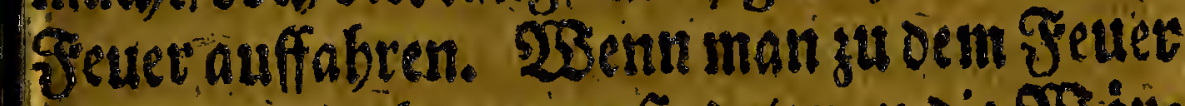

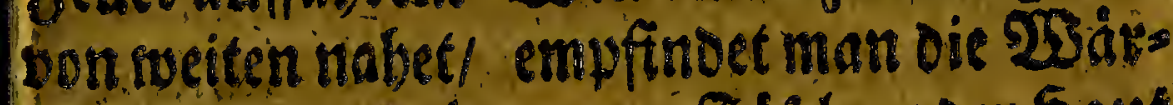

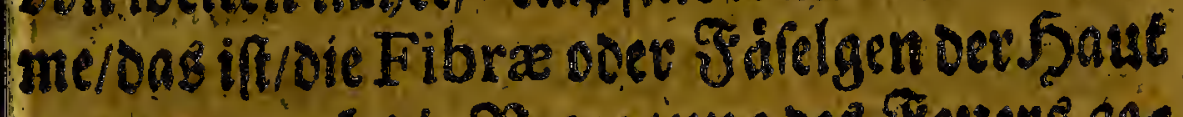

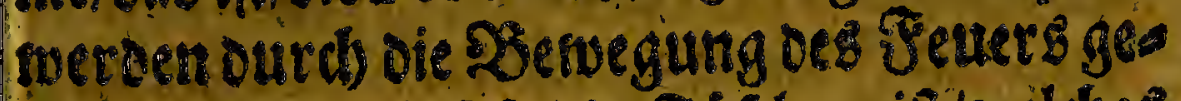

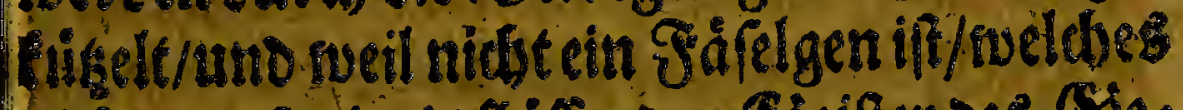

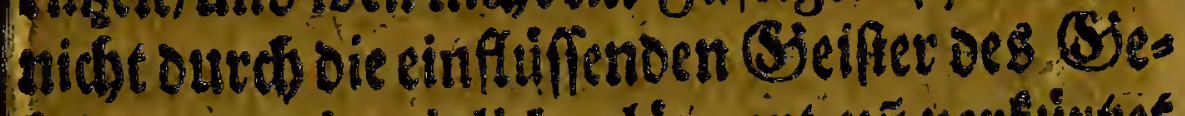

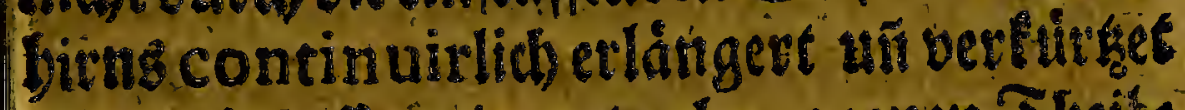
niviv/ fo fofferen Die zat ten bervogenen ?heils

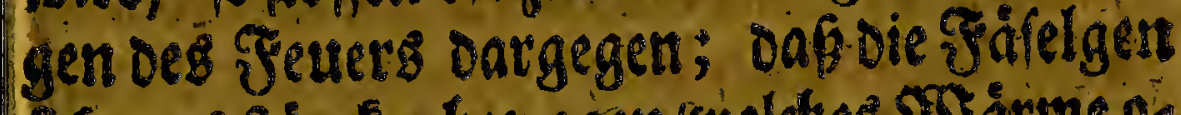

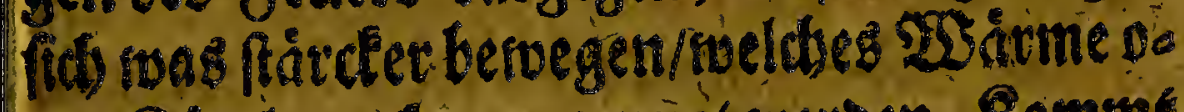
Der Ruigelung fan genennet twerden. Rommat man aber sem Jeuer etwas náber/ swiso bies

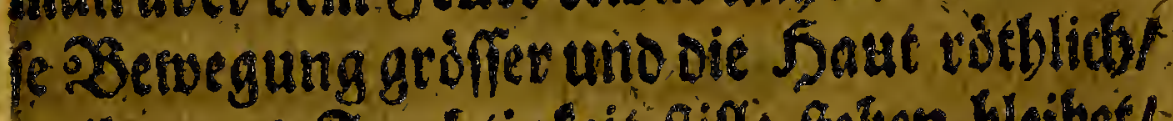

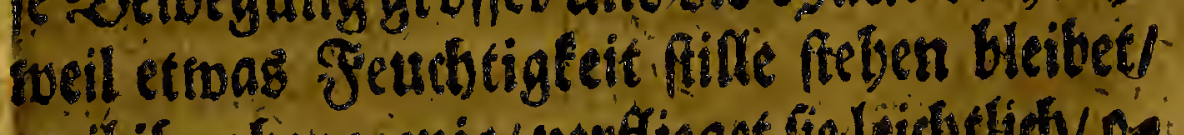

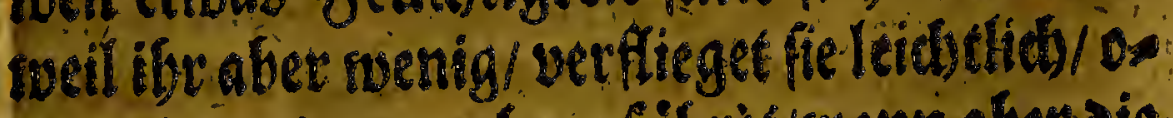

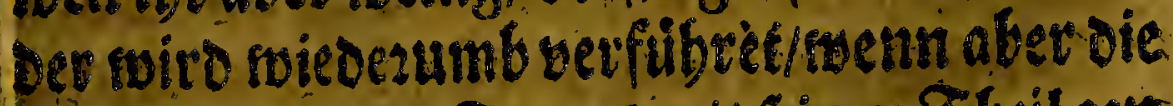

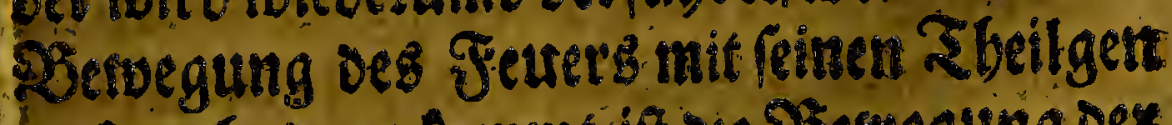

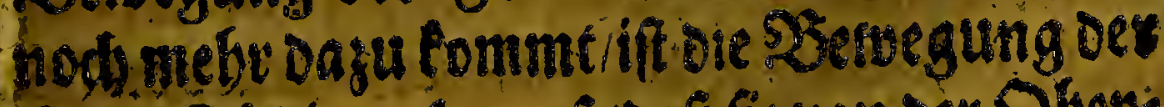

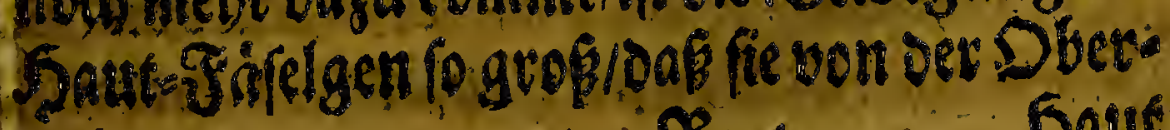
$\$ 4$ Jaut 


\section{5 (264) se}

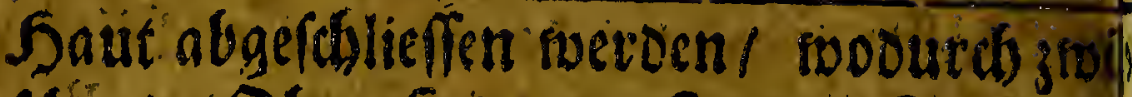

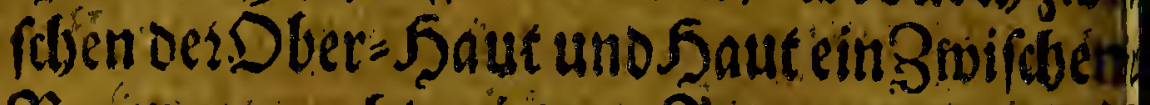
Raum gemadjt witn. Groem nun êlid

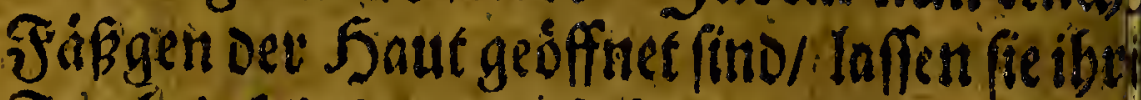

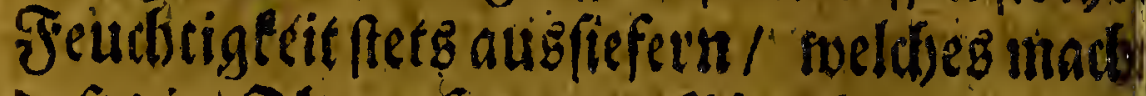

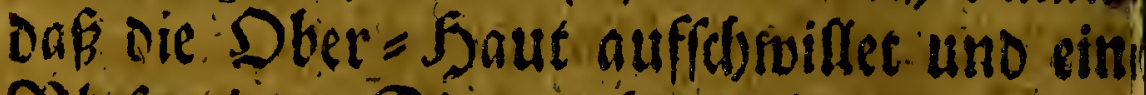
Slafe rwivo Die man bernach ourdsidgneis Def und oas 25affer becaus läft. Ceten au? foldie WBefle gebet es mit bem Spanichert

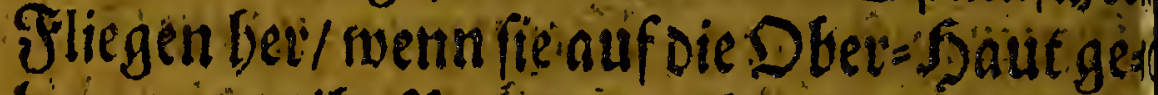

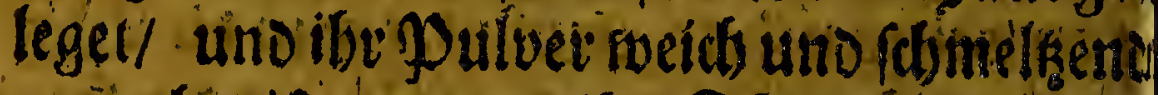

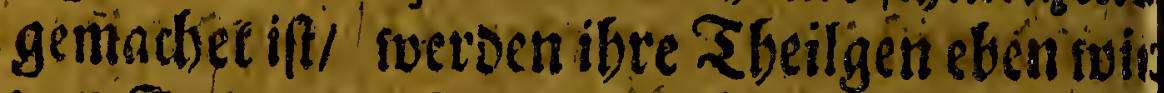

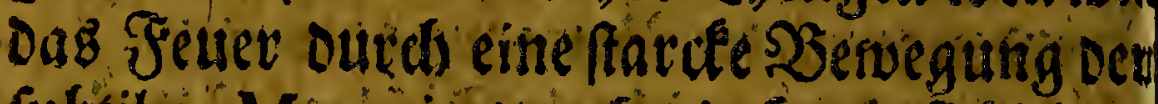

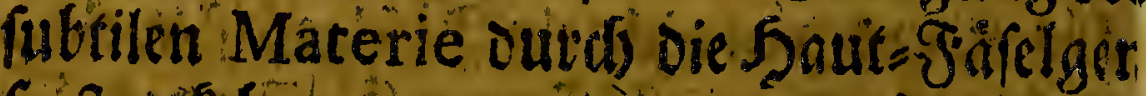

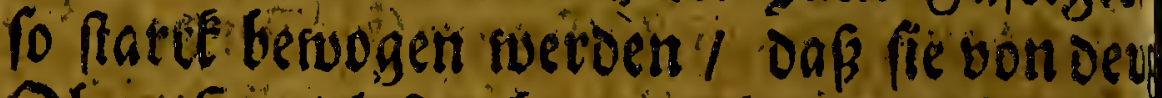

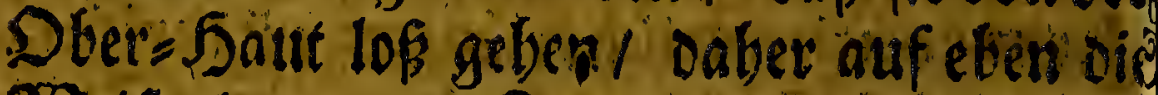

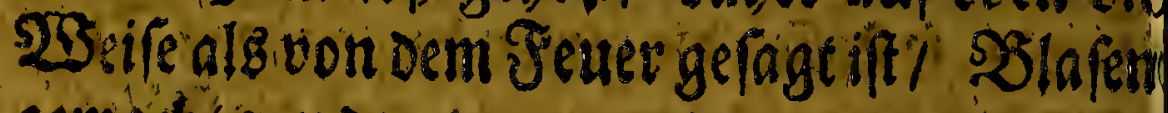
gemadbe nuerden.

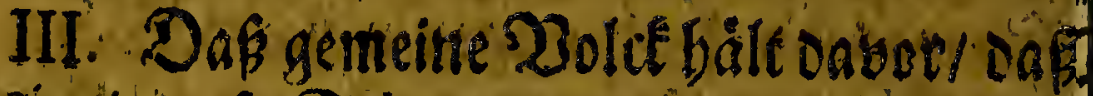

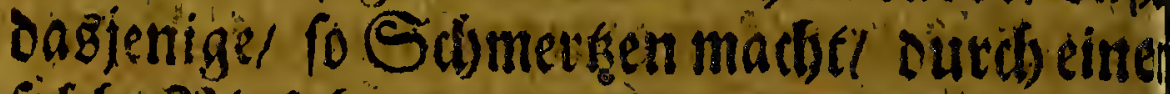

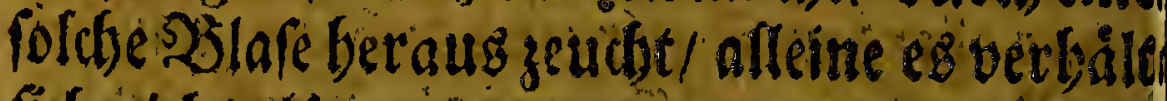

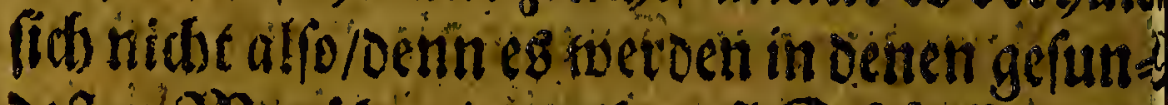
oeffen's Renidben/ der fo voll Sdjarffe nidbt

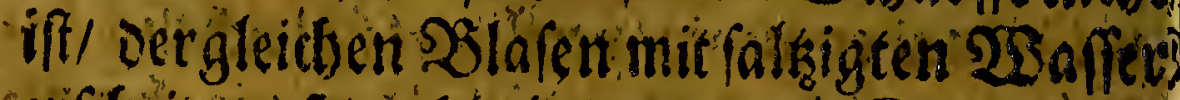

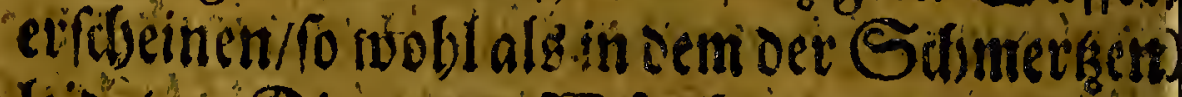

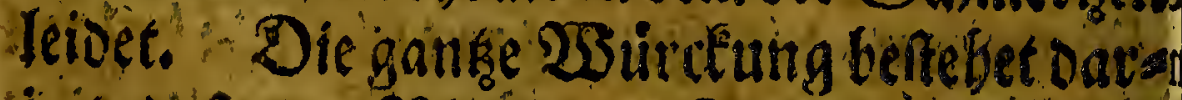

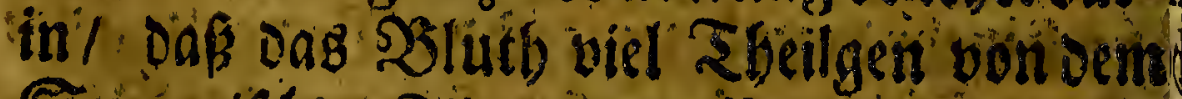
Epannifhen Fliegen empfähget/ woourdo? Daffels. 


\section{$20(266)$}

machte Deffmungen/Die nach ibrer Deffnung nichtz anoers et fooern als die Seylung/ weit fie ibre Rudifte uno Sugenden alle in Das. S3luth abgeleget baben. Diefes fanid) nich verivefren / dafs man oas Veficatorium, suenn fofon bie SBlafe gezogen/ nocherlith

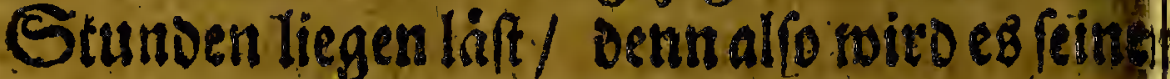
operation nodblänger bertidben/ bon des t gleichen Wuirditung fino nods etlidje (SSe of waddfe. Radices ranunculi; Flammu la, Succus albus Solfequiæ \&c. Tha thymalli, Cortex juglandis \&cc. Diefe macben auds. Bla fen rwie die Cạntharides,

V. QRanche geben die Spanifden Flie? gen ein/Den Utin uno faulen Saamen sbzuts. treiben/item Venus-Suft zuersuecken ü.215. Jes was bieraus getichtec swizo/ift bem flichtion gen uno fagrffen Salse zu zufbreiben/ nelo

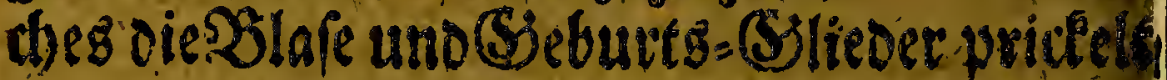
uno ganghar madjet/Derin die Materie oarin diefes fubtile Sals befolofen/uns diefellos Befwegung vermittelf Dec ez ourd oiesslat uno (Sieburto (Blieder pagiret uno foldo pritfelt / incliniren niederimb jur 2(1) fung/ worauff eine ganke Difpofition os Seites erfolger.

VI. Ez fino nod afletgand Dinge sety

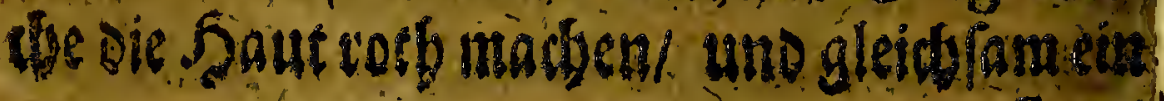




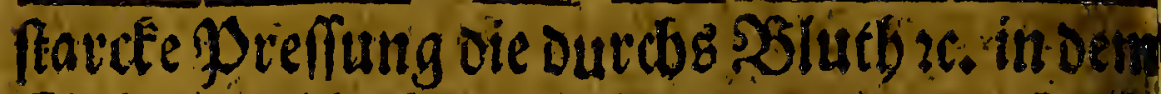

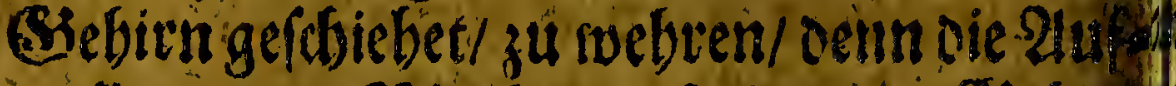
inaflung Des S3lutbes 1. To in Dem Sebirs langiam fortgieng madite die Sirse un Sibmerksen. Die Jrage ift dérorvegen/ nole

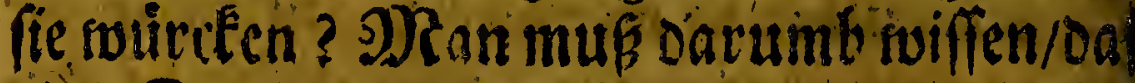
Diefe Dinge aus fdactfen uno theil's aus ob ligten uno flichtigen Tbeilgen befteferm/ uno Das diefe leichtlich ourds oie fubtile Materi gegen oie Saur angefrieben toersen /, Davoh Diefe beleidiget neerden/ swober an dem Drffe eine Jiotbe entfebet/ Deronsegen mus das Diuth bier eine groffe? Derinoerung friegen uno eine andere SEervegungl als mebr mablen gevadbt: Darneten muffert oie Servent membranen \&c. Die in bie Sbeile laif

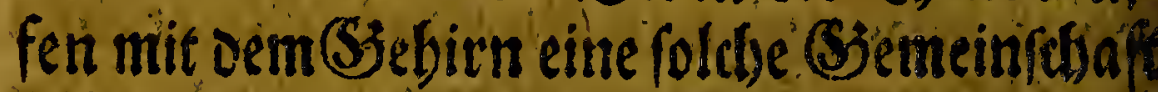
baben nole eine gefparnete Säiten/öent po

$$
\text { A }-\mathrm{C}-\mathrm{B} \text {. }
$$

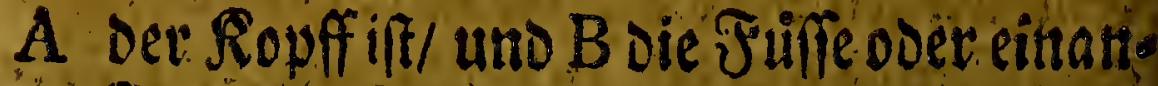

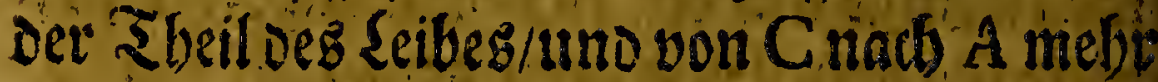
gevereft wird als ourd oie 2loern nadi C. zu fan fommen/muiffen oie membranen des

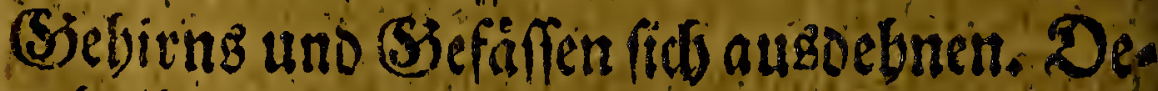
robalben madbe nadb $B$ zu eine diverfion, Damic von $C$ nad) $B$ jum wenigften fo viel ges pieft twerde als pon $C$ nach $A$ ivenn num ati $B$

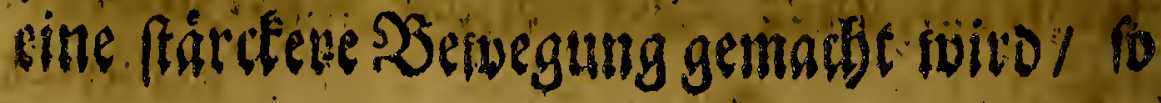




\section{की $(270)$ है}

wenn die beiffen cauftica darauf gefest bet Den/ alszum (surmpel/andie Sdiláfe oet

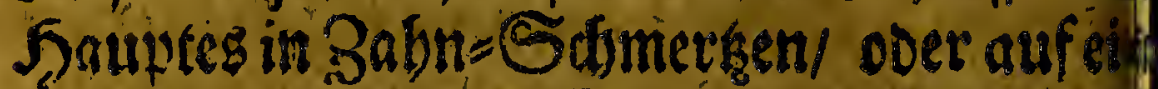
nig (Slieo gegen die (Sidft/ fogerdoiebet es

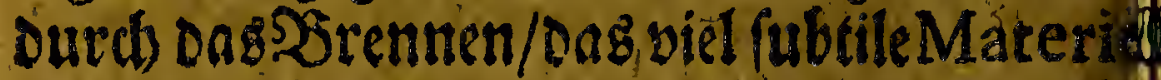

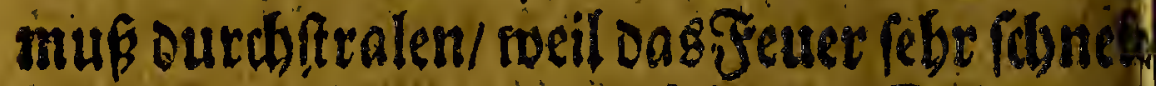
befwogen nird/Davon muifen die Sdomet. mactienten Saffe eine groffe Jొlufigfeit bed commen/onourch fie loggeben/und ous ' S bein berlaffen/alfo wurden audj Die obengenelo. ten Veficatoria. Gn Egypten forethe Prosper Alpinius, yflegen fie mits Saum 250 de oder seintwano in febr bielen frand Geiten zu brennen/gleidbroie vor allects zu ben nen Beiten Hippocratis mit fungis ooen

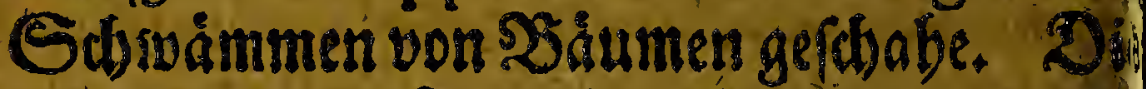
Chinefen uno Japanier aber vertidsten mit oer 250 lle von einen gerwiffen Rrout der Drtben wa achif / Dabon fe vie 250 olle mi DReffert abfdjaben uno alfo fammeln. In bier fonte man oergleidjen yon set unferfte

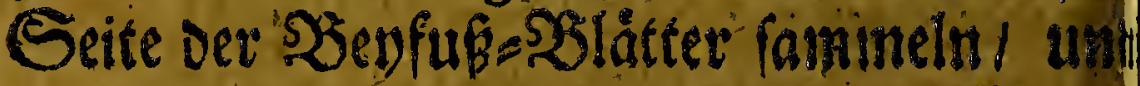
folte ids faft glnubirt oapes yon einen Rraut gemadse nitc / Dergleiden babe id aud get febett oie aub 2 Seff Stroien gebracht war/ in ovd roas brauner won Fatbe; uno iff fonsen zrueiffel defes bremnen an mebr Derfen oe

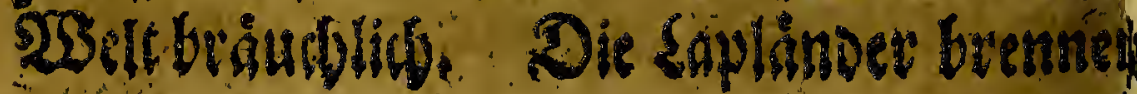




\section{He $(271)$}

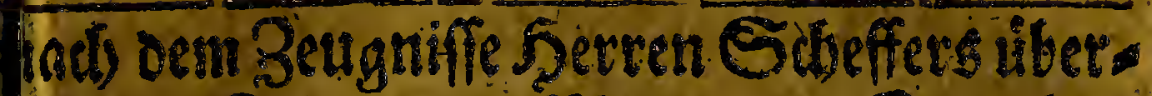
loafie Sdbmetrenfüblen / mit Sd) wims de oie an benenSircten in Sorm Der Rudien bahfen / iweldie fie an Seuer anbrenten. JEeil denn nun fo biel Nationes feon die of Srenten in braud baben / muffen fie

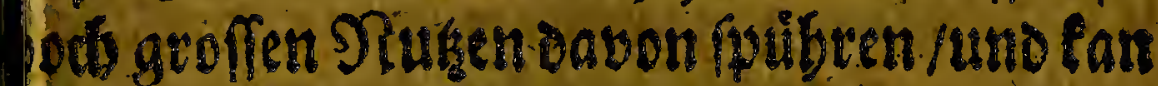

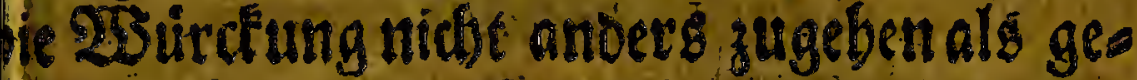
aest ift $\mathrm{cs}$ gilt aud gleids viel mit soas bot Materiefoldes gefdiebet / Denn ong Seuer durctet foldbes aflein dino nidic bie Materie amit es getbait toito / wir geber bier in etlis den (Sielegetibeiren was fadter umb/ Den

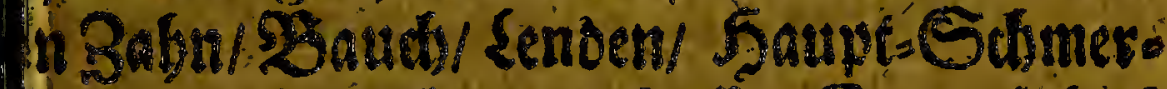
tenz. gebrauden wir beiffen Sand/fo beif - B Der spatiente bertiagen fan I item betfe uro ourdoringliche. Olea. Das 23 rennen mitCauteris over@yfen/pflegt beg. 2lbneb=

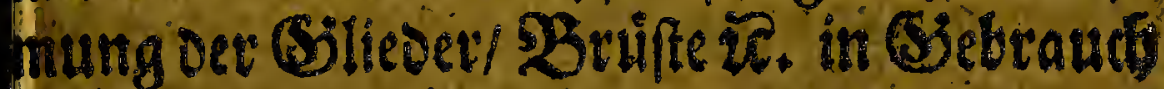
uf fent wie bey M. A urel. Severino, Sculteto uno andernmebr zureben/ foeldfe. in ifren SBidern twobl bunserterley Figuten won Szrenn: Evfen vorftellen: Es if cie te Sadie de von vorfidtigenDreifern nod hidot gat veriworffen toiv; uno oarumb nod nitis gans abfommen.

VIII. Was angefet die andere labrue

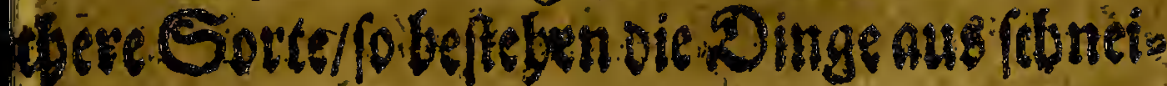




\section{มีล $(272)$ है?}

Denden backenoen und ferbenden Sheilgem uno fino bendez aus Salf ooer einen fdiarty egenden Saure beftebend/als da fino DagRuprorium commune Lapis infernalis Sublimatum, Arfenicum, Aqvofortis, Oleum antimonii, Oleum vitrio Ii \&c. Diefe gebraudtetman/menn man eto son forft olne groffe Sdomersen cine Deffet nung baben foill / neldges fonft ourch fchneiden wobl fonte vertidgtet suetDen: 2al senn etwan in Dem dunnen ober ssoifdien. Den. Drusculn ein toneng Soter liegt/ Dog

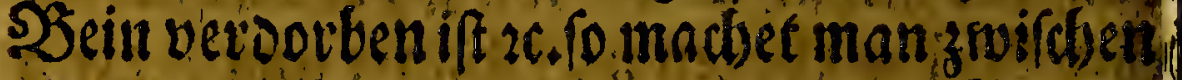
oie Mufculos mit voretroebnten SDitteln cine Deffnung/: Das ift/ fie werden auf einen. bequemen Ditb eine rueile geleget / allwo ibre, Tbeilgen/radjoem fie borber vermittelfteil

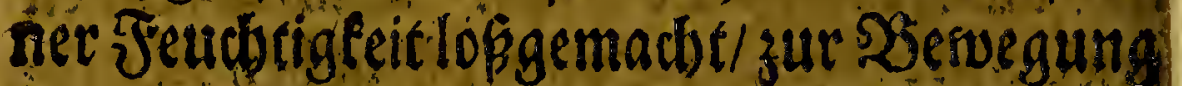
Commentuno in die 5aut uno J) Râblein bas den uno erben / ro oaf alle Fraleinferben uno ifre vorige Jeftigfeit berlieren/ soelobs.

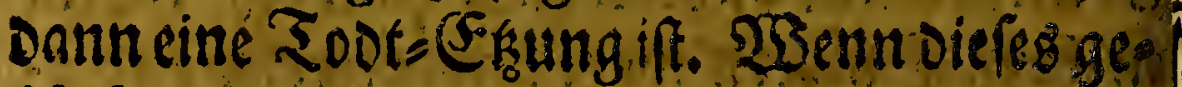
foreben/ftidje man ourd oas berforbene fo toeit al manoie Deffnung baben will bas Durd Das (Evter/ Fonfer 2c. ausláufft.

IX. Padh Dielen folget noch eine gelinoes re 21 th / oie meifteng gebraudat suiro/ oag?

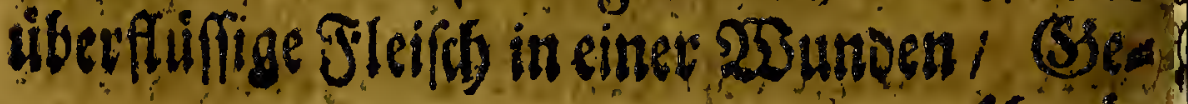




\section{स्न (273) है।}

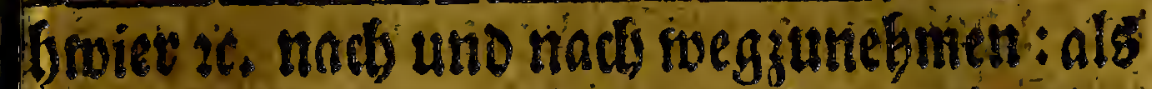
a fins die Species des Vitrioles, gebrano: ar ellaten/ rofber Pracipitat, Surinfpant

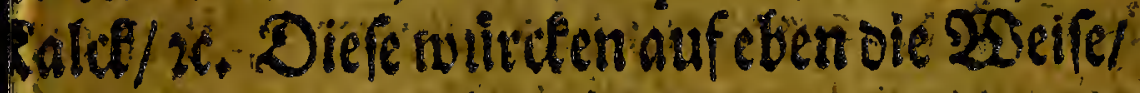
lat gelinoer uno langfaitter / und swer oent itrweder anter oic Salbengemenget/ obev

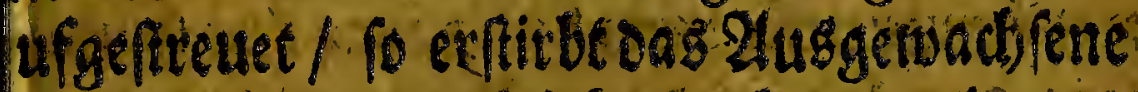
Ifobalo/ fepariret fich bertiads und twito ges eilet/ bieber geboten aud bie Dinge to eldx as Saac augfallen madten/ und diefe orit: etr out f bie Saut/ gethagen oie 23 urtelit

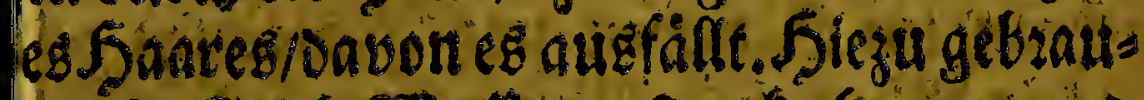
benfie Ralde-2Saffer / fat de Saugen uno

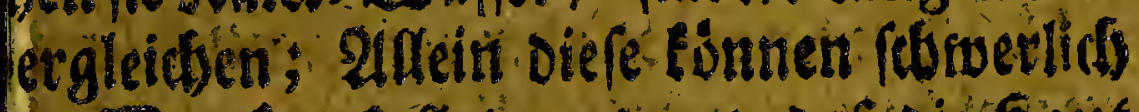
tI 2 serut geftellet werden/ Dof dic Saut licfi berlestget netoe.

\section{Dab XVI. Qgapitel. Son'Blutb = Stillungen. 1.}

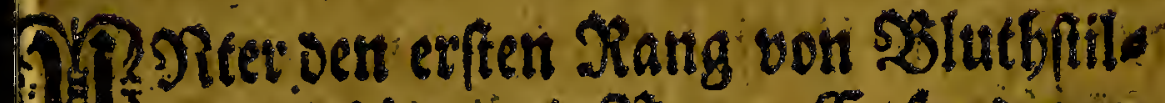

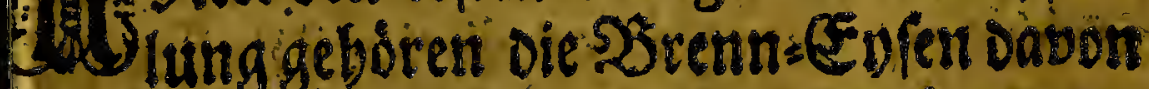
beiagt; diefe toerden gluenteinen abgenonts henen(S)liede appliciret/' oamit cine 2inse (Efchara) oafelbft toerdel unv ois Nilutbe Gefaffe feine Selegenbeit baberinofdr/oas

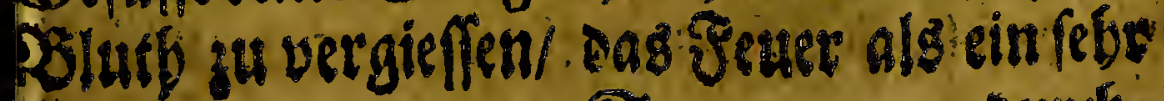




\section{$205(274)$ sto}

ourdsovinglidb uno nidst minoer betweglidbe 23séen/beftebet aus viel Alcali uno Acido, Deren F beilgen fchneidend / Gacteno und ferbeno fino/ swodurch das leidende (Ślied and gegriffen/ und deffen Táfelgen allo zerbacth uno zerferber werden/ Daf fie in fticten gebert uno ferner unbegbem fino etwas surchzulas fen Daneben witD Das S3lutb geftodfet und ? ausgetrodenet Durds oie groffe S Sise/ uno fol. des machteine barte Rinde / Dabinter / die SBlutb)=(Siefáffe firgen/ fitb) alfmáblig von Dem gebranoten Scheisen uno Burwads sen Daf fie fein SBlutb mebr fosnmen ourdslaffen.

11. Die Species aber von?Jlutbfititun= gen nel be vorifso bräudblidf 7 fino viel gelin?

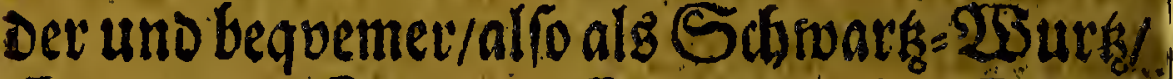
(Siranaten=Scholen/ Sjafle2lepffel/ Sdbles: ben/ Hypociftis, 2lloes/ 2trmenifden Bolus, Terra Sigillata, 2Bitriol/ Allauen/ Sbovift / Colchotar vitrioli. Diefe alfe fermmen ooer coaguliren oas S5luth ro a Det 233 unde låuffy oaruber man einen $55_{0}=$ wift binoert morein oc: ubrige Waffer oes: 23lutbe Iauffent fan: Rebf Der Coagulation aber / nerben Diefe Fbeilgen auch fo

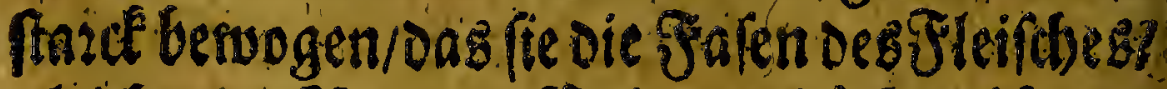
gleidy Dem Srenn - Evyfen in fucleten fobreiz Denoag fie fterben uno einen Srino zuniche 


\section{He $(275)$ S:}

laffen/ den man bernad fepariret/uno das Sielunde zubeilet.

III. (5 g wiro von einer getwiffen Dulver geragt Poeder de Sy mparhie, onê in blofs fen gemeinet/ in 2 affer folvirten Ditriol beftebet/swie oer Xitter Digby erzeblet; da er bie flutbigen sudber genommen / in sas Ditrid=2Saffer getban/ uno ons Slutbet angebalten. Dafsfoldies gefdieben ift/glaus be id swobl: allein in Warbeit bat das SElus tben Davon nidjt aufgeboret / Senn wo oie 2SSunde fefte fan zugebumben netoen/ unt fille gebalten witc/ foll sas SBlutbentrachs laften i weil algben die Siefáffe zugefoblof fen find/ uno nach Dieferm beilec die 23 unde von fids felbften. Daper ferner erzeblet/oaf Der Watiente in oer 5 Wunde Sajmerhen füb

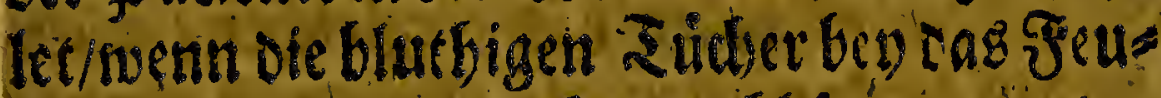
er gebalten weeben/ fan woblfeun / wo ber gotienteftch zu viel berweget bat! aber es if nidjt von dem beifen sudbe forten. Sob babe eg an mir uno andeen probiret/aber id fith De es nidd rwar/ babe aud viel bon feinen on, oern Dingen ing befunden / und fo bice der: Drtbogu noitre\%. witovidu fie wiebeclegen formen.

IV. Eben eine folde gabel in in oer sibele sis Secbindumg oeg Inftruments, Dami 


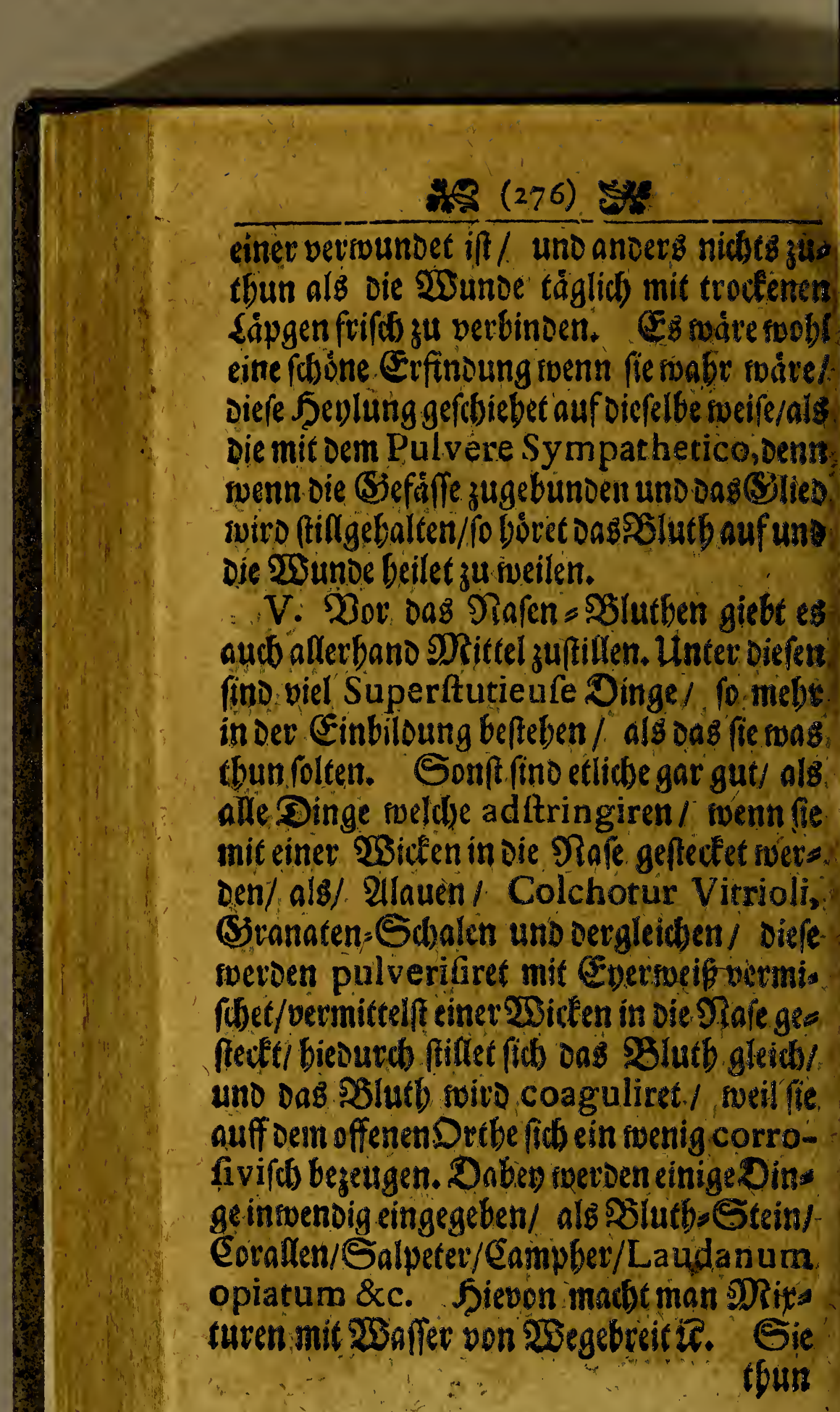




\section{มิล (277)}

t)un gnar nidbts mebr als gemein 2 Saffery Datumb oarff man mur geforten 23 affer neb men uno die obigen Dinge in geboriger Proporrion oarein mengeny fo madjen fie eir flifiger Sseblutbe / samit Daffelbe frever

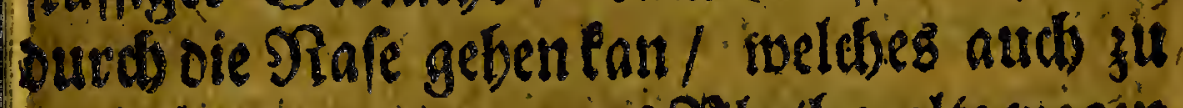
beridoten war/Denn oas SZlutb nolte wo gen feinet dick ound die Arterien nidjt parfirent.

VI. SRandie balten cine gefpaltene 802 ne/ deuyt (Rupfersplennige) odet Der gleidben/ auff die Stins:2loer / bermeinende

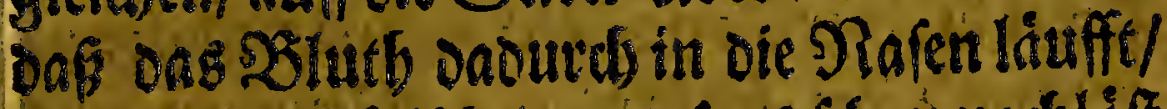

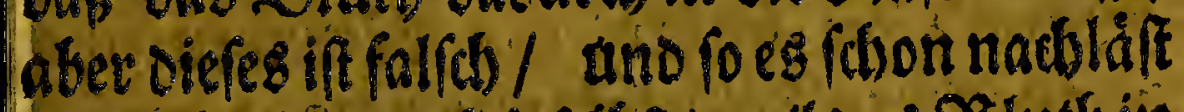

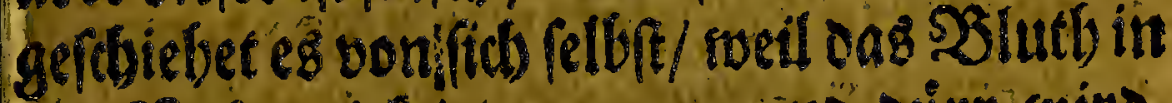
Dec SRafen bid / geconnen und ourer wito. Eg if nod eine andere Runit/wenn das Pas fenblutben ourd) Serforeden geftillet wito/

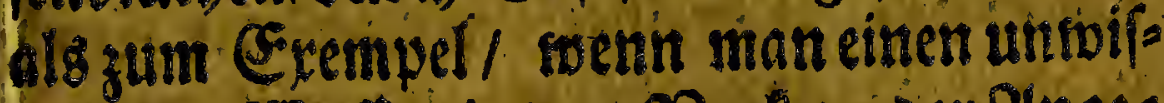
fend talt $25 a f f e r$ in oem Sacten ooer Unge

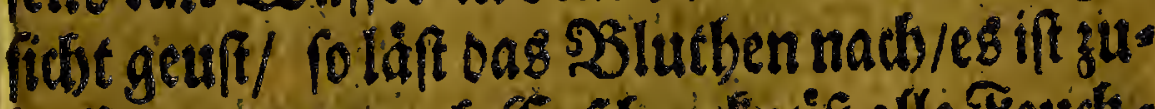

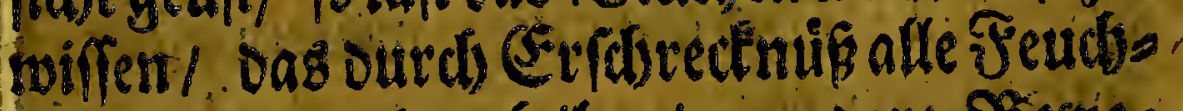
tigfeiten in unfern Seibe cine anoere. SEentes

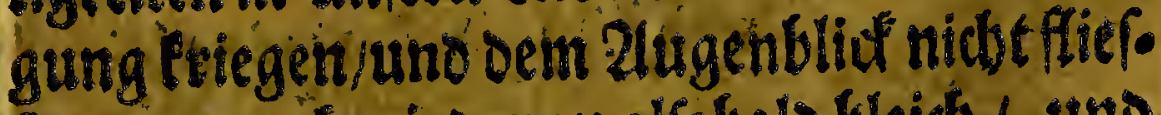
fen/ Darumb twird man alfobalo bleich/ uno wegen oec Irregularitát $f$ oaff fie fueder bit

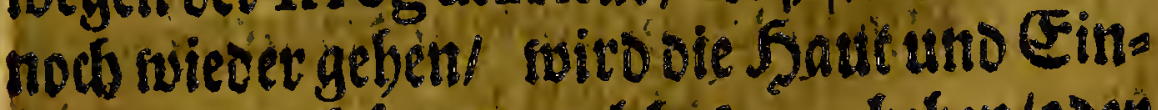
geney de ex/douttert/foreldes man beben/obet 


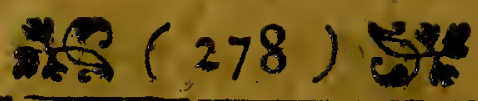

gittern nennet, Seu foldben 3uffande wivido autch oer Sauff in Der Nafé geaintert/ Daß offed

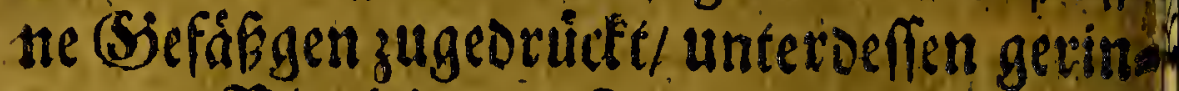
net Das $\mathfrak{B}$ /uth in oer $\mathfrak{R}$ afe und bălt an.

VII. Ferner giebt es SSlutbfithungen die in einer Imagination ooer (Einbiloung bee. fiefen / als da niederumb if Pulvis Sym-

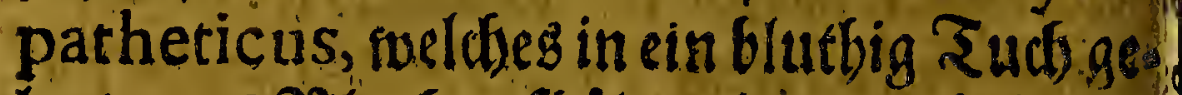
legel Das SBiutb aufbailt/ allein id habe fors?

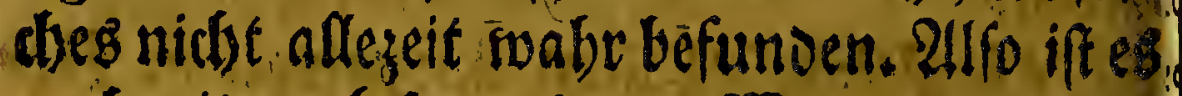

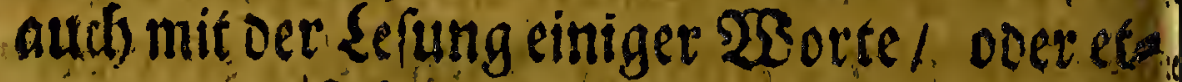
swas in oas D br zu zufcheln. Atres diefes bes. fteber in ciner Superftitieu fen. Einbilthung)

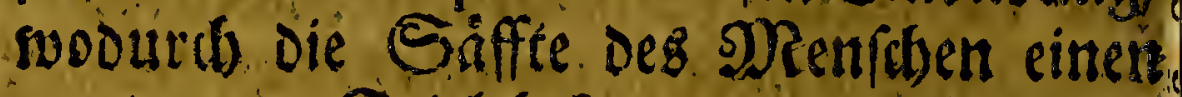
gants anoern ₹rieb befommen/Denn neer blu-

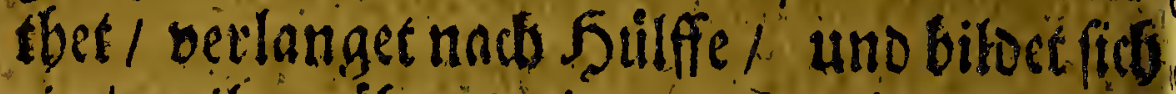

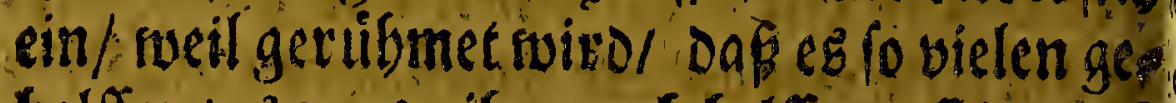
bolffen/es meroe ibm autd belffen: Jiedurdi, beformen die Sâfte cinen andern Frieb)

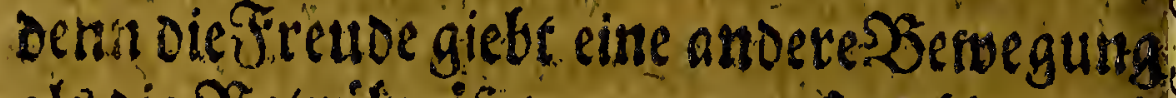

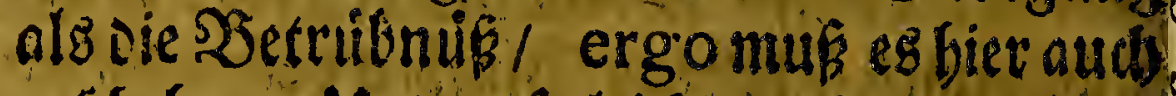
gefdeben: Utno ouf gleidse iveife curiren et. licbe das Jiekter.

\section{Das XVII. Sapitet.}

Zon 210erlaffen/ Schlag=21oet toffin/Sdrónffen/:Blutt)= Egetin zi. 


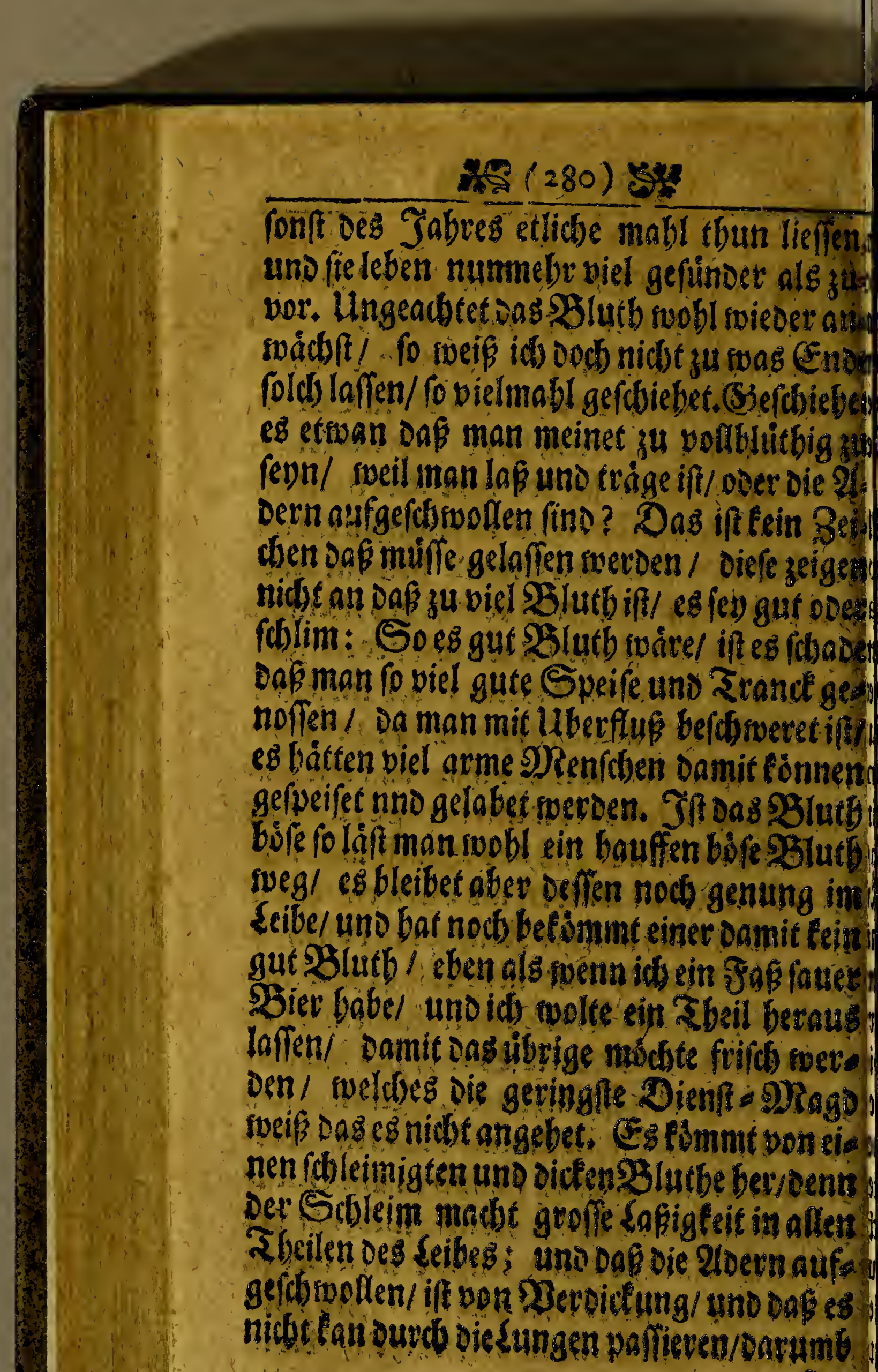

fitt 
ino fie aud beorengt uno befinoenfich nidbe uobl/ fonft folten fie baran nidit gevenden; arumb ift oas Saffen bier zu vernerffen/uño in beften nidgt zutfun / oenn es fan nidbts

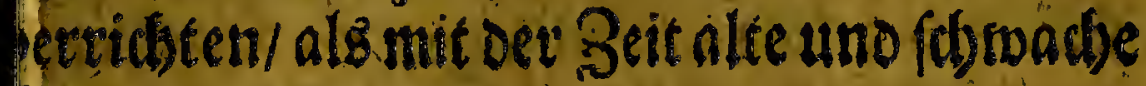
leute madben.

11. Seopd fitio Selegenbeiten / Da Das foerlaffen nobbl fan juftatten fommen. al: Senn iemano Pleuritide oser Seitenfechen aboriret/ uno oer Sdymers unetringlió fro nit lobmersffillenoen Ditteln nicht zus

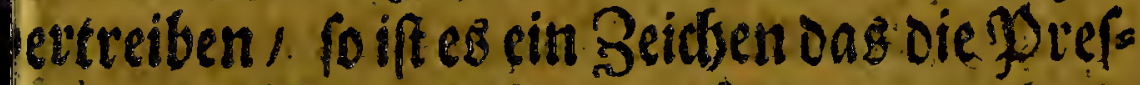

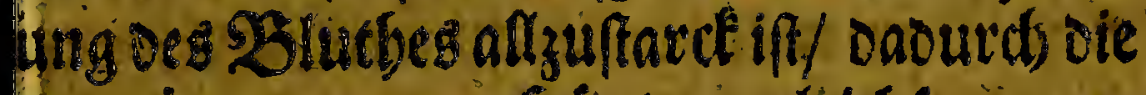
nembranen over Säutgen gleichfam von= inanoer berfen uno reiffen/ fo fan loer ges affen meroen / nidjt zu $2 B$ egnebmung oet Rtand beit/ oenn Die bleibr nobl/fondern oa =

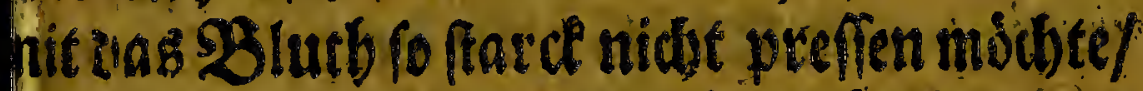
tho fo es zum eefen mable feine seidsterung ringet/ wivo es tuidst: belfen wie id foldjes ielmabl angemerctet. Sols vor mid fan fon: er.aberlaf curiren / und werben son meien Patienten nidot mebr/ fonbern weniget terfen/als bon oenen die gelafien haben. So d) es tbun laffe/ geldiebet es mebr bem praúdicio oerPatienten/Denen einfältigen him. tefenoen/ ab uno zugebencen git Dengnt: ung alb ifnen oaourd zubelfen. Untec. 


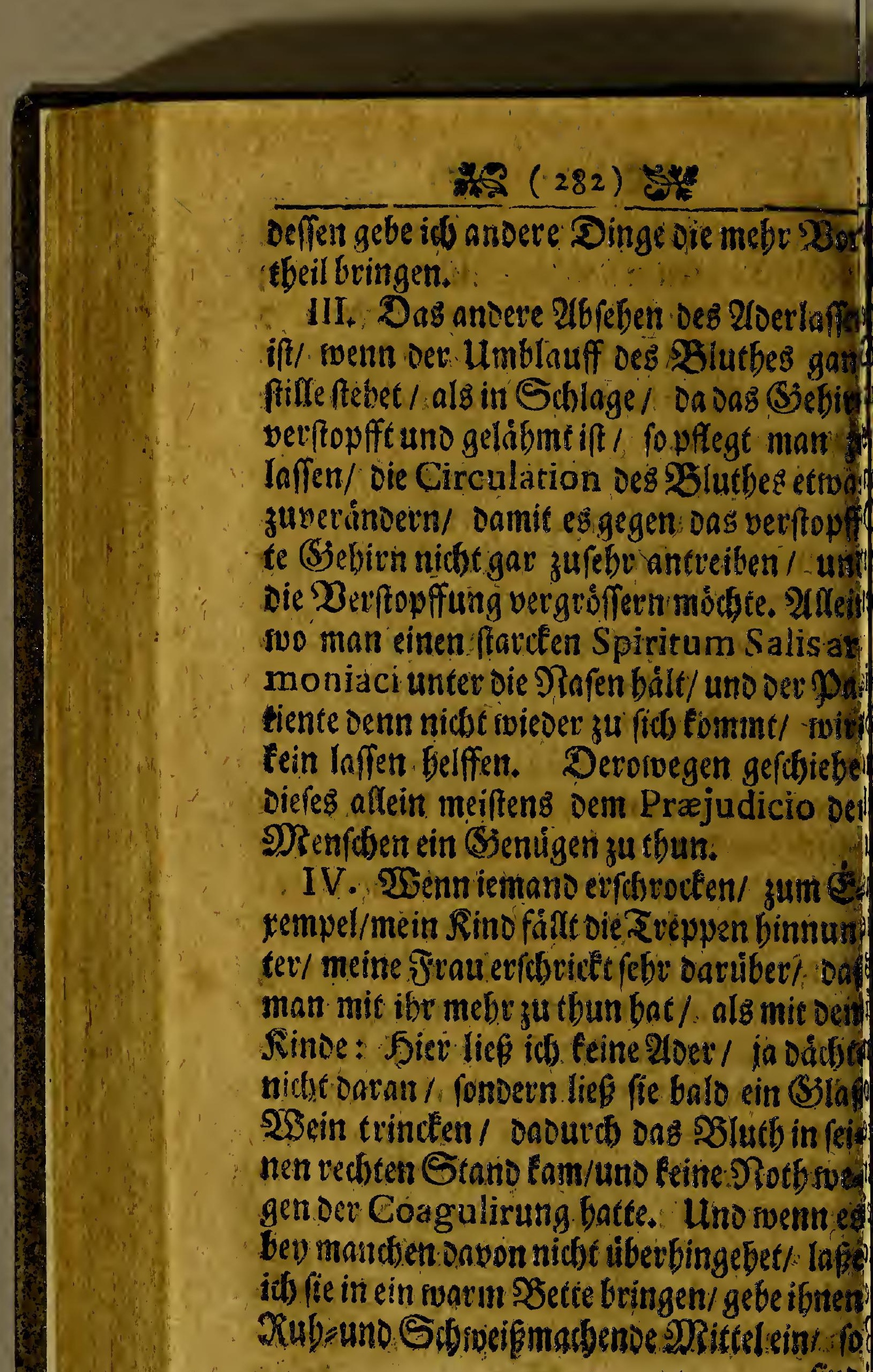




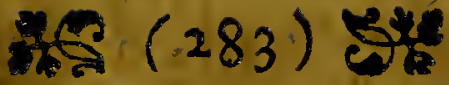

So fie oes J) orgenz erfrifdst I Dacauff laffe b fie ein Sal volatile brouchen mit Theè bet Coffè. Wo man aber ja oas \{affen ins beruffellet/ gefobiebeteba allein cem PraAdicio Der seutezu siebe. Sob babe mebr Is buncertmabl gefeben/ saßs Denen Seuten ac) sem 2loerlasen viel folimmer worsen; Derobalben mus bas saffen nidst viel belfs It unn denn ift es beffer unterlaffen/alg nads em Snffen ärger werdert.

V. Sn Xaferen und Soubeit/fonnen Olata gebraudbt werden / wornadiffe wobl onnen gezábmet soerden / allein bie braudis han oabsaffen ausb als ein fouverain JRit= 1/. uno ift es in viefen Jall wobl tridgt gants

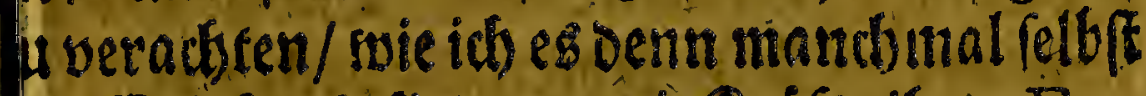

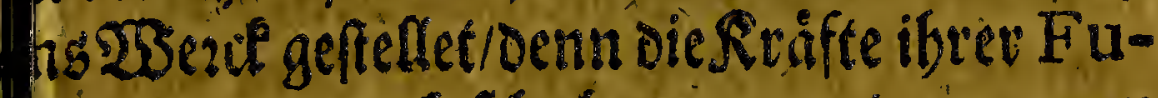
ie nird Dasureb febr benommen/wenn man s aber mit anoert Dingen verridtentam ift man diefes lieber bleiben/indem sie patio nten/swent fie bernad foieder z ommen/ fdstuad fino/ ibre ordinar Diens te/fopfern fiefófwer/zuberridgen. Diefes ino alfo die vornebmften Punceta, oabey ids bben fan / safs Das Saffen nicht ganfsu ber: daten; Salageaber/ bof sop man mit an beun Dingen belffen fan/beouffees nidst/oi Buftucht ju diefen פrittel su nebmet. 


\section{0 (284) है:}

VI. (5itlibe Medici foerden nod bis da to von iben Prajudicio regieret/mevne De/ Daf es nidgtgleith vidlif/ auts foeldber Dee das BItutb gelaffen ruerde/, baben daby sinen Unterfobeio bon \&oen gemadbe/ also Saltpt=310er/Sztut=21Der / Reber=310eh SRtuter=Qloer ic. uno oergleidhen Jabeh gen mebr. Die sloern fo bort Dem Ropf berunter nach oem Sersen geben / fan ma. fuglidi) Saupt=\$loern nennen/ uno fomm: Finen der Sabmen beffer zu. Dafich aber

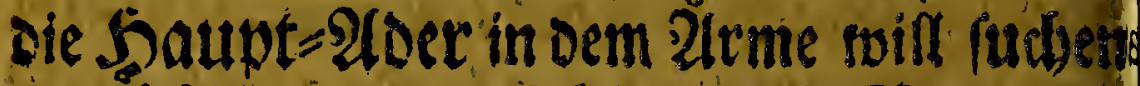
ift gefeblet/ oenn oiefe bringet ibr 53 lutb all: Dem Alrme nach Dem Serkgen/ obne mit Dent Saupte zu comuniciren. Dafelbit futbes fie audb oie SDilks Doer / onourd oie DRil bon Der Port=2loet uno Der' 2lem bott ow \$ol=2loer ibee 2loern befommen/ und ald

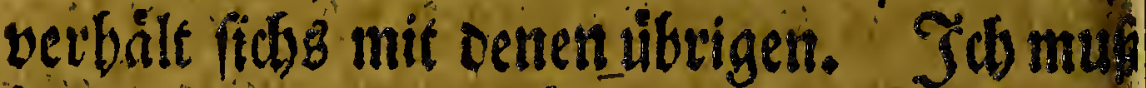
betentenoaf fie in China uns Japan wum Decliche Concepte baben/ allowo fie son Dew

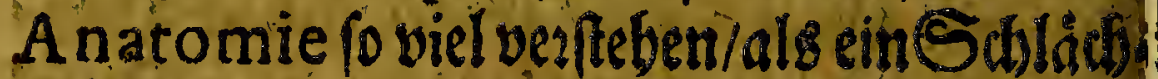
ter's Funge. Das manaber biezu sande in

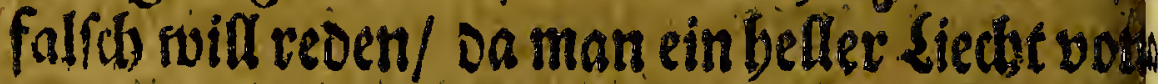
Der A natomie umo Umblauff Der Såff bat/ift febr gefeblet, isser den Itmblauff oet Saffe grunolidj berftebet/ weis teinen Un

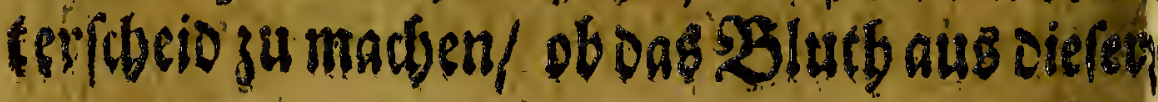




\section{$\tan (235) 5$}

E tener aloer gelaffenj toird. Wrie bat man bc bijaufb Sdhligen auf oenen Acadelen wiedercinanoer difputiret/ mas vor. dineip bat man nidbt von Dem alngefict pirdget/allein zu erforfdoen/aufroldben in in Pleuritide poer Seitenftedien zu en; Woser die Sriedjen nod Nraber ben Den befftigen Streit berdhlieffen tön: - Sa es war in Jtalien / Francereidy) Jannien/uns Deutfolans folcbe Uneinigs. hiber diefer Qveftion, Das der Magiat fidj Damit bemuiben mufe/ bif zulest oie piftigfeit ourd Die Authoritat oes Prin= mit sem blutigen Degen gefoblichtet fur:Şbeils meinten die Eloet mufte geóffuet

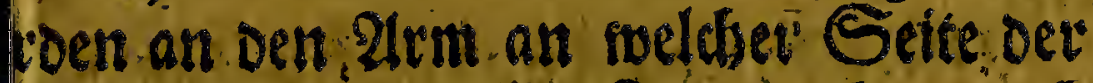
bmersen war/Die bofe Jeuclitigleicourd furtseften 23 eg abuleiten. 2Indeve wols in oem gegentberftebenoen Irm Lafien/ - Revulfion jumacten; 2ldein es iff al einciteles Seplapper/ wer die (Stelegen? t bes\{eibes fweig/batfeine Qvaftion bar:

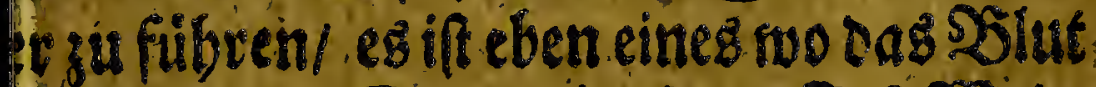
aus Jaufft; Denn ob ids ein Sap 23 ein cen oder fornen an oet einen oder andert

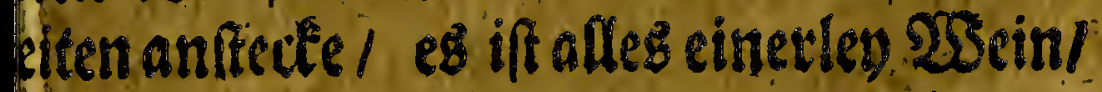
b es lauffe fo sobblaus oem cinen als dem: Dern Sodje. 
VH. Biel ineonen nodi was neues erfur Den zu baben / roent fie an ftate ber soets vorgenommen sie puls = 2loern zu laffel welches fte mit einem (Siriechitosen Term no, Arteriotomiam nennen/allein fte b geben eben auds einen freblet; Denn noier ben gefagt/ esiffeben vielaus nas vor eint

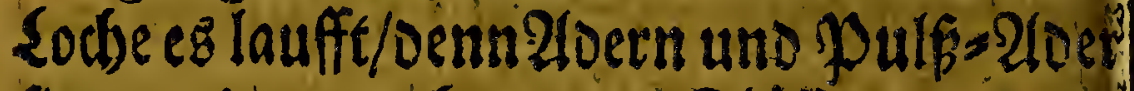
fino aneinander bangende flüffel roas einy ausgeuft/ nimbe das anbere nicder cin/ un Diefes gefiet alfo continuirlicb fo lange eim Ieber. Derowegen if siefes ene unnise in vention, Deren $2 s u n v e$ gtófiere (Siefal fan nach fid jieben als die Deffnung oer 2 ber.

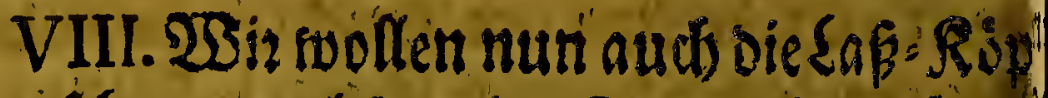
feanieber/ welctes eime Operation ift/ mar die Saut an vielen Drtben baut/ ut Dent cimen uinbgefebrten Ropff mit bre

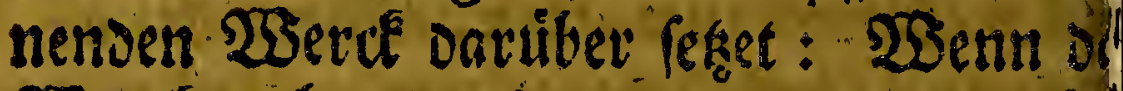

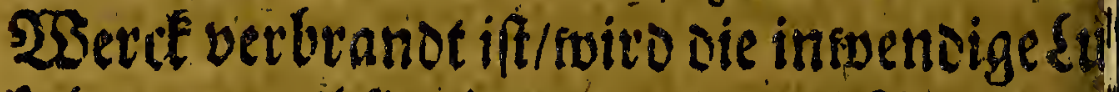
falt/und rweil fie alsoenn sweniger Paum cil nimbt/als Da fie fwarm war/ uns fein vact um oder Seoigfeit Darinnen bleiben fan/ wreft die ausiventoige Sufft/oie aus dem Roy fe gegangen warc/von aufen Die 5aut/ fo of SSluth an die Stelle fontit/ sen von oer as. gegangenen Suffe verlaffenen Drfy zu ertin. 


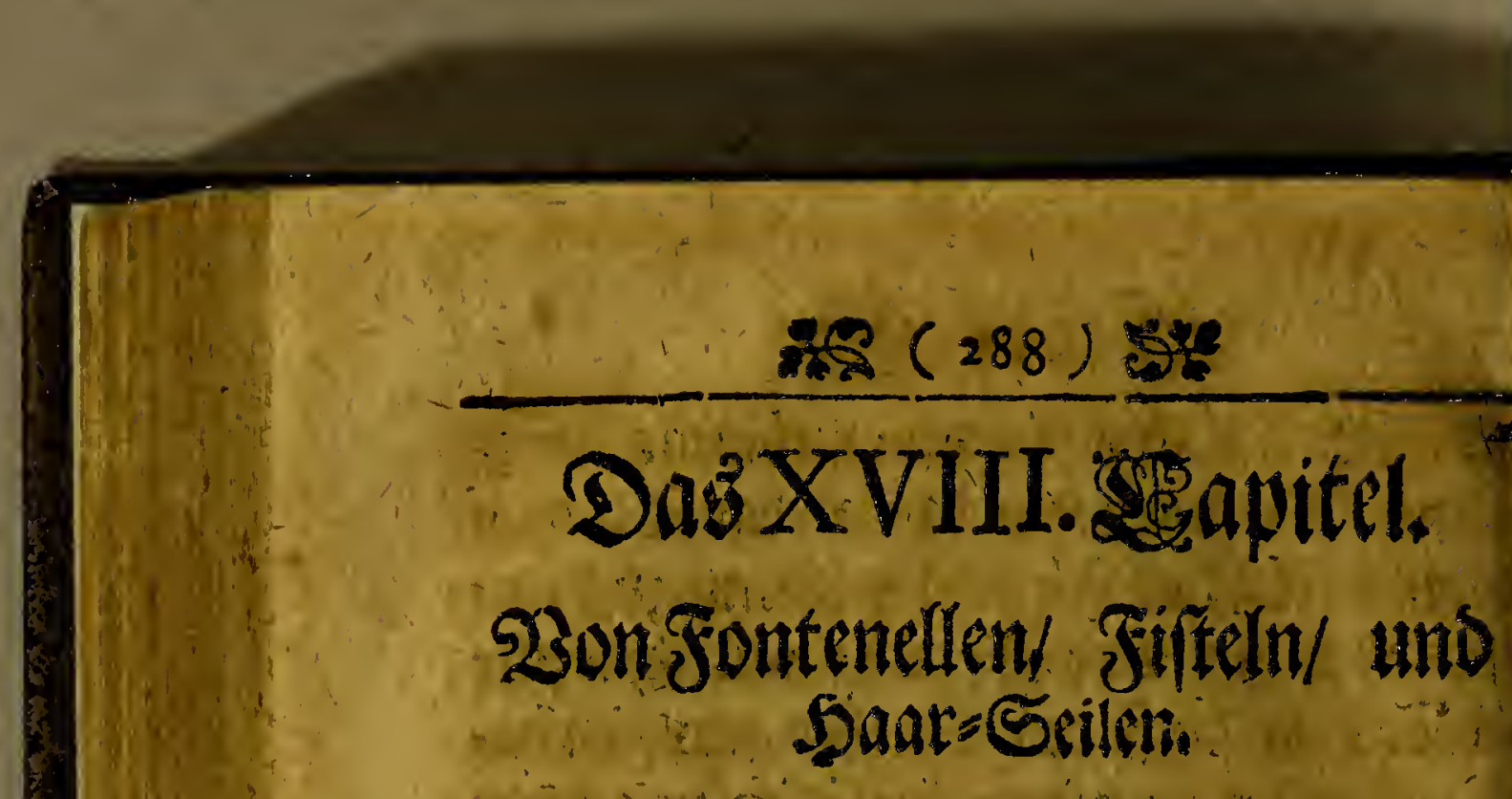

I.

STS6 babe font at bielent Drtben of

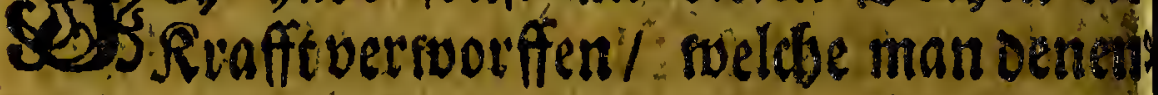
fifteln Y Fontenellen uno Saat= Seilen zus: fobreift/uno fonderlib in einen T ractat, ged nanot Het Gaft-huis der Sieken, und soeil eg hiet audb oie (Sielegenteí giebt/ thu

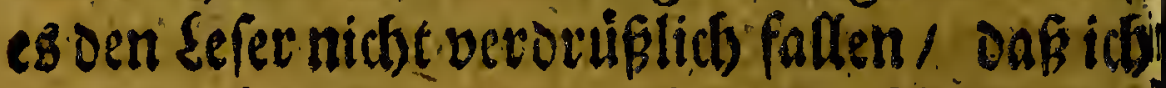
Dergleidhen Materie noch einmabl bor Den Tagbringe. Jof nerbe denen Theologis gleid feon/ welche man gerne auf oer Eantse rdelten boret/auf oie gemeinen Stinden/urt: ob fie ez fdionjum offeern tbuti) if es venen Bubjorern oods eben nicht unangenebon. Die Tifteln/auf oie 3 eife nie iff in meiner Chirurgie, bephrieben/ ober auf eine andere?

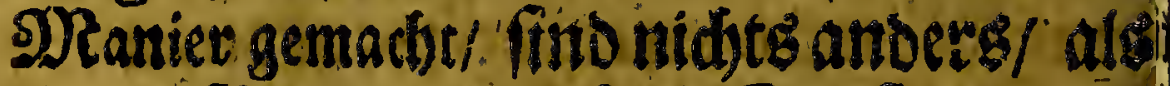
ein(Beforwier/ Durboie Runf/ andies) fen doer jenen sbeil / Jwifben ztwery Sáublein gemadt. Sieourd meinet mant Das oie Excreme nta von oem Saupte/2(w) gen/Obren/uns andern Sbetlen abgefibues 


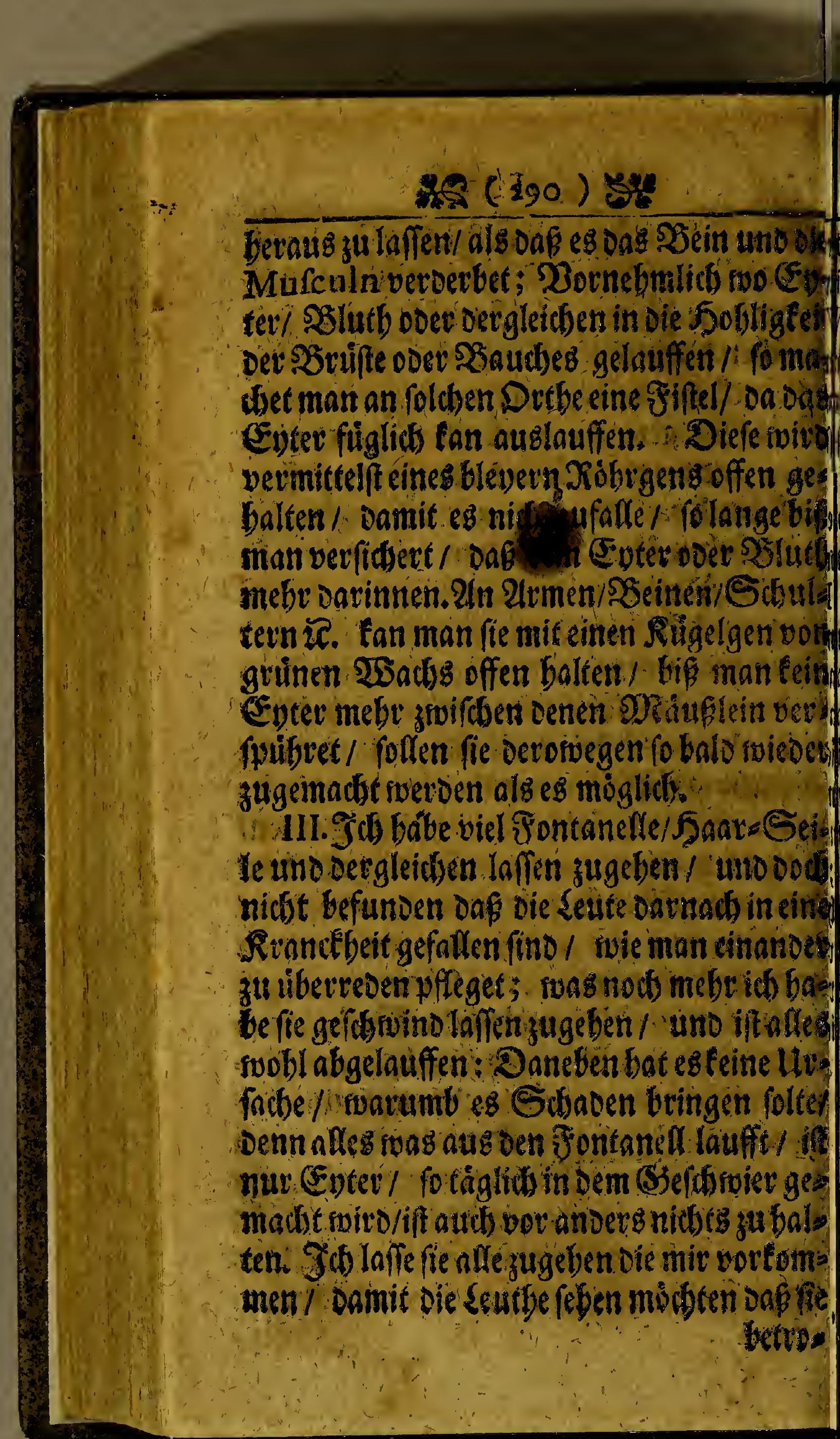




\section{मू. $(291)$ और}

trogen fino/ es nodue oenn oaferlide oie inbiloung battet daf es ibnen gut tbete.

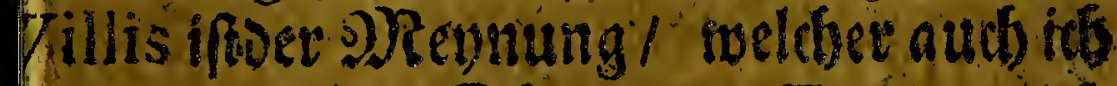
toefen / Dafie sie Säucte uno Ferment fo an bey senen Seuten gersabe swird meld se

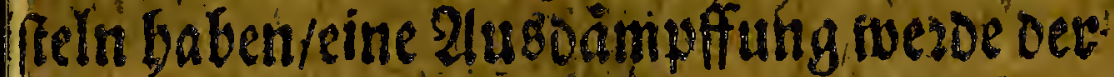
SZlutbe befindidet bofen Unteinigfeit:

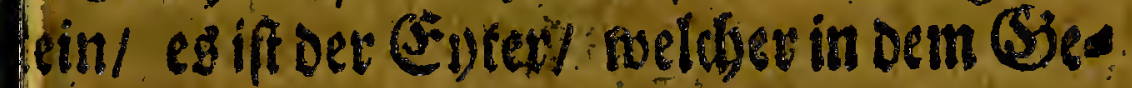
wiere aus dem Chyl wito.

IV. $23 i e$ fino vor etfiden Gabren bie Setl mitht gepeiniget worben mit. Sesung bet are Seile in oen SRaten? Wern Die dut mit einet Bangen gefaft/uno mit einett

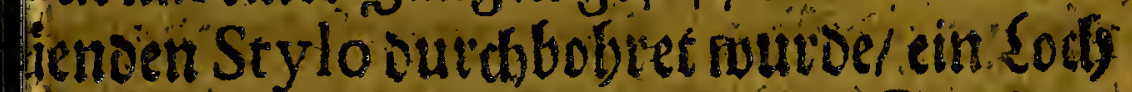

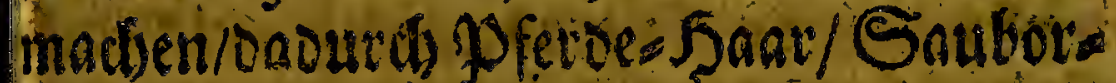
h/ ober cine feioene Sobnur gezogen nute

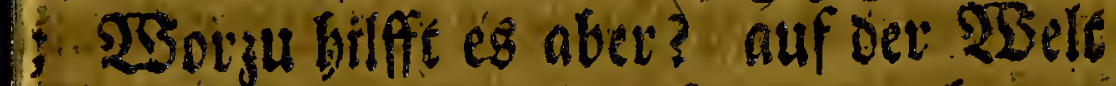
(i) 1 ' eg if aucb oie Urfache natumb man tonfänget rociter zu feben / und foldés fo Ir hidje me br vornimme. 2udh frirdsenfid le 9 atienten inetre oavor als bor diefen/ uno nicht unbilito. Diefes if eg fwas aib furblich oabon zu fagen gebabs:

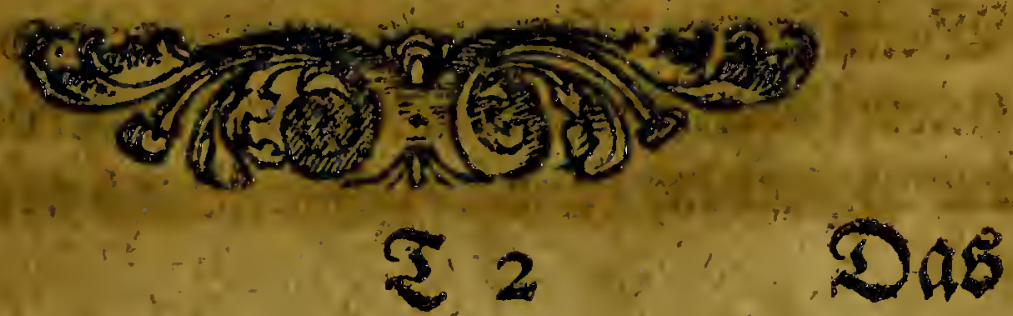




\section{Dab XIX. Saptel.}

Ronbenens) ditteln bie Surch Im gination oocr Einbildoung belfen/our Spredung etlifier $230 r t e /$ Sympathie, uno Dergleiden.

\section{1.}

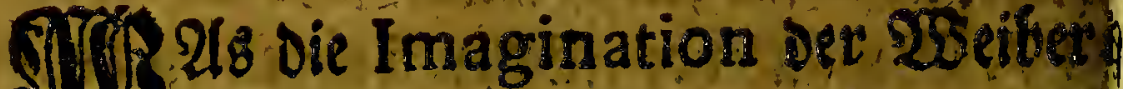
Des ibren fruidsten vermag/ if Der gan

23elt genungfam betanot/ Denn dietsglich Erfabrung giebc uns diessuirctung gu pebit uno zu greiffen. So num diefezswabo/ wo umb folte denn die Imagination in unier Sáffen nicht eine foldbe SBewegung mache Honnen oaf ein Watiente bonfeiner Rivang. bett erlofet wuiroe? Dap ourds unfeve Im gination ooer Einbiloung oie Saffece mebrere over minoere Fluipigeit betomm! iftunläugbar; nis foldhes oie Jiftorien te. ren; Bum Exempel: Deeite Frau vertie? ret ein Rino/fie gedendet nidst fo off te oara Doerfiefänget an zu beulen/ uno alle I bet Des Ingefidyts verdnoern ibr Stellung. ? 2Senn iemano vont geringer Con dition, eirem boben Stanoe for erbotien serden/

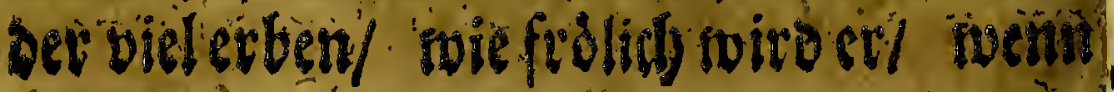




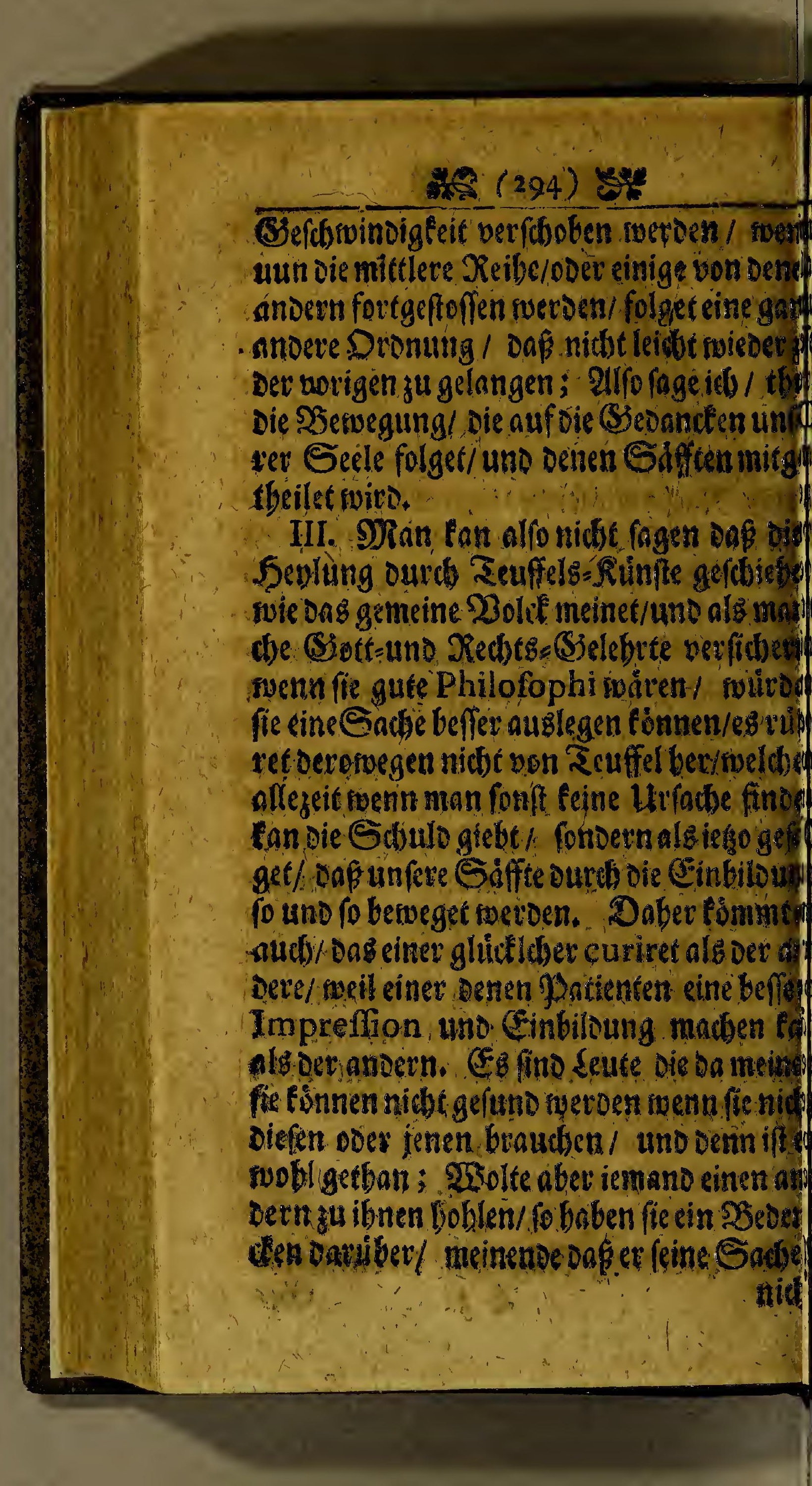




\section{Is (396) 5}

Engelano curiret/ twie Laurentius begen? get/ier ibver wiel gefeben yon afler Nation, meldebalfo curiret worden? 23 as vor ein

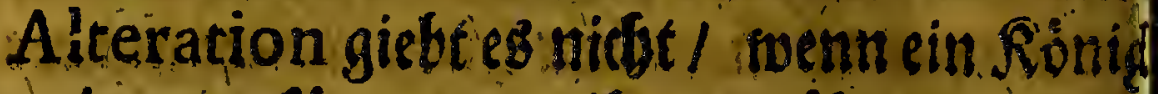

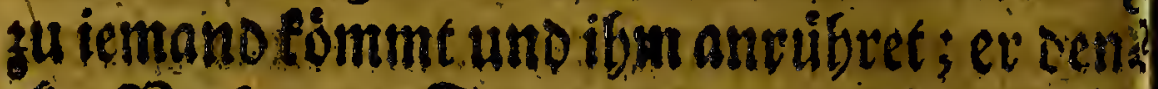

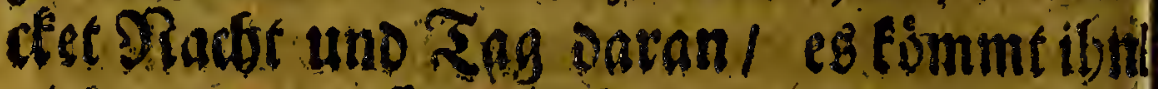

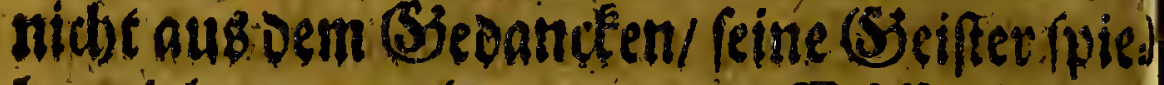
len viel ander: als zuvor / Die Sáffet trieget:

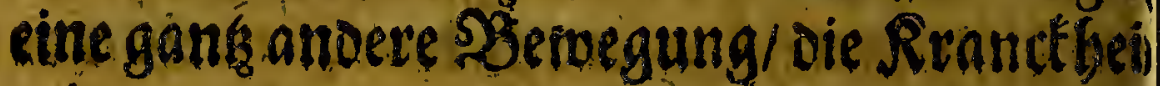
sviro nich t me bo genábret / and oer postient

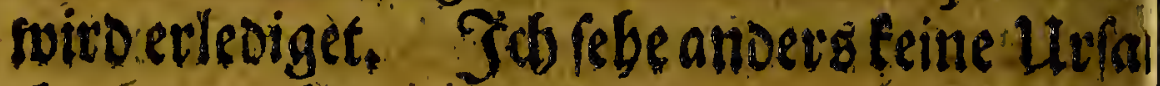
dhe fwarumbie won Harlem, Denen 2upfid Egigen eine Rlapwer geben / Dáp fre fein ander SBrod effen duirffen als Das gebettelt if / eit.

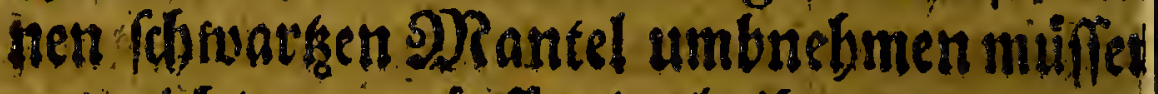
und nightresen oirffen/. alsiginer eine fold Imagination zu madjen / woDurch of

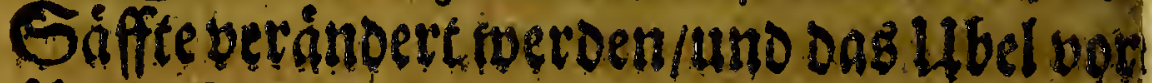
iber getbet:

$V$. Die 2 ele if vol Superftition uno 26erglauben/. menn die Imaginatio

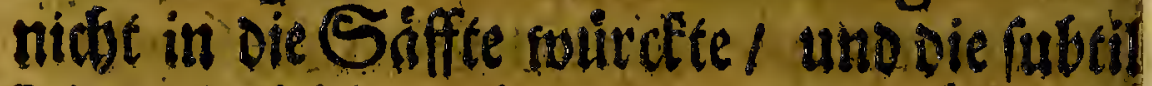
Materie foldse auf eine gerwiffe rweife beswes te. 28 enn icf oas Theatrum Sympathe

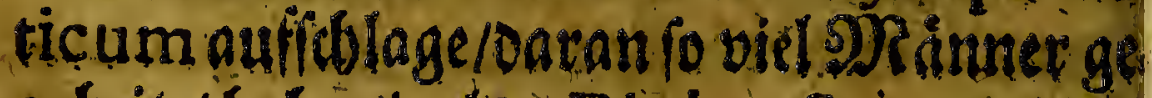
aubeitet baben/ als: Digby, Strausz, Pa pinius, Moyus, Glocenius, Robertus Helmont, Robert Fludd, Bekker, Ba 


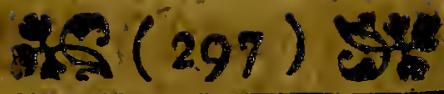

rellus, Bartholinus, Servius, Kircherus, Matthæus, Sennertus, W echelezus, Nardius, Freitagius, Conringius, Burlinus, Fracaftorius, Wecker \&c. S23as finde id) nidje bor (Sriflen von dem Pulvere Sy in pathetico, 23 affen-Salbei Geftime/pfluden uno foncioen diefes ooet fenen 5olseb/ Rrautés uno 2Butselin/auffo énen ₹ag/ Stutoente, von Influents Des Steftirns; von Figuren in Steitie gefonit. ten uno foldoe bey fid getragen; von aller: Gano Artb.Sigillis uno rwas deg plunders mebr if:.25enm nut einer ein gut SDRaulbati unv einem Pacienten folcbes uno oergleitben

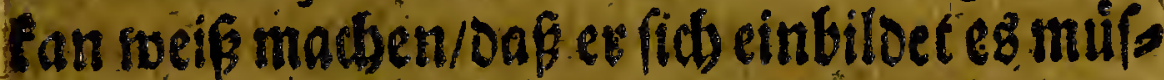

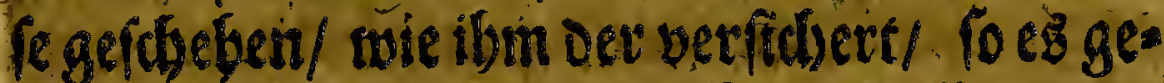
fage / to thut oiefe berbbaffige Iluffinumte rung oie eur ourd) boiclmagination nie ges fagt / Dag SJluth zu veranoern/ uno nicto bet

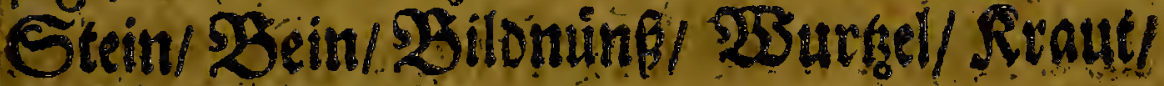

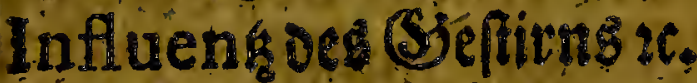

VI. Fुnar mas angebec die Influen Des Siefting / fo ift wobl mas barati/ denn wiv wiffen getwif pas Sonne uno Drano

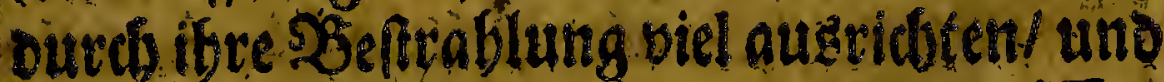

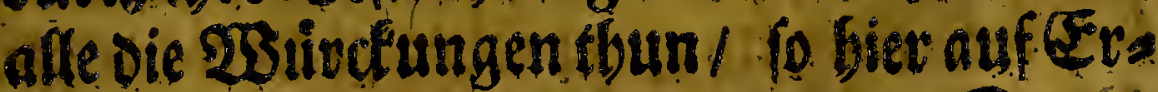
Dettgefbeben Denn weil bientrecn Stette ibre Influentien von ibret Admofphera

$$
\text { ₹ } 5
$$




\section{और (298) 38}

an bie Unfirige uberbsingen/ soarumb folte es nidbetegn fónnen/ Dap fie in unietn seibe und alfen Thbieven uno (serváchfen nicht audh ets iwas verrichsen folke: Dan man folte fagen Jupiter thut Diefes/Saturnus dafil uno oie bobern (Siefirne aufierbalb unferer admosphæra richten oaf aus//oldbes ift bisber Deo nen sie noch leben unbefant. $233 \mathrm{ag} 90 \mathrm{am}$ SRoab/2lbrabam / Salomon/ Ebrifue uc. Davon gerivuft baben/ roiffer mir nidat/ was Die Sebre Der AEgyptier von folcben Dingen argebet/ iff falfecher als fallch/ Denn fie fons ten mit afler iffere 25 eifbeic/ Pharaonis Traum nidbe auelegen / fueldies ein armer Sited bet oer umb falfaber. Sgef(d)ulöigung

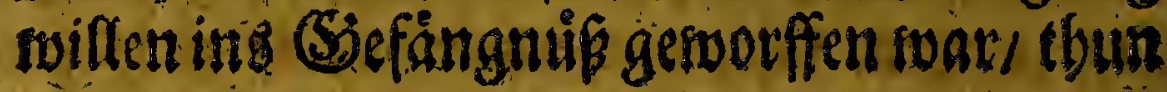
mufte.

\section{Das XX. Sapitel.}

Do man aus ber Stgur Der Sirált: tet ibre Srafte wiffer.

\section{fonne?}

I.

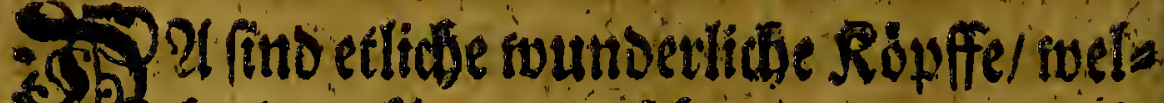

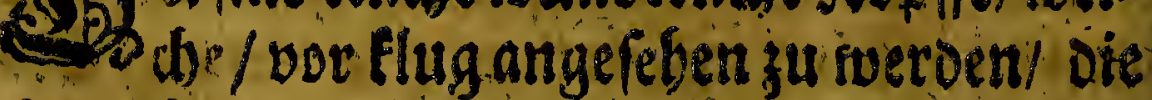
seute uferceden und fagen/ oaf mart ats bev Jigur uno Sistall Ber Rriuter siffentono 


\section{2क (299) है:}

ne wofur fe gut find it will furs zugeben/ cinige Srempel vorbringent.

1. Der MRobn foll borg Saupt gut fem weil er eine Rugel ooet Rouff præfentires; seiff aber die Jrage/ in swa vor 5aupta Rranclbeiten/ benn da firo ibr die Sulnff erfosern/ andere feinen. Vsarumb fino die Ruber/ Caftanien/ Antirrhinum \&c. nidht aud gut nor Dte Saupt = SSefdrwerutigen/ Denn das Antirrhinum if einen Tode ten=Ropff gants ábulids.

2. Die 2s zlidsen SRuffe defigniren atro Dem Kopff/ Davonfie fagen / Daf die churecli: die Sdiale: Das Pericranium; oie barte Solserne das Cranium, ons ounne Squts gen umb vie 9 tup die Sim Salugen; die Sub felbft aber als die Qvinta Effentia, Das Sebin folbfbedente. Es ift genifine rebr nabe Jergleidung. इ2ser bat aberles mabls gebsuct oaf oiele slub fo treftidi gut ver das Siebine fey; ia oie ration lebret foldoes nidt. Uno wentr es sobelfo mup aud

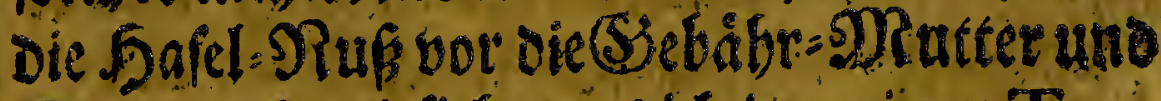
Rino gur feyn/ fobe svag ith in meinen TraEtat bon oer Fermentation, onuon gelaget Gabe.

3. Das A farum bat oie Rlebnligkeit ci nes Dannes Dbres: wie auds oie Jubasa Db. 


\section{$x$ (3) $(300)$ zot}

Dbren/ man braudbefie aber/ sie belanot

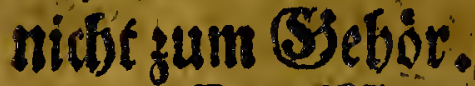

4. Die DRufcate foll oet S5abr=-DRutter gleichen/idh tan ez aber nicht feber. Soldye (Sleidsnibe wáren taufeno benzubringen!

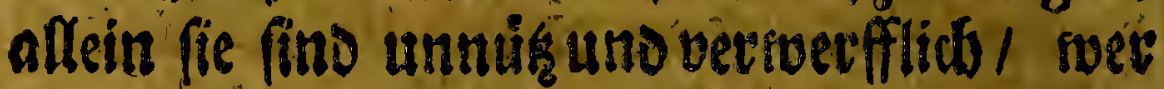
swiro an einer 2 seinrancke feben/ onf fie die Seute fan trundéten machen? Wer fan eB Des nen DRufbeln uno Sdinecten anfeben/ Daf fie Das Sauer temperiren und das SJluth verbeffern? Diefe Jabeln baben alleine ftats bev denen fo fie glauben; Jeb vor micly/ roill isnen meinen seib nicht übergebè zu curiren toent id erancebin/ nemn fie allein aus des Figur urtbeilen/ die man von auser fietet.

1I. Diefes tbun fie ricbtallein mit benen Rrautern/ fonoern madsett audb eine groffe Sileidbeit unter Der-grogen uno tleinet 25elt; Denen FGieren/Yabres:3eiten/5ime melso \{auff/guten uno bojen Sienitter. Jus mag mich nitc oiefen Dingen nicht lange auf: balket / fondern lieber fabren laffen / weil es unnotbige uno ungegrindete Specula.

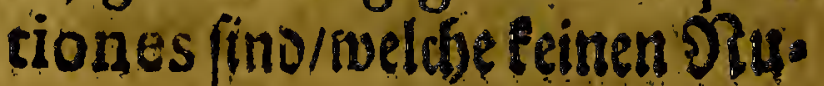
kgen bringent.

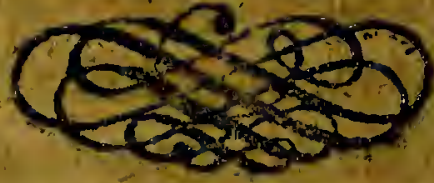

Dab. 


\section{$30(301)$ \\ Dab̉XXI. Capitel.}

Son Denen Siraffiten Des Steines Der 206ifer/Lapis Philofophorum genanpt.

\section{I.}

Arre Egen tes LapidisPhilofophorum

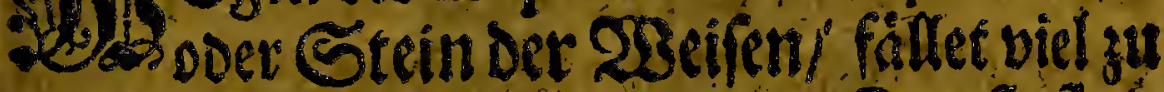
ragen: Die Suder Der Goldos Sunf//ind Sinver ber sBettel= Sunff. SBiele baben gecrachtet dag (Siolo zu fuchen aus oenen Mineralien/ ansere aus oenen Thieren/ uno

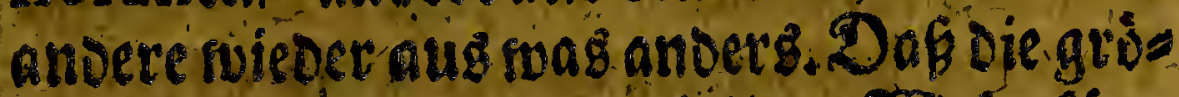
bern Metalle zu einen fubtitern 258 efen fóns

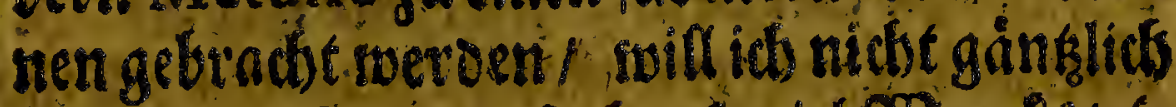

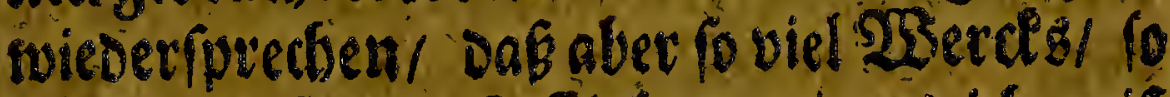

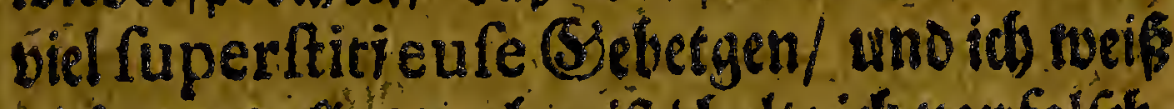

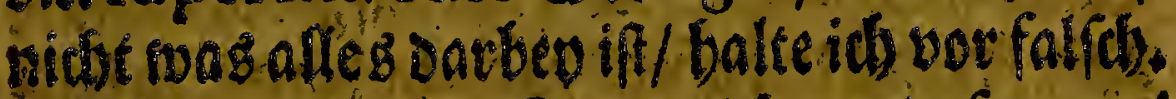
Id bate mirl bee:Runft nicht unterfangen/

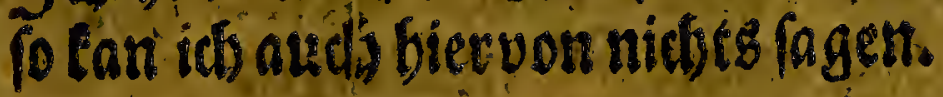

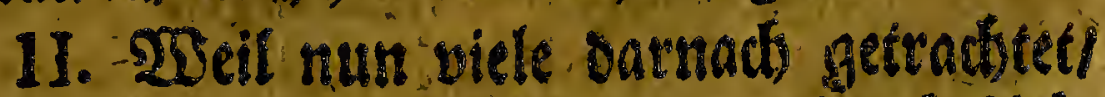

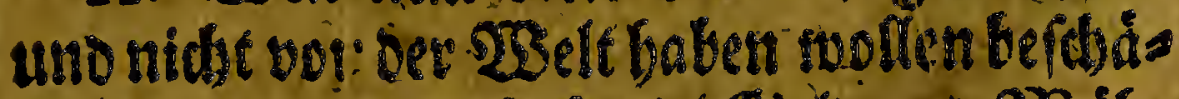

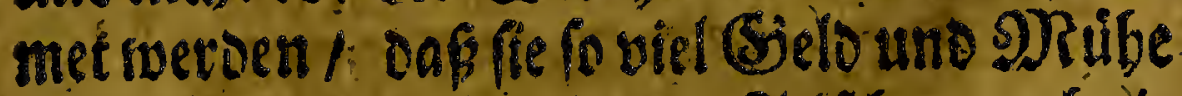

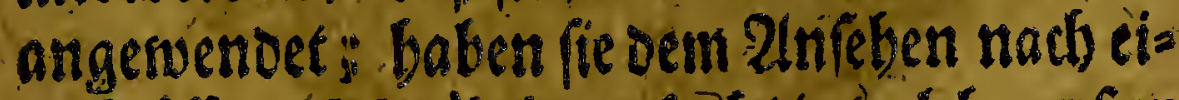
ne fráffige Medicin gefurdst/ twelche aufer 3weiffel aus bem Sioloc und andern Mineralien 


\section{ats $(302)$ है}

ralienzuerlangen if, Diefeg if num eine os dec andere Tinetur, mantomabl in ein Pulver/ooer in einter andern Form bereitet/Damit macbenfie Sdbroisen/ SStechen obet fonftes was. Diefes berfauffen fie lebr tbeues/ Denn ç. fie viel Sielo gefoftes/ fagencel oaferein grop Arcanum fey. STEnnman aber ale

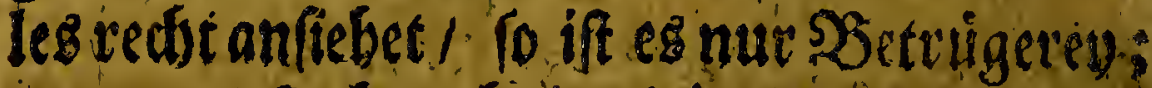
uno was fedet mebr in diefer Máterie als in

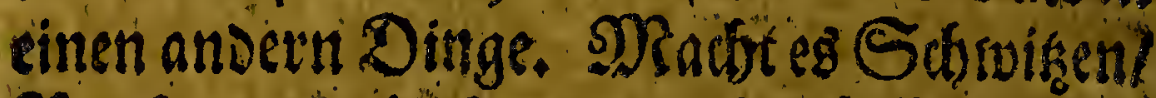
Sitechen oner fonftetwas/ fo duiffen soir ed nich erft mit groffer S)R ribe fucben/ fonoern Eonnen leidster Dabinter Eommett. Uno allo fins alfe Arcana nidste anders als Dett Drantel (Selo zu madien uno ibre fratste SEettel gu curiren. Tas will ibue Dinge ben nidge gar verworffen babert/ ich balse fie aber meinet Soutbinafiung nad bot Sdinatdsereden/ Damit fie aufgeblojen fino. Die Rtancen haben fidj nicht saran zu bin Den / man fan ifnen tobli mit menigen Roften uno beffert Dingen

$$
\text { belfent. }
$$

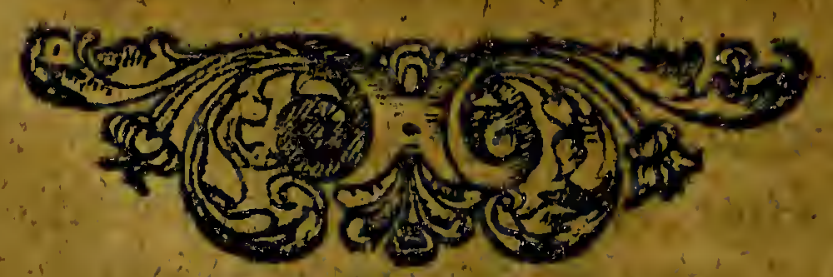




\section{मू (303) Sर \\ Das X XII. Capitt.}

\section{Sondem Sted)en Der Cbinefer uno}

Sapanter mit cinir Tadel/ auf

\section{Dem Sdjmetsbaften}

\section{Drtb.}

I.

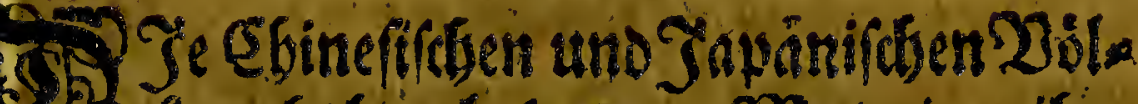
- Deter/ baben befondere Mraterien ibre Riranden zu curiren/als wir bier zu Sanoe bun/ oenn nebftifrer Moxa vamit fie brens zen wie zuvor erme bnt wowen/baben fie cine

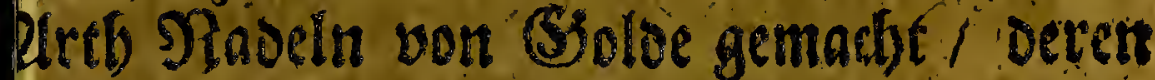
Beforteibung za feben binten an meint Fradat bon podrigra und Durd SEerren SBildtelin Ten. Rbyne M.D. und Prafejfor Chirurgie in Bata via communicitet. Deren

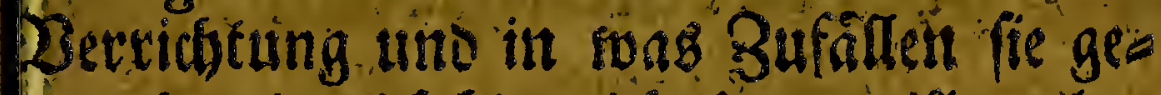

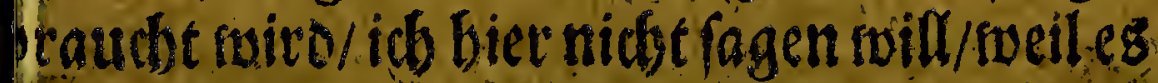

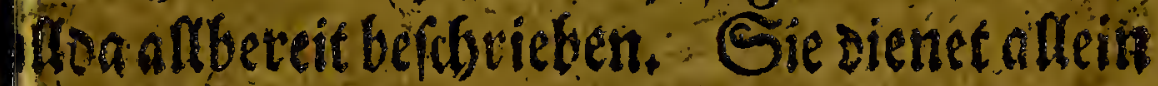
nallen Sdmiensen $\%$ und oie (B)enefung be

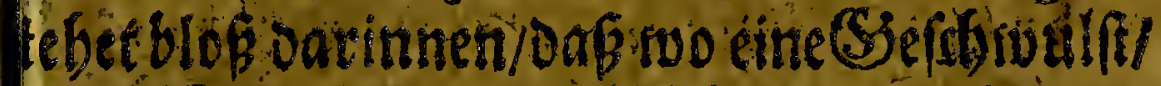

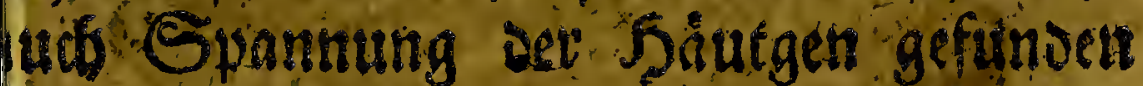
viro/ undoviefe ourds eitre Pradel fotsarffes Dreffergen ooer erruás dergleidsen gesffne vird / - gleidf beffer toiro / uns die Spanung weggebe/ fo dóp viefeg/ wris Span nung 


\section{츤 $(304)$ se}

nung madtite / feimen Unuatb ourch Die.Deffe nung auslâf. (5) ift nunoer Daf mattes biet 3u Eanoe nids aud tbut / oa man fo viel mis Scarificiren unis $\{a \mid c=$ Ropffen umbgebet;

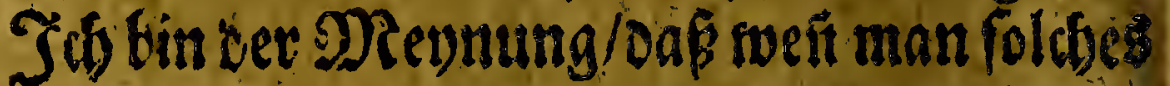
in Podagra uns andert utgeouloigent Schmerşen thác/fo viel (siefredde uno (Sie. twemmer nidfet suirde gebjret fwerden.

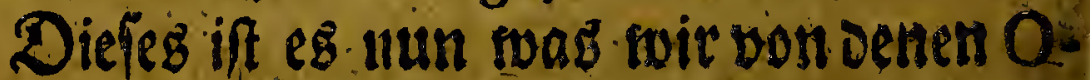
perationen bet Medicamentenlange ver

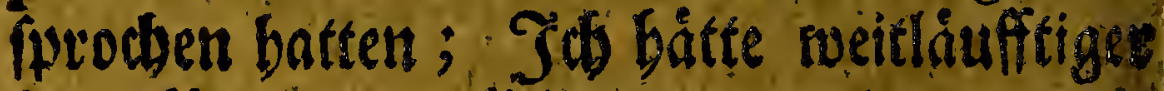
feyn fonnen/ allein wer meine anderi

Sdbriffer outciliefet, rwiro Materie ges nung finoen/ zu erfergen/ twagetruan bier mangeln módite.

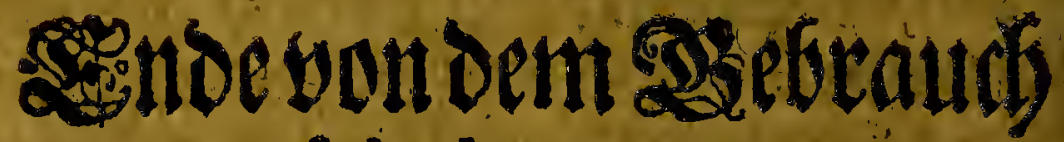 ber Medicamenten.}

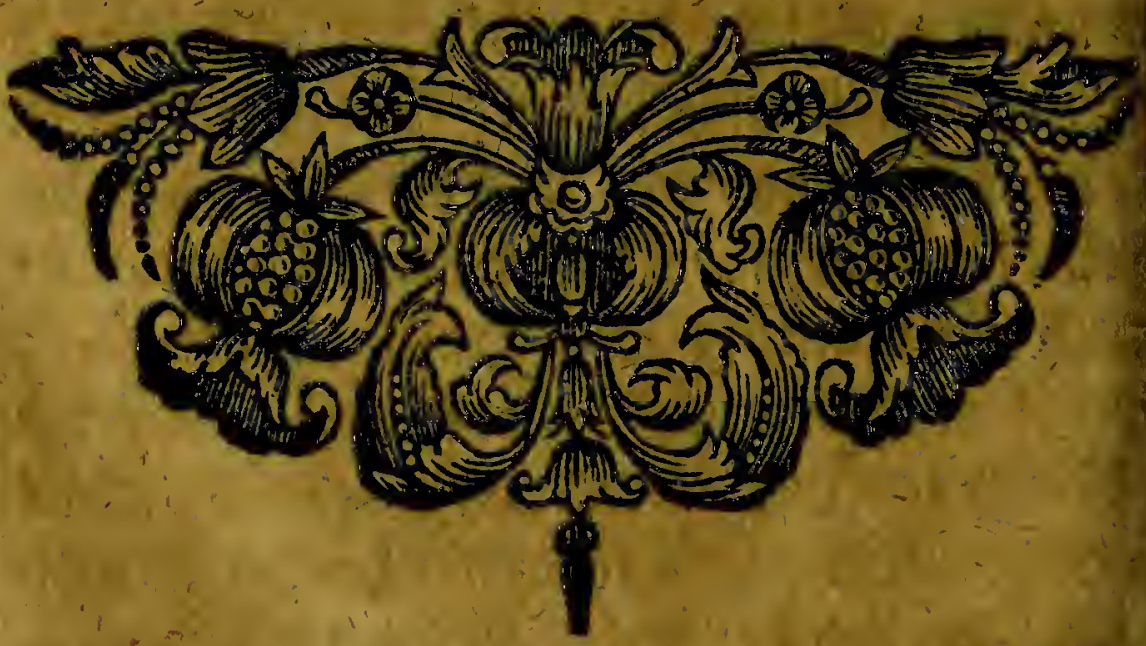


PHARMACOPOEA, Doet

\section{बำ}

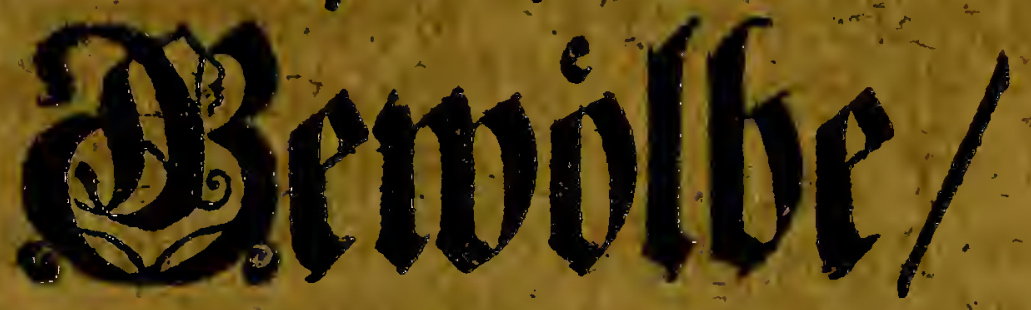

\section{Beffettet}

Deach oer VBeife der beutigen verbefierten Praxi.

$$
\mathfrak{u} \quad 1.26
$$




\section{*6 (306) S*2: \\ I. Flotbetlung}

Bon Denen einfachen Medicamenten/ woelde in einer 24potfecfen fols Ien afferviret werben.

Acori,

Allii,

Althex.

Angelicx.

Apii.

Ariftolochix $F$ - Helenii.

bacex.

Longz.

Rotundx.

Afparagi.

Bardanæ oD Lap- Imperatorix Doed

Biflo px.

Biftortæ.

Brioniz.

Cepa.

Chelidonii majo-

Chinz.

Cichorei.

Confolidæ major.

Contrayerva.

Curcumæ.
Ebuli.

Fceniculi.

minoris:

Gentianæ.

Glycyrrhiza.

Graminis.

Helleborialbi.

Nigni.

Oltrutii.

Leviftici.

Liliorum albor. Mechoacannz.

Perafitidis.

Petrofelini.

Polypodii.

Porri.

Pyrethri.

Raphani ruftican: Rhabarbari.

Rubias
Galangæ majoris 


\section{is $(307)$}

Rubia Tinctor.

Scorzonera.

\section{Taraxaci.}

Tarmentilla.

Valerianæ.

Vincetoxici.

Zalza parilla.

Zedoarix.

Zingiberis.

Stialet.

\section{Arantiorum.}

China Chine,

Cinamomi.

\section{Citri.}

Granatorum.

Gvajaci,

Macis.

Winterani obet Cinamomi. Magellanici. 5ófiger.

\section{Aloës.}

Gvajacutin.

Colubrinum.

Nephriticum.

Santalintu album.

Citrinum.

Rubrum.
Saffafras.

Blâtter u. Kräutet. Agrimonia

Alchimillat.

Althex.

Apii:

Ánethi Sum̃itates

Artemifiz.

Baccabunga.

Betonicæ.

Cardui Benedicti. Centauriiminoris Chamedryos.

Chamepytios.

Chelidonia ma joris:

Cochleariz.

Cherefolii,

Fœniculi.

Fúmaria.

Hyperici.

Hederæ terreftris.

Hyfropi.

Lavendula.

Lauri.

Lupuli,

Majoranæ.

Malvæ.

$4 z$

Matri- 
Bardanz.

Cepa.

Cannabis.

Chardamomi nónoris.

Cardui benedicti.

Carei.

Coriandri.

Cubebx.

Cumint.

Dauci.

Froniculi.

Fænugraç.

Fraxini.

Grana paradifi.

Hordeum mun datum.

Hyofeyami albi. Nigri.

Leviftici.

Lini:

Lupini.

Milii Solis.

Nafturtii.

Nigella.

Oleris atri.

Paftinace.

Papaveris albi.

Nigri.
Petrofelini.

Porri.

Raphaní.

Rutz.

Phellandri.

Santonici.

Sileris montani.

Sinapi.

Sophix.

Staphidis agriz.

Tanaceti:

Flusige Săffe.

Acetum.

Vinum.

Bietrodinete Saffel

Gúmmi uno

Sartice.

Aloë Sutccotrina. Ammóniacum. Affa foetida:

Balf Peruvianalbum.

Nigrum.

Copayvai

Benzoin.

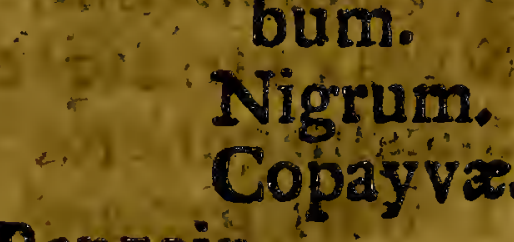

Camphora.

Colophonia.

Euforbium.

$\mathrm{U}_{3}$

Fu- 


\section{สูก (310) है?}

Fuligo famini.

Galbanum.

Gutta Gamba.

Gummï a nimæ. Arabicum.

Caranna.

Elemi.

Tacamahaca.

Lacca.

Ladanum.

Maltix.

Myrrha.

Opîm.

Opponax.

Pix.

Refina abietina.

Guajaci.

Pini.

Sacharigenera.

Sagapenum.

Sangvis Draconis

Scammoneum.

Styrax utriusq́ve.

Succinigenera.

Tartari genera.

Terebinthin. ge-

nera.

Thus. menta bon

Sbieten.

Adipum variagenera.

Album gracum.

Axungia porci.

Bufones ficcatæ.

cañcharides.

caftoreum.

cera alba.

Flava.

conchilia.

cornui cervi.

Ebur.

Felanimalium

Mel.

Millepedes.

Mofchus.

Oculi cancri \& chelæ.

Ovorum contices \&.c.

Sapo venetus.

Sepiaos.

Sperma ceti.

Stercora animali-

Tefte 


\section{$25(312) \leq 2$}

Die SBlumen aber/ pfluide man nenn fie

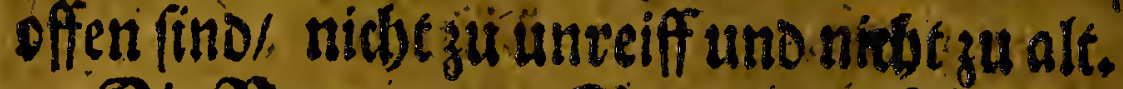

Die Secten unt Saamen miffen anc gefantmlet ineraen/ ebe dak fie reiff find/ weil fie benn in ibrer vofflen Rraffe tnods nidbe fino.

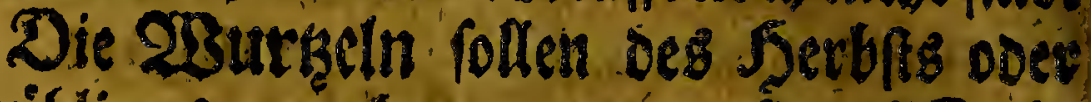
Fruiblings gegraben tweroen da fie vorf Saft

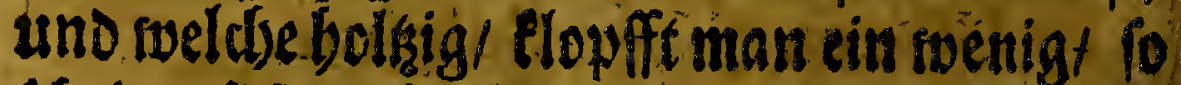

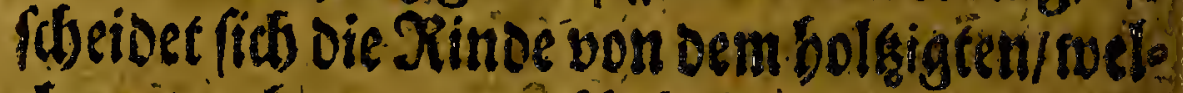
dhe getrodinet und auf betgalten swird.

CEzif am befen / menn es feur tan/ Dab man alles frictib bat/Denn oa find fie voll (s)ein frec uno ain tráfftigfien / uno beffer als oie

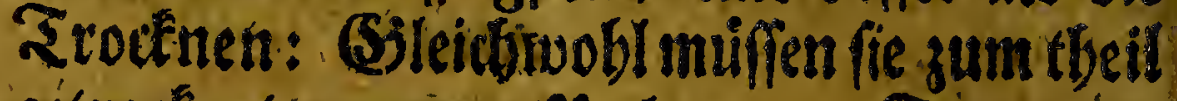
getrodínet werdetn/ goulver uno Tincturen sarausg zumadben/ audd tan man fie in 25 int

ter (dobwerlicis anders als stroden baben.

Die SRinden yon Den SBátumen mu man im Sruiblinge/ -2Binter uno Serbf iblo

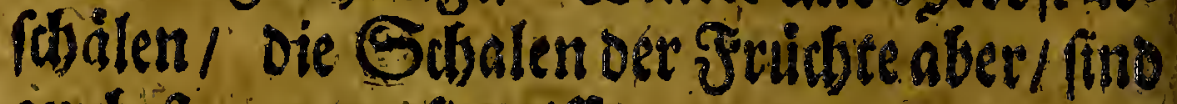
ambefien roenn fie reiff.

Die. Sólser weedern zu ver Beit eingettras.

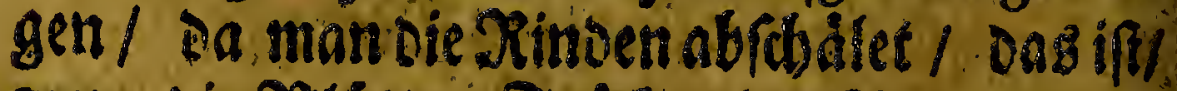

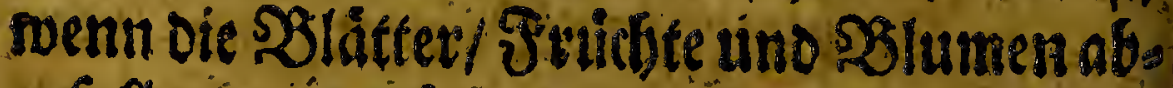

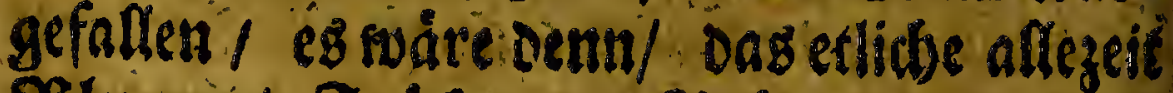
SBlunten/ Frrichte uno .2lätrer trigert/ / tönte Eeine Beit geferst roeroent.

Cz folte ieder 2lyotbecter feinen eigetren 


\section{$x(3+3)$}

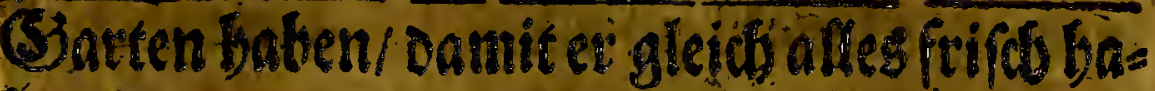
ben tonte/ Damit bie-Doctores nidsetmit bets Dorbenen/ alten / Rrafftloferi Dingen betros

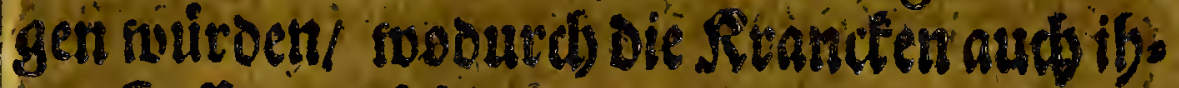
ver Soffnumg foblen.

\section{Fotfliluitg.}

\section{Bon Diffitlirten Beiftern.}

Spiritus Aromaticus f. Cardiacus.

R. Cinamomi. 3 j. R. Biminet 2. Soth.

Cariophyll. 畒. Tèglein r. Eotb.

Macis.

ORufcaten=SShitb.

Nucis Morch. Niufe iedes 2. an. $3 i j$.

Piperis : tj: geffer 1, Strup.

Cortic. Citrixo- Jrifde eitronen. cent.

Arantur. rec. an. $3 \mathrm{ij}$.

\section{Sifialen}

Pommerantsen.

Stralen iedes

$$
\text { 4. \{ot6. }
$$

OStandtiv.6.Ras,

Spiritus vini. to xij:

F. s. a. deftillatio. Difinite es fvie fidis geboret.

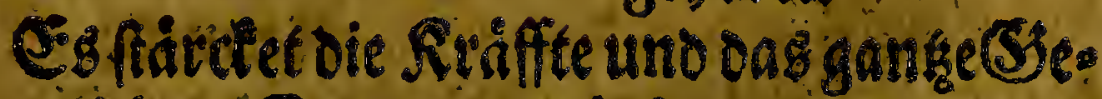
Wirne febr: Derorvegen befodert es ben Lim. Lauf oes Ssluthes une andere Saffite/ wein 


\section{$20(314)$ sै?}

man auf einmabl einen siffel ooll mit ooer of of ne 2 Bein Davon braucht.

Spiritus Diureticus.

Deet Sain=uno Sinepreibenden Spiritus. R.Rad.raphani. R.Rettig.

Ligninephrit- Griefs Sols.

ci.

Saffafras.an.zju. Saftefuniteder. Sotiv.

Succi. Hedera terreft.

Petrofelini.

Cherefoliian.

3x.

Baccar.Juniperi 2 Badjoloetb.ein h.pf. Ibf.

Samen von swile

Sem.Daucivulg.

Fraxini.

Leviftici an.

亏iij.

Thereb.Venet. lbj.

Spir. C.C. $3 j$ v.

Vini optimi. 呫就j. oen PRóbiten cefojenssatim. Stebftivel licoes 6. Sotb. Denediffer Sete Hentin . Dof Spirit, von 5itids: Sotn 8. Sotb. Sitten Ssein 6. Rannen.

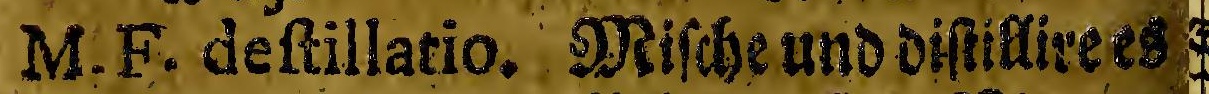
Diefeg giebr man soffel neife in Riecen 


\section{xes $(315)$ है।}

235eb/ es if aud oienlicb in 2erbaltung pes Uring falte pife uno Saamen-Flit.

Spiritus Volatilis oleofus.

R. Cinamomi jiv. R. Bimmet 8.50t5.

Caryophill.

Macis.

Cardamomi.

Sem. Carvi.

Galangx.

Zedoariz:

Angelica.

Cort. Winteranian. 噍:

Citri. Aurantiorum an. $3 \mathrm{ij}$.

Safratras. 3iij.

Spiritus Vini $16, x i j$.
Sieglein

MRurcatenblitb.

Earbemumen.

Rarbe.

Silgan.

3itwer.

Engelic.

3Ragellanifótert Bimmet iedesz. Soth.

Citronenidaletr. Pomerantigenfatas len iejes 4. Sotf. Safferag 6. Soth. Szranderwein. 6 . Rannen.

F.s.a. deftillatio biffifite es noic finge gebiret.

Diefentiquorem nitit thue bazu den teitten Sbeil Spiritus Salis armoniaci, uno oiftidirc es niederumb aufe neut.

Diefer Spiritus swito stroyffen = seife in Syaniffsen ovet andern fiffen 2 zeine ges

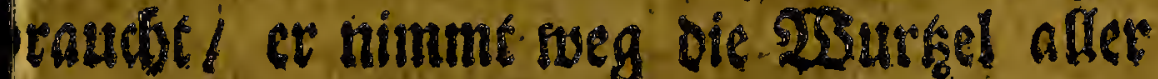

Rranct 


\section{$\sec (316) \mathrm{sis}$}

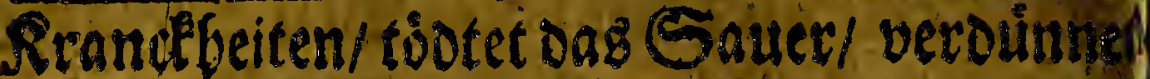

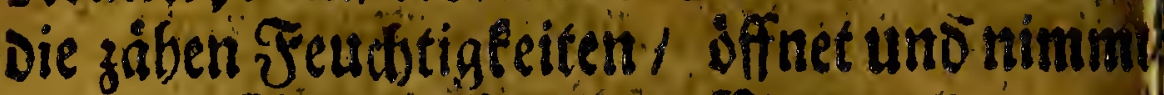
seg affe Derftopffing oer Singerveioe/ on ourci oas Silutb fluigig roito.

Spiritus Matricalis.

R. Olibani.

Myrrha.

Succini.

Maltich, an. part. æqv.

Spirit, Vini rectif. qs.
R:Olibanum. Divrctien. Xgtfeiti.

Mraftic eines fo vied als Das ander. 2libgezogenensbrane terwein fo viel ges mung ift.

Def oer 53 trandeswein vier Finger orubert gethet digere. Es treibet oie tootegrendot uno

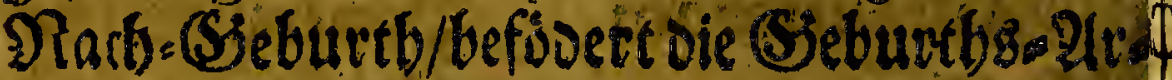
beit uno 3) Sonats. Stunden/bon r bis 2. Soth

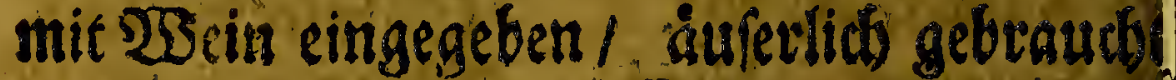
vertreibet es oie barten (S)efowulfen / nim beylet oie 33 unden.

Spiritus Carminativus,

oder $\mathfrak{Z}$ indtreibender Spiritus.

R. Rad.Angelic $R$, 2ngelic 8. Sot ऊjv.

Acori.

Imperatorix.

Galangæx an. 掉.

Fol.Rorismarini, Nofipmarien. calmus.

Deeifterti.

(Sialgan jedes r. Sots

Ma: 


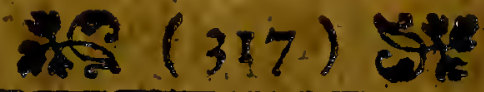

Majoranz.

Rutz.

Menthx an. $\mathrm{Mj}$.

Bacc. Juniperi.

Laurian. ̧̧ij.

em. Anifi,

Carvi.

Leviftici.

Gardemom an.zj.

Macis.

Cariophill.an. $3 \mathrm{ji}$ Cort. Aurantior.

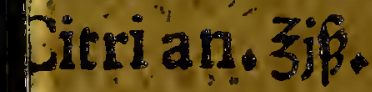

Srajoran,

Rauten.

DXingsent ledes eine Jano yoll.

23sadboldet $=25$ eren. Sorbecrent iedes 4.

$$
\text { Sotb. }
$$

2nicts.

Carben-Saamett. Siebfiofel=Saamen. Carbemume: ícoes 2. Sotb. Dircatenblitf. Neglein/ieDé 2. qv. Pomerantsen uno eitronenidsalen ise Des $3+$ Sotb).

Sấ Diefes mobl zufammen geftoffen cine Padte in 28 ranoterwein weichen uno oiftidis. ces nady dex Runft/er zertreibet Die WSinder perountmet den Sdblein 1. uno bringet die iräfte nieder; man nimme cinen siffel ogo por mit ooer obne WBeint:

Spiritus Cornu cervi, ober Situd Sourn Steif.

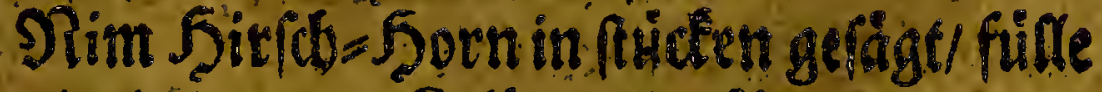
amit eine croene Rolben over Retorte/ uno veibs mit offenen J̈euer pergradus; fdreie 


\section{मी $(318)$ bु?}

De Das ftinctende Deloavon / uno Diffillit oenubrigen liqvorem mit oper obne Aro matifbe Rráuter.

Grtemperiret das Santer/madt die zod bet Fendigfeiten flifig uno if eir Uni verfals? ) Rittel in allen Rrand beiten fo wo bht zu præfervirutg als curirung/utan nimm 20. Tropffen Davon in 20 ein ooer anoern bequenien liquore, es madyet Sdwitsen uno teeibet Den 5arn mit allen Sirieg uno Sane. Qlufgleitse 2 beife rverben geniads Spiritus Eboris. Der Spiritus votb

Offium.

Fuliginis, \&c.
Soemen. ciffenbein

sueroen auf gleidbe sweife rectificiret/ fino von gleidsen Rrafften uno muiffen aus alfa gebiautit soerden.

Spiritus Salis armoniaci. R.Sal.Armon; R. Salmiac.

Tartarian.part. - 23 einftein Sals te æqv. Des gleid) viel:

Berlaf jeoes abfonoerlich in narmen 25 af fezigief es sufammen uno oiftifire es in Sat Del oen' Liqvorem reetificire/ uno bed robrees zum (Sebrautb.

(Er if gur in fobarbotidgen Rrand beifen) als ein groffes antidotum, oas Sauer und 
Shbleim jutemperiren/in Wein 10. Tro uffengegeben.

\section{Spiritus Antifcorbuticus.}

R. Fol, Cochlear. R. Euffelft. 4.16 .

Ibjv.

Rad. Raph, Syl- 2gilde Xettige 2. Ib. veft. Ibii.

Cort. Winterani, MRagethanifde Bime క̈iv.

Sem.Sinapi, ziv. Semff=Snamen ies.

Spir. Viniqs. ter 8. Sott). SStanderwein fo viel genung.

F.S. art.deftillatio diftilive es suie fifbo gebơret.

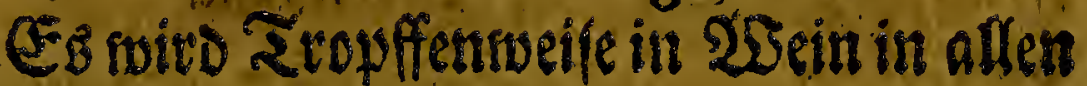
Stbarbodi gebraudit.

Spiritus Cochleariz.

R. Fol, Cochlear, R. Eoffelfer, 4. Ib. lbjv.

Spir. Vin. qs.

S5randeterwein fo vid getrung.

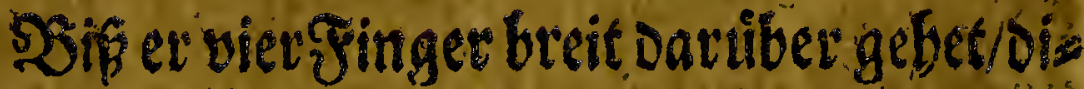

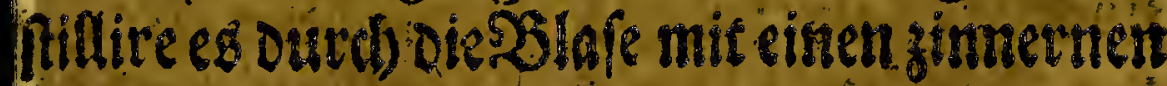
Setm.

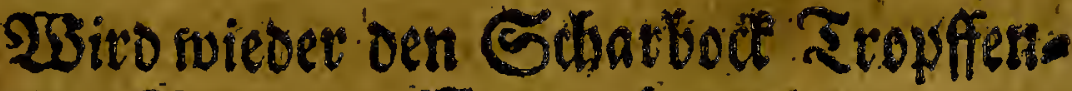
paifein SBier poer 23 ein gebraudtit. 


\section{$28(320) 3$}

. Iuf foldes sweife madbt man Dent

Spirit. Allii.

Anifi.

Aurantiorum.

Camomill vulg.

Citri.

Rom.

Carvi.

Fœniculi.

Hederx terreftr.

Juniperi.

Laurt.

Majoranz:

Meliffæ.

Menthæ.

Pulegii.

Rutæ.

Sabinz.

Salviz \&ce.
(S3cift bon Rnobs lauch.

2niep.

Domerankett:S 5. Sjemeine und Röm. Eamillen. Eitronert=Scbalen. Sarbe.

STendisel.

Siunderman,

25iatsoloer.

sorberen.

Drajorats.

Drelisen.

Nintse.

Poley.

Rauten.

Sadenbatum.

Salbey 26.

Uno alfo metoen aus allen Reáttetti)

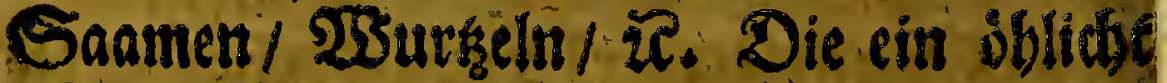
flichtig Salk/ baben Spiritus gemactst Dee

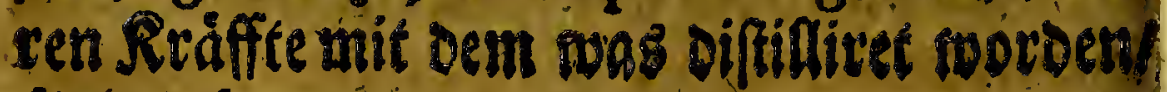
úbétrinfomumen. 


\section{is $(321)$ \\ III. stbtheilung}

2on Sincturen.

Sinctur zu purgiersin.

R. Rad.Jalappe it $\mathrm{j}$.

Spiritus Anifi.

\section{carvi ana lb.iij.}

Beuds bie Tinetur beraus / filtrive utso beruabre fielonobon ein bie orey soffel genoms men purgievert in allen (S)elegenbeiten.

4. Tinetura Errietica zunt 25 recthen.

R. Vitri Antimonii pulverif? 倨,

$$
\begin{array}{ll}
\text { cinamomi, } & 3 \text { Vini Gallici, } \\
3 \times x
\end{array}
$$

Bend bie Tinetur bargus / filtrivetmo tefuabre demligvorem, Dic Dofis it von

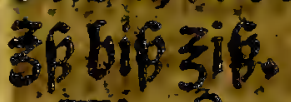

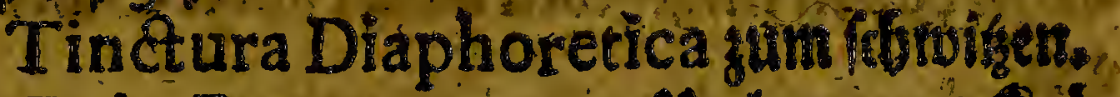

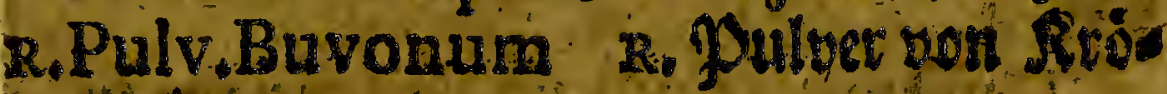
tent.

Extr. Scordii.

moontrayerva. Angelica: Zedoande. Juniperi. carde-bened. ana. ऊij.
Extraet vonkacten: Rrioblatio. Siffetroutr. 2ingeliva: Bitwet: 2radbolott. Carbelhanetitt. iedes 4 . Sot $x$ Ref. 


\section{$105(322) 58$

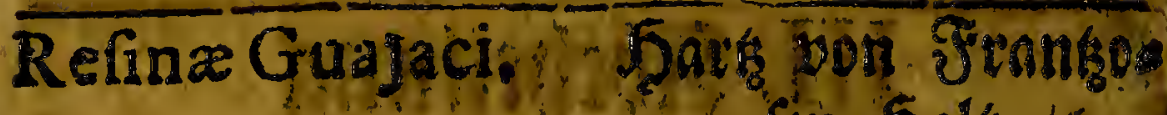

'Camphora ind Camphex. rene 5001 . Caftorei, Myrrha. , Morurehen. Crociana.3j. Saffratiedes 2. Sotb: Sal volatile cc. 张. Sals bon Sirfdo

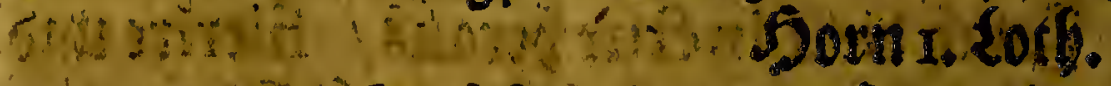
Opil Spir, dephl, Opii,2, sventl. jiv.

Olei macis deftill, Difillizt 2 Rurontent.

Cariophillian. Dieglein ieoes

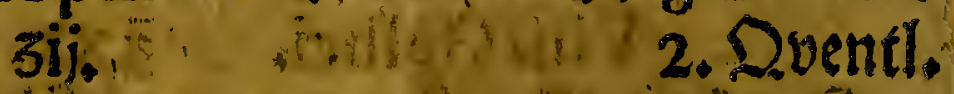

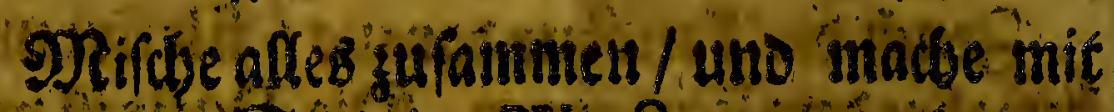

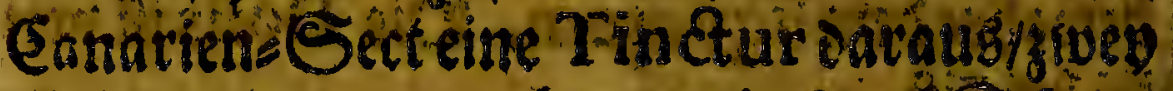
soffel oapon eingegeben mabt fart Sabriv tert.

Tinetura Anodyna, Sdhmeren ju ginien.

R. Opii thebaici $3 j$.

Sonteibe es in tleine furutgen/l laf es tros uen nerden/uno wenn eg zetrieben: Bend sie Tinetur carauz mitzxx. Spiritus volatitis oleofi, filtrire und beronbuefig zum

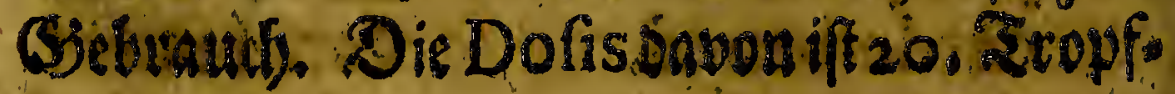
fen. 1 bost

Tin- 


\section{$615(323)$ ह5:}

(Tinctura Diuretica, 5atn zu treiben. R. Radicisononi- R. Saubediel.223ut: dis.

Eryngii ana: కij.

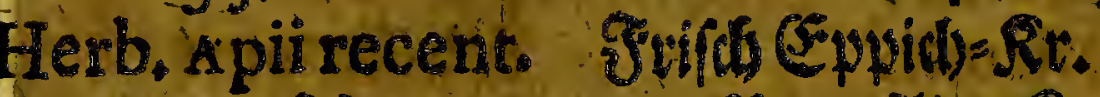

Petrofel. rec:

Dannestreu 2 ite Bel

Peterfitien Rr.

Sabina ana.

Sabenbiebesx. $\mathrm{Mij}$

Croci.

Sem. Apii.

Dauci.

Sileris mon- SRoten-Rintel iebes

$$
\text { tan.an. } 3 \text {; }
$$

Millepedum.

Baccar, Juniperi

$$
\text { an. siij. }
$$

Ligni Nephritici Sirief=50lis 4.6otb. 3ij.

Sal.Succin,vol.3j.

Spir, hedere terr.

Ib.jv.

F.. . a. Tinctura.

Safturan.

Sano vod.
Rellere Stjaben.

Sorbeenieses 6,Soth

Igttein Sals 2. Soth. Spiritus bon (Situm Derm. z. Jiant. DRacise nadib so Runft eine Tinctur tate. matr.

Woito soffelweile mit ober obne 256 in ingenommetr.

$$
x_{2} \text { Tin- }
$$




\section{-}

Tinctura Stomachica, vor vem N Nagets. R.Rad.Acori. R Rnimus. Angelic: Galangx. Helenii. Oftrutii. Zedoarite. Cort, Cinamom. Arántior.

Citri.

Macis,

Caryophillorum. an $3 i j$.

Abinth, vulg.

Majoranz.

Mentha,

Angelic.

(sialgani.

Allano.

Deiftertuuns:

Bitwer.

Bimmet.

Domerantren:Sar: Citronen: Schalen. פ) Surcatenblútb. Reglein iedes 4. \&? Rorismar, an, $\mathrm{Mj}$.

Crocii

Cardamom.

Carei,

Piperis.

Sinapi.

Myrrha.

Aloës an. 引j.

Spirit,Vini lb, xij, M.F.s.a. Tinetu-

ra.

25ermutb.

SPrajoram:

Mintşe.

Rofmarien itoes ein Santo voll.

Saffran.

Cardemimen.

Rarben.

OSfeffer.

Semf.

Novribetr.:

Aloeg jeder z. Solt.

Stranotem.6. SDR a

DRifece es uno mrictien

nads ocr Rumftit

ne Tinctur: 


\section{to $(325) \div$}

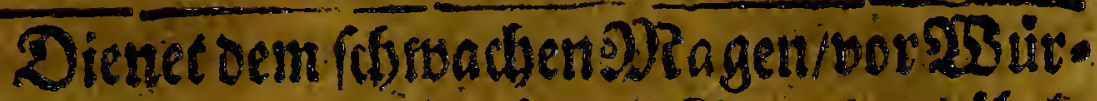
gen/ Streden/-verlobrnen Eppetit/ uble

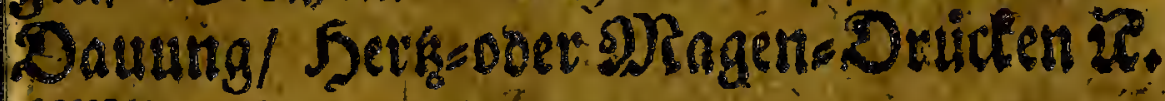
Loffelrbeife eingeriomment.

\section{Elixir Antifcorbuticum D. Bontekœ.}

R. Cinamom. 3iv. R. Simmet 8. Sotf.

Nuc. Morch. $3 \mathrm{ij}$.

Macis. 3 i.'

Coryophill. 3 :

Cort. Citri, von' 8 .' 2lepffelin. Aurantior. $n \cdot 4$

Calamiarom. Cróciana. jjif.

Spirit, Vinilb. vij.

D)ufcaten Plif 4 . Solutb 2, Soth. Neglein I. Sot6. Sobalen vonfriftaes Eitronenno. 8 . Pometantsent

$$
\text { no. } 4 \text {. }
$$

Calmug.

GansenSaftranie des. 3 . Sotb. Siranotefoein véro. tebalb DNap.

Beud oataus eine Sinctur nach oer Runft.

ssenn die sinctur abgegoffen/fo gers auf Dab úbrige nod etwas SSranotewein / Dent

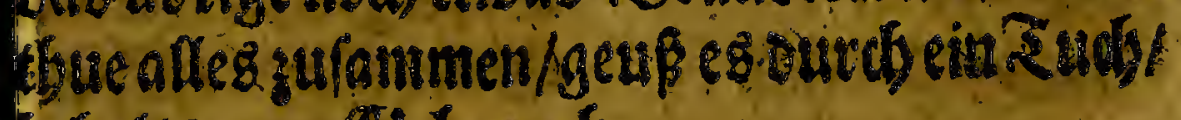
bebaltg zum. Sobraudh.

Denn wits cin Soffelwo getrominen/ fo sobt zu Prafervirung als cuxirung des Solowodt.

$$
X_{3} \quad \operatorname{Tin}
$$




\section{us $(326)$ se}

Tinctura ad Vermes vor oic Wuitmer. R.Sem. Santonici R. Somen sorišn? lb.j. ten Enpteffen x.pf. Flor. Tanaceti şlumen von Reirs jjv. farn 8. Sosf. aroci 3s. Saffran I. Soth. Vini Hifpanici Spanidsen $25 e i n$ lb.iv. 2. 2$) \operatorname{Cap}$.

F.r.a. Tinctura. Dadis oaraus citte Finctur. Davon nimme man Des DRorgens einet? siffer voll.

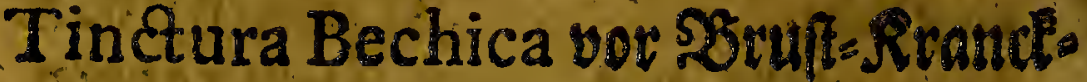
beiter):

R. Rad.Helenii, R. Zllanb=25,

Angelic,an. $3 i j$, 2ngelic,iedes 4, Rot6 croci 3j.

caphoræ 3ij.

Maftichis.

Sem, Anifi,

Saffiati 2, Rotb. Campber 2. Duent: Maftip

carvi ana. 3 j. Benzoin 3 . caftorei 3 i. Opii Siccatizs. Sinif. Cartie iedes 2. 20t5. Sesezoar r. Roth. sBibergeil I. Oventit Setroutnet Opii eitt balb Doentl. Spirit,Anifilb.viij. Inifs.(Seift 4. Naf. F. Tinctura, DRadjecine sinctu. Davon wiro untertueilen einkoffel boll ge? nommen. Tin- 
Tinctura ad Cafum, vor diéfo gefallen.

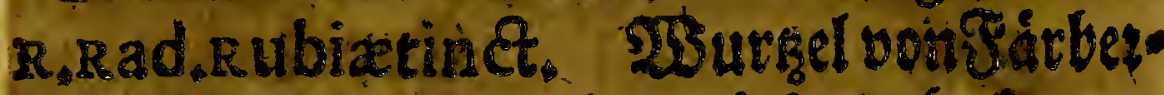

उ)

croci $3 i$.

SentiNigella, Sophize.

Nắfurtii.

Extract. Pyrola

$$
\text { an. } 36 \text { ? }
$$

Sangv. Hirci,

Spertw, cetian,3it?

Vini

Hifpanici

Ib. viij;

D. Tinctura, Deadfe cine Tin Etur.

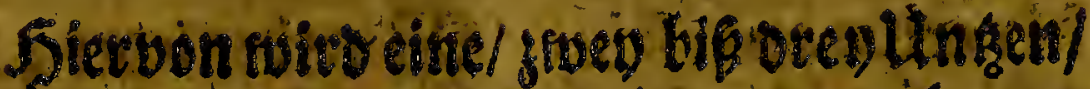
nad Selegenbeit gegehen/unb oient Eeuten fo bodi gefallen/erforocken/ uno oengleidben: Tinetura ad Menftrua provocarda. R. Rad. Angelica. R. Ingelic.

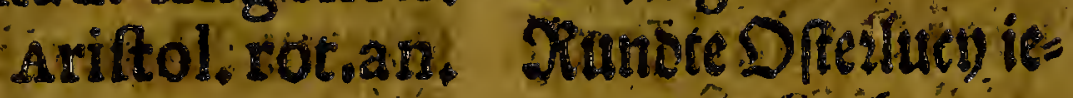
ziv, Des. Eorb.

Extr.Artemifia, Extr.'vons Sevfuf.

Matricaria.

Pulegit.:

Sabinz ana, 3 i.

Dertert.

Dolev.

Savenbauit.

teอtร 4 . Rot6.

Saffent 4 - Sotb: $x 4$ sem. 


\section{Is (328) 3*}

Sem, Anili.

Sanien vott 2niep.

Apii.

Dauci.

Leviftici.

Equid:

DRobrentimmel, Riebitidiel.

Baccas,Juniperi. Myrrhxanazij, OleilSuccini $3 j$;

Spir. Vini lb. $x$. F.f. a. Tinctur.

s3andioloerbesett. 2) Rurrbenied 8.4 .8 . Zlghtitin-Del 2 Roth. SStranterwein 5. SD: Dratic Daraura eine Tinctür.

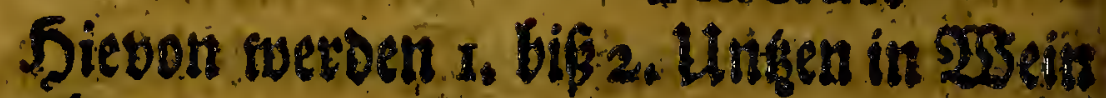
gegeben.

Tinctura ad Partum facilitandum. R. Pulv. Succini, R. pulverifitt.2igt

Myrrhix ana. Alfæ fertida 弓ii. croci. ftein. SD?turterniedes 4.8 Teufeleored 2, 90. Saftran. Spirit, cG. 3 ;.

Spirit. von Sirfer6. 2. Roth.

Vini:Optimi $3 \times x i j$ F.f.a.Tinctura.

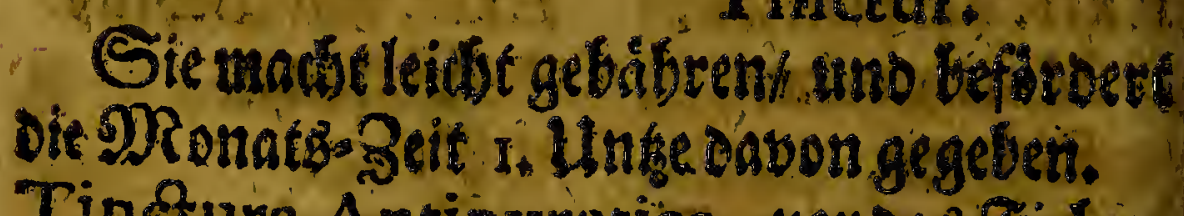
(6)uten खein $x_{*}$ D) Sieraus werde eithe Tinctur. .ntr. Tinetura Antipyretica, anc bas fieber. R.china chinx 3 ij. R. Esinä Ebind 4 . Radix Gentianx. Enksian. finc is

Herb 
称 $(330) 3$

F, f. a. Tinctura. SRodle bierbon einc Tinctur.

Diefe Tinctur bienet in allen Saudis

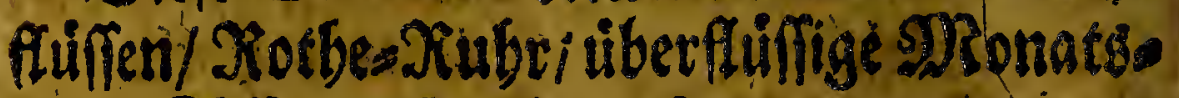
Beit/ Róffelmeife gebraudot.

Tinctura Anticolica, vor die Colica. R. Sem. levift. R. Sament bon lieb.

Carvi. Anifi.

Cort.Aurant. Flor Cam. rom. an. 3 ij.

Croci.

Macis.

Piperis ana, $3 j$.

Opii Siccati $3 i_{j}$

Spirit. Vinilb. vij.

M.F. rinetura. Lign. Saffafr. fodtel. carbe. Inies.

Somerangen-Sif. Saffaftáp. Dobinifle Camillet ieoes 4. Eoth. Saffrari. Mrufatenblutt. D.feffer ietes 2, Rot6. Setrodnet Opium 2. quentl.

Sivantert. vierdet balb פRap. Prade es zu sine Tinctur.

Tinctúra Antifcorbuticá, bor den Sdiarbodé.

R. Rad.Angelica Helenii.

R. 2ingelic,

Altano.

Cort, Arant.
Pomeransenicialen. Citri, 


\section{6 (33x) $3 x$}

Citri. Eitunendobalen.

Winterani, ORngellanifise Zimo mefiedes. Eotf.

Baccar. Lauri,

Socbern.

Crociana. 3j: Saffrartietes 2. $\Omega$.

Sem.Sinap. zit. Semf: Samen.3.R.

Spirit. Cochlearia Euffeltr. Giejpiervo

diocris nots tebalb MRap.

lb. vij.

M.F. Tinctura.
Sllifose es uno mas doe cine Tinetur. Daraus.

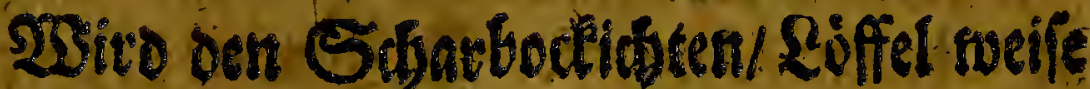
gegebet.

Tinetura Antepileptica, vor die fallento Sudje.

R.Rad. Angel, R. Angelic.

Origani cret.

Ruta.

Salviz.

Cinamom.

Caryophyll.

Cardamoman.

Croci 张.

Spirit:Lil.convall.

Rorismarini. Rogintatien iebes $x_{i}$ M.f. rinctura.
Origan. cret.

Rauten.

Salbey.

Bimmet.

Reglein.

Cardemont jebes 2: Rotb:

Saffert t. Rotb. Geif bon Dreberts bluingen. Pf. 
פ⿹丁口 alten Seuten soffelweife gegeben.

Elixir Proprietatis.

R. Aloës,

Myrrhæ.

Crociaña, Unc.j.

Spirit. Vini, Unc. $x$.

M. F. Tinetura.

Egfácutet Den DRagen/offnet oie CingervetDe/ 20. Tropfen in einen Liqvore einge? trommen oes SRougens nuidtext.

Tinctura Cordiaca, Jers Stàtung. R. Cinam.Unc,iv, R. 3immet 8. Sotb. Nucis Mofchata. DRucaten-Nuipe.

Macis.

Coryophyllor. Crociana. Unc. j. Lign.AlaesUnc.6. Spirit. Vini.

Vini Hifpan.ana.

Unc. xij.

F. M. rinetnra.

SE fódere oen Umblauff oez solutbes mo erguidect die Seifter.

Tinctura Martis, Stabl-Tinetur., R. Limat. martis R.Stabffeilt 8. Rotb: Unc.iv.

Tartari albi Unc. 'SDeiffen Iseinfein. viii.
Sieglein.

Saffranan.2 Rotb: 2loess Sols 1. Roth. S5ranterwein. Spanifhen ssein i. $\$$ f. 


\section{मूल $(333)$ है}

Aqva commun. Gemein 30 afier 4 . Ib. viij. Nap.

Rodie alles mitemattoce bif cs trocen mito/ setud Die Sinctur betang mit Strante. wein/ Dap tibrige fode niedec mit 23 affer. biß es troden wiro / geuf oie vorige Sinctur barauff un tbue foldes fu oreven mablem filtrite uno bemabre

Es nirb eine balbe Untse vabon eingege ben toieder alle Detfouffungen.

Tinctur Antimonii, Finctur von Spieß̈? (s)lấ.

R. Salis Tartan. R. Wreinftein Salk. Antimonitpulve- Spiéso(Sing putoe. verif ana part. vifiretiedes gleiff aqual. vil.

Raf es sulammen etliche Stumben lang it

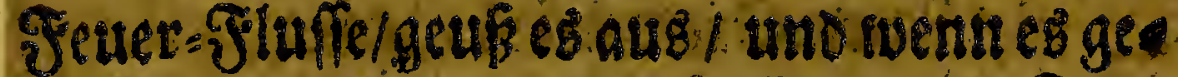
foffen fo zeud mit Alcochol vinibie Sime ctur berauş.

Sie dienet in langeniengeti frand feitem Detfopftungen/uno Sdbarbod zo. Trovfo ferin Spannifben 2 ein eitigerommen. Tinitura Stccini, Sinctur von Rgffeit, R. Succini albi R. Sulvertitifneife pulviunc.jv. Spir: vin. dephlegm. Unc.xiv. ren 2geffein. 8. Statcen Szranote wein $x$. Roffel. Salis 


\section{$29\left(334^{\prime}\right)$ set}

Salis armoniaci Spiritus mon Salk

Unc, f.

M.F.rinctura, SRadje Damit time

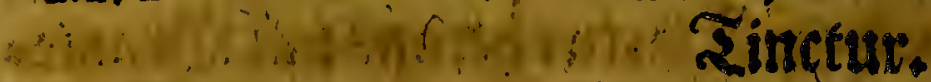

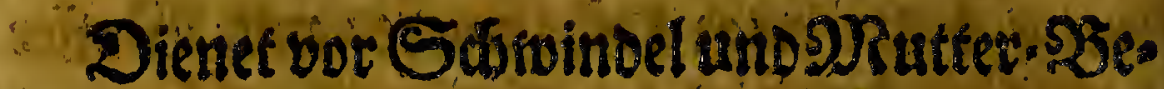
pchinerung/ Tropffen=sweife in wein einges nomiten.

Tinetura Croci, Saftun SEents. R. Croci opt. R. SiangenSaffran Unc.ij.

Spiritus vini opt. Giten Sscantenvein Unc. $x$. F. Tinctura.

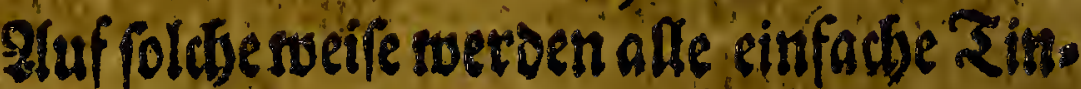
cturengemactit ats:

- Tinctura Gummi Ammoniaci.

Arantiorum. Affe Fctidx. Caftoræi.

- Catechu. Citri. Galangx. Galbani. Gentianz.
Gvajaci. Ligni Albes. colubrini. Saffaftas. Sabinze. Sem.Santonici. Zedooria. 


\section{$95(335)$ एक \\ IV. Sletbeilung. 23on' Extracten. \\ Extractum purgans,}

R. Pulp $x$ Colocynthidis Unc, if. Agaricialbi.

Heliebori nigri ana Unc. $\mathrm{i}$ :

Mradse aus oiferm cine Sinctur mit.

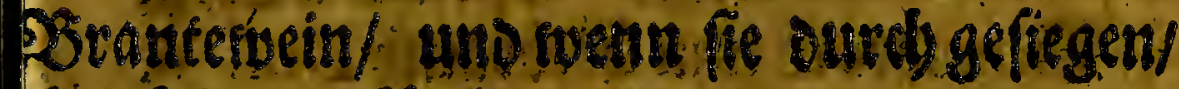
Whue folgense Pulver saju.

R. Aloës opt.Unc. ii.

Scammon, pulv. Unc.;

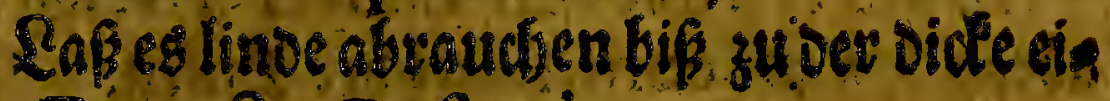
treg Extracts. Dofis 3i.

Extractum Diaphoreticum, Sdjweifs. tweibenber Extract.

R.Rad, Angel.

Contrayerv.

Petafitidis.

Vinceroxician.

Unc.iv.

Ligni

\section{Unc. viii.}

Saffafias:Une,vi

Herb.card, sened. scordii.

Cent. mip ana. Ib. ii.
R. Engelic.

Ssiffruartsel.

Peftitentsow.

Stimaltento, ieves 8. Roth.

Sunntsofers 5 . 5 lis.16 Rotf.

Safiafrafs.8.Rofb. Eardebenedicten. 0 Scorbient.

Taufens=(Stiloent?

ieves $2 . \Omega P F$.

Bac. 


\section{ํำ $(335)$ है}

Baccar.Laurilb.ij. Rorbetn.2. \$f.

Croci.'Unc.iji. Safferan. 4, Rotr:

2lus diefen Dingen zeuds mit nuarmen

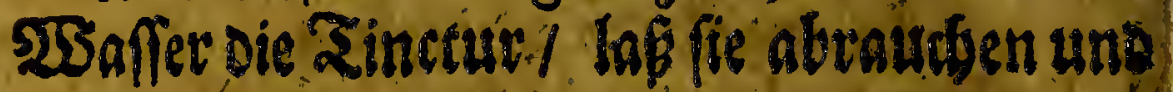
thue dazu:

Sal, vol, C6, Unc. ij, Sirfobbotm Sal 4 . lote

Opii Siccat.Une. B. Opium 1. \&oth! Berwabre sum sebratid Dor. 7 \%.

Extrąum Emeticum SEred Exirac.

R. Succil.decocti Tabaci q.v. Iaftes abrauchen zur confilten oer piffen.

Dofis gr. $v$.

Extraetum Anodynum, Sdjmersial. lenioer Extract.

R. Opii Siccati R. Sutrouttet OpiUnc, ij.

crociUnc.ij. Saffran iedes 4.8. Betob mit suarmen $25 a f f e r$ oje Sinctur baraus / rautbe a ab zur Dide cine EX tracts, thue oaju:

Sal. CC.

Sinfb5oun Sals.

Olei Cinamom a- Bimmete Del, ieveds. na 3ij. quentl.

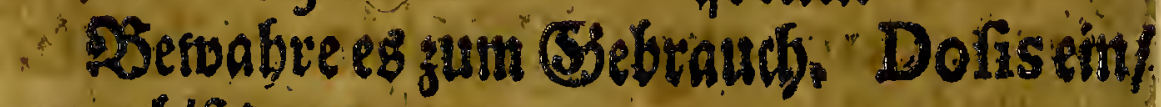
gewey bis oreng gran. 


\section{$48(337) 5$}

Extracturn Diuretičum, 5antrectiben? Dev Extraet.

R. Ligni Nephri- R. Siriefbols.8.\&. ticiUnc. jv.

Herb.Petrofelin. Heder terreft. Sabinæ ana. M. vi.

Sem:Petrofel.

Deterfilien.

Siundermats.

Sadenbaumirder 6. Santo volf.

Sileris mont. Sem sileris montani,

Baccar, Juniperi Rorbeeren iedes 4 . ana Unc.ij. Sotb.

Rodje alles in cinet guten qoantităt 25 aps fer / raudbe ab a fu cinen soeidfen Extract mische oarunter: Millepedum, Iereb. Coct. ana

Unc, ij.

Reflerráaben. (SSetoriten Serpene tbin ietes 4. Rotb. Balfam. Copayva. Unic.

F. Fa. Extractum. Dófis jy.

Extractum Carminativum, 25 indeceis betner Extract.

R. Rad.Acori. Angel.

Oftrutii,
R. RaImus.

Angelic.

Deifterip.

?) 

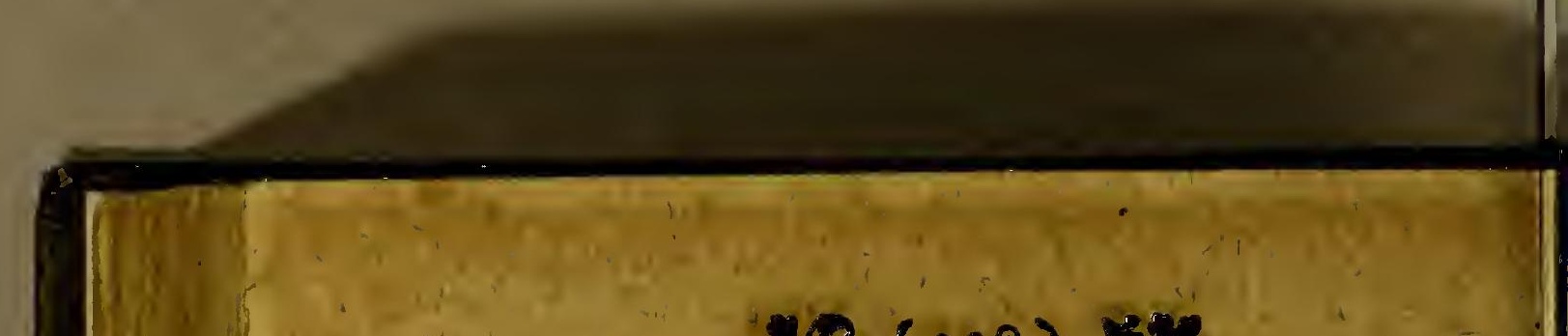

Cort. Arantior.

Citri.

Macis.

Fl. Cammon.rom. sem.Carvi.

Dauci.

Anifi.

Fœniculi:

siler, moni, ana.

Unc. ij.

Baccar,Juniperi.

Lauri.

Piperis nigri ana.

Unc.iij.

Rodhe es mit wiaffer / Ins Das ourctigefié gene zu oer oide eines (Extracts abiaudiem Dofis 3 i. bi 3 .

Extractum Stomachicum; NRegen: Exrract.

R. Aloës,

Myrrhx.

Crociana part $x$.

Zeudb mit Soranterwein die Tinctur bar aug/ / 'audse gelinte ab bi zuc confiftens eines Crtracts Dor: $)$.

Extractum Deobitruens, Eriffuenoes Extraet. R.Gum. Ammon:

Fomernngsen= $\mathrm{Sth}^{\circ}$ Eitronenichaler. DR Rircatenblitban. Rómicbe Camidlens Carbe. Sobrentuminel. 2ninér. Fenchel: Sil. mont, Sem, iel Deg 4 . Sotb. $53 a d b o l o e r b e r n$. Rorbern. Pfeffereórnes iebes 6. Rotb. 


\section{क्ष $(339)$ है}

Guajaci. ana.Unc,

Vitrioli martis.

Aloës ana. 3ij.

M.

Dor. Ji.

\section{Extract. Croci.}

R. Croci qv.

उetch oie Sinctue mit conarmet 25 affer

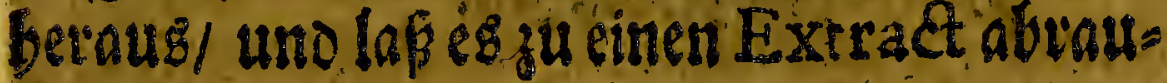
disen.

allo madist man

Extract A cori. Zedoaria.

Angelica. Cardui Benedicti.

Rhabarbari. Scordii.

Senze. Ghelidoni maj.

Galangx. Euphorbii \&c.

\section{V. stotferilung. \\ Bon Electuariis.}

Electuarium Purgans, Pungienen Rativerge.

R. Fol Sennx.lb.

Rhei electi.

Jalappá.

Mechoacan. ana.Tb.

Prun. St. Carharin.lb. vi.

Raf alte grtoen sage lang in fievent beife. ten Wafferfteben/ foche es mondinablauf (3) 2 fits 


\section{Is $(340)$ s.}

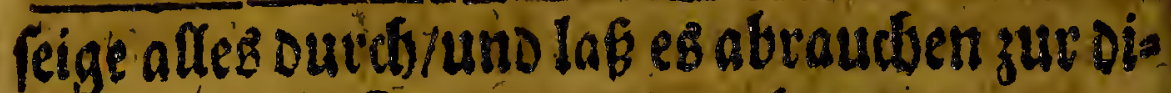
de eimes Eleetuarii, zule

scammon, pulv. Unc. fi,

Pulv, Cinamom. Unc.j.

Caryophyllor.

Macis ana.sii.

M.F.Eleđtuar.

Dofis von 7 i. bifs ziif.

Electuarium Emeticum?

R. Fol. Afari.

Gratiolæ.

Nicotiana.

Cort, Samb. med.

an. $16 . i$.
R. SBlátter bon Şa feliwurisel.

(Sottes SSenao.

Tabac.

Ritlere Ninde von Solunder ieces: pf.

Paflul. min. co- Nleime Rofinen. 3. rinth, lb, iij.

of.

Rapés in genung a mer Dvantitat fietent

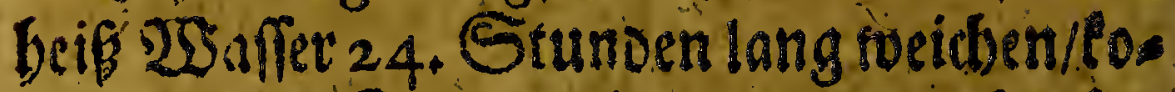
dhe es etlid) Stunoenlang/ Das out gefies gene lấ zue dicfe sinesElectuarii abrau. d)en. Sulesgt thuse onzu:

Pulv. Macis, Mufcaten-S3lutb 2, Unc.j. \&otb. Dofis; 3 . 


\section{มิ $(341)$ ste}

Antidotum Sudoriferum, gum Sofotigen.

R Pulv. Bufon. R.Pulved von gee ficc.

itrodittetenirioten.

Extr.Scordii.

Angel.

Zedoar.

Extr. von Eadhen: Rnoblatuds.

Juniperi:

êngelic.

Bitruer.

Wadbolberbern.

01. macis Expref. Angepreft 20ufos an.zij. ten . D\&l iesis 4 . Eotb.

Ref. Grajaci.

Camphore.

Caftorei.

Myrrhæ.

Crocian.Unc.j.

Sal. vol.CC. Uc.p.

Opii Prap. Jiv.

Ol. ma cis deft.

Sark oon Jrhntso pen $=50$ ols.

Camplect.

Riefergeil.

MRturtben.

Saffranietes 2.?.

Jirfdsborn=Sals 1 .

Roth.

Dräparit Opium. jvi

Diftilite SMarcaten.

Del.

Coryoph. an. Negleit =Del it 3ij.

bes 2. quentl.

Vini Canariniqs, Canazien = Sect 10 viel genuting.

$$
\text { (3) SNifge }
$$




\section{0 ( 342$)$ st}

Spirde folcbeg tis es die dicte bat eines Electuarii Dor. 3j. madbet fard Sd roin sen uno if eim S) ittel tor alles (S3iff.

Eleetuárium Diurericum, Den Satn zu beforsern.

R. Bacc. Iuniperi R. Wactioloerberten rec.lb.iv. 4. $\mathfrak{P f}$.

Ligni nephritici, Srief Sols I. Df. lb. j.

Sem. Petrofel.

Daùci.

pererfilien-Samen: Sobren = Rumunels. Samen.

Sileris mont. Sileris montanianalb. f. Gamen. lb. f.

Rocbe Diefer mit 2 affer Sier uno

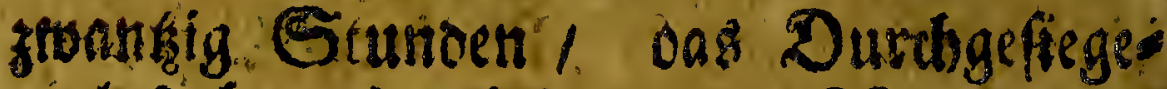
ne lafiabraudien fif zut confiften sines Elect uarii, thucoazu:

Millepedum Unc. Rellerfoben $4 . \Omega$ ij.

Conchenilior.zj. Conchenil 2. Reth. Dofis siij:

Electuarium stomachicum, vorn Diagen.

RRad Acori . Ralmuf. $\begin{array}{ll}\text { Angel. } & \text { Sngelic. } \\ \text { Galang. } & \text { Sialgan. } \\ \text { Zedoar. } & \text { Sitnot. }\end{array}$ 


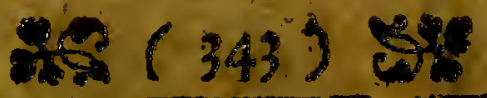

Zingiberis.

Oftrutii ana. Meiferwiedes $4 . \Omega$

Unc. if.

Piperis nigri.

Bacc. Lauri.

Myrrha.

Aloës.

Croci ana Unc.j. Saffran ieder 2. \&.

Stof alles zu einen Pulver uno vermis fore es mit fo viet Spannifoten 2 ? Die Dict: bat eines Ele etuarii, mergenods Darunter:

Olei macis deftill. Diffiltirt S) Pufcaten

Unc. P. .

Dolis 3j.

Pfeffer.

Sorbern.

Murrber.

2lloes.

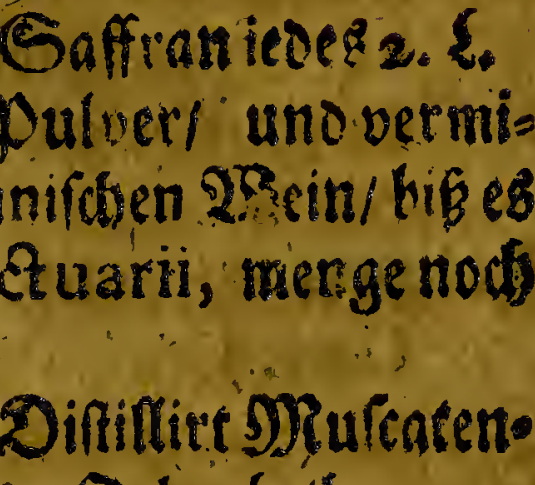

Delis fotb.

Electuarium Bechicum, Brufto

Satroerge.

R. Rad. Glycyrh. R. Sulfebors.4. $P$ P. rec.ti.iv.

Sem. Anifi.

Carduisened. Envoebenedicten.S. ana th. j.

Paf.min.lb.ij. Klem.Xofinen.3. $\$$.

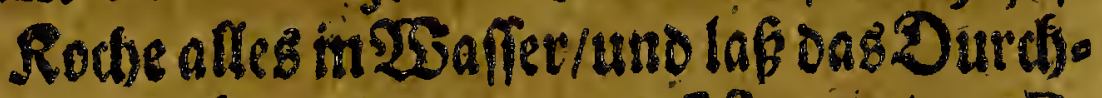
gefiegene abraudbet zatr confiftentseines Electuarii, thue Dazu

Pulv. Zingib.

Sieftoffenen Yngrue?.

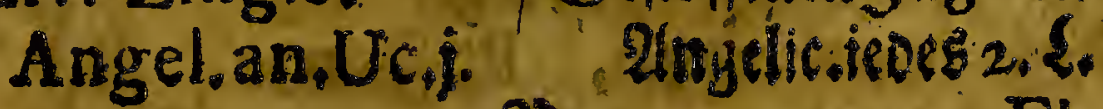
$3_{4}$ FI. 


\section{9 (344) Ste}

Fl. Benzoin Uc, $\beta$.

Fl. Bénzoin.i rot

Opii præp. 36.

Preparirt Opitm ein balb Dvent.

M.F, Elect.

Dor. Unc. f.

Niactse allez zu cinet: Satwerge.

Electuarium Antiforburicum, boun Scharboit.

R. Cortic. Winte- R, Magellaniforen rani. 3immet.

Baccar.Laur, ana. Sorberniedes 4.5ot. Unc.ij.

Croci.

Eaffratr.

Macis,

Mruca tenbluté.

Caryophyll. ana, Nieglein iedeb 2. Sorf; Unc. i.

Stopáfles tlar uno tbue dasu:

Olei Cochlear Soffelfr.Del r. Soty. Unc. $\beta$.

Cinam. 3 i. Vini Hifpanici qq

Simmeto Del $y j$.

Epantildben 525 ein fo biel nòthig.

F. Electuarium. Nactse еร zน⿰ Zat.

Dor. $3 \mathrm{ij}$. werge.

Electarium cardiacum, ftadtentente Satnerge.

R. Cinam: acuti.' R, Simmet 2. Sotb. Unce.t. Regleit. 


\section{$x(345)$}

Caryoph.

Macis.

Crociana.Unc. $\beta$. Morchi.

Ambra grif.

Olei Cinam.

Caryoph.

Macis.

Cort. Citri non

puer.

Arantion non putrid.an.38. Vini Malvalic,q.f.

F.Elect.
NReglein.

Dufcatenbutith.

Saffran iedes i. E Morchi.

Ilmbea.

Del vor Bimmet.

Regleits.

Murcatert.

Citronen.

Someraitgen iev. cin balb Qu. DRaluafietifo viel gea nung. Madie es zut Sats nuérge.

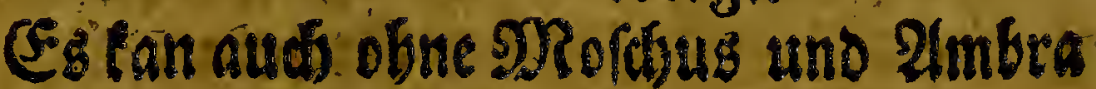
bereitet iverten. Dofis zij.

Electuarium acidum temperans. Sat suerge die Salueve zu dampffen.

Rim Equies: Salas mir 4. tbeilen Salpes ter gebrand:

Xotbe Corallets.

Rrabgi2tuget.

Jirfaborn.Sall:

Saffran.

Gum. Ammon. teved Unc.f. Pulver von Salfa parilli Unc.jvi 


\section{$89(346)$ 3\% \\ S)Ralvafier qs. \\ SRiche eह yu siner Satwerge. \\ Dol. sij.}

Electuar adftringens, anbaltenoe Satwerge.

R. Cortic durior, R. Die harten Scha: a mygdal.Unc. lemoon MRariodin iv. 8. Sotb.

- Ovor.Unc.j. Enerfulden 2. Soth. Cinamom. Bunmet.

Nucis Mofenat. \$Rufoten=Mils.

Carbon. Suberis Roblen von Pantofe anaUnc.ij. $\mathrm{fel}=50 \mathrm{llg}$ ieder 4.5

TincturæCatech. TincturaCatechu q. . $_{\text {. }}$

M. F. Elect. fo viel genung. DRache Daraus eine Entruerge.

In fratulen SRonat = Stup uno Durcfo. fall. 3ij.

\section{VI. alfthetiung Bon Dultwer. Pulvis Purgans.}

R. Scammoni. Une $\beta$. Antimonil crudi. Crem tartariana 3 it.

M. 


\section{$29(347) 58$}

Rinvern giebe man gr.vi. altert Seuten 3 it, bif 3j. auth finnen einfache Pulves aflerviret merven von A lö, Senna, Gutta gamba, Rhabarb. \&c.

Pulvis Emericus, Stredsapulver. R. Cryfall. Tartari. Unc. ij. Vitri Antimonii. Unc. $\beta$.

Rodie eg etlidie Stunden in genung men 2Baffer/ filtrire uno Eruftalifiere es nadi Det Runft.

Davon pflegen 6. 6ip 8. gr. gegebengu seroen/ radocem die Riaffe find.

Dergleiden $53 \mathrm{red}$ - M) Wittel fan man aud bereiten von bloffen Sabac/, Turbith minerale, Mercurio vita uno vergleidsett. Pulvis Sudoriferus, Sundeif = Pulver. R. Opii crudi \& R. Robe gettodenes ficcariUnc. 6 . Croci. Unc.j. Baccar.Lauri. Ariftol.fabac. Scordii: Opium r. Sotb. Salis abfinth vel. Card. Bened. an.

Unc.ii.

M. F. Pulvis. Eaffian 2. Soth. Sorbern. Dferlucy. Sadoen= Rnoblaurb; 25 ermutb oost. Satdebenedict.Sark. jeoes 4 : Sorb. Mrifose pulbet. SRan giebt alten Eentgen auf einmabr t). 


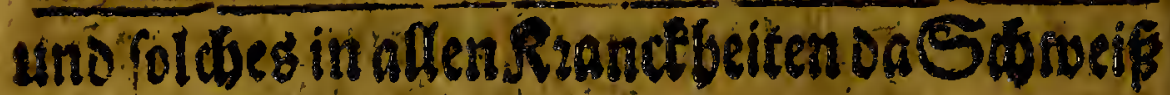
exfodert nute.

Pulv. Diureticus, sannfetheno pulder. R. Calc, Ottreor $\pi$ Gébronde 24 . Unc. ij.

Sabinze. Rer-Sulden $4 \Omega$

Baccar.Juniperi.

Sem. Apii.

Perrofelini.

Sadenbaum.

23 atbolserbert.

Eppid Somen.

Milleped.an.Uci. Rellerfónbenicoss 2. sotb:

M.F.Pulvis.

s)ifbe ef zu cinet Pulber.

Dienet bor sem Stein/ Decbaltung oes

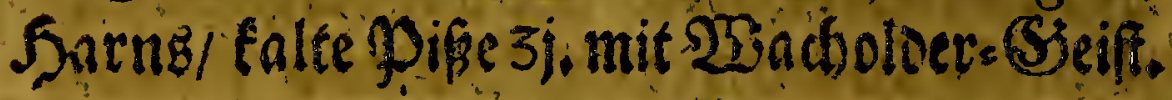
Pulvis Stomachicus, SRagen Mulver: R. Galangx, , R. Sialgant. Acori.

Oftrutii.

Bac. Lauri.

Piper nigr.

Myrrha.

Antimon,crudia -

na part. æqual.

M, F. Pulv.
Ealmufp.

SReiftertours.

Sortern.

Sobwartien Pfeffet. SRurben.

Spies: SHap iever gleidaviel.

SRiche es zu sinen Sulbet.

Es roiro táglid sin Quettlein oavon ge geber 
geben temperiret ons Sauev/gertheilet Den Sotein/ uno fubret ab.

Pulvis Antifeorbütius, vorn Shatbod. R. Vitriolichaly - - R. Bitr bon Stah!

bis Unc. B.

Oculi cancri.

Antimon crudi Xob Spiefiglafiedes anaUnc.ii.

M. F. Pulvis.
1. Eotb.

Arebge Plugen.

4.5065.

9)ifue es anet Dutuer.

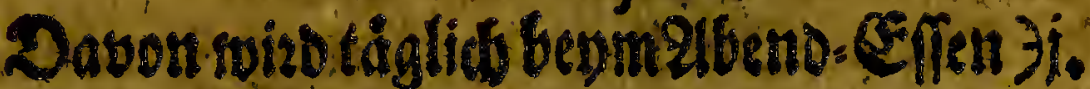
snit SBier aingenommen.

Pulvis Anodynus, Sdimerffifleno pulver.

R. Croci, Unc.ij, R. Saffran 4.Rotb: Opii ficcatijij.

Sietrocinet Opium

Morchizi.

aij.

Móchin abencl.

Amb.grif, 3 .

Amber. $)$.

M. F, Puly.

Shidse es su einet Oulter.

Gigniro in afferbano Sdbmersen Hi. ges braudht/ uno wenn er befftig bip ouf 31 . po- es W3eiber find/goldse feinet Morchum veto tragen/tan man ibn weglaffen

Pulvis Bechicus, 8 ruft-Bulver. R. Sulphuris cru- R. Roben Sdiwef di.

fol. 
croci.

Benzoin,

Saffran.

Zingiberis,

Sem. anifi.

Papav.

SSentsor.

Tnguet.

Intes.

DRabr:Samen.

Sperm. Cetian 36.

M.F. Pulvis.

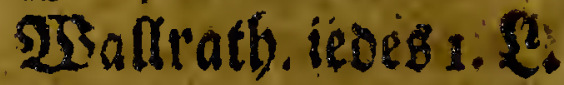

Mifde co zu cinen Pulvet:

Siervon mag man táglich 3i. nefimen nebft etruas Spannidjen 2 sein.

Pulvis Cardiacus, fátroleno pulver.

R. Rad, Angel.

cinamom.

Macis.

caryophyll.

cardern. min.

Mangaritar. ana.

Unc. $\beta_{\text {. }}$

Ambrægrif. 狧.

M.F.Pulvis.
R. 2ngelic.

Biminet,

SRucatenblutb.

Sieglein.

Eardemumet.

Perlen iedeg 1. loth.

$21 \mathrm{mbra} .38$.

S)iricbe es zu einen

pulver.

(E. swito Davon bis auf 3). eingegebery

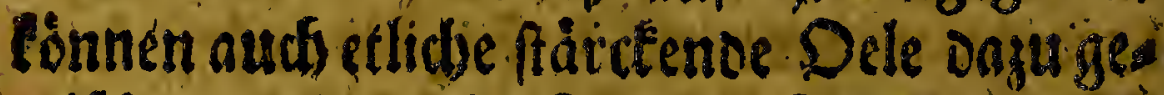

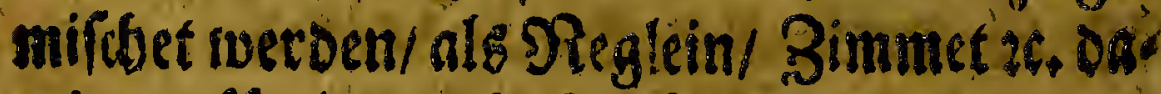
mit Dag Pulver oeffo fráffiger sperde.

Pulvis ad Vermes.

R. Seminis Santonici Unc, ij. Mercurii dulcis. Jiv.

M.F. Pulvis Dofis if Jif big $3 i$. 


\section{$\operatorname{xis}(351) 58$}

- Pulvis Dentifririus, zam 3åfnen. R. Lapid. Púmicis. cryft. Tartari.

Tartari Vitriolati,an,part. æqval, M. F. Pulvis,

Damit reibet man zuneeilen die Zålfne

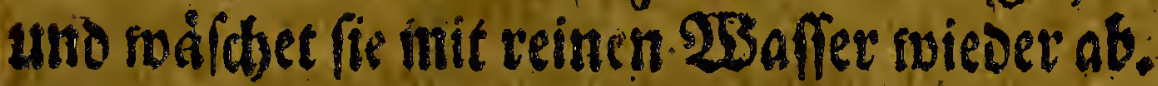

Pulvis aditringens, anthaltend pulvet. R.Rad, Biftort. R. Natterwursel. cort.Granator. Sivanafenichalen. cornu cervi ufti (Sebrano Siridbbics an.partizqv. Des gleidiviel. M, F. Pulvis.

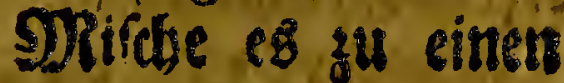
Poulver.

Die Dofis' if von 3i. bif 3j. in Durdi. foll/ allzuftateten 9)tonatb-FYlus/iberfhigis oer Xeinigung Der Wódbnetin/ fiflet auds

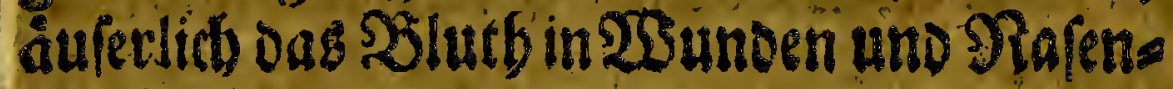
Elutber.

Unter bie Dulver gebireti aud folgenoet. ดी ถี:

Ruptorium.

Sal. Abfinthii.

cardui Benedicti. cryittalli Tartari.

Tartarus Vitriolatus.

Lapis Brunella. 


\section{และ $(352)-5$}

Mercurius pracipitatus albus.

\section{Ruber.}

Vitriolum martis.

Crocus martis.

Salia volatilia. Cornu Cervi.

Eboris.

Urina:

- Dulver yon einfacten geftoffenen Dinget

$$
\text { ic. }
$$

Diefe uno oergleidsen fino genungfam in

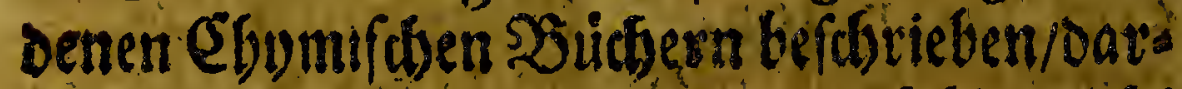
amb id iffe praparationes audb bier nidfi exft vorbringen nill.

\section{Rotfeilumy. 3on Diffitllitten Delen.}

Die Dlitaten roelde in cinter 2lpotbectent ju aflerviren deftilliret nerden fino fol gende:

Ol. Anifi. Del bon Zlnief. Baccar,Juniperi. 2 Wadboloerbern.

Carvi.

Caryophyllor. Camphoræ.

Calamiaromatici

Cort.Aurantior.

Cinamomi. Citri.
Rimme!.

Deglein. Eampher.

Calmuif:

Pomerantsenfchalen. Bimmet. Eitronenfobaler. 
Therebinth \&c. Serpintbinx.

Don Jercn Praparationibus suiflio audis nidts ecroibnen/ toeilfie fattfam in oes nen Ebumifcsen SBtidern zufinder. Wusgeprefte Deléfrio:

\section{Amygdalarum, Del bon S) Parioeln.}

Cáuri.

Lini:

Macis:

Mofchata.

Olivarum \&c.

Sorbern.

Reinfämen.

Muccateriblitb.

S)Rucatez: SRuf.

Dliven ic.

Diefe fommen theils aus Dft Ynoient theilg aus sevant/ Jtalien xc. Theils norven bier zu Sanoe gemádgt bie SRanier foldse gu: bereiten if bev seneri $A$ uthoribus guftroest. Olea per Infufionum.

Olēum Allii.

Chamomilli.

IIyperici.

Lumbriterreft.
Del von Rnoblaudy. Camillen:

Gobanneste.

Xiegenourmet

3 . Mentha. 


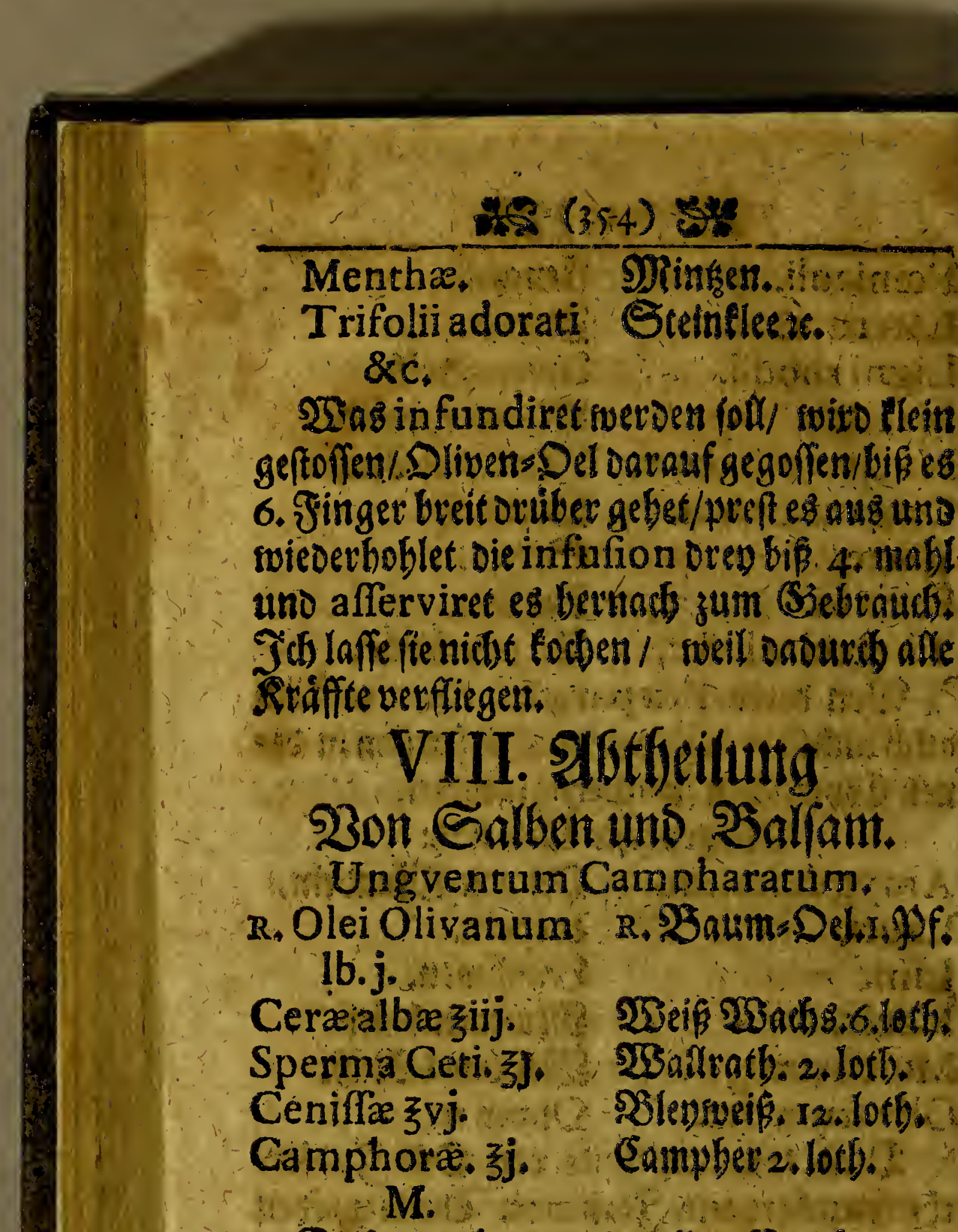

Diefes ift bequerr in alfen Entzunouns gen/: finfet die Schmertsen/ Geilet diesslase fen uno oem Sdfmersen Des abgetriebenen Saut.

Ung ventum 2 Egyptiacum:

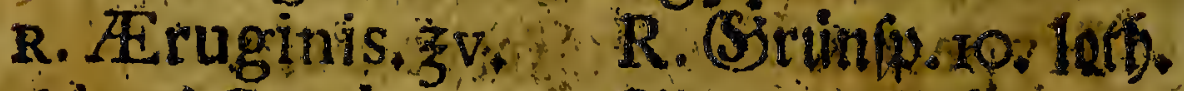
Alun Crud gio 2tanenz loth

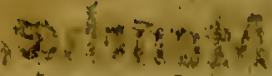




\section{as (355) 50}

Mell optimi.3xiv. 5omig 28. 1otb: Acetifortis zvij... Esig 14. loth.

Safiez zu einer Salbenfodben. Es reints

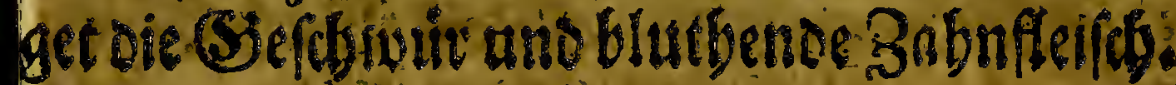

Balfam. Vulnerarium.

R. Balf Peruviàni,

Copoyva analb,

Olei Petre ziij.

Maftichislb. $\lambda_{+}, \mathrm{l} q . \mathrm{f}_{0}$

$\mathrm{M}_{\text {. }}$

Diefe Salbe bevlet die frifticn wernoento

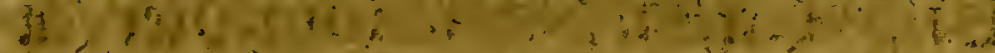

Ungventum Detergens, teinigende

$$
\text { sito Salbe. }
$$

R. Refina:

Terebinth.

Cerre cirr. ana.

Une.

Lythargyr.Uc,iij:

Ariftol. long:

Maftichis.

Galbani.

Myrrhaan. Uc.j.

etruginis sii.
R. Satk:

Eerpentbin.

(Sielb Wadb iedes t. Sotb.

(\$)letbe 6. Sorf).

Sange Dftetucy,

MRaftix:

(sialbani.

Mprrbeniedes 2. \&. Strinfpar 2 quent?

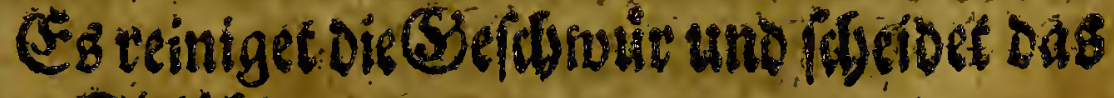
boterteifio. 
Ungventum Epuloticum,

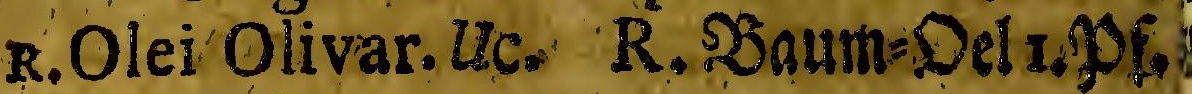
\& $x$ ivj.

Ceræa albæ

2sei $25 a c t$

Plumbi ufti.

SBley=2(d)e.

Terræ vitrioli.

ceruffa.

Terra vitrioli.

Litargyran.uc,iv (Sletbeienes 8. Rotb. M.F.Ungven-

tum.

Dadfe daraus eine Solbe.

Es madjet 5 aut wadsfen/uno eufullet die gereinigten Sseforour mit Fleifd:

Ungventum Nervinum, Slecben:

\$. Salbe.

R. Olei Laurini R. EooreDe!.

Terebinth. Fexpenthins.D.

Terra an.ll.iij (5n=Deliedes 6. $\Omega$

iN Nuc. Morchat. 2lusgepreft Dlurca-

expreff.

Styracis calam.

Pingucd.expedi-

bus urfi qfi

M.F.ungvent. Driche esur Sals

Dienet wieber alfe Sabmung / vertente Silicoer/ uno allen (Siebredien ver Nerben. Ungentum Bafilicum

R. ceræ flavie.

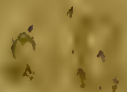
beri.

R.Selfe Wadte.

Etocar

SEiren = Sdomals fo viclgenung. R. 


\section{$\operatorname{sis}(357) 5 \pi$}

Picis naval.

Sdhiffepect).

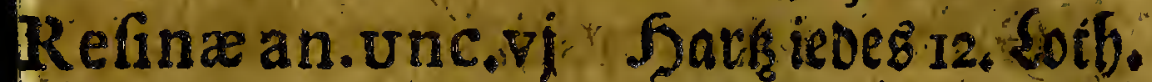

Oleiolivar, lb.1. Saum Del I. \$PF. Myrrhæ. Maftich.

Thuris an. vc. iij. Detenud iedes 6.S.

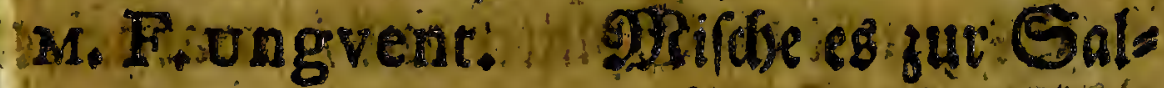
ben.

Es teiniget die Sefthtoriv/unb mactet oie Bicfornutreif.

\section{IX. abtbillung. 2Jon PSflaftern.}

Emplaftrum camphoratum.

R. Olei olivarum 1 b. iij.

Lithargyrii auri Ib. ij.

- Roche es mit Regen-235affer gut bide eis regp fiafters ftet mit cinen Spatcl ünbges Hifret: thue oagu:

Axu g porci mund. Ib. if. camphor, unc ij.

Sperm, ceti.unc.jol a

F.S. A. Emplaftrum.

Silft bor das Pooggra/madjet die Saut badsfen 1 uno berteribet offters oie $S_{e}$ 6) nulfen uno Entginoungen.

Emplaftrum de Gummis. R. Enpl. Supradefcripti. lb.j. 
ceræflava.

Gummi Ammon:

Galbani.

Refin. Pini.

Terebinth ana unc,$i j$.

3 M. F. Eimpl.

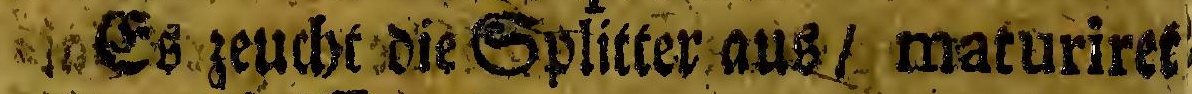
uno mactot Ejtet:

wh Emplaftrum Citatrifans.

R. Lapid, Calam. R. (Salmevftein.

ceruffa.'

SSleusueif́?

Lithargyrii.

Tutia.

Silbere(s)lat.

Thuris.

Sildot.

Maftichis.

20everaud.

Myrrha.

DRaftix.

camphor ana. Campleriedes 4. \&. Whanc. ij.

Terebinth. Uc,iv, Serpentfin 8. Eoth.

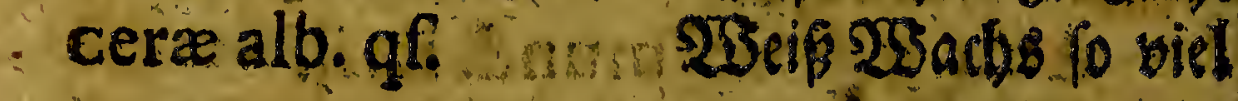

4 genung.

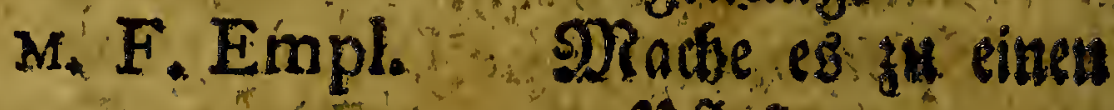

Hin Emplaftrum Croceum.

Riceracitr. Re (Sielbe 23 adis.

Colophon.

Colopbanen:

Picis nav ana. Sduiffopediedeg 12. vac.yj. 20 . 


\section{2. $(359)$ है:}

Crocioptimi uc.ij. Sáfran 4. Soth. Galbanl uncivi Galbanum 8.Rotf. Maftichis. Saftix:

Myrrha. Sorverten.

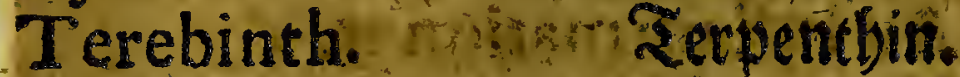

Thuris an vcif 23 estaud ledes 4.5

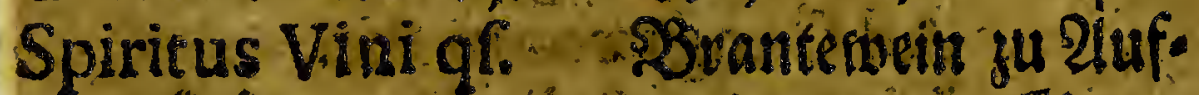

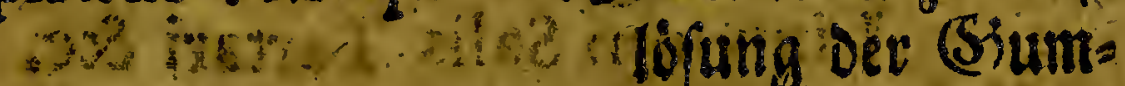

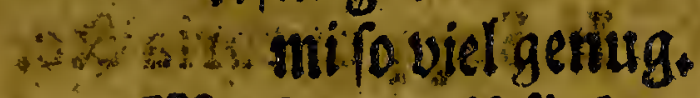
F. Empl. Dradje ein Offafter. Emplaftrum de Mercurio. R. Axungia Por- $R$. Sdyweinefone ci.lb.j. Pf.

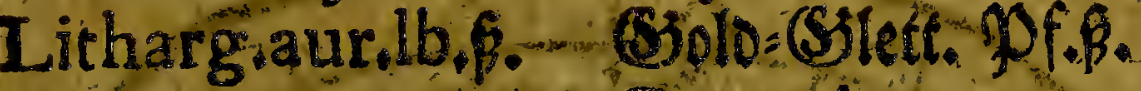
G.Ammon Gum.Ammon. Therebinth. Serpentbin. Mercurii vivi ana, sebeno Doudfiber. unc.ij. ieoes 4. Roth. M.F.f.a.Empl Drifo ç nads oet Runft on cinte Dflafter.

\section{X. stotferlung.}

Bonbereitetenखafiem bie officinal rent éstren.

SRan fan in einer fpotbecten alfegeit in soceitfobfthalten folgende 25 affer:

$$
34 \quad \text { Aqva }
$$


$\frac{\operatorname{2a}(360) 3 t}{\text { Aqvacalcis. }}$

Gucta I. Stillatitia fimplex.

Salfa.

Ophthalmica.

Ad Inflammationes.

$3+$ Ulcera detergens.

Ad Gangranam \&c.
Lixivium Salis Tartari \&c.

It: Suppolitoria \&c.

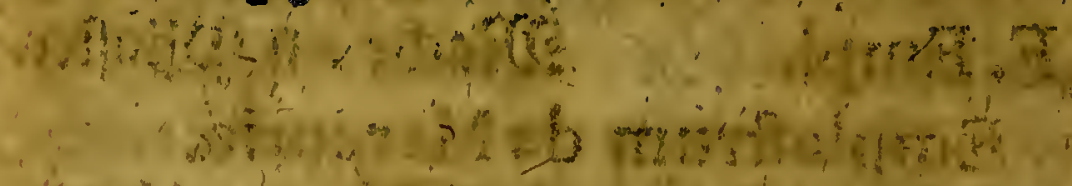

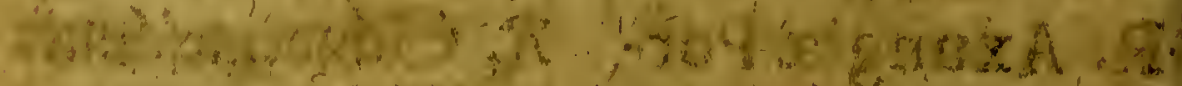

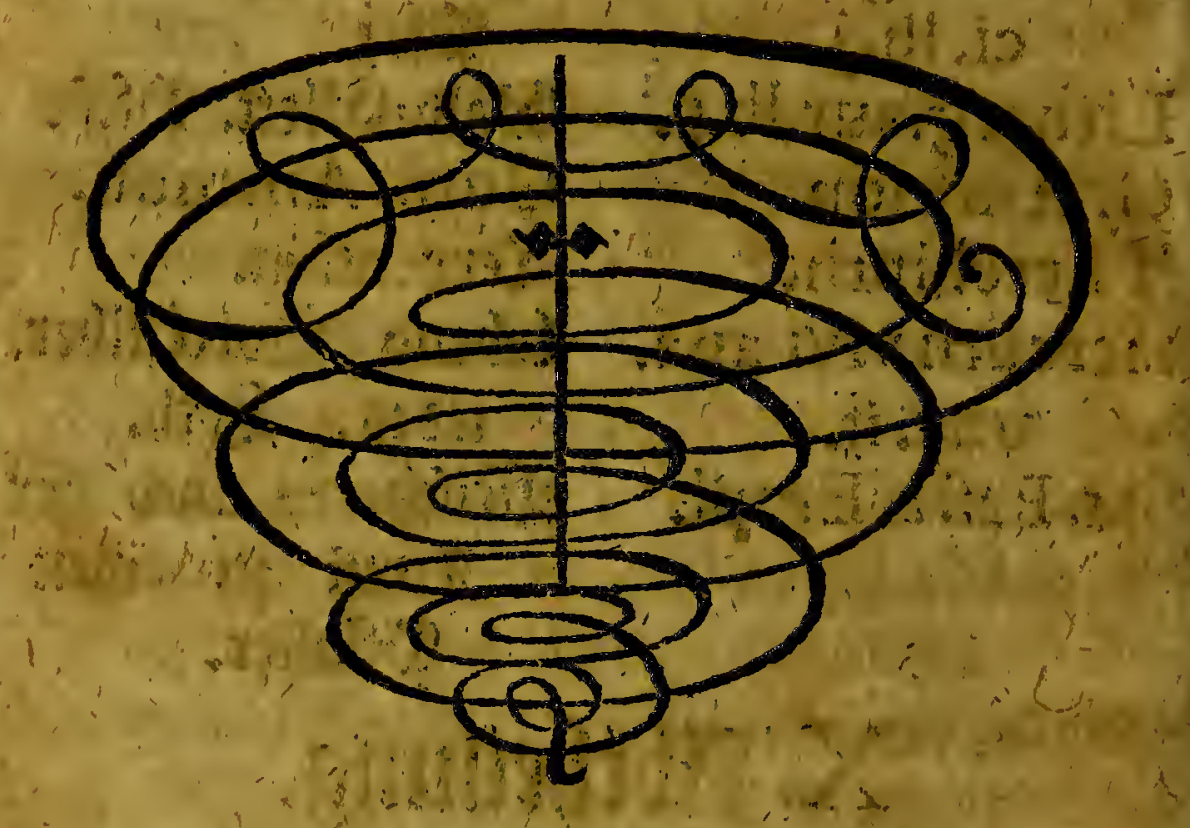

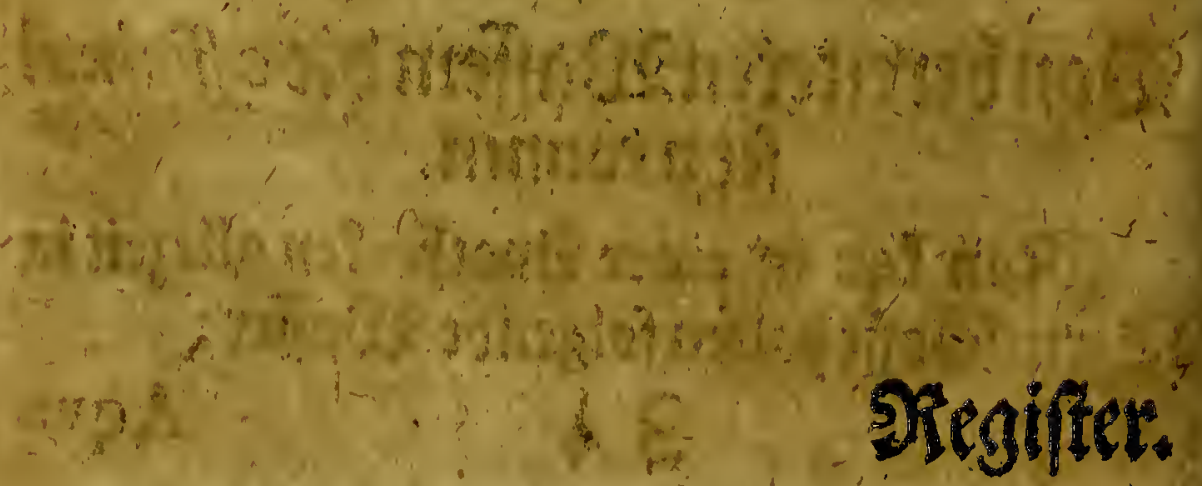




\section{9: :0: Be: \\ Tegifter:}

\section{2toertafen}

$$
\text { 2. }
$$

. ob es in 2 ergifftung bienlid

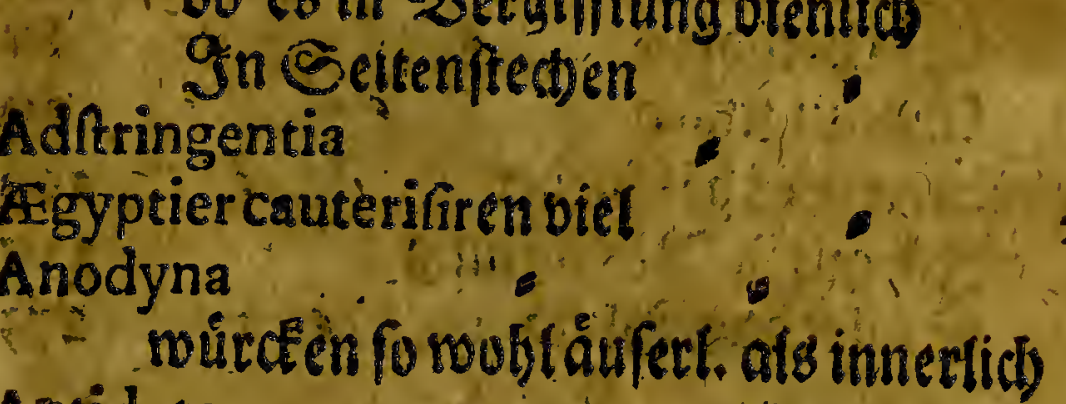

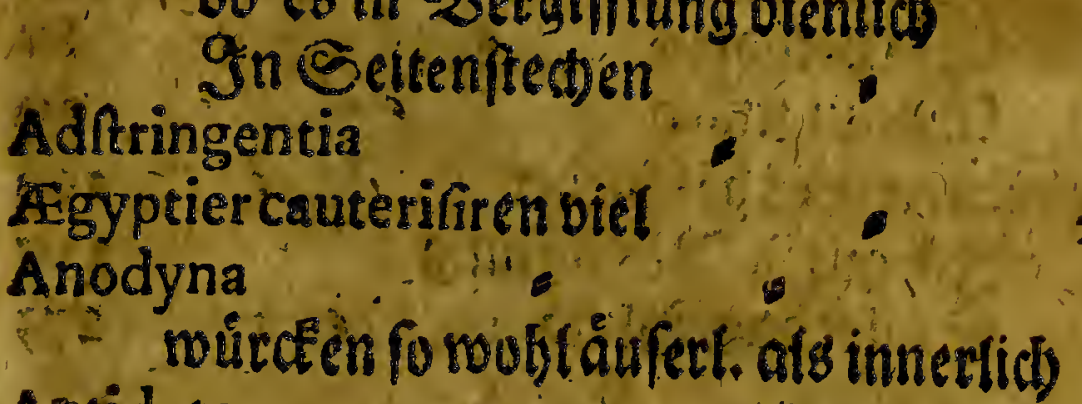

Antidota

Âteriotomia

Atrenuantia

Attraheritia

Tufas Dureb Qnewbren curiret

$\mathfrak{B}$.

138

208

23 aucjogrimmennaci Dem Purgiecen

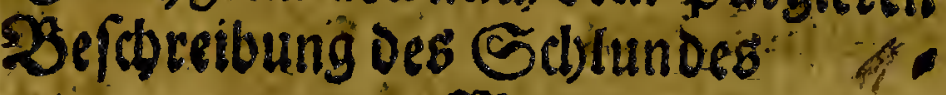

Sienen baben ein Slábgen bey dem Stachel

Dolaren wie fre werden

Blutiofillen

Filutb $=$ sgel

Bred = Dittel wovon fie gemadif werden 82 Sommen ins Viluth, ibid. 2 Berdenzu Purgangen $\quad 40$ 23ie fie operiren 82.84 25 redsen Eummit einerifob werer an alb Den an. Dern

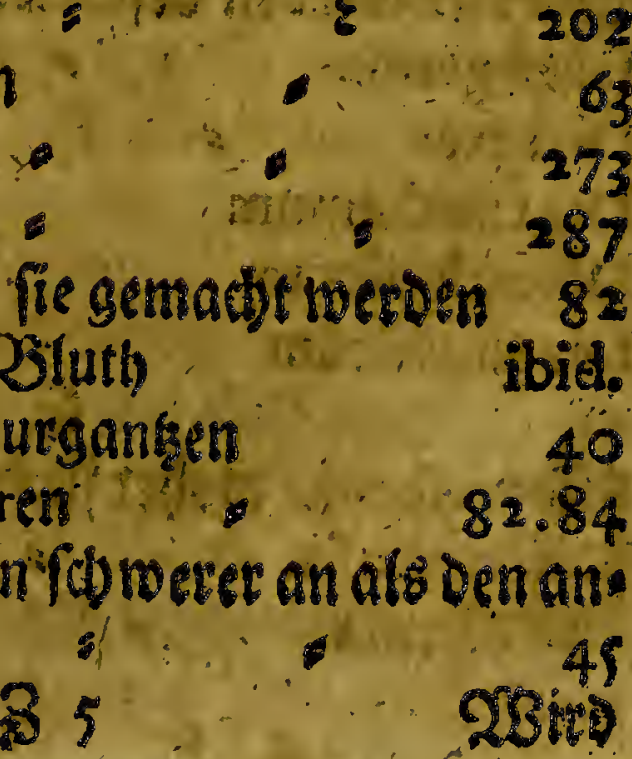


2Biro Durds reben uno búren getoiffe Dingelit theils Speifen erivect, 50 Der Sedwangern

as SBocebienlid $\quad 78.87$ 230verboten $\quad 80$ c2siejuftillen $\quad 90$ Scennen/mie es gefdiebet 369 Sft gut in 2 ergiftung Etillet Das SBluth

273 c.

Catholica Medicamenta

Carminantia :

Cauftica

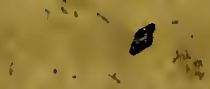

II

Shinefen freden mit einer Dapel

Cicatrizantia

Eluftice

3.

252

237

303

245

2Sojufie oienen

Coffee

30.32

Confeciio Hamech

Correctoria

Sorrufioifh Sifft

D.

Dármer roctoen beforieben

Detergentia

Diabetes

Dia Carthámum

Diacatholicum

Diaphonicum
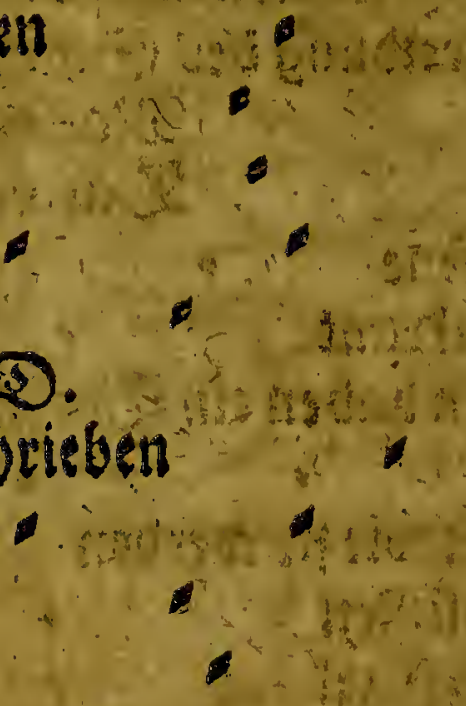

33

124 36

26

$-\quad \ln 202$

13.

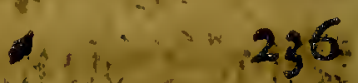

I.17.

, 
Biefie núcicen

Emplaftica

Epiceraltica

Epulotica

Crrweidjende SRittel.

iren

Erforoctenezu curiren

Efig aufpie Brúfte gelegt ift fobalio

$\mathfrak{F}$.

Fermentation woher fie lommt

Fike Galse

Fleifdmacbenoes 9 inge

Flect:Firbei

Sontanelle

Siff:

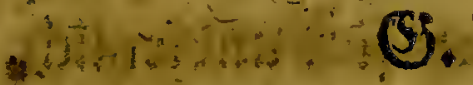

(s).

Deben Matécieift bislerley

$23 a 6$ es ift und wie es witcet Db les tin iunfarm seibe ergeuget noerde Sun शatternuno Ed Llangen getrun gen foraoet uidsts $\quad 208$

Segens Siffit

S5ulo ob es rob was operiret . $=165$ Gradus Der Medicamenten

220 S.

Saar Seile $\quad 288$ Sartleibigmoletes tommt $\quad 4 \cdot 7.31 .32$ Sarn wie er gefammlet wiro Sarnes Derbaltung wober fie fomtnt.

Sarnen narumbesnotbig $\quad 204$ Sarnwas er feg "

DB er auber oem vedenttidjen (S)ange noch einen babe 
Gelyet bey sinen beffer algoem andern t. 0 117 Hiera fimplex $\quad 3 x$ Hirudines,, 287

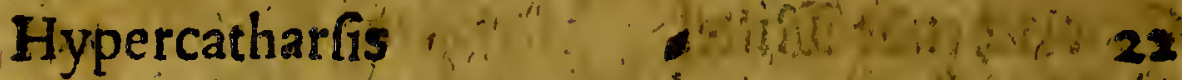
Hyofcyamus.

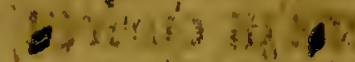

Imaginations Cur. Gucten. g. ji

292 Influeng̨ Der Sterne.

Scandbeiten baben zoey Saupta Urfadien. $8 \%$... " 170 Súblende Medicamenta fino undienlicf. 224 sráke

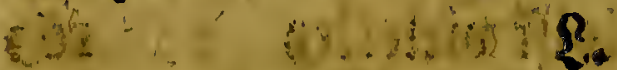

1258

Lapis Philofophorum. -301

Materie prima.

$$
\text { 7ons son. }
$$

Q.". Secundà. Magnes affenicalis $\quad-\quad 210$ Magens= Befcbreibung. $\quad 4.5$ Mafer,flecte. Maturantia. , 242 Medicamenta roas fie fins. Wie vielerley 21rtb.

Mercurialia. tora y y

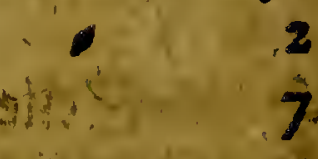
Mild) vermelorende und verninoend Mitte?

Mithridates fónig. 246. Moxà. Dronatlidje scinigung zube fobern.

$$
\text { - } \begin{array}{r}
246 \\
216
\end{array}
$$
270 251 


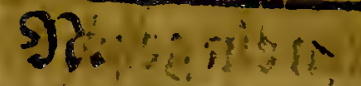

Darens Butuen $\quad 276$ Durch Nufaten 3 lutb erwect. 254 Narcotica. , 137 Dimmegifd Bier. o on,$\quad$ i2s Siefenwoheres Eommt: , $\quad 187$ gief-5) Dittel. 183 Bertreiben die Sopff=Schmensen. 189 Sabn:Eomersen. 190 Fallenoe Cudit. Edilucten. 192 Sefodern dic Sebutt. - 195 O.

Opium Dienet vor allzu vieleg\$ Durgieren. 40

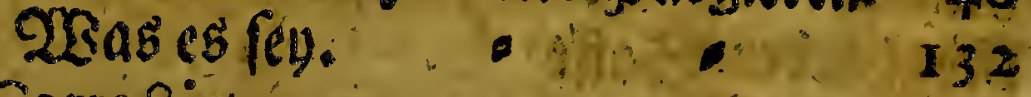
Opii Correctio.

Opium mie es folaffen madit. 139 Srubet Den Soldneif. $\quad 142$ Sertbeile oie (Sefdomulf. ibid. Sitgut vor Entuinoung. $\quad 343$ Deffen Sampff nicht fluablich. $\quad 146$ Oft: Indianifjer Stein. . . 217

Pectoralia. ग. :

Dep. Yfiafter. ation 235

Oietermanein gemiffer fif $\quad \begin{array}{r}210 \\ 196\end{array}$ Justen flect: a an 257 Principia cosporum. ßurgieren was es fev.

Wurganken mobon fie gemadit werben. $\quad 9$

Gino féine Specifica. It Drenerten Surten. ibid. 
Eollen ein geboirig vehiculum baben. 18 Surganęen murcten Durd bloffes smeber

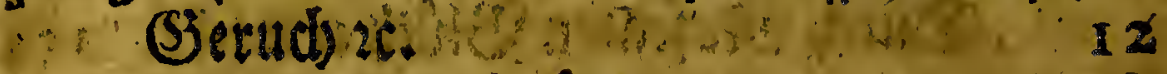
Jn20erngefpright. $\quad 23$ Pulvis Sympatheticus. 275 Fil i. D.

Doectfiber woraus egeffebet 176 द. STite caperiret ibid. Siub in Soantitát cingenomtnen fobabet 1. niddts

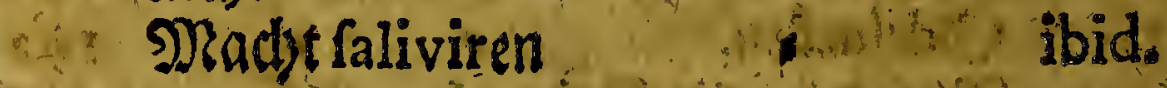
Whe he pis.

Rarefacintia

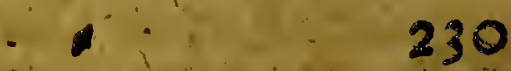

Sinube (1)

Sil)abarber: Sofft Sieiffendes Shittel Sieinigenue Singe Repellentia Sios nober er Eśmmt Rubificantia $+1$.

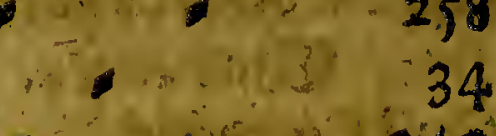
S.

Salivatoria

Galmioc a. $-10242$ Salpeter woraus ec beftehet - Sat Eeinen Schroefel Eamen vermefirende Mrittel Dermindernde Dittel Sauter temperirende Mittel 2rie fie núcten Sarcotua

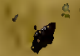
$=2,30$ 180 186
267 Scincus - , -

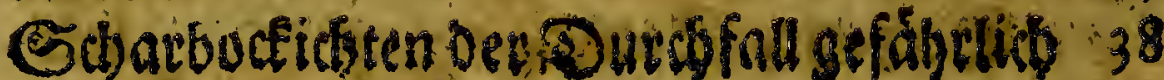
Sunabuctiflect: 
Colbeffen Sd)laff: Mittel Sinobielentev 132 Coblaffubertreiben $\quad$ I46 Solleimmas elley $17 \mathrm{r}$ Coblein jertheilende Mnittel 172 Gellundersseforeibung $\quad$ Solinupfi Sabad , 185.185 Deflen Mi Schrouffien Solimeif was és 286 Sdbriéb und Sarn fino miteinander felye bers in: tono. Sdimam mieer $93 i$ iro praferviret -1 Iry fitig 205 .

\section{(a)}




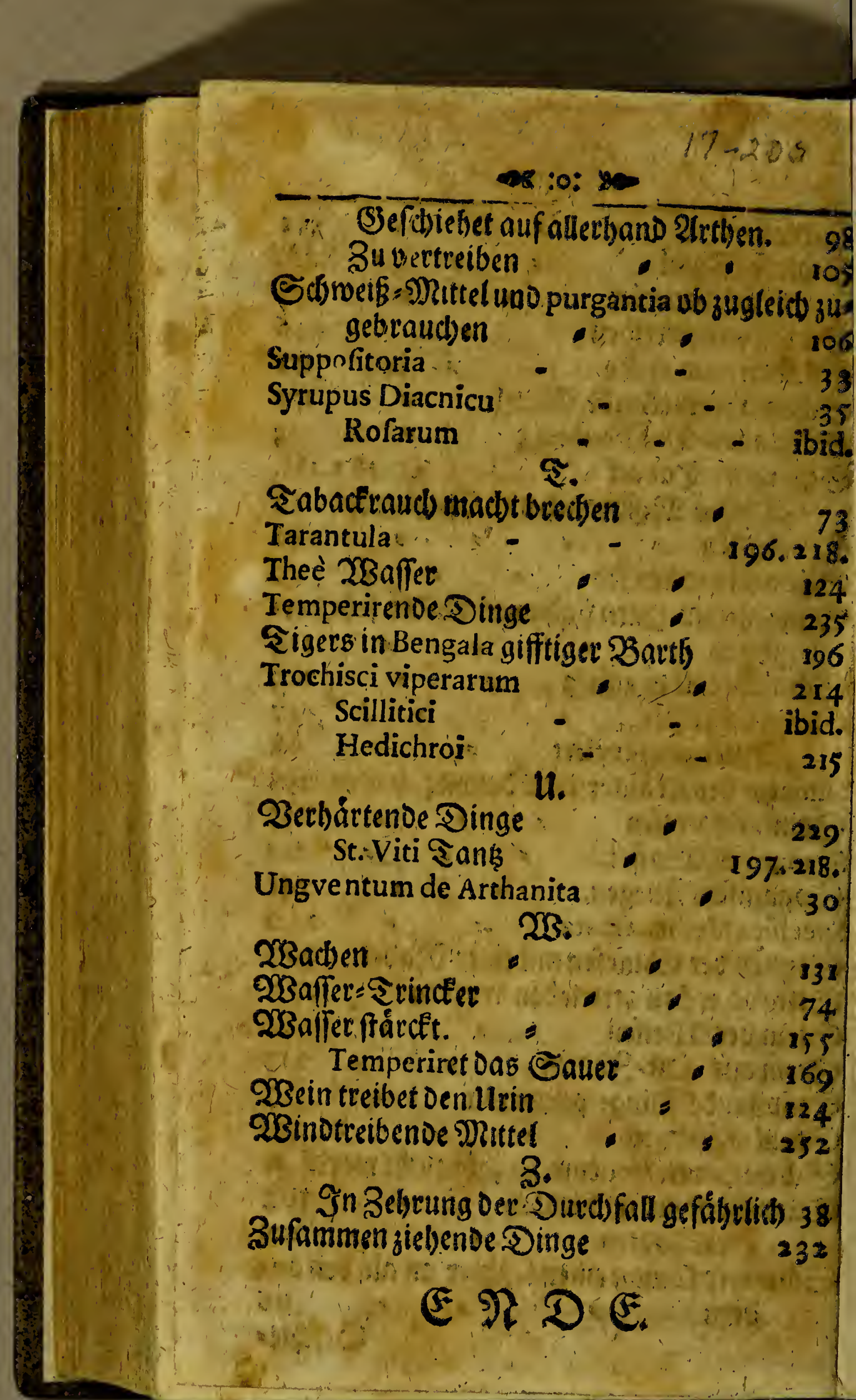




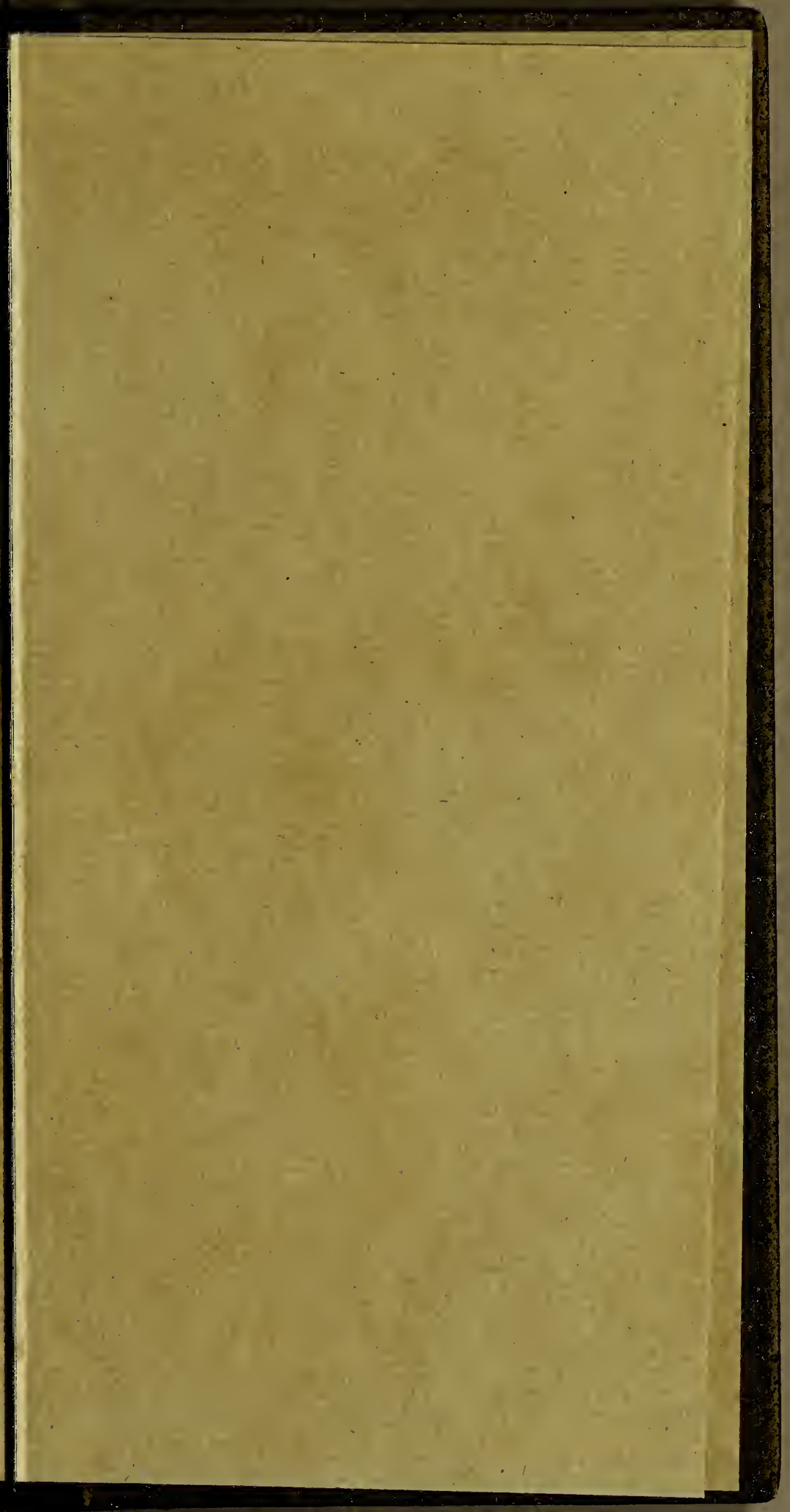


$\frac{\text { Ahlm }}{x ! b}$ 
F 690

$B 642 \mathrm{~V}$ 


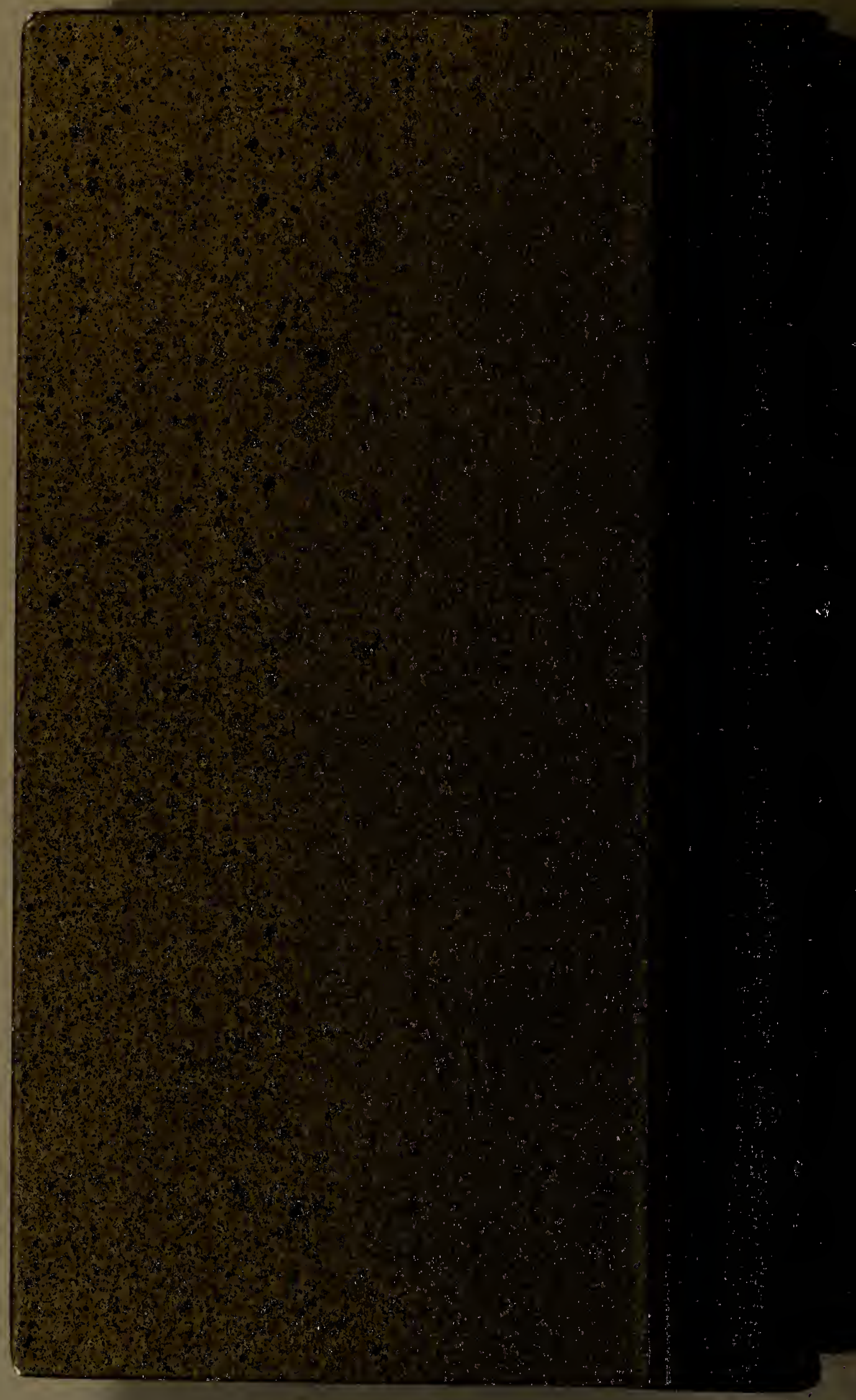

